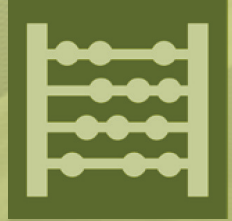

\title{
axioms
}

Nonlinear Analysis and Optimization with Applications

Edited by

Wei-Shih Du, Liang-Ju Chu, Fei He and Radu Precup

Printed Edition of the Special Issue Published in Axioms 
Nonlinear Analysis and Optimization with Applications 



\section{Nonlinear Analysis and Optimization with Applications}

Editors

Wei-Shih Du

Liang-Ju Chu

Fei He

Radu Precup 


\section{Editors}

Wei-Shih Du

Department of Mathematics,

National Kaohsiung Normal

University

Taiwan

Radu Precup

Department of Mathematics,

Babeş-Bolyai University

Romania
Liang-Ju Chu

Department of Mathematics,

National Taiwan Normal

University

Taiwan
Fei $\mathrm{He}$

School of Mathematical Sciences, Inner Mongolia University

China

Editorial Office

MDPI

St. Alban-Anlage 66

4052 Basel, Switzerland

This is a reprint of articles from the Special Issue published online in the open access journal Axioms (ISSN 2075-1680) (available at: https://www.mdpi.com/journal/axioms/special_issues/nonlinear_ analysis).

For citation purposes, cite each article independently as indicated on the article page online and as indicated below:

LastName, A.A.; LastName, B.B.; LastName, C.C. Article Title. Journal Name Year, Volume Number, Page Range.

ISBN 978-3-0365-2045-2 (Hbk)

ISBN 978-3-0365-2046-9 (PDF)

(C) 2021 by the authors. Articles in this book are Open Access and distributed under the Creative Commons Attribution (CC BY) license, which allows users to download, copy and build upon published articles, as long as the author and publisher are properly credited, which ensures maximum dissemination and a wider impact of our publications.

The book as a whole is distributed by MDPI under the terms and conditions of the Creative Commons license CC BY-NC-ND. 


\section{Contents}

Preface to "Nonlinear Analysis and Optimization with Applications" . . . . . . . . . vii

Junjian Zhao, and Wei-Shih Du

New Generalized Ekeland'sVariational Principle, Critical Point Theorems and Common Fuzzy

Fixed Point Theorems Induced by Lin-Du's Abstract Maximal Element Principle

Reprinted from: Axioms 2021, 10, 11, doi:10.3390/axioms10010011 . . . . . . . . . . . . . . .

Jaeyoo Choy, Hahng-Yun Chu and Ahyoung Kim

A Remark for the Hyers-Ulam Stabilities on $n$-Banach Spaces

Reprinted from: Axioms 2021, 10, 2, doi:10.3390/axioms10010002 . . . . . . . . . . . . . . .

Hammed Anuoluwapo Abass and Lateef Olakunle Jolaoso

An Inertial Generalized Viscosity Approximation Method for Solving Multiple-Sets Split

Feasibility Problems and Common Fixed Point of Strictly Pseudo-Nonspreading Mappings

Reprinted from: Axioms 2021, 10, 1, doi:10.3390/axioms10010001 . . . . . . . . . . . . . 21

Chibueze Christian Okeke and Lateef Olakunle Jolaoso

A Self-Adaptive Shrinking Projection Method with an Inertial Technique for Split Common Null

Point Problems in Banach Spaces

Reprinted from: Axioms 2020, 9, 140, doi:10.3390/axioms9040140 . . . . . . . . . . . . . 39

Simon Gluzman

Nonlinear Approximations to Critical and Relaxation Processes

Reprinted from: Axioms 2020, 9, 126, doi:10.3390/axioms9040126 . . . . . . . . . . . . . 59

Nopparat Wairojjana, Mudasir Younis, Habib ur Rehman, Nuttapol Pakkaranang and Nattawut Pholasa

Modified Viscosity Subgradient Extragradient-Like Algorithms for Solving Monotone Variational Inequalities Problems

Reprinted from: Axioms 2020, 9, 118, doi:10.3390/axioms9040118 . . . . . . . . . . . . . . . 977

David Levin

Reconstruction of Piecewise Smooth Multivariate Functions from Fourier Data

Reprinted from: Axioms 2020, 9, 88, doi:10.3390/axioms9030088 . . . . . . . . . . . . 117

Erdal Karapınar, Mujahid Abbas, Sadia Farooq

A Discussion on the Existence of Best Proximity Points That Belong to the Zero Set

Reprinted from: Axioms 2020, 9, 19, doi:10.3390/axioms9010019 . . . . . . . . . . . . 137

Cristian Chifu and Erdal Karapınar

Admissible Hybrid $\mathcal{Z}$-Contractions in $b$-Metric Spaces

Reprinted from: Axioms 2020, 9, 2, doi:10.3390/axioms9010002 . . . . . . . . . . . . 153

Praveen Agarwal, Abd-Allah Hyder, M. Zakarya, Clemente Cesarano and Dario Assante

Exact Solutions for a Class of Wick-Type Stochastic (3+1)-Dimensional Modified

Benjamin-Bona-Mahony Equations

Reprinted from: Axioms 2019, 8, 134, doi:10.3390/axioms8040134 . . . . . . . . . . . . . . 171

Karim Chaira, Mustapha Kabil, Abdessamad Kamouss and Samih Lazaiz

Best Proximity Points for Monotone Relatively Nonexpansive Mappings in Ordered Banach Spaces

Reprinted from: Axioms 2019, 8, 121, doi:10.3390/axioms8040121 . . . . . . . . . . . . . 187 



\section{Preface to "Nonlinear Analysis and Optimization with Applications"}

Nonlinear analysis has wide and significant applications in many areas of mathematics, including functional analysis, variational analysis, nonlinear optimization, convex analysis, nonlinear ordinary and partial differential equations, dynamical system theory, mathematical economics, game theory, signal processing, control theory, data mining, and so forth. Optimization problems have been intensively investigated, and various feasible methods in analyzing convergence of algorithms have been developed over the last half century.

This book focuses on the connection between nonlinear analysis and optimization as well as their applications to integrate basic science into the real world. It consists of eleven papers covering a number of new ideas, concepts, methods, applications and current research problems. The Guest Editors would like to sincerely thank all the authors for their valuable contributions. There are still many fundamental and important questions that remain unanswered, promising a great future for these fields. We are sure that these extremely valuable papers in this book will interest readers and will stimulate new research work, and open new perspectives over some specific problems and applications.

Finally, we would like to express our hearty thanks to the editors of the journal Axioms, particularly Assistant Editor Luna Shen, for their great support throughout the editing process of the Special Issue for Axioms and its present MDPI Reprint Book.

Wei-Shih Du, Liang-Ju Chu, Fei He, Radu Precup Editors 

Article

\title{
New Generalized Ekeland's Variational Principle, Critical Point Theorems and Common Fuzzy Fixed Point Theorems Induced by Lin-Du's Abstract Maximal Element Principle
}

\author{
Junjian Zhao ${ }^{1}$ and Wei-Shih Du ${ }^{2, *}$ \\ 1 School of Mathematical Sciences, Tiangong University, Tianjin 300387, China; zhaojunjian@tiangong.edu.cn \\ 2 Department of Mathematics, National Kaohsiung Normal University, Kaohsiung 82444, Taiwan \\ * Correspondence: wsdu@mail.nknu.edu.tw
}

check for

updates

Citation: Zhao, J.; Du, W.-S. New Generalized Ekeland's Variational Principle, Critical Point Theorems and Common Fuzzy Fixed Point Theorems Induced by Lin-Du's Abstract Maximal Element Principle. Axioms 2021, 10, 11. https://doi.org/ 10.3390/axioms 10010011

Received: 19 December 2020

Accepted: 17 January 2021

Published: 20 January 2021

Publisher's Note: MDPI stays neutral with regard to jurisdictional clai$\mathrm{ms}$ in published maps and institutional affiliations.

Copyright: $@ 2021$ by the authors. Licensee MDPI, Basel, Switzerland. This article is an open access article distributed under the terms and conditions of the Creative Commons Attribution (CC BY) license (https:// creativecommons.org/licenses/by/ $4.0 /)$.
Abstract: In this paper, by applying the abstract maximal element principle of Lin and Du, we present some new existence theorems related with critical point theorem, maximal element theorem, generalized Ekeland's variational principle and common (fuzzy) fixed point theorem for essential distances.

Keywords: maximal element; fixed point; sizing-up function; $\mu$-bounded quasi-ordered set; critical point; fuzzy mapping; Ekeland's variational principle; Caristi's fixed point theorem; Takahashi's nonconvex minimization theorem; essential distance

MSC: 47H04; 47H10; 58E30

\section{Introduction}

Maximal element principle (MEP, for short) is a fascinating theory that has a wide range of applications in many fields of mathematics. Various generalizations in different directions of maximal element principle have been investigated by several authors, see [1-8] and references therein. Lin and Du [3,4,7] introduced the concepts of the sizing-up function and $\mu$-bounded quasi-ordered set to define sufficient conditions for a nondecreasing sequence on a quasi-ordered set to have an upper bound and used them to establish an abstract MEP.

Definition 1 (see $[3,4,7])$. Let $E$ be a nonempty set. A function $\mu: 2^{E} \rightarrow[0,+\infty]$ defined on the power set $2^{E}$ of $E$ is called sizing-up if it satisfies the following properties

( $\mu 1) \mu(\varnothing)=0$;

( $\mu 2) \mu(C) \leq \mu(D)$ if $C \subseteq D$.

Definition 2 (see $[3,4,7]$ ). Let $E$ be a nonempty set and $\mu: 2^{E} \rightarrow[0,+\infty]$ a sizing-up function. A multivalued map $T: E \rightarrow 2^{E}$ with nonempty values is said to be of type $(\mu)$ if for each $x \in E$ and $\epsilon>0$, there exists a $y=y(x, \epsilon) \in T(x)$ such that $\mu(T(y)) \leq \epsilon$.

Definition 3 (see $[3,4,7])$. A quasi-ordered set $(E, \lesssim)$ with a sizing-up function $\mu: 2^{E} \rightarrow$ $[0,+\infty]$, in short $(E, \lesssim, \mu)$, is said to be $\mu$-bounded if every $\lesssim$-nondecreasing sequence $z_{1} \lesssim z_{2} \lesssim$ $\cdots \lesssim z_{n} \lesssim z_{n+1} \lesssim \cdots$ in E satisfying

$$
\lim _{n \rightarrow+\infty} \mu\left(\left\{z_{n}, z_{n+1}, \cdots\right\}\right)=0
$$

has an upper bound.

The following abstract maximal element principle of Lin and $\mathrm{Du}$ is established in $[3,4,7]$. 
Theorem 1. Let $(E, \lesssim, \mu)$ be a $\mu$-bounded quasi-ordered set with a sizing-up function $\mu: 2^{E} \rightarrow$ $[0,+\infty]$. For each $x \in E$, let $S: E \rightarrow 2^{E}$ be defined by $S(x)=\{y \in E: x \lesssim y\}$. If $S$ is of type $(\mu)$, then for each $z_{0} \in E$, there exists a nondecreasing sequence $z_{0} \lesssim z_{1} \lesssim z_{2} \lesssim \cdots$ in $E$ and $v \in$ E such that

(i) $v$ is an upper bound of $\left\{z_{n}\right\}_{n=0}^{\infty}$;

(ii) $S(v) \subseteq \bigcap_{n=1}^{+\infty} S\left(z_{n}\right)$;

(iii) $\mu\left(\cap_{n=1}^{+\infty} S\left(z_{n}\right)\right)=\mu(S(v))=0$.

Ekeland's variational principle $[9,10]$ is a very important tool for the study of approximate solutions approximate solutions of nonconvex minimization problems.

Theorem 2. (Ekeland's variational principle) Let $(M, d)$ be a complete metric space and $f: M \rightarrow$ $(-\infty,+\infty]$ be a proper lower semicontinuous and bounded below function. Let $\varepsilon>0$ and $u \in M$ with $f(u)<+\infty$. Then there exists $v \in M$ such that

(a) $f(v)+\varepsilon d(u, v) \leq f(u)$;

(b) $\quad f(z)+\varepsilon d(v, z)>f(v)$ for all $z \in M$ with $z \neq v$.

In 1976, Caristi [11] established the following famous fixed point theorem:

Theorem 3. (Caristi's fixed point theorem) Let $(M, d)$ be a complete metric space and $f: M \rightarrow$ $(-\infty,+\infty]$ be a proper lower semicontinuous and bounded below function. Suppose that $T: M \rightarrow$ $M$ is selfmapping, satisfying

$$
f(T z)+d(z, T z) \leq f(z)
$$

for each $z \in M$. Then there exists $w \in M$ such that $T w=w$.

In 1991, Takahashi [12] proved the following nonconvex minimization theorem:

Theorem 4. (Takahashi's nonconvex minimization theorem) Let $(M, d)$ be a complete metric space and $f: M \rightarrow(-\infty,+\infty]$ be a proper lower semicontinuous and bounded below function. Suppose that for any $x \in M$ with $f(x)>\inf _{z \in M} f(z)$, there exists $y_{x} \in M$ with $y_{x} \neq x$ such that

$$
f\left(y_{x}\right)+d\left(x, y_{x}\right) \leq f(x) .
$$

Then there exists $w \in M$ such that $f(w)=\inf _{z \in M} f(z)$.

It is well known that Caristi's fixed point theorem, Takahashi's nonconvex minimization theorem and Ekeland's variational principle are logically equivalent; for detail, one can refer to [3,6-8,13-24]. Many authors have devoted their attention to investigating generalizations and applications in various different directions of the well-known fixed point theorems (see, e.g., [3-8,12-31] and references therein). By using Theorem 1, Du proved several versions of generalized Ekeland's variational principle and maximal element principle and established their equivalent formulations in complete metric spaces, for detail, see $[3,4]$.

In this paper, we present some new existence theorems related with critical point theorem, generalized Ekeland's variational principle, maximal element principle, and common (fuzzy) fixed point theorem for essential distances by applying Theorem 1 .

\section{Preliminaries}

Let $E$ be a nonempty set. A fuzzy set in $E$ is a function of $E$ into $[0,1]$. Let $\mathcal{F}(E)$ be the family of all fuzzy sets in $E$. A fuzzy mapping on $E$ is a mapping from $E$ into $\mathcal{F}(E)$. This enables us to regard each fuzzy map as a two variable function of $E \times E$ into $[0,1]$. Let $F$ be a fuzzy mapping on $E$. An element $a$ of $E$ is said to be a fuzzy fixed point of $F$ if $F(a, a)=1$ (see, e.g., [4]). Let $\Gamma: E \rightarrow 2^{E}$ be a multivalued mapping. A point $x \in E$ is called to be a critical point (or stationary point or strict fixed point) [4] of $\Gamma$ if $\Gamma(v)=\{v\}$. 
Let $E$ be a nonempty set and " $\lesssim$ " a quasi-order (preorder or pseudo-order; that is, a reflexive and transitive relation) on $E$. Then $(E, \lesssim)$ is called a quasi-ordered set. An element $v$ in $E$ is called a maximal element of $E$ if there is no element $x$ of $E$, different from $v$, such that $v \lesssim x$; that is, $v \lesssim w$ for some $w \in E$ implies that $v=w$. Let $(E, \lesssim)$ be a quasi-ordered set. A sequence $\left\{x_{n}\right\}_{n \in \mathbb{N}}$ is called $\lesssim$-nondecreasing (resp. $\lesssim$-nonincreasing) if $x_{n} \lesssim x_{n+1}$ (resp. $x_{n+1} \lesssim x_{n}$ ) for each $n \in \mathbb{N}$.

Let $(X, d)$ be a metric space. A real valued function $\varphi: X \rightarrow \mathbb{R}$ is lower semicontinuous (in short l.s.c) (resp. upper semicontinuous, in short u.s.c) if $\{x \in X: \varphi(x) \leq r\}$ (resp. $\{x \in X: \varphi(x) \geq r\})$ is closed for each $r \in \mathbb{R}$. A real-valued function $f: X \rightarrow(-\infty,+\infty]$ is said to be proper if $f \not \equiv+\infty$. Recall that a function $p: X \times X \rightarrow[0,+\infty)$ is called a $w$-distance $[17,23]$, if the following are satisfied

(w1) $p(x, z) \leq p(x, y)+p(y, z)$ for any $x, y, z \in X$;

(w2) For any $x \in X, p(x, \cdot): X \rightarrow[0,+\infty)$ is l.s.c.;

(w3) For any $\varepsilon>0$, there exists $\delta>0$ such that $p(z, x) \leq \delta$ and $p(z, y) \leq \delta$ imply $d(x, y) \leq \varepsilon$.

The concept of $\tau$-function was introduced and studied by Lin and Du as follows. A function $p: X \times X \rightarrow[0, \infty)$ is said to be a $\tau$-function $[4,13,15,20,22,24,25]$, if the following conditions hold

( $\tau 1) ~ p(x, z) \leq p(x, y)+p(y, z)$ for all $x, y, z \in X$;

( $\tau 2)$ If $x \in X$ and $\left\{y_{n}\right\}$ in $X$ with $\lim _{n \rightarrow \infty} y_{n}=y$ such that $p\left(x, y_{n}\right) \leq c$ for some $c=$ $c(x)>0$, then $p(x, y) \leq c$;

( $\tau 3)$ For any sequence $\left\{x_{n}\right\}$ in $X$ with $\lim _{n \rightarrow \infty} \sup \left\{p\left(x_{n}, x_{m}\right): m>n\right\}=0$, if there exists a sequence $\left\{y_{n}\right\}$ in $X$ such that $\lim _{n \rightarrow+\infty} p\left(x_{n}, y_{n}\right)=0$, then $\lim _{n \rightarrow+\infty} d\left(x_{n}, y_{n}\right)=0$;

( $\tau 4)$ For $x, y, z \in X, p(x, y)=0$ and $p(x, z)=0$ imply $y=z$.

It is worth mentioning that a $\tau$-function is nonsymmetric in general. It is known that any metric $d$ is a $w$-distance and any $w$-distance is a $\tau$-function, but the converse is not true, see [24] for more detail.

Lemma 1 (see $[15,16,26])$. If condition $(\tau 4)$ is weakened to the following condition $(\tau 4)^{\prime}$ :

$(\tau 4)^{\prime}$ for any $x \in X$ with $p(x, x)=0$, if $p(x, y)=0$ and $p(x, z)=0$, then $y=z$, then $(\tau 3)$ implies $(\tau 4)^{\prime}$.

The concept of essential distance was introduced by Du [15] in 2016.

Definition 4 (see [15]). Let $(X, d)$ be a metric space. A function $p: X \times X \rightarrow[0,+\infty)$ is called an essential distance if conditions $(\tau 1),(\tau 2)$, and $(\tau 3)$ hold.

Remark 1. It is obvious that any $\tau$-function is an essential distance. By Lemma 1, we know that if $p$ is an essential distance, then condition $(\tau 4)^{\prime}$ holds.

The following known result is very crucial in our proofs.

Lemma 2 (see [4]). Let $(X, d)$ be a metric space and $p: X \times X \rightarrow[0,+\infty)$ be a function. Assume that $p$ satisfies the condition ( $\tau 3)$. If a sequence $\left\{x_{n}\right\}$ in $X$ with $\lim _{n \rightarrow \infty} \sup \left\{p\left(x_{n}, x_{m}\right): m>\right.$ $n\}=0$, then $\left\{x_{n}\right\}$ is a Cauchy sequence in $X$.

\section{Main Results}

Lemma 3. Let $(M, d)$ be a metric space and $p: M \times M \rightarrow[0,+\infty)$ be a function satisfying $p(x, x)=0$ for all $x \in M$ and $p(x, z) \leq p(x, y)+p(y, z)$ for any $x, y, z \in M$. Suppose that the extended real-valued function $L: M \times M \rightarrow(-\infty,+\infty]$ satisfies the following assumptions

(i) $L(x, x) \leq 0$ for all $x \in M$;

(ii) $L(x, z) \leq L(x, y)+L(y, z)$ for all $x, y, z \in M$; 
(iii) For each $x \in M, y \rightarrow L(x, y)$ is l.s.c.;

(iv) $\left\{x \in X: \inf _{y \in M} L(x, y)>-\infty\right\} \neq \varnothing$.

Define a binary relation $\lesssim$ on $M$ by

$$
x \lesssim y \Longleftrightarrow L(x, y)+p(x, y) \leq 0 .
$$

Then $\lesssim$ is a quasi-order.

Proof. Clearly, $x \lesssim x$ for all $x \in M$. If $x \lesssim y$ and $y \lesssim z$, then

$$
L(x, y)+p(x, y) \leq 0
$$

and

$$
L(y, z)+p(y, z) \leq 0
$$

By (ii), we get

$$
L(x, z)+p(x, z) \leq L(x, y)+L(y, z)+p(x, y)+p(y, z) \leq 0,
$$

which shows that $x \lesssim z$. Hence $\lesssim$ is a quasi-order.

Lemma 4. Let $(M, d), p, L$, and $\lesssim$ be the same as in Lemma 3. Assume that for each $x \in M$, the function $y \rightarrow p(x, y)$ is l.s.c. Define $G: M \rightarrow 2^{M}$ by

$$
G(x)=\{y \in M: x \lesssim y\} \text { for } x \in M .
$$

Then the following hold

(a) $G(x)$ is nonempty and closed for each $x \in M$;

(b) $G(y) \subseteq G(x)$ for each $y \in G(x)$.

Proof. Obviously, the conclusion (a) holds. To see (b), let $y \in G(x)$. Then $x \lesssim y$. We claim that $G(y) \subseteq G(x)$. Given $z \in G(y)$. Thus $y \lesssim z$. By the transitive relation, we get $x \lesssim z$ which means $z \in G(x)$. Hence $G(y) \subseteq G(x)$.

The following theorem is one of the main results of this paper.

Theorem 5. Let $(M, d)$ be a metric space and $p$ be an essential distance on $M$ with $p(x, \cdot)$ is l.s.c. for each $x \in M$ and $p(a, a)=0$ for all $a \in M$. Suppose that $L, \lesssim$ and $G$ be the same as in Lemmas 3 and 4. If,

$$
p(y, x) \leq p(x, y) \text { for all } y \in G(x),
$$

then the following hold:

(a) $G$ is of type $\left(\mu_{p}\right)$ where $\mu_{p}(D):=\sup \{p(x, y): x, y \in D\}$ for $D \subseteq M$;

(b) If $M$ is $\lesssim$-complete, then $\left(M, \lesssim, \mu_{p}\right)$ is a $\mu_{p}$-bounded quasi-ordered set.

Proof. We first show that $G$ is of type $\left(\mu_{p}\right)$. Let $x \in M$ and $\epsilon>0$ be given. Then there exists $n_{0}=n_{0}(\varepsilon) \in \mathbb{N}$, such that $2^{-n_{0}}<\frac{\epsilon}{2}$. Define a function $\kappa: M \rightarrow[-\infty,+\infty]$ by

$$
\kappa(x)=\inf _{y \in G(x)} L(x, y) .
$$

Let $y \in G(x)$. If $\kappa(x)=-\infty$, then $0 \leq p(x, y)<-\kappa(x)$. Otherwise, if $\kappa(x)>-\infty$, then

$$
p(x, y) \leq-L(x, y) \leq-\kappa(x) .
$$

Hence we conclude

$$
0 \leq p(x, y) \leq-\kappa(x) \text { for all } y \in G(x) .
$$


Set $x_{1}:=x \in M$. Thus one can choose $x_{2} \in G\left(x_{1}\right) \subseteq M$, such that

$$
L\left(x_{1}, x_{2}\right) \leq \kappa\left(x_{1}\right)+\frac{1}{2} .
$$

Let $k \in \mathbb{N}$ and assume that $x_{k} \in M$ is already known. Then, one can choose $x_{k+1} \in G\left(x_{k}\right)$ such that

$$
L\left(x_{k}, x_{k+1}\right) \leq \kappa\left(x_{k}\right)+\frac{1}{2^{k}} .
$$

Hence, by induction, we obtain a nondecreasing sequence $x_{1} \lesssim x_{2} \lesssim \cdots$ in $M$ such that $x_{n+1} \in G\left(x_{n}\right)$ and

$$
L\left(x_{n}, x_{n+1}\right) \leq \kappa\left(x_{n}\right)+\frac{1}{2^{n}} \quad \text { for all } n \in \mathbb{N} .
$$

By Lemma 4, we have $G\left(x_{n+1}\right) \subseteq G\left(x_{n}\right)$ for all $n \in \mathbb{N}$. So it follows that

$$
\begin{aligned}
\kappa\left(x_{n+1}\right) & =\inf _{y \in G\left(x_{n+1}\right)} L\left(x_{n+1}, y\right) \\
& \geq \inf _{y \in G\left(x_{n}\right)} L\left(x_{n+1}, y\right) \\
& \geq \inf _{y \in G\left(x_{n}\right)}\left[L\left(x_{n}, y\right)-L\left(x_{n}, x_{n+1}\right)\right] \\
& =\kappa\left(x_{n}\right)-L\left(x_{n}, x_{n+1}\right) .
\end{aligned}
$$

Combining (2) with (3), we obtain

$$
\kappa\left(x_{n+1}\right)+\frac{1}{2^{n}} \geq 0,
$$

and hence

$$
0 \leq-\kappa\left(x_{n+1}\right) \leq \frac{1}{2^{n}}<\frac{\epsilon}{2} \text { for all } n \geq n_{0} .
$$

Put $w=x_{n_{0}+1}$. Thus $w \in G(x)$ and

$$
0 \leq-\kappa(w)<\frac{\epsilon}{2}
$$

If $G(w)$ is a singleton set, then $\mu_{p}(G(w))=0 \leq \epsilon$. Assume that $G(w)$ is not a singleton set. Let $u, v \in G(w)$. By our hypothesis, we have $p(u, w) \leq p(w, u)$. So, by (1), we obtain

$$
\begin{aligned}
p(u, v) & \leq p(u, w)+p(w, v) \\
& \leq-2 \kappa(w) \\
& <\epsilon
\end{aligned}
$$

which implies

$$
\mu_{p}(G(w))=\sup \{p(u, v): u, v \in G(w)\} \leq \epsilon .
$$

Therefore $G$ is of type $\left(\mu_{p}\right)$. Finally, we prove (b). Let $\alpha_{1} \lesssim \alpha_{2} \lesssim \cdots$ be a $\lesssim-$ nondecreasing sequence in $M$ satisfying $\lim _{n \rightarrow+\infty} \mu_{p}\left(\left\{\alpha_{n}, \alpha_{n+1}, \cdots\right\}\right)=0$. Since

$$
\begin{aligned}
0 & =\lim _{n \rightarrow+\infty} \mu_{p}\left(\left\{\alpha_{n}, \alpha_{n+1}, \cdots\right\}\right) \\
& =\lim _{n \rightarrow+\infty} \sup \left\{p(u, v): u, v \in\left\{\alpha_{n}, \alpha_{n+1}, \cdots\right\}\right\},
\end{aligned}
$$

we get

$$
\lim _{n \rightarrow+\infty} \sup \left\{p\left(\alpha_{n}, \alpha_{m}\right): m>n\right\}=0 .
$$


So, by applying Lemma 2, we show that $\left\{\alpha_{n}\right\}$ is a nondecreasing Cauchy sequence in $M$. By the $\lesssim$-completeness of $M$, there exists $\beta \in M$ such that $\alpha_{n} \rightarrow \beta$ as $n \rightarrow+\infty$. We claim that $\beta$ is an upper bound of $\left\{\alpha_{n}\right\}_{n=1}^{+\infty}$. For each $n \in \mathbb{N}$, since $\alpha_{m} \in G\left(\alpha_{n}\right)$ for all $m \geq n$ and $\alpha_{n} \rightarrow \beta$, by the closedness of $G\left(\alpha_{n}\right)$, we have $\beta \in G\left(\alpha_{n}\right)$ or $\alpha_{n} \lesssim \beta$ for all $n \in \mathbb{N}$. Therefore $\beta$ is an upper bound of $\left\{\alpha_{n}\right\}$ and hence $\left(M, \lesssim, \mu_{p}\right)$ is a $\mu_{p}$-bounded quasi-ordered set. The proof is completed.

The following result is immediate from Theorem 5 and Lemmas 3 and 4 .

Corollary 1. Let $(M, d)$ be a metric space and $p$ be an essential distance on $M$ with $p(x, \cdot)$ a l.s.c. for each $x \in M$ and $p(a, a)=0$ for all $a \in M$. Suppose that the extended real-valued function $f: M \rightarrow(-\infty,+\infty]$ is proper, l.s.c. and bounded below. Let $\varepsilon>0$. Define a binary relation $\lesssim(\varepsilon, f, p)$ on $M$ by

$$
x \lesssim(\varepsilon, f, p) y \Longleftrightarrow \varepsilon p(x, y) \leq f(x)-f(y) .
$$

Let $\Gamma: M \rightarrow 2^{M}$ be defined by

$$
\Gamma(x)=\{y \in M: x \lesssim(\varepsilon, f, p) y\} \text { for } x \in M .
$$

Then the following hold:

(a) $\lesssim_{(\varepsilon, f, p)}$ is a quasi-order;

(b) For each $x \in M, \Gamma(x)$ is closed;

(c) $\Gamma$ is of type $\left(\mu_{p}\right)$ where $\mu_{p}(D):=\sup \{p(x, y): x, y \in D\}$ for $D \subset M$;

(d) If $M$ is complete, then $\left(M, \lesssim(\varepsilon, f, p), \mu_{p}\right)$ is a $\mu_{p}$-bounded quasi-ordered set.

Proof. Define $L: M \times M \rightarrow(-\infty, \infty]$ by

$$
L(x, y)=\frac{1}{\varepsilon}(f(y)-f(x)) .
$$

Then the following hold

- $\quad x \lesssim_{(\varepsilon, f, p)} y \Longleftrightarrow L(x, y)+p(x, y) \leq 0$;

- $L(x, x)=0$ for all $x \in M$;

- $\quad L(x, z)=L(x, y)+L(y, z)$ for all $x, y, z \in M$;

- $\quad$ For each $x \in M, y \rightarrow L(x, y)$ is l.s.c.;

- $\left\{x \in X: \inf _{y \in M} L(x, y)>-\infty\right\} \neq \varnothing$.

Therefore, applying Theorem 5 and Lemmas 3 and 4, we show the desired conclusions.

By applying Theorem 5, we obtain a new result related to common fuzzy fixed point theorem, critical point theorem, maximal element principle and generalized Ekeland's variational principle for essential distances.

Theorem 6. Let $(M, d)$ be a complete metric space. Suppose that $p, L, \lesssim$, and $G$ be the same as in Theorem 5. Let $I$ be any index set. For each $i \in I$, let $F_{i}$ be a fuzzy mapping on $M$. Assume that for each $(i, x) \in I \times M$, there exists $y_{(i, x)} \in G(x)$ such that $F_{i}\left(x, y_{(i, x)}\right)=1$. Then for every $\varepsilon>0$ and for every $u \in M$, there exists $v \in M$ such that

(a) $v$ is a maximal element of $(M, \lesssim)$;

(b) $G(v)=\{v\}$;

(c) $L(u, v)+p(u, v) \leq 0$;

(d) $L(v, x)+p(v, x)>0$ for all $x \in M$ with $x \neq v$;

(e) $F_{i}(v, v)=1$ for all $i \in I$.

Proof. By applying Theorem $5, G$ is of type $\left(\mu_{p}\right)$ and $\left(M, \lesssim, \mu_{p}\right)$ is a $\mu_{p}$-bounded quasiordered set, where

$$
\mu_{p}(D):=\sup \{p(x, y): x, y \in D\} \quad \text { for } D \subseteq M
$$


Let $u \in M$ be given. Put $u_{0}:=u$. Since $G$ is of type $\left(\mu_{p}\right)$, by Theorem 1 , there exists a $\lesssim$-nondecreasing sequence $u_{0} \lesssim u_{1} \lesssim u_{2} \lesssim \cdots$ in $M$ and $v \in M$ such that

(i) $v$ is an upper bound of $\left\{u_{n}\right\}_{n=0}^{+\infty}$;

(ii) $\mu_{p}(G(v))=0$.

From (i), we prove (c). Next, we claim that $G(v)=\{v\}$. Let $z \in G(v)$. By $(\mu 2)$ and (ii), we have

$$
p(v, z)=\mu_{p}(\{v, z\}) \leq \mu_{p}(G(v))=0,
$$

which deduces $p(v, z)=0$. Since $p(v, v)=0$, by Lemma 1 , we get $z=v$. Therefore $G(v)=\{v\}$ and, equivalency, (d) holds. For each $(i, v) \in I \times M$, due to $G(v)=\{v\}$ and our hypothesis, there exists $y_{(i, v)}:=v \in G(v)$ such that $F_{i}(v, v)=F_{i}\left(v, y_{(i, v)}\right)=1$. So (e) is true. Finally, we verify (a). If $v \lesssim w$ for some $w \in W$, then $w \in G(v)=\{v\}$, which implies $v=w$. Hence $v$ is a maximal element of $(M, \lesssim)$. The proof is completed.

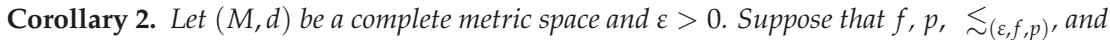
$\Gamma$ be the same as in Corollary 1 . Let $I$ be any index set. For each $i \in I$, let $F_{i}$ be a fuzzy mapping on $M$. Assume that for each $(i, x) \in I \times M$, there exists $y_{(i, x)} \in \Gamma(x)$ such that $F_{i}\left(x, y_{(i, x)}\right)=1$. Then for every $u \in M$, there exists $v \in M$ such that

(a) $v$ is a maximal element of $(M, \lesssim(\varepsilon, f, p))$;

(b) $\Gamma(v)=\{v\}$;

(c) $f(v)+\varepsilon p(u, v) \leq f(u)$;

(d) $f(z)+\varepsilon p(v, z)>f(v)$ for all $z \in M$ with $z \neq v$;

(e) $F_{i}(v, v)=1$ for all $i \in I$.

Proof. Define $L: M \times M \rightarrow(-\infty,+\infty]$ by

$$
L(x, y)=\frac{1}{\varepsilon}(f(y)-f(x)) .
$$

Then,

$$
x \lesssim_{(\varepsilon, f, p)} y \Longleftrightarrow L(x, y)+p(x, y) \leq 0 .
$$

So the desired conclusions follow from Theorem 6 immediately.

Let $(M, d)$ be a metric space and $T: M \rightarrow 2^{M}$ be a multivalued mapping with nonempty values. Then we can define a fuzzy mapping $K$ on $M$ by

$$
K(x, y)=\chi_{T(x)}(y),
$$

where $\chi_{A}$ is the characteristic function for an arbitrary set $A \subset M$. Note that

$$
K(x, y)=1 \Longleftrightarrow y \in T(x) .
$$

The following new result related to critical point theorem, generalized Ekeland's variational principle, maximal element principle, and common fixed point theorem for essential distances can be established by Theorem 6 immediately.

Theorem 7. Let $(M, d)$ be a complete metric space. Suppose that $p, L, \lesssim$, and $G$ are the same as in Theorem 5. Let $I$ be any index set. For each $i \in I$, let $T_{i}: M \rightarrow 2^{M}$ be a multivalued mapping with nonempty values such that for each $(i, x) \in I \times M$, there exists $y_{(i, x)} \in T_{i}(x) \cap G(x)$. Then for every $\varepsilon>0$ and for every $u \in M$, there exists $v \in M$ such that

(a) $v$ is a maximal element of $(M, \lesssim)$;

(b) $G(v)=\{v\}$;

(c) $L(u, v)+p(u, v) \leq 0$;

(d) $L(v, x)+p\left(v_{i}, x_{i}\right)>0$ for all $x \in M$ with $x \neq v$; 
(e) $v$ is a common fixed point for the family $\left\{T_{i}\right\}_{i \in I}$.

Corollary 3. Let $(M, d)$ be a complete metric space and $\varepsilon>0$. Suppose that $f, p, \lesssim(\varepsilon, f, p)$, and $\Gamma$ be the same as in Corollary 1. Let I be any index set. For each $i \in I$, let $T_{i}: M \rightarrow 2^{M}$ be a multivalued mapping with nonempty values such that for each $(i, x) \in I \times M$, there exists $y_{(i, x)} \in T_{i}(x) \cap \Gamma(x)$. Then for every $u \in M$, there exists $v \in M$ such that

(a) $v$ is a maximal element of $(M, \lesssim(\varepsilon, f, p)$;

(b) $\Gamma(v)=\{v\}$;

(c) $f(v)+\varepsilon p(u, v) \leq f(u)$;

(d) $f(z)+\varepsilon p(v, z)>f(v)$ for all $z \in M$ with $z \neq v$;

(e) $v$ is a common fixed point for the family $\left\{T_{i}\right\}_{i \in I}$.

Finally, the following simple example is given to illustrate Corollary 3.

Example 1. Let $M=[-1,1]$ with the metric $d(x, y)=|x-y|$ for $x, y \in M$. Then $(M, d)$ is $a$ complete metric space. Let $T_{1}, T_{2}: M \rightarrow 2^{M}$ be defined by $T_{1} x=\left\{\frac{1}{2} x\right\}$ and $T_{2} x=\left\{\frac{1}{3} x\right\}$ for $x \in M$. Clearly, 0 is the unique common fixed point of $T_{1}$ and $T_{2}$. Let $f: M \rightarrow \mathbb{R}$ by $f(x)=|x|$ for $x \in M$. Define a binary relation $\lesssim(1, f, d)$ on $M$ by

$$
x \lesssim(1, f, d) y \Longleftrightarrow d(x, y) \leq f(x)-f(y)
$$

Then $\lesssim(1, f, d)$ is a quasi-order and

$$
\Gamma(x)=\{y \in M: x \lesssim(1, f, d), y\}=\{y \in M: d(x, y) \leq f(x)-f(y)\} \neq \varnothing .
$$

It is easy to see that for each $x \in M$, we have

$$
d\left(x, \frac{1}{2} x\right)=f(x)-f\left(\frac{1}{2} x\right)
$$

and

$$
d\left(x, \frac{1}{3} x\right)=f(x)-f\left(\frac{1}{3} x\right) .
$$

Hence $\frac{1}{2} x \in T_{1} x \cap \Gamma(x)$ and $\frac{1}{3} x \in T_{2} x \cap \Gamma(x)$ for any $x \in M$. Therefore, all the assumptions of Corollary 3 are satisfied. By applying Corollary 3 , for every $u \in M$, we can obtain $v \in M$ (in fact, $v=0$ ) such that

(a) 0 is a common fixed point for $T_{1}$ and $T_{2}$;

(b) 0 is a maximal element of $(M, \lesssim(1, f, d))$;

(c) $\Gamma(0)=\{0\}$;

(d) $f(0)+d(u, 0) \leq f(u)$;

(e) $f(z)+d(0, z)>f(0)$ for all $z \in M$ with $z \neq 0$.

\section{Remark 2.}

(a) Theorems 5-7 and Corollaries 1-3 improve and generalize some of the existence results on the topic in the literature, see, e.g., $[3,4,8,17,23,24]$ and references therein;

(b) Following the same argument as in the proof of [16], one can establish the equivalence of Ekeland's variational principle Caristi's fixed point theorem and Takahashi's nonconvex minimization theorem for essential distances.

\section{Conclusions}

Maximal element principle is a significant theory and has already been proposed and investigated its potential applications in several areas of mathematics. In this paper, by applying the abstract maximal element principle of Lin and $\mathrm{Du}$, we present some new existence theorems related with common (fuzzy) fixed point theorem, maximal element 
theorem, critical point theorem and generalized Ekeland's variational principle for essential distances.

Author Contributions: Writing-original draft, J.Z. and W.-S.D. Both authors have read and agreed to the published version of the manuscript.

Funding: The first author is partially supported by the Natural Science Foundation of Tianjin city, China (Grant No. 18JCYBJC16300). The second author is partially supported by Grant No. MOST 109-2115-M-017-002 of the Ministry of Science and Technology of the Republic of China.

Institutional Review Board Statement: Not applicable.

Informed Consent Statement: Not applicable.

Data Availability Statement: Not applicable.

Acknowledgments: The authors wish to express their deep thanks to the anonymous referees for their valuable suggestions and comments.

Conflicts of Interest: The authors declare no conflict of interest.

\section{References}

1. Bishop, E.; Phelps, R.R. The support functionals of a convex set. In Proceedings of Symposia in Pure Mathematics; American Mathematical Society: Providence, RI, USA, 1963; Volume VII, pp. 27-35.

2. Brézis, H.; Browder, F.E. A general principle on ordered sets in nonlinear functional analysis. Adv. Math. 1976, $21,355-364$. [CrossRef]

3. Du, W.-S. On some nonlinear problems induced by an abstract maximal element principle. J. Math. Anal. Appl. 2008, 347, 391-399. [CrossRef]

4. Du, W.-S. Critical point theorems for nonlinear dynamical systems and their applications. Fixed Point Theory Appl. 2010, 2010, 246382. [CrossRef]

5. Granas, A.; Horvath, C.D. On the order-theoretic Cantor theorem. Taiwan. J. Math. 2000, 4, 203-213. [CrossRef]

6. Kang, B.G.; Park, S. On generalized ordering principles in nonlinear analysis. Nonlinear Anal. Theory Methods Appl. 1990, 14, 159-165. [CrossRef]

7. Lin, L.-J.; Du, W.-S. From an abstract maximal element principle to optimization problems, stationary point theorems and common fixed point theorems. J. Glob. Optim. 2010, 46, 261-271. [CrossRef]

8. Park, S. On generalizations of the Ekeland-type variational principles. Nonlinear Anal. Theory Methods Appl. 2000, 39, 881-889. [CrossRef]

9. Ekeland, I. Remarques sur les problémes variationnels. CR Acad. Sci. Paris Ser. A-B 1972, 275, 1057-1059.

10. Ekeland, I. Nonconvex minimization problems. Bull. Am. Math. Soc. 1979, 1, 443-474. [CrossRef]

11. Caristi, J. Fixed point theorems for mappings satisfying inwardness conditions. Trans. Am. Math. Soc. 1976, $215,241-251$. [CrossRef]

12. Takahashi, W. Existence theorems generalizing fixed point theorems for multivalued mappings. In Fixed Point Theory and Applications, Pitmam Research Notes in Mathematics Series; Théra, M.A., Baillon, J.B., Eds.; Longmam Sci. Tech.: Harlow, UK, 1991; Volume 252, pp. 397-406.

13. Du, W.-S. On Caristi type maps and generalized distances with applications. Abstr. Appl. Anal. 2013, 2013, 407219. [CrossRef]

14. Du, W.-S. On Caristi-type mappings without lower semicontinuity assumptions. J. Fixed Point Theory Appl. 2015, 17, 733-752. [CrossRef]

15. Du, W.-S. On generalized Caristi's fixed point theorem and its equivalence. Nonlinear Anal. Differ. Equ. 2016, 4, 635-644. [CrossRef]

16. Du, W.-S. Some generalizations of fixed point theorems of Caristi type and Mizoguchi-Takahashi type under relaxed conditions. Bull. Braz. Math. Soc. New Ser. 2019, 50, 603-624. [CrossRef]

17. Kada, O.; Suzuki, T.; Takahashi, W. Nonconvex minimization theorems and fixed point theorems in complete metric spaces. Math. Japon. 1996, 44, 381-391.

18. Khamsi, M.A.; Kirk, W.A. An introduction to metric spaces and fixed point theory. In Pure and Applied Mathematics; WileyInterscience: New York, NY, USA, 2001.

19. Kirk, W.A.; Shahzad, N. Fixed Point Theory in Distance Spaces; Springer: Cham, Switzerland, 2014.

20. Lin, L.-J.; Du, W.-S. Ekeland's variational principle, minimax theorems and existence of nonconvex equilibria in complete metric spaces. J. Math. Anal. Appl. 2006, 323, 360-370. [CrossRef]

21. Lin, L.-J.; Du, W.-S. Some equivalent formulations of generalized Ekeland's variational principle and their applications. Nonlinear Anal. 2007, 67, 187-199. [CrossRef]

22. Lin, L.-J.; Du, W.-S. On maximal element theorems, variants of Ekeland's variational principle and their applications. Nonlinear Anal. 2008, 68, 1246-1262. [CrossRef]

23. Takahashi, W. Nonlinear Functional Analysis; Yokohama Publishers: Yokohama, Japan, 2000. 
24. Włodarczyk, K.; Plebaniak, R. Maximality principle and general results of Ekeland and Caristi types without lower semicontinuity assumptions in cone uniform spaces with generalized pseudodistances. Fixed Point Theory Appl. 2010, 2010, 175453. [CrossRef]

25. Du, W.-S. New existence results and generalizations for coincidence points and fixed points without global completeness. Abstr. Appl. Anal. 2013, 2013, 214230. [CrossRef]

26. Jiang, B.; Huang, H.; Du, W.-S. New generalized Mizoguchi-Takahashi's fixed point theorems for essential distances and $e^{0}$-metrics. Mathematics 2019, 7, 1224. [CrossRef]

27. Jovanović, R.Ž.; Sretenović, A.A.; Živković, B.D. Ensemble of various neural networks for prediction of heating energy consumption. Energy Build. 2015, 94, 189-199. [CrossRef]

28. Mitrović, Z.D.; Hussain, A.; de la Sen, M.; Radenović, S. On best approximations for set-valued mappings in G-convex spaces. Mathematics 2020, 8, 347. [CrossRef]

29. Todorčević, V. Harmonic Quasiconformal Mappings and Hyperbolic Type Metrics; Springer Nature Switzerland AG: Cham, Switzerland, 2019.

30. Du, W.-S. A note on cone metric fixed point theory and its equivalence. Nonlinear Anal. Theory Methods Appl. 2010, 72, 2259-2261. [CrossRef]

31. Du, W.-S. Some new results and generalizations in metric fixed point theory. Nonlinear Anal. Theory Methods Appl. 2010, 73, 1439-1446. [CrossRef] 


\title{
A Remark for the Hyers-Ulam Stabilities on $n$-Banach Spaces
}

\author{
Jaeyoo Choy ${ }^{1}$, Hahng-Yun Chu ${ }^{2, *}$ and Ahyoung Kim ${ }^{2}$ \\ 1 School of Mathematics, KIAS, 85 Hoegi-ro, Dongdaemun-Gu, Seoul 02455, Korea; choy@kias.re.kr \\ 2 Department of Mathematics, Chungnam National University, 79 Daehak-ro, Yuseong-Gu, \\ Daejeon 34134, Korea; aykim@cnu.ac.kr \\ * Correspondence: hychu@cnu.ac.kr
}

check for updates

Citation: Choy, J.; Chu, H.-Y.; Kim, A. A Remark for the Hyers-Ulam Stabilities on $n$-Banach Spaces. Axioms 2021, 10, 2. https://doi.org/ 10.3390/axioms 10010002

Received: 6 November 2020 Accepted: 17 December 2020 Published: 29 December 2020

Publisher's Note: MDPI stays neutral with regard to jurisdictional claims in published maps and institutional affiliations.

Copyright: (c) 2020 by the authors. Licensee MDPI, Basel, Switzerland. This article is an open access article distributed under the terms and conditions of the Creative Commons Attribution (CC BY) license (https: / / creativecommons.org / licenses/by/4.0/).
Abstract: In this article, we deal with stabilities of several functional equations in $n$-Banach spaces. For a surjective mapping $f$ into a $n$-Banach space, we prove the generalized Hyers-Ulam stabilities of the cubic functional equation and the quartic functional equation for $f$ in $n$-Banach spaces.

Keywords: $n$-Banach space; cubic mappings; quartic mappings; the generalized Hyers-Ulam stability

\section{Introduction}

A question of the stability of functional equations concerning group homomorphisms was first raised by S. M. Ulam in 1940 [1]. In the next year, a partial affirmative answer to the question of Ulam was given by D. H. Hyers [2] for additive mappings on Banach spaces. Hyers' theorem was generalized by T. Aoki [3] for additive mapping. In 1978, Rassias [4] provided a generalization of the theorem for linear mappings by allowing the Cauchy differences to be unbounded. Subsequently, the result of Rassias' theorem was generalized by P. Găvruta [5], allowing the Cauchy difference controlled by a general unbounded function which is called the generalized Hyers-Ulam stability. On the other hand, Rassias and Šemrl found an example of a continuous real-valued function from $\mathbb{R}$ for which the Hyers-Ulam stability does not occur. See [6].

Let $X$ and $Y$ be real vector spaces and $f: X \rightarrow Y$ a mapping. For a cubic function $f(x)=c x^{3}(c \in \mathbb{R}, X=Y=\mathbb{R}), f$ clearly satisfies the following functional equation

$$
f(x+2 y)+3 f(x)=3 f(x+y)+f(x-y)+6 f(y) .
$$

For this reason, it is natural that Equation (1) is called a cubic functional equation and every solution of Equation (1) is also called a cubic function. The general solution for Equation (1) was solved by J. M. Rassias [7] for a mapping from a real normed space to a Banach space. Jun et al. [8] proved that the cubic functional Equation (1) is equivalent to the following functional equation

$$
f(2 x+y)+f(2 x-y)=2 f(x+y)+2 f(x-y)+12 f(x) .
$$

In [9], Chu et al. extended the cubic functional equation to the following generalized form

$$
\begin{aligned}
& f\left(\sum_{j=1}^{n-1} x_{j}+2 x_{n}\right)+f\left(\sum_{j=1}^{n-1} x_{j}-2 x_{n}\right)+\sum_{j=1}^{n-1} f\left(2 x_{j}\right) \\
& =2 f\left(\sum_{j=1}^{n-1} x_{j}\right)+4 \sum_{j=1}^{n-1}\left(f\left(x_{j}+x_{n}\right)+f\left(x_{j}-x_{n}\right)\right),
\end{aligned}
$$

where $n \geq 2$ is an integer, and they also proved the generalized Hyers-Ulam stability. The stability problem for cubic functional equations has been extensively investigated by many mathematicians (see [10-12].) 
In [13], J. M. Rassias introduced the functional equation as follows:

$$
f(2 x+y)+f(2 x-y)=4 f(x+y)+4 f(x-y)+24 f(x)-6 f(y)
$$

It is obvious that $f(x)=x^{4}$ is a solution of Equation (3), so we call Equation (3) a quartic functional equation. Chung and Sahoo [14] investigated the general solution of (3) and A. Najati [15] proved the generalized Hyers-Ulam stability for the quartic functional Equation (3) using the idea of Găvruta [5]. The stability results of quartic functional equations can be found in several other papers (see [16-18].) There are a number of papers and research monographs regarding various generalizations and applications of the generalized Hyers-Ulam stability of several functional equations. See [19-22]. Park investigated the generalized Hyers-Ulam stability for additive mappings, Jensen mappings and quadratic mappings in 2-Banach spaces in [23,24].

Misiak $[25,26]$ introduced the notion of $n$-normed spaces which is one of the generalizations of normed spaces and 2-normed spaces. For more information of the phase spaces, we refer to the papers [27-30]. Recently, Chu et al. [31] studied the generalized Hyers-Ulam stabilities of the Cauchy functional equations, the Jensen functional equations and the quadratic functional equations on $n$-Banach spaces. In [32], Brzdęk and Ciepliński proved a fixed point theorem for operator acting on a class of functions with values in an $n$-Banach space. For study of the Hyers-Ulam stability, the extension to $n$-Banach spaces is valuable in terms of development of the field of functional equations.

Motivated by results in [31,32], we focus on the generalized Hyers-Ulam stabilities of several functional equationss-in detail, the cubic functional equation expressed as Equation (2) and the quartic functional equation expressed as Equation (3) on $n$-Banach spaces. We prove the generalized Hyers-Ulam stabilities of the functional equations on the spaces.

The contents of paper: In Section 2, we recall definitions and lemma in $n$-Banach spaces to investigate the generalized Hyers-Ulam stabilities on the spaces. In Section 3, we investigate the generalized Hyers-Ulam stability problem in $n$-Banach spaces. The problems for the generalized Hyers-Ulam stability related on the cubic functional equation in $n$-Banach spaces are studied in Section 3.1. We also deal with applications of the stabilities for the functional equations on the spaces. In Section 3.2, we focus on the the quartic functional equation and prove the generalized stability on the $n$-Banach spaces.

\section{Preliminaries}

In this section, we recall definitions and lemma in $n$-Banach spaces as a preliminary step toward the main theorems.

Definition $1([25,26])$. Let $X$ be a real linear space with $\operatorname{dim} X \geq n$ and $\|\cdot, \cdots, \cdot\|: X^{n} \rightarrow \mathbb{R}$ be a function. Then $(X,\|\cdot, \cdots, \cdot\|)$ is called a linear $n$-normed space if

$$
\begin{aligned}
& \left(n N_{1}\right)\left\|x_{1}, \cdots, x_{n}\right\|=0 \Leftrightarrow x_{1}, \cdots, x_{n} \text { are linearly dependent; } \\
& \left(n N_{2}\right)\left\|x_{1}, \cdots, x_{n}\right\|=\left\|x_{j_{1}}, \cdots, x_{j_{n}}\right\| \text { for every permutation }\left(j_{1}, \cdots, j_{n}\right) \text { of }(1, \cdots, n) ; \\
& \left(n N_{3}\right)\left\|\alpha x_{1}, \cdots, x_{n}\right\|=|\alpha|\left\|x_{1}, \cdots, x_{n}\right\| ; \\
& \left(n N_{4}\right)\left\|x+y, x_{2}, \cdots, x_{n}\right\| \leq\left\|x, x_{2}, \cdots, x_{n}\right\|+\left\|y, x_{2}, \cdots, x_{n}\right\|
\end{aligned}
$$

for all $\alpha \in \mathbb{R}$ and all $x, y, x_{1}, \cdots, x_{n} \in X$. The function $\|\cdot, \cdots, \cdot\|$ is called an $n$-norm on $X$.

Definition 2 ([31]). Let $\left\{x_{\ell}\right\}$ be a sequence in a linear n-normed space $X$. The sequence $\left\{x_{\ell}\right\}$ is said to be $n$-convergent in $X$ if there exists an element $x \in X$ such that

$$
\lim _{\ell \rightarrow \infty}\left\|x_{\ell}-x, y_{2}, \cdots, y_{n}\right\|=0
$$

for all $y_{2}, \cdots, y_{n} \in X$. In this case, we say that a sequence $\left\{x_{\ell}\right\}$ converges to the limit $x$, simply denoted by $\lim _{\ell \rightarrow \infty} x_{\ell}=x$ with a slight abuse of notation. 
Definition 3 ([31]). A sequence $\left\{x_{\ell}\right\}$ in a linear n-normed space $X$ is called an $n$-Cauchy sequence if for any $\varepsilon>0$, there exists $N \in \mathbf{N}$ such that for all $s, t \geq N,\left\|x_{s}-x_{t}, y_{2}, \cdots, y_{n}\right\|<\varepsilon$ for all $y_{2}, \cdots, y_{n} \in X$. For convenience, we will write $\lim _{s, t \rightarrow \infty}\left\|x_{s}-x_{t}, y_{2}, \cdots, y_{n}\right\|=0$ for an $n$-Cauchy sequence $\left\{x_{\ell}\right\}$. An n-Banach space is defined to be a linear n-normed space in which every $n$-Cauchy sequence is n-convergent.

The following lemma is a useful toolbox for a linear n-normed space.

Lemma 1 ([31]). Let $(X,\|\cdot, \cdots, \cdot\|)$ be a linear $n$-normed space and $x \in X$. Then

(1) If $\left\|x, x_{2}, \cdots, x_{n}\right\|=0$ for all $x_{2}, \cdots, x_{n} \in X$, then $x=0$.

(2) $\quad\left\|x, x_{2}, \cdots, x_{n}\right\|-\left\|y, x_{2}, \cdots, x_{n}\right\| \mid \leq\left\|x-y, x_{2}, \cdots, x_{n}\right\|$ for all $x, y, x_{2}, \cdots, x_{n} \in X$.

(3) if a sequence $\left\{x_{m}\right\}$ is convergent in $X$, then

$$
\lim _{m \rightarrow \infty}\left\|x_{m}, y_{2}, \ldots, y_{n}\right\|=\left\|\lim _{m \rightarrow \infty} x_{m}, y_{2}, \ldots, y_{n}\right\|
$$

for all $y_{2}, \ldots, y_{n} \in X$.

From now on, let $X$ be a real linear space and let $(Z,\|\cdot, \cdots, \cdot\|)$ be an $n$-Banach space unless otherwise stated.

\section{Main Results}

In this section, we present the generalized Hyers-Ulam stabilities for the several functional equations in $n$-Banach spaces. We solve the problems for the stabilities and consider applications of the results in $n$-Banach spaces.

\subsection{Stability of the Cubic Functional Equation}

We start this subsection by investigating the generalized Hyers-Ulam stability for the cubic functional Equation (2) in $n$-Banach spaces. For convenience, we use the the notation $D_{f}(x, y)$ as follows:

$$
D_{f}(x, y):=f(2 x+y)+f(2 x-y)-2 f(x+y)-2 f(x-y)-12 f(x)
$$

for all $x, y \in X$. If $D_{f}(x, y)=0$, then the function $f$ is a solution of the cubic functional equation. Thus, $D_{f}(x, y)$ is an approximate remainder of the functional Equation (2) and acts as a perturbation of the equation. We use this approximate remainder to solve the generalized Hyers-Ulam stability for the cubic functional equation in $n$-Banach spaces.

Now, in the following theorem, we present a solution of stability for the cubic funtional equation in the spaces.

Theorem 1. Let $\varphi: X^{n+1} \rightarrow \mathbb{R}^{+}$be a function such that

$$
\sum_{i=0}^{\infty} \frac{\varphi\left(2^{i} x, 0, x_{2}, \cdots, x_{n}\right)}{8^{i}}<\infty, \lim _{n \rightarrow \infty} \frac{\varphi\left(2^{n} x, 2^{n} y, x_{2}, \ldots, x_{n}\right)}{8^{n}}=0
$$

for all $x, y, x_{2}, \ldots, x_{n} \in X$. Suppose that a function $f: X \rightarrow Z$ be a surjective mapping satisfying

$$
\left\|D_{f}(x, y), z_{2}, \ldots, z_{n}\right\| \leq \varphi\left(x, y, x_{2}, \ldots, x_{n}\right)
$$

for all $x, y, x_{2}, \ldots, x_{n} \in X$, where $z_{i}=f\left(x_{i}\right)$ for each $i=2, \ldots, n$. Then there is a unique cubic mapping $C: X \rightarrow Z$ such that

$$
\left\|f(x)-C(x), z_{2}, \ldots, z_{n}\right\| \leq \frac{1}{16} \sum_{i=0}^{\infty} \frac{\varphi\left(2^{i} x, 0, x_{2}, \ldots, x_{n}\right)}{8^{i}}
$$


for all $x, x_{2}, \ldots, x_{n} \in X$, where $z_{i}=f\left(x_{i}\right)$ for each $i=2, \ldots, n$.

We call the function $f$ the pseudo-cubic function for the error function $\varphi$, and the solution function $C$ is the cubic function induced from the pseudo-cubic function $f$.

Proof. Let $z_{i}=f\left(x_{i}\right)(i=2,3, \ldots, n)$. First, take $y=0$ in (4) to have

$$
\left\|\frac{f(2 x)}{8}-f(x), z_{2}, \ldots, z_{n}\right\| \leq \frac{1}{16} \varphi\left(x, 0, x_{2}, \ldots, x_{n}\right)
$$

for all $x, x_{2}, \ldots, x_{n} \in X$. Replacing $x$ by $2 x$ in (6) and dividing by 8 , we obtain

$$
\left\|\frac{f\left(2^{2} x\right)}{8^{2}}-f(x), z_{2}, \ldots, z_{n}\right\| \leq \frac{1}{16}\left[\varphi\left(x, 0, x_{2}, \ldots, x_{n}\right)+\frac{\varphi\left(2 x, 0, x_{2}, \ldots, x_{n}\right)}{8}\right]
$$

for all $x, x_{2}, \ldots, x_{n} \in X$. Using the induction on $n$, we get that

$$
\left\|\frac{f\left(2^{n} x\right)}{8^{n}}-f(x), z_{2}, \cdots, z_{n}\right\| \leq \frac{1}{16} \sum_{i=0}^{n-1} \frac{\varphi\left(2^{i} x, 0, x_{2}, \ldots, x_{n}\right)}{8^{i}}
$$

for all $x, x_{2}, \ldots, x_{n} \in X$. For $0 \leq m<n$, divide inequality (8) by $8^{m}$ and also replace $x$ by $2^{m} x$ to find that

$$
\begin{aligned}
\left\|\frac{f\left(2^{n} 2^{m} x\right)}{8^{n+m}}-\frac{f\left(2^{m} x\right)}{8^{m}}, z_{2}, \ldots, z_{n}\right\| & =\frac{1}{8^{m}}\left\|\frac{f\left(2^{n} 2^{m} x\right)}{8^{n}}-f\left(2^{m} x\right), z_{2}, \ldots, z_{n}\right\| \\
& \leq \frac{1}{16 \cdot 8^{m}} \sum_{i=0}^{n-1} \frac{\varphi\left(2^{i} 2^{m} x, 0, x_{2}, \ldots, x_{n}\right)}{8^{i}} \\
& \leq \frac{1}{16} \sum_{i=m}^{n-1} \frac{\varphi\left(2^{i} x, 0, x_{2}, \ldots, x_{n}\right)}{8^{i}}
\end{aligned}
$$

for all $x, x_{2}, \ldots, x_{n} \in X$. We then obtain

$$
\lim _{m, n \rightarrow \infty}\left\|\frac{f\left(2^{n} 2^{m} x\right)}{8^{n+m}}-\frac{f\left(2^{m} x\right)}{8^{m}}, z_{2}, \ldots, z_{n}\right\|=0
$$

for all $x_{2}, \ldots, x_{n} \in X$. Since $f$ is surjective, by Lemma 1 , the sequence $\left\{\frac{1}{8^{n}} f\left(2^{n} x\right)\right\}$ is an $n$-Cauchy sequence in $Z$. Therefore, we may define a mapping $C: X \rightarrow Z$ by

$$
C(x):=\lim _{n \rightarrow \infty} \frac{1}{8^{n}} f\left(2^{n} x\right)
$$

for all $x \in X$. By letting $n \rightarrow \infty$ in (8), we arrive at the formula (5). To show that the mapping $C: X \rightarrow Z$ satisfies Equation (2), replace $x, y$ with $2^{n} x, 2^{n} y$, respectively, in (4) and divide by $8^{n}$; then it follows that

$$
\begin{array}{r}
8^{-n} \| f\left(2^{n}(2 x+y)+f\left(2^{n}(2 x-y)-2 f\left(2^{n}(x+y)\right)-2 f\left(2^{n}(x-y)-12 f\left(2^{n} x\right), z_{2}, \ldots, z_{n} \|\right.\right.\right. \\
\leq 8^{-n} \varphi\left(2^{n} x, 2^{n} y, x_{2}, \ldots, x_{n}\right)
\end{array}
$$

for all $x, x_{2}, \ldots, x_{n} \in X$. Taking the limit as $n \rightarrow \infty$ in (9), we immediately obtain that the mapping $C$ satisfies (2).

Now, let $D: X \rightarrow Z$ be another cubic mapping satisfying (5). Then we have

$$
\begin{aligned}
\left\|C(x)-D(x), z_{2}, \ldots, z_{n}\right\| & =8^{-n}\left\|C\left(2^{n} x\right)-D\left(2^{n} x\right), z_{2}, \ldots, z_{n}\right\| \\
& \leq 8^{-n}\left(\left\|C\left(2^{n} x\right)-f\left(2^{n} x\right), z_{2}, \ldots, z_{n}\right\|+\left\|f\left(2^{n} x\right)-D\left(2^{n} x\right), z_{2}, \ldots, z_{n}\right\|\right) \\
& \leq \frac{1}{8} \sum_{i=0}^{\infty} \frac{\varphi\left(2^{i} 2^{n} x, 0, x_{2}, \ldots, x_{n}\right)}{8^{n+i}}
\end{aligned}
$$


which tends to zero as $k \rightarrow \infty$ for all $x, z_{2}, \ldots, z_{n} \in X$. By Lemma 1 , we conclude that $C(x)=D(x)$ for all $x \in X$. This completes the proof of the theorem.

As an application of Theorem 1, we obtain a stability of Equation (2) in the following corollary.

Corollary 1. Assume that $(X,\|\cdot\|)$ is a real normed space and that $(Z,\|\cdot, \cdots, \cdot\|)$ is a linear $n$-normed space. Let $\theta \in[0, \infty), p, q, r \in(0, \infty)$ and $p, q<3$ and let $f: X \rightarrow Z$ be a surjective mapping satisfying

$$
\left\|D_{f}(x, y), z_{2}, \ldots, z_{n}\right\| \leq \theta\left(\|x\|^{p}+\|y\|^{q}\right)\left\|x_{2}\right\|^{r} \cdots\left\|x_{n}\right\|^{r}
$$

for all $x, y, x_{2}, \ldots, x_{n} \in X$, where $z_{i}=f\left(x_{i}\right)$ for each $i=2, \ldots, n$. Then there is a unique cubic mapping $C: X \rightarrow Z$ such that

$$
\left\|f(x)-C(x), z_{2}, \ldots, z_{n}\right\| \leq \frac{\theta}{16-2^{p+1}}\|x\|^{p}\left\|x_{2}\right\|^{r} \cdots\left\|x_{n}\right\|^{r}
$$

for all $x, x_{2}, \ldots, x_{n} \in X$, where $z_{i}=f\left(x_{i}\right)$ for each $i=2, \ldots, n$.

Proof. The assertion follows from Theorem 1 by setting

$$
\varphi\left(x, y, x_{2}, \cdots, x_{n}\right)=\theta\left(\|x\|^{p}+\|y\|^{q}\right)\left\|x_{2}\right\|^{r} \cdots\left\|x_{n}\right\|^{r}
$$

for all $x, y, x_{2}, \ldots, x_{n} \in X$.

In the next theorem we also deal with a solution of the cubic functional equation in $n$-Banach spaces under different conditions. Next, we investigate the change of conditions for the pseudo-cubic function $f$ and the error function $\varphi$, and also obtain a stability of Equation (2) in the following theorem (compare with Theorem 1).

Theorem 2. Let $\varphi: X^{n+1} \rightarrow \mathbb{R}^{+}$be a function such that

$$
\sum_{i=0}^{\infty} 8^{i} \varphi\left(\frac{x}{2^{i+1}}, 0, x_{2}, \ldots, x_{n}\right)<\infty, \lim _{n \rightarrow \infty} 8^{n} \varphi\left(\frac{x}{2^{n}}, \frac{y}{2^{n}}, x_{2}, \ldots, x_{n}\right)=0
$$

for all $x, y, x_{2}, \ldots, x_{n} \in X$, where $z_{i}=f\left(x_{i}\right)$ for each $i=2, \ldots, n$. Suppose that a function $f: X \rightarrow Z$ be a surjective mapping satisfying (4). Then there is a unique cubic mapping $C: X \rightarrow Z$ such that

$$
\left\|f(x)-C(x), z_{2}, \ldots, z_{n}\right\| \leq \frac{1}{2} \sum_{i=0}^{\infty} 8^{i} \varphi\left(\frac{x}{2^{i+1}}, 0, x_{2}, \ldots, x_{n}\right)
$$

for all $x, x_{2}, \ldots, x_{n} \in X$, where $z_{i}=f\left(x_{i}\right)$ for each $i=2, \ldots, n$.

Proof. Let $z_{i}=f\left(x_{i}\right)$ for each $i=2, \ldots, n$. Take $x=\frac{x}{2}$ in (6) and multiply by eight to have

$$
\left\|f(x)-8 f\left(\frac{x}{2}\right), z_{2}, \ldots, z_{n}\right\| \leq \frac{1}{2} \varphi\left(\frac{x}{2}, 0, x_{2}, \ldots, x_{n}\right)
$$

for all $x, x_{2}, \ldots, x_{n} \in X$. By replacing $x$ with $\frac{x}{2}$ in (11) and multiplying by eight, we get

$$
\left\|f(x)-8^{2} f\left(\frac{x}{2^{2}}\right), z_{2}, \ldots, z_{n}\right\| \leq \frac{1}{2} \varphi\left(\frac{x}{2}, 0, x_{2}, \ldots, x_{n}\right)+4 \varphi\left(\frac{x}{2^{2}}, 0, x_{2}, \ldots, x_{n}\right)
$$

for all $x, x_{2}, \ldots, x_{n} \in X$. Then we can find a unique cubic mapping $C: X \rightarrow Z$ defined by

$$
C(x):=\lim _{n \rightarrow \infty} 8^{n} f\left(\frac{x}{2^{n}}\right)
$$


for all $x \in X$, as in the proof of Theorem 4. This completes the proof.

Using the above theorem, we immediately get the following corollary.

Corollary 2. Assume that $(X,\|\cdot\|)$ is a real normed space and that $(Z,\|\cdot, \cdots, \cdot\|)$ is a linear $n$-normed space. Let $\theta \in[0, \infty), p, q, r \in(0, \infty)$, and $p, q>3$. Let $f: X \rightarrow Z$ be a surjective mapping satisfying

$$
\left\|D_{f}(x, y), z_{2}, \ldots, z_{n}\right\| \leq \theta\left(\|x\|^{p}+\|y\|^{q}\right)\left\|x_{2}\right\|^{r} \cdots\left\|x_{n}\right\|^{r}
$$

for all $x, y, x_{2}, \ldots, x_{n} \in X$, where $z_{i}=f\left(x_{i}\right)$ for each $i=2, \ldots, n$. Then there is a unique cubic mapping $C: X \rightarrow Z$ such that

$$
\left\|f(x)-C(x), z_{2}, \ldots, z_{n}\right\| \leq \frac{\theta}{2^{p+1}-16}\|x\|^{p}\left\|x_{2}\right\|^{r} \cdots\left\|x_{n}\right\|^{r}
$$

for all $x, x_{2}, \ldots, x_{n} \in X$, where $z_{i}=f\left(x_{i}\right)$ for each $i=2, \ldots, n$.

Proof. The proof follows from Theorem 2 with

$$
\varphi\left(x, y, x_{2}, \cdots, x_{n}\right)=\theta\left(\|x\|^{p}+\|y\|^{q}\right)\left\|x_{2}\right\|^{r} \cdots\left\|x_{n}\right\|^{r}
$$

for all $x, y, x_{2}, \ldots, x_{n} \in X$.

\subsection{Stability of the Quartic Functional Equation}

In this subsection, we discuss the generalized Hyers-Ulam stability of the quartic functional Equation (3) in $n$-Banach spaces. For $x, y \in X$, we define $E_{f}(x, y)$ given by

$$
E_{f}(x, y):=f(2 x+y)+f(2 x-y)-4 f(x+y)-4 f(x-y)-24 f(x)+6 f(y) .
$$

The difference $E_{f}(x, y)$ means an approximate remainder of the functional Equation (3). equation.

Now we provide important consequences for the stability of the quartic functional

Theorem 3. Let $\varphi: X^{n+1} \rightarrow \mathbb{R}^{+}$be a function such that

$$
\sum_{i=0}^{\infty} 2^{-4 k} \varphi\left(2^{i} x, 0, x_{2}, \ldots, x_{n}\right)<\infty \text { and } \lim _{n \rightarrow \infty} 2^{-4 n} \varphi\left(2^{n} x, 2^{n} y, x_{2}, \ldots, x_{n}\right)=0,
$$

for all $x, y, x_{2}, \ldots, x_{n} \in X$. Suppose that a function $f: X \rightarrow Z$ be a surjective mapping satisfying

$$
\left\|E_{f}(x, y), z_{2}, \ldots, z_{n}\right\| \leq \delta+\varphi\left(x, y, x_{2}, \ldots, x_{n}\right)
$$

for all $x, y, x_{2}, \ldots, x_{n} \in X$, where $z_{i}=f\left(x_{i}\right)$ for each $i=2, \ldots, n$, where $\delta \geq 0$. Then there is a unique quartic mapping $T: X \rightarrow Z$ such that

$$
\left\|f(x)-T(x), z_{2}, \ldots, z_{n}\right\| \leq \frac{1}{30} \delta+\frac{1}{32} \sum_{i=0}^{\infty} 2^{-4 k} \varphi\left(2^{i} x, 0, x_{2}, \ldots, x_{n}\right)+\frac{1}{5}\left\|f(0), z_{2}, \ldots, z_{n}\right\|
$$

for all $x, x_{2}, \ldots, x_{n} \in X$, where $z_{i}=f\left(x_{i}\right)$ for each $i=2, \ldots, n$.

From now on we call the function $f$ the pseudo-quartic function for $\varphi$, and the solution function $T$ is the quartic function induced from the pseudo-quartic function $f$.

Proof. Let $z_{i}=f\left(x_{i}\right)$ for each $i=2, \ldots, n$. By letting $y=0$ in (12), we get

$$
\left\|2^{-4} f(2 x)-f(x)+\frac{3}{16} f(0), z_{2}, \ldots, z_{n}\right\| \leq \frac{1}{32} \delta+\frac{1}{32} \varphi\left(x, 0, x_{2}, \ldots, x_{n}\right),
$$


for all $x, x_{2}, \ldots, x_{n} \in X$. If we replace $x$ by $2^{n-1} x$ in (14) and divide both sides of (14) by $2^{4 n-4}$, we have that

$$
\left\|2^{-4 n} f\left(2^{n} x\right)-2^{4-4 n} f\left(2^{n-1 x}\right)+3 \cdot 2^{-4 n} f(0), z_{2}, \ldots, z_{n}\right\| \leq \frac{\delta}{2^{4 n+1}}+\frac{1}{2^{4 n+1}} \varphi\left(2^{n-1} x, 0, x_{2}, \ldots, x_{n}\right)
$$

for all $x, x_{2}, \ldots, x_{n} \in X$ and integers $n \geq 1$. Therefore, for all integers $0 \leq m<n$, we obtain

$$
\begin{aligned}
& \left\|\sum_{k=m+1}^{n}\left[2^{-4 k} f\left(2^{k} x\right)-2^{4-4 k} f\left(2^{k-1} x\right)+3 \cdot 2^{-4 k} f(0)\right], z_{2}, \ldots, z_{n}\right\| \\
& \leq \sum_{k=m+1}^{n}\left\|\left[2^{-4 k} f\left(2^{k} x\right)-2^{4-4 k} f\left(2^{k-1} x\right)+3 \cdot 2^{-4 k} f(0)\right], z_{2}, \ldots, z_{n}\right\| \\
& \leq \delta \sum_{k=m+1}^{n} 2^{-4 k-1}+\sum_{k=m+1}^{n} 2^{-4 k-1} \varphi\left(2^{k-1} x, 0, x_{2}, \ldots, x_{n}\right)
\end{aligned}
$$

and so

$$
\begin{aligned}
& \left\|2^{-4 n} f\left(2^{n} x\right)-2^{-4 m} f\left(2^{m} x\right), z_{2}, \ldots, z_{n}\right\| \\
& \leq 3 \cdot\left\|f(0), z_{2}, \ldots, z_{n}\right\| \sum_{k=m+1}^{n} 2^{-4 k}+\delta \sum_{k=m+1}^{n} 2^{-4 k-1}+\sum_{k=m+1}^{n} 2^{-4 k-1} \varphi\left(2^{k-1} x, 0, x_{2}, \ldots, x_{n}\right)
\end{aligned}
$$

for all $x, x_{2}, \ldots, x_{n} \in X$. In a similar way as in the proof of Theorem 1 , we can show that the sequence $\left\{2^{-4 n} f\left(2^{n} x\right)\right\}$ is an $n$-Cauchy sequence in $Z$ for all $x \in X$. Define a mapping $T: X \rightarrow Z$ by

$$
T(x):=\lim _{n \rightarrow \infty} 2^{-4 n} f\left(2^{n} x\right)
$$

for all $x \in X$. By (15), we have the inequality (13). It follows from (12) that

$$
\begin{aligned}
& \left\|T(2 x+y)+T(2 x-y)-4 T(x+y)-4 T(x-y)-24 T(x)+6 T(y), z_{2}, \ldots, z_{n}\right\| \\
& =\lim _{n \rightarrow \infty} 2^{-4 n} \| f\left(2^{n}(2 x+y)\right)+f\left(2^{n}(2 x-y)\right)-4 f\left(2^{n}(x+y)\right)-4 f\left(2^{n}(x-y)\right) \\
& -24 f\left(2^{n} x\right)+6 f\left(2^{n} y\right), z_{2}, \ldots, z_{n} \| \\
& \leq \lim _{n \rightarrow \infty} 2^{-4 n} \varphi\left(2^{n} x, 2^{n} y, x_{2}, \cdots, x_{n}\right)=0
\end{aligned}
$$

for all $x, y, x_{2}, \ldots, x_{n} \in X$. This implies that $T: X \rightarrow Z$ is a quartic mapping. Let $Q: X \rightarrow Z$ be another quartic mapping satisfying (13). Therefore we have

$$
\begin{aligned}
\left\|T(x)-Q(x), z_{2}, \ldots, z_{n}\right\| & =\lim _{n \rightarrow \infty} 2^{-4 n}\left\|f\left(2^{n} x\right)-Q\left(2^{n} x\right), z_{2}, \ldots, z_{n}\right\| \\
& \leq \lim _{n \rightarrow \infty} 2^{-4 n}\left(\frac{1}{30} \delta+\frac{1}{32} \widetilde{\varphi}\left(2^{n} x\right)+\frac{1}{5}\left\|f(0), z_{2}, \ldots, z_{n}\right\|\right) \\
& =\frac{1}{32} \lim _{n \rightarrow \infty} \sum_{k=n}^{\infty} 2^{-4 k} \varphi\left(2^{k} x, 0, x_{2}, \ldots, x_{n}\right)=0
\end{aligned}
$$

for all $x, y, x_{2}, \ldots, x_{n} \in X$. It follows from Lemma 1 that $T(x)=Q(x)$ for all $x \in X$. This proves the uniqueness of $T$.

Now we show a simple application of Theorem 3 to obtain a stability of Equation (3).

Corollary 3. Assume that $(X,\|\cdot\|)$ is a real normed space and that $(Z,\|\cdot, \cdots, \cdot\|)$ is a linear $n$-normed space. Let $\varepsilon, \delta, \theta, \in[0, \infty), p, q, r \in(0, \infty)$ and $p, q<4$. Let $f: X \rightarrow Z$ be a surjective mapping satisfying

$$
\left\|E_{f}(x, y), z_{2}, \ldots, z_{n}\right\| \leq \delta+\left(\varepsilon\|x\|^{p}+\theta\|y\|^{q}\right)\left\|x_{2}\right\|^{r} \cdots\left\|x_{n}\right\|^{r}
$$


for all $x, y, z_{2}, \ldots, z_{n} \in X$, where $z_{i}=f\left(x_{i}\right)$ for each $i=2, \ldots, n$. Then there is a unique quartic mapping $T: X \rightarrow Z$ such that

$$
\left\|f(x)-T(x), z_{2}, \ldots, z_{n}\right\| \leq \frac{\delta}{24}+\frac{\varepsilon}{32-2^{p+1}}\|x\|^{p}\left\|x_{2}\right\|^{r} \cdots\left\|x_{n}\right\|^{r}
$$

for all $x, x_{2}, \ldots, x_{n} \in X$, where $z_{i}=f\left(x_{i}\right)$ for each $i=2, \ldots, n$.

Proof. It is a consequence of Theorem 3 with

$$
\varphi\left(x, y, x_{2}, \ldots, x_{n}\right)=\left(\varepsilon\|x\|^{p}+\theta\|y\|^{q}\right)\left\|x_{2}\right\|^{r} \cdots\left\|x_{n}\right\|^{r}
$$

for all $x, y, x_{2}, \ldots, x_{n} \in X$.

Next we consider the changes of conditions of the pseudo-quartic function and the error function, and we find a solution of the quartic functional equation in $n$-Banach spaces. We prove the existence of a solution of Equation (3) in $n$-Banach spaces.

Theorem 4. Let $\varphi: X^{n+1} \rightarrow \mathbb{R}^{+}$be a function such that

$$
\sum_{i=0}^{\infty} 2^{4 i} \varphi\left(\frac{x}{2^{i+1}}, 0, x_{2}, \cdots, x_{n}\right)<\infty, \lim _{n \rightarrow \infty} 2^{4 n} \varphi\left(\frac{x}{2^{n}}, \frac{y}{2^{n}}, x_{2}, \ldots, x_{n}\right)=0,
$$

for all $x, y, x_{2}, \ldots, x_{n} \in X$. Suppose that a function $f: X \rightarrow Z$ be a surjective mapping satisfying

$$
\left\|E_{f}(x, y), z_{2}, \ldots, z_{n}\right\| \leq \varphi\left(x, y, x_{2}, \ldots, x_{n}\right)
$$

for all $x, y, x_{2}, \ldots, x_{n} \in X$, where $z_{i}=f\left(x_{i}\right)$ for each $i=2, \ldots, n$. Then there is a unique quartic mapping $T: X \rightarrow Z$ such that

$$
\left\|f(x)-T(x), z_{2}, \ldots, z_{n}\right\| \leq \frac{1}{2} \sum_{i=0}^{\infty} 2^{4 i} \varphi\left(\frac{x}{2^{i+1}}, 0, x_{2}, \ldots, x_{n}\right)
$$

for all $x, x_{2}, \ldots, x_{n} \in X$, where $z_{i}=f\left(x_{i}\right)$ for each $i=2, \ldots, n$.

Proof. It follows from (16) that $\varphi(0,0)=0$. Thus, we have $f(0)=0$ from (17). By letting $y=0$ in (17), we get

$$
\left\|2^{-4} f(2 x)-f(x), z_{2}, \ldots, z_{n}\right\| \leq \frac{1}{32} \varphi\left(x, 0, x_{2}, \ldots, x_{n}\right)
$$

for all $x, x_{2}, \ldots, x_{n} \in X$, where $z_{i}=f\left(x_{i}\right)$ for each $i=2, \ldots, n$. Through replacing $x$ by $\frac{x}{2}$ in (19) and multiplying by $2^{4}$, we obtain

$$
\left\|f(x)-2^{4} f\left(\frac{x}{2}\right), z_{2}, \ldots, z_{n}\right\| \leq \frac{1}{2} \varphi\left(\frac{x}{2}, 0, x_{2}, \ldots, x_{n}\right)
$$

for all $x, x_{2}, \ldots, x_{n} \in X$, where $z_{i}=f\left(x_{i}\right)$ for each $i=2, \ldots, n$. By (20), we have

$$
\left\|f(x)-2^{8} f\left(\frac{x}{2^{2}}\right), z_{2}, \ldots, z_{n}\right\| \leq \frac{1}{2} \varphi\left(\frac{x}{2}, 0, x_{2}, \ldots, x_{n}\right)+2^{3} \varphi\left(\frac{x}{2^{2}}, 0, x_{2}, \ldots, x_{n}\right)
$$

for all $x, x_{2}, \ldots, x_{n} \in X$, where $z_{i}=f\left(x_{i}\right)$ for each $i=2, \ldots, n$. As in the proof of Theorem 3 , we can find a unique quartic mapping $T: X \rightarrow Z$ defined by

$$
T(x):=\lim _{n \rightarrow \infty} 2^{4 n} f\left(\frac{x}{2^{n}}\right)
$$

for all $x \in X$. This completes the proof. 
As an application of Theorem 1, we obtain a stability of Equation (2) in the following corollary.

Corollary 4. Assume that $(X,\|\cdot\|)$ is a real normed space and that $(Z,\|\cdot, \cdots, \cdot\|)$ is a linear $n$-normed space. Let $\varepsilon, \theta \in[0, \infty), p, q, r \in(0, \infty)$ and $p, q>4$. Let $f: X \rightarrow Z$ be a surjective mapping satisfying

$$
\left\|E_{f}(x, y), z_{2}, \ldots, z_{n}\right\| \leq\left(\varepsilon\|x\|^{p}+\theta\|y\|^{q}\right)\left\|x_{2}\right\|^{r} \cdots\left\|x_{n}\right\|^{r}
$$

for all $x, y, x_{2}, \ldots, x_{n} \in X$, where $z_{i}=f\left(x_{i}\right)$ for each $i=2, \ldots, n$. Then there is a unique quartic mapping $T: X \rightarrow Z$ such that

$$
\left\|f(x)-T(x), z_{2}, \ldots, z_{n}\right\| \leq \frac{\varepsilon}{2^{p+1}-32}\|x\|^{p}\left\|x_{2}\right\|^{r} \cdots\left\|x_{n}\right\|^{r}
$$

for all $x, x_{2}, \ldots, x_{n} \in X$, where $z_{i}=f\left(x_{i}\right)$ for each $i=2, \ldots, n$.

Proof. It is a direct consequence of Theorem 4 with

$$
\varphi\left(x, y, x_{2}, \ldots, x_{n}\right)=\left(\varepsilon\|x\|^{p}+\theta\|y\|^{q}\right)\left\|x_{2}\right\|^{r} \cdots\left\|x_{n}\right\|^{r}
$$

for all $x, y, x_{2}, \ldots, x_{n} \in X$.

\section{Conclusions}

In this paper, we considered the cubic functional equation and quartic functional equation in $n$-Banach spaces. We dealt with stabilities of the functional equations in $n$-Banach spaces. For a surjective mapping $f$ into an $n$-Banach space, called a pseudocubic function or a pseudo-quartic function, we solved the stability problem for the cubic functional equations and the quartic functional equations for $f$, as we demonstrated the existence of the solutions of the functional equations. As applications, we got the solutions of the generalized Hyers-Ulam stabilities under the changes of conditions of the pseudofunctions and the error functions. Our results about the equations in $n$-Banach spaces are a new approach and are key extensions for the study of functional equations, where the novelty of our results lies.

Author Contributions: Conceptualization, Investigation and Methodology: J.C., H.-Y.C. and A.K. All authors have read and agreed to the published version of the manuscript.

Funding: This work was supported by research fund of Chungnam National University.

Acknowledgments: The authors are deeply grateful to the referees whose remarks helped to improve the manuscript.

Conflicts of Interest: The authors declare no conflicts of interest.

\section{References}

1. Ulam, S.M. A Collection of the Mathematical Problems; Interscience Publ.: New York, NY, USA, 1960.

2. Hyers, D.H. On the stability of the linear functional equation. Proc. Natl. Acad. Sci. USA 1941, 27, 222-224. [CrossRef] [PubMed]

3. Aoki, T. On the stability of the linear transformation in Banach Spaces. J. Math. Soc. Japan 1950, 2, 64-66. [CrossRef]

4. Rassias, T.M. On the stability of the linear mapping in Banach spaces. Proc. Am. Math. Soc. 1978, 72, 297-300. [CrossRef]

5. Găvruta, P. A generalization of the Hyers-Ulam-Rassias Stability of Approximately Additive Mappings. J. Math. Anal. Appl. 1994, 184, 431-436. [CrossRef]

6. Rassias, T.M.; Šemrl, P. On the behavior of mappings which do not satisfy Hyers-Ulam stability. Proc. Am. Math. Soc. 1992, 114, 989-993. [CrossRef]

7. Rassias, J.M. On the stability of the Euler-Lagrange functional equation. Chin. J. Math. 1992, 20, 185-190.

8. Jun, K.; Kim, H. The generalized Hyers-Ulam-Rassias stability of a cubic functional equation. J. Math. Anal. Appl. 2002, 274, 867-878. [CrossRef]

9. Chu, H.-Y.; Kang, D.S. On the stability of an n-dimensional cubic functional equation. J. Math. Anal Appl. 2007, 325, 595-607. [CrossRef] 
10. Chu, H.-Y.; Kim, A.; Yoo, S. On the stability of the generalized cubic set-valued functional equation. Appl. Math. Lett. 2014, 37, 7-14. [CrossRef]

11. Jun, K.; Kim, H. Stability Problem for Jensen-type Functional Equations of Cubic Mappings. Acta Math. Sin. 2006, 22, 1781-1788. [CrossRef]

12. Jun, K.; Kim, H. On the stability of Euler-Lagrange type cubic mappings in quasi-Banach spaces. J. Math. Anal. Appl. 2007, 332, 1335-1350. [CrossRef]

13. Rassias, J.M. Solution of the Ulam stability problem for quartic mappings. Glas. Mat. Ser. III 1999, 34, $243-252$.

14. Chung, J.K.; Sahoo, P.K. On the general solution of a quartic functional equation. Bull. Korean Math. Soc. 2003, 40, 565-576. [CrossRef]

15. Najati, A. On the stability of a quartic functional equation. J. Math. Anal. Appl. 2008, 340, 569-574. [CrossRef]

16. EL-Fassi, I. Approximate solution of radical quartic functional equation related to additive mapping in 2-Banach spaces. J. Math. Anal. Appl. 2017, 455, 2001-2013. [CrossRef]

17. Lee, S.; Im, S.; Hwang, I. Quartic functional equations. J. Math. Anal. Appl. 2005, 307, 387-394. [CrossRef]

18. Pinelas, S.; Govindan, V.; Tamilvanan, K. Stability of a quartic functional equation. J. Fixed Point Theory Appl. 2018, 20, 148. [CrossRef]

19. Forti, G.-L. Hyers-Ulam stability of functional equations in several variables. Aequationes Math. 1995, 50, 143-190. [CrossRef]

20. Khan, A.; Khan, H.; Gómez-Aguilar, J.F.; Abdeljawad, T. Existence and Hyers-Ulam stability for a nonlinear singular fractional differential equations with Mittag-Leffler kernel. Chaos Solitons Fractals 2019, 127, 422-427. [CrossRef]

21. Liu, K.; Wang, J.; Zhou, Y.; O'Regan, D. Hyers-Ulam stability and existence of solutions for fractional differential equations with Mittag-Leffler kernel. Chaos Solitons Fractals 2020, 132, 109534. [CrossRef]

22. Rassias, T.M. On the stability of functional equations and a problem of Ulam. Acta Appl. Math. 2000, 62, 23-130. [CrossRef]

23. Chung, S.; Park, W. Hyers-Ulam stability of functional equations in 2-Banach spaces. Int. J. Math. Anal. 2012, 6, 951-961.

24. Park, W. Approximate additive mappings in 2-Banach spaces and related topics. J. Math. Anal. Appl. 2011, 376, 193-202. [CrossRef]

25. Misiak, A. $n$-inner product spaces. Math. Nachr. 1989, 140, 299-319. [CrossRef]

26. Misiak, A. Orthogonality and orthogonormality in $n$-inner product spaces. Math. Nachr. 1989, 143, 249-261. [CrossRef]

27. Chu, H.-Y.; Choi, S.K.; Kang, D.S. Mappings of conservative distances in linear n-normed spaces. Nonlinear Anal. 2009, 70, 1168-1174. [CrossRef]

28. Chu, H.-Y.; Ku, S.-H. A Mazur-Ulam problem in non-Archimedean n-normed spaces. J. Inequal. Appl. 2013, 2013, 34. [CrossRef]

29. Chu, H.-Y.; Lee, K.; Park, C. On the Aleksandrov problem in linear n-normed spaces. Nonlinear Anal. 2004, 59, 1001-1011.

30. Chu, H.-Y.; Park, C.; Moslehian, M.S. On the Cauchy-Rassias inequality and linear n-inner product preserving mappings. Math. Inequal. Appl. 2006, 9, 121-133.

31. Chu, H.-Y.; Kim, A.; Park, J. On the Hyers-Ulam stabilities of functional equations on $n$-Banach spaces. Math. Nachr. 2016, 289, 1177-1188. [CrossRef]

32. Brzdęk, J.; Ciepliński, K. A fixed point theorem in n-Banach spaces and Ulam stability. J. Math. Anal. Appl. 2019, 470, 632-646. [CrossRef] 
Article

\title{
An Inertial Generalized Viscosity Approximation Method for Solving Multiple-Sets Split Feasibility Problems and Common Fixed Point of Strictly Pseudo-Nonspreading Mappings
}

\author{
Hammed Anuoluwapo Abass ${ }^{1,2}$ and Lateef Olakunle Jolaoso ${ }^{3, *}$ \\ 1 School of Mathematics, Statistics and Computer Science, University of KwaZulu-Natal, \\ Durban 4001, South Africa; hammedabass548@gmail.com \\ 2 DSI-NRF Center of Excellence in Mathematical and Statistical Sciences (CoE-MaSS), \\ Johannesburg 2193, South Africa \\ 3 Department of Mathematics and Applied Mathematics, Sefako Makgatho Health Sciences University, \\ P.O. Box 94, Pretoria 0204, South Africa \\ * Correspondence: lateef.jolaoso@smu.ac.za
}

check for updates

Citation: Abass, H.A.; Jolaoso, L.O An Inertial Generalized Viscosity Approximation Method for Solving Multiple-Sets Split Feasibility Problems and Common Fixed Point of Strictly Pseudo-Nonspreading Mappings. Axioms 2021, 10, 1. https:/ /dx.doi.org/ 10.3390/axioms 10010001

Received: 30 November 2020 Accepted: 14 December 2020 Published: 24 December 2020

Publisher's Note: MDPI stays neutral with regard to jurisdictional claims in published maps and institutional affiliations.

\section{Copyright: (c) 2020 by the authors. Li-} censee MDPI, Basel, Switzerland. This article is an open access article distributed under the terms and conditions of the Creative Commons Attribution (CC BY) license (https: / / creativecommons.org / licenses/by/4.0/).

\begin{abstract}
In this paper, we propose a generalized viscosity iterative algorithm which includes a sequence of contractions and a self adaptive step size for approximating a common solution of a multiple-set split feasibility problem and fixed point problem for countable families of $k$-strictly pseudononspeading mappings in the framework of real Hilbert spaces. The advantage of the step size introduced in our algorithm is that it does not require the computation of the Lipschitz constant of the gradient operator which is very difficult in practice. We also introduce an inertial process version of the generalize viscosity approximation method with self adaptive step size. We prove strong convergence results for the sequences generated by the algorithms for solving the aforementioned problems and present some numerical examples to show the efficiency and accuracy of our algorithm. The results presented in this paper extends and complements many recent results in the literature.
\end{abstract}

Keywords: multiple-sets split feasibility problem; strictly pseudocontractive mappings; nonexpansive mappings; viscossity iterative scheme; fixed point problem

\section{Introduction}

The problem of finding a point in the intersection of closed and convex subsets in real Hilbert spaces has appeared severally in diverse areas of mathematics and physical sciences. This problem is commonly referred to as the Convex Feasibility Problem (shortly, CFP), and finds its applications in various disciplines such as image restoration, computer tomograph and radiation therapy treatment planning, see [1]. A generalization of the CFP is the Split Feasibility Problem (SFP) which was introduced by Censor and Elfving [2] and defined as finding a point in a nonempty closed convex set, whose image under a bounded operator is in another set. Mathematically, the SFP can be formulated as:

$$
\text { find } x^{*} \in C \text { such that } A x^{*} \in Q \text {, }
$$

where $C$ and $Q$ are nonempty closed convex subsets of $\mathbb{R}^{N}$ and $\mathbb{R}^{M}$ respectively, and $A$ is a given matrix of dimension $N \times M$. The SFP also models inverse problems arising from phase retrieval and intensity modulated radiation therapy [2]. Censor et al. [3] further introduced another generalization of the CFP and SFP called the Multiple Set Split Feasibility Problem (MSSFP) which is formulated as

$$
\text { find } x^{*} \in C:=\cap_{i=1}^{k} C_{i} \text { such that } A x^{*} \in Q:=\cap_{j=1}^{t} Q_{j},
$$

where $k \geq 1$ and $r \geq 1$ are given integers, $A$ is a given $M \times N$ real matrix with $A^{*}$ its transpose, $\left\{C_{i}\right\}_{i=1}^{k}$ and $\left\{Q_{j}\right\}_{j=1}^{t}$ are nonempty closed convex subsets of $\mathbb{R}^{N}$ and $\mathbb{R}^{M}$, 
respectively. Observe that when $k=r=1$, the MSSFP reduces to SFP (1). In this paper, we focus on the MSSFP in a unified framework. We denote the set of solutions of (2) by $\Omega$ and assume that $\Omega$ is consistent (i.e., nonempty). It is well known that the MSSFP is equivalent to the following minimization problem:

$$
\min \left\{\frac{1}{2}\left\|x-P_{C}(x)\right\|^{2}+\frac{1}{2}\left\|A x-P_{Q}(A x)\right\|^{2}\right\},
$$

where $P_{C}$ and $P_{Q}$ are the orthogonal projections onto $C$ and $Q$ respectively. For solving (3), Censor et al. [3] defined a proximity function $p(x)$ for measuring the distance of a point to all sets as follows:

$$
p(x):=\frac{1}{2} \sum_{i=1}^{k} \alpha_{i}\left\|x-P_{C_{i}}(x)\right\|^{2}+\frac{1}{2} \sum_{j=1}^{t} \beta_{j}\left\|A x-P_{Q_{j}}(A x)\right\|^{2}
$$

where $\alpha_{i}>0, \beta_{j}>0 \forall i$ and $j$ respectively, and $\sum_{i=1}^{k} \alpha_{i}+\sum_{j=1}^{t} \beta_{j}=1$. It is easy to see that

$$
\nabla p(x):=\sum_{i=1}^{k} \alpha_{i}\left(x-P_{C_{i}}(x)\right)+\sum_{j=1}^{t} \beta_{j} A^{*}\left(I-P_{Q}\right) A x .
$$

Censor et al. [3] also introduced the following projection method for solving the MSSFP:

$$
x_{n+1}=P_{\Omega}\left(x_{n}-s \nabla p\left(x_{n}\right)\right),
$$

where $s$ is a positive scalar. They further proved the weak convergence of (5) under the condition that the stepsize $s$ satisfies

$$
0<s_{L} \leq s \leq s_{\mu}<\frac{2}{L}
$$

where $L=\sum_{i=1}^{k} \alpha_{i}+\rho\left(A^{*} A\right) \sum_{j=1}^{t} \beta_{j}$ is the Lipschitz constant of $\nabla p$. A major setback of (5) is the fact that the algorithm used a fixed stepsize which is restricted by the Lipschitz constant (this depends on the largest eigenvalue of the matrix $A^{*} A$ ). Computing the largest eigenvalue of $A^{*} A$ is usually difficult and its conservation results in slow convergence. More so, note that the projection onto the sets $C$ and $Q$ are often difficult to calculate when the sets are not simple. This can also result in the complication of (5). Several efforts have been made in order to find best appropriate modifications of (5) without the setbacks in infinite dimensional real Hilbert spaces. For instance, Zhao and Yang [4] introduced a new projection method such that the stepsize $s$ is selected via an Armijo line search technique for solving the MSSFP. However, this line search process required extra inner iteration for obtaining a suitable stepsize. The authors in [5] also introduced a self-adaptive projection method which requires the computation of the stepsize directly without any inner iteration. More so, López et al. [6] introduced a relaxed projection method with a fixed stepsize and proved a weak convergence result for solving the MSSFP. He et al. [7] further combined a Halpern iterative scheme with the relaxed projection method and proved a strong convergence result for solving the MSSFP. Recently, Suantai et al. [8] introduced an inertial relaxed projection method with a self-adaptive stepsize for solving the MSSFP. Also, Wen et al. [9] introduced a cyclic-simultaneous projection method and proved weak convergence result for solving the MSSFP.

Constructing iterative schemes with a faster rate of convergence are usually of great interest. The inertial-type algorithm which originated from the equation for an oscillator with damping and conservative restoring force has been an important tool employed in improving the performance of algorithms and has some nice convergence characteristics. In general, the main feature of the inertial-type algorithms is that we can use the previous 
iterates to construct the next one. Since the introduction of the inertial-like algorithm, many authors combined the inertial term $\left[\theta_{n}\left(x_{n}-x_{n-1}\right)\right]$ together with different kinds of iterative algorithms, including Mann, Kranoselski, Halpern, Viscosity, to mention a few, to approximate solutions of fixed point problems and optimization problems. Most authors were able to prove weak convergence results while few proved strong convergence results. Polyak [10] was the first author to propose the heavy ball method, Alvarez and Attouch [11] employed this to the setting of a general maximal monotone operator using the Proximal Point Algorithm (PPA), which is called the inertial PPA, and is of the form:

$$
\left\{\begin{array}{l}
y_{n}=x_{n}+\theta_{n}\left(x_{n}-x_{n-1}\right) \\
x_{n+1}=\left(I+r_{n} B\right)^{-1} y_{n}, n>1
\end{array}\right.
$$

They proved that if $\left\{r_{n}\right\}$ is non-decreasing and $\left\{\theta_{n}\right\} \subset[0,1)$ with

$$
\sum_{n=1}^{+\infty} \theta_{n}\left\|x_{n}-x_{n-1}\right\|^{2}<+\infty,
$$

then the Algorithm (6) converges weakly to a zero of a maximal monotone operator $B$. More precisely, condition (7) is true for $\theta_{n}<\frac{1}{3}$. Here $\theta_{n}$ is an extrapolation factor. Other initial-type algorithms can be found in, for instance [12-17].

Motivated by the works of Wen et al. [9] and López et al. [6], in this paper, we introduce a general viscosity relaxed projection method with inertial process for solving the MSSFP with the fixed point of strictly pseudo-nonspreading mappings in real Hilbert spaces. The stepsize of our algorithm is selected self-adaptively in each iteration and its convergence does not involve prior estimate of the matrix $A^{*} A$. More so, we define some sublevel sets whose projections can be calculated explicitly using the formula in [18]. The general viscosity approximation method guarantees strong convergence of the sequences generated by the algorithm. This improves the weak convergence results proved in $[6,9,19]$. We further provide some numerical experiments to illustrate the performance and accuracy of our algorithm. Our results improve and complement the results of [6-9,19-24] and many other results in this direction.

\section{Preliminaries}

We state some known and useful results which will be needed in the proof of our main theorem. In the sequel, we denote strong and weak convergence by " $\rightarrow$ " and " $\rightarrow$ ", respectively.

Let $C$ be a nonempty closed convex subset of a real Hilbert space $H$ with inner product $\langle.,$.$\rangle and norm \|$.$\| . Let S: C \rightarrow C$ be a nonlinear mapping and $F(S)=\{x \in C: S x=x\}$ be the set of all fixed points of $S$.

A mapping $S: C \rightarrow C$ is called

1. nonexpansive, if

$$
\|S x-S y\| \leq\|x-y\|, \forall x, y \in C ;
$$

2. quasi-nonexpansive, if $F(S)$ is nonempty, and

$$
\|S x-p\| \leq\|x-p\|, \forall p \in F(S) ;
$$

3. nonspreading [25], if

$$
2\|S x-S y\|^{2} \leq\|S x-y\|^{2}+\|S y-x\|^{2}, \forall x, y \in C ;
$$


4. $\quad k$-strictly pseudo-nonspreading in terms of Browder-Petryshyn [26], if there exists $k \in[0,1)$ such that

$$
\|S x-y\|^{2} \leq\|x-y\|^{2}+k\|x-S x-(y-S y)\|^{2}+2\langle x-S x, y-S y\rangle, \forall x . y \in C .
$$

Remark 1. (a) If $S: C \rightarrow C$ is a nonspreading mapping with $F(S) \neq \varnothing$, then $S$ is quasinonexpansive and $F(S)$ is closed and convex.

(b) It is also clear that every nonspreading mapping is $k$-strictly pseudo-nonspreading with $k=0$, but the converse is not true, see example 3 in [27].

Lemma 1. [27] Let $T: C \rightarrow C$ be a $k$-strictly pseudo-nonspreading mapping with $k \in[0,1)$. Denote $T_{\beta}:=\beta I+(1-\beta) T$, where $\beta \in[k, 1)$, then

(a) $F(T)=F\left(T_{\beta}\right)$,

(b) the following inequality holds:

$$
\left\|T_{\beta} x-T_{\beta} y\right\|^{2} \leq\|x-y\|^{2}+\frac{2}{1-\beta}\left\langle x-T_{\beta} x, y-T_{\beta} y\right\rangle, \forall x, y \in C ;
$$

(c) $T_{\beta}$ is a quasi-nonexpansive mapping.

Lemma 2. [28] Let $C \subset H$ be nonempty, closed and convex set. Then, $\forall x, y \in H$ and $z \in C$

1. $\left\langle x-P_{C} x, z-P_{C} x\right\rangle \leq 0$,

2. $\left\|P_{C} x-P_{C} y\right\|^{2} \leq\left\langle P_{C} x-P_{C} y, x-y\right\rangle$,

3. $\left\|P_{C} x-z\right\|^{2} \leq\|x-z\|^{2}-\left\|P_{C} x-x\right\|^{2}$.

Lemma 3. [29] Let $H$ be a real Hilbert space and $\left\{x_{i}\right\}_{i \geq 1}$ be a bounded sequence in $H$. For $\alpha_{i} \in(0,1)$ such that $\sum_{i=1}^{\infty} \alpha_{i}=1$, the following identity holds

$$
\left\|\sum_{i=1}^{\infty} \alpha_{i} x_{i}\right\|^{2}=\sum_{i=1}^{\infty} \alpha_{i}\left\|x_{i}\right\|^{2}-\sum_{1 \leq i<j<\infty} \alpha_{i} \alpha_{j}\left\|x_{i}-x_{j}\right\|^{2} .
$$

More so, from Lemma 3, we get the following result.

Lemma 4. [30] For all $x_{1}, x_{2}, \ldots, x_{n} \in H$, the following inequality holds:

$$
\left\|\sum_{i=1}^{n} \lambda_{i} x_{i}\right\|^{2}=\sum_{i=1}^{n} \lambda_{i}\left\|x_{i}\right\|^{2}-\frac{1}{2} \sum_{i, j=1}^{n} \lambda_{i} \lambda_{j}\left\|x_{i}-x_{j}\right\|^{2}, n \geq 2,
$$

where $\lambda_{i} \in[0,1], i=1,2, \ldots, n, \sum_{i=1}^{n} \lambda_{i}=1$.

Lemma 5. [27] Let $C$ be a closed convex subset of $H, T: C \rightarrow C$ be a k-strictly pseudononspreading mapping with $F(T) \neq \varnothing$. If $\left\{x_{n}\right\}$ is a sequence in $C$ which converges weakly to $p$ and $\left\{(I-T) x_{n}\right\}$ converges strongly to $q$, then $(I-T) p=q$. In particular, if $q=0$, then $p=T p$.

Lemma 6. [31] Let $\left\{a_{n}\right\}$ be a sequence of nonegative real numbers $\left\{\gamma_{n}\right\}$ be a sequence of real numbers in $(0,1)$ with conditions $\sum_{n=1}^{\infty} \gamma_{n}=\infty$ and $\left\{d_{n}\right\}$ be a sequence of real numbers. Assume that

$$
a_{n+1} \leq\left(1-\gamma_{n}\right) a_{n}+\gamma_{n} d_{n}, n \geq 1
$$

If $\lim \sup _{k \rightarrow \infty} d_{n_{k}} \leq 0$ for every subsequence $\left\{a_{n_{k}}\right\}$ of $\left\{a_{n}\right\}$ satisfying the condition: $\lim \inf _{k \rightarrow \infty}\left(a_{n_{k+1}}-a_{n_{k}}\right) \geq 0$, then $\lim _{n \rightarrow \infty} a_{n}=0$.

\section{Main Results}

In this section, we present our iterative algorithm and its convergence result. 
Let $H_{1}$ and $H_{2}$ be real Hilbert spaces, $C$ be a nonempty, closed and convex subset of a real Hilbert space $H$ and $\left\{g_{n}\right\}$ be a sequence of $\left\{\sigma_{n}\right\}$-contractive self maps of $H$ with $\liminf _{n \rightarrow \infty} \sigma_{n} \leq \lim \sup _{n \rightarrow \infty} \sigma_{n}=\sigma_{\mu}<1$. Suppose that $\left\{g_{n}(x)\right\}$ is uniformly convergent to $\{g(x)\}$ for any $x \in D$, where $D$ is a bounded subset of $C$, let $S_{m}: H_{1} \rightarrow H_{1}$, be a countable family of $k_{m}$-strictly pseudo-nonspreading mapping with $k:=\sup _{m \geq 1} k_{m} \in(0,1)$ and $S_{m, \beta}:=\beta I+(1-\beta) S_{m}$, where $\beta \in[k, 1)$, and $m \in \mathbb{N} \backslash\{0\}$.

Before we state our algorithm, we assume that the following conditions hold:

(A1) The set $C_{i}$ is given by $C_{i}=\left\{x \in H_{1}: c_{i}(x) \leq 0\right\}$ where $c_{i}: H_{1} \rightarrow \mathbb{R}(i=1,2 \ldots, k)$ are convex functions. Also, the set $Q_{j}$ is given by $Q_{j}=\left\{y \in H_{2}: q_{j}(y) \leq 0\right\}$ $(j=1,2, \ldots, t)$ are convex functions. In addition, we assume that both $c_{i}$ and $q_{j}$ are subdifferentiable on $H_{1}$ and $H_{2}$ respectively and $\partial c_{i}$ and $\partial q_{j}$ are bounded operators.

(A2) For any $x \in H_{1}$ and $y \in H_{2}$, at least one subgradient $\xi_{i} \in \partial c_{i}(x)$ and $\eta_{j} \in \partial q_{j}(y)$ can be calculated, where $\partial c_{i}(x)$ and $\partial q_{j}(y)$ denote the subdifferentials of $c_{i}$ and $q_{j}$ at $x$ and $y$ respectively, i.e.,

$$
\partial c_{i}(x)=\left\{\xi_{i} \in H_{1}: c_{i}(z) \geq c_{i}(x)+\left\langle\xi_{i}, z-x\right\rangle \quad \forall z \in H_{1}\right\},
$$

and

$$
\partial q_{j}(y)=\left\{\eta_{j} \in H_{2}: q_{j}(u) \geq q_{j}(y)+\left\langle\eta_{j}, u-y\right\rangle \quad \forall u \in H_{2}\right\} .
$$

(A3) We set $C_{i}^{n}$ and $Q_{j}^{n}$ as the half-spaces defined by

$$
C_{i}^{n}=\left\{x \in H_{1}: c_{i}\left(x_{n}\right)+\left\langle\xi_{i}^{n}, x-x_{n}\right\rangle \leq 0\right\},
$$

where $\xi_{n}^{n} \in \partial c_{i}\left(x_{n}\right)(i=1,2, \ldots, k)$ and

$$
Q_{j}^{n}=\left\{y \in H_{2}: q_{j}\left(A x_{n}\right)+\left\langle\eta_{j}^{n}, y-A x_{n}\right\rangle \leq 0\right\},
$$

where $\eta_{j}^{n} \in \partial q_{j}\left(A x_{n}\right)(j=1,2, \ldots, t)$.

(A4) We define the proximity function by

$$
f_{n}(x)=\frac{1}{2} \sum_{j=1}^{t} \lambda_{j}\left\|A x-P_{Q_{j}^{n}}(A x)\right\|^{2},
$$

where $\lambda_{j}>0 \forall 1 \leq j \leq t$. Then the gradient of $f_{n}(x)$ is given by

$$
\nabla f_{n}(x)=\sum_{j=1}^{t} \lambda_{j} A^{*}\left(I-P_{Q}^{n}\right)(A x) .
$$

(A5) The control sequences $\left\{\alpha_{n}\right\},\left\{w_{i}\right\},\left\{\gamma_{n, m}\right\}$ and $\left\{\rho_{n}\right\}$ are chosen such that

$$
\begin{array}{ll}
- & \left\{\alpha_{n}\right\} \subset(0,1), \lim _{n \rightarrow+\infty} \alpha_{n}=0, \sum_{n=1}^{+\infty} \alpha_{n}=+\infty ; \\
- & \left\{\gamma_{n, m}\right\} \subset(0,1), \liminf _{n \rightarrow+\infty} \gamma_{n, 0} \gamma_{n, m}>0, \sum_{m=0}^{+\infty} \gamma_{n, m}=1 ; \\
\text { - } & \left\{w_{i}\right\} \subseteq[0,1] \text { with } \sum_{i=1}^{+\infty} w_{i}=1 ; \\
\text { - } & \left\{\rho_{n}\right\} \subset(0,4) \text { and } \liminf _{n \rightarrow+\infty} \rho_{n}\left(4-\rho_{n}\right)>0 .
\end{array}
$$

We now present our algorithm as follows:

First we show that the sequence $\left\{x_{n}\right\}$ generated by Algorithm 1 is bounded.

Lemma 7. Suppose the solution set $\Gamma=\left\{\Omega \cap \bigcap_{m=1}^{\infty} F\left(S_{m}\right)\right\} \neq \varnothing$ and $\left\{x_{n}\right\}$ is the sequence generated by Algorithm 1. Then $\left\{x_{n}\right\}$ is bounded. 


\section{Algorithm 1: GVA}

Step 0: Select the initial point $x_{1} \in H$ and the sequences $\left\{\alpha_{n}\right\},\left\{w_{i}\right\},\left\{\gamma_{n, m}\right\},\left\{\rho_{n}\right\}$ such that Assumption (A5) is satisfied. Set $n=1$.

Step 1: Given the $n$th iterate (i.e., $x_{n}, n \geq 0$ ), if $\nabla f_{n}\left(x_{n}\right)=0$, STOP. Otherwise, compute

$$
y_{n}=\sum_{i=1}^{k} \omega_{i} P_{C_{i}^{n}}\left(x_{n}-\tau_{n} \nabla f_{n}\left(x_{n}\right)\right),
$$

where the stepsize $\tau_{n}$ is defined by

$$
\tau_{n}=\frac{\rho_{n} f_{n}\left(x_{n}\right)}{\left\|\nabla f_{n}\left(x_{n}\right)\right\|^{2}}
$$

Step 2: Compute

$$
x_{n+1}=\alpha_{n} g_{n}\left(x_{n}\right)+\left(1-\alpha_{n}\right)\left(\gamma_{n, 0} y_{n}+\sum_{m=1}^{\infty} \gamma_{n, j} S_{m, \beta} y_{n}\right) .
$$

Step 3: Set $n \leftarrow n+1$ and return to Step 1 .

Proof. Let $x^{*} \in \Gamma$ and $w_{n}=\gamma_{n, 0} y_{n}+\sum_{m=1}^{\infty} \gamma_{n, m} S_{m, \beta} y_{n}$. By applying the nonexpansivity property of the projection mapping and Lemma 4 , we have

$$
\begin{aligned}
\left\|y_{n}-x^{*}\right\|^{2} & =\left\|\sum_{i=1}^{k} \omega_{i} P_{C_{i}^{n}}\left(x_{n}-\tau_{n} \nabla f_{n}\left(x_{n}\right)\right)-x^{*}\right\|^{2} \\
& \leq\left\|x_{n}-\tau_{n} \nabla f_{n}\left(x_{n}\right)-x^{*}\right\|^{2} \\
& =\left\|x_{n}-x^{*}\right\|^{2}-2 \tau_{n}\left\langle\nabla f_{n}\left(x_{n}\right), x_{n}-x^{*}\right\rangle+\left\|\tau_{n} \nabla f_{n}\left(x_{n}\right)\right\|^{2} .
\end{aligned}
$$

Also from Lemma 2, we obtain

$$
\begin{aligned}
\left\langle\nabla f_{n}\left(x_{n}\right), x_{n}-x^{*}\right\rangle & =\left\langle\sum_{j=1}^{t} \lambda_{j} A^{*}\left(I-P_{Q_{j}^{n}}\right) A x_{n}, x_{n}-x^{*}\right\rangle \\
& =\sum_{j=1}^{t} \lambda_{j}\left\langle\left(I-P_{Q_{j}^{n}}\right) A x_{n}, A x_{n}-P_{Q_{j}^{n}}\left(A x_{n}\right)\right\rangle+\sum_{j=1}^{t} \lambda_{j}\left\langle\left(I-P_{Q_{j}^{n}}\right) A x_{n}, P_{Q_{j}^{n}}\left(A x_{n}\right)-A x^{*}\right\rangle \\
& \geq \sum_{j=1}^{t} \lambda_{j}\left\|A x_{n}-P_{Q_{j}^{n}}\left(A x_{n}\right)\right\|^{2} \\
& =2 f_{n}\left(x_{n}\right) .
\end{aligned}
$$

On substituting (9) into (8), we have

$$
\begin{aligned}
\left\|y_{n}-x^{*}\right\|^{2} & \leq\left\|x_{n}-x^{*}\right\|^{2}-4 \tau_{n} f_{n}\left(x_{n}\right)+\left\|\tau_{n} \nabla f_{n}\left(x_{n}\right)\right\|^{2} \\
& =\left\|x_{n}-x^{*}\right\|^{2}-\rho_{n}\left(4-\rho_{n}\right) \frac{f_{n}^{2}\left(x_{n}\right)}{\left\|\nabla f_{n}\left(x_{n}\right)\right\|^{2}} \\
& \leq\left\|x_{n}-x^{*}\right\|^{2} .
\end{aligned}
$$


More so from Lemma 1, we get

$$
\begin{aligned}
\left\|w_{n}-x^{*}\right\| & =\left\|\gamma_{n, 0} y_{n}+\sum_{m=1}^{\infty} \gamma_{n, m} S_{m, \beta} y_{n}-x^{*}\right\| \\
& \leq \gamma_{n, 0}\left\|y_{n}-x^{*}\right\|+\sum_{m=1}^{\infty} \gamma_{n, m}\left\|S_{m, \beta} y_{n}-x^{*}\right\| \\
& \leq \gamma_{n, 0}\left\|y_{n}-x^{*}\right\|+\sum_{m=1}^{\infty} \gamma_{n, m}\left\|y_{n}-x^{*}\right\| \\
& =\left\|y_{n}-x^{*}\right\| .
\end{aligned}
$$

Therefore from (10) and (11), we have

$$
\begin{aligned}
\left\|x_{n+1}-x^{*}\right\| & =\left\|\alpha_{n} g_{n}\left(x_{n}\right)+\left(1-\alpha_{n}\right) w_{n}-x^{*}\right\| \\
& \leq \alpha_{n}\left\|g_{n}\left(x_{n}\right)-x^{*}\right\|+\left(1-\alpha_{n}\right)\left\|w_{n}-x^{*}\right\| \\
& \leq \alpha_{n} \sigma_{n}\left\|x_{n}-x^{*}\right\|+\left(1-\alpha_{n}\right)\left\|x_{n}-x^{*}\right\|+\alpha_{n}\left\|g_{n}\left(x^{*}\right)-x^{*}\right\| \\
& \leq\left(1-\alpha_{n}\left(1-\sigma_{n}\right)\right)\left\|x_{n}-x^{*}\right\|+\alpha_{n}\left(1-\sigma_{n}\right) \frac{\left\|g_{n}\left(x^{*}\right)-x^{*}\right\|}{1-\sigma_{n}} \\
& \vdots \\
& \leq \max \left\{\left\|x_{n}-x^{*}\right\|, \frac{\left\|g_{n}\left(x^{*}\right)-x^{*}\right\|}{1-\sigma_{n}}\right\} .
\end{aligned}
$$

Since $\left\{g_{n}\right\}$ is uniformly convergent on $D$, it follows that $\left\{g_{n}\left(x^{*}\right)\right\}$ is bounded. Thus, there exists a positive constant $M$, such that $\left\|g_{n}\left(x^{*}\right)-x^{*}\right\| \leq M$. By induction, we obtain

$$
\left\|x_{n}-x^{*}\right\| \leq \max \left\{\left\|x_{1}-x^{*}\right\|, \frac{M}{1-\sigma_{\mu}}\right\} \text {. }
$$

Hence, $\left\{x_{n}\right\}$ is bounded. Consequently $\left\{S_{m, \beta} x_{n}\right\},\left\{g_{n}\left(x_{n}\right)\right\},\left\{y_{n}\right\}$ and $\left\{w_{n}\right\}$ are all bounded.

We now give our main convergence theorem.

Theorem 1. Suppose that $\Gamma=\left\{\Omega \cap \bigcap_{m=1}^{\infty} F\left(S_{m}\right)\right\} \neq \varnothing$ and Assumptions (A1)-(A5) hold. Then, the sequence $\left\{x_{n}\right\}$ generated by Algorithm 1 converges strongly to point $z \in P_{\Gamma}$ which is a unique solution of the variational inequality

$$
\left\langle g(z)-z, x^{*}-z\right\rangle \leq 0, \forall x^{*} \in P_{\Gamma} .
$$


Proof. From Lemma 1 (c), Lemma 3 and (10), we have

$$
\begin{aligned}
\left\|w_{n}-x^{*}\right\|^{2} & =\left\|\gamma_{n, 0} y_{n}+\sum_{m=1}^{\infty} \gamma_{n, m} S_{m, \beta} y_{n}-x^{*}\right\|^{2} \\
& =\gamma_{n, 0}\left\|y_{n}-x^{*}\right\|^{2}+\sum_{m=1}^{\infty} \gamma_{n, m}\left\|S_{m, \beta} y_{n}-x^{*}\right\|^{2}-\sum_{m=1}^{\infty} \gamma_{n, 0} \gamma_{n, m}\left\|y_{n}-S_{m, \beta} y_{n}\right\| \\
& -\sum_{1 \leq m<r<\infty} \gamma_{n, m} \gamma_{n, r}\left\|S_{m, \beta}^{m}-S_{m, \beta}^{r}\right\|^{2} \\
& \leq \gamma_{n, 0}\left\|y_{n}-x^{*}\right\|^{2}+\sum_{m=1}^{\infty} \gamma_{n, m}\left\|y_{n}-x^{*}\right\|^{2}-\sum_{m=1}^{\infty} \gamma_{n, 0} \gamma_{n, m}\left\|y_{n}-S_{m, \beta} y_{n}\right\| \\
& =\left\|y_{n}-x^{*}\right\|^{2}-\sum_{m=1}^{\infty} \gamma_{n, 0} \gamma_{n, m}\left\|y_{n}-S_{m, \beta} y_{n}\right\| \\
& \leq\left\|x_{n}-x^{*}\right\|^{2}-\rho_{n}\left(4-\rho_{n}\right) \frac{f_{n}^{2}\left(x_{n}\right)}{\left\|\nabla f_{n}\left(x_{n}\right)\right\|^{2}}-\sum_{m=1}^{\infty} \gamma_{n, 0} \gamma_{n, m}\left\|y_{n}-S_{m, \beta} y_{n}\right\| . \quad(12)
\end{aligned}
$$

Now, from (10) and (12), we have that

$$
\begin{aligned}
\left\|x_{n+1}-x^{*}\right\|^{2} & =\left\|\alpha_{n} g_{n}\left(x_{n}\right)+\left(1-\alpha_{n}\right) w_{n}-x^{*}\right\|^{2} \\
& \leq\left(1-\alpha_{n}\right)^{2}\left\|w_{n}-x^{*}\right\|^{2}+2 \alpha_{n}\left\langle x_{n+1}-x^{*}, g_{n}\left(x_{n}\right)-x^{*}\right\rangle \\
& \leq\left(1-\alpha_{n}\right)^{2}\left\|x_{n}-x^{*}\right\|^{2}-\left(1-\alpha_{n}\right) \rho_{n}\left(4-\rho_{n}\right) \frac{f_{n}^{2}\left(x_{n}\right)}{\left\|\nabla f_{n}\left(x_{n}\right)\right\|} \\
& -\left(1-\alpha_{n}\right) \sum_{m=1}^{\infty} \gamma_{n, 0} \gamma_{n, m}\left\|y_{n}-S_{m, \beta} y_{n}\right\|+2 \alpha_{n}\left\langle x_{n+1}-x^{*}, g_{n}\left(x_{n}\right)-x^{*}\right\rangle \\
& =\left(1-\alpha_{n}\right)^{2}\left\|x_{n}-x^{*}\right\|^{2}+\alpha_{n}\left(2\left\langle x_{n+1}-x^{*}, g_{n}\left(x_{n}\right)-x^{*}\right\rangle\right) .
\end{aligned}
$$

Putting $d_{n}=2\left\langle x_{n+1}-x^{*}, g_{n}\left(x_{n}\right)-x^{*}\right\rangle$, in view of Lemma 5, we need to prove that $\lim \sup _{k \rightarrow \infty} d_{n_{k}} \leq 0$ for every $\left\{\left\|x_{n_{k}}-x^{*}\right\|\right\}$ of $\left\{\left\|x_{n}-x^{*}\right\|\right\}$ satisfying the condition

$$
\liminf _{k \rightarrow+\infty}\left\{\left\|x_{n_{k+1}}-x^{*}\right\|-\left\|x_{n_{k}}-x^{*}\right\|\right\} \geq 0 .
$$

To show this, suppose that $\left\{\left\|x_{n_{k}}-x^{*}\right\|\right\}$ is a subsequence of $\left\{\left\|x_{n}-x^{*}\right\|\right\}$ such that (14) holds. Then

$$
\begin{aligned}
& \liminf _{k \rightarrow+\infty}\left(\left\|x_{n_{k+1}}-x^{*}\right\|^{2}-\left\|x_{n_{k}}-x\right\|^{2}\right) \\
& \quad=\liminf _{k \rightarrow \infty}\left(\left(\left\|x_{n_{k+1}}-x^{*}\right\|^{2}-\left\|x_{n_{k}}-x^{*}\right\|^{2}\right)\left(\left\|x_{n_{k+1}}-x^{*}\right\|+\left\|x_{n_{k}}-x^{*}\right\|\right)\right) \geq 0 .
\end{aligned}
$$

Now, using (12), we have that

$$
\begin{aligned}
\limsup _{k \rightarrow+\infty}\left(\left(1-\alpha_{n_{k}}\right) \sum_{m=1}^{+\infty} \gamma_{n_{k}, 0} \gamma_{n_{k}, m}\left\|y_{n_{k}}-S_{m, \beta} y_{n_{k}}\right\|\right) & \leq \limsup _{k \rightarrow+\infty}\left(\left(1-\alpha_{n_{k}}\right)\left\|x_{n_{k}}-x^{*}\right\|^{2}-\left\|x_{n_{k}+1}-x^{*}\right\|^{2}\right. \\
& \left.+2 \alpha_{n_{k}}\left\langle x_{n_{k+1}}-x^{*}, g_{n_{k}}\left(x_{n_{k}}\right)-x^{*}\right\rangle\right) \\
& \leq \limsup _{k \rightarrow+\infty}\left(\left\|x_{n_{k}}-x^{*}\right\|^{2}-\left\|x_{n_{k}+1}-x^{*}\right\|^{2}\right) \\
& +\limsup _{k \rightarrow+\infty}\left(2 \alpha_{n_{k}}\left\langle x_{n_{k}+1}-x^{*}, g_{n_{k}}\left(x_{n_{k}}\right)-x^{*}\right\rangle\right) \\
& =-\liminf _{k \rightarrow+\infty}\left(\left\|x_{n_{k}+1}-x^{*}\right\|^{2}-\left\|x_{n_{k}}-x^{*}\right\|^{2}\right) \leq 0 .
\end{aligned}
$$


Hence

$$
\lim _{k \rightarrow+\infty}\left\|y_{n_{k}}-S_{m, \beta} y_{n_{k}}\right\|=0 .
$$

Please note that

$$
\begin{aligned}
\left\|S_{m, \beta} y_{n}-y_{n}\right\| & =\left\|\beta y_{n}+(1-\beta) S_{m} y_{n}-y_{n}\right\| \\
& =(1-\beta)\left\|S_{m} y_{n}-y_{n}\right\| .
\end{aligned}
$$

Then it follows that

$$
\left\|S_{m} y_{n}-y_{n}\right\|=\frac{1}{1-\beta}\left\|S_{m, \beta} y_{n}-y_{n}\right\| \rightarrow 0 .
$$

Furthermore, using (12) and following the same approach as in (15), we also have that

$$
\rho_{n}\left(4-\rho_{n}\right) \frac{f_{n}^{2}\left(x_{n_{k}}\right)}{\left\|\nabla f\left(x_{n_{k}}\right)\right\|^{2}} \leq \rho_{n_{k}}\left(4-\rho_{n_{k}}\right) \frac{f_{n}^{2}\left(x_{n_{k}}\right)}{\left\|\nabla f\left(x_{n_{k}}\right)\right\|^{2}} \rightarrow 0, \text { as } k \rightarrow \infty .
$$

This implies that

$$
\sum_{n=0}^{+\infty} \frac{f_{n}^{2}\left(x_{n_{k}}\right)}{\left\|\nabla f\left(x_{n_{k}}\right)\right\|^{2}}<+\infty
$$

Since $\nabla f_{n}$ is Lipschitz continuous and $\left\{x_{n}\right\}$ is bounded, so $\left\{\nabla f_{n}\left(x_{n}\right)\right\}$ is also bounded. Hence from (18), we can conclude that

$$
\lim _{k \rightarrow+\infty} \frac{1}{2} \sum_{j=1}^{t} \lambda_{j}\left\|A x_{n_{k}}-P_{Q_{j}^{n}}\left(A x_{n_{k}}\right)\right\|^{2}=0,
$$

which also implies that

$$
\lim _{k \rightarrow+\infty}\left\|A x_{n_{k}}-P_{Q_{j}^{n}}\left(A x_{n_{k}}\right)\right\|=0, \text { for } j=1,2, \ldots, t .
$$

Since $\partial q_{j}$ is bounded on bounded sets, there exists $\eta$ such that $\left\|\eta_{j}^{n}\right\| \leq \eta \forall j$. Please note that $P_{Q_{j}^{n}} A x_{n} \in Q_{j}^{n}$, thus we get

$$
\begin{aligned}
q\left(A x_{n_{k}}\right) & \leq\left\langle\eta_{j}^{n_{k}}, A x_{n_{k}}-P_{Q_{j}^{n_{k}}} A x_{n_{k}}\right\rangle \\
& \leq\left\|\eta_{j}^{n_{k}}\right\| \cdot\left\|A x_{n_{k}}-P_{Q_{j}^{n_{k}}} A x_{n_{k}}\right\| \\
& \leq \eta\left\|A x_{n_{k}}-P_{Q_{j}^{n_{k}}} A x_{n_{k}}\right\| \rightarrow 0 \text { as } k \rightarrow+\infty .
\end{aligned}
$$

Since $\left\{x_{n}\right\}$ is bounded and $C$ is closed and convex, we can suppose that the subsequence $\left\{x_{n_{k}}\right\}$ of $\left\{x_{n}\right\}$ converges weakly to $\bar{x} \in C$. We now show that $\bar{x} \in \Omega$. By the weakly lower semicontinuity of $q_{j}$ and boundedness of $A$, we have

$$
q_{j}(A \bar{x}) \leq \liminf _{k \rightarrow+\infty} q_{j}\left(A x_{n_{k}}\right) \leq 0 .
$$

Then $A \bar{x} \in Q_{j}, j=1,2, \ldots, t$. This implies that $A \bar{x} \in \bigcap_{j=1}^{t} Q_{j}$. Next we show that $\bar{x} \in \bigcap_{i=1}^{k} C_{i}$. 
Let $u_{n}=x_{n}-\tau_{n} \nabla f_{n}\left(x_{n}\right)$. Since $\left\{u_{n}\right\},\left\{w_{n}\right\}$ and $\left\{y_{n}\right\}$ are bounded, there exist subsequences $\left\{u_{n_{k}}\right\},\left\{w_{n_{k}}\right\}$ and $\left\{y_{n_{k}}\right\}$ which all converges to $\bar{x}$. Using (10), we have that

$$
\left\|u_{n}-x^{*}\right\|^{2} \leq\left\|x_{n}-x^{*}\right\|^{2}-\rho_{n_{k}}\left(4-\rho_{n_{k}}\right) \frac{f_{n}^{2}\left(x_{n_{k}}\right)}{\left\|\nabla f\left(x_{n_{k}}\right)\right\|^{2}} .
$$

By applying Lemma 2 (iii), we have that

$$
\begin{aligned}
\sum_{i=1}^{k} \omega_{i}\left\|P_{C_{i}^{n}}\left(u_{n_{k}}\right)-u_{n_{k}}\right\|^{2} & \leq\left\|u_{n_{k}}-x^{*}\right\|^{2}-\sum_{i=1}^{k} \omega_{i}\left\|P_{C_{i}}\left(u_{n_{k}}\right)-x^{*}\right\|^{2} \\
& \leq\left\|x_{n_{k}}-x^{*}\right\|^{2}-\left\|y_{n_{k}}-x^{*}\right\|^{2} \\
& \leq\left\|x_{n_{k}}-x^{*}\right\|^{2}-\left\|w_{n_{k}}-x^{*}\right\|^{2} \\
& =\left\|x_{n_{k}}-x^{*}\right\|^{2}-\left\|x_{n_{k}+1}-x^{*}\right\|^{2}+\left\|x_{n_{k}+1}-x^{*}\right\|^{2}-\left\|w_{n_{k}}-x^{*}\right\|^{2} \\
& \leq\left\|x_{n_{k}}-x^{*}\right\|^{2}-\left\|x_{n_{k}+1}-x^{*}\right\|^{2}+\alpha_{n_{k}}\left\|g_{n_{k}}\left(x_{n_{k}}\right)-x^{*}\right\|^{2} \\
& +\left(1-\alpha_{n_{k}}\right)\left\|w_{n_{k}}-x^{*}\right\|^{2}-\left\|w_{n_{k}}-x^{*}\right\|^{2} .
\end{aligned}
$$

By taking lim sup as $k \rightarrow+\infty$ on both sides of (22) and following the same argument as in (15), we have that

$$
\lim _{k \rightarrow+\infty}\left\|P_{C_{i}^{n}}\left(u_{n_{k}}\right)-u_{n_{k}}\right\|=0=\lim _{k \rightarrow \infty}\left\|y_{n_{k}}-u_{n_{k}}\right\| .
$$

Also, from the definition of $u_{n_{k}}=x_{n_{k}}-\tau_{n_{k}} \nabla f\left(x_{n_{k}}\right)$, we have from (19) that

$$
\lim _{k \rightarrow+\infty}\left\|u_{n_{k}}-x_{n_{k}}\right\|=0 .
$$

Using (23) and (24), we obtain that

$$
\lim _{k \rightarrow+\infty}\left\|P_{C_{i}^{n}}\left(u_{n_{k}}\right)-x_{n_{k}}\right\|=0 .
$$

Since $\partial c_{i}$ is bounded on bounded sets, there exists $\xi$ such that $\left\|\xi_{i}^{n}\right\| \leq \xi \forall i$. Thus,

$$
\begin{aligned}
c_{i}\left(x_{n_{k}}\right) & \leq\left\langle\xi_{i}^{n_{k}}, x_{n_{k}}-P_{C_{i}}^{n_{k}}\left(x_{n_{k}}\right)\right\rangle \\
& \leq \xi\left(\left\|x_{n_{k}}-u_{n_{k}}\right\|+\| u_{n_{k}}-P_{C_{i} n_{k}}\left(x_{n_{k}}\right)\right) \rightarrow 0 .
\end{aligned}
$$

By the lower semicontinuity of $c_{i}$, we have

$$
c_{i}(\bar{x}) \leq \liminf _{k \rightarrow+\infty} c_{i}\left(x_{n_{k}}\right) \leq 0 .
$$

Hence $\bar{x} \in C_{i}$ for $i=1,2, \ldots, k$, which implies that $\bar{x} \in \bigcap_{i=1}^{k} C_{i}$. Hence $\bar{x} \in \Omega$. Furthermore, we have from (23) and (24) that

$$
\lim _{k \rightarrow+\infty}\left\|y_{n_{k}}-x_{n_{k}}\right\|=0 .
$$

Then, from the demiclosedness of $k$-strictly pseudo-nonspreading mappings (Lemma 5), (16) and (25), we obtain $\bar{x} \in \bigcap_{m=1}^{\infty} F\left(S_{m}\right)$. Therefore, $\bar{x} \in \Gamma$.

Next is to prove that $\left\{x_{n}\right\}$ converges strongly to $z \in \Gamma$. Also, (16), we have

$$
\lim _{k \rightarrow+\infty}\left\|w_{n_{k}}-y_{n_{k}}\right\|=0 .
$$


More so, from (25) and (26), we obtain

$$
\lim _{k \rightarrow+\infty}\left\|w_{n_{k}}-x_{n_{k}}\right\|=0 .
$$

From (27), we obtain

$$
\left\|x_{n_{k}+1}-x_{n_{k}}\right\| \leq \alpha_{n_{k}}\left\|g_{n_{k}}\left(x_{n_{k}}\right)-x_{n_{k}}\right\|+\left(1-\alpha_{n_{k}}\right)\left\|w_{n_{k}}-x_{n_{k}}\right\| .
$$

Next is to prove that the $\lim \sup _{k \rightarrow+\infty}\left\langle x_{n_{k}+1}-x^{*}, g_{n}\left(x_{n}\right)-x^{*}\right\rangle \leq 0$. Indeed, take a subsequence $\left\{x_{n_{k}}\right\}$ of $\left\{x_{n}\right\}$ such that $x_{n_{k}} \rightarrow z$. Hence, we have

$$
\limsup _{n \rightarrow+\infty}\left\langle g\left(x^{*}\right)-x^{*}, x_{n}-x^{*}\right\rangle=\lim _{k \rightarrow+\infty}\left\langle g\left(x^{*}\right)-x^{*}, x_{n_{k}}-x^{*}\right\rangle .
$$

Since $g_{n}(x)$ is uniformly convergent on $\mathrm{D}$, we have that

$$
\lim _{n \rightarrow+\infty}\left(g_{n}\left(x^{*}\right)-x^{*}\right)=g\left(x^{*}\right)-x^{*} .
$$

Now, from (28) and Lemma 4 (i), we obtain

$$
\lim _{k \rightarrow+\infty}\left\langle g\left(x^{*}\right)-x^{*}, x_{n_{k}}-x^{*}\right\rangle=\left\langle g\left(x^{*}\right)-x^{*}, z-x^{*}\right\rangle \leq 0 .
$$

Using Schwartz's inequality, we have

$$
\limsup _{k \rightarrow+\infty}\left\langle x_{n_{k}+1}-x^{*}, g_{n_{k}}\left(x^{*}\right)-x^{*}\right\rangle \leq \lim _{k \rightarrow+\infty}|| x_{n_{k}+1}-x^{*}\|\| g_{n_{k}}\left(x^{*}\right)-g\left(x^{*}\right) \|+\limsup _{k \rightarrow+\infty}\left\langle x_{n_{k}+1}-x^{*}, g\left(x^{*}\right)-x^{*}\right\rangle .
$$

By the boundedness of $\left\{x_{n}\right\}, g_{n}(x) \rightarrow g(x)$, then by (28) and (29), we have

$$
\limsup _{k \rightarrow+\infty}\left\langle x_{n_{k}+1}-x^{*}, g_{n_{k}}\left(x^{*}\right)-x^{*}\right\rangle \leq 0 .
$$

Applying (30) and Lemma 5 in (13), we obtain that $\left\{x_{n}\right\}$ converges to $z$. This completes the proof.

Next, we give a generalized viscosity approximation method with inertial term which can be regard as a procedure for speeding up the convergence properties of Algorithm 1. In addition to Assumptions (A1)-(A5), we choose a sequence $\left\{\epsilon_{n}\right\} \subset(0, \epsilon)$ with $\epsilon \in[0,1)$ and

$$
\lim _{n \rightarrow \infty} \frac{\epsilon_{n}}{\alpha_{n}}=0
$$

Remark 2. From (31) and Step 1, it is easy to see that $\lim _{n \rightarrow \infty} \frac{\theta_{n}}{\alpha_{n}}\left\|x_{n}-x_{n-1}\right\|=0$. Indeed, we have $\theta_{n}|| x_{n}-x_{n-1}|| \leq \epsilon_{n}$ for each $n \geq 1$, which together with (31) implies that

$$
\liminf _{n \rightarrow+\infty} \frac{\theta_{n}}{\alpha_{n}}\left\|x_{n}-x_{n-1}\right\|=0 \leq \lim _{n \rightarrow+\infty} \frac{\epsilon_{n}}{\alpha_{n}}=0 .
$$

Lemma 8. Suppose the solution set $\Gamma=\left\{\Omega \cap \bigcap_{m=1}^{+\infty} F\left(S_{m}\right)\right\} \neq \varnothing$ and $\left\{x_{n}\right\}$ is the sequence generated by Algorithm 2. Then $\left\{x_{n}\right\}$ is bounded.

Proof. Let $x^{*} \in \Gamma$, using Step 1, we get

$$
\begin{aligned}
\left\|a_{n}-x^{*}\right\| & =\left\|x_{n}+\theta_{n}\left(x_{n}-x_{n-1}\right)-x^{*}\right\| \\
& \leq\left\|x_{n}-x^{*}\right\|+\theta_{n}\left\|x_{n}-x_{n-1}\right\| \\
& =\left\|x_{n}-x^{*}\right\|+\alpha_{n} \cdot \frac{\theta_{n}}{\alpha_{n}}\left\|x_{n}-x_{n_{1}}\right\| .
\end{aligned}
$$




\section{Algorithm 2: IGVA}

Step 0: Select the initial points $x_{0}, x_{1} \in H$ and the sequences $\left\{\alpha_{n}\right\},\left\{w_{i}\right\},\left\{\gamma_{n, m}\right\},\left\{\rho_{n}\right\},\left\{\epsilon_{n}\right\}$ such that Assumption

(A5) and (31) are satisfied. Set $n=1$.

Step 1: Given the $(n-1)$ th and $n$th iterates (i.e., $x_{n-1}$ and $x_{n}, n \geq 1$ ). Choose $\theta_{n}$ such that $0 \leq \theta_{n} \leq \bar{\theta}_{n}$, where

$$
\bar{\theta}_{n}= \begin{cases}\min \left\{\theta, \frac{\epsilon_{n}}{\left\|x_{n}-x_{n-1}\right\|}\right\}, & \text { if } x_{n} \neq x_{n-1}, \\ \theta, & \text { otherwise, }\end{cases}
$$

where $\theta>0$. Compute

$$
a_{n}=x_{n}+\theta_{n}\left(x_{n}-x_{n-1}\right),
$$

and

$$
y_{n}=\sum_{i=1}^{k} \omega_{i} P_{C_{i}^{n}}\left(a_{n}-\tau_{n} \nabla f_{n}\left(a_{n}\right)\right),
$$

where the stepsize $\tau_{n}$ is defined by

$$
\tau_{n}=\frac{\rho_{n} f_{n}\left(a_{n}\right)}{\left\|\nabla f_{n}\left(a_{n}\right)\right\|^{2}}
$$

Step 2: Compute

$$
x_{n+1}=\alpha_{n} g_{n}\left(x_{n}\right)+\left(1-\alpha_{n}\right)\left(\gamma_{n, 0} y_{n}+\sum_{m=1}^{\infty} \gamma_{n, j} S_{m, \beta} y_{n}\right) .
$$

Step 3: Set $n \leftarrow n+1$ and return to Step 1 .

By the condition $\frac{\theta_{n}}{\alpha_{n}}|| x_{n}-x_{n-1}|| \rightarrow 0$, there exists a constant $M_{1}>0$ such that $\frac{\theta_{n}}{\alpha_{n}}|| x_{n}-x_{n-1}|| \rightarrow 0 \leq M_{1}, \forall n \geq 1$. Following similar argument as in the prove of (10) in Algorithm 1, we have

$$
\left\|y_{n}-x^{*}\right\| \leq\left\|a_{n}-x^{*}\right\| .
$$

Also as in (11), putting $w_{n}=\gamma_{n, 0} y_{n}+\sum_{m=1}^{\infty} \gamma_{n, m} S_{m, \beta} y_{n}$, then we get

$$
|| w_{n}-x^{*}|| \leq \| y_{n}-x^{*}||
$$

Then, it follows from (32), (33) and (34) that

$$
\left\|w_{n}-x^{*}\right\|=\left\|x_{n}-x^{*}\right\|+\alpha_{n} M_{1} .
$$

Thus, we have

$$
\begin{aligned}
\left\|x_{n+1}-x^{*}\right\| & =\left\|\alpha_{n} g_{n}\left(x_{n}\right)+\left(1-\alpha_{n}\right) w_{n}-x^{*}\right\| \\
& \leq \alpha_{n}\left\|g_{n}\left(x_{n}\right)-x^{*}\right\|+\left(1-\alpha_{n}\right)\left\|w_{n}-x^{*}\right\| \\
& \leq \alpha_{n} \sigma_{n}\left\|x_{n}-x^{*}\right\|+\left(1-\alpha_{n}\right)\left[\left\|x_{n}-x^{*}\right\|+\alpha_{n} M_{1}\right]+\alpha_{n}\left\|g_{n}\left(x^{*}\right)-x^{*}\right\| \\
& =\left(1-\alpha_{n}\left(1-\sigma_{n}\right)\right)\left\|x_{n}-x^{*}\right\|+\alpha_{n}\left(1-\sigma_{n}\right) \frac{\left\|g_{n}\left(x^{*}\right)-x^{*}\right\|}{1-\sigma_{n}}+\alpha_{n}\left(1-\alpha_{n}\right) M_{1} \\
& =\left(1-\alpha_{n}\left(1-\sigma_{n}\right)\right)\left\|x_{n}-x^{*}\right\|+\alpha_{n}\left(1-\sigma_{n}\right)\left[\frac{\left\|g_{n}\left(x^{*}\right)-x^{*}\right\|}{1-\sigma_{n}}+\left(1-\alpha_{n}\right) \frac{M_{1}}{1-\sigma_{n}}\right] \\
& \vdots \\
& \leq \max \left\{\left\|x_{n}-x^{*}\right\|, \frac{\left\|g_{n}\left(x^{*}\right)-x^{*}\right\|+M_{1}}{1-\sigma_{n}}\right\} .
\end{aligned}
$$


Since $\left\{g_{n}\right\}$ is uniformly convergence on $\mathrm{D}$, it follows that $\left\{g_{n}\left(x^{*}\right)\right\}$ is bounded. Thus, there exists a positive constant $M_{2}$ such that $\left\|g_{n}\left(x^{*}\right)-x^{*}\right\| \leq M_{2}$. Thus, it follows by induction that

$$
\left\|x_{n}-x^{*}\right\| \leq \max \left\{\left\|x_{1}-x^{*}\right\|, \frac{M_{1}+M_{2}}{1-\sigma_{\mu}}\right\} .
$$

Therefore $\left\{x_{n}\right\}$ is bounded.

Theorem 2. Suppose that $\Gamma=\left\{\Omega \cap \cap_{m=1}^{\infty} F\left(S_{m}\right)\right\} \neq \varnothing$ and Assumptions (A1)-(A5) with (31) hold. Then, the sequence $\left\{x_{n}\right\}$ generated by Algorithm 2 converges strongly to point $z \in P_{\Gamma}$ which is a unique solution of the variational inequality

$$
\left\langle g(z)-z, x^{*}-z\right\rangle \leq 0, \forall x^{*} \in P_{\Gamma} .
$$

Proof. Let $x^{*} \in \Gamma$, then we have from Step 1 that

$$
\begin{aligned}
\left\|a_{n}-x^{*}\right\|^{2} & =\left\|x_{n}+\theta_{n}\left(x_{n}-x_{n-1}\right)-x^{*}\right\|^{2} \\
& =\left\|\left(x_{n}-x^{*}\right)+\theta_{n}\left(x_{n}-x_{n-1}\right)\right\|^{2} \\
& =\left\|x_{n}-x^{*}\right\|^{2}+2 \theta_{n}\left\langle x_{n}-x^{*}, x_{n}-x_{n-1}\right\rangle+\theta_{n}^{2}\left\|x_{n}-x_{n_{1}}\right\|^{2} \\
& \leq\left\|x_{n}-x^{*}\right\|^{2}+2 \theta_{n}\left\|x_{n}-x^{*}\right\|\left\|x_{n}-x_{n-1}\right\|+\theta_{n}^{2}\left\|x_{n}-x_{n-1}\right\|^{2} \\
& \leq\left\|x_{n}-x^{*}\right\|^{2}+\theta_{n}\left\|x_{n}-x_{n-1}\right\|\left[2|| x_{n}-x^{*}\left\|+\theta_{n}\right\| x_{n}-x_{n-1} \|\right] \\
& \leq\left\|x_{n}-x^{*}\right\|^{2}+\theta_{n}\left\|x_{n}-x_{n-1}\right\| M_{3},
\end{aligned}
$$

where $M_{3}=\sup _{n>1}\left\{2|| x_{n}-x^{*} \|+\theta_{n}|| x_{n}-x_{n-1}||\right\}$.

Similarly as in (12), we get

$$
\begin{aligned}
\left\|w_{n}-x^{*}\right\|^{2} & \leq\left\|a_{n}-x^{*}\right\|^{2} \\
& =\left\|x_{n}-x^{*}\right\|+\theta_{n}\left\|x_{n}-x_{n-1}\right\| M_{3} .
\end{aligned}
$$

Using Step 1, we have that

$$
\left\|a_{n}-x_{n}\right\|=\alpha_{n} \cdot \frac{\theta_{n}}{\alpha_{n}}|| x_{n}-x_{n-1} \| \rightarrow 0, \text { as } n \rightarrow \infty .
$$

Now, from (36), we have that

$$
\begin{aligned}
\left\|x_{n+1}-x^{*}\right\| & =\left\|\alpha_{n} g_{n}\left(x_{n}\right)+\left(1-\alpha_{n}\right) w_{n}-x^{*}\right\|^{2} \\
& =\left(1-\alpha_{n}\right)^{2}\left\|w_{n}-x^{*}\right\|^{2}+2 \alpha_{n}\left\langle x_{n+1}-x^{*}, g_{n}\left(x_{n}\right)-x^{*}\right\rangle \\
& =\left(1-\alpha_{n}\right)^{2}\left\|x_{n}-x^{*}\right\|^{2}+\left(1-\alpha_{n}\right) \theta_{n}\left\|x_{n}-x_{n-1}\right\| M_{3}+2 \alpha_{n}\left\langle x_{n+1}-x^{*}, g_{n}\left(x_{n}\right)-x^{*}\right\rangle \\
& =\left(1-\alpha_{n}\right)^{2}\left\|x_{n}-x^{*}\right\|^{2}+\alpha_{n}\left(1-\alpha_{n}\right) \frac{\theta_{n}}{\alpha_{n}}\left\|x_{n}-x_{n-1}\right\| M_{3}+2 \alpha_{n}\left\langle x_{n+1}-x^{*}, g_{n}\left(x_{n}\right)-x^{*}\right\rangle \\
& \leq\left(1-\alpha_{n}\right)\left\|x_{n}-x^{*}\right\|^{2}+\alpha_{n}\left[\left(1-\alpha_{n}\right) \frac{\theta_{n}}{\alpha_{n}}\left\|x_{n}-x_{n-1}\right\| M_{3}+2\left\langle x_{n+1}-x^{*}, g_{n}\left(x_{n}\right)-x^{*}\right\rangle\right] .
\end{aligned}
$$

Next is to show that the $\lim \sup _{k \rightarrow+\infty}\left\langle x_{n_{k}+1}-x^{*}, g_{n}\left(x_{n}\right)-x^{*}\right\rangle \leq 0$.

Indeed, take a subsequence $\left\{x_{n_{k}}\right\}$ of $\left\{x_{n}\right\}$ such that $x_{n_{k}} \rightarrow z$. Hence, we have

$$
\limsup _{n \rightarrow+\infty}\left\langle g\left(x^{*}\right)-x^{*}, x_{n}-x^{*}\right\rangle=\lim _{k \rightarrow+\infty}\left\langle g\left(x^{*}\right)-x^{*}, x_{n_{k}}-x^{*}\right\rangle .
$$

Since $g_{n}(x)$ is uniformly convergent on $D$, we have that

$$
\lim _{n \rightarrow+\infty}\left(g_{n}\left(x^{*}\right)-x^{*}\right)=g\left(x^{*}\right)-x^{*} .
$$


Now, from (28) and Lemma 4 (i), we obtain

$$
\lim _{k \rightarrow+\infty}\left\langle g\left(x^{*}\right)-x^{*}, x_{n_{k}}-x^{*}\right\rangle=\left\langle g\left(x^{*}\right)-x^{*}, z-x^{*}\right\rangle \leq 0 .
$$

By applying Schwartz's inequality, we get

$$
\limsup _{k \rightarrow+\infty}\left\langle x_{n_{k}+1}-x^{*}, g_{n_{k}}\left(x^{*}\right)-x^{*}\right\rangle \leq \lim _{k \rightarrow+\infty}\left\|x_{n_{k}+1}-x^{*}\right\|\left\|g_{n_{k}}\left(x^{*}\right)-g\left(x^{*}\right)\right\|+\limsup _{k \rightarrow+\infty}\left\langle x_{n_{k}+1}-x^{*}, g\left(x^{*}\right)-x^{*}\right\rangle .
$$

By the boundedness of $\left\{x_{n}\right\}, g_{n}(x) \rightarrow g(x)$, then by (28) and (39), we have

$$
\limsup _{k \rightarrow+\infty}\left\langle x_{n_{k}+1}-x^{*}, g_{n_{k}}\left(x^{*}\right)-x^{*}\right\rangle \leq 0
$$

On substituting (40) in (38), we obtain that $\left\{x_{n}\right\}$ converges strongly to $x^{*}$. This completes the proof.

\section{Numerical Example}

In this section, we give some numerical experiments to illustrate the performance of our algorithms with respect to some other algorithms in the literature. All computation are carried out using Lenovo PC with the following specification: Intel(R)core i7-600, CPU 2.48GHz, RAM 8.0GB, MATLAB version 9.5 (R2019b).

Example 1. We consider the MSSFP where $H_{1}=\mathbb{R}^{N}$ and $H_{2}=\mathbb{R}^{M}, A: \mathbb{R}^{N} \rightarrow \mathbb{R}^{M}$ is given by $A(x)=\mathbb{G}_{M \times N}(x)$, where $\mathbb{G}_{M \times N}$ is a $M \times N$ matrix. The closed convex sets $C_{i}(i \in\{1, \ldots, k\})$ of $\mathbb{R}^{N}$ are given by

$$
C_{i}=\left\{x=\left(x_{1}, \ldots, x_{N}\right)^{T} \in \mathbb{R}^{N}: c_{i}(x) \leq 0\right\}
$$

where $c_{i}(x)=\left\|x-d_{i}\right\|^{2}-p_{i}^{2}$ such that $p_{i}=p$, where is a positive real number and $d_{i}=$ $\left(x_{1, i}, \ldots, x_{N, i}\right)^{T}=(0, \ldots, 0, i-1)^{T} \in \mathbb{R}^{N}$ for each $i=1,2, \ldots, k$. Also, $Q_{j}(j \in\{1, \ldots, t\})$ is defined by

$$
Q_{j}=\left\{y \in \mathbb{R}^{M}: q_{j}(y) \leq 0\right\},
$$

where $q_{j}(y)=\frac{1}{2} y^{T} B_{j} y+b_{j}^{T} y+c_{j}, j=1,2, \ldots, k, B_{j}$ is a Hessian matrix, $b_{j}$ and $c_{j}$ are vectors generated randomly. For each $i \in\{1, \ldots, k\}$ and $j \in\{1, \ldots, t\}$ the subdifferentials are given by

$$
\partial c_{i}\left(x_{n}\right)= \begin{cases}\left\{\frac{x_{n}-d_{i}}{\left\|x_{n}-d_{i}\right\|}\right\} & \text { if } x_{n}-d_{i} \neq 0, \\ \left\{a_{i} \in \mathbb{R}^{N}:\left\|a_{i}\right\| \leq 1\right\} & \text { otherwise, }\end{cases}
$$

and $\partial q_{j}\left(A x_{n}\right)=\left\{\left(b_{1, j}, \ldots, b_{M, j}\right)^{T}\right\}$. Please note that the projection

$$
P_{C_{i}^{n}}\left(x_{n}\right)=\operatorname{argmin}\left\{\left\|x-x_{n}\right\|: x \in C_{i}^{n}\right\},
$$

where $C_{i}^{n}=\left\{x \in H_{1}: c_{i}\left(x_{n}\right) \leq\left\langle\xi_{i}^{n}, x_{n}-x\right\rangle\right\}$ which is equivalent to the following quadratic programming problem

$$
\begin{cases}\text { minimize } & \frac{1}{2} x^{T} \overline{\mathcal{H}} x+\overline{\mathcal{B}}_{n}^{T} x+\bar{c}, \\ \text { subject to } & \overline{\mathcal{D}}_{i, n}(x) \leq \overline{\mathcal{F}}_{i},\end{cases}
$$

where $\overline{\mathcal{H}}=2 I_{M \times N}, \overline{\mathcal{B}}_{n}=-2 x_{n}, \bar{c}=\left\|x_{n}\right\|^{2}, \overline{\mathcal{D}}_{i, n}=\bar{\xi}_{i}^{n}=\left[\bar{\xi}_{i, 1}, \ldots, \bar{\xi}_{i, N}^{n}\right], \overline{\mathcal{F}}_{i}=p_{i}^{2}-\| x_{n}-$ $d_{i} \|^{2}+\left\langle\bar{\xi}_{i}^{n}, x_{n}\right\rangle$. The Problem (41) as well as the projection onto $Q_{j}^{n}$ can effectively be solved using Optimization Toolbox solver 'quadprog' in MATLAB. We defined the mapping $S_{m}: \mathbb{R}^{N} \rightarrow \mathbb{R}^{N}$ by

$$
S_{m} x= \begin{cases}\left(x_{1}, x_{2}, \ldots, x_{i}, \ldots\right) & \text { if } \prod_{i=1}^{\infty} x_{i}<0 \\ \left(-2 x_{1},-2 x_{2}, \ldots,-2 x_{i}, \ldots\right) & \text { if } \prod_{i=1}^{\infty} x_{i} \geq 0 .\end{cases}
$$


It is easy to see that $S_{m}$ is $\frac{1}{3}$-strictly pseudo-nonspreading. For each $n \in \mathbb{N}$ and $m \geq 0$, let $\left\{\gamma_{n, m}\right\}$ be defined by

$$
\gamma_{n, m}= \begin{cases}\frac{1}{b^{m+1}}\left(\frac{n}{n+1}\right), & n \geq m+1, \\ 1-\frac{n}{n+1} \sum_{k=1}^{n} \frac{1}{b^{k}} & n=m, \\ 0 & n<m,\end{cases}
$$

where $b>1$. For simplicity, we consider the case for which $k=t$ and compare the performance of Algorithm 1, Algorithm 2 and Algorithm (42) of Wen et al. [9] using various dimension of $N$. We choose $b=5, g_{n}(x)=\frac{x}{4}, \beta=0.2, \alpha_{n}=\frac{1}{\sqrt{n+1}}, \epsilon_{n}=\frac{1}{n+1}, \theta=0.01, \rho_{n}=\frac{n}{n+1}, w_{i}=\frac{1}{k}$. Similarly, for Algorithm (42) of Wen et al. [9], we take $\rho_{n}=\frac{n}{n+1}$ and $w_{i}=\frac{1}{k}$. The initial points $x_{0}, x_{1}$ and the matrices $\mathbb{G}_{M \times N}$ are generated randomly for the following values of $N$ and $M$ :

Case I: $N=4$ and $M=10$;

Case II: $N=10$ and $M=5$;

Case III: $N=10$ and $M=10$;

Case IV: $N=15$ and $M=20$.

We use $E_{n}=\left\|x_{n+1}-x_{n}\right\|<10^{-4}$ as stopping criterion and plot the graphs of $E_{n}$ against number of iterations. The numerical results are shown in Table 1 and Figure 1.

Table 1. Computation result for Example 1.

\begin{tabular}{|c|c|c|c|c|}
\hline & & Algorithm 1 & Algorithm 2 & Wen et al. alg. \\
\hline \multirow[t]{2}{*}{ Case I } & No of Iter. & 28 & 13 & 48 \\
\hline & CPU time (s) & 0.1731 & 0.2499 & 0.4600 \\
\hline \multirow[t]{2}{*}{ Case II } & No of Iter. & 29 & 14 & 50 \\
\hline & CPU time (s) & 0.1693 & 0.1523 & 0.4719 \\
\hline \multirow[t]{2}{*}{ Case III } & No of Iter. & 30 & 14 & 51 \\
\hline & CPU time (s) & 0.1702 & 0.1971 & 0.4222 \\
\hline \multirow[t]{2}{*}{ Case IV } & No of Iter. & 30 & 14 & 53 \\
\hline & CPU time (s) & 0.1932 & 0.2240 & 0.5702 \\
\hline
\end{tabular}
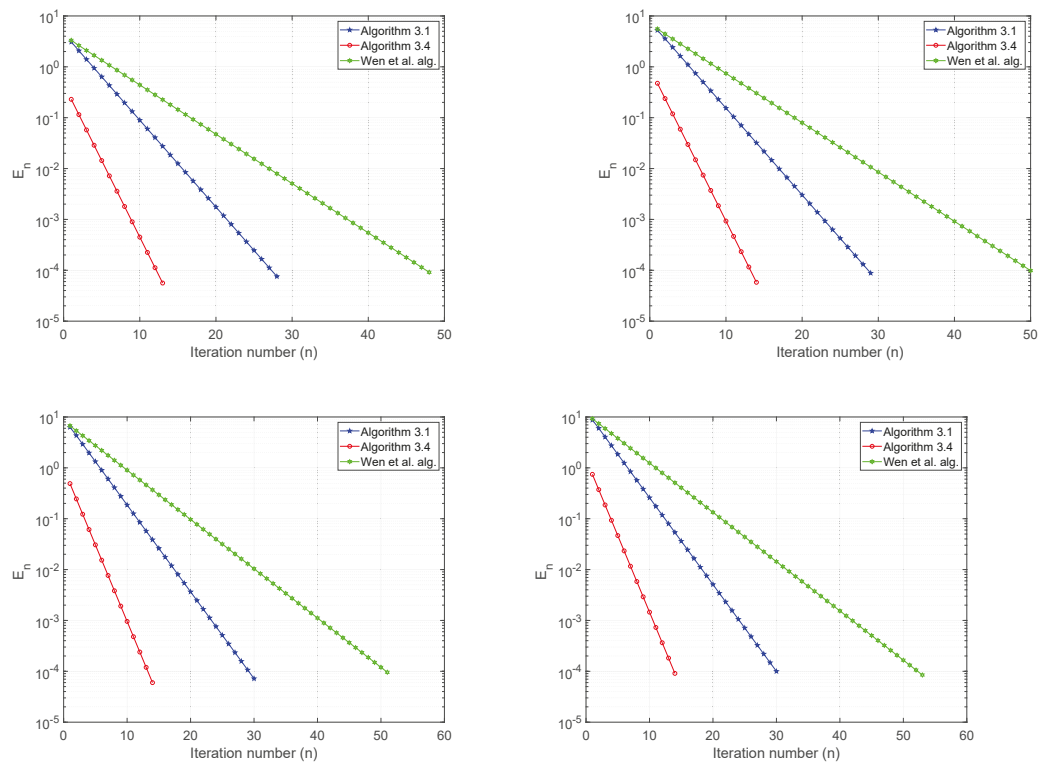

Figure 1. Example 1, Case I-Case IV; Top-Bottom. 
Finally, we present an example in infinite dimensional Hilbert spaces.

Example 2. Let $H_{1}=H_{2}=L^{2}([0,1])$ with norm $\|x\|=\left(\int_{0}^{1}|x(t)|^{2} d t\right)^{1 / 2}$ and the inner product $\langle x, y\rangle=\int_{0}^{1} x(t) y(t) d t$. We defined the nonempty, closed convex sets $C=\left\{x \in L^{2}([0,1])\right.$ : $\left.\left\langle x(t), 3 t^{2}\right\rangle=0\right\}$ and $Q=\left\{y \in L^{2}([0,1]):\langle y, t / 3\rangle \geq-1\right\}$. We defined the linear operator $A: L^{2}([0,1]) \rightarrow L^{2}([0,1])$ by $(A x)(t)=x(t)$. The projection onto $C$ and $Q$ are given by

$$
P_{C}(x(t))=\left\{\begin{array}{lll}
x(t)-\frac{\left\langle x(t), 3 t^{2}\right\rangle}{\left\|3 t^{2}\right\|^{2}} 3 t^{2} & \text { if } & \left\langle x(t), 3 t^{2}\right\rangle \neq 0, \\
x(t), & \text { if } & \left\langle x(t), 3 t^{2}\right\rangle=0,
\end{array}\right.
$$

and

$$
P_{Q}(y(t))=\left\{\begin{array}{lll}
y(t)-\frac{\left\langle y(t), \frac{-t}{3}\right\rangle}{\left\|-\frac{t}{3}\right\|^{2}}\left(\frac{-t}{3}\right), & \text { if } & \left\langle y(t), \frac{-t}{3}\right\rangle<-1, \\
y(t) & \text { if } & \left\langle y(t), \frac{-t}{3}\right\rangle \geq-1 .
\end{array}\right.
$$

We consider the MSSFP where $k=t=1, C_{i}=C, Q_{j}=Q, S_{m}=I$ (identity mapping) and $m=4$. We compare our Algorithm 2 with the CQ-type algorithm (Algorithm 3.1) of Vinh et al. [20]. For Algorithm 2, we take $g_{n}(x)=\frac{x}{8}, \beta=0.5, w_{i}=1, \alpha_{n}=\frac{1}{n+1}, \epsilon_{n}=\frac{1}{(n+1)^{2}}$, and $\gamma_{n, m}=\frac{1}{5}$ for $m=0,1, \ldots, 4$. Also, for Vinh et al. alg, we take $\rho_{n}=\frac{n}{n+1}$ and $\beta_{n}=\frac{1}{n+1}$. We use $E_{n}=\frac{1}{2}\left\|A x_{n}-P_{Q}\left(A x_{n}\right)\right\|^{2}<10^{-4}$ as stopping criterion and test the algorithms for the following initial points:

Case I: $x_{0}=\exp (-2 t), x_{1}=t^{3} \sin (3 t) / 3$,

Case II: $x_{0}=t^{2}+2 t-1, x_{1}=(\cos (2 t)+\sin (3 t)) / 5$,

Case III: $x_{0}=2 t \cos (-3 t), x_{1}=4 \sin (2 t)$,

Case IV: $x_{0}=\exp (2 t) / 2, x_{1}=t^{3}+3 t-1$.

The numerical results are reported in Table 2 and Figure 2.

Table 2. Computation result for Example 2.

\begin{tabular}{cccc}
\hline & & Algorithm 2 & Vinh et al. alg. \\
\hline Case I & No of Iter. & 4 & 9 \\
& CPU time (s) & 0.4563 & 1.3020 \\
\hline Case II & No of Iter. & 5 & 10 \\
& CPU time (s) & 1.5565 & 3.1576 \\
\hline Case III & No of Iter. & 9 & 13 \\
& CPU time (s) & 0.8736 & 2.4094 \\
\hline Case IV & No of Iter. & 8 & 12 \\
& CPU time (s) & 0.7985 & 1.1278 \\
\hline
\end{tabular}



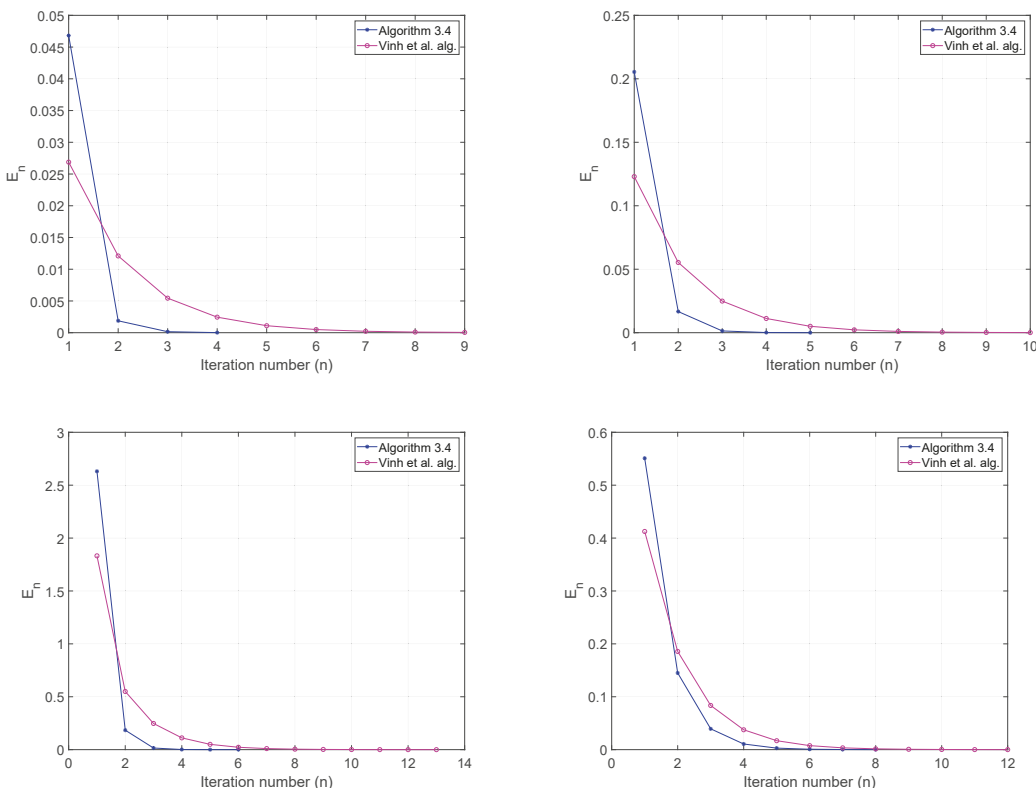

Figure 2. Example 2, Case I-Case IV; Top-Bottom.

\section{Conclusions}

In this paper, we introduce a generalized viscosity approximation method with selfadaptive stepsize for finding common solution of multiple set split feasibility problem and fixed point of a countable family of $k$-strictly pseduononspreading mappings in real Hilbert spaces. We also introduce a generalized viscosity approximation method with inertial and self-adaptive stepsize for solving the underlying problem. We prove strong convergence results for the sequences generated the algorithms under some mild conditions. We also provide some numerical example to show the performance of the proposed methods with respect to some other methods in the literature. These results improve and compliment several other results (e.g., [6-9,20]) in the literature.

Author Contributions: Conceptualization, H.A.A. and L.O.J.; methodology, H.A.A. and L.O.J.; software, H.A.A. and L.O.J.; validation, H.A.A. and L.O.J.; formal analysis, H.A.A. and L.O.J.; writing-original draft preparation, H.A.A. and L.O.J.; writing-review and editing, H.A.A. and L.O.J.; supervision, H.A.A. and L.O.J.; project administration, H.A.A. and L.O.J.; funding acquisition, H.A.A. and L.O.J. All authors have read and agreed to the published version of the manuscript.

Funding: This research is funded by the Mathematical research fund at the Sefako Makgatho Health Sciences University.

Acknowledgments: The authors acknowledge with thanks, the Department of Mathematics and Applied Mathematics at the Sefako Makgatho Health Sciences University for making their facilities available for the research.

Conflicts of Interest: The authors declare no conflicts of interest.

\section{References}

1. Combettes, P.L. The Convex Feasibility Problem in Image Recovery in Hawkes P, Editor Advance. Advances in Imaging and Electron Physics; Academic Press: New York, NY, USA, 1996; Volume 95, pp. 155-270.

2. Censor, Y.; Elfving, T. A multiprojection algorithm using Bregman projection in a product space. Numer. Algor. 1994, 8, 221-239. [CrossRef] 
3. Censor, Y.; Elfving, T.; Kopf, N.; Bortfeld, T. The multiple-sets split feasibility problem and its application for inverse problem. Inverse Probl. 2005, 21, 2071-2084. [CrossRef]

4. Zhang, J.L.; Yang, Q.Z. Self adaptive projection methods for the multiple-sets split feasibility problem. Inverse Probl. 2011, 27, 035009. [CrossRef]

5. Zhang, J.L.; Yang, Q.Z. A simple projection method for solving multiple-sets split feasibility problem. Inverse Probl. Sci. Eng. 2013, 21, 537-546.

6. Lopez, G.; Martin-Marquez, V.; Wang, F.H.; Xu, H.K. Solving the split feasibility problem without prior knowledge of matrix norms. Inverse Probl. 2012, 28, 8, Article ID: 085004. [CrossRef]

7. He, S.; Zhao, Z.; Luo, B. A relaxed self-adaptive CQ algorithm for the multiple-sets split feasibility problem. Optimization 2015, 64, 1907-1918. [CrossRef]

8. Suantai, S.; Pholasa, N.; Cholamjiak, P. Relaxed CQ algorithms involving the inertial technique for multiple-sets split feasibility problem. Rev. R. Acad. Cienc. Exactas Fis Nat. Ser. A Mat. 2019, 113, 1081-1097. [CrossRef]

9. Wen, M.; Peng, J.; Tang, Y. A cyclic and simultaneous iterative method for solving the multiple-sets split feasibility problem. J. Optim. Theory Appl. 2015, 166, 844-860. [CrossRef]

10. Polyak, B.T. Some methods of speeding up the convergence of iterates methods. USSR Comput. Math. Phys. 1964, 4, 1-17. [CrossRef]

11. Alvarez, F.; Attouch, H. An Inertial proximal method for maximal monotone operators via discretization of a nonlinear oscillator with damping. Set-Valued Anal. 2001, 9,3-11. [CrossRef]

12. Abass, H.A.; Aremu, K.O.; Jolaoso, L.O.; Mewomo, O.T. An inertial forward-backward splitting method for approximating solutions of certain optimization problem. J. Nonlinear Funct. Anal. 2020, 2020, 6.

13. Abass, H.A.; Izuchukwu, C.; Mewomo, O.T.; Dong, Q.L. Strong convergence of an inertial forward-backward splitting method for accretive operators in real Banach space. Fixed Point Theory 2020, 21, 397-412. [CrossRef]

14. Jolaoso; Taiwo, L.O.; Alakoya, A.; Mewomo, T.O. A self adaptive inertial subgradient extragradient algorithm for variational inequality and common fixed point of multivalued mappings in Hilbert spaces. Demonstr. Math. 2019, 52, 183-203. [CrossRef]

15. Jolaoso, L.O.; Abass, H.A.; Mewomo, O.T. A viscosity-proximal gradient method with inertial extrapolation for solving certain minimization problems in Hilbert space. Arch. Math. 2019, 55, 167-194. (In English) [CrossRef]

16. Jolaoso, L.O.; Alakoya, T.O.; Taiwo, A.; Mewomo, O.T. An inertial extragradient method via viscoscity approximation approach for solving equilibrium problem in Hilbert spaces. Optimization 2020. [CrossRef]

17. Jolaoso, L.O.; Aphane, M. A self-adaptive inertial subgradient extragradient method for pseudomonotone equilibrium and common fixed point problems. Fixed Point Theory Appl. 2020, 2020, 9. [CrossRef]

18. Fukushima, M. A relaxed projection method for variational inequality. Math. Program 1986, 35, 58-70. [CrossRef]

19. Wang, J.; Hu, Y.; Yu, C.K.W.; Zhuang, Y. A family of projection gradient methods for solving the multiple set split feasibility problems. J. Optim. Theory Appl. 2019, 183, 520-534. [CrossRef]

20. Vinh, N.T.; Cholamjiak, P.; Suantai, S. A new CQ algorithm for solving split feasibility problems in Hilbert spaces. Bull. Malys. Math. Sci. Soc. 2019, 42, 2517-2534. [CrossRef]

21. Abass, H.A.; Ogbuisi, F.U.; Mewomo, O.T. Common solution of split equilibrium problem with no prior knowledge of operator norm. UPB Sci. Bull. Ser. A 2018, 80, 175-190.

22. Abass, H.A.; Okeke, C.C.; Mewomo, O.T. On split equality mixed equilibrium and fixed point problems of generalized $k_{i}$-strictly pseudo-contractive multivalued mappings. Dyn. Contin. Discret. Impuls. syst. Ser. B Appl. Algorithms 2018, 25, 369-395.

23. Duan, P.; He, S. Generalized viscosity approximation methods for nonexpansive mapping. Fixed Point Theory Appl. 2014, 2014, 68. [CrossRef]

24. Moudafi, A. Viscosity approximation methods for fixed points problems. J. Math. Anal. Appl. 2000, 241, 46-55. [CrossRef]

25. Koshaka, F.; Takahashi, W. Existence and approximation of fixed points of firmly nonexpansive-type mappings in Banach spaces. SIAM J. Optim. 2008, 19, 824-835.

26. Browder, F.E.; Petryshyn, W.V. Construction of fixed points of nonlinear mappings in Hilbert space. J. Math. Anal. Appl. 1967, 20, 197-228. [CrossRef]

27. Zhao, T.; Chang, S.S. Weak and strong convergence theorems for strictly pseudo-nonspreading mappings and equilibrium problem in Hilbert spaces. Abstr. Appl. Anal. 2013, 2013, 169206. [CrossRef]

28. Bauschke, H.H.; Borwein, J.M. On projection algorithm for solving convex feasibility problems. SIAM Rev. 1996, $38,367-426$. [CrossRef]

29. Chidume, C.E.; Okpala, M.E. Fixed point iteration for a countable family of multivalued strictly pseudocontractive-type mappings. SpringerPlus 2015, 4, 506. [CrossRef]

30. Bauschke, H.H.; Combettes, P.L. Convex Analysis and Monotone Operator Theory in Hilbert Spaces; Springer: London, UK, 2011.

31. Saejung, S.; Yotkaew, P. Approximation of Zeros of inverse strongly monotone operators in Banach spaces. Nonlinear Anal. Theory Methods Appl. 2012, 75, 742-750. [CrossRef] 
Article

\title{
A Self-Adaptive Shrinking Projection Method with an Inertial Technique for Split Common Null Point Problems in Banach Spaces
}

\author{
Chibueze Christian Okeke ${ }^{1}$, Lateef Olakunle Jolaoso ${ }^{2, *}$ and Regina Nwokoye ${ }^{3}$ \\ 1 Department of Mathematics, Faculty of Science and Engineering, University of Eswatini, Private Bag 4, \\ Kwaluseni M201, Eswatini; ccokeke@uniswa.sz \\ 2 Department of Mathematics and Applied Mathematics, Sefako Makgatho Health Sciences University, \\ P.O. Box 94, Pretoria 0204, South Africa \\ 3 Department of Mathematics, Faculty of Physical Sciences, University of Nigeria Nsukka, Nsukka 410001, \\ Nigeria; regina.nwokoye@unn.edu.ng \\ * Correspondence: lateef.jolaoso@smu.ac.za
}

Received: 27 October 2020; Accepted: 20 November 2020; Published: 2 December 2020

\begin{abstract}
In this paper, we present a new self-adaptive inertial projection method for solving split common null point problems in $p$-uniformly convex and uniformly smooth Banach spaces. The algorithm is designed such that its convergence does not require prior estimate of the norm of the bounded operator and a strong convergence result is proved for the sequence generated by our algorithm under mild conditions. Moreover, we give some applications of our result to split convex minimization and split equilibrium problems in real Banach spaces. This result improves and extends several other results in this direction in the literature.
\end{abstract}

Keywords: split common null point; strong convergence; resolvent; metric resolvent; split minimization problem; split equilibrium problem; Banach space

\section{Introduction}

Let $H_{1}$ and $H_{2}$ be real Hilbert spaces and $C$ and $Q$ be nonempty, closed and convex subsets of $H_{1}$ and $H_{2}$, respectively. We consider the Split Common Null Point Problem (SCNPP) which was introduced by Byrne et al. [1] as follows:

$$
\text { Find } z \in H_{1} \text { such that } z \in A^{-1}(0) \bigcap T^{-1}\left(B^{-1}(0)\right) \text {, }
$$

where $A: H_{1} \rightarrow 2^{H_{1}}$ and $B: H_{2} \rightarrow 2^{H_{2}}$ are maximal monotone operators and $T: H_{1} \rightarrow H_{2}$ is a linear bounded operator. The solution set of SCNPP (1) is denoted by $\Omega$. The SCNPP contains several important optimization problems such as split feasibility problem, split equilibrium problem, split variational inequalities, split convex minimization problem, split common fixed point problems, etc., as special cases (see, e.g., [1-5]). Due to their importance, several researchers have studied and proposed various iterative methods for finding its solutions (see, e.g., [1,4-9]). In particular, Byrne et al. [1] introduced the following iterative scheme for solving SCNPP in real Hilbert spaces:

$$
\left\{\begin{array}{l}
x_{0} \in H_{1}, \lambda>0, \\
x_{n+1}=J_{\lambda}^{A}\left(x_{n}+\lambda T^{*}\left(J_{\lambda} B\right) T x_{n}\right), \quad n \geq 0,
\end{array}\right.
$$


where $J_{\lambda}^{A} x=(I+\lambda A)^{-1} x$, for all $x \in H_{1}$. They also proved that the sequence $\left\{x_{n}\right\}$ generated by (2) converges weakly to a solution of SCNPP provided the step size $\lambda$ satisfies

$$
\lambda \in\left(0, \frac{2}{L}\right),
$$

where $L$ is the spectral radius of $T$. Furthermore, Kazmi and Rizvi [10] proposed a viscosity method which converges strongly to a solution of (1) as follows:

$$
\left\{\begin{array}{l}
x_{0} \in H_{1}, \lambda>0, \\
u_{n}=J_{\lambda}^{A}\left(x_{n}+\lambda T^{*}\left(J_{\lambda}^{B}-I\right) A x_{n}\right), \\
x_{n+1}=\alpha_{n} f\left(x_{n}\right)+\left(1-\alpha_{n}\right) S u_{n}, \quad n \geq 0,
\end{array}\right.
$$

where $\left\{\alpha_{n}\right\} \subset(0,1)$ satisfies some certain conditions and $S: H_{1} \rightarrow H_{1}$ is a nonexpansive mapping. It is important to emphasize that the convergence of (4) is achieved with the aid of condition (3). Other similar results can be found, for instance, in [11,12] (and references therein). However, it is well known that the norm of bounded linear operator is very difficult to find (or at least estimate) (see [13-15]). Hence, it becomes necessary to find iterative methods whose step size selection does not require prior estimate of the norm of the bounded linear operator. Recently, some authors have provided breakthrough results in the framework of real Hilbert spaces (see, e.g., [13-15]).

On the other hand, Takahashi $[8,16]$ extends the study of SCNPP (1) to uniformly convex and smooth Banach spaces as follows: Let $E_{1}$ and $E_{2}$ be uniformly convex and uniformly smooth real Banach spaces with dual $E_{1}^{*}$ and $E_{2}^{*}$, respectively, and $T: E_{1} \rightarrow E_{2}$ be a bounded linear operator. Let $A: E_{1} \rightarrow 2^{E_{1}^{*}}$ and $B: E_{2} \rightarrow 2^{E_{2}^{*}}$ be maximal monotone operators such that $A^{-1}(0) \neq \varnothing$, $B^{-1}(0) \neq \varnothing$ and $Q_{\mu}$ is a metric resolvent operator with respect to $B$ and parameter $\mu>0$. Takahashi and Takahashi [17] introduced the following shrinking projection method for solving SCNPP in uniformly convex and smooth Banach spaces:

$$
\left\{\begin{array}{l}
x_{1} \in C, \mu_{1}>0 \\
z_{n}=x_{n}-J_{\lambda_{n}} J_{E_{1}}^{-1} T^{*} J_{E_{2}}\left(T x_{n}-Q_{\mu_{n}} T x_{n}\right), \\
C_{n+1}=\left\{z \in C_{n}:\left\langle z_{n}-z, J_{E_{1}}\left(x_{n}-z_{n}\right)\right\rangle \geq 0\right\} \\
x_{n+1}=P_{C_{n+1}} x_{1}, \quad \text { for all } n \in \mathbb{N}
\end{array}\right.
$$

where $J_{E_{i}}$ are the normalized duality mapping with respect to $E_{i}$ for $i=1,2$ (defined in the next section). They proved a strong convergence result with the condition that the step size satisfies

$$
0<a \leq \lambda_{n}\|T\|^{2}<b<1 \text { and } 0<c \leq \mu_{n} \quad \text { for all } n \in \mathbb{N} \text {. }
$$

Furthermore, Suantai et al. [18] introduced a new iterative scheme for solving SCNPP in a real Hilbert space $H$ and a real Banach space $E$ as follows:

$$
\left\{\begin{array}{l}
x_{1} \in H \\
y_{n}=J_{\lambda_{n}}^{A}\left(x_{n}+\lambda_{n} T^{*} J_{E}\left(Q_{\mu_{n}}-I\right) T x_{n}\right), \\
x_{n+1}=\alpha_{n} f\left(x_{n}\right)+\beta_{n} x_{n}+\gamma_{n} y_{n}, \quad n \geq 1
\end{array}\right.
$$

where $\left\{\alpha_{n}\right\},\left\{\beta_{n}\right\},\left\{\gamma_{n}\right\} \subset(0,1)$ such that $\alpha_{n}+\beta_{n}+\gamma_{n}=1$ and $f: H \rightarrow H$ is a contraction mapping. They also proved a strong convergence result under the condition that the step size satisfies

$$
0<\lambda_{n}\|T\|^{2}<2
$$


Recently, Takahashi [19] introduced a new hybrid method with generalized resolvent operators for solving the SCNPP in real Banach spaces as follows:

$$
\left\{\begin{array}{l}
z_{n}=J^{-1}\left(J_{E} x_{n}-r_{n} T^{*}\left(J_{F} T x_{n}-J_{F} Q_{\mu_{n}} T x_{n}\right)\right) \\
y_{n}=J_{\lambda_{n}} z_{n} \\
C_{n}=\left\{z \in E: 2\left\langle x_{n}-z, J_{E} x_{n}-J_{E} z_{n}\right\rangle \geq r_{n} \varphi_{F}\left(T x_{n}, Q_{\mu_{n}} T x_{n}\right)\right\} \\
D_{n}=\left\{z \in E:\left\langle y_{n}-z, J_{E} z_{n}-J_{E} y_{n}\right\rangle \geq 0\right\} \\
Q_{n}=\left\{z \in E:\left\langle x_{n}-z, J_{E} x_{1}-J_{E} x_{n}\right\rangle \geq 0\right\} \\
x_{n+1}=\Pi_{C_{n} \cap D_{n} \cap Q_{n}} x_{1}, \text { for all } n \in \mathbb{N} .
\end{array}\right.
$$

He also proved that the sequence generated by Algorithm (7) converges strongly to a solution of SCNPP provided the step sizes satisfy

$$
0<a \leq r_{n} \leq \frac{1}{\|T\|^{2}} \text {, and } 0<b \leq \lambda_{n}, \mu_{n} \text { for all } n \in \mathbb{N} .
$$

It is evident that the above methods and other similar ones (see, e.g., $[6,9,20]$ ) require prior knowledge of the operator norm, which is very difficult to find. Thus, the following natural question arises.

Problem 1. Can we provide a new iterative method for solving SCNPP in real Banach spaces such that the step size does not require prior estimate of the norm of the bounded linear operator?

Let us also mention the inertial extrapolation process which is considered as a means of speeding up the rate of convergence of iterative methods. This technique was first introduced by Polyak [21] as a heavy-ball method of a two-order time dynamical system and has been employed by many authors recently (see, e.g., [22-27]). Moreover, Dong et al. [27] introduced a modified inertial hybrid algorithm for approximating the fixed points of non-expansive mappings in real Hilbert spaces as follows:

$$
\left\{\begin{array}{l}
x_{0}, x_{1} \in C \\
w_{n}=x_{n}+\theta_{n}\left(x_{n}-x_{n-1}\right) \\
z_{n}=\left(1-\beta_{n}\right) w_{n}+\beta_{n} T w_{n} \\
C_{n}=\left\{x \in C:\left\|z_{n}-x\right\|^{2} \leq\left\|x_{n}-x\right\|^{2}\right\} \\
Q_{n}=\left\{x \in C:\left\langle x_{n}-x, x_{0}-x_{n}\right\rangle \geq 0\right\} \\
x_{n+1}=P_{C_{n} \cap Q_{n}} x_{0}
\end{array}\right.
$$

where $\left\{\theta_{n}\right\} \subset\left[a_{1}, a_{2}\right], a_{1} \in(-\infty, 0], a_{2} \in[0,+\infty),\left\{\beta_{n}\right\} \subset(0,1)$ are suitable parameters.

More recently, Cholamjiak et al. [28] introduced an inertial forward-backward algorithm for finding the zeros of sum of two monotone operators in Hilbert spaces as follows:

$$
\left\{\begin{array}{l}
x_{0}, x_{1} \in H, r_{n}>0 \\
y_{n}=x_{n}+\theta_{n}\left(x_{n}-x_{n-1}\right) \\
z_{n}=\alpha_{n} y_{n}+\left(1-\alpha_{n}\right) T y_{n} \\
v_{n}=\beta_{n} z_{n}+\left(1-\beta_{n}\right) J_{r_{n}}^{B}\left(I-r_{n} A\right) z_{n} \\
C_{n+1}=\left\{v \in C_{n}:\left\|v_{n}-v\right\|^{2} \leq\left\|x_{n}-v\right\|^{2}+K_{n}\right\} \\
x_{n+1}=P_{C_{n+1}} x_{1}, n \geq 1
\end{array}\right.
$$


where $K_{n}=2 \theta_{n}^{2}\left\|x_{n}-x_{n-1}\right\|-2 \theta_{n}\left\langle x_{n}-z, x_{n-1}-x_{n}\right\rangle, J_{r_{n}}^{B}=\left(I+r_{n} B\right)^{-1},\left\{\theta_{n}\right\} \subset[0, \theta]$ for some $\theta \in[0,1)$ and $\left\{\alpha_{n}\right\},\left\{\beta_{n}\right\}$ are sequences in $[0,1]$. The authors proved that the sequence $\left\{x_{n}\right\}$ generated by (9) converges strongly to a solution $x \in(A+B)^{-1}(0)$ under some mild conditions.

Motivated by the above results, in this paper, we aim to provide an affirmative answer to Problem 1. We introduce a new inertial shrinking projection method for solving SCNPP in $p$-uniformly convex and uniformly smooth real Banach spaces. The algorithm is designed such that its step size is determined by a self-adaptive technique and its convergence does not require prior knowledge of the norm of the bounded operator. We also prove a strong convergence result and provide some applications of our main theorem to solving other nonlinear optimization problems. This result improves and extends the results in $[6,8,9,11,12,16,19,20]$ and many other recent results in the literature.

\section{Preliminaries}

Let $E$ be a real Banach space with dual $E^{*}$ and norm $\|\cdot\|$. We denote the duality pairing between $f \in E$ and $g^{*} \in E^{*}$ as $\left\langle f, g^{*}\right\rangle$. The weak and strong convergence of $\left\{x_{n}\right\} \subset E$ to $a \in E$ are denoted by $x_{n} \rightarrow a$ and $x_{n} \rightarrow a$, respectively, $\forall$ by "for all" and $\Leftrightarrow$ by "if and only if". The function $\delta_{E}:[0,2] \rightarrow$ $[0,1]$ defined by

$$
\delta_{E}(\alpha)=\inf \left\{1-\frac{\|f+g\|}{2}:\|f\|=1=\|g\|,\|f-g\| \geq \alpha\right\}
$$

is called the modulus of convexity of $E$. The Banach space $E$ is said to be uniformly convex if $\delta_{E}(\alpha)>0$. If there exists a constant $C_{p}>0$ such that $\delta_{E}(\alpha) \geq C_{p} \alpha^{p}$ for any $\alpha \in(0,2]$, then we say $E$ is $p$-uniformly convex. In addition, the function $\rho_{E}(\beta):[0, \infty) \rightarrow[0,+\infty)$ defined by

$$
\rho_{E}(\beta)=\left\{\frac{\|f+\beta g\|+\|f-\beta g\|}{2}-1:\|f\|=\|g\|=1\right\}
$$

is called the modulus of smoothness of $E$. The Banach space $E$ is said to be uniformly smooth if $\lim _{\beta \rightarrow+\infty} \frac{\rho_{E}(\beta)}{\beta}=0$. If there exists a constant $D_{q}>0$ such that $\rho_{E}(\beta) \leq D_{q} \beta^{q}$ for any $\beta>0$, then $E$ is called $q$-uniformly smooth Banach space. Let $1<q \leq 2 \leq p$ with $\frac{1}{p}+\frac{1}{q}=1$. We Remark that a Banach space $E$ is $p$-uniformly convex if and only if its dual $E^{*}$ is $q$-uniformly smooth. Examples of $q$-uniformly smooth Banach spaces include Hilbert spaces, $L_{q}$ (or $l_{p}$ ) spaces, $1<p<\infty$ and the Sobolev spaces, $W_{m}^{p}, 1<p<\infty$ (see [29]). Moreover, the Hilbert spaces are uniformly smooth while

$$
L_{p}\left(\text { or } l_{p}\right) \text { or } W_{m}^{p} \text { is }\left\{\begin{array}{l}
p-\text { uniformly smooth if } 1<p \leq 2 \\
2-\text { uniformly smooth if } p \geq 2 .
\end{array}\right.
$$

Let $\varphi: \mathbb{R}^{+} \rightarrow \mathbb{R}^{+}$be a continuous strictly increasing function. $\varphi$ is called a gauge function if

$$
\varphi(0)=0, \quad \lim _{t \rightarrow \infty} \varphi(t)=+\infty
$$

The duality mapping with respect to $\varphi$, i.e., $J_{\varphi}: E \rightarrow E^{*}$ is defined by

$$
J_{\varphi}(x)=\left\{j \in E^{*}:\langle x, j\rangle=\|x\|\|j\|_{*},\|j\|_{*}=\varphi(\|x\|)\right\}, \quad x \in E .
$$

When $\varphi(t)=t$, then we call $J_{\varphi}=J$ a normalized duality mapping. In addition, if $\varphi(t)=t^{p-1}$ where $p>1$, then, $J_{\varphi}=J_{p}$ is called a generalized duality mapping defined by

$$
J_{p}(u)=\left\{f \in E^{*}:\langle u, f\rangle=\|u\|\|f\|_{*},\|f\|_{*}=\|u\|^{p-1}\right\}, \quad x \in E .
$$

In the sequel, $C$ is a nonempty closed convex subset of $E$ and $F(T)=\{x \in C: T x=x\}$ is the set of fixed point of $T: C \rightarrow C$. 
Definition 1. Ref. [30] Let $E$ be a Banach space, $J_{\varphi}: E \rightarrow E^{*}$ a duality mapping with gauge function $\varphi$, and $C$ a nonempty subset of E. A mapping $T: C \rightarrow E$ is said to be

(i) $\varphi$-firmly non-expansive if

$$
\left\langle T u-T v, J_{\varphi}(T u)-J_{\varphi}(T v)\right\rangle \leq\left\langle T u-T v, J_{\varphi}(u)-J_{\varphi}(v)\right\rangle
$$

for all $u, v \in C$.

(ii) $\varphi$-firmly quasi-non-expansive if $F(T) \neq \varnothing$ and

$$
\left\langle T u-z, J_{\varphi}(u)-J_{\varphi}(T u)\right\rangle \geq 0
$$

for all $u$ in $C$ and $z$ in $F(T)$.

Definition 2. Given a Gâteaux differentiable and convex function $f: E \rightarrow \mathbb{R}$, the function

$$
\Delta_{f}(u, v):=f(v)-f(u)-\left\langle f^{\prime}(u), v-u\right\rangle, \text { for all } u, v \in E
$$

is called the Bregman distance of $u$ to $v$ with respect to the function $f$.

Moreover, since $J_{E}^{p}$ is the derivative of the function $f_{p}(u)=\frac{1}{p}\|u\|^{p}$, in that case, the Bregman distance with respect to $f_{p}$ becomes

$$
\begin{aligned}
\Delta_{p}(u, v) & =\frac{1}{q}\|u\|^{p}-\left\langle J_{E}^{p} u, v\right\rangle+\frac{1}{p}\|v\|^{p} \\
& =\frac{1}{p}\left(\|v\|^{p}-\|u\|^{p}\right)+\left\langle J_{E}^{p} u, u-v\right\rangle \\
& =\frac{1}{q}\left(\|u\|^{p}-\|v\|^{p}\right)-\left\langle J_{E}^{p} u-J_{E}^{p} v, v\right\rangle .
\end{aligned}
$$

Remark 1. It follows from the Definition of $\Delta_{p}$ that

$$
\Delta_{p}(u, v)=\Delta_{p}(u, z)+\Delta_{p}(z, v)+\left\langle z-v, J_{E}^{p} u-J_{E}^{p} z\right\rangle, \text { for all } u, v, z \in E,
$$

and

$$
\Delta_{p}(u, v)+\Delta_{p}(v, u)=\left\langle u-v, J_{E}^{p} u-J_{E}^{p} v\right\rangle, \text { for all } u, v, z \in E .
$$

Although the Bregman is not symmetrical, it however has the following relationship with $\|\cdot\|$ distance:

$$
\alpha\|u-v\|^{p} \leq \Delta_{p}(u, v) \leq\left\langle u-v, J_{E}^{p} u-J_{E}^{p} v\right\rangle, \text { for all } u, v \in E, \alpha>0 .
$$

This indicates that Bregman distance is non-negative.

Definition 3. The Bregman projection mapping $\Pi_{C}: E \rightarrow C$ is defined by

$$
\Pi_{C} u=\arg \min _{v \in C} \Delta_{p}(u, v), \text { for all } u \in E .
$$

The Bregman projection can also be characterized by the following inequality

$$
\left\langle J_{E}^{p} u-J_{E}^{p} \Pi_{C} u, z-\Pi_{C} u\right\rangle \leq 0 \text {, for all } z \in C,
$$


This is equivalent to

$$
\Delta_{p}\left(\Pi_{C} u, z\right) \leq \Delta_{p}(u, z)-\Delta_{p}\left(u, \Pi_{C} u\right), \text { for all } z \in C
$$

Lemma 1. Ref. [31] Let E be a q-uniformly smooth Banach space with q-uniformly smoothness constant $c_{q}>0$. For any $u, v \in E$, the following inequality holds:

$$
\|u-v\|^{q} \leq\|u\|^{q}-q\left\langle v, J_{E}^{q} u\right\rangle+c_{q}\|v\|^{q} .
$$

Definition 4. A mapping $T: C \rightarrow C$ is said to be closed or has a closed graph if a sequence $\left\{x_{n}\right\} \subset C$ converges strongly to a point $x \in C$ and $T x_{n} \rightarrow y$, then $T x=y$.

Lemma 2. Ref. [29] It is known that the generalized duality has the following properties:

(I) $J_{E}^{p}(x)$ is nonempty bounded closed and convex, for any $x \in E$.

(II) If $E$ is a reflexive Banach space, then $J_{E}^{p}$ is a mapping from $E$ onto $E^{*}$.

(III) If E is smooth Banach space, then $J_{E}^{p}$ single valued.

(IV) If E is a uniformly smooth Banach space, then $J_{E}^{p}$ is norm-to-norm uniformly continuous on each bounded subset of $E$.

Lemma 3. Ref. [32] For any $\left\{x_{n}\right\} \subset E,\left\{t_{n}\right\} \subset(0,1)$ with $\sum_{n=1}^{N} t_{n}=1$, the following inequality holds:

$$
\Delta_{p}\left(J_{E^{*}}^{q},\left(\sum_{n=1}^{N} t_{n} J_{E}^{p}\left(x_{n}\right)\right), x\right) \leq \sum_{n=1}^{N} t_{n} \Delta_{p}\left(x_{n}, x\right) \text { for all } x \in E \text {. }
$$

We now define some important operators which play key role in our convergence analysis.

Definition 5. Let $A: E \rightarrow 2^{E^{*}}$ be a multi-valued mapping. We define the effective domain of $A$ by $\mathbb{D}(A)=$ $\{x \in E: A x \neq 0\}$ and range of $A$ by $\Re(A)=\bigcup_{x \in \mathbb{D}(A)} A x$. The operator $A$ is said to be monotone if $\left\langle x-y, u^{*}-v^{*}\right\rangle \geq 0$ for all $x, y \in \mathbb{D}(A), u^{*} \in A x$ and $v^{*} \in A y$. When the graph of $A$ is not properly contained in the graph of any other monotone operator, then we say that $A$ is maximally monotone.

Let $E$ be a smooth, strictly convex, and reflexive Banach space and $A: E \rightarrow 2^{E^{*}}$ be a maximal monotone operator. The metric resolvent operator with respect to $A$ is defined by $Q_{r}^{\varphi}(u)=\left(I+r J_{\varphi}^{-1} A\right)^{-1}(u)$. It is easy to see that

$$
0 \in J_{\varphi}\left(Q_{r}^{\varphi}(u)-u\right)+r A Q_{r}^{\varphi}(u),
$$

and $F\left(Q_{r}^{\varphi}\right)=A^{-1} 0$ for all $r>0$ (see, e.g., [20]). Moreover, by the monotonicity of $A$, we can show that

$$
\left\langle Q_{r}^{\varphi}(u)-Q_{r}^{\varphi}(v), J_{\varphi}\left(u-Q_{r}^{\varphi}(u)\right)-J_{\varphi}\left(v-Q_{r}^{\varphi}(v)\right)\right\rangle \geq 0
$$

for all $u, v \in E$. In addition, if $A^{-1} 0 \neq \varnothing$, then

$$
\left\langle Q_{r}^{\varphi}(u)-z, J_{\varphi}\left(u-Q_{r}^{\varphi}(u)\right)\right\rangle \geq 0
$$

for all $u \in E$ and $z \in A^{-1} 0$. In the case $\varphi(t)=t^{p-1}$ with $p \in(1,+\infty)$, we denote $Q_{r}^{\varphi}$ by $Q_{r}=\left(I+r J_{p}^{-1} A\right)^{-1}$ (see, e.g., [33]).

Proposition 1. Ref. [30] Let $A: E \rightarrow 2^{E^{*}}$ be an operator satisfying the following range condition

$$
\mathbb{D}(A) \subset C \subset J_{\varphi}^{-1} \Re\left(J_{\varphi}+\lambda A\right) \text { for all } \lambda>0 .
$$

Define the $\varphi$-resolvent operator $R_{\lambda}^{\varphi}: C \rightarrow 2^{E}$ associated with operator $A$ by

$$
R_{\lambda}^{\varphi}(x)=\left\{z \in X: J_{\varphi}(x) \in\left(J_{\varphi}+\lambda A\right) z\right\}, \quad x \in C .
$$


Then, for any $u \in C$ and $\lambda>0$, we see that

$$
\begin{aligned}
0 \in A u & \Leftrightarrow J_{\varphi}(u) \in\left(J_{\varphi}+\lambda A\right) u \\
& \Leftrightarrow u \in\left(J_{\varphi}+\lambda A\right)^{-1} J_{\varphi}(u) \\
& \Leftrightarrow u \in F\left(R_{\lambda}^{\varphi}\right) .
\end{aligned}
$$

Proposition 2. Ref. [30] Let $C$ be a nonempty, closed, and convex subset of a reflexive, strictly convex Banach space $E$ and let $J_{\varphi}: E \rightarrow E^{*}$ be the duality mapping with gauge $\varphi$. Let $A: E \rightarrow 2^{E^{*}}$ be a monotone operator satisfying the condition $\mathbb{D} \subset C \subset J_{\varphi}^{-1} \Re\left(J_{\varphi}+\lambda A\right)$, where $\lambda>0$. Let $R_{\lambda}^{\varphi}$ be a resolvent operator of $A$; then,

(a) $R_{\lambda}^{\varphi}$ is $\varphi$-firmly non-expansive mapping from $C$ into $C$.

(b) $\quad F\left(R_{\lambda}^{\varphi}\right)=A^{-1} 0$.

Let $E$ be a uniformly convex and smooth Banach space. Let $A$ be a monotone operator of $E$ into $2^{E^{*}}$. From Browder [34], we know that $A$ is maximal if and only if, for any $r>0$,

$$
\Re\left(J_{\varphi}+r A\right)=E^{*}
$$

\section{Remark 2.}

(i) The smoothness and strict convexity of E ensures that $R_{\lambda}^{\varphi, A}$ is single-valued. In addition, the range condition ensure that $R_{\lambda}^{\varphi}$ single-valued operator from $C$ into $\mathbb{D}(A)$. In other words,

$$
R_{\lambda}(x)^{\varphi}(x)=\left(J_{\varphi}+\lambda A\right)^{-1} J_{\varphi}(x), \text { for all } x \in C .
$$

(ii) When $A$ is maximal monotone, the range condition holds for $C=\overline{\mathbb{D}(A)}$.

In the sequel, we denote $R_{\lambda}^{\varphi}$ by $R_{\lambda}=\left(J_{p}+\lambda A\right)^{-1} J_{p}$ for convenience.

Let $E$ and $F$ be real Banach spaces and let $T: E \rightarrow F$ be a bounded linear. The dual (adjoint) operator of $T$, denoted by $T^{*}$, is a bounded linear operator defined by $T^{*}: F^{*} \rightarrow E^{*}$

$$
\left\langle T^{*} \bar{y}, x\right\rangle:=\langle\bar{y}, T x\rangle, \quad \text { for all } x \in E, \bar{y} \in F^{*}
$$

and the equalities $\left\|T^{*}\right\|=\|T\|$ and $\aleph\left(T^{*}\right)=\Re(T)^{\perp}$ are valid, where $\Re(T)^{\perp}:=\left\{x^{*} \in F^{*}:\left\langle x^{*}, u\right\rangle=\right.$ 0 , for all $u \in \Re(T)\}$ (see [35,36] for more details on bounded linear operators and their duals).

Lemma 4. Ref. [9] Let $E$ and $F$ be uniformly convex and smooth Banach spaces, Let $T: E \rightarrow F$ be a bounded linear operator with the adjoint operator $T^{*}$. Let $R_{\lambda}$ be the resolvent operator associated with a maximal monotone operator $A$ on $E$ and let $Q_{r}$ be a metric resolvent associated with a maximal monotone operator $B$ on $F$. Assume that $A^{-1} 0 \cap T^{-1}\left(B^{-1} 0\right) \neq \varnothing$. Let $\lambda, \mu, r>0$ and $z \in E$. Then, the following are equivalent:

(a) $z=R_{\lambda}\left(J_{E^{*}}^{q}\left(J_{E}^{p}(z)-\mu T^{*} J_{F}^{p}(T z-Q-r T z)\right)\right) ;$ and

(b) $z \in A^{-} 0 \cap T^{-1}\left(B^{-1} 0\right)$.

\section{Main Results}

In this section, we present our algorithm and its convergence analysis. In the sequel, we assume that the following assumption hold.

(i) $E_{1}$ and $E_{2}$ are two $p$-uniformly convex and uniformly smooth real Banach spaces.

(ii) $T: E_{1} \rightarrow E_{2}$ is a bounded linear operator with $T \neq 0$ with adjoint $T^{*}: E_{2}^{*} \rightarrow E_{1}^{*}$.

(iii) $A: E_{1} \rightarrow 2^{E_{1}^{*}}$ and $B: E_{2} \rightarrow 2^{E_{2}^{*}}$ are maximal monotone operators.

(iv) $R_{\lambda}$ is the resolvent operator associated with $A$ and $Q_{r}$ is the metric resolvent operator associated with $B$. 
In addition, we denote by $J_{E_{1}}^{p}$ and $J_{E_{2}}^{p}$ the duality mappings of $E_{1}$ and $E_{2}$, respectively, while $J_{E_{1}^{*}}^{q}$ is the duality mapping of $E_{1}^{*}$. It is worth mentioning that, when $E_{1}^{*}$ and $E_{2}^{*}$ are two $q$-uniformly smooth and uniformly convex Banach spaces, $J_{E_{1}}^{p}=\left(J_{E_{1}^{*}}^{q}\right)^{-1}$ where $1<q \leq 2 \leq p<+\infty$ with $\frac{1}{p}+\frac{1}{q}=1$.

Algorithm SASPM: Given initial values $x_{0}, x_{1} \in C_{1}=E_{1}$, the sequence $\left\{x_{n}\right\}$ generated by the following iterative algorithm:

$$
\left\{\begin{array}{l}
w_{n}=J_{E_{1}^{*}}^{q}\left[J_{E_{1}}^{p} x_{n}+\theta_{n} J_{E_{1}}^{p}\left(x_{n}-x_{n-1}\right)\right] \\
z_{n}=J_{E_{1}^{*}}^{q}\left[J_{E_{1}}^{p}\left(w_{n}\right)-\rho_{n} \frac{f^{p-1}\left(w_{n}\right)}{\left\|T^{*}\left(J_{E_{2}}^{p}\left(T w_{n}-Q_{r_{n}} T w_{n}\right)\right)\right\|^{p}} T^{*} J_{E_{1}}^{p}\left(T w_{n}-Q_{r_{n}} T w_{n}\right)\right] \\
y_{n}=J_{E_{1}}^{q}\left(\alpha_{n} J_{E_{1}}^{p} z_{n}+\left(1-\alpha_{n}\right) J_{E_{1}}^{p} R_{\lambda_{n}} z_{n}\right) \\
C_{n+1}=\left\{u \in C_{n}: \Delta_{p}\left(y_{n}, u\right) \leq \Delta_{p}\left(z_{n}, u\right) \leq \Delta_{p}\left(w_{n}, u\right)\right\} \\
x_{n+1}=\Pi_{C_{n+1}} x_{0}
\end{array}\right.
$$

where $\left\{r_{n}\right\},\left\{\lambda_{n}\right\} \subset(0, \infty), \Pi_{C_{n+1}}$ is a Bregman projection of $E_{1}$ onto $C_{n+1}$, the sequence of real number $\left\{\alpha_{n}\right\} \subset[a, b] \subset(0,1)$ and $\left\{\theta_{n}\right\} \subset[c, d] \subset(-\infty,+\infty), f\left(w_{n}\right):=\frac{1}{p}\left\|\left(I-Q_{r_{n}}\right) T w_{n}\right\|^{p}$, and $\left\{\rho_{n}\right\} \subset$ $(0,+\infty)$ satisfying

$$
\liminf _{n \rightarrow+\infty} \rho_{n}\left(p-C_{q} \frac{\rho_{n}^{q-1}}{q}\right)>0 .
$$

To prove the convergence analysis of Algorithm SASPM, we first prove some useful results.

Lemma 5. Let $E_{1}$ be a p-uniformly convex and uniformly smooth real Banach space, and $C_{1}=E_{1}$. Then, for any sequence $\left\{y_{n}\right\},\left\{z_{n}\right\}$ and $\left\{w_{n}\right\}$ in $E_{1}$, the set

$$
C_{n+1}=\left\{u \in C_{n}: \Delta_{p}\left(y_{n}, u\right) \leq \Delta_{p}\left(z_{n}, u\right) \leq \Delta_{p}\left(w_{n}, u\right)\right\}
$$

is closed and convex for each $n \geq 1$.

Proof. First, since $C_{1}=E_{1}, C_{1}$ is closed and convex. Then, we assume that $C_{n}$ is a closed and convex. For each $u \in C_{n}$, by the definition of the function $\Delta_{p}$, we have

$$
\Delta_{p}\left(y_{n}, u\right) \leq \Delta_{p}\left(z_{n}, u\right) \text { if and only if } 2\left\langle J_{E_{1}}^{p} z_{n}-J_{E_{1}}^{p} y_{n}, u\right\rangle \leq \frac{1}{q}\left(\left\|z_{n}\right\|^{p}-\left\|y_{n}\right\|^{p}\right),
$$

and

$$
\Delta_{p}\left(z_{n}, u\right) \leq \Delta_{p}\left(w_{n}, u\right) \text { if and only if } 2\left\langle J_{E_{1}}^{p} w_{n}-J_{E_{1}}^{p} z_{n}, u\right\rangle \leq \frac{1}{q}\left(\left\|w_{n}\right\|^{p}-\left\|z_{n}\right\|^{p}\right) .
$$

Hence, we know that $C_{n+1}$ is closed. In addition, we easily prove that $C_{n+1}$ is convex. The proof is completed.

Lemma 6. Let $E_{1}, E_{2}, T T^{*} A, B$, and $J_{E_{1}}^{p}, J_{E_{2}}^{p} \cdot J_{E_{2}}^{p} J_{E_{1}^{*}}^{q}$ be the same as above such that Conditions (1)-(4) are satisfied. If $Y=\left\{z: z \in A^{-1} 0 \cap T^{-1}\left(B^{-1} 0\right)\right\}$, then $Y \subseteq C_{n}$ for any $n \geq 1$.

Proof. If $Y=\varnothing$, it is obvious that $Y \subseteq C_{n}$. Conversely, for any $z \in \Gamma$, according to Lemma 3 and using the fact that the resolvent $R_{\lambda_{n}}$ is non-expansive, we easily obtain

$$
\begin{aligned}
\Delta_{p}\left(y_{n}, z\right) & =\Delta_{p}\left(J_{E_{1}^{*}}^{q}\left(\alpha_{n} J_{E_{1}}^{p} z_{n}+\left(1-\alpha_{n}\right) J_{E_{1}}^{p} R_{\lambda_{n}} z_{n}\right), z\right) \\
& \leq \alpha_{n} \Delta_{p}\left(z_{n}, z\right)+\left(1-\alpha_{n}\right) \Delta_{p}\left(R_{\lambda_{n}} z_{n}, z\right) \\
& \leq \Delta_{p}\left(z_{n}, z\right) .
\end{aligned}
$$


From (20), let $u_{n}=J_{E_{1}}^{p}\left(w_{n}\right)-\rho_{n} \frac{f^{p-1}\left(w_{n}\right)}{\left\|g\left(w_{n}\right)\right\|^{p}} g\left(w_{n}\right)$ for all $n \geq 1$, where $g\left(w_{n}\right)=T^{*} J_{E_{1}}^{p}\left(T w_{n}-\right.$ $\left.Q_{r_{n}} T w_{n}\right)$. We see from Lemma 1 that

$$
\begin{aligned}
\left\|u_{n}\right\|_{E_{1}^{*}}^{q} & =\left\|J_{E_{1}}^{p}\left(w_{n}\right)-\rho_{n} \frac{f^{p-1}\left(w_{n}\right)}{\left\|g\left(w_{n}\right)\right\|^{p}} g\left(w_{n}\right)\right\|_{E_{1}^{*}}^{q} \\
& \leq\left\|w_{n}\right\|^{p}-q \rho_{n} \frac{f^{p-1}\left(w_{n}\right)}{\left\|g\left(w_{n}\right)\right\|^{p}}\left\langle w_{n}, g\left(w_{n}\right)\right\rangle+c_{q} \rho_{n}^{q} \frac{f^{(p-1) q}\left(w_{n}\right)}{\left\|g\left(w_{n}\right)\right\|^{p q}}\left\|g\left(w_{n}\right)\right\|^{q} \\
& =\left\|w_{n}\right\|^{p}-q \rho_{n} \frac{f^{p-1}\left(w_{n}\right)}{\left\|g\left(w_{n}\right)\right\|^{p}}\left\langle w_{n}, g\left(w_{n}\right)\right\rangle+c_{q} \rho_{n}^{q} \frac{f^{p}\left(w_{n}\right)}{\left\|g\left(w_{n}\right)\right\|^{p}} .
\end{aligned}
$$

Then, by (16) and (22), we get

$$
\begin{aligned}
\Delta_{p}\left(z_{n}, z\right) \leq & \Delta_{p}\left(J_{E_{1}}^{p}\left(u_{n}\right), z\right) \\
= & \frac{\|z\|^{p}}{p}+\frac{1}{q}\left\|J_{E_{1}}^{p}\left(u_{n}\right)\right\|^{p}-\langle z, u\rangle \\
= & \frac{\|z\|^{p}}{p}+\frac{1}{q}\left\|u_{n}\right\|^{(q-1) p}-\left\langle z, u_{n}\right\rangle \\
= & \frac{\|z\|^{p}}{p}+\frac{1}{q}\left\|u_{n}\right\|^{(q-1)} \frac{q}{(q-1)}-\left\langle z, u_{n}\right\rangle \\
= & \frac{\|z\|^{p}}{p}+\frac{1}{q}\left\|u_{n}\right\|^{q}-\left\langle z, u_{n}\right\rangle \\
= & \frac{\|z\|^{p}}{p}+\frac{1}{q}\left\|u_{n}\right\|^{q}-\left\langle z, J_{E_{1}}^{p}\left(w_{n}\right)\right\rangle+\rho_{n} \frac{f^{p-1}\left(w_{n}\right)}{\left\|g\left(w_{n}\right)\right\|^{p}}\left\langle z, g\left(w_{n}\right)\right\rangle \\
\leq & \frac{\|z\|^{p}}{p}+\frac{1}{q}\left(\left\|w_{n}\right\|^{p}-q \rho_{n} \frac{f^{p-1}\left(w_{n}\right)}{\left\|g\left(w_{n}\right)\right\|^{p}}\left\langle w_{n}, g\left(w_{n}\right)\right\rangle+c_{q} \rho_{n}^{q} \frac{f^{p}\left(w_{n}\right)}{\left\|g\left(w_{n}\right)\right\|^{p}}\right) \\
& -\left\langle z, J_{E_{1}}^{p}\left(w_{n}\right)\right\rangle+\rho_{n} \frac{f^{p-1}\left(w_{n}\right)}{\left\|g\left(w_{n}\right)\right\|^{p}}\left\langle z, g\left(w_{n}\right)\right\rangle \\
= & \frac{\|z\|^{p}}{p}+\frac{\left\|w_{n}\right\|^{p}}{q}-\left\langle z, J_{E_{1}}^{p}\left(w_{n}\right)\right\rangle+\frac{c_{q} \rho_{n}^{q}}{q} \frac{f^{p}\left(w_{n}\right)}{\left\|g\left(w_{n}\right)\right\|^{p}}+\rho_{n} \frac{f^{p-1}\left(w_{n}\right)}{\left\|g\left(w_{n}\right)\right\|^{p}}\left\langle z-w_{n}, g\left(w_{n}\right)\right\rangle \\
= & \Delta_{p}\left(w_{n}, z\right)+\frac{c_{q} \rho_{n}^{q}}{q} \frac{f^{p}\left(w_{n}\right)}{\left\|g\left(w_{n}\right)\right\|^{p}}+\rho_{n} \frac{f^{p-1}\left(w_{n}\right)}{\left\|g\left(w_{n}\right)\right\|^{p}}\left\langle z-w_{n}, g\left(w_{n}\right)\right\rangle
\end{aligned}
$$

On the other hand, observe that

$$
\begin{aligned}
\left\langle g\left(w_{n}\right), z-w_{n}\right\rangle & =\left\langle T^{*} J_{E_{2}}^{p}\left(I-Q_{r_{n}} T w_{n}\right), z-w_{n}\right\rangle \\
& =\left\langle J_{E_{2}}^{p}\left(I-Q_{r_{n}} T w_{n}\right), T z-T w_{n}\right\rangle \\
& =\left\langle J_{E_{2}}^{p}\left(w_{n}\right)\left(I-Q_{r_{n}}\right) T w_{n}, Q_{r_{n}} T w_{n}-T w_{n}\right\rangle+\left\langle J_{E_{2}}^{p}\left(I-Q_{r_{n}}\right) T w_{n}, T z-Q_{r_{n}} T w_{n}\right\rangle \\
& \leq-\left\|\left(I-Q_{r_{n}}\right) T w_{n}\right\|^{p}=-p f\left(w_{n}\right) .
\end{aligned}
$$

By using (23) and (24), we get

$$
\Delta_{p}\left(z_{n}, z\right) \leq \Delta_{p}\left(w_{n}, z\right)+\left(\frac{c_{q} \rho_{n}^{q}}{q}-\rho_{n} p\right) \frac{f^{p}\left(w_{n}\right)}{\left\|g\left(w_{n}\right)\right\|^{p}},
$$

which implies by our assumption that

$$
\Delta_{p}\left(z_{n}, z\right) \leq \Delta_{p}\left(w_{n}, z\right)
$$

From (21) and (26), we have that $z \in C_{n+1}$, that is, $Y \subseteq C_{n}$, for all $n \geq 1$. 
Theorem 1. Let $E_{1}, E_{2} T, T^{*}, A, B$, and $J_{E_{1}}^{p}, J_{E_{2}}^{p} J_{E_{1}^{*}}^{q}$ be the same as above such that Conditions (1)-(4) are satisfied. If $\mathrm{Y}=\left\{z: z \in A^{-1} 0 \cap T^{-1}\left(B^{-1} 0\right)\right\} \neq \varnothing$, then the sequence generated by Algorithm (20) converges strongly to a point $z=\Pi_{Y} x_{0} \in \mathrm{Y}$.

Proof. By Lemmas 5 and 6 , we know that $\Pi_{C_{n+1}} x_{0}$ is well defined and $Y \subset C_{n}$. According to Algorithm (20), we know that $x_{n}=\Pi_{C_{n}} x_{0}$ and $x_{n+1}=\Pi_{C_{n+1}} x_{0}$ for each $n \geq 1$. Using $\mathrm{Y} \subset C_{n}$ and (16), we have

$$
\Delta_{p}\left(x_{0}, x_{n}\right)=\Delta_{p}\left(x_{0}, \Pi_{C_{n}} x_{0}\right) \leq \Delta_{p}\left(x_{0}, z\right) z \in \mathrm{Y}, \forall n \geq 1
$$

It implies that $\left\{\Delta_{p}\left(x_{0}, x_{n}\right)\right\}$ is bounded. Reusing (16), we also have

$$
\begin{aligned}
\Delta_{p}\left(x_{n}, x_{n+1}\right)=\Delta_{p}\left(\Pi_{C_{n}} x_{0}, x_{n+1}\right) & \leq \Delta_{p}\left(x_{0}, x_{n+1}\right)-\Delta_{p}\left(x_{0}, \Pi_{C_{n}} x_{0}\right) \\
& =\Delta_{p}\left(x_{0}, x_{n+1}\right)-\Delta_{p}\left(x_{0}, x_{n}\right) .
\end{aligned}
$$

It follows that $\left\{\Delta_{p}\left(x_{0}, x_{n+1}\right)\right\}$ is nondecreasing. Hence, the limit $\lim _{n \rightarrow+\infty} \Delta_{p}\left(x_{0}, x_{n}\right)$ exists, and

$$
\lim _{n \rightarrow+\infty} \Delta_{p}\left(x_{n}, x_{n+1}\right)=0
$$

It follows from (13) that

$$
\lim _{n \rightarrow+\infty}\left\|x_{n+1}-x_{n}\right\|=0
$$

For some positive $m, n$ with $m \geq n$, we have $x_{m}=\Pi_{C_{m}} x_{1} \subseteq C_{n}$. Using (16), we obtain

$$
\begin{aligned}
\Delta_{p}\left(x_{n}, x_{m}\right)=\Delta_{p}\left(\Pi_{C_{n}} x_{0}, x_{m}\right) & \leq \Delta_{p}\left(x_{0}, x_{m}\right)-\Delta_{p}\left(x_{0}, \Pi_{C_{n}} x_{0}\right) \\
& =\Delta_{p}\left(x_{0}, x_{m}\right)-\Delta_{p}\left(x_{0}, x_{n}\right) .
\end{aligned}
$$

Since the limit $\lim _{n \rightarrow+\infty} \Delta_{p}\left(x_{0}, x_{n}\right)$ exists, it follows from (31) that $\lim _{n \rightarrow+\infty} \Delta_{p}\left(x_{n}, x_{m}\right)=0$ and $\lim _{n \rightarrow+\infty}\left\|x_{n}-x_{m}\right\|=0$. Therefore, $\left\{x_{n}\right\}$ is Cauchy sequence. Further, there exists a point $x^{*} \in C$ such that $x_{n} \rightarrow x^{*}$.

From Algorithm (20), Definition 2, and Lemma 1, we have

$$
\begin{aligned}
\Delta_{p}\left(w_{n}, z\right)= & \frac{1}{q}\left\|J_{E_{1}^{*}}^{p}\left(J_{E_{1}}^{p} x_{n}+\theta_{n} J_{E_{1}}^{p}\left(x_{n}-x_{n-1}\right)\right)\right\|^{p}+\frac{1}{p}\|z\|^{p} \\
& -\left\langle J_{E_{1}}^{p} x_{n}+\theta_{n} J_{E_{1}}^{p}\left(x_{n}-x_{n-1}\right), z\right\rangle \\
= & \frac{1}{q}\left\|J_{E_{1}}^{p} x_{n}+\theta_{n} J_{E_{1}}^{p}\left(x_{n}-x_{n-1}\right)\right\|^{q}+\frac{1}{p}\|z\|^{p} \\
& -\left\langle J_{E_{1}}^{p} x_{n}+\theta_{n} J_{E_{1}}^{p}\left(x_{n}-x_{n-1}\right), z\right\rangle \\
\leq & \frac{1}{q}\left\|J_{E_{1}}^{p} x_{n}\right\|^{q}+\frac{1}{p}\|z\|^{p}-\left\langle J_{E_{1}}^{p} x_{n}, x^{*}\right\rangle-\theta_{n}\left\langle J_{E_{1}}^{p}\left(x_{n}-x_{n-1}\right), z\right\rangle \\
& +\theta_{n}\left\langle J_{E_{1}}^{p}\left(x_{n}-x_{n-1}\right), x_{n}\right\rangle+\frac{c_{q}\left(\theta_{n}\right)^{q}}{q}\left\|J_{E_{1}}^{p}\left(x_{n}-x_{n-1}\right)\right\|^{q} \\
= & \frac{1}{q}\left\|x_{n}\right\|^{q}+\frac{1}{p}\|z\|^{p}-\left\langle J_{E_{1}}^{p} x_{n}, x^{*}\right\rangle-\theta_{n}\left\langle J_{E_{1}}^{p}\left(x_{n}-x_{n-1}\right), z\right\rangle \\
& +\theta_{n}\left\langle J_{E_{1}}^{p}\left(x_{n}-x_{n-1}\right), x_{n}\right\rangle+\frac{c_{q}\left(\theta_{n}\right)^{q}}{q}\left\|J_{E_{1}}^{p}\left(x_{n}-x_{n-1}\right)\right\|^{q} \\
= & \Delta_{p}\left(x_{n}, z\right)+\theta_{n}\left\langle J_{E_{1}}^{p}\left(x_{n}-x_{n-1}\right), x_{n}-x^{*}\right\rangle+\frac{c_{q}\left(\theta_{n}\right)^{q}}{q}\left\|x_{n}-x_{n-1}\right\|^{p} .
\end{aligned}
$$


By virtue of Remark 1 and the definition of $w_{n}$, we know

$$
\begin{aligned}
\Delta_{p}\left(w_{n}, z\right) & =\Delta_{p}\left(w_{n}, x_{n}\right)+\Delta_{p}\left(x_{n}, z\right)+\left\langle x_{n}-z, J_{E_{1}}^{p} w_{n}-J_{E_{1}}^{p} x_{n}\right\rangle \\
& =\Delta_{p}\left(w_{n}, x_{n}\right)+\Delta_{p}\left(x_{n}, z\right)+\theta_{n}\left\langle x_{n}-z, J_{E_{1}}^{p}\left(x_{n}-x_{n-1}\right)\right\rangle .
\end{aligned}
$$

By (32) and (33), we get $\Delta_{p}\left(w_{n}, x_{n}\right) \leq \frac{c_{q}\left(\theta_{n}\right)^{q}}{q}\left\|x_{n}-x_{n-1}\right\|^{p}$. Then, using (13) and (30) and the boundedness of the sequence $\left\{\theta_{n}\right\}$, we can obtain

$$
\lim _{n \rightarrow+\infty}\left\|w_{n}-x_{n}\right\|=0 .
$$

Using a similar method, we can get

$$
\Delta_{p}\left(w_{n}, x_{n+1}\right)=\Delta_{p}\left(w_{n}, x_{n}\right)+\Delta_{p}\left(x_{n}, x_{n+1}\right)+\left\langle x_{n}-x_{n+1}, J_{E_{1}}^{p} w_{n}-J_{E_{1}}^{p} x_{n}\right\rangle .
$$

By setting $n \rightarrow+\infty$, we have

$$
\lim _{n \rightarrow+\infty}\left\|w_{n}-x_{n+1}\right\|=0 .
$$

Since $x_{n+1}=\Pi_{C_{n+1}} x_{0} \in C_{n+1} \subseteq C_{n}$, we have

$$
\Delta_{p}\left(y_{n}, x_{n+1}\right) \leq \Delta_{p}\left(z_{n}, x_{n+1}\right) \leq \Delta_{p}\left(w_{n}, x_{n+1}\right) .
$$

According to (35), we obtain

$$
\lim _{n \rightarrow+\infty} \Delta_{p}\left(y_{n}, x_{n+1}\right)=0, \lim _{n \rightarrow+\infty} \Delta_{p}\left(z_{n}, x_{n+1}\right)=0,
$$

which implies that $\lim _{n \rightarrow+\infty}\left\|y_{n}-x_{n+1}\right\|=0, \lim _{n \rightarrow+\infty}\left\|z_{n}-x_{n+1}\right\|=0$. Hence,

$$
\left\|x_{n}-z_{n}\right\| \leq\left\|x_{n+1}-x_{n}\right\|+\left\|x_{n+1}-z_{n}\right\| \rightarrow 0, \text { as } n \rightarrow+\infty,
$$

and

$$
\left\|y_{n}-z_{n}\right\| \leq\left\|x_{n+1}-y_{n}\right\|+\left\|x_{n+1}-z_{n}\right\| \rightarrow 0, \text { as } n \rightarrow+\infty .
$$

We also get from (34) and (37) that

$$
\left\|w_{n}-z_{n}\right\| \leq\left\|w_{n}-x_{n}\right\|+\left\|x_{n}-z_{n}\right\| \rightarrow 0, \text { as } n \rightarrow \infty .
$$

As $J_{E_{1}}^{p}$ is norm to norm uniformly continuous on a bounded subset of $E_{1}$, we obtain

$$
\left\|J_{E_{1}}^{p}\left(w_{n}\right)-J_{E_{1}}^{p}\left(z_{n}\right)\right\| \rightarrow 0 \text {, as } n \rightarrow+\infty .
$$

Since $E_{1}$ is a $p$-uniformly convex and uniformly smooth real Banach space, then $J_{E_{1}}^{p}$ is uniformly norm-to-norm continuous. Thus, it follows from Algorithm (20) and real number sequence $\left\{\alpha_{n}\right\}$ in $[a, b] \subset(0,1)$ that

$$
\lim _{n \rightarrow+\infty}\left\|J_{E_{1}}^{p} R_{\lambda_{n}} z_{n}-J_{E_{1}}^{p} z_{n}\right\|=0=\lim _{n \rightarrow+\infty} \frac{1}{1-\alpha_{n}}\left\|J_{E_{1}}^{p} y_{n}-J_{E_{1}}^{p} z_{n}\right\|=0,
$$


which also implies that $\lim _{n \rightarrow+\infty}\left\|R_{\lambda_{n}} z_{n}-z_{n}\right\|=0$. From (25), and $z$ being in $Y$, we get

$$
\begin{aligned}
\Delta_{p}\left(z_{n}, z\right) & \leq \Delta_{p}\left(w_{n}, z\right)+\rho_{n}\left(\frac{c_{q} \rho_{n}^{q-1}}{q}-p\right) \frac{f^{p}\left(w_{n}\right)}{\left\|g\left(w_{n}\right)\right\|^{p}} \\
& =\Delta_{p}\left(w_{n}, z\right)-\rho_{n}\left(p-\frac{c_{q} \rho_{n}^{q-1}}{q}\right) \frac{f^{p}\left(w_{n}\right)}{\left\|g\left(w_{n}\right)\right\|^{p}} .
\end{aligned}
$$

This implies that

$$
\begin{aligned}
\rho_{n}\left(p-\frac{c_{q} \rho_{n}^{q-1}}{q}\right) \frac{f^{p}\left(w_{n}\right)}{\left\|g\left(w_{n}\right)\right\|^{p}} & \leq \Delta_{p}\left(w_{n}, z\right)-\Delta_{p}\left(z_{n}, z\right) \\
& =\frac{1}{q}\left\|w_{n}\right\|^{p}-\frac{1}{q}\left\|z_{n}\right\|^{p}-\left\langle J_{E_{1}}^{p} w_{n}-J_{E_{1}}^{p} z_{n}, z\right\rangle \\
& =\Delta_{p}\left(w_{n}, z_{n}\right)+\left\langle J_{E_{1}}^{p} w_{n}-J_{E_{1}}^{p} z_{n}, z_{n}-z\right\rangle \\
& \leq\left(\left\|w_{n}-z_{n}\right\|+\left\|z_{n}-z\right\|\right)\left\|J_{E_{1}}^{p} w_{n}-J_{E_{1}}^{p} z_{n}\right\| .
\end{aligned}
$$

By setting of $n \rightarrow+\infty$, the right-hand side of the last inequality tends to 0 . This implies that

$$
\rho_{n}\left(p-\frac{c_{q} \rho_{n}^{q-1}}{q}\right) \frac{f^{p}\left(w_{n}\right)}{\left\|g\left(w_{n}\right)\right\|^{p}} \rightarrow 0, n \rightarrow+\infty .
$$

Since $\liminf _{n \rightarrow+\infty} \rho_{n}\left(p-c_{q} \frac{\rho_{n}^{q-1}}{q}\right)>0$, we get

$$
\frac{f^{p}\left(w_{n}\right)}{\left\|g\left(w_{n}\right)\right\|^{p}} \rightarrow 0, n \rightarrow+\infty
$$

and hence

$$
\frac{f\left(w_{n}\right)}{\left\|g\left(w_{n}\right)\right\|^{p}} \rightarrow 0, n \rightarrow+\infty
$$

Furthermore, since $\left\{g\left(w_{n}\right)\right\}$ is bounded, we obtain from (42) that

$$
\begin{aligned}
0 \leq g\left(w_{n}\right) & =\left\|g\left(w_{n}\right)\right\| \frac{f\left(w_{n}\right)}{\left\|g\left(w_{n}\right)\right\|} \\
& \leq M_{1} \frac{f\left(w_{n}\right)}{\left\|g\left(w_{n}\right)\right\|} \rightarrow 0, n \rightarrow+\infty,
\end{aligned}
$$

for some $M_{1}>0$. Therefore,

$$
\lim _{n \rightarrow+\infty} f\left(w_{n}\right)=0
$$

Hence,

$$
\lim _{n \rightarrow+\infty}\left\|\left(I-Q_{r_{n}}\right) T w_{n}\right\|=0
$$

In addition,

$$
\left\|T^{*} J_{E_{2}}^{p}\left(I-Q_{r_{n}}\right) T w_{n}\right\| \leq\|T\|\left\|\left(I-Q_{r_{n}}\right) T w_{n}\right\| \rightarrow 0, n \rightarrow+\infty .
$$

Since $\left\|x_{n}-w_{n}\right\| \rightarrow 0$, as $n \rightarrow+\infty$, there exists a subsequence $\left\{x_{n_{j}}\right\}$ of $\left\{x_{n}\right\}$ such that $x_{n_{j}} \rightarrow$ $w \in E_{1}$, as well as $\left\|x_{n}-w_{n}\right\| \rightarrow 0$, as $n \rightarrow+\infty$ there exists a subsequence $\left\{w_{n_{j}}\right\}$ of $\left\{w_{n}\right\}$ such that 
$w_{n_{j}} \rightarrow w \in E_{1}$. From $\left\|T w_{n}-Q_{r_{n}} T w_{n}\right\| \rightarrow 0$ and by the boundedness and linearity of $T$, we have $T w_{n_{j}} \rightarrow T w$ and $Q_{r_{j}} T w_{n_{j}} \rightarrow T w$. Since $Q_{r_{n}}$ is a metric resolvent on $B$ for $r_{n}>0$, we have

$$
\frac{J_{E_{2}}^{p}\left(T w_{n}-Q_{r_{n}} T w_{n}\right)}{r_{n}} \in B Q_{r_{n}} T w_{n}
$$

for all $n \in \mathbb{N}$, thus we obtain

$$
0 \leq\left\langle v-Q_{n_{j}} T w_{n_{j}} T w_{n_{j}}, v^{*}-\frac{J_{E_{2}}^{p}\left(T w_{n_{j}}-Q_{n_{n_{j}}} T w_{n_{j}}\right)}{r_{n_{j}}}\right\rangle
$$

for all $\left(v, v^{*}\right) \in B$. It follows that

$$
0 \leq\left\langle v-T w, v^{*}-0\right\rangle
$$

for all $\left(v, v^{*}\right) \in B$. Since $B$ is maximal monotone, $T w \in B^{-1} 0$ and hence $w \in T^{-1}\left(B^{-1} 0\right)$.

Let $b_{n}=R_{\lambda_{n}} z_{n}$ and $k_{n}=T w_{n}-Q_{r_{n}} T w_{n} \forall n \in \mathbb{N}$

$$
\begin{aligned}
& b_{n}=J_{\lambda_{n}}\left(J_{E_{1}^{*}}^{q}\left(J_{E_{1}}^{p}\left(w_{n}\right)-\lambda_{n} T^{*} J_{E_{2}}^{p}\left(k_{n}\right)\right)\right) \\
\Longleftrightarrow & b_{n}=\left(J_{E_{1}}^{p}+\lambda_{n} A\right)^{-1} J_{E_{1}}^{p}\left(J_{E_{1}^{*}}^{q}\left(J_{E_{1}}^{p}\left(w_{n}\right)-\lambda_{n} T^{*} J_{E_{2}}^{p}\left(k_{n}\right)\right)\right) \\
\Longleftrightarrow & b_{n}=\left(J_{E_{1}}^{p}+\lambda_{n} A\right)^{-1}\left(J_{E_{1}}^{p}\left(w_{n}\right)-\lambda_{n} T^{*} J_{E_{2}}^{p}\left(k_{n}\right)\right) \\
\Longleftrightarrow & J_{E_{1}}^{p}\left(w_{n}\right)-\lambda_{n} T^{*} J_{E_{2}}^{p}\left(k_{n}\right) \in J_{E_{1}}^{p}\left(b_{n}\right)+\lambda_{n} A b_{n} \\
\Longleftrightarrow & \frac{J_{E_{1}}^{p}\left(w_{n}\right)-J_{E_{1}}^{p}\left(b_{n}\right)}{\lambda_{n}}-T^{*} J_{E_{2}}^{p}\left(k_{n}\right) \in A b_{n} .
\end{aligned}
$$

Note that

$$
\begin{aligned}
\left\|J_{E_{1}}^{p}\left(w_{n}\right)-J_{E_{1}}^{p}\left(b_{n}\right)\right\| & =\left\|J_{E_{1}}^{p}\left(w_{n}\right)-J_{E_{1}}^{p}\left(R_{\lambda_{n}} z_{n}\right)\right\| \\
& \leq\left\|J_{E_{1}}^{p}\left(w_{n}\right)-J_{E_{1}}^{p}\left(z_{n}\right)\right\|+\left\|J_{E_{1}}^{p}\left(z_{n}\right)-J_{E_{1}}^{p}\left(R_{\lambda_{n}} z_{n}\right)\right\| \rightarrow 0, n \rightarrow+\infty .
\end{aligned}
$$

By the monotonicity of $A$, it follows that

$$
0 \leq\left\langle v-b_{n}, v^{*}-\frac{J_{E_{1}}^{p}\left(w_{n}\right)-J_{E_{1}}^{p}\left(b_{n}\right)}{\lambda_{n}}+T^{*} J_{E_{2}}^{p}\left(k_{n}\right)\right\rangle
$$

for all $\left(v, v^{*}\right) \in A$. Then,

$$
0 \leq\left\langle v-b_{n}, v^{*}-\frac{J_{E_{1}}^{p}\left(w_{n_{i}}\right)-J_{E_{1}}^{p}\left(b_{n_{i}}\right)}{\lambda_{n_{i}}}+T^{*} J_{E_{2}}^{p}\left(k_{n_{i}}\right)\right\rangle .
$$

Since $b_{n_{i}} \rightarrow w,(40)$ and (43), it follows that $0 \leq\left\langle v-w, v^{*}-0\right\rangle$ and hence $w \in A^{-1} 0$. This concludes that $w \in A^{-1} 0 \cap T^{-1}\left(B^{-1} 0\right)$. Then, from (28) and (20), we have

$$
\left\langle J_{E_{1}}^{p} x_{0}-J_{E_{1}}^{p} x_{n}, p-x_{n}\right\rangle, \text { for all } p \in \mathrm{Y} .
$$

By setting $n \rightarrow+\infty$ in (44), we obtain

$$
\left\langle J_{E_{1}}^{p} x_{0}-J_{E_{1}}^{p} x^{*}, p-x^{*}\right\rangle \leq 0 \text {, for all } p \in \mathrm{Y} .
$$

Again, from (15), we have $x^{*}=\Pi_{Y} x_{0}$. Definitely, we obtain that $\left\{x_{n}\right\}$ generated by Algorithm (20) strongly converges to $x^{*}=\Pi_{Y} x_{0} \in \mathrm{Y}$. The proof is completed. 
As a corollary of Theorem 1 , when $E_{1}$ and $E_{2}$ reduces to Hilbert spaces, the function $\Delta_{p}$ is equal to $\frac{1}{2}\|x-y\|^{2}$ and the Bregman projection $\Pi_{C}$ is equivalent to the metric projection $P_{C}$. Then, we obtain the following result.

Theorem 2. Let $H_{1}$ and $H_{2}$ be Hilbert spaces, $A: H_{1} \rightarrow 2^{H_{1}}$ and $B: H_{2} \rightarrow 2^{H_{2}}$ be maximal monotone operators, $T: H_{1} \rightarrow H_{2}$ be a bounded linear operator with $T \neq 0$, and $T^{*}: H_{2} \rightarrow H_{1}$ be the adjoint of $T$. Let $R_{\lambda}$ be the resolvent operator associated with a maximal monotone operator $A$ on $H_{1}$ and $Q_{r}$ be metric resolvent associated with a maximal monotone operator $B$ on $H_{2}$. Suppose that $\mathrm{Y}=A^{-1} 0 \cap T^{-1}\left(B^{-1} 0\right) \neq \varnothing$. For fixed $x_{0} \in H_{1}$, let $\left\{x_{n}\right\}_{n=0}^{+\infty}$ be iteratively generated by $x_{1} \in H_{1}$ and

$$
\left\{\begin{array}{l}
w_{n}=x_{n}+\theta_{n}\left(x_{n}-x_{n-1}\right) \\
z_{n}=w_{n}-\rho_{n} \frac{f\left(w_{n}\right)}{\left\|T^{*}\left(I-Q_{r_{n}}\right) T w_{n}\right\|^{2}}\left[T^{*}\left(I-Q_{r_{n}}\right) T w_{n}\right] \\
y_{n}=\alpha_{n} z_{n}+\left(1-\alpha_{n}\right) R_{\lambda_{n}} z_{n} \\
C_{n+1}=\left\{u \in C_{n}:\left\|y_{n}-u\right\| \leq\left\|z_{n}-u\right\| \leq\left\|w_{n}-u\right\|\right\} \\
x_{n+1}=P_{C_{n+1}} x_{0}
\end{array}\right.
$$

where $P_{C_{n+1}}$ is the metric projection of $H_{1}$ onto $C_{n+1}$, the sequence of real numbers, $\left\{\alpha_{n}\right\} \subset[a, b] \subset(0,1)$ and $\left\{\theta_{n}\right\} \subset[c, d] \subset(-\infty,+\infty) . f\left(w_{n}\right):=\frac{1}{2}\left\|\left(I-Q_{r_{n}}\right) T w_{n}\right\|^{2}$, and $\left\{\rho_{n}\right\} \in(0,4)$. Then, the sequence $\left\{x_{n}\right\}$ generated by (46) converges strongly to a point $z_{0}=P_{Y} x_{0} \in \mathrm{Y}$.

\section{Applications}

In this section, we provide some applications of our result to solving other nonlinear optimization problems.

\subsection{Application to Minimization Problem}

First, we consider an application of our result to convex minimization problem in real Banach space $E$. Let $\vartheta: E \rightarrow(-\infty,+\infty]$ be a proper, convex and lower semicontinuous function. The convex minimization problem is to find $x \in E$ such that

$$
\vartheta(x) \leq \vartheta(y), \quad \text { for all } y \in E
$$

The set of minimizer of $\vartheta$ is denoted by Argmin $\vartheta$. The subdifferential of $\partial \vartheta$ of $\vartheta$ is defined as follows

$$
\partial \vartheta(u)=\left\{w \in E^{*}: \vartheta(u)+\langle v-u, w\rangle \leq \vartheta(u), \text { for all } v \in E\right\},
$$

for all $u \in E$. From Rockafellar [37], it is known that $\partial \vartheta$ is a maximal monotone operator. Let $C$ be a nonempty, closed, and convex subset of $E$ and let $i_{C}$ be the indicator function of $C$ i.e.,

$$
i_{C}(u)= \begin{cases}0, & u \in C \\ \infty, & u \notin C\end{cases}
$$

Then, $i_{C}$ is a proper, convex, and lower semicontinuous function on $E$. Thus, the subdifferential $\partial_{i_{C}}$ of $i_{C}$ is a maximal monotone operator. Then, we can define the resolvent $R_{\lambda}$ of $\partial_{i_{C}}$ for $\lambda>0$ i.e.,

$$
R_{\lambda} u=\left(J_{p}+\lambda \partial_{i_{C}}\right)^{-1} J_{p} u
$$


for all $u \in E$ and $p \in(1,+\infty)$. We have that for any $x \in E$ and $u \in C$

$$
\begin{aligned}
u=R_{\lambda} x \quad & \text { if and only if } J_{p} x \in J_{p} u+\lambda \partial_{i_{C}} u \\
& \text { if and only if } \frac{1}{\lambda}\left(J_{p} x-J_{p} u\right) \in \partial_{i_{C}} u \\
& \text { if and only if } i_{C} y \geq\left\langle y-u, \frac{1}{\lambda}\left(J_{p} x-J_{p} u\right)\right\rangle+i_{C} u \text { for all } y \in C \\
& \text { if and only if } 0 \geq\left\langle y-u, \frac{1}{\lambda}\left(J_{p} x-J-p u\right)\right\rangle \text {, for all } y \in C \\
& \text { if and only if }\left\langle y-u, J_{p} x-J_{p} u\right\rangle \leq 0, \text { for all } x \in C \\
& \text { if and only if } u=\Pi_{C} x .
\end{aligned}
$$

Let $E_{1}$ and $E_{2}$ be real Banach spaces and $\vartheta: E_{1} \rightarrow(-\infty,+\infty]$ and $\xi: E_{2} \rightarrow(-\infty,+\infty]$ be proper, lower semicontinuous, and convex functions such that $\operatorname{Argmin} \vartheta \neq \varnothing$ and $\operatorname{Argmin} \xi \neq \varnothing$. Consider the Split Proximal Feasibility Problem (SPFP) defined by: Find $x \in E_{1}$ such that

$$
x \in \operatorname{Argmin} \vartheta \text { and } A x \in \operatorname{Argmin} \xi
$$

where $\operatorname{Argmin} \vartheta:=\left\{\bar{x} \in E_{1}: \vartheta(\bar{x}) \leq \vartheta(x), \quad\right.$ for all $\left.x \in E_{1}\right\}$, and Argmin $\xi=\left\{\bar{y} \in E_{2}: \xi(\bar{y}) \leq\right.$ $\xi(y)$, for all $\left.y \in E_{2}\right\}$. We denote the solution set of (47) by $\Omega$. The PSFP is a generalization of the split feasibility problem and has been studied extensively by many authors in real Hilbert space (see, e.g., [38-42]).

By setting $A=\partial \vartheta$ and $B=\partial \xi$, we obtain a strong convergence result for solving (47) in real Banach spaces.

Theorem 3. Let $E_{1}$ be a p-uniformly convex and uniformly smooth Banach space and $E_{2}$ be a uniformly convex smooth Banach space. Let $\vartheta$ and $\xi$ be proper, lower semicontinuous, and convex functions of $E_{1}$ into $(-\infty,+\infty]$ and $E_{2}$ into $(-\infty,+\infty]$ such that $(\partial \vartheta)^{-1} 0 \neq \varnothing$ and $(\partial \xi)^{-1} 0 \neq \varnothing$, respectively. Let $T: E_{1} \rightarrow E_{2}$ be a bounded linear operator such that $T \neq 0$ and let $T^{*}$ be the adjoint operator $T$. Suppose that $\Omega \neq \varnothing$. For fixed $x_{0} \in E_{1}$, let $\left\{x_{n}\right\}_{n=0}^{\infty}$ be iteratively generated by $x_{1} \in E_{1}$ and

$$
\left\{\begin{array}{l}
w_{n}=J_{E_{1}^{*}}^{q}\left[J_{E_{1}}^{p} x_{n}+\theta_{n} J_{E_{1}}^{p}\left(x_{n}-x_{n-1}\right)\right] \\
v_{n}=\arg \min _{y \in E_{2}}\left\{\xi(y)+\frac{1}{\mu_{n}}\|y\|^{2}-\frac{1}{\mu_{n}}\left\langle y, J_{E_{2}}^{p} T w_{n}\right\rangle\right\} \\
z_{n}=J_{E_{1}^{*}}^{q}\left[J_{E_{1}}^{p}\left(w_{n}\right)-\rho_{n} \frac{f^{p-1}\left(w_{n}\right)}{\| T^{*}\left(J_{E_{2}}^{p}\left(T w_{n}-v_{n}\right) \|^{p}\right)} T^{*} J_{E_{1}}^{p}\left(T w_{n}-v_{n}\right)\right] \\
u_{n}=\arg \min _{x \in E_{1}}\left\{\vartheta(x)+\frac{1}{\sigma_{n}}\|x\|^{2}-\frac{1}{\sigma_{n}}\left\langle x, J_{E_{2}}^{p} z_{n}\right\rangle\right\} \\
y_{n}=J_{E_{1}}^{q}\left(\alpha_{n} J_{E_{1}}^{p} z_{n}+\left(1-\alpha_{n}\right) J_{E_{1}}^{p} u_{n}\right) \\
C_{n+1}=\left\{u \in C_{n}: \Delta_{p}\left(y_{n}, u\right) \leq \Delta_{p}\left(z_{n}, u\right) \leq \Delta_{p}\left(w_{n}, u\right)\right\} \\
x_{n+1}=\Pi_{C_{n+1}} x_{0}
\end{array}\right.
$$

where $\left\{\sigma_{n}\right\},\left\{\mu_{n}\right\} \subset(0,+\infty), \Pi_{C_{n+1}}$ is a Bregman projection of $E_{1}$ onto $C_{n+1}$, the sequence of real number $\left\{\alpha_{n}\right\} \subset$ $[a, b] \subset(0,1)$ and $\left\{\theta_{n}\right\} \subset[c, d] \subset(-\infty,+\infty), f\left(w_{n}\right):=\frac{1}{p}\left\|T w_{n}-v_{n}\right\|^{p}$, and $\left\{\rho_{n}\right\} \subset(0,+\infty)$ satisfies

$$
\liminf _{n \rightarrow+\infty} \rho_{n}\left(p-C_{q} \frac{\rho_{n}^{q-1}}{q}\right)>0 .
$$

where $c_{q}$ is the uniform smoothness coefficient of $E_{1}$. Then, $x_{n} \rightarrow z_{0} \in(\partial \vartheta)^{-1} 0 \cap T^{-1}\left((\partial \xi)^{-1} 0\right)$, where $z_{0}:=\Pi_{(\partial \vartheta)^{-1} 0 \cap T^{-1}\left((\partial \xi)^{-1} 0\right)} x_{0}$ 
Proof. We know from [43] that

$$
v_{n}=\arg \min _{y \in E_{2}}\left\{\xi(y)+\frac{1}{2 \mu_{n}}\|y\|^{2}-\frac{1}{\mu_{n}}\right\}\left\langle y, J_{E_{2}}^{p} T w_{n}\right\rangle
$$

is equivalent to

$$
0 \in(\partial \xi) x_{n}+\frac{1}{\mu_{n}} J_{E_{2}}^{p} x_{n}-\frac{1}{\mu_{n}} J_{E_{2}}^{p} T w_{n}
$$

From this, we have $J_{E_{2}}^{p} T w_{n} \in J_{E_{2}}^{p} v_{n}+\mu_{n}(\partial \xi) v_{n}$ i.e., $v_{n}=Q_{r_{n}} T w_{n}$. Similarly, we have that

$$
u_{n}=\arg \min _{x \in E_{1}}\left\{\vartheta(x)+\frac{1}{2 \sigma_{n}}\|x\|^{2}-\frac{1}{\sigma_{n}}\left\langle x, J_{E_{1}}^{p} z_{n}\right\rangle\right\}
$$

is equivalent to $u_{n}=R_{\lambda_{n}} z_{n}$. Using Theorem 1 , we get the conclusion.

\subsection{Application to Equilibrium Problem}

Let $C$ be a nonempty closed and convex subset of a Banach space $E$ and let $G: C \times C \rightarrow \mathbb{R}$ be a bifunction. For solving the equilibrium problem, we assume that $G$ satisfies the following conditions:

(A1) $G(x, x)=0, \forall x \in C$.

(A2) $G$ is monotone, i.e., $G(x, y)+G(y, x) \leq 0$ for any $x, y \in C$.

(A3) $G$ is upper-hemicontinuous, i.e., for each $x, y, z \in C$,

$$
\limsup _{t \rightarrow 0^{+}} G(t z+(1-t) x, y) \leq G(x, y) .
$$

(A4) $G(x, 0)$ is convex and lower semicontinuous for each $x \in C$.

The equilibrium problem is to find $x^{*} \in C$ such that

$$
G\left(x^{*}, y\right) \geq 0 \text { for all } y \in C .
$$

The set of solution of this problem is denoted by $E P(G)$.

Lemma 7. [44] Let $g: E \rightarrow(-\infty,+\infty$ ] be super coercive Legendre function, $G$ be a bifunction of $C \times C$ into $\mathbb{R}$ satisfying Conditions (A1)-(A4), and $x \in E$. Define a mapping $S_{G}^{g}: E \rightarrow C$ as follows:

$$
S_{G}^{g}(x)=\{z \in C: G(z, y)+\langle y-z, \nabla g(z)-\nabla g(x)\rangle \geq 0 \text { for all } y \in C\} .
$$

Then,

(i) $\operatorname{dom}_{G}^{g}=E$.

(ii) $S_{G}^{g}$ is single-valued.

(iii) $S_{G}^{s}$ is a Bregman firmly nonexpansive operator.

(iv) The set of fixed point of $S_{G}^{f}$ is the solution set of the corresponding equilibrium problem, i.e., $F\left(S_{G}^{g}\right)=E P(G)$.

(v) $\operatorname{EP}(G)$ is closed and convex.

(vi) For all $x \in E$ and for all $u \in F\left(S_{G}^{g}\right)$, we have

$$
D_{g}\left(u, S_{G}^{g}(x)\right)+D_{g}\left(S_{G}^{g}(x), x\right) \leq D_{g}(u, x) .
$$


Proposition 3. [45] Let $g: E \rightarrow(-\infty,+\infty$ ] be a super coercive Legendre Frécht differentiable and totally convex function. Let $C$ be a closed and convex subset of $E$ and assume that the bifunction $G: C \times C \rightarrow \mathbb{R}$ satisfies the Conditions (A1)-(A4). Let $A_{G}$ be a set-valued mapping of E into $2^{E^{*}}$ defined by

$$
A_{G}(x)=\left\{\begin{array}{l}
\left\{z \in E^{*}: G(x, y) \geq\langle y-x, z\rangle \text { for all } y \in C\right\}, \quad x \in C \\
\varnothing, \quad x \in E-C .
\end{array}\right.
$$

Then, $A_{G}$ is a maximal monotone operator, $\operatorname{EP}(G)=A_{G}^{-1}(0)$ and $S_{G}^{g}=R_{A_{G}}^{g}$.

Let $E_{1}$ and $E_{2}$ real Banach spaces and $C$ and $Q$ be nonempty, closed, and convex subsets of $E_{1}$ and $E_{2}$, respectively. Let $G_{1}: C \times C \rightarrow \mathbb{R}$ and $G_{2}: Q \times Q \rightarrow \mathbb{R}$ be bifunctions satisfying Conditions (A1)-(A4) and $T: E_{1} \rightarrow E_{2}$ be a bounded linear operator. We consider the Split Equilibrium Problem (SEP) defined by: Find $x \in C$ such that

$$
x \in \operatorname{EP}\left(G_{1}\right) \text { and } \quad T x \in E P\left(G_{2}\right) .
$$

The SEP was introduced by Moudafi [46] and has been studied by many authors for Hilbert and Banach spaces (see, e.g., [47-50]). We denote the set of solution of (49) by $\operatorname{SEP}\left(G_{1}, G_{2}\right)$.

Setting $A=A_{G_{1}}$ and $B=A_{G_{2}}$ in Algorithm (20), Lemma 7, and Proposition 3, we obtain a strong convergence result for solving SEP in real Banach spaces.

Theorem 4. Let $E_{1}$ be a p-uniformly convex and uniformly smooth Banach space, $E_{2}$ be a uniformly smooth Banach space, and $C$ and $Q$ be nonempty closed subsets of $E_{1}$ and $E_{2}$, respectively. Let $G: C \times C \rightarrow \mathbb{R}$ and $H: Q \times Q \rightarrow \mathbb{R}$ be bifunctions satisfying Conditions (A1)-(A4) and $g: E_{1} \rightarrow \mathbb{R}$ and $h: E_{2} \rightarrow \mathbb{R}$ be super coercive Legendre functions which are bounded, uniformly Frechet differentiable, and totally convex on bounded subset of $E_{2}$. Let $T: E_{1} \rightarrow E_{2}$ be a bounded linear operator with $T \neq 0$ and $T^{*}: E_{2}^{*} \rightarrow E_{1}^{*}$ be the adjoint of $T$. Suppose that $\operatorname{SEP}\left(G_{1}, G_{2}\right) \neq \varnothing$ for fixed $x_{0} \in E_{1}$, let $\left\{x_{n}\right\}_{n=0}^{\infty}$ be iteratively generated by $x_{1} \in E_{1}$, and

$$
\left\{\begin{array}{l}
w_{n}=J_{E_{1}^{*}}^{q}\left[J_{E_{1}}^{p} x_{n}+\theta_{n} J_{E_{1}}^{p}\left(x_{n}-x_{n-1}\right)\right], \\
z_{n}=J_{E_{1}^{*}}^{q}\left[J_{E_{1}}^{p}\left(w_{n}\right)-\rho_{n} \frac{f^{p-1}\left(w_{n}\right)}{\| T^{*}\left(J_{E_{2}}^{p}\left(T w_{n}-S_{H_{n}}^{h} T w_{n}\right) \|^{p}\right)} T^{*} J_{E_{1}}^{p}\left(T w_{n}-S_{H_{n}}^{h} T w_{n}\right)\right] \\
y_{n}=J_{E_{1}}^{q}\left(\alpha_{n} J_{E_{1}}^{p} z_{n}+\left(1-\alpha_{n}\right) J_{E_{1}}^{p} S_{G_{n}}^{g} z_{n}\right), \\
C_{n+1}=\left\{u \in C_{n}: \Delta_{p}\left(y_{n}, u\right) \leq \Delta_{p}\left(z_{n}, u\right) \leq \Delta_{p}\left(w_{n}, u\right)\right\} \\
x_{n+1}=\Pi_{C_{n+1}} x_{0}
\end{array}\right.
$$

where $\left\{H_{n}\right\}$ and $\left\{G_{n}\right\} \subset(0,+\infty), f\left(w_{n}\right)=\frac{1}{p}\left\|\left(I-S_{H_{n}}^{h}\right) T u_{n}\right\|^{p}, \Pi_{C_{n+1}}$ is a Bregman projection of $E_{1}$ onto $C_{n+1}$, the sequence of real number $\left\{\alpha_{n}\right\} \subset[a, b] \subset(0,1)$ and $\left\{\theta_{n}\right\} \subset[c, d] \subset(-\infty,+\infty)$, and $\left\{\rho_{n}\right\} \subset(0,+\infty)$ satisfies

$$
\liminf _{n \rightarrow+\infty} \rho_{n}\left(p-C_{q} \frac{\rho_{n}^{q-1}}{q}\right)>0
$$

where $c_{q}$ is the uniform smoothness coefficient of $E_{1}$. Then, $x_{n} \rightarrow z_{0} \in \Pi_{S E P\left(G_{1}, G_{2}\right)} x_{0}$.

\section{Conclusions}

In this paper, we introduce a new inertial shrinking projection method for solving the split common null point problem in uniformly convex and uniformly smooth real Banach spaces. The algorithm is designed such that its step size does not require prior knowledge of the norm of the bounded linear operator. A strong convergence result is also proved under some mild 
conditions. We further provide some applications of our result to other nonlinear optimization problems. We highlight our contributions in this paper as follow:

1. A significant improvement in this paper is that a self-adaptive technique is introduced for selecting the step size such that a strong convergence result is proved without prior knowledge of the norm of the bounded linear operator. This improves the results in $[6,8,9,11,12,16,19,20]$ and other important results in this direction.

2. The result in this paper extends the results in $[4,5,10,11]$ and several other results on solving split common null point problem from real Hilbert spaces to real Banach spaces.

3. The strong convergence result in this paper is more desirable in optimization theory (see, e.g., [51]).

Author Contributions: Conceptualization, C.C.O.; methodology, C.C.O. and L.O.J.; software, C.C.O. and L.O.J.; validation, C.C.O., L.O.J. and R.N.; formal analysis, C.C.O.and L.O.J.; writing-original draft preparation, C.C.O. and L.O.J.; writing-review and editing, C.C.O., L.O.J. and R.N.; supervision, L.O.J.; project administration, C.C.O.; funding acquisition, L.O.J. All authors have read and agreed to the published version of the manuscript.

Funding: This research is funded by the Mathematical research fund at the Sefako Makgatho Health Sciences University.

Conflicts of Interest: The authors declare no conflict of interest.

\section{References}

1. Byrne, C.; Censor, Y.; Gibali, A.; Reich, S. The split common null point problem. J. Nonlinear Convex Anal. 2012, 13, 759-775.

2. Bruck, R.E.; Reich, S. Nonexpansive projections and resolvents of accretive operators in Banach spaces. Houst. J. Math. 1977, 3, 459-470.

3. Censor, Y.; Elfving, T. A multiprojection algorithm using Bregman projections in a product space. Numer. Algorithms 1994, 8, 221-239. [CrossRef]

4. Censor, Y.; Segal, A. The split common fixed-point problem for directed operators. J. Convex Anal. 2009, $16,587-600$.

5. Muodafi, A. The split common fixed point problem for demicontractive mappings. Inverse Probl. 2010, 26, 055007. [CrossRef]

6. Alofi, A.S.; Alsulami, S.M.; Takahashi, W. Strongly convergent iterative method for the split common null point problem in Banach spaces. J. Nonlinear Convex Anal. 2016, 17, 311-324.

7. Alsulami, S.M.; Takahashi, W. The split common null problem for maximal monotone mappings in Hilbert spaces and applications in Banach spaces. J. Nonlinear Convex Anal. 2014, 15, 793-808.

8. Takahashi, W. The split common null point problem in Banach space. Arch. Math. 2015, 104, 357-365. [CrossRef]

9. Suantai, S.; Shehu, Y.; Cholamjiak, P. Nonlinear iterative methods for solving the split common null point problem in Banach spaces. Optim. Method Softw. 2019, 34, 853-874. [CrossRef]

10. Kazmi, K.R.; Rizvi, S.H. An iterative method for split variational inclusion problem and fixed point problem for a nonexpansive mapping. Optim. Lett. 2014, 8, 1113-1124. [CrossRef]

11. Jailoka, P.; Suantai, S. Split null point problems and fixed point problems for demicontractive multivalued mappings. Meditter. J. Math. 2018, 15, 204. [CrossRef]

12. Sitthithakerngkiet, K.; Deepho, J.; Kumam, P. Convergence analysis of a general iterative algorithm for finding a common solution o split variational inclusion and optimization problems. Numer. Algorithms 2018, 79, 801-824. [CrossRef]

13. Dong, Q.L.; He, S.; Zhao, J. Solving the split equality problem without prior knowledge of operator norms. Optimization 2015, 64, 1887-1906. [CrossRef]

14. Zhao, J. Solving split equality fixed-point problem of quasi-nonexpansive mappings without prior knowledge of operators norms. Optimization 2015, 64, 2619-2630. [CrossRef]

15. Zhao, J.; Zhang, H. Solving split common fixed-point problem of firmly quasi-nonexpansive mappings without prior knowledge of operators norms. Abstr. Appl. Anal. 2014, 389689

16. Takahashi, W. The split common null point problem in two Banach spaces. J. Nonlinear Convex Anal. 2015, $16,2343-2350$. 
17. Takahashi, S.; Takahashi, W. The split common null point problem and the shrinking projection method in Banach spaces. Optimization 2016, 65, 281-287. [CrossRef]

18. Suantai, S.; Srisap, K.; Naprang, N.; Mamat, M.; Yundon, V.; Cholamjiak, P. Convergence Theorems for finding split common null point problem in Banach spaces. Appl. Gen. Topol. 2017, 18, 345-360. [CrossRef]

19. Takahashi, W. The split common null point problem for generalized resolvents in two banach spaces. Numer. Algorithms 2017, 75, 1065-1078. [CrossRef]

20. Takahashi, W. The split feasibility problem in Banach spaces. Nonlinear Convex Anal. 2014, 15, 1349-1355.

21. Polyak, B.T. Some methods of speeding up the convergence of iteration methods. USSR Comput. Math. Phys. 1964, 4, 1-17. [CrossRef]

22. Alvarez, F.; Attouch, H. An inertial proximal method for maximal monotone operators via discretization of a nonlinear oscillator with damping. Set Valued Anal. 2001, 9, 3-11. [CrossRef]

23. Attouch, H.; Peypouquet, J.; Redont, P. A dynamical approach to an inertial forward-backward algorithm for convex minimization. SIAM J. Optim. 2014, 24, 232-256. [CrossRef]

24. Bot, R.I.; Csetnek, E.R.; Hendrich, C. Inertial Douglas-Rachford splitting for monotone inclusion. Appl. Math. Comput. 2015, 256, 472-487.

25. Bot, R.I.; Csetnek, E.R. An inertial alternating direction method of multipliers. Minimax Theory Appl. 2016, 1, 29-49.

26. Bot, R.I.; Csetnek, E.R. An inertial forward-backward-forward primal-dual splitting algorithm for solving monotone inclusion problems. Numer. Algebra 2016, 71, 519-540. [CrossRef]

27. Dong, Q.L.; Yuan, H.B.; Cho, Y.J.; Rassias, T.M. Modified inertial Mann algorithm and inertial CQ-algorithm for nonexpansive mappings. Optim. Lett. 2018, 12, 87-102. [CrossRef]

28. Cholamjiak, W.; Pholasa, N.; Suantai, S. A modified inertial shrinking projection method for solving inclusion problems and quasi nonepansive multivalued mappings. Comput. Appl. Math. 2018, 34, 5750-5774. [CrossRef]

29. Cioranescu, I. Geometry of Banach Spaces, Duality Mappings and Nonlinear Problems; Kluwer: Doradrecht, The Netherlands, 1990; Volume 62.

30. Kuo, L.-W.; Sahu, D.R. Bregman distance and strong convergence of proximal-type algorithms. Abstr. Appl. Anal. 2003, 2003, 590519. [CrossRef]

31. Xu, H.K. Inequalities in Banach spaces with applications. Nonlinear Anal. 1991, 16, 1127-1138. [CrossRef]

32. Shehu, Y.; Ogbuisi, F.; Iyiola, O. Convergence analysis of an iterative algorithm for fixed point problems and split feasibility problems in certain Banach spaces. Optimization 2016, 65, 299-323. [CrossRef]

33. Aoyama, K.; Kohsaka, F.; Takahashi, W. Three generalizations of firmly nonexpansive mappings: Their relations and continuity properties. J. Nonlinear Convex Anal. 2009, 10, 131-147.

34. Browder, F.E. Nonlinear maximal monotone operators in Banach space. Math. Ann. 1968, 175, 89-113. [CrossRef]

35. Dunford, N.; Schwartz, J.T. Linear Operators I; Willey Interscience: New York, NY, USA, 1958.

36. Takahashi, W. Nonlinear Functional Analysis; Yokohama Publishers: Yokohama, Japan, 2000.

37. Rockafellar, R.T. On the maximality of sums of nonlinear monotone operators. Trans. Am. Math. Soc. 1970, 149, 75-88. [CrossRef]

38. Muodafi, A.; Thakur, B.S. Solving proximal split feasibility problems without prior knowledge of operator norms. Optim. Lett. 2014, 8, 2099-2110. [CrossRef]

39. Shehu, Y.; Cai, G.; Iyiola, O.S. Iterative approximation of solutions for proximal split feasibility problems. Fixed Point Theory Appl. 2015, 2015, 123. [CrossRef]

40. Shehu, Y.; Ogbuisi, F.U. Convergence analysis for proximal split feasibility problems and fixed point problems. J. Appl. Math. Comput. 2015, 48, 221-239. [CrossRef]

41. Pant, R.; Okeke, C.C.; Izuchukwu, C. Modified viscosity implicit rules for proximal split feasibility problem. J. Appl. Math. Comput. 2020. [CrossRef]

42. Yao, Y.; Yao, Z.; Abdou, A.; Cho, Y. Self-adaptive algorithms for proximal split feasibility problems and strong convergence analysis. Fixed Point Theory Appl. 2015, 2015, 205. [CrossRef]

43. Barbu, V. Nonlinear Semigroups and Differential Equations in Banach Spaces. Editura Acad. R. S. R., Bucuresti; Springer: Berlin/Heidelberg, Germany, 1976.

44. Reich, S.; Sabach, S. Two strong convergence Theorems for a proximal method in reflexive Banach spaces. Numer. Funct. Anal. Optim. 2010, 31, 22-44. [CrossRef] 
45. Sabach, S. Products of finitely many resolvents of maximal monotone mappings in reflexive banach spaces. SIAM J. Optim. 2011, 21, 1289-1308. [CrossRef]

46. Moudafi, A. Split monotone variational inclusions. J. Optim. Theory Appl. 2011, 150, 275-283. [CrossRef]

47. Kazmi, K.R.; Rizvi, S.H. Iterative approximation of a common solution of a split equilibrium problem, a variational inequality problem and a fixed point problem. J. Egypt. Math. Soc. 2013, 21, 44-51. [CrossRef]

48. Jolaoso, L.O.; Oyewole, O.K.; Okeke, C.C.; Mewomo, O.T. A unified algorithm for solving split generalized mixed equilibrium problem and fixed point of nonspreading mapping in Hilbert space. Demonstr. Math. 2018, 51, 211-232. [CrossRef]

49. Jolaoso, L.O.; Karahan, I. A general alternative regularization method with line search technique for solving split equilibrium and fixed point problems in Hilbert space. Comput. Appl. Math. 2020, 30. [CrossRef]

50. Okeke, C.C.; Jolaoso, L.O.; Isiogugu, F.O.; Mewomo, O.T. Solving split equality equilibrium and fixed point problems in Banach spaces without prior knowledge of operator norm. J. Nonlinear Convex Analy. 2019, 20, 661-683.

51. Bauschke, H.H.; Combettes, P.L. A weak-to-strong convergence principle for Féjer-monotone methods in Hilbert spaces. Math. Oper. Res. 2001, 26, 248-264. [CrossRef]

Publisher's Note: MDPI stays neutral with regard to jurisdictional claims in published maps and institutional affiliations.

(C) 2020 by the authors. Licensee MDPI, Basel, Switzerland. This article is an open access article distributed under the terms and conditions of the Creative Commons Attribution (CC BY) license (http://creativecommons.org/licenses/by/4.0/). 


\title{
Article \\ Nonlinear Approximations to Critical and Relaxation Processes
}

\author{
Simon Gluzman \\ Materialica+ Research Group, Bathurst St. 3000, Apt. 606, Toronto, ON M6B 3B4, Canada; gluz@sympatico.ca \\ Received: 5 September 2020; Accepted: 22 October 2020; Published: 28 October 2020

Abstract: We develop nonlinear approximations to critical and relaxation phenomena, complemented by the optimization procedures. In the first part, we discuss general methods for calculation of critical indices and amplitudes from the perturbative expansions. Several important examples of the Stokes flow through 2D channels are brought up. Power series for the permeability derived for small values of amplitude are employed for calculation of various critical exponents in the regime of large amplitudes. Special nonlinear approximations valid for arbitrary values of the wave amplitude are derived from the expansions. In the second part, the technique developed for critical phenomena is applied to relaxation phenomena. The concept of time-translation invariance is discussed, and its spontaneous violation and restoration considered. Emerging probabilistic patterns correspond to a local breakdown of time-translation invariance. Their evolution leads to the time-translation invariance complete (or partial) restoration. We estimate the typical time extent, amplitude and direction for such a restorative process. The new technique is based on explicit introduction of origin in time as an optimization parameter. After some transformations, we arrive at the exponential and generalized exponential-type solutions (Gompertz approximants), with explicit finite time scale, which is only implicit in the initial parameterization with polynomial approximation. The concept of crash as a fast relaxation phenomenon, consisting of time-translation invariance breaking and restoration, is advanced. Several COVID-related crashes in the time series for Shanghai Composite and Dow Jones Industrial are discussed as an illustration.

Keywords: critical index; relaxation time; time-translation invariance breaking and restoration; market crash; COVID-19; Gompertz approximants

\section{Introduction}

Let the function $\Phi(x)$ of a real variable $x \in[0, \infty)$ be defined by some rather complicated problem. The variable $x>0$ can represent, e.g., a coupling constant or concentration of particles. Of course, one should strive to find an exact solution to the problem [1,2]. Among such exact solutions one can find the solution to the celebrated Kondo problem and its thermodynamics. In a number of cases important for optical applications, such as Bessel beams and its generalizations [3], one can find an intriguing physics already within the linear wave equation. In optics, there are a variety of exact solutions: spatial, temporal, dark optical solitons and breathers all follow from the celebrated nonlinear Schrödinger equation and its modifications [4]. The so-called spatiotemporal $X$-waves, another type of the closed-form solutions are being studied as well (see, e.g., [5]).

What if such a problem does not allow for an explicit solution for the sought function? Let us assume that some kind of perturbation theory is still possible to develop, so that it generates formal power series about the point $x=x_{0}=0, \Phi(x)=\sum_{n=0}^{\infty} c_{n} x^{n}$, for the function in question [6]. The perturbation methods can generate the series (often slowly) convergent for all $x$ smaller than the radius of convergence, or the series divergent for all $x$, except $x=0$. 
That is, for smooth function $\Phi(x)$ [7], we have the asymptotic power series [7,8],

$$
\Phi(x) \sim \sum_{n=0}^{\infty} c_{n} x^{n}
$$

Our task is to recast the series (2) into some convergent expressions by means of a nonlinear analytical constructs, the so-called approximants. When literally all of the terms in divergent series are known, one can invoke Euler of Borel summation [8]. Even for convergent series there is still a problem of how to continue the expansion outside of radius of convergence [9], where the approximants could be useful.

However, in realistic problems, only a few terms on the RHS of (1) can be calculated, and applying various approximants is the only available analytical option for the truncated series (5) and (A6). The approximants are conditioned to be asymptotically equivalent to the series (1), truncated at some finite number $k$. However, the approximants are able to generate an additional infinite number of coefficients, approximating unknown exact coefficients. Determination of the best approximant is grounded solely on the empirical, numerical convergence [9], of the sequences of approximants.

One can always attempt to extrapolate the perturbative results by means of the Padé approximants $P_{M, N}(x)[6,9]$. The Padé approximants $P_{M, N}$ can be understood as the ratio of two polynomials $P_{M}(x)$ and $Q_{N}(x)$ of the order $M$ and $N$, respectively. The diagonal Padé approximant of order $N$ corresponds to the case of $M=N$. Conventionally, $Q_{N}(0)=1$. The coefficients of the polynomials are derived directly from the asymptotic equivalence with the given power series for the sought function $\Phi(x)$. Sometimes, when there is a need to stress the role of $\Phi(x)$, we write Pade Approximant $[\Phi[x], n, m]$.

The Padé approximant might possess a pole associated with a finite critical point, but can only produce an integer critical index. While usually critical indices are not integers. The same concerns the large-variable behavior where the power of $x$ produced from extrapolation with some form of Padé approximants is always an integer. Unfortunately, solutions to many problems exhibit irrational functional behavior. Such a behavior cannot be properly described by the standard rational Padé approximants. However, it would be highly desirable to modify somehow the familiar technique of Padé approximants in order to take into account the irrational behavior. Such modification can be performed by separating the sought modification of the Padé approximants into two factors [10]. The first factor is to be expressed as an iterated root or factor approximant [11,12]. It is specifically designed to take care of the irrational part of the solution. The second factor is simply a diagonal Padé approximant, and it is supposed to take care of the rational part of the solution. We arrive thus to the corrected Padé approximants. They appear to be applicable to a larger class of problems, even when the standard Padé technique is not applicable [11].

Many examples of application of the Padé approximants as well as their theoretical modifications, can be found in [13], including some important applications to aerodynamics and boundary layer problems [14]. The so-called two-point Padé is applied for interpolation, when in addition to the expansion about $x_{0}=0$, given by (1), additional information is available and contained in the asymptotic power series expansion about $x=\infty, \Phi(x) \sim \sum_{n=0}^{\infty} b_{n} x^{-n}$ [8]. The two-point Padé approximant has the same form as the standard Padé approximant, but with the coefficients expressed through $c_{n}$ and $b_{n}$.

The idea of combining information coming from the different limits appear to be fruitful and can be exploited for different types of approximants and various forms of asymptotic expansions $[11,12,15,16]$. Various self-similar approximants also allow extrapolating and interpolating between the small-variable and large-variable asymptotic expansions, as discussed recently in [16]. The key to the success is to introduce the so-called control functions to allow "to sew" the two limit-cases together in the form most natural for each concrete problem [11,12,15-17]. The example of such an approach is brought up in Appendix B. Although the expansions for small and large couplings are very bad, the resulting approximants are in a good agreement with the numerical data. 
There are four main technical approaches to the approximants constructions, all aimed to optimize their performance. The first approach is conventional, also called accuracy-through order. It is based on progressive improvement of quality of approximants with adding new information through the higher-order coefficients, with the approximants becoming more and more complex. It is exemplified in construction of Pad'e and Euler super-exponential approximants [8], factor, root and additive approximants $[11,12,16]$. The latter "cluster" of approximations was derived based on the ideas of self-similar approximation theory, a close relative of the field-theoretic renormalization group [17]. The property of self-similarity is discussed in Section 3.1.

The second approach leads to corrected approximants. The idea is to ensure the correct form of the solution already in the starting approximation with some initial parameters. The initial parameters should be corrected by asymptotically matching with the truncated series/polynomial regressions in increasing orders. Thus, instead of increasing the order of approximation, one can correct the parameters of the initial approximation [11,12]. The form of the solution is not getting more complex, but the parameters take more and more complex form with increasing order.

In the third approach, predominantly adopted in Section 3, we keep the form and order of approximants the same in all orders, but let the series/regressions evolve into higher orders. Independent on the order of regression, we construct the same approximant, based on the first-order terms solely, only with the parameters changing with increasing order of regression. In the framework of such effective first-order theories, we employ exponential approximants and their extensions.

In the fourth approach, the critical index is treated as a vital part of optimization procedure. The critical index plays the role of a control parameter, to be determined from the optimization procedure described in Section 2.2, following Gluzman and Yukalov [18]. Different optimization techniques based on introduction of control parameters were proposed in $[19,20]$.

The problems arising in approximation theory can vary. Note that, for a recovery problem, when measurements of the sought function are given for some finite set of points, there is Prony's method available, with the sought function represented as sums of polynomial or exponential functions combined with periodic functions [21]. For approximation of a continuous function on the interval $x \in[0,1]$, one can use Bernstein polynomials [22]. However, the two methods do not allow for inclusion of the asymptotic information.

Prony's and Bernstein methods are numerical and work only for interpolation problems. The latter method was further adapted to the region $x \in[0, \infty)$, and applied in [23]. The technique of Cioslowski [23] allows for incorporation of the asymptotic information. The technique of self-similar roots [24] allows us to solve the same problems as in [23], but without resorting to fitting [23,24].

Our methods are analytical, user-friendly and applicable to the most difficult extrapolation problem $[11,12,16]$, involving explicit calculation of various critical indices and amplitudes, with novel applications to finding relaxation times. However, our methods remain applicable also for various interpolation problems $[11,12,16,24]$ (see also Appendix B).

It is likely impossible to find the same approximant to be the best for each and every realistic problem. Based on the same asymptotic information, such as series coefficients, thresholds, critical indices, and correction to scaling indices, one can construct not only Padé but quite a few different approximants, such as corrected Padé, additive, DLog-additive, etc. [12,16]. It is feasible that for each problem one can find an optimal different approximant. We think that the idea behind the method of corrected approximants $[11,12,16]$, is the most progressive, since it allows to combine the strength of a few methods together and proceed, in the space of approximations, with piece-wise construction of the approximation sequences, as pointed out recently by Gluzman [16].In the following sections, we present a more expended description of the concept of approximants, applied now both to critical and relaxation phenomena, extending the earlier work of Chapter 1 of the book [12]. 


\section{Critical Index and Relaxation Time}

The function $\Phi(x)$ of a real variable $x$ exhibits critical behavior, with a critical index $\alpha$, at a finite critical point $x_{c}$, when

$$
\Phi(x) \simeq A\left(x_{c}-x\right)^{\alpha}, \text { as } x \rightarrow x_{c}-0 .
$$

The definition covers the case of negative index when function can tend to infinity, or the sought function can tend to zero if the index is positive. Sometimes, the values of critical index and critical point are known from some sources, and the problem consists in finding the critical amplitude $A$, as extensively exemplified in [11].

The case when critical behavior occur at infinity,

$$
\Phi(x) \simeq A x^{\alpha}, \text { as } x \rightarrow \infty,
$$

can be analyzed similarly. It can be understood as the particular case with the critical point positioned at infinity.

Critical phenomena are ubiquitous [18], ranging from the field theory to hydrodynamics. It is vital to explain related critical indices theoretically. Regrettably, for realistic physical systems, one can as a rule learn only its behavior at small variable,

$$
\Phi(x) \simeq \Phi_{k}(x) \text {, as } x \rightarrow 0,
$$

which follows form some perturbation theory. The function $\Phi_{k}(x)$ is approximated by an expansion

$$
\Phi_{k}(x)=1+\sum_{n=1}^{k} c_{n} x^{n} .
$$

Most often one finds that such expansions give numerically divergent results, valid only for very small or very large $x$ (see Appendix B). Constructively, the expansion is treated as a polynomial of the order $k$. Sometimes, theoretically, one even has a convergent series, resulting in a rather good numerically convergent, truncated polynomial approximations (A6). However, there is still a problem of extrapolating outside of the region of numerical convergence, where the critical behavior sets in. Three examples of such type are given in Appendix A, based on the results of Chapter 7 of the book [12].

The discussion below traces the basic ideas from Chapter 1 of the book [12]. One can always express the critical index directly by using its definition, and find it as the limit of explicitly expressed approximants. For instance, critical index can be estimated from a standard representation as the following derivative

$$
\mathcal{B}_{a}(x)=\partial_{x} \log (\Phi(x)) \simeq \frac{-\alpha}{x_{c}-x}
$$

as $x \rightarrow x_{c}$, thus defining the critical index as the residue in the corresponding single pole. The pole corresponds to the critical point $x_{c}$. The critical index corresponds to the residue

$$
\alpha=\lim _{x \rightarrow x_{c}}\left(x-x_{c}\right) \mathcal{B}_{a}(x)
$$

To the $D L o g$-transformed series $\mathcal{B}_{a}(x)$ one is bound to apply the Padé approximants [6]. Moreover, the whole table of Padé approximants can be constructed [9], That is, the DLog Padé method does not lead to a unique algorithm for finding critical indices. procedure. Basically, different values are produced by different Padé approximants. Then, it is not clear which of these estimates to prefer. The standard approach consists in applying a diagonal Padé approximants [6]. 
When a function, at asymptotically large variable, behaves as in (3), then the critical exponent can be defined similarly, by means of the $D \log$ transformation. It is represented by the limit

$$
\alpha=\lim _{x \rightarrow \infty} x \mathcal{B}_{a}(x)
$$

Assume that the small-variable expansion for the function $B_{a}(x)$ is given. In order for the critical index to be finite, it is necessary to take only the approximants behaving as $x^{-1}$ as $x \rightarrow \infty$. It leaves us no choice but to select the non-diagonal $P_{n, n+1}(x)$ approximants, so that the corresponding approximation $\alpha_{n}$ is finite. One can also apply, in place of Padé, some different approximants $[12,16]$. The examples of application of the DLog Padeé methods are given in Appendix A, based on the results first obtained in Chapter 1 of the book [12].

To simplify and standardize calculations different, and more powerful, approximants, called self-similar factor approximants, are introduced in [25]. The singular solutions emerging from factor approximants correspond to critical points and phase transitions [25], including also the case of singularity located at $\infty$. When the series is long, one would expect that the accuracy is going to improve with increasing numbers of terms. Sometimes, an optimum is achieved for some finite number of terms, reflecting the asymptotic nature of the underlying series. It is very difficult to improve the quality of results produced by the factor approximants, when the series are short. Some suggestions on such improvement were advanced by Gluzman [12].

In some simple but rather important cases of ODEs, the factor approximants allow to restore exact solutions, such a bell soliton, kink soliton, logistic equation solution and instanton-type solution [26]. However, as pointed out in the Introduction, such cases are quite special, and only an approximate solution could be found in many important cases [26,27]. More information about various methods of calculating critical index, amplitude and critical point can be found in $[11,12,16]$.

\subsection{Relaxation Time}

Consider the case of relaxation behavior when a function at asymptotically large variable decays as

$$
\Phi(t) \simeq A \exp \left(\frac{t}{\tau}\right) \quad(t \rightarrow \infty)
$$

with negative $\tau$. Formally, the relaxation time is $-\tau$. It can be found as the limit

$$
\frac{1}{\tau}=\lim _{t \rightarrow \infty} \frac{d}{d t} \ln \Phi(t)
$$

As in the case of critical behavior considered above, the small-variable expansion for the function is given by the sum $\Phi_{k}(t)$. The effective relaxation time can be expressed in terms of the small-variable expansion as follows,

$$
\frac{1}{\tau_{k}(t)}=\frac{d}{d t} \ln \Phi_{k}(t)
$$

It can be expanded in powers of $t$, leading to

$$
\tau_{k}(t)=\sum_{n=0}^{k} b_{n} t^{n} .
$$

The coefficients $b_{n}$ are easily expressed through $c_{n}$ of the original series (1). Let us apply to the obtained expansion the self-similar or Padé approximants, That is, we have to derive an approximant $\tau_{k}^{*}(t)$ whose limit

$$
\tau_{k}^{*}(t) \rightarrow \text { const } \quad(t \rightarrow \infty)
$$


gives the relaxation time

$$
\tau_{k}^{*}=\lim _{t \rightarrow \infty} \tau_{k}^{*}(t)
$$

In such approach, the amplitude $A$ does not enter the consideration. In practice, one can indeed construct the approximants with such required behavior. The complete approximant for the sought function $\Phi(t)$ denoted below as $E(t, r)$, can be constructed as well. Even some ad hoc forms satisfying some general symmetry requirements can be suggested, as in Section 3.

As an illustration, let us find $\tau_{k}^{*}(t)$ in explicit form under some simple assumptions concerning its asymptotic behaviors. Assume simply that there are two distinct exponential behaviors for short and long times with two different $\tau_{1}, \tau_{2}$, and the transition from short to long time behavior also occurs at the duration of some third characteristic time $\tau_{3}=-\beta_{3}^{-1}$. The characteristic times can be found from the short-time expansion. The simple approximation to the effective relaxation time, expressed in second order of (12), can be written down in the spirit of Yukalov and Gluzman [28] as follows:

$$
\tau_{2}^{*}(t)^{-1}=\beta_{2}+\left(\beta_{1}-\beta_{2}\right) \exp \left(\beta_{3} t\right)
$$

so that for negative $\beta_{3}$ we have $\tau_{2}^{*}(0)^{-1}=\beta_{1}, \tau_{2}^{*}(\infty)^{-1}=\beta_{2}$.

In the theory of reliability, the failure (hazard) rate or mortality force [29] is analogous to the inverse effective relaxation time, and the model of the type of formula (13) is known as the Gompertz-Makeham law of mortality.

The complete approximant corresponding to (13) is reconstructed after elementary integration

$$
F(t)=A \exp \left(\frac{\left(\beta_{1}-\beta_{2}\right) \exp \left(\beta_{3} t\right)}{\beta_{3}}+\beta_{2} t\right),
$$

with all unknown constituents of (13) expressed explicitly, from the asymptotic equivalence with the power-series,

$$
\begin{aligned}
& A=c_{0} \exp \left(\frac{\left(c_{1}{ }^{2}-2 c_{0} c_{2}\right)^{3}}{4\left(3 c_{0}^{2} c_{3}-3 c_{0} c_{1} c_{2}+c_{1}\right)^{2}}\right), \quad \beta_{1}=\frac{c_{1}}{c_{0}}, \quad \beta_{2}=\frac{6 c_{0}^{2} c_{1} c_{3}-4 c_{0}^{2} c_{2}^{2}-2 c_{0} c_{1}^{2} c_{2}+c_{1}^{4}}{2 c_{0}\left(3 c_{0} c_{3}-3 c_{0} c_{1} c_{2}+c_{1}^{3}\right)}, \\
& \beta_{3}=\frac{2\left(3 c_{0}{ }^{2} c_{3}-3 c_{0} c_{1} c_{2}+c_{1}{ }^{3}\right)}{c_{0}\left(2 c_{0} c_{2}-c_{1}{ }^{2}\right)} .
\end{aligned}
$$

Most interesting, as $\beta_{2}=0$ the linear decay (growth) term in the formula for $F(t)$ disappears, we arrive in different notations to the Gompertz function (54),

$$
G(t)=A \exp \left(\frac{\beta_{1} \exp \left(\beta_{3} t\right)}{\beta_{3}} t\right),
$$

employed in calculations of Gluzman [30]. In this case, we have the effective relaxation time decaying (growing) exponentially with time.In Section 3, we apply this method of finding the effective relaxation time for time series.

\subsection{Critical Index as Control Parameter. Optimization Technique}

The function's critical behavior follows from extrapolating the asymptotic expansion (1) to finite or large values of the variable. Such an extrapolation can be accomplished by means of a direct technique just discussed above. However, its successful application requires knowledge of a large number of terms in the expansion. However, it is also possible to obtain rather good estimates for the critical indices from a small number of terms in the asymptotic expansion [12,18]. To this end, we can employ the self-similar root approximants given by (17). The external power $m_{k}$ is to be determined here from additional conditions. More detailed explanations and more examples can be found in the book [12]. 
The self-similar root approximant has the following general form [15],

$$
\mathcal{R}_{k}^{*}\left(x, m_{k}\right)=\left(\left(\left(1+\mathcal{P}_{1} x\right)^{m_{1}}+\mathcal{P}_{2} x^{2}\right)^{m_{2}}+\ldots+\mathcal{P}_{k} x^{k}\right)^{m_{k}} .
$$

In principle, all the parameters may be found from asymptotic equivalence with a given power series.

The large-variable power $\alpha$ in Equation (3) could be compared with the large-variable behavior of the root approximant (17),

$$
\mathcal{R}_{k}^{*}\left(x, m_{k}\right) \simeq A_{k} x^{k m_{k}}
$$

where

$$
A_{k}=\left(\left(\left(\mathcal{P}_{1}^{m_{1}}+\mathcal{P}_{2}\right)^{m_{2}}+\mathcal{P}_{3}\right)^{m_{3}}+\ldots+\mathcal{P}_{k}\right)^{m_{k}} .
$$

This comparison yields the relation $k m_{k}=\alpha$, defining the external power $m_{k}=\frac{\alpha}{k}$, when $\alpha$ is known. This way of defining the external power is used when the root approximants are applied for interpolation. The root approximants (17) are applied in Appendix B, in the context of interpolation problem, for construction of accurate formulas valid for all values of $x$.

Consider an exceptionally difficult situation of an extrapolation problem: the large-variable behavior of the function is not known and $\alpha$ is not given. In addition, the critical behavior can happen at a finite value $x_{c}$ of the variable $x$. The method for calculating the critical index $\alpha$ by employing the self-similar root approximants was developed by Gluzman and Yukalov [18].

In such approach, we construct several root approximants $R_{k}^{*}\left(x, m_{k}\right)$, and the external power $m_{k}$ plays the role of a control function. The sequence of approximants is considered as a trajectory of a dynamical system. The approximation order $k$ plays the role of discrete time. A discrete-time dynamical system or the approximation cascade consists of the sequence of approximants. The cascade velocity is defined by Euler discretization formula [31-33]

$$
V_{k}\left(x, m_{k}\right)=\mathcal{R}_{k+1}^{*}\left(x, m_{k}\right)-\mathcal{R}_{k}^{*}\left(x, m_{k}\right)+\left(m_{k+1}-m_{k}\right) \frac{\partial}{\partial m_{k}} \mathcal{R}_{k}^{*}\left(x, m_{k}\right) .
$$

The effective limit of the sequence of approximants corresponds to the fixed point of the cascade. Based on just a few approximants, the cascade velocity has to decrease. In such a sense, the sequence appears to be convergent. The control functions $m_{k}=m_{k}(x)$, have to minimize the absolute value of the cascade velocity

$$
\left|V_{k}\left(x, m_{k}(x)\right)\right|=\min _{m_{k}}\left|V_{k}\left(x, m_{k}\right)\right| .
$$

A finite critical point $x_{k}^{c}$, in the $k$ th approximation, is to be obtained from Equation (17) by imposing the condition on the critical behavior expressed by (2),

$$
\left[\mathcal{R}_{k}^{*}\left(x_{k}^{c}, m_{k}\right)\right]^{1 / m_{k}}=0 \quad\left(0<x_{k}^{c}<\infty\right) .
$$

Its finite solution is denoted as $x_{k}^{c}=x_{k}^{c}\left(m_{k}\right)$.

The critical index in the $k$ th approximation is given by the limit

$$
\alpha_{k}=\lim _{x \rightarrow x_{k}^{c}} m_{k}(x)
$$

In the case of the critical behavior at infinity, when $x_{c} \sim \infty$, the critical index is

$$
\alpha=k \lim _{x \rightarrow \infty} m_{k}(x), \text { as } x_{c} \sim \infty .
$$


Thus, to find the critical indices, the control functions $m_{k}(x)$ have to be found. The minimization of the cascade velocity (50) is complicated. Equation (21) contains two control functions, $m_{k+1}$ and $m_{k}$. Nevertheless, the problem can be resolved.

This can be done in two ways. The first constructive approach notices that $m_{k+1}$ should be close to $m_{k}$. Then, we arrive to to the minimal difference condition

$$
\min _{m_{k}}\left|\mathcal{R}_{k+1}^{*}\left(x, m_{k}\right)-\mathcal{R}_{k}^{*}\left(x, m_{k}\right)\right| \quad(k=1,2, \ldots) .
$$

One should typically find a solution $m_{k}=m_{k}(x)$ of the simpler equation

$$
\mathcal{R}_{k+1}^{*}\left(x, m_{k}\right)-\mathcal{R}_{k}^{*}\left(x, m_{k}\right)=0 .
$$

The control functions $m_{k}$, characterizing the critical behavior of $\Phi(x)$, become the numbers $m_{k}\left(x_{c}\right)$. We simply write $m_{k}=m_{k}\left(x_{c}\right)$.

In the vicinity of a finite critical point, the function $\mathcal{R}_{k}^{*}$ behaves as

$$
\mathcal{R}_{k}^{*}\left(x, m_{k}\right) \simeq\left(1-\frac{x}{x_{k}^{c}}\right)^{m_{k}} \text {, as } x \rightarrow x_{k}^{c}-0 .
$$

The condition (25) is expressed as follows,

$$
x_{k+1}^{c}\left(m_{k}\right)-x_{k}^{c}\left(m_{k}\right)=0 \quad\left(0<x_{k}^{c}<\infty\right) .
$$

For the critical behavior at infinity, it is expedient to introduce the control function

$$
\mathrm{s}_{k}=k m_{k}
$$

The large-variable behavior reads as

$$
\mathcal{R}_{k}^{*}\left(x, \mathrm{~s}_{k}\right) \simeq A_{k}\left(\mathrm{~s}_{k}\right) x^{\mathrm{s}_{k}}, \text { as } x \rightarrow \infty
$$

As a result, the minimal difference condition is reduced to the equation

$$
A_{k+1}\left(\mathrm{~s}_{k}\right)-A_{k}\left(\mathrm{~s}_{k}\right)=0 \text {, as } x_{k}^{c} \sim \infty \text {. }
$$

The alternative equation for the control functions also follows from the minimal velocity condition (21), and is called the minimal derivative condition

$$
\min _{k}\left|\frac{\partial}{\partial m_{k}} \mathcal{R}_{k}^{*}\left(x, m_{k}\right)\right| \quad(k=1,2, \ldots),
$$

In practice, we have to solve the equation

$$
\frac{\partial}{\partial m_{k}} \mathcal{R}_{k}^{*}\left(x, m_{k}\right)=0 .
$$

To apply this condition, we have first to extract from the function its non-divergent parts. If the critical point is finite, one can study the residue of the function $\partial \log \mathcal{R}_{k}^{*} / \partial m_{k}$, expressed as

$$
\lim _{x \rightarrow x_{k}^{c}}\left(x_{k}^{c}-x\right) \frac{\partial}{\partial m_{k}} \log \mathcal{R}_{k}^{*}\left(x, m_{k}\right)=m_{k} \frac{\partial x_{k}^{c}}{\partial m_{k}} .
$$


Thus, from Equation (32), we arrive to the condition

$$
\frac{\partial x_{k}^{c}}{\partial m_{k}}=0 \quad\left(0<x_{k}^{c}<\infty\right) .
$$

When the critical behavior occurs at infinity, then we can consider the limiting form of the amplitude and reduce Equation (32) to the form

$$
\frac{\partial A_{k}\left(s_{k}\right)}{\partial s_{k}}=0, \text { as } x_{k}^{c} \sim \infty .
$$

The final estimate for the critical index is given by a simple average of the minimal difference and minimal derivative results.

The technique reviewed in Section 2.2, following Chapter 1 of the book [12], turned out to be useful in calculating the critical properties of the classical analog of the graphene-type composites with varying concentration of vacancies [34].

In the next subsection, we give some examples, first presented in Chapter 1 of the book [12]. More information and details can also be found in Chapter 7 of the book [12].

\subsection{Examples: Permeability in the Two-Dimensional Channels}

In the cases considered below, we deal with a unique theoretical opportunity to attack the problem of critical exponent and criticality in general, directly from the solution of the hydrodynamic Stokes problem. Let us consider as example the case of the two-dimensional channel bounded by the surfaces $z= \pm b(1+\epsilon \cos x)$, as explained in Appendix A. Here, $\epsilon$ is termed waviness.

The permeability behaves critically [12], That is, it tends to zero as

$$
K(\epsilon) \sim\left(\epsilon_{\mathcal{C}}-\epsilon\right)^{\varkappa}, \text { as } \epsilon \rightarrow \epsilon_{C}-0
$$

with $\epsilon_{c}=1, \varkappa=\frac{5}{2}$. The permeability as a function of the waviness can be derived in the form of an expansion in powers of $\epsilon$ [35]. In the particular case of $b=0.5$, the permeability can be found explicitly as

$$
K(\epsilon) \simeq 1-3.14963 \epsilon^{2}+4.08109 \epsilon^{4}, \text { as } \epsilon \rightarrow 0 .
$$

By setting $\epsilon_{c}=1$, and changing the variable $y=\frac{\epsilon^{2}}{1-\epsilon^{2}}$, one can move the critical point to infinity.

The critical index is calculated as explained above and in [18]. From the minimal-difference condition we find $\varkappa_{1}=2.184$, with an error $12.6 \%$. From the minimal derivative condition, we obtain $\varkappa_{2}=2.559$, with an error $2.37 \%$. The final answer $\varkappa^{*}$ is given by the average of two solutions $\varkappa^{*}=2.372 \pm 0.19$.

In another particular case considered in Chapter 1 of the book [12], for $b=0.25$, the permeability expands as follows,

$$
K(\epsilon) \simeq 1-3.03748 \epsilon^{2}+3.54570 \epsilon^{4}, \text { as } \epsilon \rightarrow 0 .
$$

Setting $\epsilon=1$, and using the same technique as above the approximations for critical index are found, so that $\varkappa_{1}=2.342$, and $\varkappa_{2}=2.743$. Finally, $\varkappa^{*}=2.543 \pm 0.2$.

Let us also consider some examples of the numerical convergence of root approximants in high-orders, first presented in Chapter 1 of the book [12]. The technique is applied for calculating critical index $\varkappa$. It seems instructive to consider the same two cases of permeability $K(\epsilon)$, but with higher-order terms, up to 16th order inclusively.

The numerical form of the corresponding expansions can be found in Appendix A (see expansions (A8) and (A14)). Concretely, we construct the iterated root approximants

$$
\mathcal{R}_{k}^{*}(y)=\left(\left(\left(\left(1+\mathcal{P}_{1} y\right)^{2}+\mathcal{P}_{2} y^{2}\right)^{3 / 2}+\mathcal{P}_{3} y^{3}\right)^{4 / 3}+\ldots+\mathcal{P}_{k} y^{k}\right)^{\alpha / k}
$$


The parameters $\mathcal{P}_{j}$ have to be found from the asymptotic equivalence with the expansions. The permeability has the required critical asymptotic forms

$$
\mathcal{R}_{k}^{*}(y) \simeq A_{k} y^{\alpha}, \text { as } y \rightarrow \infty
$$

The amplitudes $A_{k}=A_{k}\left(\alpha_{k}\right)$ are found explicitly as

$$
A_{k}=\left(\left(\left(\mathcal{P}_{1}^{2}+\mathcal{P}_{2}\right)^{3 / 2}+\mathcal{P}_{3}\right)^{4 / 3}+\ldots+\mathcal{P}_{k}\right)^{\alpha / k}
$$

To define the critical index $\alpha_{k}$, we analyze the differences

$$
\Delta_{k n}\left(\alpha_{k}\right)=A_{k}\left(\alpha_{k}\right)-A_{n}\left(\alpha_{k}\right)
$$

From the sequences $\Delta_{k n}=0$, we find the related sequences of approximate values $\alpha_{k}$ for the critical indices.

Although it is possible to investigate different sequences of the conditions $\Delta_{k n}=0$, the most natural from is presented by the sequences of $\Delta_{k, k+1}=0$ and $\Delta_{k 8}=0$, with $k=1,2,3,4,5,6,7$.

The results for $b=\frac{1}{2}$ are shown in Table 1 . We observe good numerical convergence of the approximations $\alpha_{k} \equiv \varkappa_{k}$, to the value $\varkappa=\frac{5}{2}$.

Similar results, presented in Table 2 (for $b=\frac{1}{4}$ ), again demonstrate rather good numerical convergence of the approximate critical indices to the value $\varkappa=\frac{5}{2}$.

Comparison of the results for different parameters $b$ allows us to think that the critical index does not depend on parameter $b$. In both examples considered above, the convergence sets in rather quickly.

The DLog Padé method appears to bring convergent sequences and consistent expressions for permeability as well. Further details can be found in Appendix A. The results obtained from the two different methods well agree with each other. A similar comparison was made by Gluzman and co-authors [34] for the effective conductivity of graphene-type composites.

Table 1. Walls can touch $(b=1 / 2)$. The problems described in Appendices A and A.1. Critical indices for the permeability $\varkappa_{k}$ obtained from the optimization conditions (41). There is rather good numerical convergence to the number $5 / 2$.

\begin{tabular}{ccc}
\hline$\varkappa_{k}$ & $\Delta_{k+1}\left(\varkappa_{k}\right)=0$ & $\Delta_{k 8}\left(\varkappa_{k}\right)=0$ \\
\hline$\varkappa_{1}$ & 2.18445 & 2.39678 \\
\hline$\varkappa_{2}$ & 2.68311 & 2.52028 \\
\hline$\varkappa_{3}$ & 2.48138 & 2.49208 \\
\hline$\varkappa_{4}$ & 2.49096 & 2.49692 \\
\hline$\varkappa_{5}$ & 2.5012 & 2.49982 \\
\hline$\varkappa_{6}$ & 2.49935 & 2.499 \\
\hline$\varkappa_{7}$ & 2.49861 & 2.49861 \\
\hline
\end{tabular}


Table 2. Walls can touch $(b=1 / 4)$. The problems described in the Appendices A and A.2. Critical indices $\varkappa_{k}$ are found from the optimization conditions (41). There is a good numerical convergence of the sequences to the value $5 / 2$.

\begin{tabular}{ccc}
\hline$\varkappa_{k}$ & $\Delta_{k+1}\left(\varkappa_{k}\right)=0$ & $\Delta_{k 8}\left(\varkappa_{k}\right)=0$ \\
\hline$\varkappa_{1}$ & 2.34165 & 2.452 \\
\hline$\varkappa_{2}$ & 2.52463 & 2.50542 \\
\hline$\varkappa_{3}$ & 2.4976 & 2.49933 \\
\hline$\varkappa_{4}$ & 2.49941 & 2.50004 \\
\hline$\varkappa_{5}$ & 2.50028 & 2.50033 \\
\hline$\varkappa_{6}$ & 2.50032 & 2.50036 \\
\hline$\varkappa_{7}$ & 2.50041 & 2.50041 \\
\hline
\end{tabular}

Consider a different case of permeability $K(\epsilon)$ (see Appendixes A and A.3). The results were first obtained in Chapter 1 of the book [12]. For the parallel sinusoidal two-dimensional channel when the walls would not touch, the permeability remains finite. It is expected to decay as a power-law as the waviness $\epsilon$ becomes large,

$$
K(\epsilon) \sim \epsilon^{v}, \text { as } \epsilon \rightarrow \infty,
$$

with negative index $v$.

In the expansion of $K(\epsilon)$ in small parameter $\epsilon^{2}$, we retain the same number of terms as in the previous two examples. The numerical values of the corresponding coefficients can be found in Appendix A ( see expression (A16)). The results of calculations are presented in Table 3 (for $b=\frac{1}{2}$ ). They show rather good numerical convergence, especially in the last column, to the value -4 . The sequence, based on the DLog Padé method, is convergent as well (see Appendixes A and A.3).

Table 3. Walls can not touch. Case of $b=1 / 2$. Critical indices for the permeability for the problems in Appendixes A and A.3, obtained from the optimization conditions $\Delta_{k n}\left(v_{k}\right)=0$. The sequences demonstrate reasonably good numerical convergence to the value $v=-4$.

\begin{tabular}{ccc}
\hline$v_{k}$ & $\Delta_{k+1}\left(v_{k}\right)=\mathbf{0}$ & $\boldsymbol{\Delta}_{k 8}\left(v_{k}\right)=\mathbf{0}$ \\
\hline$v_{1}$ & -6 & -4.36 \\
\hline$v_{2}$ & -4.04 & -4.1 \\
\hline$v_{3}$ & n.a. & -4.13 \\
\hline$v_{4}$ & -4.09 & -4.05 \\
\hline$v_{5}$ & -3.97 & -4.03 \\
\hline$v_{6}$ & n.a. & -4.08 \\
\hline$v_{7}$ & -3.94 & -3.94 \\
\hline
\end{tabular}

More information on the problems of critical permeability, can be found in Appendix A. The three problems considered above are studied by applying the DLog Padé method of Section 2 to calculate the critical index for permeability. The computations complement and confirm the results for critical index, obtained above from the optimization technique. The optimization technique works better for short truncated series, converging more quickly, while the $D \log$ Padé method is easier to apply for very long series. In addition, the DLog Padé method, as well as the Padé method, when its application is appropriate, allows us to compute the critical amplitudes. 


\section{Relaxation Phenomena in Time Series}

For the phenomenon to occur, the basic underlying symmetry must be broken. While studying the phenomenon it is important to distinguish between an explicit symmetry breaking when governing equations are not invariant under the desired symmetry and spontaneous symmetry breaking, without presence of any asymmetric cause [36]. When successful, the approach based on broken global symmetries leads to understanding of the key phenomena of magnetism, superconductivity and superfluidity. On the other hand, when some global inherent symmetry can be recognized in physical quantities, we arrive to the gloriously successful theory of critical phenomena and vital extensions of perturbation results in quantum field theories, jointly called renormalization group (RG) $[17,37]$. In a nutshell, we suggest below how to apply symmetry considerations and RG-inspired methods to the sharp moves which occur in time series, with the most notable examples given by stock market crashes.

Assume that numerical data on the time series variable (e.g., price) $s$ is given for some time $t$ segment. Typically, one considers $N+1$ values $s\left(t_{0}\right), s\left(t_{1}\right) \ldots, s\left(t_{N}\right)$, for $N+1$ given at equidistant successive moments in time $t=t_{j}$, with $j=0,1,2 \ldots, N$ [38].

In the study of time series, one is interested in the extrapolated to future value of $s$. In financial mathematics, one is particularly interested in the predicted value of log return [38,39],

$$
R\left(t_{N}+\delta t\right)=\ln \left(\frac{s\left(t_{N}+\delta t\right)}{s\left(t_{N}\right)}\right) .
$$

One can see from the definition that we are really interested in the quantity $\mathbf{S}=\ln (s)$, to be called return. Let us place the origin at the very beginning of the time interval, setting also $t_{0}=0$. Naturally, one is interested in the value of $\mathbf{S}\left(t_{N}+\delta t\right)$, allowing to find $R\left(t_{N}+\delta t\right)$ at a later time. Since the approach developed in $[30,38]$ is invariant with regard to the time unit choice, we consider temporal points of the dataset as integer, while considering the actual time variable as continuous.

Modern physics when applied to financial theory is concerned with ergodicity violations [40-43]. Ergodicity violations may be understood as a manifestation of a non-stationarity, or violation of time-invariance of random process. Metastable phases in condensed matter also defy ergodicity over long observation timescales. In special quantum systems of ultracold atoms, spontaneous breaking of time-translation symmetry causes the formation of temporal crystalline structures [44]. The concept of a spontaneously broken time-translation invariance can be useful for time series in application to market dynamics, as first suggested in [38]. According to Andersen, Gluzman and Sornette [38], the window of forecasting of time series describing market evolution emerges due to a spontaneous breaking/restoration of the continuous time-translation invariance, dictated by relative probabilities of the evolution patterns [45]. In turn, the probabilities are derived from the stability considerations.

The notion of probability introduced in [45] is not based on the same conventional statistical ensemble probability for a collection of people, but it is closer to the time probability, concerned with a single person living through time (see Gell-Mann and Peters [42] and Taleb [43]). Probabilistic trading patterns correspond to local breakdown of time-translation invariance. Their evolution leads to the time-translation symmetry complete (or partial) restoration. We need to estimate typical time, amplitude and direction for such a restorative process. Thus, we are not confined to a binary outcome as in [38] but attempt to estimate also the magnitude of the event.

According to Hayek [46], markets are mechanisms for collecting vast amounts of information held by individuals and synthesizing it into a useful data point [46,47], e.g., price of the stock market index dependent on time. Conversely, consolidation of knowledge is done via prices and arbitrageurs (Taleb on Hayek).

A catastrophic downward acceleration regime in the time series is known as crash [48]. Time series representing market price dynamics in the vicinity of crisis (crash, melt-up), could be treated as a self-similar evolution, because of the prevalence of the collective coherent behavior of many trading, interacting agents $[45,49]$, including humans and machine algorithms. The dominant 
collective slow mode corresponding to such behavior, develops according to some law, formalized as a time-invariant, self-similar evolution. Away from crisis, there is a superposition of collective coherent mode (generalized trend) and of a stochastic incoherent behavior of the agents $[39,45]$. We do not attempt here to write down a generic evolution equation of behind the time series pertaining to market dynamics. Instead. we consider, locally in time, some trial functions—approximants-in the form inspired by the solutions to some well-known evolution equations. The approximants are designed to respect or violate self-similarity. If in physics the relation of phenomenon and symmetry violation is understood, in econophysics such connection is far from being clear. However, to realize the promise of econophysics [50], on a consistent basis and at par with physics achievements, one has to identify and study the phenomenon from the relevant symmetry viewpoint. Our primary goal here is not forecasting/timing the crash, but studying the crash as a particular phenomenon created by spontaneous, time-translation symmetry breaking/restoration.

Since the market dynamics is believed to be formed by a crowd (herd) behavior of many interacting agents, there are ongoing attempts to create empirical, binary-type prediction markets functioning on such principle, or mini Wall Streets [47]. Prediction markets often work pretty well, however there are many cases when they give wrong prediction or do not make any predictions at all. Such special set-ups are already very useful in reaching understanding that market crowds are correct only if they express a sufficient diversity of opinion. Otherwise, the market crowd can have a collective breakdown, i.e., is fallible, as expected by Soros [48]. In our understanding, such breakdowns amount to breaking of time-translation invariance. Restoration of the time-translation invariance-in theory-may be attributed to a small proportion of the traders having either superior information or market intellect [47]. Data from a survey conducted with high income and institutional investors show that they "generally exaggerated assessments of the risk of a stock market crash, and that these assessments are influenced by the news stories, especially front page stories, that they read" [51]. The division into two (at least) groups can be seen in the very parallel existence of future and spot markets for the same asset, such as S\&P 500 index, with the futures market working $24 \mathrm{~h}$. It is believed that a lot of the daily crashes, or melt-up days, start overnight. It is not that arbitrage is not effective, the spot market is just closed overnight, while the futures market operates in a discovery mode.

\subsection{Self-Similarity and Time Translation Invariance}

According to Isaac Newton and Murray Gell-Mann, the laws of nature are somehow self-similar. The laws of Newtonian mechanics are invariant with respect to the Galilean group, expressing Galileo's principle of relativity [52]. The group includes time-translation invariance, or else the laws of classical mechanics are self-similar.

What should be the underlying symmetry for price dynamics? Mind that in normal times the average price trajectory is exponential, because of the compounding interests, and we enjoy an almost constant return (or price growth rate) [53]. Indeed, let $s_{t_{0}}$ be an underlying security (index) price at $t=t_{0}$. Let $F_{t}^{P}$ be the fair value of the future requiring a risk associated expected return $\beta$ [43]. Then (see, e.g., [43]), expected forward price $F_{t}^{P}=s_{t_{0}} \exp \left(\beta\left(t-t_{0}\right)\right.$. For example, a share of a stock would be correctly priced with the expected return calculated as the return of a risk-free money market fund minus the payout of the asset, being a continuous dividend for a stock [43]. Thus, rather simple and natural exponential estimates are constantly made for stocks and the alike. The formula for the forward price is self-similar, or time-translation invariant, as explained below.

However, as noted in $[48,53]$, prices often significantly deviate from such a simple description. Bubbles can be formed, as well as other presumed patterns of technical analysis. Asset prices strongly deviate from the fundamental value over significant intervals of time. The fundamental value is not truly observable, making definition of such intervals somewhat elusive. There are some very real mechanisms in work, acting to increase and even accelerate the deviation from fundamental value. The causes of deviation could be "option hedging, portfolio insurance strategies, leveraging and margin requirements, imitation and herding behavior", as is the authoritative opinion expressed in [48,53]. 
Recall also that meaningful technical analysis starts from recasting the time series data using some polynomial representation to serve as the expansion [38]. The regression is constructed in standard fashion by minimizing mean-square deviation, with the effective result that the high-frequency component of the price is getting average out. Then, one can consider self-similarity in averages [49]. Indeed, the standard polynomial regressions are invariant under time-translation, retaining their form after arbitrary selection of origin of time with simple redefinition of all parameters. The position of origin in time can be explicitly introduced into the regression formula and included into the coefficients, but actual results of calculations with any arbitrary chosen origin will remain the same. Such property can be expressed as some symmetry.

We put forward the idea that it is the onset of broken time-translation invariance that signifies the birth of a bubble, or of some other temporal pattern preceding a crash. End of pattern corresponds to the restoration of time-translation invariance, partially or fully. Our task is to express this idea in quantitative terms by making explicit transformation from the regression-based technical analysis to the valuation formula in the exponential form, taking into account strong deviations from the standard valuation formulae.

Assume that a time series dynamics is predominantly governed by its own internal laws. This is the same as to write down a self-similar evolution for the marker price $s$ [54], meaning that, for arbitrary shift $\tau$, one can see that

$$
s(t+\tau, a)=s(t, s(\tau, a)),
$$

with the initial condition $s(0, a)=a[55,56]$. The value of the self-similar function $s$ in the moment $t+\tau$ with given initial condition, is the same as in the moment $t$, with the initial condition shifted to the value of $s$ in the moment $\tau$.

When $t$ stands for true time, the property of self-similarity means the time-translation invariance. Formally understood, Equation (43) gives a background for the field-theoretical RG, with addition of some perturbation expansion for the sought quantity, which should be resummed in accordance with self-similarity expressed in the form of ODE [55-57]. The time-translation invariance expressed by (43) means that the law for price evolution exists and remains unchanged with time, with proper transformation of the initial conditions [52]. The role of perturbation expansion when price dynamics is concerned, is accomplished by meaningful technical analysis, by recasting data in the form of some polynomial representation [38]. There is no formal difference in treating polynomials and expansions, as already mentioned in Section 2.

Consider first the simplest case of technical analysis. The linear function can be formally considered as the function of time and initial condition $a$, namely $s_{1}(t, a)=a+b t$, and $s_{1}(0, a)=a$. The linear function (regression) is self-similar, or time-translation invariant, as can be checked directly, by substitution into (43).

Through some standard procedure, let us obtain the linear regression on the data around the origin $t_{0}=0$, so that

$$
s_{0,1}(t)=a_{1}+b_{1} t
$$

Note that the position of origin is arbitrary, and it can be moved to arbitrary position given by real number $r$, so that

$$
s_{r, 1}(t)=A_{1}(r)+B_{1}(r)(t-r),
$$

with new and different coefficients. It turns out that the coefficients are related as follows

$$
A_{1}(r)=a_{1}+b_{1} r, B_{1}(r)=b_{1}
$$

so that

$$
s_{r, 1}(t) \equiv s_{0,1}(t)
$$

By shifting the origin, we create an $r$-dependent form of the linear regression $s_{r, 1}$, which can be used constructively. Thus, instead of a single regression we have its $r$-replicas, equivalent to the original 
form of regression, and all replicas respect time-translation symmetry. In such a sense, one can speak about replica symmetry. Of course, we would like to avoid such redundancy in data parameterization and to find the origin(s) by imposing some optimal conditions (see Section 3.2).

The position of origin in time can be explicitly introduced into the regression formula and included into the coefficients, but actual results of calculations with any arbitrary chosen origin will remain the same. Such property can be expressed as some symmetry. However, intuitively, one would expect that the result of extrapolation with chosen predictors should be dependent on the point of origin $r$. Indeed, various patterns such as "heads and shoulders", "cup-with-handle", " hockey stick", etc., considered by technical analysts do depend on where the point of origin is placed. In physics, the point of origin (Big Bang) plays a fundamental role. We should find a way to break the replica symmetry.

As discussed above, it is exponential shapes that are natural in pricing. Exponential function

$$
E(t, a)=a \exp (\beta t)
$$

with initial condition $a$ and arbitrary $\beta$ satisfy functional self-similarity as well as the linear functions. It can be replicated as

$$
\begin{aligned}
& E_{r}(t)=\alpha(r) \exp (\beta(t-r)), \\
& \alpha(r)=a \exp (\beta r) .
\end{aligned}
$$

Having $\beta$ dependent on $r$ is going to violate the time-translation and replica symmetry. Instead of a global time-translation invariance, we have a set of $r$ local "laws" near each point of origin. However, having $r$ in Formula (44) fixed, by imposing some additional condition, or just being integrated out, should restore the global time-translation invariance completely as long as the exponential function is considered. Moreover, stability of the exponential function is measured by the exponential function with the same symmetry (see Formula (46)). Not only is exponential function time-translation invariant, but the expected return $\beta$ has the same property. For exponential functions, the expected (predicted) value of return per unit time exactly equals $\beta$.

Another simple rational function, known as hyperbolic discounting function [58], $H(t, a)=\frac{1}{a^{-1}+b t}$, where $a$ is the initial condition and $b$ is arbitrary, is time-translation invariant. Note that shifted exponential function $E_{s}(t, a)=c+(a-c) \exp (b t)$, with initial condition $a$ and arbitrary $b$ and $c$, is invariant under time-translation as well.

Another interesting symmetry is shape invariance [59], meaning

$$
F_{t+\tau}^{P}=\mathrm{m} F_{t}^{P}
$$

and an exponential function is shape invariant with $\mathrm{m}=\exp (\beta \tau)$, leaving the expected return unchanged. Keep in mind that our task is to calculate $\beta$ from the time series. In principle, one can think about breaking/restoration of shape invariance, as a guide for construction of the concrete scheme for calculations.

A critical phenomenon, an underlying symmetry of the formula for the observable, is scaling

$$
\phi_{\lambda t}=\Lambda \phi_{t}
$$

where $\Lambda=\phi_{\lambda}$. The class of power laws, $\phi_{t}=t^{\alpha}$, with critical index $\alpha$, is scaling-invariant. The central task is to calculate $c$. The statistical renormalization group formulated by Wilson [37] explains well the critical index in equilibrium statistical systems. When information on the critical index is encoded in some perturbation expansion, one can use resummation ideas to extract the index, even for short expansions and for non-equilibrium systems [11,12,18]. Some of the methods are discussed in the preceding section (see also [12,16]).

Working with power-law functions will not leave the return unchanged. However, one can envisage the scheme with broken scaling invariance, as an alternative to the former schemes. The log-periodic solutions extend the simple scaling [60] and are extensively employed in the form of a 
sophisticated seven-parametric fit to long historical dataset [53], as well as of its extensions [61]. The fit is tuned for prediction of the crossover point to a crash, understood as catastrophic downward acceleration regime [48]. However, one cannot exclude the possibility of the solutions with different time symmetries (scaling and time-invariance, for instance) competing to win over, or to coexist, all measured in terms of their stability characteristics.

Our primary concern is the crash per se, not the regime preceding it. We start analyzing crashes with the polynomial approximation that respects time-translation symmetry, have the symmetry broken, and then restored (completely or partially), by means of some optimization. Such sequence ends with a non-trivial outcome: $\beta$ becomes renormalized $\beta(r)$, with $r$ being found using the optimization procedure(s) defined below. We discuss in Section 2.1 a general technique for correcting $\beta$ directly, which accounts for higher order terms in regression, making it time-dependent.

In [38], the framework for technical analysis of time series was developed, based on second-degree regression and asymptotically equivalent exponential approximants, with some rudimentary, implicit breaking of the symmetry. We intend to go to higher-degree regressions and develop a consistent technique for explicit symmetry breaking with its subsequent restoration. According to textbooks, the fourth order should be considered as "high". Taleb (see footnote on p. 53 in [43]) also considered models with five parameters as more than sufficient.

\subsection{Optimization, Approximants, Multipliers}

Higher-order regressions allow for replica symmetry. For instance, the quadratic regression $s_{0,2}(t)=a_{2}+b_{2} t+c_{2} t^{2}$ can be replicated as follows:

$$
s_{r, 2}(t)=A_{2}(r)+B_{2}(r)(t-r)+C_{2}(r)(t-r)^{2}
$$

with

$$
A_{2}(r)=a_{2}+b_{2} r+c_{2} r^{2}, B_{2}(r)=b_{2}+2 c_{2} r, C_{2}(r)=c_{2} .
$$

With such transformed parameters, we find that $s_{r, 2}(t) \equiv s_{0,2}(t)$. In fact, one can still formulate self-similarity analogous to (43), but in vector form with increased number of parameters/initial conditions in place of $a$ [57]. However, if only the linear part of quadratic regression, or trend, is taken into account, we return to the conventional functional self-similarity $\equiv$ time-translation invariance, discussed above extensively.

Such effective linear/trend approach to higher-order regressions allows applying the same idea at all orders and observe how the exponential structures change with increasing regression order. Note that, in the course of trading, a common pattern is trend following, which appears to be a collective, self-reinforcing motion that, intuitively, lends itself to a self-similar description. Indeed, some participants are waiting for a market confirmation of the trend before acting on it, which in turn acts as a confirmation for others. Having a universal model explaining this dynamics (if not predicting it) would be quite useful.

To take into account the dependence on origin, the replica symmetry has to be broken. Breaking of the symmetry means the dependence on origin of actual extrapolations with non-polynomial predictors. As the primary predictors, we suggest the simplest exponential approximants considered as the function of origin $r$ and time,

$$
E_{1}^{*}(t, r)=A(r) \exp \left(\frac{B(r)}{A(r)}(t-r)\right),
$$

independent on the order of polynomial regression. The approximants (45) are constructed by requiring an asymptotic equivalence with the linear part of chosen polynomial regression. If the extrapolations $E_{1}^{*}\left(t_{N}+\delta t, r\right)$ are made by each of the approximants, they appear to be different for various $r$, meaning breaking of the replica symmetry and of the time-translation symmetry. Passage from polynomials to exponential functions leads to emergence of the continuous spectrum of relaxation (growth) times. 
To compare the approximants quality, one can look at their stability. Stability of the approximants is characterized by the so-called multipliers defined as the variation derivative of the function with respect to some initial approximation function [45]. Following Yukalov and Gluzman [62], one can take the linear regression as zero approximation and find the multiplier

$$
M_{1}^{*}(t, r)=\exp \left(\frac{B(r)}{A(r)}(t-r)\right) .
$$

The simple structure of multipliers (46) allows avoiding appearance of spurious zeroes which often complicate analysis with more complex approximants/multipliers.

Because of the multiplicity of solutions, embodied in their dependence on origin, it is both natural and expedient to introduce probability for each solution. As explained in [45], one can introduce

$$
\text { Probability } \propto\left|M_{1}^{*}(t, r)\right|^{-1},
$$

with proper normalization, as shown below in Formula (48). Probability appears to be of a pure dynamic origin and is expressed only from the time series itself. When the approximants and multipliers of the first order are applied to the starting terms of the quadratic, third- or fourth-order regression, we are confined to effective first-order models, with velocity parameter from [38] dependent also on higher-order coefficients and origin.

To make extrapolation with approximants (45), one has still to know the origin. In other words, the time-translation symmetry has to be restored completely or partially, so that a specific predictor with specifically selected origin, or as close as possible to a time-translation invariant form, is devised. Fixing unique origin also selects unique relaxation (growth) time, during which the price is supposed to find a time-translation invariant state.

Exponential functions are chosen above because they are invariant under time translation. Any shift in origins is absorbed by the pre-exponential amplitude and does not influence the return $R$. A similar in spirit view that broken symmetries have to be restored in a correct theory was expressed by Duguet and Sadoudi [63].

In the approach predominantly adopted in this section, we keep the form and order of approximants the same in all orders, but let the series/regressions evolve into higher orders. Independent of the order of regression, we construct the same approximant, based only on the first-order terms, only with parameters changing with increasing order of regression. In the framework of the effective first-order theories, we employ exponential approximants.

Consider the value of origin as an optimization parameter [30]. To find it and restore the time-translation symmetry, we have to impose an additional condition directly on the exponential predictors with known last closing price,

$$
E_{1}^{*}\left(t_{N}, r\right)=s_{N}
$$

One has to solve the latter equation to find the particular origin(s) $r=r^{*}$. In this case, we consider a discrete spectrum of origins, consisting of several isolated values. To avoid double-counting when the last closing price enters both regression and optimization, one can determine the regression parameters in the segment limited from above by $t_{N-1}, s_{N-1}$. Alternatively, one can consider the two ways to define regression parameters and choose the one which leads to more stable solutions. Unless otherwise stated, we consider that such comparison was performed and the most stable way was selected.

The extrapolation for the price is simply $s\left(t_{N}+\delta t\right)=E_{1}^{*}\left(t_{N}+\delta t, r^{*}\right)$. The condition imposed by Equation (47) is natural, because then a first-order approximation to Formula (42), $R \approx \frac{s\left(t_{N}+\delta t\right)-s\left(t_{N}\right)}{s\left(t_{N}\right)}$, is recovered (see, e.g., [39]), as one would expect intuitively. 
The procedure embodied in (47), leads to a radical reduction of the set of $r$-predictors to just a few. Set of predictors and corresponding to each multiplier, define the probabilistic, poor man's order book. Instead of an unknown to us true numbers of buy and sell orders, we calculate a priori probabilities for the price going up or down and corresponding levels. Target price is estimated through weighted averaging developed in [45,62], in its concrete form (48) given below.

For the sake of uniqueness, one can simply choose the most stable result among such conditioned predictors. One can also consider extrapolation with a weighted average of all such selected solutions. With $1 \leq M \leq 6$ solutions, their weighted average $\bar{E}_{1}$ for the time $t_{N}+\delta t$ is given as follows,

$$
\bar{E}_{1}\left(t_{N}+\delta t\right)=\frac{\sum_{k=1}^{M} E_{1}^{*}\left(t_{N}+\delta t, r_{k}^{*}\right)\left|M_{1}^{*}\left(t_{N}+\delta t, r_{k}^{*}\right)\right|^{-1}}{\sum_{k=1}^{M}\left|M_{1}^{*}\left(t_{N}+\delta t, r_{k}^{*}\right)\right|^{-1}} .
$$

Within the discrete spectrum, we can find solutions with varying degrees of adherence to the original data. They can follow data rather closely or be loosely defined by the parameters of regression. The former could be called "normal" solutions, and tend to be less stable, with multipliers $\sim 1$, but the latter are "anomalous" solutions, since they cut through the data and typically are the most stable with small multipliers. Anomalous solutions are crashes (meltdowns) and melt-ups. The typical situation with the solutions in the discrete spectrum is presented in Figure 1. The novel feature introduced through (48) is that averaging is performed over all approximants of the same order, compatible with constraints expressed by (47).

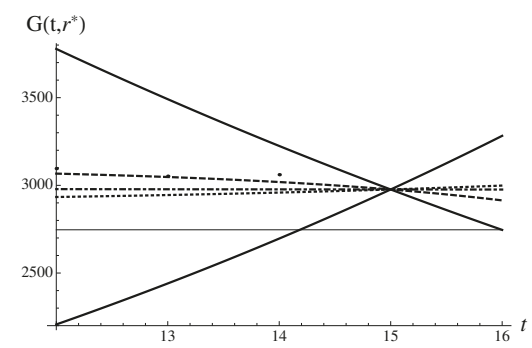

Figure 1. All Gompertz approximants corresponding to the discrete spectrum, i.e., solutions to (56) are shown. The most stable downward and less stable upward solutions are shown with solid lines. Three additional solutions are shown as well. The solution shown with the dashed line is closest to the data. The "no-change", practically flat solution, is shown with a dot-dashed line. Another solution, corresponding to moderate growth, is shown with a dotted line. The level $s_{16}=2746.61$ is shown with black line. Several historical data points are shown as well.

One can also integrate out the dependence on origin $r$, considered as a continuous variable, by applying an averaging technique of weighted fixed points suggested in [45]. The dependence on origin enters the integration limit through parameter $T$. Integration can be performed numerically for the simplest exponential predictors according to the formula

$$
I(t, T)=\frac{\int_{t_{0}-T}^{t_{N}+T} E_{1}^{*}(X, t)\left|M_{1}^{*}(X, t)\right|^{-1} d X}{\int_{t_{0}-T}^{t_{N}+T}\left|M_{1}^{*}(X, t)\right|^{-1} d X}
$$

To optimize the integral, we have to impose an additional condition on the weighted average/integral. It is natural to force it to pass precisely through the last historical point.

$$
I\left(t_{N}, T\right)=s\left(t_{N}\right),
$$


and solve the latter equation to find the integration limit $T=T^{*}$. The sought extrapolation value for the price $s$ is simply $I\left(t_{N}+\delta t, T^{*}\right)$. We prefer to take into account the broadest possible region of integration. Under such conditions, if and when the solution to (50) exists, it is unique. The value of $s_{N}$ may enter the consideration twice: in the regression parameters and in the optimization condition (50). To avoid counting twice the last known value $s_{N}$, one can slightly different definition

$$
I(t, T)=\frac{\int_{t_{0}-T}^{t_{N-1}+T} E_{1}^{*}(X, t)\left|M_{1}^{*}(X, t)\right|^{-1} d X}{\int_{t_{0}-T}^{t_{N-1}+T}\left|M_{1}^{*}(X, t)\right|^{-1} d X} .
$$

As an additional condition to find origin, one can also consider the minimal difference requirement on the lowest order predictors, as first suggested in [49]. Such approach is analogous to the technique discussed in Section 2.2. However, instead of a critical index, we calculate relaxation time. To this end, one has to construct the second order super-exponential approximant

$$
\begin{aligned}
& E_{2}^{*}(t, r)=A(r) \exp \left(\frac{B(r)(t-r) \exp \left(\frac{C(r)(t-r) \tau(r)}{B(r)}\right)}{A(r)}\right), \\
& \tau(r)=1-\frac{B(r)^{2}}{2 A(r) C(r)},
\end{aligned}
$$

and minimize its difference with the simplest exponential approximant in the time of interest $t_{N}+\delta t$. Namely, one has to find all roots of the equation

$$
\exp \left(\frac{C(r) \tau(r)\left(t_{N}+\delta t-r\right)}{B(r)}\right)=1,
$$

with respect to real variable $r$. Corresponding multiplier

$$
M_{2}^{*}(t, r)=\frac{1}{B(r)} \frac{\partial E_{2}^{*}(t, r)}{\partial t},
$$

can be found as well.

The discrete spectrum optimization seems to be the most natural and transparent. Our goal is to find the approximants and probabilistic distributions in the last available historical point of time series. Crashes are attributed to the stable solutions with large negative $r$, meaning that the origin of time has to be moved to the deep past to explain the crash in near future. Preliminary results of Gluzman [30] suggest that, in the overwhelming majority of cases, a crash is preceded by similar, asymmetric probability pattern(s), of the type shown in the figures below. As noted in [51], Kahneman and Tversky explained that people tend to judge current events by their similarity to memories of representative events.

There are also additional solutions with multipliers of the order of unity, coming from the region of moderate $r$, and it is often possible to find some rather stable upward solution for large positive $r$. One can think that, for such stable time series as describing population dynamics, only the region of moderate $r$ gives relevant solutions, while for time series describing price dynamics all types of solutions may exist simultaneously.

Within our approach to constructing approximants, one can also try to exploit the second order terms in regression. Instead of exponential approximants, one should try some other, higher-order approximants, but with time-translation invariance property. Such approximants are presented below. They are considered ad hoc, because they can be written in closed form only in special, low-order situations. It is not feasible to extend them systematically into arbitrary high order. Hence, our interest in special forms with desired symmetry. Sometimes, it is even not possible to find stable solutions with a single approximant, but it is still possible with corrected approximants. 
Recall that exponential function can be obtained as the solution to simple linear first-order ODE. In the search for second-order approximants with time-translation invariance, we turned to some explicit formulas, emerging in the course of solving some first-order ODE with added nonlinear term with arbitrary positive power, which generalizes ODE for simple exponential growth. It is known as Bertalanffy-Richards (BR) growth model $[64,65]$. Among its solutions in the case of second-order nonlinear term, there is a celebrated logistic function [64],

$$
L(t)=\frac{1}{q_{2}+\frac{\left(1-q_{1} q_{2}\right) \exp \left(-q_{0} t\right)}{q_{1}}},
$$

where $q_{1}$ is the initial condition. The logistic function is widely used to describe population growth phenomena and is also known to be the solution to the logistic equation of growth. The logistic function written in the form $L\left(t, q_{1}\right)$, dependent on the initial condition $L\left(0, q_{1}\right)=q_{1}$, with arbitrary $q_{0}, q_{2}$, is time-translation invariant. One can also introduce the second-order logistic approximant which generalizes logistic function [30]. In addition to describing situations with saturation at infinity, the logistic approximant include also the case of so-called finite-time singularity, which makes it redundant, since such solutions were axiomatically excluded from the price dynamics [38].

Another solution to the BR model in the case when the nonlinear term has power only slightly differing from unity, is known as Gompertz function [64],

$$
G(t)=g_{0} \exp \left(g_{1} \exp \left(g_{2} t\right)\right)
$$

used to describe growth (relaxation, decay) phenomena. However, as we demonstrate in Section 2.1, it is possible to explain $G(t)$ directly from the resummation technique leading to Formula (16), without resorting to BR. Relaxation (growth) time behaves exponentially with time. The Gompertz function is log-time-translation invariant.

One can consider the second order Gompertz approximant. It simply generalizes the Gompertz function. Namely, one can find Gompertz approximant in the following form

$$
\begin{aligned}
& G(t, r)=g_{0}(r) \exp \left(g_{1}(r) \exp \left(g_{2}(r)(t-r)\right)\right), \\
& g_{0}(r)=A(r) e^{-g_{1}(r)}, \quad g_{1}(r)=\frac{B(r)}{A(r) g_{2}(r)}, \quad g_{2}(r)=\frac{2 A(r) C(r)-B(r)^{2}}{A(r) B(r)},
\end{aligned}
$$

with the multiplier

$$
M_{G}(t, r)=\frac{g_{0}(r) g_{1}(r) g_{2}(r) e^{\left(g_{1}(r) e^{g_{2}(r)(t-r)}+g_{2}(r)(t-r)\right)}}{B(r)}
$$

The Gompertz approximant, of course, is not limited to the situations with saturation at infinity, as it can also describe very fast decay (growth) at infinity.

With $r$ to be found from some optimization procedure, the return $R$ generated by Gompertz approximant is time-translation invariant and has a compact form

$$
R(\delta t)=g_{1}(r) \exp \left(g_{2}(r)\left(t_{N}-r\right)\right)\left(\exp \left(g_{2}(r) \delta t\right)-1\right)
$$

For small $\delta t$, it becomes particularly transparent:

$$
R(\delta t) \approx g_{1}(r) g_{2}(r) \exp \left(g_{2}(r)\left(t_{N}-r\right)\right) \times \delta t \equiv \frac{\delta t}{\tau\left(T_{N}, r\right)},
$$

with the pre-factor giving the return per unit time. The inverse return per unit time has the physical meaning of the effective time for growth (relaxation)

$$
\beta(t, r)^{-1} \equiv \tau(t, r)=\left(g_{1}(r) g_{2}(r)\right)^{-1} \exp \left(g_{2}(r)(r-t)\right),
$$


considered at the moment $t=T_{N}$. Here, we employ the the effective relaxation (growth) time (see Section 2.1),

$$
\tau(t)=\left(\frac{d}{d t} \ln G(t)\right)^{-1},
$$

and replicate it. We find that the return for Gompertz approximant is solely determined by relaxation time

$$
\mathbf{S}(t, r)=\frac{1}{\tau(t, r)},
$$

allowing to express the log return in a compact form

$$
R(\delta t)=\mathbf{S}\left(t_{N}+\delta t, r\right)-\mathbf{S}\left(t_{N}, r\right)
$$

Thus, the return for Gompertz approximant appears as purely dynamic quantity, not involving any consent about equilibrium, fundamental value, etc. If relaxation time is found from the data to be very large as it should be close to equilibrium conditions [66], we have no potential for returns, i.e., near-equilibrium yields dull, everyday mundane events that are repetitive and lend themselves to statistical generalizations [48]. If relaxation time is anticipated to be very short, we have potentially huge returns. The far-from-equilibrium conditions give rise to unique, historic events [48], or to some very fast relaxation events/crashes. The latter condition makes real markets fragile [67].

Gompertz approximant can go at infinity faster or slower than exponential, and in some important examples such differences amounting to a few percent, can be detected. The function $g_{0}(r)$, could be called a gauge function for the price, expressing arbitrariness of choice of the price unit, as it does not enter the return. The time-translation invariance of return and gauge invariance for the price are considered very desirable in price model formulation [38], both properties are pertinent to exponential and Gompertz approximations for the price temporal dynamics.

We are interested in market prices on a daily level, and consider only significant market price drops/crashes with magnitude more than 5.5\%. Such magnitude is selected to be comparable to the typical yearly return of Dow Jones Industrial Average index. Typically, a 2\% daily move is considered as big, but not at the times of various turmoils.

It is widely accepted in practical finance that asset price moves in response to unexpected fundamental information. The information can be identified as well as the tone, positive versus negative. It is found that news arrival is concentrated among days with large return movements, positive or negative [68]. Spontaneously emerging narratives, a simple story or easily expressed explanation of events, might be considered as largely exogenous shocks to the aggregate economy [51]. Simply put, one should analyze what people are talking about in the search for the source of economic fluctuations. Moreover, as in true epidemics governed by evolutionary biology, mutations in narratives spring up randomly, and if contagious generate unpredictable changes in the economy [51]. As noted by Harmon et al. [69], panic on the market can be due to external shocks or self-generated nervousness.

It is argued [70] that cause and effect can be cleanly disentangled only in the case of exogenous shocks, as it is only needed to select some interesting set of shocks to which price is likely to respond. Effects of positive and negative oil price shocks on the stock price need not be symmetric. In macroeconomics, it is even accepted that only positive changes in the price of oil have important effects. Periods dominated by oil price shocks are reasonably easy to identify, and they can indeed be considered as exogenous as well as, often, strong, although difficult to model. Oil price shocks are the leading alternative to monetary shocks and may very well have similar effects [70].

Our goal here is not to forecast/timing the crash, but to study the crash as a particular phenomenon created by spontaneous, time-translation symmetry breaking/restoration. In essence, we ask the following questions:

1. What probabilistic pattern would an observer see the day before crash, 
2. What would be the market reaction (expressed through the index), if we are aware that a Swan of some color has already arrived?

In our opinion, in the presence of a Swan, understood as a shock of unspecified strength, the problem simplifies, because of a reduced set of outcomes, dominated by the most extreme, very stable downward solution. Consider that, in natural sciences, most efforts are dedicated to creating a correct experimental setup. Studying reaction to shock is the only current viable substitute for clean experimental conditions.

\subsection{Examples}

Consider as example a $7.72 \%$ drop in the value of Shanghai Composite index related to the first COVID-19 crash, which occurred on 3 February 2020. With $N=15$, as recommended in [38], the following data points are available,

$$
\begin{gathered}
s_{0}=3085.2, s_{1}=3083.79, s_{2}=3083.41, s_{3}=3104.8, s_{4}=3066.89, s_{5}=3094.88, \\
s_{6}=3092.29, s_{7}=3115.57, s_{8}=3106.82, s_{9}=3090.04, s_{10}=3074.08, s_{11}=3075.5, \\
s_{12}=3095.79, s_{13}=3052.14, s_{14}=3060.75, s_{15}=2976.53 .
\end{gathered}
$$

The value of $s_{16}=2746.61$ is to be "predicted". From the whole set of daily data, we employ only several values of the closing price. Such coarse-grained description of the time series may be justified if one is interested in the phenomenon not dependent on the fine details, such as crash. In the examples presented below, we keep the number of data points per quartic regression parameter in the range 3-4. Lower order calculations can be found in [30]. Here, we show only the quartic regression

$$
s_{0,4}(t)=a_{4}+b_{4} t+c_{4} t^{2}+d_{4} t^{3}+f_{4} t^{4}
$$

and based on it optimize approximants and multipliers. It can be replicated as follows:

$$
s_{r, 4}(t)=A_{4}(r)+B_{4}(r)(t-r)+C_{4}(r)(t-r)^{2}+D_{4}(r)(t-r)^{3}+F_{4}(r)(t-r)^{4},
$$

with

$$
\begin{gathered}
A_{4}(r)=a_{4}+b_{4} r+c_{4} r^{2}+d_{4} r^{3}+f_{4} r^{4}, B_{4}(r)=b_{4}+2 c_{4} r+3 d_{4} r^{2}+4 f_{4} r^{3}, \\
C_{4}(r)=c_{4}+3 d_{4} r+6 f_{4} r^{2}, \quad D_{4}(r)=d_{4}+4 f_{4} r, \quad F_{4}(r)=f_{4} .
\end{gathered}
$$

With such transformed parameters, we have $s_{r, 4}(t) \equiv s_{0,4}(t)$.

Within the data shown in Figure 2, one can discern competing trends. First, let us show the data compared to the regression. There are two obvious trends, "up" and "down", as can be seen in Figure 2.

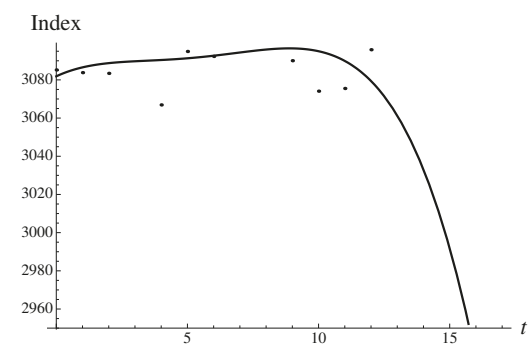

Figure 2. COVID-19, Shanghai Composite, 3 February 2020. Fourth-order regression is shown against data points. 
Our analysis indeed finds highly probable solutions of both types, with the downward trend developing into fast exponential decay. Let us analyze the typical approximant and multiplier dependencies on origin, for fixed time $t=t_{N}$. The inverse multiplier is shown as a function of the origin $r$ in Figure 3 as well as the first-order approximant.
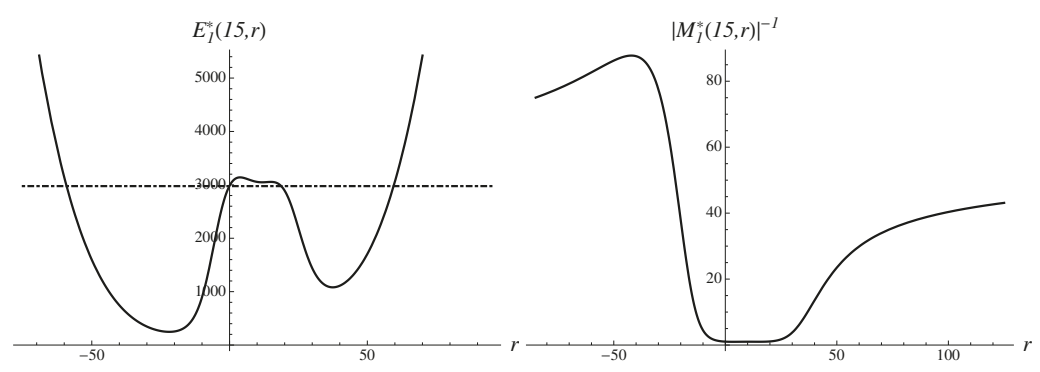

Figure 3. Shanghai Composite, 3 February 2020. Calculations with fourth-order regression. The inverse multiplier is shown as a function of the origin $r$ at $t=T_{N}, N=15$. The first-order approximant is shown in a separate figure. Level $s_{15}$ is shown as well, with dot-dashed line.

There are two uneven humps in the probabilistic inverse multiplier, suggesting that large negative and large positive $r$ dominate, with more weight put on the negative region. Such dependence on $r$ manifests the time-translation invariance violation, which should be lifted by finding appropriate origin. More details on the example can be found in [30]. Below, we discuss only the fourth-order calculations.

The results of extrapolation by method expressed by Equation (47) is given as

$$
E_{1}^{*}(16)=2804.32, \quad M_{1}^{*}(16)=0.0113494,
$$

with relative percentage error of $2.1 \%$. There is also a less stable "upward" solution

$$
E_{1}^{*}(16)=3211.95, \quad M_{1}^{*}(16)=0.0363796,
$$

in agreement with intuitive picture based on naive data analysis. There are also two additional solutions in between with multipliers close to 1 . They do not affect averages much, but in real time the metastable solutions, similar to the metastable phases in condensed matter, may show up under special conditions. Metastable solutions when realized violate the principle of maximal stability over the observation timescale, complicating or even negating a unique forecast, based on weighted averages or the most stable solution.

Calculation of the discrete spectrum can be extended to different approximants. For instance, one can also construct the second-order Gompertz approximant introduced above, and solve the following equation on origins:

$$
G\left(t_{N}, r\right)=s\left(t_{N}\right) \text {. }
$$

The most stable Gompertz approximant gives the most accurate estimate

$$
G(16)=2746.05, \quad M_{G}(16)=0.001539,
$$

with a very small error of $0.02 \%$. There are altogether five solutions to (56), in the discrete spectrum, as shown in Figure 1.

Thus, the Gompertz approximant of second order with log-time-translation invariance gives better results than symmetric exponential approximant $E_{1}^{*}$. Although Taleb's Black Swan did seem to materialize, the short-time stock market response was not different than in somewhat comparable instances of crashes brought up in [30], making it look like a Grey Swan. Indeed, it is plausible that the 
holiday season in China played the role here. It also helped our cause, effectively pinpointing the day for crash. One can think that all solutions, except the most extreme downward solution, were simply not considered.

Consider several most spectacular examples of crashes from the tumultuous spring and summer of 2020, caused by combination of economic causes such as oil anti-shock and COVID-19 related, enormous disruptions-a rare constellation of Two Swans of Gray coming together! There was a month long delay until DJ crashed. All three conspicuous crashes from March 2020 can be considered as an exponentially accelerated decay.

Black Monday I. Drop in DJ Industrial of $7.79 \%$ was caused by the shock from coronavirus, to the value of $s_{19}=23,851$, on 9 March 2020 (Black Monday I), as demonstrated in Figure 4 . The data and the components defining spectrum of scenarios are presented.

Again, there are two asymmetric humps in the probabilistic space, and the region of large negative $r$ dominates. The extrapolation by the most stable solution results in the following result,

$$
E_{1}^{*}(19)=24257.9, M_{1}^{*}(19)=0.00629791,
$$

of $1.7 \%$. There is also less stable by order of magnitude "upward" solution, as well as four additional solutions in between with multipliers of the order of unity. Using the same methodology, we obtain Gompertz approximant, and find that it gives rather good extrapolation

$$
G(19)=23669.1, M_{G}(19)=0.000805813,
$$

with a very small multiplier, and shows accuracy of $0.76 \%$. There is also an upward solution, by order of magnitude less stable. Averaging the two solutions improves the estimate to the error of only $0.52 \%$.

Black Thursday. Drop of $9.99 \%$, to the level of $s_{16}=21,200.6$ on 12 March 2020 (Black Thursday), is also believed to be caused by the coronavirus-shock. In this case, we use the standard dataset with $N=15$ and the third-order regression to see the typical pattern shown in Figure 5.

There is again a marked asymmetry on the graphs for the components in the probabilistic space, as the region of large negative $r$ prevails. The extrapolation by the most stable solution gives

$$
E_{1}^{*}(16)=22,237.1, M_{1}^{*}(16)=0.0371606,
$$

bringing the numerical error $4.89 \%$. There is also a much less stable "upward" solution. Using the same methodology for finding the discrete spectrum, we obtain Gompertz approximant, and find that it gives rather good result

$$
G(16)=21,800.2, M_{G}(16)=0.00997846,
$$

with a very small multiplier and an accuracy of $2.83 \%$. There is also an additional solution, even slightly more stable, leading to a super-fast decay almost to zero. Such scenario, obviously, is absent in calculations with pure exponential approximants. 


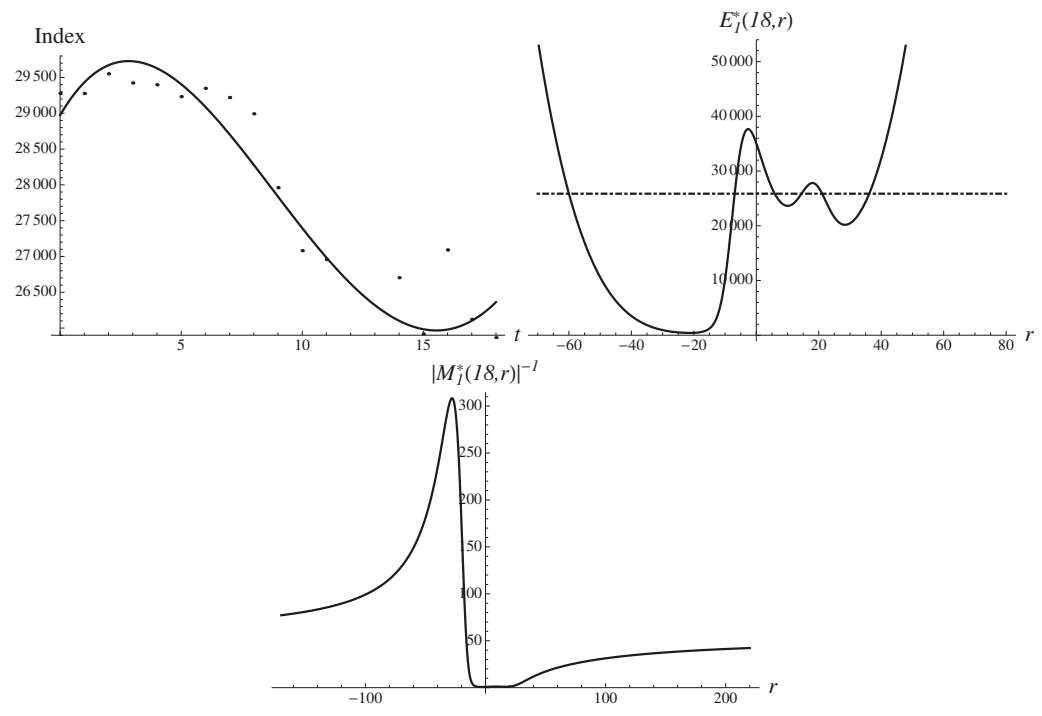

Figure 4. Black Monday I. Pattern in DJ Industrial index preceding 9 March 2020 Non-monotonous decay pattern reminds of a hockey stick. Fourth-order regression is shown against data points. The inverse multiplier is shown as a function of the origin $r$ at $t=T_{N}, N=18$. The first-order approximant is shown in separate figures. Level $s_{18}=25,864.8$ is shown with a dot-dashed line.
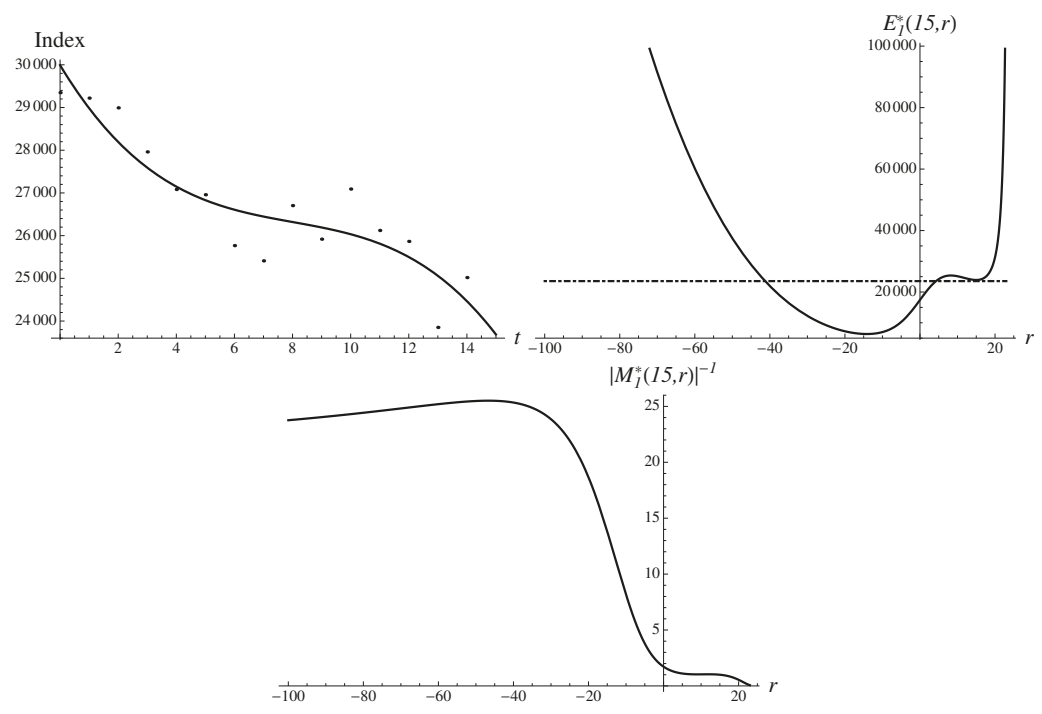

Figure 5. Black Thursday. Pattern in DJ Industrial index preceding 12 March 2020. Monotonous decay pattern. Third-order regression is shown against data points. The inverse multiplier is shown as a function of the origin $r$ at $t=T_{N}, N=15$. The first-order approximant is shown in separate figures. Level $s_{15}=23,553.2$ is shown with a dot-dashed line.

Black Monday II. Consider also the massive crash of 12.93\%, to the value of $s_{16}=20,188.5$ on 16 March 2020 (Black Monday II), caused also by oil anti-shock. Because the USA is the largest producer of oil, the big drop in oil prices (anti-shock) caused an effect typically attributed to oil shock. In this 
case, we again use the dataset of standard length with $N=15$, to see the typical pattern shown in Figure 6. It demonstrates the data, approximant and multiplier.

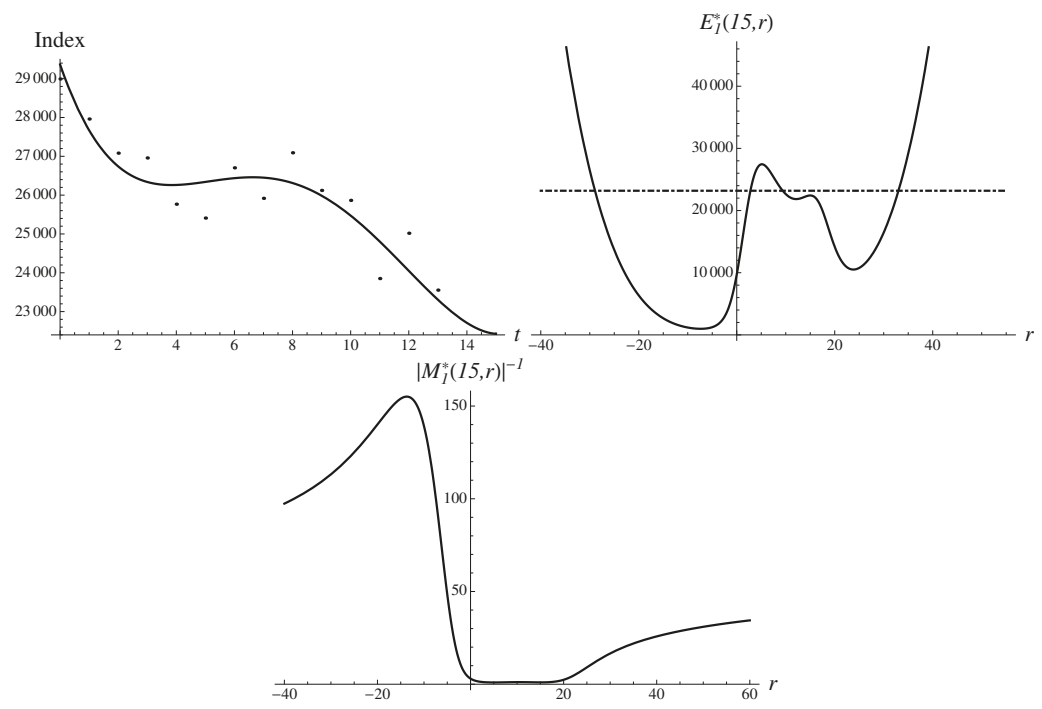

Figure 6. Black Monday II. Pattern in DJ Industrial index preceding 16 March 2020. Non-monotonous decay pattern. Fourth-order regression is shown against data points. The inverse multiplier is shown as a function of the origin $r$ at $t=T_{N}, N=15$. The first-order approximant is shown in separate figures. Level $s_{15}=23,185.6$ is shown with a dot-dashed line.

There are two typical asymmetric humps in the probabilistic space, and the region of large negative $r$ dominates. The extrapolation by the most stable solution gives the following values,

$$
E_{1}^{*}(16)=20,810.7, M_{1}^{*}(16)=0.00777882,
$$

bringing the numerical error of $3.08 \%$. There is also much less stable "upward" solution,

$$
E_{1}^{*}(16)=27,387, \quad M_{1}^{*}(16)=0.058839,
$$

as well two additional solutions in between, with multipliers of the order of unity. Using the same optimization methodology, we obtain Gompertz approximant, and find extrapolations

$$
G(16)=19,987.4, M_{G}(16)=0.00100679 \text {, }
$$

with accuracy of $0.996 \%$.

Fear of second wave of coronavirus.Bubble configuration corresponds to the price (index) going up monotonously, with rapid change of direction at some point, during the time scale of order of the time-series resolution. The growth finally becomes unsustainable. The crash of 11 June 2020 had started overnight. The index dropped to $s_{17}=25,128.2$, corresponding to a mini-crash of $6.9 \%$. For the the dataset of length $N=16$, we observe almost a perfect bubble, as shown in Figure 7. It demonstrates the data, approximant and multiplier as functions of origin. 


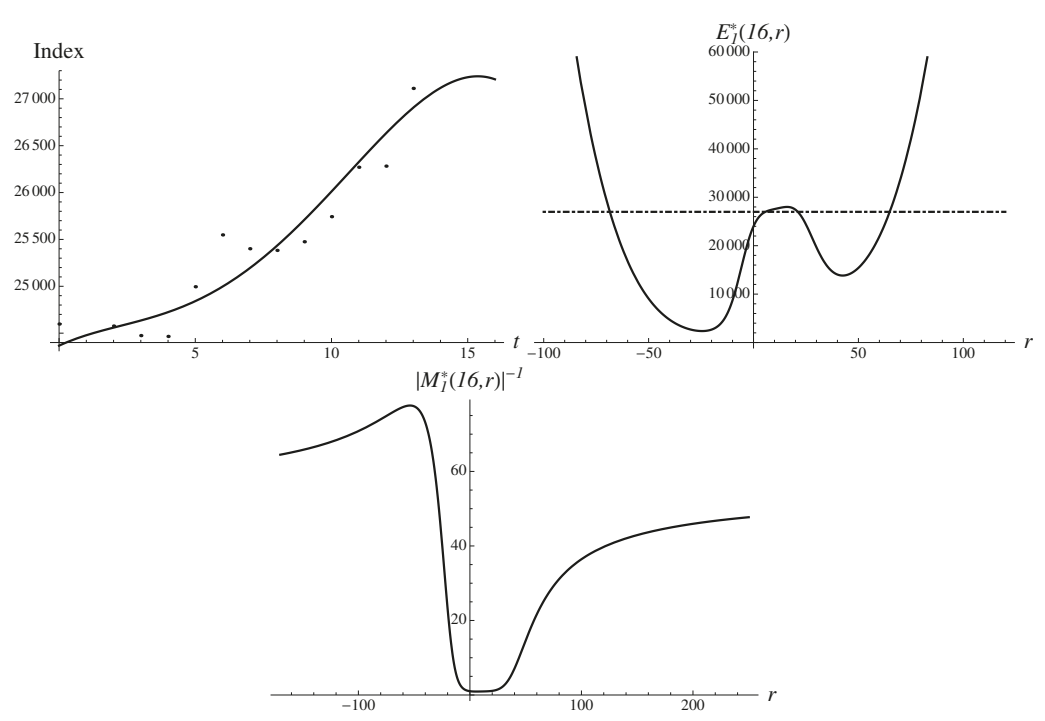

Figure 7. Temporal bubble in Dow Jones Industrial index, preceding mini-crash of 11 June 2020. Fourth-order regression is shown against data points. The first-order approximant and multiplier are shown in separate figures. Level $s_{16}=26,990$ is shown with a dot-dashed line.

There is also a marked asymmetry in the probabilistic space, and the region of large negative $r$ dominates. In the current case, the pattern appeared before the very day of crash and evolved into the mini-crash due to the overnight shock.

Extrapolation by the most stable solution results in

$$
E_{1}^{*}(17)=25,641, M_{1}^{*}(17)=0.0124981,
$$

bringing the error of $2.04 \%$. There is also less stable "upward" solution,

$$
E_{1}^{*}(17)=28,814.7, \quad M_{1}^{*}(17)=0.0435021,
$$

as well two additional solutions in between with multipliers of the order of unity.

Similar calculations with Gompertz approximant, give better estimate for the crash,

$$
G(17)=25,189.9, \quad M_{G}(17)=0.00169455,
$$

with error of just $0.25 \%$. One can think that fear of a second coronavirus wave leads to self-generated nervousness, leading to panic [69], having the net result of a shock. Bubbles are quite rare patterns in DJ index and more typical to Shanghai Composite [30].

\subsection{Comments}

Many more examples of various notable crashes can be found in [30]. They were selected to exemplify market reaction to various shocks, including $9 / 11$, Fukushima disaster, US entrance to the Great War, death of Chinese leader Deng Xiaoping, Friday the 13th, flash crash, etc. and to demonstrate similarity of early panics with coronavirus recession. Despite their different "geometry", different temporal patterns preceding crashes exhibit probabilistic distributions analogous in their main features, with significant difference only in the region of moderate $r$, but with analogous structure for large negative and positive origins. Crashes are attributed to the stable solutions with large negative $r$, meaning that the origin of time has to be moved to the deep past to explain the crash in the near future. 
Preliminary results of Gluzman [30] suggest that, in the overwhelming majority of cases, a crash is preceded by similar, asymmetric probability pattern(s), of the type shown in figures of this section.

Exponential and Gompertz approximants are found to work rather well, despite (or possibly due to) their simplicity. Unlike all other approximants, they give very clear graphic snapshots of the probabilistic space. Besides, their application is grounded in the exponential form of any future contract, with a transparent interpretation to the renormalized trend parameter $\beta(t, r)$, as expected return per unit time, equivalent to inverse relaxation (growth) time.

Our theory explains or at least gives a hint why making predictions about the future is so notoriously difficult. Instead of a unique, ironclad solution to the problem, we advocate finding all solutions and interpreting them as bounds, as plainly illustrated in Figure 1. Bounds are given different strengths, a priori determined by multipliers. Reality is not completely confined to reaching the most stable bound, but various metastable bounds can be realized as well, blurring the picture and complicating emergent time dynamics.

After applying some arguments concerned with broken/restored time-invariance, we come to the exponential solution with explicit finite time scale, which was only implicit in initial parameterization with polynomial regressions. In condensed matter physics and field theory, there is a key Meissner-Higgs mechanism for generating mass or, equivalently, for creating some typical space scale from original fields through broken symmetry technique (see, e.g., [71]). Relatively recently, the concept was confirmed, culminating in the discovery of the Higgs boson. Our approach to market price evolution is by all means inspired by the Meissner-Higgs effect. However, instead of a mass of mind-boggling elementary particles, we have a mundane, but highly sought after return per unit time.

Funding: This research received no external funding.

Conflicts of Interest: The authors declare no conflict of interest.

\section{Appendix A. Critical Index Calculations with Padé and DLog Pad'e Techniques}

For low Reynolds numbers $R$, the flow of a viscous fluid through a channel is described by the well-known Darcy's law. The Darcy law describes a linear relation between the average pressure gradient $\overline{\nabla p}$ and the average velocity $\bar{u}$ along the pressure gradient [72]. It is given as follows,

$$
|\overline{\nabla p}|=\frac{\eta}{K} \bar{u}
$$

where $K$ stands for the permeability and $\eta$ is the dynamic viscosity of the fluid. The definition of permeability simply characterizes the amount of viscous fluid flow through a porous medium per unit time and unit area when a unit macroscopic pressure gradient is applied to the system [12]. The classical Poiseuille flow is a classic example, which yields the Darcy's law. It unfolds in the channel bounded by two parallel planes separated by a distance $2 b$, generated by an average pressure gradient $\overline{\nabla p}$. The flow profile is known to be parabolic when the Reynolds number is small. When the channel is "wavy", i.e., not straight and when the Reynolds number is not negligible, additional terms appear in this relation.

Darcy law holds in the interesting cases of the Stokes flow through a channel with two-dimensional and three-dimensional wavy walls. The enclosing wavy walls are described by the analytical expressions, including the amplitude of waviness. The amplitude is proportional to the mean clearance of the channel and is multiplied by the small dimensionless parameter $\epsilon$.

We briefly discuss below the main steps of the derivation leading to the expansions for permeability, as obtained by Mityushev, Malevich and Adler. In Ref. [35], a general asymptotic 
analysis was applied to a Stokes flow in curvilinear three-dimensional channel. It is bounded by walls of rather general shape described as follows

$$
\begin{aligned}
& z=S^{+}\left(x_{1}, x_{2}\right) \equiv b\left(1+\epsilon T\left(x_{1}, x_{2}\right)\right) \\
& z=S^{-}\left(x_{1}, x_{2}\right) \equiv-b\left(1+\epsilon B\left(x_{1}, x_{2}\right)\right) .
\end{aligned}
$$

The formally small dimensionless parameter $\epsilon \geq 0$ is considered. It is introduced in such a way to allow the general shape to be recast as the geometric perturbation around the straight channel. The expansion then is accomplished around the straight channel considered as zero-approximation. Such approach builds on an original work by Pozrikidis [73].

In $[12,35]$, arbitrary profiles $S^{ \pm}\left(x_{1}, x_{2}\right)$ were explored. It was assumed only that they satisfy some natural conditions, such as

$$
\left|T\left(x_{1}, x_{2}\right)\right| \leqslant 1 \text { and }\left|B\left(x_{1}, x_{2}\right)\right| \leqslant 1 .
$$

The infinite differentiability is assumed for the functions $T\left(x_{1}, x_{2}\right)$ and $B\left(x_{1}, x_{2}\right)$. Such assumption was made in order to calculate velocities and permeability, and to solve an emerging cascade of boundary value problems for the Stokes equations in a straight channel [35]. Influence of the curvilinear edges on flow is of significant theoretical interest. It illustrates the mechanism of viscous flow under different geometrical conditions.

To make our paper self-consistent, we bring below some general information about the mathematical formulation of the problem and some permeability definitions. Let $\mathbf{u}=\mathbf{u}\left(x_{1}, x_{2}, x_{3}\right)$ be the velocity vector, and $p=p\left(x_{1}, x_{2}, x_{3}\right)$ the pressure. The flow of a viscous fluid through a channel is considered under condition that the Reynolds number is small and the Stokes flow approximation is valid. The fluid is governed by the Stokes equations. The solution $\mathbf{u}$ of the Stokes equations is sought within the class of functions periodic with period $2 L$ both in variable $x_{1}$ and in variable $x_{2}$.

Let also $u$ be the $x$-component of $\mathbf{u}$. Let also an overall external gradient pressure $\overline{\nabla p}$ be applied along the $x_{1}$-direction. It corresponds to a constant jump $2 L \overline{\nabla p}$ along the $x_{1}$-axis of the periodic cell. Then, the permeability of the channel in the $x_{1}$-direction $K_{x_{1}}(\epsilon)$ is defined as the result of integration,

$$
K_{x_{1}}(\epsilon)=-\frac{\mu}{\overline{\nabla p}|\tau|} \int_{-L}^{L} \int_{-L}^{L} d x_{1} d x_{2} \int_{S^{-}\left(x_{1}, x_{2}\right)}^{S^{+}\left(x_{1}, x_{2}\right)} u\left(x_{1}, x_{2}, x_{3}\right) d x_{3} .
$$

Here, $|\tau|$ stands for the volume of the unit cell $Q$ of the channel. The sought $K_{x_{1}}(\epsilon)$ in (A5) is expressed explicitly as a function in $\epsilon$. More precisely, we are interested in the ratio $K=K(\epsilon)$ of the dimensional permeability for the curvilinear channel and permeability of the Poiseuille flow.

Most important for our methodology, the formulae of Mityushev, Malevich and Adler [35] determine the coefficients of a Taylor series expansion for the permeability

$$
K(\epsilon)=\sum_{n=0}^{\infty} c_{n} \epsilon^{n},
$$

with the normalization with respect to the dimensional permeability for the of the Poiseuille flow. In practical computations, $K(\epsilon)$ is approximated by means of the truncation, leading to the Taylor polynomial of the order $k$

$$
K_{k}(\epsilon)=\sum_{n=0}^{k} c_{n} \epsilon^{n} .
$$

The domain of application of this formula appears to be restricted. The corresponding Taylor series are divergent for larger $\epsilon$. 
Appendix A.1. Symmetric Sinusoidal Two-Dimensional Channel: Walls Can Touch

Mityushev, Malevich and Adler [35] considered the following bounded two-dimensional channel

$$
z=b(1+\epsilon \cos x), \quad z=-b(1+\epsilon \cos x) .
$$

The expansion for permeability was found up to $O\left(\epsilon^{32}\right)$, and for $b=0.5$. This example is popular among the researchers, as is documented in [35]. The following truncated polynomial for the permeability as the function of "waviness" parameter $\epsilon$ was presented,

$$
\begin{aligned}
& K_{30}(\epsilon)= \\
& 1-3.14963 \epsilon^{2}+4.08109 \epsilon^{4}-3.48479 \epsilon^{6}+2.93797 \epsilon^{8}-2.56771 \epsilon^{10}+ \\
& 2.21983 \epsilon^{12}-1.93018 \epsilon^{14}+1.67294 \epsilon^{16}-1.45302 \epsilon^{18}+1.26017 \epsilon^{20}- \\
& 1.09411 \epsilon^{22}+0.949113 \epsilon^{24}-0.823912 \epsilon^{26}+0.714804 \epsilon^{28}-0.620463 \epsilon^{30} \\
& +O\left(\epsilon^{32}\right) .
\end{aligned}
$$

On the other hand, for larger $\epsilon$, a lubrication approximation $K_{l}$ was discussed by Adler [72]. It is motivated by the solution in the case of two cylinders of different radii that are almost in contact with one another along a line. As $\epsilon \rightarrow \epsilon_{c}=1$, we arrive to the following power-law

$$
K_{l} \simeq \frac{8 \sqrt{2} \sqrt{b^{4}}(\epsilon-1)^{5 / 2}}{9 \pi}
$$

It has the general critical form, with the critical index for permeability $\varkappa=5 / 2$. The critical amplitude can be extracted as well, so that $A=\frac{8 \sqrt{2} b^{2}}{9 \pi}$. In the case under consideration, we calculate $A=0.100035$.

The reasons for failure of lubrication approximation are explained in [35,72], as well as in [12]. In a nutshell, the main assumption of the lubrication approximation is that the velocity has a parabolic profile. Even for the plane channels [35], the lubrication approximation gives correct results only for channels in which the mean surface is sufficiently close to a plane and for small value of $\epsilon$.

In what follows, we completely avoid the lubrication approximation by following the approach of Gluzman [12] (Chapter 7). The technique of approximants allows approaching the critical region, when the walls nearly touch, only based on the expansion (A8).

As an input, we have the polynomial approximation (A8) of the function $K(\epsilon)$. We intend to to calculate the critical index and amplitude(s) of the asymptotically equivalent approximants in the vicinity of the threshold $\epsilon=\epsilon_{c}=1$. When such extrapolation problem is solved, one can proceed with an interpolation problem. In the latter case, assuming that the critical behavior is known in advance, one can derive the compact formula for all $\epsilon$ (see Chapter 7, [12]).

Let us calculate the index and amplitude for the critical behavior written in general form

$$
K(\epsilon) \simeq A\left(\epsilon_{c}-\epsilon\right)^{\varkappa}, \text { as } \epsilon \rightarrow \epsilon_{c}-0,
$$

with unknown index and amplitude.

Let us first apply the transformation,

$$
z=\frac{\epsilon}{1-\epsilon} \Leftrightarrow \epsilon=\frac{z}{z+1},
$$

to the series (A8). The transformation makes technical application of the different approximants more convenient. 
To the transformed series $M_{1}(z)$, let us apply the $D \log$ transformation and obtain the transformed series $M(z)$. In terms of $M(z)$ one can readily obtain the sequence of Padé approximations $\varkappa_{n}$ for the critical index $\varkappa$. Namely, we obtain the sequence of values

$$
\varkappa_{n}=-\lim _{z \rightarrow \infty}(z \text { PadeApproximant }[M[z], n, n+1]),
$$

as described in Section 2. The approximations for the critical index generated by the sequence of Padé approximants, converge nicely to the value $5 / 2$, as shown below,

$$
\begin{gathered}
\varkappa_{1}=2.57972, \quad \varkappa_{2}=2.30995, \quad \varkappa_{3}=2.47451, \quad \varkappa_{4}=2.49689, \\
\varkappa_{5}=2.4959, \quad \varkappa_{6}=2.49791, \quad \varkappa_{7}=2.49923, \quad \varkappa_{8}=2.50113, \\
\varkappa_{9}=2.50028, \varkappa_{10}=2.49783, \varkappa_{11}=2.49778, \varkappa_{12}=2.49829, \varkappa_{13}=2.49836 .
\end{gathered}
$$

This result well agrees with estimates by the optimization technique of Section 2.3.

If $B_{n}(z)=$ PadeApproximant $[M[z], n, n+1]$, then one can also find the approximation for permeability

$$
K_{n}^{*}(\epsilon)=\exp \left(\int_{0}^{\frac{\epsilon}{\epsilon_{c}-\epsilon}} B_{n}(z) d z\right)
$$

and compute the corresponding amplitude

$$
A_{n}=\lim _{\epsilon \rightarrow \epsilon_{c}}\left(\epsilon_{c}-\epsilon\right)^{-\varkappa_{n}} K_{n}^{*}(\epsilon) .
$$

The typical value of amplitude could be found as $A_{9}=3.7758$. It appears to be by order of magnitude larger than the value deduced from the lubrication approximation. Now, let us fix the critical index to a value of $5 / 2$, obtained from the extrapolation procedure. Now, one can calculate $A$ using the standard Padé technique, finding the value of 3.77188. The latter result turns out to be very close to the value just found above from the extrapolation.

It was illustrated by Gluzman [12] (Chapter 7) how the lubrication approximation approximation breaks down even in a close vicinity of $\epsilon_{c}$. The truncated polynomial is applicable only for small and moderately large $\epsilon$, breaking down for larger $\epsilon$ in the vicinity of the critical point. But the final formula derived by means of factor approximant is qualitatively correct for all $\epsilon$. Obviously, the standard Padé approximants are not able to capture the non-trivial power-law in the vicinity of critical point $\epsilon_{c}$.

Appendix A.2. Symmetric Sinusoidal Two-Dimensional Channel: Example 2

Let us again consider the channel bounded by the surfaces (A7), but with different parameter, $b=0.25$. The truncated polynomial $K(\epsilon)$ was obtained by Mityushev, Malevich and Adler [35] as well,

$$
\begin{aligned}
& K(\epsilon)= \\
& 1-3.03748 \epsilon^{2}+3.54570 \epsilon^{4}-2.33505 \epsilon^{6}+1.35447 \epsilon^{8}-0.83303 \epsilon^{10} \\
& +0.49762 \epsilon^{12}-0.30350 \epsilon^{14}+0.18185 \epsilon^{16}-0.11083 \epsilon^{18}+0.06636 \epsilon^{20} \\
& -0.04051 \epsilon^{22}+0.02419 \epsilon^{26} 0.00880 \epsilon^{28}-0.00544 \epsilon^{30}+ \\
& O\left(\epsilon^{32}\right) .
\end{aligned}
$$

Again, as in the previous example, we follow Chapter 7 from the book [12], where the case was researched in great detail. Using Formula (A11), we found an excellent convergence in the sequence of estimates for the index,

$$
\begin{aligned}
& \varkappa_{1}=2.64456, \quad \varkappa_{2}=2.41346, \quad \varkappa_{3}=2.49488, \quad \varkappa_{4}=2.49992, \\
& \varkappa_{5}=2.49991, \quad \varkappa_{6}=2.50026, \quad \varkappa_{7}=2.50068, \quad \varkappa_{8}=2.50087,
\end{aligned}
$$




$$
\begin{gathered}
\varkappa_{9}=2.50086, \quad \varkappa_{10}=2.50063, \quad \varkappa_{11}=2.50063, \quad \varkappa_{12}=2.50086, \\
\varkappa_{13}=2.50087, \quad \varkappa_{14}=2.50068, \quad \varkappa_{15}=2.50026,
\end{gathered}
$$

leading to the same value for the index as above, $\varkappa=5 / 2$. This result agrees with estimates by the optimization technique of Section 2.3. Clearly, the standard Padé technique fails.

The value of amplitude is estimated as well, as $A_{15}=3.77362$. Both amplitude and index appear to be independent on the parameter $b$, suggesting a universal regime in the vicinity of $\epsilon_{c}$.

Interpolating with the known critical index, one can calculate the amplitude $A$, using standard Padé technique, finding again the very close value of $A \approx 3.77316$. As in the previous example, the lubrication approximation approximation breaks down even in a close vicinity of $\epsilon_{c}$. The truncated polynomial is applicable only for small and moderately large $\epsilon$, breaking down for larger $\epsilon$ in the vicinity of the critical point. However, the final formula derived by means of factor approximant is qualitatively correct for all $\epsilon$ (for more details, see Chapter 7, [12]).

The critical index, amplitude and overall behavior of permeability in the vicinity of $\epsilon_{\mathcal{C}}$, practically do not depend on the parameter $b$ [12].

\section{Appendix A.3. Parallel Sinusoidal Two-Dimensional Channel. Walls Can Not Touch}

Let us proceed with the case principally different from the two cases just studied. Consider the channel bounded by the surfaces

$$
z=b(1+\epsilon \cos x), \quad z=-b(1-\epsilon \cos x)
$$

with $b=0.5$ [35]. It is not possible for the walls to touch, and permeability remains finite but expected to decay as a power-law as $\epsilon$ becomes large. Instead of a critical transition from permeable to non-permeable phase, we have a non-critical transition, or crossover, as defined in [15]. The crossover is from high to low permeability and unravels with increasing parameter $\epsilon$. The crossover can still be characterized by the power-law, as one can study corresponding critical index at large $\epsilon$. Eddies are not expected in such channels even for very large $\epsilon$ [35]. However, for large $b$, eddies are not excluded [35].

The truncated series expansion for the permeability were calculated up to $O\left(\epsilon^{32}\right)$,

$$
\begin{aligned}
& K_{30}(\epsilon)= \\
& 1-2.53686 \times 10^{-1} \epsilon^{2}+4.28907 \times 10^{-2} \epsilon^{4}-5.46188 \times 10^{-3} \epsilon^{6} \\
& +4.54695 \times 10^{-4} \epsilon^{8}+9.0656 \times 10^{-6} \epsilon^{10}-1.41572 \times 10^{-5} \epsilon^{12}+3.76584 \times 10^{-6} \epsilon^{14} \\
& -6.72021 \times 10^{-7} \epsilon^{16}+7.58331 \times 10^{-8} \epsilon^{18}+2.34495 \times 10^{-9} \epsilon^{20}-4.59993 \times 10^{-9} \epsilon^{22} \\
& +1.88446 \times 10^{-9} \epsilon^{24}-8.6005 \times 10^{-11} \epsilon^{26}+3.34156 \times 10^{-9} \epsilon^{28}+1.63748 \times 10^{-9} \epsilon^{30} .
\end{aligned}
$$

In this case, it is well understood that the velocity is analytic in $\epsilon$ in the disk $|\epsilon|<\epsilon_{0}$. Therefore, one can deduce that (A16) is valid for $\epsilon<\epsilon_{0}$, where $\epsilon_{0}$ is of order $\frac{1}{b \chi}$, with $\chi$ being the maximal wave number of $T\left(x_{1}, x_{2}\right)$ and $B\left(x_{1}, x_{2}\right)$. However, to extend $K(\epsilon)$ for $\epsilon \geqslant \epsilon_{0}$, it was suggested to apply the Padé approximation to the polynomial (A16), which agrees with it up to $O\left(\epsilon^{32}\right)$.

The Padé approximant of the order $(10,20)$, denoted here as $K_{10,20}(\epsilon)$, was first developed by Mityushev, Malevich and Adler [35]. Its explicit expression can also be found in Chapter 7 of the book [12]. This approximant gives $K_{10,20}(\epsilon) \sim \epsilon^{-10}$, as $\epsilon \rightarrow \infty$. One can think then that the permeability decays as

$$
K(\epsilon) \simeq B \epsilon^{v}
$$

as $\epsilon \rightarrow \infty$, with the critical index $v$ different from the estimate given by $K_{10,20}(\epsilon)$. Calculation of the critical index $v$ was accomplished in Chapter 7 of the book [12].

Assuming that the small-variable expansion for the function is given by the truncated sum $K_{30}(\epsilon)$ (A16), we can find the corresponding small-variable expression for the effective critical exponent which 
equals $\epsilon \frac{d}{d \epsilon} \log K_{30}(\epsilon)$. By applying to the obtained series, the method of Padé approximants, as in two previous examples, the sought approximate expression for the critical exponent

$$
v_{k}=\lim _{\epsilon \rightarrow \infty} \epsilon P_{k, k+1}(\epsilon),
$$

can be computed dependent on the approximation order $k$. Application of the method to the truncated power series (A16), is straightforward and suggests strongly the value of $v=-4$, as can be seen in Figure A1. This result agrees with estimates by the optimization technique of Section 2.3. Clearly, the Padé estimate mentioned above fails. The amplitude $B$, corresponding to $k=14$, is equal to 44.5872 .

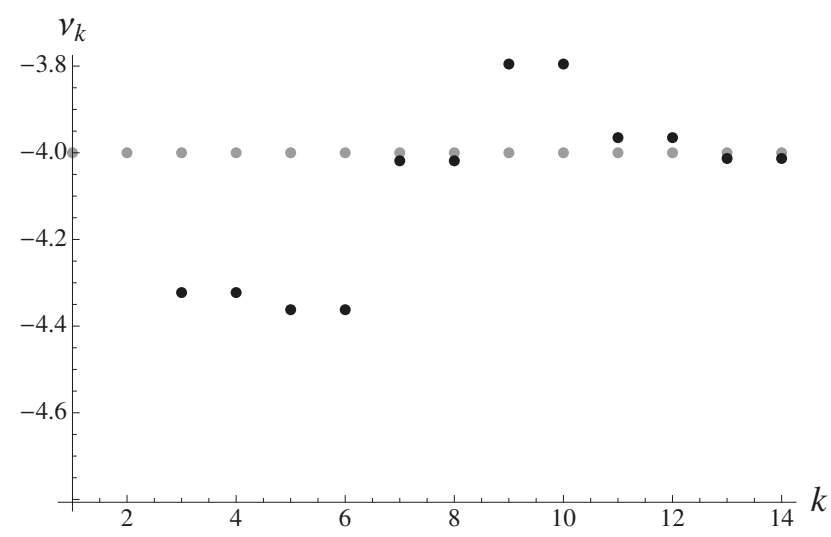

Figure A1. The index $v$ at infinity, is shown dependent on the approximation number $k$. The values found by computing (A17) are shown with black circles. They are compared with the most plausible value of -4 (shown with gray circles).

Assume now that $v=-4$ and construct the sequence of Padé approximants $P_{n, n+4}$ for the original truncated polynomial (A16). There is a convergence in the approximation sequence for the amplitude $B$. One can safely assume that it converges to the value of 43.2. The sequence is shown in Figure A2.

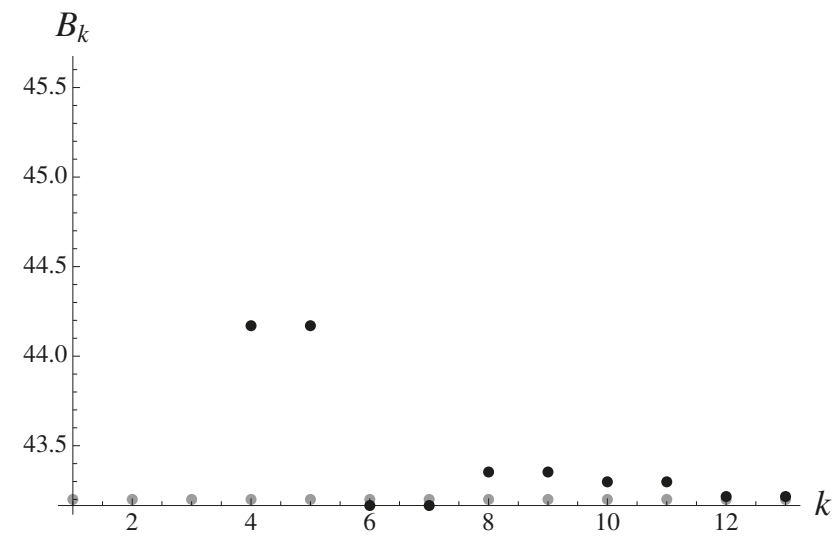

Figure A2. The amplitude $B$ dependence on approximation number $k$ is shown with black circles. One can see the convergence to the value of 43.2 , shown with squares. 


\section{Appendix B. Example of Interpolation with Root Approximants: One-Dimensional Bose Gas}

Lieb and Liniger [74] considered a one-dimensional Bose gas with contact interactions. The ground-state energy of the gas can be written as a weak-coupling expansion, with respect to the coupling parameter $g[75,76]$, as

$$
E(g) \simeq g-\frac{4}{3 \pi} g^{3 / 2}+\frac{1.29}{2 \pi^{2}} g^{2}-0.017201 g^{5 / 2},
$$

as $g \rightarrow 0$, In the strong-coupling limit, as $g \rightarrow \infty$, we have the following expression [75,76]

$$
E(g) \simeq \frac{\pi^{2}}{3}\left(1-\frac{4}{g}+\frac{12}{g^{2}}\right)
$$

In what follows, $E_{3+3}^{*}(g)$ assimilates the three coefficients from weak and strong coupling expansions, while $E_{4+3}^{*}(g)$ is based on all four terms from the weak-coupling side.

The accuracy of the root approximants (17)

$$
E_{3+3}^{*}(g)=\frac{\pi^{2}}{\sqrt[5]{\frac{585.383}{g^{5}}+\left(\frac{388.171}{g^{4}}+\left(\frac{164.914}{g^{3}}+\left(\frac{37.3454}{g^{2}}+\left(\frac{8.12698}{g}+1\right)^{3 / 2}\right)^{5 / 4}\right)^{7 / 6}\right)^{9 / 8}}},
$$

and

$$
E_{4+3}^{*}(g)=\frac{\pi^{2}}{\sqrt[6]{\frac{1267.86}{8^{6}}+\left(\frac{1548.85}{8^{5}}+\left(\frac{811.495}{8^{4}}+\left(\frac{254.699}{8^{3}}+\left(\frac{45.6531}{g^{2}}+\left(\frac{8.8658}{g}+1\right)^{3 / 2}\right)^{5 / 4}\right)^{7 / 6}\right)^{9 / 8}\right)^{11 / 10}}},
$$

turns out to be good. The approximants are constructed from "right-to-left". i.e., we self-similarly connect a known asymptotic expansion at the right boundary of the interval with a known asymptotic form at the left boundary.

In Table A1, they are compared to the extensive numerical data obtained by Dunjko and Olshanii $E_{D O}$ [77]. The Padé-estimates, $E_{P}$, are also presented. The Padé approximant $P_{3,5}(\sqrt{g})$ reads as follows:

$$
P_{3,5}(\sqrt{g})=\frac{g\left(0.285957 g^{3 / 2}-0.177533 g+0.355474 \sqrt{g}+1\right)}{0.455734 g^{3 / 2}+0.0869206 g^{5 / 2}-0.0539636 g^{2}+0.0881093 g+0.779887 \sqrt{g}+1}
$$

Table A1. Ground-state energy of Lieb-Liniger model, for the varying dimensionless parameter $g$, in different approximations: Root approximants $E_{3+3}^{*}(g), E_{4+3}^{*}(g)$, numerical data $E_{D O}$, and the Padé approximant $E_{P}$.

\begin{tabular}{ccccc}
\hline$g$ & $E_{3+3}$ & $E_{4+3}$ & $E_{D O}$ & $E_{P}$ \\
\hline 0.00509427 & 0.00494169 & 0.00494163 & 0.00494165 & 0.00494136 \\
\hline 0.0250691 & 0.0234269 & 0.0234247 & 0.0234254 & 0.0234125 \\
\hline 0.100428 & 0.0875959 & 0.0875605 & 0.0875748 & 0.0872792 \\
\hline 0.49294 & 0.361757 & 0.361368 & 0.361639 & 0.35512 \\
\hline 1.00361 & 0.640965 & 0.640137 & 0.640920 & 0.622859 \\
\hline 1.98395 & 1.04466 & 1.04325 & 1.04474 & 1.01247 \\
\hline 5.122 & 1.78912 & 1.78751 & 1.78888 & 1.76111 \\
\hline 6.02566 & 1.92249 & 1.92102 & 1.92206 & 1.89836 \\
\hline 10.0214 & 2.31276 & 2.31188 & 2.31229 & 2.30062 \\
\hline 20.0175 & 2.7248 & 2.72454 & 2.72458 & 2.72169 \\
\hline 51.4117 & 3.04855 & 3.04853 & 3.04852 & 3.04825 \\
\hline 277.602 & 3.24297 & 3.24297 & 3.24297 & 3.24927 \\
\hline
\end{tabular}


It should become completely clear from observing Figure A3 that the problem of interpolation is neither simple nor superficial. The asymptotic expressions for small and large couplings have little in common with each other. Although the expansions (A18) and (A19) appear to work only for very small and very large coupling constants, the deduced approximants work rather well. More examples of interpolation with various self-similar approximants can be found in [16].

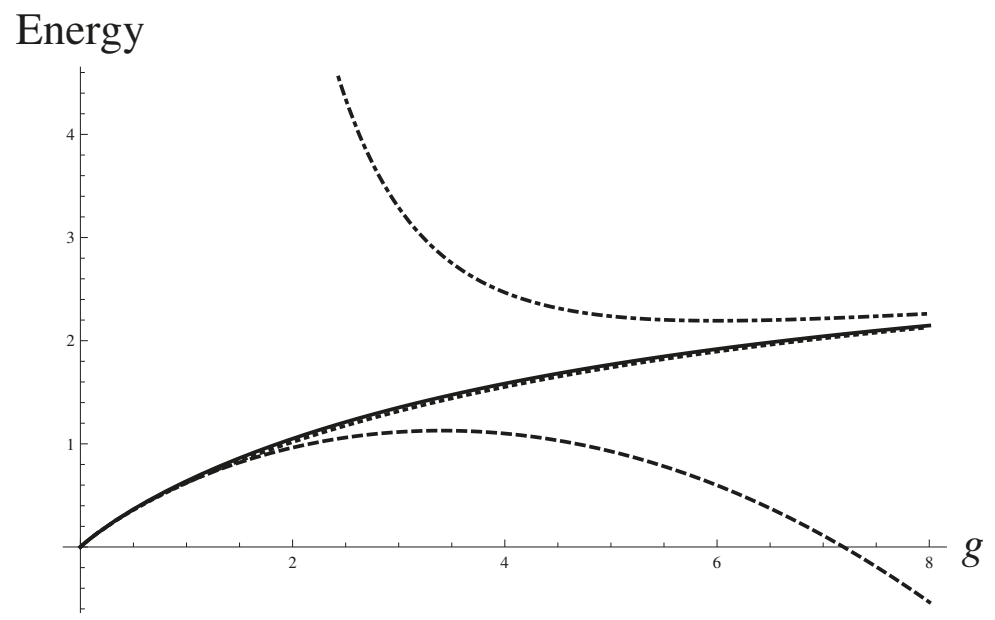

Figure A3. The interpolation with root approximant (A20) is shown with solid line, while the Padé approximant is shown with dotted line. The weak- (dashed) and strong-coupling (dot-dashed) expansions are shown as well.

\section{References}

1. Baxter, R.J. Exactly Solved Models in Statistical Mechanics; Academic Press: Cambridge, MA, USA, 1989.

2. Izyumov, Y.A.; Skryabin, Y.N. Statistical Mechanics of Magnetically Ordered Systems; Springer: Berlin, Germany, 1988.

3. Mendoza-Hernández, J.; Arroyo-Carrasco, M.; Iturbe-Castillo, M.; Chávez-Cerda, S. Laguerre-Gauss beams versus Bessel beams showdown: Peer comparison. Opt. Lett. 2015, 40, 3739-3742. [CrossRef] [PubMed]

4. Taylo, J.R. Optical Solitons: Theory and Experiment; Cambridge University Press: Cambridge, UK, 1992.

5. Valiulis, G.; Dubietis, A.; Piskarskas, A. Optical parametric amplification of chirped X pulses. Phys. Rev. A 2008, 77, 043824. [CrossRef]

6. Baker, G.A. Padé approximant. Scholarpedia 2012, 7, 9756. [CrossRef]

7. Hunter, J.K. Asymptotic Analysis and Singular Perturbation Theory; UC Davis: Davis, CA, USA, 2004.

8. Bender, C.M.; Orszag, S.A. Advanced Mathematical Methods for Scientists and Engineers. In Asymptotic Methods and Perturbation Theory; Springer: New York, NY, USA, 1999.

9. Baker, G.A.; Graves-Moris, P. Padé Approximants; Cambridge University: Cambridge, UK, 1996.

10. Gluzman, S.; Yukalov, V.I. Self-similarly corrected Pad'e approximants for indeterminate problem. Eur. Phys. J. Plus 2016, 131, 340-361. [CrossRef]

11. Gluzman, S.; Mityushev, V.; Nawalaniec, W. Computational Analysis of Structured Media; Academic Press: Cambridge, MA, USA, 2017.

12. Dryga's, P.; Gluzman, S.; Mityushev, V.; Nawalaniec, W. Applied Analysis of Composite Media; Woodhead Publishing: Sawston, UK, 2020.

13. Andrianov, I.; Awrejcewicz, J.; Danishevs'kyy, V.; Ivankov, S. Asymptotic Methods in the Theory of Plates with Mixed Boundary Conditions; John Wiley \& Sons: Hoboken, NJ, USA, 2014.

14. Andrianov, I.; Shatrov, A. Padé Approximation to Solve the Problems of Aerodynamics and Heat Transfer in the Boundary Layer; IntechOpen: London, UK, 2020. [CrossRef] 
15. Gluzman, S.; Yukalov, V.I. Unified approach to crossover phenomena. Phys. Rev. E 1998, 58, 4197-4209. [CrossRef]

16. Gluzman, S. Padé and Post-Padé Approximations for Critical Phenomena. Symmetry 2020, 12, 1600. [CrossRef]

17. Yukalov, V.I. Interplay between Approximation Theory and Renormalization. Phys. Part. Nuclei 2019, 50, 141-209. [CrossRef]

18. Gluzman, S.; Yukalov, V.I. Critical indices from self-similar root approximants. Eur. Phys. J. Plus 2017, 132, 535. [CrossRef]

19. Gluzman, S.; Yukalov, V.I. Self-Similar Power Transforms in Extrapolation Problems. J. Math. Chem. 2006, 39, 47-56. [CrossRef]

20. Yukalov, V.I.; Gluzman, S. Optimization of Self-Similar Factor Approximants. Mol. Phys. 2009, 107, 2237-2244. [CrossRef]

21. Sauer, T. Prony's method: An old trick for new problems. Snapshots Modern Math. Oberwolfach 2018, 4, 1-11.

22. Bernstein, S. Démonstration du théoréme de Weierstrass fondée sur le calcul des probabilités. Comm. Kharkov Math. Soc. 1912, 13, 1-2.

23. Cioslowski, J. Robust interpolation between weak-and strong-correlation regimes of quantum systems. J. Chem. Phys. 2012, 136, 044109. [CrossRef] [PubMed]

24. Gluzman, S.; Yukalov, V.I. Effective summation and interpolation of series by self-similar root approximants. Mathematics 2015, 3, 510-526. [CrossRef]

25. Gluzman, S.; Yukalov, V.I.; Sornette, D. Self-similar factor approximants. Phys. Rev. E 2003, 67, 026109. [CrossRef]

26. Yukalova, E.P.; Yukalov, V.I.; Gluzman, S. Solution of differential equations by self-similar factor approximants. Ann. Phys. 2008, 323, 3074-3090. [CrossRef]

27. Gluzman, S.; Yukalov, V.I. Self-similarly corrected Pade approximants for nonlinear equations. Int. J. Mod. Phys. B 2019, 33, 1950353. [CrossRef]

28. Yukalov, V.I.; Gluzman, S. Self-similar exponential approximants. Phys. Rev. E 1998, 58, 1359-1382. [CrossRef]

29. Gavrilov, L.A.; Gavrilova, N.S. The reliability theory of aging and longevity. J. Theor. Biol. 2001, 213, 427-453. [CrossRef] [PubMed]

30. Gluzman, S. Market crashes and time-translation invariance. Quant. Tech. Anal. 2020, doi:10.13140/RG.2.2.22623.07842/1. [CrossRef]

31. Yukalov, V.I. Statistical mechanics of strongly nonideal systems. Phys. Rev. A 1990, 42, 3324-3334. [CrossRef] [PubMed]

32. Yukalov, V.I. Method of self-similar approximations. J. Math. Phys. 1991, 32, 1235-1239. [CrossRef]

33. Yukalov, V.I. Stability conditions for method of self-similar approximations. J. Math. Phys. 1992, 33, $3994-4001$. [CrossRef]

34. Drygaś, P.; Filishtinski, L.A.; Gluzman, S.; Mityushev, V. Conductivity and elasticity of graphene-type composites. In 2D and Quasi-2D Composite and Nano Composite Materials, Properties and Photonic Applications; McPhedran, R., Gluzman, S., Mityushev, V., Rylko, N., Eds.; Elsevier: Amsterdam, The Netherlands, 2020; Chapter 8, pp. 193-231.

35. Malevich, A.E.; Mityushev, V.V.; Adler, P.M. Stokes flow through a channel with wavy walls. Acta Mech. 2006, 182, 151-182. [CrossRef]

36. Brading, K.; Castellani, E.; Teh, N. Symmetry and symmetry breaking. In The Stanford Encyclopedia of Philosophy; Winter 2017 Edition; Zalta Edward, N., Ed.; SEP: Standford, CA, USA, 2017.

37. Ma, S. Theory of Critical Phenomena; Benjamin: London, UK, 1976.

38. Andersen, J.V.; Gluzman, S.; Sornette, D. General framework for technical analysis of market prices. Europhys. J. B 2000, 14, 579-601.

39. Fliess, M.; Join, C. A mathematical proof of the existence of trends in financial time series. arXiv 2009, arXiv:0901.1945v1.

40. Peters, O. Optimal leverage from non-ergodicity. Quant. Fin. 2011, 11, 593-602. [CrossRef]

41. Peters, O.; Klein, M. Ergodicity breaking in geometric Brownian motion. Phys. Rev. Lett. 2013, 110, 100603. [CrossRef]

42. Peters, O.; Gell-Mann, M. Evaluating gambles using dynamics. Chaos 2016, 26, 023103. [CrossRef] 
43. Taleb, N.N. Statistical Consequences of Fat Tails (Technical Incerto Collection). 2020. Available online: https: //www.academia.edu/download/59794771/Technical_Incerto_Vol_1.pdf (accessed on 26 October 2020).

44. Sacha, K. Modeling spontaneous breaking of time-translation symmetry. Phys. Rev. A 2015, 91, 033617. [CrossRef]

45. Yukalov, V.I.; Gluzman, S. Weighted fixed points in self-similar analysis of time series. Int. J. Mod. Phys. B 1999, 13, 1463-1476. [CrossRef]

46. Hayek, F.A. The use of knowledge in society. Am. Econ. Rev. 1945, 35, 519-530.

47. Mann, A. Market forecasts. Nature 2017, 538, 308-310. [CrossRef] [PubMed]

48. Soros, G. Fallibility, reflexivity, and the human uncertainty principle. J. Econ. Methodol. 2013, 20, 309-329. doi:10.1080/1350178X.2013.859415 [CrossRef]

49. Gluzman, S.; Yukalov, V.I. Renormalization group analysis of October market crashes. Mod. Phys. Lett. B 1998, 12, 75-84. [CrossRef]

50. Buchanan, M. What has econophysics ever done for us? Nat. Phys. 2013, 9, 317. [CrossRef]

51. Shiller, R.J. Narrative economics. Am. Econ. Rev. 2017, 107, 967-1004. [CrossRef]

52. Arnold, V.I. Mathematical Methods of Classical Mechanics; Springer-Verlag: Berlin, Germany, 1989.

53. Zhang, Q.; Zhang, Q.; Sornette, S. Early warning signals of financial crises with multi-scale quantile regressions of log-periodic power law singularities. PLOS ONE 2016, 11, e0165819. [CrossRef]

54. Gluzman, S.; Yukalov, V.I. Booms and crashes of self-similar markets. Mod. Phys. Lett. B 1998, 12, 575-587. [CrossRef]

55. Bogoliubov, N.N.; Shirkov, D.V. Quantum Fields; Benjamin-Cummings Pub. Co.: San Francisco, CA, USA, 1982.

56. Shirkov, S.V. The renormalization group, the invariance principle, and functional self-similarity. Sov. Phys. Dokl. 1982, 27, 197-199.

57. Kröger, H. Fractal geometry in quantum mechanics, field theory and spin systems. Phys. Rep. 2000, 323, 81-181. [CrossRef]

58. Adamou, A.; Berman, Y.; Mavroyiannisz, D.; Peters, O. Microfoundations of Discounting. arXiv 2019, arXiv:1910.02137v2.

59. Bougie, J.; Gangopadhyaya, A.; Mallow, J.; Rasinariu, C. Supersymmetric quantum mechanics and solvable models. Symmetry 2012, 4, 452-473. [CrossRef]

60. Gluzman, S.; Sornette, D. Log-periodic route to fractal functions. Phys. Rev. E 2002, 65, 036142. [CrossRef] [PubMed]

61. Lynch, C.; Mestel, B. Logistic model for stock market bubbles and anti-bubbles. Int. J. Theor. Appl. Financ. 2017, 20, 1750038. [CrossRef]

62. Yukalov, V.I.; Gluzman, S. Extrapolation of power series by self-similar factor and root approximants. Int. J. Mod. Phys. B 2004, 18, 3027-3046. [CrossRef]

63. Duguet, T.; Sadoudi, J. Breaking and restoring symmetries within the nuclear energy density functional method. J. Phys. G Nucl. Part. Phys. 2010, 37, 064009. [CrossRef]

64. Lei, Y.C.; Zhang, S.Y. Features and partial derivatives of Bertalanffy-Richards growth model in forestry. Nonlinear Anal. Model. Control 2004, 9, 65-73. [CrossRef]

65. Richards, F.J. A flexible growth function for empirical use. J. Exp. Bot. 1959, 10, 290-301. [CrossRef]

66. Gluzman, S.; Karpeev, D. Perturbative expansions and critical phenomena in random structured media. In Modern Problems in Applied Analysis; Drygaś, P., Rogosin, S., Eds.; Birkhauser: Basel, Switzerland, 2017; pp. 117-134.

67. Sandhu, R.; Georgiou, T.; Tannenbaum, A. Market Fragility, Systemic Risk, and Ricci Curvature. arXiv 2015, arXiv1505.05182v1.

68. Boudoukh, J.; Feldman, R.; Kogan, S.; Richardson, M. Which News Moves Stock Prices? A Textual Analysis. NBER Working Paper No. 18725 January 2012. Available online: https:/ / www.nber.org/papers/w18725 (accessed on 26 October 2020).

69. Harmon, D.; Lagi, M.; de Aguiar, M.A.M.; Chinellato, D.D.; Braha, D.; Epstein, I.R.; Bar-Yam, Y. Anticipating economic market crises using measures of collective panic. PLoS ONE 2015, 10, e0131871. [CrossRef] [PubMed]

70. Bernanke, B.S.; Gertler, M.; Watson, M. Systematic monetary policy and the effects of oil price shocks. Brook. Pap. Econ. Act. 1997, 1, 91-157. [CrossRef] 
71. Kleinert, H. Vortex origin of tricritical point in Ginzburg-Landau theory. Europhys. Lett. 2006, 74, 889-895. [CrossRef]

72. Adler, P.M. Porous Media. Geometry and Transport, 2nd ed.; Butterworth-Heinemann: New York, NY, USA, 1992.

73. Pozrikidis, C. Creeping flow in two-dimensional channel. J. Fluid Mech. 1987, 180, 495-514. [CrossRef]

74. Lieb, E.H.; Liniger, S. Exact analysis of an interacting Bose gas: The general solution and the ground state. Phys. Rev. 1963, 13, 1605-1616. [CrossRef]

75. Yukalov, V.I.; Girardeau, M.D. Fermi-Bose mapping for one-dimensional Bose gases. Laser Phys. Lett. 2005, 2, 375-382.

[CrossRef]

76. Yukalov, V.I.; Yukalova, E.P.; Gluzman, S. Extrapolation and interpolation of asymptotic series by self-similar approximants. J. Math. Chem. 2010, 47, 959-983. [CrossRef]

77. Dunjko, V.; Olshanii, M. Available online: http://physics.usc.edu/olshanii/DIST/ (accessed on 26 October 2020).

Publisher's Note: MDPI stays neutral with regard to jurisdictional claims in published maps and institutional affiliations.

(C) 2020 by the authors. Licensee MDPI, Basel, Switzerland. This article is an open access article distributed under the terms and conditions of the Creative Commons Attribution (CC BY) license (http:/ / creativecommons.org/licenses/by/4.0/). 
Article

\title{
Modified Viscosity Subgradient Extragradient-Like Algorithms for Solving Monotone Variational Inequalities Problems
}

\author{
Nopparat Wairojjana ${ }^{1}$, Mudasir Younis ${ }^{2}$, Habib ur Rehman ${ }^{3}$, Nuttapol Pakkaranang ${ }^{3}$ and \\ Nattawut Pholasa ${ }^{4, *}$ \\ 1 Applied Mathematics Program, Faculty of Science and Technology, Valaya Alongkorn Rajabhat University \\ under the Royal Patronage (VRU), 1 Moo 20 Phaholyothin Road, Klong Neung, Klong Luang, \\ Pathumthani 13180, Thailand; nopparat@vru.ac.th \\ 2 Department of Applied Mathematics, UIT-Rajiv Gandhi Technological University \\ (University of Technology of M.P.), Bhopal 462033, India; mudasiryouniscuk@gmail.com \\ 3 Department of Mathematics, Faculty of Science, King Mongkut's University of Technology Thonburi \\ (KMUTT), Bangkok 10140, Thailand; habib.rehman@mail.kmutt.ac.th (H.u.R.); \\ nuttapol.pak@mail.kmutt.ac.th (N.P.) \\ 4 School of Science, University of Phayao, Phayao 56000, Thailand \\ * Correspondence: nattawut_math@hotmail.com
}

Received: 19 August 2020; Accepted: 7 October 2020; Published: 15 October 2020

check for updates

\begin{abstract}
Variational inequality theory is an effective tool for engineering, economics, transport and mathematical optimization. Some of the approaches used to resolve variational inequalities usually involve iterative techniques. In this article, we introduce a new modified viscosity-type extragradient method to solve monotone variational inequalities problems in real Hilbert space. The result of the strong convergence of the method is well established without the information of the operator's Lipschitz constant. There are proper mathematical studies relating our newly designed method to the currently state of the art on several practical test problems.
\end{abstract}

Keywords: projection methods; strong convergence; extragradient method; monotone mapping; variational inequalities

\section{Introduction}

Assume that $\mathcal{C}$ is a nonempty, closed and convex subset of a real Hilbert space $\mathbb{H}$, and $\mathbb{R}$ and $\mathbb{N}$ are the sets of real numbers and natural numbers, respectively. In this paper, we consider the classical variational inequalities problems [1,2] (in short, $V I(F, \mathcal{C})$ ) and the solution set of variational inequalities problem represent by $\operatorname{SVI}(F, \mathcal{C})$. Assume that $F$ is an operator $F: \mathbb{H} \rightarrow \mathbb{H}$ and the variational inequalities problem for an operator $F: \mathbb{H} \rightarrow \mathbb{H}$ is defined in the following way:

$$
\text { Find } u^{*} \in \mathcal{C} \text { such that }\left\langle F\left(u^{*}\right), y-u^{*}\right\rangle \geq 0, \forall y \in \mathcal{C} \text {. }
$$

The problem (1) is well defined and equivalent to solve the following fixed point problem:

$$
\text { Find a point } u^{*} \in \mathcal{C} \text { such that } u^{*}=P_{\mathcal{C}}\left[u^{*}-\zeta F\left(u^{*}\right)\right],
$$

for some $0<\zeta<\frac{1}{L}$ where $L$ is the Lipschitz constant of the operator $F$. We assume that the followings conditions have been satisfied:

(b1) The solution set is represented by $\operatorname{SVI}(F, \mathcal{C})$ and it is nonempty; 
(b2) An operator $F: \mathbb{H} \rightarrow \mathbb{H}$ is monotone-i.e.,

$$
\left\langle F\left(u_{1}\right)-F\left(u_{2}\right), u_{1}-u_{2}\right\rangle \geq 0, \forall u_{1}, u_{2} \in \mathcal{C} ;
$$

(b3) $F$ is Lipschitz continuous if there exists $L>0$, such that

$$
\left\|F\left(u_{1}\right)-F\left(u_{2}\right)\right\| \leq L\left\|u_{1}-u_{2}\right\|, \forall u_{1}, u_{2} \in \mathcal{C}
$$

The variational inequalities theory is a useful technique for investigating a large number of problems in physics, economics, engineering and optimization theory. It was firstly introduced by Stampacchia [1] in 1964 and also well established that the problem (1) is an important problem in nonlinear analysis. It is an advantageous mathematical model that puts together several topics of applied mathematics, such as the network equilibrium problems, the necessary optimality conditions, the systems of non-linear equations and the complementarity problems [3-7].

The projection method and its modified version methods are crucial for finding the numerical solutions of variational inequality problems. Many studies have been suggested and researched different types of projection methods to solve the variational inequalities problem (see for more details [8-18]) and others, as in [19-28]. The simplistic methodology is the gradient method for which only one projection on a feasible set is required. A convergence of the method, however, requires strong monotonicity on F. To prevent the strong monotonicity hypothesis, Korpelevich [8] and Antipin [29] introduced the following extragradient method.

$$
\left\{\begin{array}{l}
u_{n} \in \mathcal{C} \\
v_{n}=P_{\mathcal{C}}\left[u_{n}-\zeta F\left(u_{n}\right)\right], \\
u_{n+1}=P_{\mathcal{C}}\left[u_{n}-\zeta F\left(v_{n}\right)\right]
\end{array}\right.
$$

for some $0<\zeta<\frac{1}{L}$. The subgradient extragradient algorithm was recently developed by Censor et al. [10] to resolve problem (1) in real Hilbert space. Their method has the form of

$$
\left\{\begin{array}{l}
u_{n} \in \mathcal{C} \\
v_{n}=P_{\mathcal{C}}\left[u_{n}-\zeta F\left(u_{n}\right)\right] \\
u_{n+1}=P_{\mathbb{H}_{n}}\left[u_{n}-\zeta F\left(v_{n}\right)\right]
\end{array}\right.
$$

where $0<\zeta<\frac{1}{L}$ and $\mathbb{H}_{n}=\left\{z \in \mathbb{H}:\left\langle u_{n}-\zeta F\left(u_{n}\right)-v_{n}, z-v_{n}\right\rangle \leq 0\right\}$.

In this article, motivated by the methods in $[10,30,31]$ and the viscosity method [14] we introduce a new viscosity subgradient-extragradient algorithm to solve variational inequality problems involving monotone operators in Hilbert space. It is important to note that, our proposed algorithm operates more effectively than the existing ones. Particularly in comparison to the results of Yang et al. [30], our algorithm operates efficiently in most situations. Analogously to the results of Yang et al. [30], proof of the convergence of Algorithm 1, it is not compulsory to have the information of the Lipschitz constant of the operator $F$. The proposed algorithm could be seen as a modification of the methods that are found in $[8,10,30,31]$. Under mild conditions, a strong convergence theorem was proven to be associated with the proposed method. Numerical experimental studies have been shown that the new method considers being more effective than the current ones in [30].

The rest of the article is arranged in the following way: Section 2 provides a few definitions and basic results that are used throughout the paper. Section 3 contains the main algorithm and convergence theorem. Section 4 includes the numerical results that illustrate the algorithmic efficacy of the introduced method. 


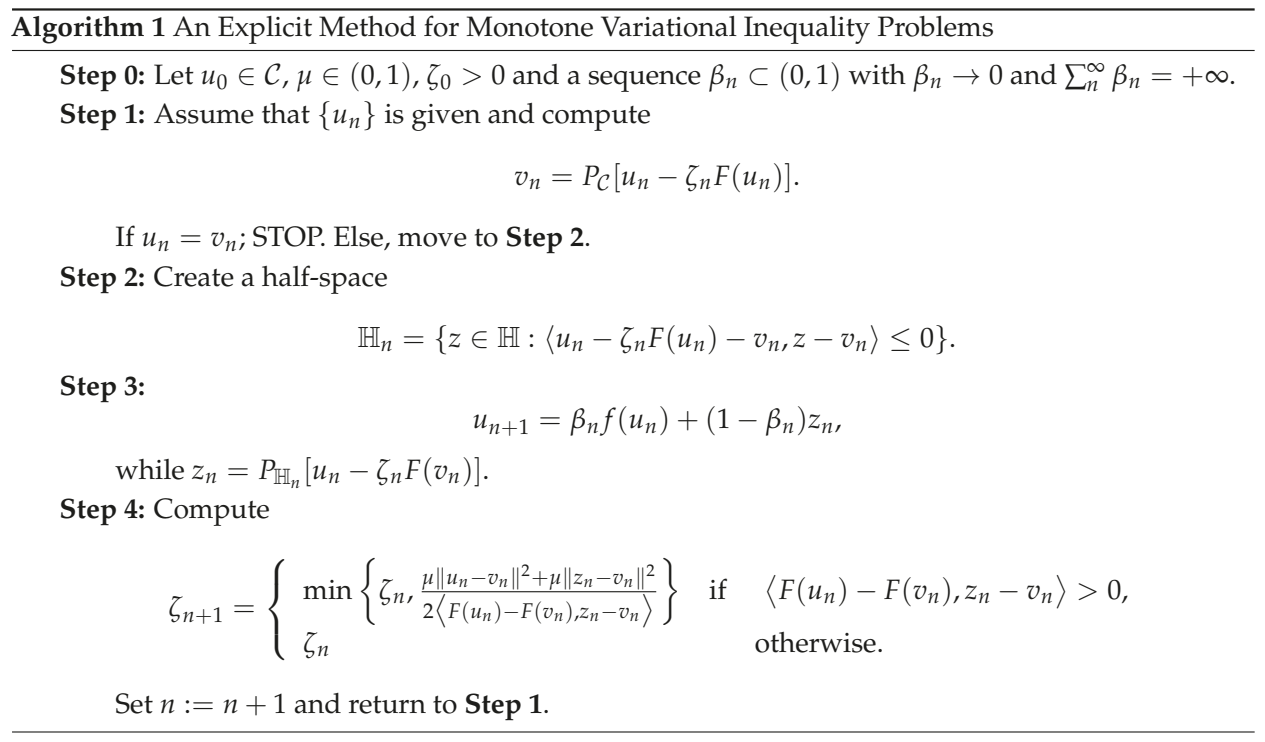

\section{Background}

A metric projection $P_{\mathcal{C}}\left(u_{1}\right)$ for $u_{1} \in \mathbb{H}$ onto a closed and convex subset $\mathcal{C}$ of $\mathbb{H}$ is defined by

$$
P_{\mathcal{C}}\left(u_{1}\right)=\arg \min \left\{\left\|u_{2}-u_{1}\right\|: u_{2} \in \mathcal{C}\right\} .
$$

Lemma 1 ([32]; Page 31). For $u, v \in \mathbb{H}$ and $a \in \mathbb{R}$, then the following relationship holds.

(i). $\|a u+(1-a) v\|^{2}=a\|u\|^{2}+(1-a)\|v\|^{2}-a(1-a)\|u-v\|^{2}$.

(ii). $\|u+v\|^{2} \leq\|u\|^{2}+2\langle v, u+v\rangle$.

Lemma 2 ([32,33]). Assume $\mathcal{C}$ be a nonempty, closed and convex subset of a real Hilbert space $\mathbb{H}$ and let $P_{\mathcal{C}}: \mathbb{H} \rightarrow \mathcal{C}$ be a metric projection from $\mathbb{H}$ onto $\mathcal{C}$. Then:

(i). Let $u_{1} \in \mathcal{C}$ and $u_{2} \in \mathbb{H}$

$$
\left\|u_{1}-P_{\mathcal{C}}\left(u_{2}\right)\right\|^{2}+\left\|P_{\mathcal{C}}\left(u_{2}\right)-u_{2}\right\|^{2} \leq\left\|u_{1}-u_{2}\right\|^{2} .
$$

(ii). $u_{3}=P_{\mathcal{C}}\left(u_{1}\right)$ if and only if

$$
\left\langle u_{1}-u_{3}, u_{2}-u_{3}\right\rangle \leq 0, \forall u_{2} \in \mathcal{C}
$$

(iii). For $u_{2} \in \mathcal{C}$ and $u_{1} \in \mathbb{H}$

$$
\left\|u_{1}-P_{\mathcal{C}}\left(u_{1}\right)\right\| \leq\left\|u_{1}-u_{2}\right\|
$$

Lemma 3 ([34]). Assume that $\left\{\chi_{n}\right\}$ is a sequence of non-negative real numbers such that

$$
\chi_{n+1} \leq\left(1-\alpha_{n}\right) \chi_{n}+\alpha_{n} \delta_{n}, \forall n \in \mathbb{N},
$$

where $\left\{\alpha_{n}\right\} \subset(0,1)$ and $\left\{\delta_{n}\right\} \subset \mathbb{R}$ meet with the following criteria:

$$
\lim _{n \rightarrow \infty} \alpha_{n}=0, \sum_{n=1}^{\infty} \alpha_{n}=\infty, \text { and } \limsup _{n \rightarrow \infty} \delta_{n} \leq 0
$$


Then, $\lim _{n \rightarrow \infty} \chi_{n}=0$.

Lemma 4 ([35]). Assume that $\left\{\chi_{n}\right\}$ is a sequence of real numbers such that there is a subsequence $\left\{n_{i}\right\}$ of $\{n\}$ such that $\chi_{n_{i}}<\chi_{n_{i+1}}$ for all $i \in \mathbb{N}$. Then, there is a non decreasing sequence $m_{k} \subset \mathbb{N}$ such that $m_{k} \rightarrow \infty$ as $k \rightarrow \infty$, and the following conditions are fullfilled by all (sufficiently large) numbers $k \in \mathbb{N}$ :

$$
\chi_{m_{k}} \leq \chi_{m_{k+1}} \text { and } \chi_{k} \leq \chi_{m_{k+1}} .
$$

In fact, $m_{k}=\max \left\{j \leq k: \chi_{j} \leq \chi_{j+1}\right\}$.

Lemma 5 ([36]). Assume that $\mathcal{C}$ is a nonempty closed convex set in $\mathbb{H}$ and an operator $F: \mathcal{C} \rightarrow \mathbb{H}$ is monotone and continuous. Then, $u^{*}$ is a solution of the problem (1) if and only if $u^{*}$ is a solution of the following problem:

Find $x \in \mathcal{C}$ such that $\langle F(y), y-x\rangle \geq 0, \forall y \in \mathcal{C}$.

\section{Algorithm and Corresponding Strong Convergence Theorem}

We provide a method consisting of two convex minimization problems through a viscosity and an explicit stepsize formula which are being used to enhance the rate of convergence the iterative sequence and to make the method independent of the Lipschitz constant $L$. The detailed method is given below:

Remark $1 . \mathbb{H}_{n}$ is a half-space and so $\mathbb{H}_{n}$ is a closed and convex set in $\mathbb{H}$.

Lemma 6. The sequence $\left\{\zeta_{n}\right\}$ is decreasing monotonically with a lower bound $\min \left\{\frac{\mu}{L}, \zeta_{0}\right\}$ and converges to $\zeta>0$.

Proof. From the sequence $\left\{\tau_{n}\right\}$, we see that this sequence is monotone and nonincreasing. It is given that $F$ is Lipschitz-continuous with $L>0$. Let $\left\langle F\left(u_{n}\right)-F\left(v_{n}\right), z_{n}-v_{n}\right\rangle>0$, such that

$$
\begin{aligned}
\frac{\mu\left(\left\|u_{n}-v_{n}\right\|^{2}+\left\|z_{n}-v_{n}\right\|^{2}\right)}{2\left\langle F\left(u_{n}\right)-F\left(v_{n}\right), z_{n}-v_{n}\right\rangle} & \geq \frac{2 \mu\left\|u_{n}-v_{n}\right\|\left\|z_{n}-v_{n}\right\|}{2\left\|F\left(u_{n}\right)-F\left(v_{n}\right)\right\|\left\|z_{n}-v_{n}\right\|} \\
& \geq \frac{2 \mu\left\|u_{n}-v_{n}\right\|\left\|z_{n}-v_{n}\right\|}{2\left\|u_{n}-v_{n}\right\|\left\|z_{n}-v_{n}\right\|} \\
& \geq \frac{\mu}{L} .
\end{aligned}
$$

The above discussion implies that the sequence $\left\{\zeta_{n}\right\}$ has a lower bound $\min \left\{\frac{\mu}{L}, \zeta_{0}\right\}$. Moreover, there exists number $\zeta>0$, such that $\lim _{n \rightarrow \infty} \zeta_{n}=\zeta$.

Lemma 7. Assume that an operator $F: \mathcal{C} \rightarrow \mathbb{H}$ satisfies the conditions (b1)-(b3). For each $u^{*} \in S V I(F, \mathcal{C}) \neq$ $\varnothing$, we have

$$
\left\|z_{n}-u^{*}\right\|^{2} \leq\left\|u_{n}-u^{*}\right\|^{2}-\left(1-\frac{\mu \zeta_{n}}{\zeta_{n+1}}\right)\left\|u_{n}-v_{n}\right\|^{2}-\left(1-\frac{\mu \zeta_{n}}{\zeta_{n+1}}\right)\left\|z_{n}-v_{n}\right\|^{2}
$$

Proof. Let consider the following

$$
\begin{aligned}
\left\|z_{n}-u^{*}\right\|^{2}= & \left\|P_{\mathbb{H}_{n}}\left[u_{n}-\zeta_{n} F\left(v_{n}\right)\right]-u^{*}\right\|^{2} \\
= & \left\|P_{\mathbb{H}_{n}}\left[u_{n}-\zeta_{n} F\left(v_{n}\right)\right]+\left[u_{n}-\zeta_{n} F\left(v_{n}\right)\right]-\left[u_{n}-\zeta_{n} F\left(v_{n}\right)\right]-u^{*}\right\|^{2} \\
= & \left\|\left[u_{n}-\zeta_{n} F\left(v_{n}\right)\right]-u^{*}\right\|^{2}+\left\|P_{\mathbb{H}_{n}}\left[u_{n}-\zeta_{n} F\left(v_{n}\right)\right]-\left[u_{n}-\zeta_{n} F\left(v_{n}\right)\right]\right\|^{2} \\
& +2\left\langle P_{\mathbb{H}_{n}}\left[u_{n}-\zeta_{n} F\left(v_{n}\right)\right]-\left[u_{n}-\zeta_{n} F\left(v_{n}\right)\right],\left[u_{n}-\zeta_{n} F\left(v_{n}\right)\right]-u^{*}\right\rangle .
\end{aligned}
$$


From the assumption that $u^{*} \in S V I(F, \mathcal{C}) \subset \mathcal{C} \subset \mathbb{H}_{n}$, we have

$$
\begin{aligned}
\left\|P_{\mathbb{H}_{n}}\left[u_{n}-\zeta_{n} F\left(v_{n}\right)\right]-\left[u_{n}-\zeta_{n} F\left(v_{n}\right)\right]\right\|^{2} & +\left\langle P_{\mathbb{H}_{n}}\left[u_{n}-\zeta_{n} F\left(v_{n}\right)\right]-\left[u_{n}-\zeta_{n} F\left(v_{n}\right)\right],\left[u_{n}-\zeta_{n} F\left(v_{n}\right)\right]-u^{*}\right\rangle \\
= & \left\langle\left[u_{n}-\zeta_{n} F\left(v_{n}\right)\right]-P_{\mathbb{H}_{n}}\left[u_{n}-\zeta_{n} F\left(v_{n}\right)\right], u^{*}-P_{\mathbb{H}_{n}}\left[u_{n}-\zeta_{n} F\left(v_{n}\right)\right]\right\rangle \leq 0,
\end{aligned}
$$

implies that

$$
\begin{aligned}
& \left\langle P_{\mathbb{H}_{n}}\left[u_{n}-\zeta_{n} F\left(v_{n}\right)\right]-\left[u_{n}-\zeta_{n} F\left(v_{n}\right)\right],\left[u_{n}-\zeta_{n} F\left(v_{n}\right)\right]-u^{*}\right\rangle \\
& \leq-\left\|P_{\mathbb{H}_{n}}\left[u_{n}-\zeta_{n} F\left(v_{n}\right)\right]-\left[u_{n}-\zeta_{n} F\left(v_{n}\right)\right]\right\|^{2} .
\end{aligned}
$$

Now, using the Equation (4) implies that

$$
\begin{aligned}
\left\|z_{n}-u^{*}\right\|^{2} & \leq\left\|u_{n}-\zeta_{n} F\left(v_{n}\right)-u^{*}\right\|^{2}-\left\|P_{\mathbb{H}_{n}}\left[u_{n}-\zeta_{n} F\left(v_{n}\right)\right]-\left[u_{n}-\zeta_{n} F\left(v_{n}\right)\right]\right\|^{2} \\
& \leq\left\|u_{n}-u^{*}\right\|^{2}-\left\|u_{n}-z_{n}\right\|^{2}+2 \zeta_{n}\left\langle F\left(v_{n}\right), u^{*}-z_{n}\right\rangle .
\end{aligned}
$$

Given that $u^{*}$ is a solution of $V I(F, \mathcal{C})$, we get

$$
\left\langle F\left(u^{*}\right), y-u^{*}\right\rangle \geq 0, \forall y \in \mathcal{C} .
$$

Due to the monotonicity of $F$ on $\mathcal{C}$, we can obtain

$$
\left\langle F\left(v_{n}\right)-F\left(u^{*}\right), v_{n}-u^{*}\right\rangle \geq 0, \forall y \in \mathcal{C} .
$$

Since $v_{n} \in \mathcal{C}$, it follows that

$$
\left\langle F\left(v_{n}\right), v_{n}-u^{*}\right\rangle \geq 0
$$

Thus, we have

$$
\left\langle F\left(v_{n}\right), u^{*}-z_{n}\right\rangle=\left\langle F\left(v_{n}\right), u^{*}-v_{n}\right\rangle+\left\langle F\left(v_{n}\right), v_{n}-z_{n}\right\rangle \leq\left\langle F\left(v_{n}\right), v_{n}-z_{n}\right\rangle .
$$

From (7) and (11), we get

$$
\begin{aligned}
\left\|z_{n}-u^{*}\right\|^{2} & \leq\left\|u_{n}-u^{*}\right\|^{2}-\left\|u_{n}-z_{n}\right\|^{2}+2 \zeta_{n}\left\langle F\left(v_{n}\right), v_{n}-z_{n}\right\rangle \\
& =\left\|u_{n}-u^{*}\right\|^{2}-\left\|u_{n}-v_{n}+v_{n}-z_{n}\right\|^{2}+2 \zeta_{n}\left\langle F\left(v_{n}\right), v_{n}-z_{n}\right\rangle \\
& \leq\left\|u_{n}-u^{*}\right\|^{2}-\left\|u_{n}-v_{n}\right\|^{2}-\left\|v_{n}-z_{n}\right\|^{2}+2\left\langle u_{n}-\zeta_{n} F\left(v_{n}\right)-v_{n}, z_{n}-v_{n}\right\rangle .
\end{aligned}
$$

Note that $z_{n}=P_{\mathbb{H}_{n}}\left[u_{n}-\zeta_{n} F\left(v_{n}\right)\right]$ and by the definition of $\zeta_{n+1}$, we have

$$
\begin{aligned}
& 2\left\langle u_{n}-\zeta_{n} F\left(v_{n}\right)-v_{n}, z_{n}-v_{n}\right\rangle \\
& =2\left\langle u_{n}-\zeta_{n} F\left(u_{n}\right)-v_{n}, z_{n}-v_{n}\right\rangle+2 \zeta_{n}\left\langle F\left(u_{n}\right)-F\left(v_{n}\right), z_{n}-v_{n}\right\rangle \\
& \leq \frac{2 \zeta_{n}}{\zeta_{n+1}} \zeta_{n+1}\left\langle F\left(u_{n}\right)-F\left(v_{n}\right), z_{n}-v_{n}\right\rangle \leq \frac{\zeta_{n}}{\zeta_{n+1}}\left[\mu\left\|u_{n}-v_{n}\right\|^{2}+\mu\left\|z_{n}-v_{n}\right\|^{2}\right] .
\end{aligned}
$$

From expression (12) and (13), we obtain

$$
\begin{aligned}
& \left\|z_{n}-u^{*}\right\|^{2} \\
& \leq\left\|u_{n}-u^{*}\right\|^{2}-\left\|u_{n}-v_{n}\right\|^{2}-\left\|v_{n}-z_{n}\right\|^{2}+\frac{\zeta_{n}}{\zeta_{n+1}}\left[\mu\left\|u_{n}-v_{n}\right\|^{2}+\mu\left\|z_{n}-v_{n}\right\|^{2}\right] \\
& \leq\left\|u_{n}-u^{*}\right\|^{2}-\left(1-\frac{\mu \zeta_{n}}{\zeta_{n+1}}\right)\left\|u_{n}-v_{n}\right\|^{2}-\left(1-\frac{\mu \zeta_{n}}{\zeta_{n+1}}\right)\left\|z_{n}-v_{n}\right\|^{2} .
\end{aligned}
$$


Theorem 1. Assume that an operator $F: \mathcal{C} \rightarrow \mathbb{H}$ satisfies the conditions (b1)-(b3) and $u^{*}$ belongs to solution set $\operatorname{SVI}(F, \mathcal{C})$. Then, the sequences $\left\{u_{n}\right\},\left\{v_{n}\right\}$ and $\left\{z_{n}\right\}$ generated by Algorithm 1 strongly converge to $u^{*}$.

Proof. Claim 1: The sequence $\left\{u_{n}\right\}$ is bounded in $\mathbb{H}$.

From Lemma 7, we have

$$
\left\|z_{n}-u^{*}\right\|^{2} \leq\left\|u_{n}-u^{*}\right\|^{2}-\left(1-\frac{\mu \zeta_{n}}{\zeta_{n+1}}\right)\left\|u_{n}-v_{n}\right\|^{2}-\left(1-\frac{\mu \zeta_{n}}{\zeta_{n+1}}\right)\left\|z_{n}-v_{n}\right\|^{2}
$$

Since $\zeta_{n} \rightarrow \zeta$, then exits a fixed number $\epsilon \in(0,1-\mu)$ such that

$$
\lim _{n \rightarrow \infty}\left(1-\frac{\mu \zeta_{n}}{\zeta_{n+1}}\right)=1-\mu>\epsilon>0 .
$$

Thus, there is a finite number $N_{1} \in \mathbb{N}$ such that

$$
\left(1-\frac{\mu \zeta_{n}}{\zeta_{n+1}}\right)>\epsilon>0, \forall n \geq N_{1}
$$

Thus, from (15), we obtain

$$
\left\|z_{n}-u^{*}\right\|^{2} \leq\left\|u_{n}-u^{*}\right\|^{2}, \forall n \geq N_{1}
$$

Let $u^{*} \in S V I(F, \mathcal{C})$. By definition of the sequence $\left\{u_{n+1}\right\}$ and due to contraction $f$ with constant $\rho \in[0,1)$ and $n \geq N_{1}$, we obtain

$$
\begin{aligned}
\left\|u_{n+1}-u^{*}\right\| & =\left\|\beta_{n} f\left(u_{n}\right)+\left(1-\beta_{n}\right) z_{n}-u^{*}\right\| \\
& =\left\|\beta_{n}\left[f\left(u_{n}\right)-u^{*}\right]+\left(1-\beta_{n}\right)\left[z_{n}-u^{*}\right]\right\| \\
& =\left\|\beta_{n}\left[f\left(u_{n}\right)+f\left(u^{*}\right)-f\left(u^{*}\right)-u^{*}\right]+\left(1-\beta_{n}\right)\left[z_{n}-u^{*}\right]\right\| \\
& \leq \beta_{n}\left\|f\left(u_{n}\right)-f\left(u^{*}\right)\right\|+\beta_{n}\left\|f\left(u^{*}\right)-u^{*}\right\|+\left(1-\beta_{n}\right)\left\|z_{n}-u^{*}\right\| \\
& \leq \beta_{n} \rho\left\|u_{n}-u^{*}\right\|+\beta_{n}\left\|f\left(u^{*}\right)-u^{*}\right\|+\left(1-\beta_{n}\right)\left\|z_{n}-u^{*}\right\| .
\end{aligned}
$$

Consider the expressions (17) and (18) and $\beta_{n} \subset(0,1)$, we have

$$
\begin{aligned}
\left\|u_{n+1}-u^{*}\right\| & \leq \beta_{n} \rho\left\|u_{n}-u^{*}\right\|+\beta_{n}\left\|f\left(u^{*}\right)-u^{*}\right\|+\left(1-\beta_{n}\right)\left\|u_{n}-u^{*}\right\| \\
& =\left[1-\beta_{n}+\rho \beta_{n}\right]\left\|u_{n}-u^{*}\right\|+\beta_{n}(1-\rho) \frac{\left\|f\left(u^{*}\right)-u^{*}\right\|}{(1-\rho)} \\
& \leq \max \left\{\left\|u_{n}-u^{*}\right\|, \frac{\left\|f\left(u^{*}\right)-u^{*}\right\|}{(1-\rho)}\right\} \\
& \leq \max \left\{\left\|u_{N_{1}}-u^{*}\right\|, \frac{\left\|f\left(u^{*}\right)-u^{*}\right\|}{(1-\rho)}\right\} .
\end{aligned}
$$

Finally, we deduce that the sequence $\left\{u_{n}\right\}$ is bounded.

Claim 2: If $\lim _{n \rightarrow \infty}\left\|u_{n}-v_{n}\right\|=0$, then, as a subsequence, $\left\{u_{n_{k}}\right\}$ of $\left\{u_{n}\right\}$ such that $\left\{u_{n_{k}}\right\} \rightarrow u^{*} \in$ $\operatorname{SVI}(F, \mathcal{C})$ as $k \rightarrow \infty$. 
The reflexivity of $\mathbb{H}$ and the boundedness of $\left\{u_{n}\right\}$ imply that there exists a subsequence $\left\{u_{n_{k}}\right\}$ such that $\left\{u_{n_{k}}\right\} \rightarrow u^{*} \in \mathbb{H}$ as $k \rightarrow \infty$. It is sufficient to prove that $u^{*} \in S V I(F, \mathcal{C})$. Due to $\lim _{n \rightarrow \infty} \| u_{n}-$ $v_{n} \|=0$, we also have $\left\{v_{n_{k}}\right\} \rightarrow u^{*}$ as $k \rightarrow \infty$. In addition, the fact that

$$
v_{n_{k}}=P_{\mathcal{C}}\left[u_{n_{k}}-\zeta_{n_{k}} F\left(u_{n_{k}}\right)\right]
$$

that is equivalent to

$$
\left\langle u_{n_{k}}-\zeta_{n_{k}} F\left(u_{n_{k}}\right)-v_{n_{k}}, y-v_{n_{k}}\right\rangle \leq 0, \forall y \in \mathcal{C} .
$$

That is, we have

$$
\left\langle u_{n_{k}}-v_{n_{k}}, y-v_{n_{k}}\right\rangle \leq \zeta_{n_{k}}\left\langle F\left(u_{n_{k}}\right), y-v_{n_{k}}\right\rangle, \forall y \in \mathcal{C} .
$$

From the monotonicity condition on $F$, we have

$$
\left\langle F\left(u_{n_{k}}\right)-F(y), u_{n_{k}}-y\right\rangle \geq 0, \forall y \in \mathcal{C},
$$

that is

$$
\left\langle F(y), y-u_{n_{k}}\right\rangle \geq\left\langle F\left(u_{n_{k}}\right), y-u_{n_{k}}\right\rangle, \forall y \in \mathcal{C} .
$$

Combining expressions (20) and (21), we obtain

$$
\begin{aligned}
0 & \leq\left\langle v_{n_{k}}-u_{n_{k}}, y-v_{n_{k}}\right\rangle+\zeta_{n_{k}}\left\langle F\left(u_{n_{k}}\right), y-v_{n_{k}}\right\rangle \\
& =\left\langle v_{n_{k}}-u_{n_{k}}, y-v_{n_{k}}\right\rangle+\zeta_{n_{k}}\left\langle F\left(u_{n_{k}}\right), y-u_{n_{k}}\right\rangle+\zeta_{n_{k}}\left\langle F\left(u_{n_{k}}\right), u_{n_{k}}-v_{n_{k}}\right\rangle \\
& \leq\left\langle v_{n_{k}}-u_{n_{k}}, y-v_{n_{k}}\right\rangle+\zeta_{n_{k}}\left\langle F(y), y-u_{n_{k}}\right\rangle+\zeta_{n_{k}}\left\langle F\left(u_{n_{k}}\right), u_{n_{k}}-v_{n_{k}}\right\rangle,
\end{aligned}
$$

for all $y \in \mathcal{C}$, since $\lim _{k \rightarrow \infty} \zeta_{n_{k}}=\zeta>0$ (see Lemma 6) and the sequence $\left\{u_{n}\right\}$ is bounded in $\mathbb{H}$. As $\lim _{n \rightarrow \infty}\left\|u_{n}-v_{n}\right\|=0$, and pass the limit in (22) as $k \rightarrow \infty$, we obtain

$$
\left\langle F(y), y-u^{*}\right\rangle \geq 0, \forall y \in \mathcal{C}
$$

Apply the well-known Minty Lemma 5, this is what we infer: $u^{*} \in S V I(F, \mathcal{C})$.

Claim 3: The sequence $\left\{u_{n}\right\}$ is strong convergent in $\mathbb{H}$.

The strong convergence of the sequence $\left\{u_{n}\right\}$ is as follows. The continuity and monotonicity of the operator $F$ and the Minty lemma gives that $S V I(F, \mathcal{C})$ is a closed and convex set (see $[37,38]$ for more details). As mapping $f$ is a contraction, so is $P_{S V I(F, \mathcal{C})} \circ f$. By using the Banach contraction principle to guarantee that an unique element exists, $u^{*} \in S V I(F, \mathcal{C})$, such that

$$
u^{*}=P_{S V I(F, \mathcal{C})}\left(f\left(u^{*}\right)\right) \text {. }
$$

Hence, we have

$$
\left\langle f\left(u^{*}\right)-u^{*}, y-u^{*}\right\rangle \geq 0, \forall y \in S V I(F, \mathcal{C})
$$


Now, considering $u_{n+1}=\beta_{n} f\left(u_{n}\right)+\left(1-\beta_{n}\right) z_{n}$, and using Lemma 1 (i) and Lemma 7 , we have

$$
\begin{aligned}
\left\|u_{n+1}-u^{*}\right\|^{2}= & \left\|\beta_{n} f\left(u_{n}\right)+\left(1-\beta_{n}\right) z_{n}-u^{*}\right\|^{2} \\
= & \left\|\beta_{n}\left[f\left(u_{n}\right)-u^{*}\right]+\left(1-\beta_{n}\right)\left[z_{n}-u^{*}\right]\right\|^{2} \\
= & \beta_{n}\left\|f\left(u_{n}\right)-u^{*}\right\|^{2}+\left(1-\beta_{n}\right)\left\|z_{n}-u^{*}\right\|^{2}-\beta_{n}\left(1-\beta_{n}\right)\left\|f\left(u_{n}\right)-z_{n}\right\|^{2} \\
\leq & \beta_{n}\left\|f\left(u_{n}\right)-u^{*}\right\|^{2}+\left(1-\beta_{n}\right)\left[\left\|u_{n}-u^{*}\right\|^{2}-\left(1-\frac{\mu \zeta_{n}}{\zeta_{n+1}}\right)\left\|u_{n}-v_{n}\right\|^{2}\right. \\
& \left.-\left(1-\frac{\mu \zeta_{n}}{\zeta_{n+1}}\right)\left\|z_{n}-v_{n}\right\|^{2}\right]-\beta_{n}\left(1-\beta_{n}\right)\left\|f\left(u_{n}\right)-z_{n}\right\|^{2} \\
\leq & \beta_{n}\left\|f\left(u_{n}\right)-u^{*}\right\|^{2}+\left\|u_{n}-u^{*}\right\|^{2}-\left(1-\beta_{n}\right)\left(1-\frac{\mu \zeta_{n}}{\zeta_{n+1}}\right)\left[\left\|z_{n}-v_{n}\right\|^{2}+\left\|u_{n}-v_{n}\right\|^{2}\right] .
\end{aligned}
$$

The remainder of the proof can be divided into two cases:

Case 1: Assume that there is a fixed number $N_{2} \in \mathbb{N}\left(N_{2} \geq N_{1}\right)$ such that

$$
\left\|u_{n+1}-u^{*}\right\| \leq\left\|u_{n}-u^{*}\right\|, \forall n \geq N_{2}
$$

Thus, $\lim _{n \rightarrow \infty}\left\|u_{n}-u^{*}\right\|$ exists and let $\lim _{n \rightarrow \infty}\left\|u_{n}-u^{*}\right\|=l$. By using expression (25), we have

$$
\begin{aligned}
& \left(1-\beta_{n}\right)\left(1-\frac{\mu \zeta_{n}}{\zeta_{n+1}}\right)\left[\left\|z_{n}-v_{n}\right\|^{2}+\left\|u_{n}-v_{n}\right\|^{2}\right] \\
& \leq \beta_{n}\left\|f\left(u_{n}\right)-u^{*}\right\|^{2}+\left\|u_{n}-u^{*}\right\|^{2}-\left\|u_{n+1}-u^{*}\right\|^{2} .
\end{aligned}
$$

Due to the existence of $\lim _{n \rightarrow \infty}\left\|u_{n}-u^{*}\right\|=l$, and $\beta_{n} \rightarrow 0$, we obtain

$$
\lim _{n \rightarrow \infty}\left\|u_{n}-v_{n}\right\|=\lim _{n \rightarrow \infty}\left\|z_{n}-v_{n}\right\|=0
$$

It follows that

$$
\lim _{n \rightarrow \infty}\left\|u_{n}-z_{n}\right\| \leq \lim _{n \rightarrow \infty}\left\|u_{n}-v_{n}\right\|+\lim _{n \rightarrow \infty}\left\|v_{n}-z_{n}\right\|=0
$$

Hence, we obtain

$$
\begin{aligned}
\left\|u_{n+1}-u_{n}\right\| & =\left\|\beta_{n} f\left(u_{n}\right)+\left(1-\beta_{n}\right) z_{n}-u_{n}\right\| \\
& =\left\|\beta_{n}\left[f\left(u_{n}\right)-u_{n}\right]+\left(1-\beta_{n}\right)\left[z_{n}-u_{n}\right]\right\| \\
& \leq \beta_{n}\left\|f\left(u_{n}\right)-u_{n}\right\|+\left(1-\beta_{n}\right)\left\|z_{n}-u_{n}\right\| \rightarrow 0 .
\end{aligned}
$$

The sequence $\left\{u_{n}\right\}$ is bounded and implies that the sequences $\left\{v_{n}\right\}$ and $\left\{z_{n}\right\}$ are also bounded. Thus, we can take a subsequence $\left\{u_{n_{k}}\right\}$ of $\left\{u_{n}\right\}$ such that $\left\{u_{n_{k}}\right\}$ converges weakly to some $\hat{u} \in \mathcal{C}$ and

$$
\begin{aligned}
& \limsup _{n \rightarrow \infty}\left\langle f\left(u^{*}\right)-u^{*}, u_{n}-u^{*}\right\rangle \\
& =\limsup _{k \rightarrow \infty}\left\langle f\left(u^{*}\right)-u^{*}, u_{n_{k}}-u^{*}\right\rangle=\left\langle f\left(u^{*}\right)-u^{*}, \hat{u}-u^{*}\right\rangle \leq 0 .
\end{aligned}
$$

We have $\lim _{n \rightarrow \infty}\left\|u_{n+1}-u_{n}\right\|=0$. It means that

$$
\begin{aligned}
& \limsup _{n \rightarrow \infty}\left\langle f\left(u^{*}\right)-u^{*}, u_{n+1}-u^{*}\right\rangle \\
& \leq \limsup _{k \rightarrow \infty}\left\langle f\left(u^{*}\right)-u^{*}, u_{n+1}-u_{n}\right\rangle+\limsup _{k \rightarrow \infty}\left\langle f\left(u^{*}\right)-u^{*}, u_{n}-u^{*}\right\rangle \leq 0 .
\end{aligned}
$$


From Lemma 7 and Lemma 1 (ii) $\left(\forall n \geq N_{2}\right)$, we obtain

$$
\begin{aligned}
& \left\|u_{n+1}-u^{*}\right\|^{2} \\
& =\left\|\beta_{n} f\left(u_{n}\right)+\left(1-\beta_{n}\right) z_{n}-u^{*}\right\|^{2} \\
& =\left\|\beta_{n}\left[f\left(u_{n}\right)-u^{*}\right]+\left(1-\beta_{n}\right)\left[z_{n}-u^{*}\right]\right\|^{2} \\
& \leq\left(1-\beta_{n}\right)^{2}\left\|z_{n}-u^{*}\right\|^{2}+2 \beta_{n}\left\langle f\left(u_{n}\right)-u^{*},\left(1-\beta_{n}\right)\left[z_{n}-u^{*}\right]+\beta_{n}\left[f\left(u_{n}\right)-u^{*}\right]\right\rangle \\
& =\left(1-\beta_{n}\right)^{2}\left\|z_{n}-u^{*}\right\|^{2}+2 \beta_{n}\left\langle f\left(u_{n}\right)-f\left(u^{*}\right)+f\left(u^{*}\right)-u^{*}, u_{n+1}-u^{*}\right\rangle \\
& =\left(1-\beta_{n}\right)^{2}\left\|z_{n}-u^{*}\right\|^{2}+2 \beta_{n}\left\langle f\left(u_{n}\right)-f\left(u^{*}\right), u_{n+1}-u^{*}\right\rangle+2 \beta_{n}\left\langle f\left(u^{*}\right)-u^{*}, u_{n+1}-u^{*}\right\rangle \\
& \leq\left(1-\beta_{n}\right)^{2}\left\|z_{n}-u^{*}\right\|^{2}+2 \beta_{n} \rho\left\|u_{n}-u^{*}\right\|\left\|u_{n+1}-u^{*}\right\|+2 \beta_{n}\left\langle f\left(u^{*}\right)-u^{*}, u_{n+1}-u^{*}\right\rangle \\
& \leq\left(1+\beta_{n}^{2}-2 \beta_{n}\right)\left\|u_{n}-u^{*}\right\|^{2}+2 \beta_{n} \rho\left\|u_{n}-u^{*}\right\|^{2}+2 \beta_{n}\left\langle f\left(u^{*}\right)-u^{*}, u_{n+1}-u^{*}\right\rangle \\
& =\left(1-2 \beta_{n}\right)\left\|u_{n}-u^{*}\right\|^{2}+\beta_{n}^{2}\left\|u_{n}-u^{*}\right\|^{2}+2 \beta_{n} \rho\left\|u_{n}-u^{*}\right\|^{2}+2 \beta_{n}\left\langle f\left(u^{*}\right)-u^{*}, u_{n+1}-u^{*}\right\rangle \\
& =\left[1-2 \beta_{n}(1-\rho)\right]\left\|u_{n}-u^{*}\right\|^{2}+2 \beta_{n}(1-\rho)\left[\frac{\beta_{n}\left\|u_{n}-u^{*}\right\|^{2}}{2(1-\rho)}+\frac{\left\langle f\left(u^{*}\right)-u^{*}, u_{n+1}-u^{*}\right\rangle}{1-\rho}\right] .
\end{aligned}
$$

It follows (32) that

$$
\limsup _{n \rightarrow \infty}\left[\frac{\beta_{n}\left\|u_{n}-u^{*}\right\|^{2}}{2(1-\rho)}+\frac{\left\langle f\left(u^{*}\right)-u^{*}, u_{n+1}-u^{*}\right\rangle}{1-\rho}\right] \leq 0 .
$$

Choose $n \geq N_{3} \in \mathbb{N}\left(N_{3} \geq N_{2}\right)$ large enough such that $2 \beta_{n}(1-\rho)<1$. Now, by using expressions (33) and (34) and applying Lemma 3, conclude that $\left\|u_{n}-u^{*}\right\| \rightarrow 0$, as $n \rightarrow \infty$.

Case 2: Assume that there is a subsequence $\left\{n_{i}\right\}$ of $\{n\}$ such that

$$
\left\|u_{n_{i}}-u^{*}\right\| \leq\left\|u_{n_{i+1}}-u^{*}\right\|, \forall i \in \mathbb{N} .
$$

Thus, by Lemma 4 there is a sequence $\left\{m_{k}\right\} \subset \mathbb{N}$ as $\left\{m_{k}\right\} \rightarrow \infty$, such that

$$
\left\|u_{m_{k}}-u^{*}\right\| \leq\left\|u_{m_{k+1}}-u^{*}\right\| \text { and }\left\|u_{k}-u^{*}\right\| \leq\left\|u_{m_{k+1}}-u^{*}\right\|, \forall k \in \mathbb{N} .
$$

Similar to case 1 and from (25), we obtain

$$
\begin{aligned}
& \left(1-\beta_{m_{k}}\right)\left(1-\frac{\mu \zeta_{m_{k}}}{\zeta_{m_{k}+1}}\right)\left[\left\|z_{m_{k}}-v_{m_{k}}\right\|^{2}+\left\|u_{m_{k}}-v_{m_{k}}\right\|^{2}\right] \\
& \leq \beta_{m_{k}}\left\|f\left(u_{m_{k}}\right)-u^{*}\right\|^{2}+\left\|u_{m_{k}}-u^{*}\right\|^{2}-\left\|u_{m_{k}+1}-u^{*}\right\|^{2}
\end{aligned}
$$

Due to $\beta_{m_{k}} \rightarrow 0$, and $\left(1-\frac{\mu \zeta_{m_{k}}}{\zeta_{m_{k}+1}}\right) \rightarrow 1-\mu$, we deduce the following:

$$
\lim _{n \rightarrow \infty}\left\|u_{m_{k}}-v_{m_{k}}\right\|=\lim _{k \rightarrow \infty}\left\|z_{m_{k}}-v_{m_{k}}\right\|=0 .
$$

It follows that

$$
\lim _{k \rightarrow \infty}\left\|u_{m_{k}}-z_{m_{k}}\right\| \leq \lim _{k \rightarrow \infty}\left\|u_{m_{k}}-v_{m_{k}}\right\|+\lim _{k \rightarrow \infty}\left\|v_{m_{k}}-z_{m_{k}}\right\|=0
$$

Similar to case 1 , we can easily obtain that

$$
\lim _{k \rightarrow \infty}\left\|u_{m_{k+1}}-u_{m_{k}}\right\|=0, \quad \text { and } \quad \limsup _{k \rightarrow \infty}\left\langle f\left(u^{*}\right)-u^{*}, u_{m_{k}+1}-u^{*}\right\rangle \leq 0 .
$$


By using (35) and the same argument as in (33), we have

$$
\begin{aligned}
& \left\|u_{m_{k}+1}-u^{*}\right\|^{2} \\
& =\left[1-2 \beta_{m_{k}}(1-\rho)\right]\left\|u_{m_{k}}-u^{*}\right\|^{2}+2 \beta_{m_{k}}(1-\rho)\left[\frac{\beta_{m_{k}}\left\|u_{m_{k}}-u^{*}\right\|^{2}}{2(1-\rho)}+\frac{\left\langle f\left(u^{*}\right)-u^{*}, u_{m_{k}+1}-u^{*}\right\rangle}{1-\rho}\right] \\
& \leq\left[1-2 \beta_{m_{k}}(1-\rho)\right]\left\|u_{m_{k}+1}-u^{*}\right\|^{2}+2 \beta_{m_{k}}(1-\rho)\left[\frac{\beta_{m_{k}}\left\|u_{m_{k}}-u^{*}\right\|^{2}}{2(1-\rho)}+\frac{\left\langle f\left(u^{*}\right)-u^{*}, u_{m_{k}+1}-u^{*}\right\rangle}{1-\rho}\right] .
\end{aligned}
$$

It follows that

$$
\left\|u_{m_{k}+1}-u^{*}\right\|^{2} \leq \frac{\beta_{m_{k}}\left\|u_{m_{k}}-u^{*}\right\|^{2}}{2(1-\rho)}+\frac{\left\langle f\left(u^{*}\right)-u^{*}, u_{m_{k}+1}-u^{*}\right\rangle}{1-\rho} .
$$

Due to $\beta_{m_{k}} \rightarrow 0$ as $k \rightarrow \infty$, and $\limsup _{k \rightarrow \infty}\left\langle f\left(u^{*}\right)-u^{*}, u_{m_{k}+1}-u^{*}\right\rangle \leq 0$, we obtain

$$
\left\|u_{m_{k}+1}-u^{*}\right\|^{2} \rightarrow 0, \text { as } k \rightarrow \infty .
$$

Finally, the inequality

$$
\lim _{n \rightarrow \infty}\left\|u_{k}-u^{*}\right\|^{2} \leq \lim _{n \rightarrow \infty}\left\|u_{m_{k}+1}-u^{*}\right\|^{2} \leq 0
$$

Consequently, $u_{n} \rightarrow u^{*}$. This completes the proof of the theorem.

\section{Numerical Illustrations}

The experimental results are discussed in this section to illustrate the efficacy of our proposed Algorithm 1 (m-EgA3) compared to Algorithm 1 (m-EgA1) in [30] and Algorithm 2 (m-EgA2) in [30].

Example 1. Consider the HpHard problem which is taken from [39] and considered by many authors for numerical tests (see [40-42]), where $F: \mathbb{R}^{m} \rightarrow \mathbb{R}^{m}$ is an operator defined by $F(u)=M u+q$ with $q \in \mathbb{R}^{m}$ and

$$
M=N N^{T}+B+D,
$$

where $N$ is an $m \times m$ matrix, $B$ is an $m \times m$ skew-symmetric matrix and $D$ is an $m \times m$ positive definite diagonal matrix. The feasible set is defined by

$$
\mathcal{C}=\left\{u \in \mathbb{R}^{m}: Q u \leq b\right\}
$$

where $Q$ is an $100 \times m$ matrix and $b$ is a nonnegative vector in $\mathbb{R}^{m}$. It is clear that $F$ is monotone and Lipschitz continuous with $L=\|M\|$. For $q=0$, the solution set of the corresponding variational inequality is $\operatorname{VI}(\mathcal{C}, F)=$ $\{0\}$. In this experiment, we take the initial point $u_{0}=(1,1, \cdots, 1)$ and $D_{n}=\left\|u_{n}-v_{n}\right\| \leq T O L=10^{-3}$. Moreover, the control parameters $\zeta_{0}=\frac{0.7}{L}$ and $\mu=0.9$ for Algorithm 1 (m-EgA1) in [30]; $\zeta_{0}=\frac{0.7}{L}, \mu=0.9$ and $\beta_{n}=\frac{1}{30(k+2)}$ for Algorithm $2(m-E g A 2)$ in [30]; $\zeta_{0}=\frac{0.7}{L}, \mu=0.9, \beta_{n}=\frac{1}{n+4}$ and $f(u)=\frac{u}{2}$ for Algorithm 1 ( $m$-EgA3). The numerical results of all methods have been reported in Figures 1-8 and Table 1. 


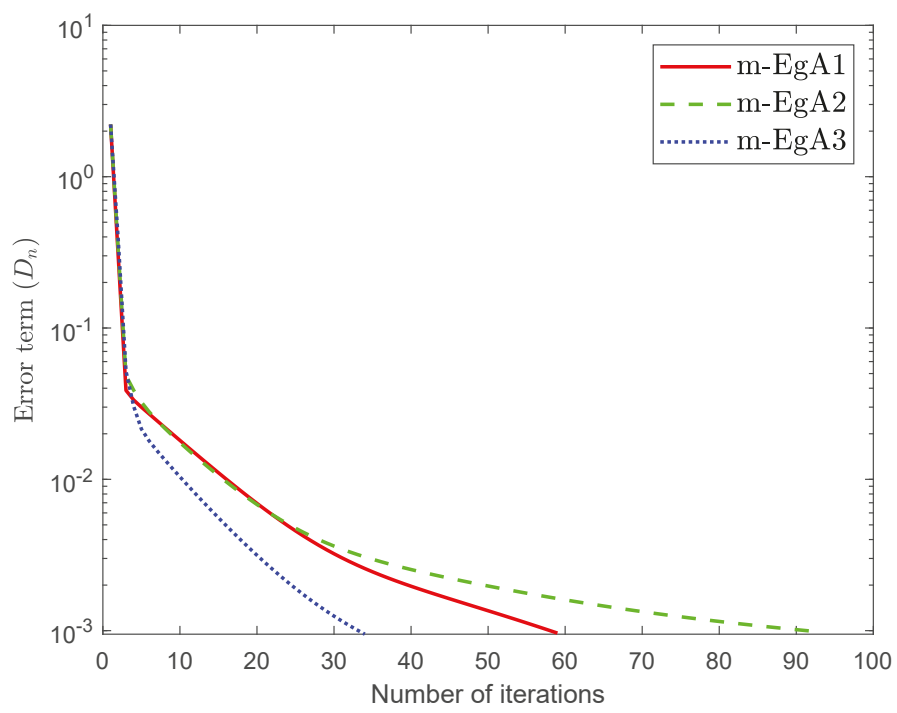

Figure 1. Numerical behaviour of Algorithm 1 compared to Algorithm 1 in [30] and Algorithm 2 in [30] for Example 1, when $m=5$.

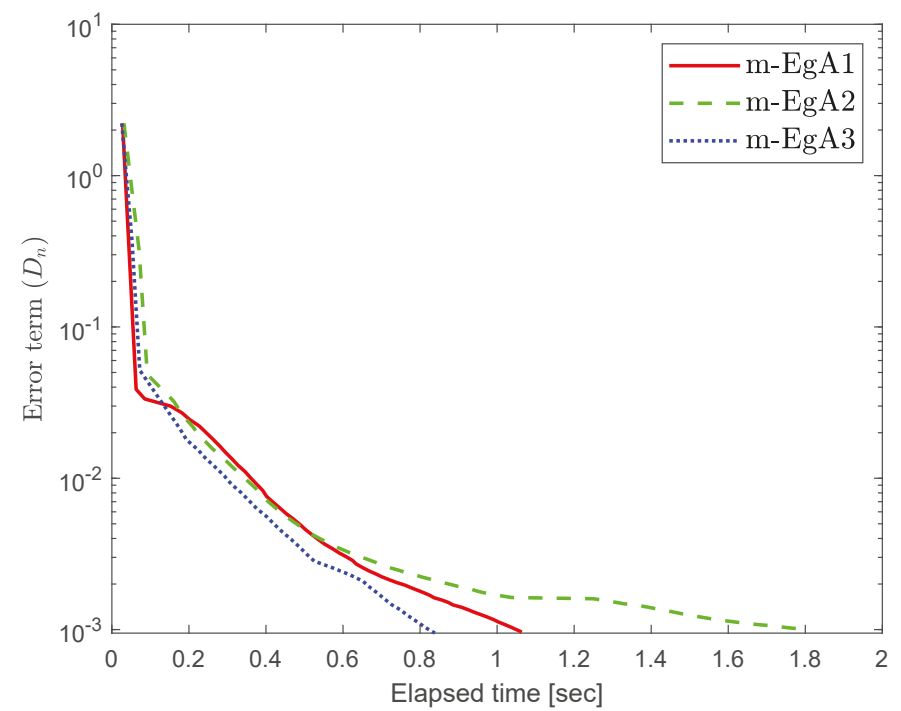

Figure 2. Numerical behaviour of Algorithm 1 compared to Algorithm 1 in [30] and Algorithm 2 in [30] for Example 1, when $m=5$. 


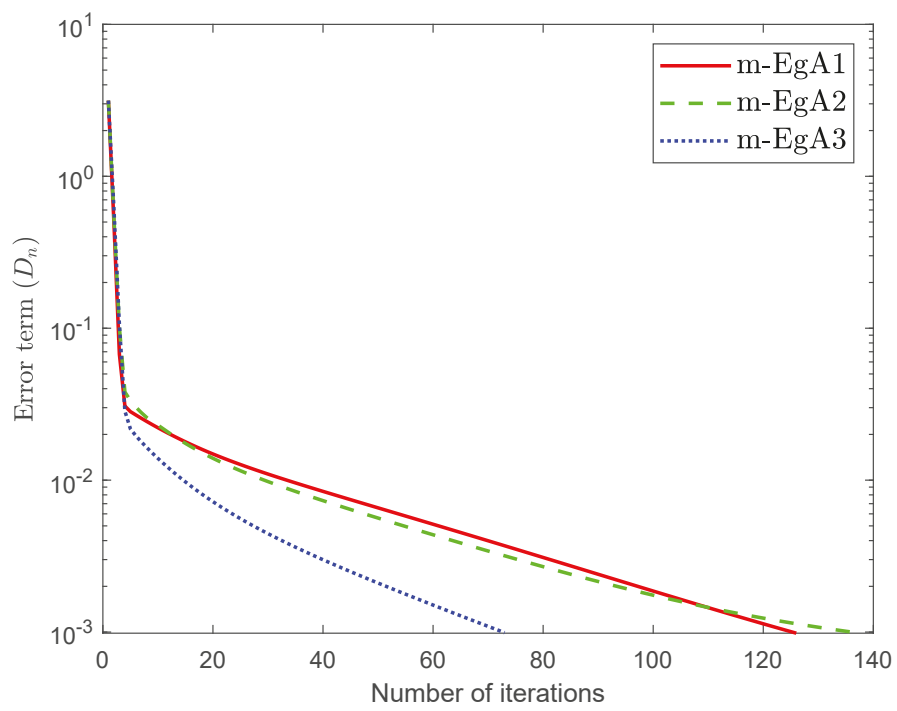

Figure 3. Numerical behaviour of Algorithm 1 compared to Algorithm 1 in [30] and Algorithm 2 in [30] for Example 1, when $m=10$.

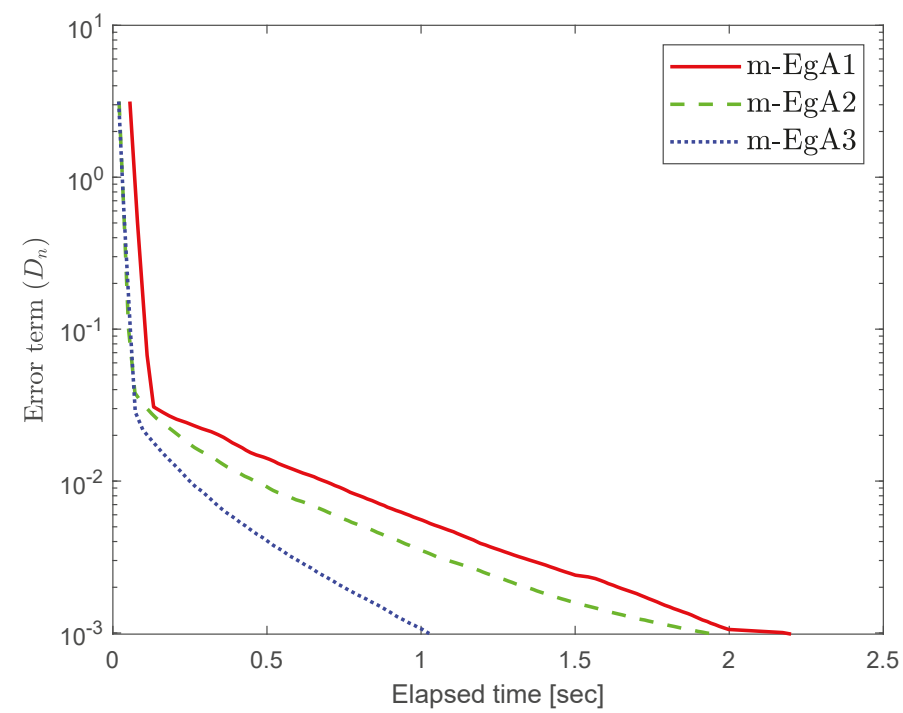

Figure 4. Numerical behaviour of Algorithm 1 compared to Algorithm 1 in [30] and Algorithm 2 in [30] for Example 1, when $m=10$. 


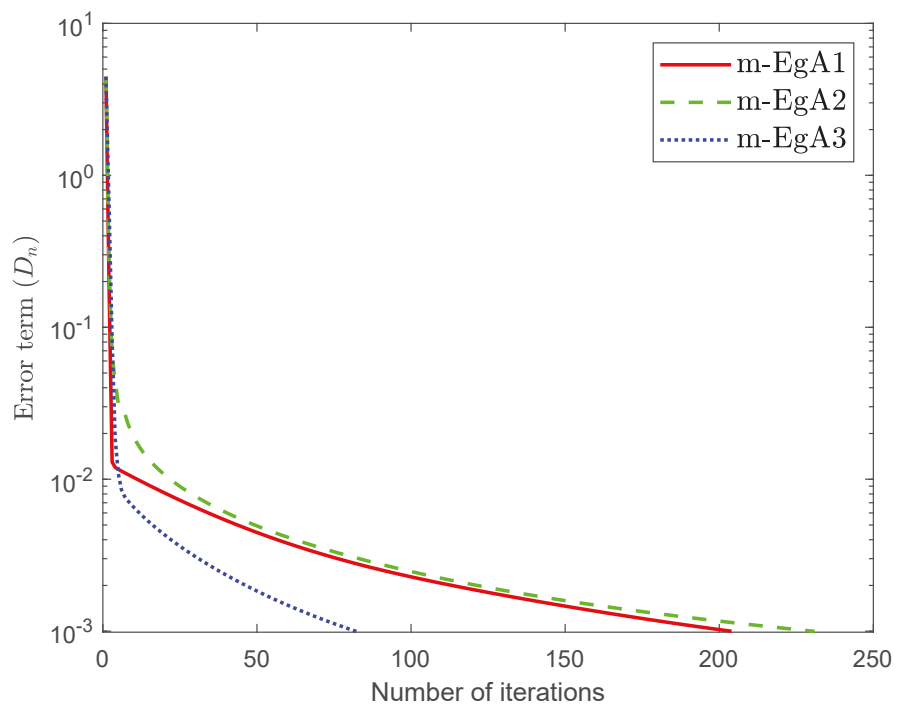

Figure 5. Numerical behaviour of Algorithm 1 compared to Algorithm 1 in [30] and Algorithm 2 in [30] for Example 1, when $m=20$.

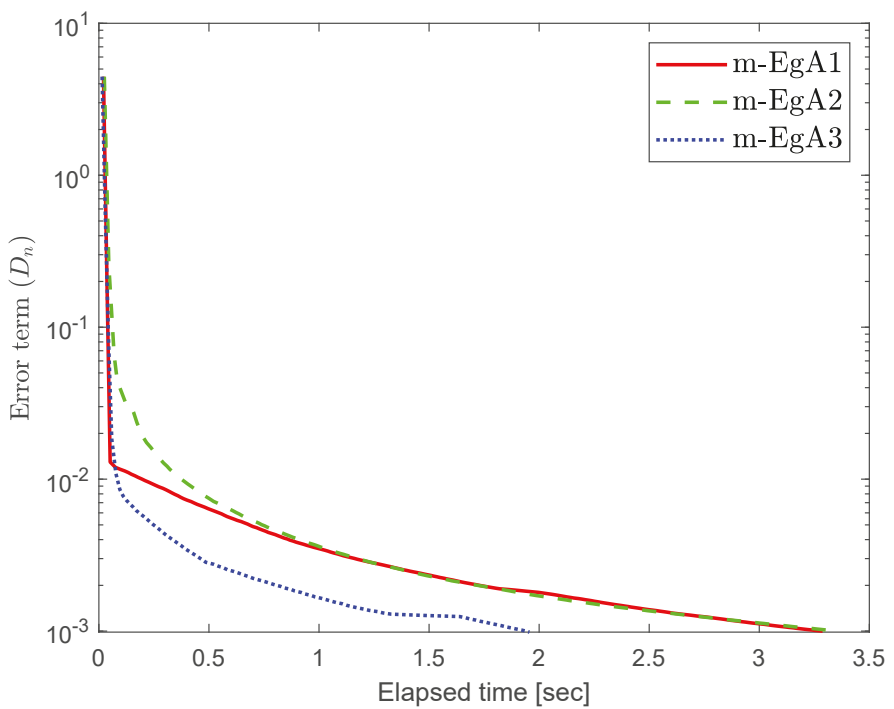

Figure 6. Numerical behaviour of Algorithm 1 compared to Algorithm 1 in [30] and Algorithm 2 in [30] for Example 1, when $m=20$. 


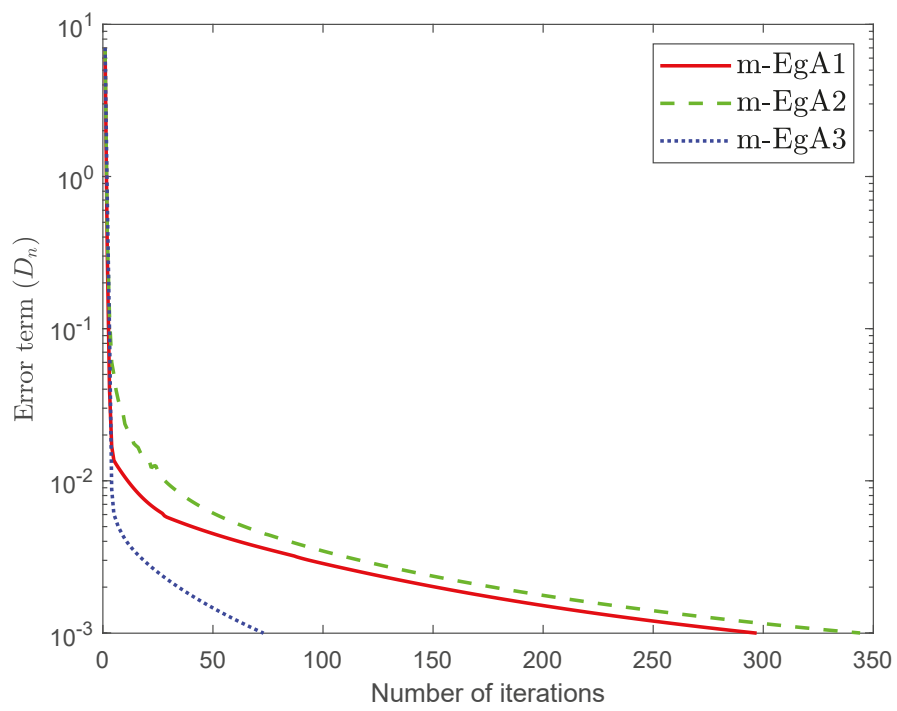

Figure 7. Numerical behaviour of Algorithm 1 compared to Algorithm 1 in [30] and Algorithm 2 in [30] for Example 1, when $m=50$.

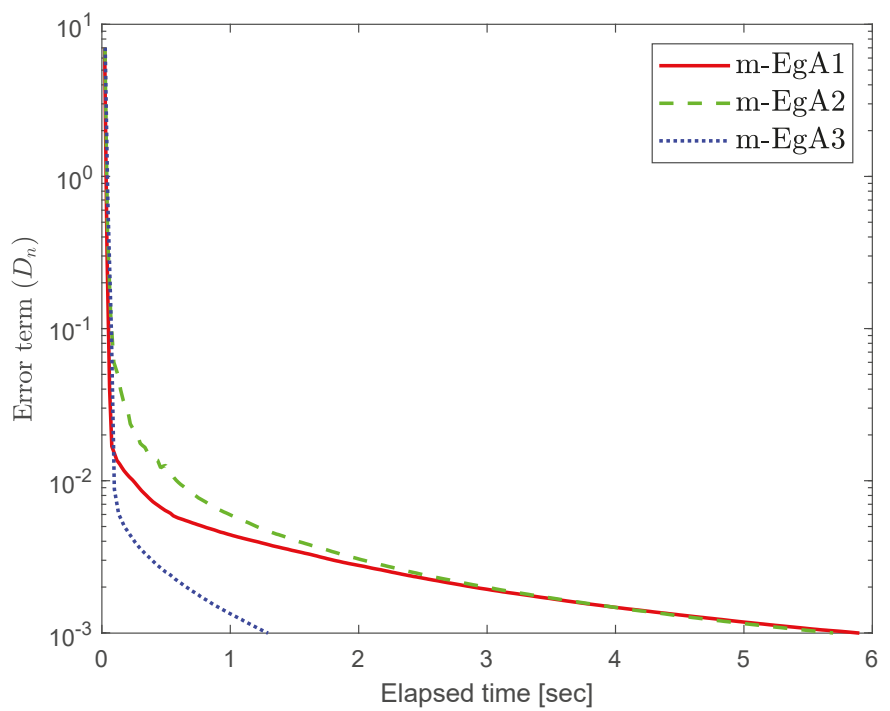

Figure 8. Numerical behaviour of Algorithm 1 compared to Algorithm 1 in [30] and Algorithm 2 in [30] for Example 1, when $m=50$. 
Table 1. Numerical results numeric values for Figures 1-8.

\begin{tabular}{ccccccc}
\hline & \multicolumn{2}{c}{ m-EgA1 [30] } & \multicolumn{2}{c}{ m-EgA2 [30] } & \multicolumn{2}{c}{ m-EgA3 } \\
\cline { 2 - 7 } m & Iter. & Time & Iter. & Time & Iter. & Time \\
\hline 5 & 59 & 1.0641 & 92 & 1.8107 & 34 & 0.8386 \\
10 & 126 & 2.2007 & 137 & 1.9408 & 73 & 1.0267 \\
20 & 204 & 3.2879 & 231 & 3.3654 & 83 & 11.9559 \\
50 & 297 & 5.8990 & 344 & 5.6944 & 73 & 1.2942 \\
\hline
\end{tabular}

Example 2. Assume that $\mathbb{H}=L^{2}([0,1])$ is a Hilbert space with an inner product

$$
\langle u, v\rangle=\int_{0}^{1} u(t) v(t) d t, \forall u, v \in \mathbb{H},
$$

and the induced norm is

$$
\|u\|=\sqrt{\int_{0}^{1}|u(t)|^{2} d t}
$$

Let $\mathcal{C}:=\left\{u \in L^{2}([0,1]):\|u\| \leq 1\right\}$ be the unit ball and $F: \mathcal{C} \rightarrow \mathbb{H}$ is defined by

$$
F(u)(t)=\int_{0}^{1}(u(t)-H(t, s) f(u(s))) d s+g(t),
$$

where

$$
H(t, s)=\frac{2 t s e^{(t+s)}}{e \sqrt{e^{2}-1}}, \quad f(u)=\cos (u), \quad g(t)=\frac{2 t e^{t}}{e \sqrt{e^{2}-1}} .
$$

We can see in [41], that $F$ is Lipschitz-continuous with Lipschitz constant $L=2$ and monotone. Figures 9-11 and Table 2 show the numerical results by taking different initial values $u_{0}$ and $\epsilon=10^{-3}$. In this experiment, we take the different initial points $u_{0}$ and $D_{n}=\left\|u_{n}-v_{n}\right\| \leq T O L=10^{-3}$. Moreover, the control parameters $\zeta_{0}=\frac{0.6}{L}$ and $\mu=0.45$ for Algorithm 1 (m-EgA1) in [30]; $\zeta_{0}=\frac{0.6}{L}, \mu=0.45$ and $\beta_{n}=\frac{1}{100(k+2)}$ for Algorithm $2(m-E g A 2)$ in [30]; $\zeta_{0}=\frac{0.6}{L}, \mu=0.45, \beta_{n}=\frac{1}{n+2}$ and $f(u)=\frac{u}{3}$ for Algorithm 1 ( $m$-EgA3).
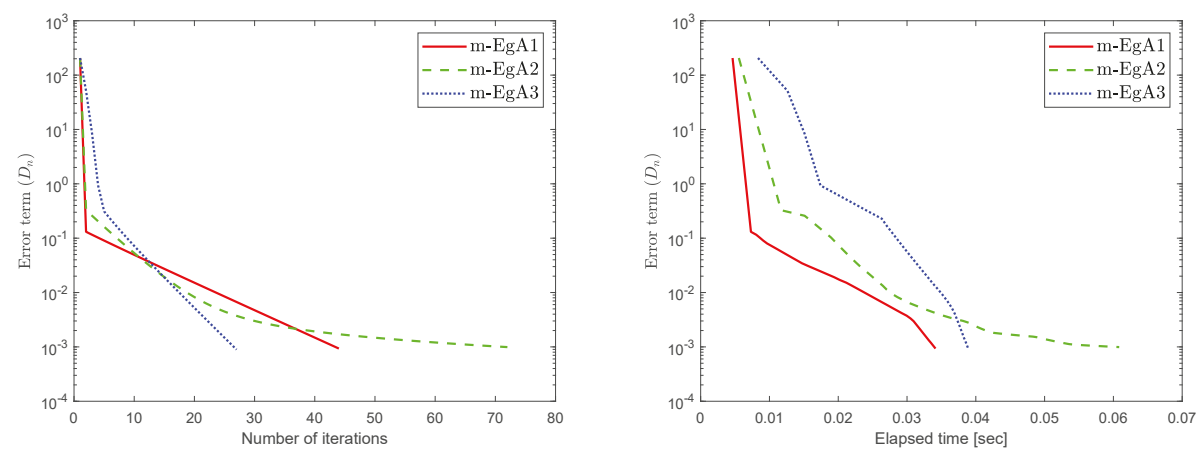

Figure 9. Numerical behaviour of Algorithm 1 compared to Algorithm 1 in [30] and Algorithm 2 in [30] for Example 1, when $u_{0}=t$. 

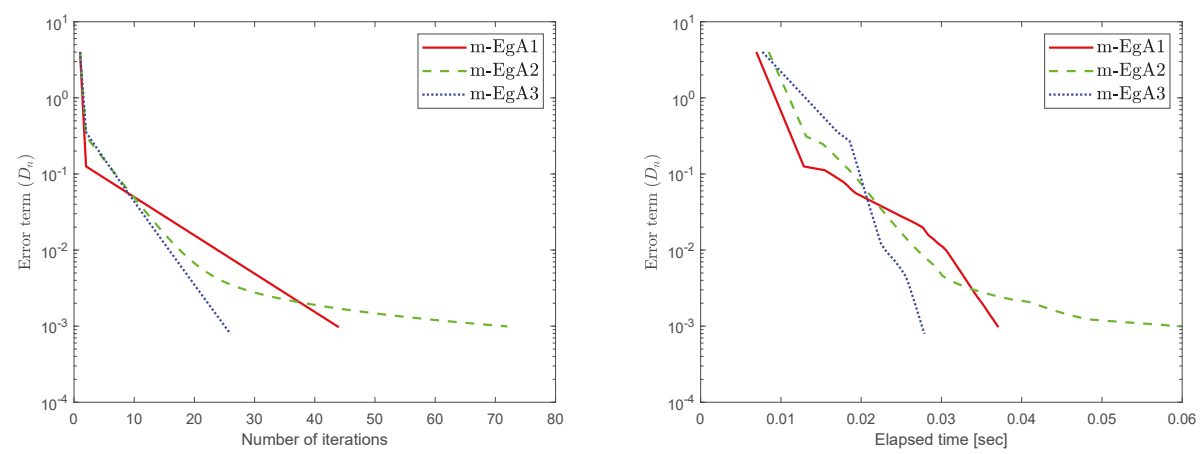

Figure 10. Numerical behaviour of Algorithm 1 compared to Algorithm 1 in [30] and Algorithm 2 in [30] for Example 1, when $u_{0}=\sin (t)$.
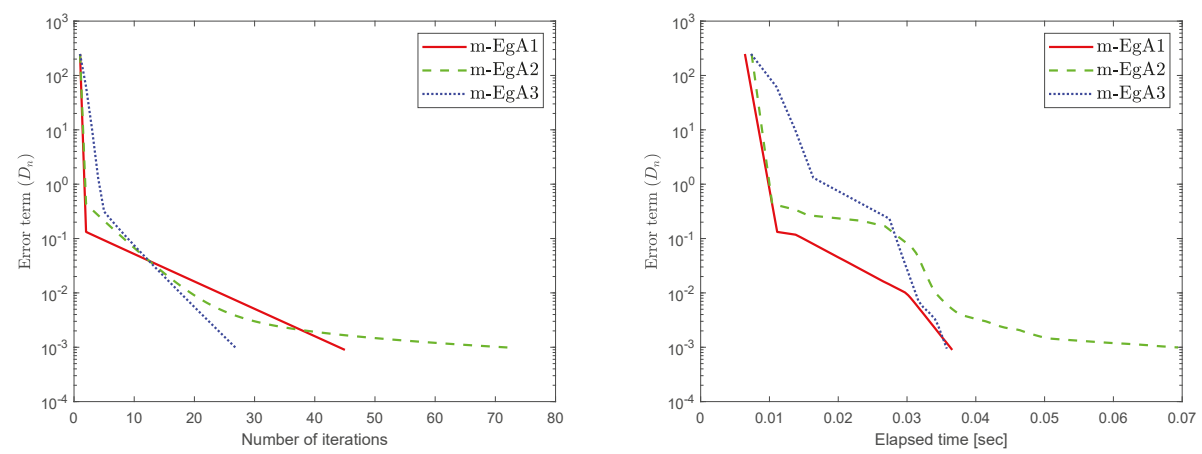

Figure 11. Numerical behaviour of Algorithm 1 compared to Algorithm 1 in [30] and Algorithm 2 in [30] for Example 1, when $u_{0}=\cos (t)$.

Table 2. Numerical comparison values for Figures 1-8.

\begin{tabular}{ccccccc}
\hline & \multicolumn{2}{c}{ m-EgA1 [30] } & \multicolumn{2}{c}{ m-EgA2 [30] } & \multicolumn{2}{c}{ m-EgA3 } \\
\cline { 2 - 7 }$u_{\mathbf{0}}$ & Iter. & Time & Iter. & Time & Iter. & Time \\
\hline$t$ & 44 & 0.0342 & 72 & 0.0609 & 27 & 0.0390 \\
$\sin (t)$ & 44 & 0.0876 & 72 & 0.0569 & 40 & 0.0569 \\
$\cos (t)$ & 45 & 0.0366 & 72 & 0.0358 & 27 & 0.0358 \\
\hline
\end{tabular}

Example 3. Let $F: \mathbb{R}^{2} \rightarrow \mathbb{R}^{2}$ is defined by

$$
F\left(\begin{array}{l}
u_{1} \\
u_{2}
\end{array}\right)=\left(\begin{array}{c}
u_{1}+u_{2}+\sin \left(u_{1}\right) \\
-u_{1}+u_{2}+\sin \left(u_{2}\right)
\end{array}\right), \quad \forall\left(\begin{array}{l}
u_{1} \\
u_{2}
\end{array}\right) \in \mathbb{R}^{2}
$$

and $\mathcal{C}$ is taken as

$$
\mathcal{C}=\left\{u=\left(u_{1}, u_{1}\right)^{T} \in \mathbb{R}^{2}: 0 \leq u_{i} \leq 10, i=1,2\right\}
$$

This problem was proposed in [43], where F is L-Lipschitz continuous with Lipschitz constant $L=\sqrt{10}$ and monotone. In this experiment, we take the different initial points $u_{0}$ and $D_{n}=\left\|u_{n}-v_{n}\right\| \leq T O L$. Moreover, the control parameters $\zeta_{0}=\frac{0.7}{L}$ and $\mu=0.50$ for Algorithm 1 (m-EgA1) in [30]; $\zeta_{0}=\frac{0.7}{L}, \mu=0.50$ and $\beta_{n}=\frac{1}{100(n+2)}$ for Algorithm $2(m-E g A 2)$ in [30]; $\zeta_{0}=\frac{0.7}{L}, \mu=0.50, \beta_{n}=\frac{1}{100(n+2)}$ and $f(u)=\frac{u}{4}$ for Algorithm 1 ( $m$-EgA3). Table 3 reports the numerical results by using different tolerance and initial points. 
Table 3. Numerical behaviour of Algorithm 1 compared to Algorithm 1 in [30] and Algorithm 2 in [30] for Example 3 by using different initial points $u_{0}$.

\begin{tabular}{|c|c|c|c|c|c|c|c|c|c|}
\hline & $\begin{array}{c}\text { TOL } \\
u_{0}\end{array}$ & $\begin{array}{l}0.01 \\
\text { Iter. }\end{array}$ & $\begin{array}{c}0.001 \\
\text { Iter. }\end{array}$ & $\begin{array}{c}0.0001 \\
\text { Iter. }\end{array}$ & $\begin{array}{c}0.00001 \\
\text { Iter. }\end{array}$ & $\begin{array}{c}0.01 \\
\text { Time }\end{array}$ & $\begin{array}{l}0.001 \\
\text { Time }\end{array}$ & $\begin{array}{c}0.0001 \\
\text { Time }\end{array}$ & $\begin{array}{c}0.00001 \\
\text { Time }\end{array}$ \\
\hline \multicolumn{10}{|l|}{ Algorithm 1 in [30] } \\
\hline & {$[10,20]^{T}$} & 29 & 41 & 83 & 277 & 0.4668 & 0.6234 & 1.5395 & 3.0415 \\
\hline & {$[-10,-10]^{T}$} & 45 & 57 & 117 & 345 & 0.9234 & 1.1440 & 1.7387 & 3.4382 \\
\hline & {$[10,20]^{T}$} & 59 & 71 & 143 & 389 & 1.0806 & 1.4264 & 1.8271 & 3.9269 \\
\hline \multicolumn{10}{|l|}{ Algorithm 2 in [30] } \\
\hline & {$[10,20]^{T}$} & 31 & 42 & 87 & 290 & 0.4743 & 0.5981 & 1.4921 & 3.2051 \\
\hline & {$[-10,-10]^{T}$} & 45 & 61 & 115 & 360 & 0.8976 & 1.2081 & 1.5891 & 3.7891 \\
\hline & {$[10,20]^{T}$} & 69 & 73 & 151 & 407 & 1.2711 & 1.3910 & 2.0810 & 4.1981 \\
\hline \multicolumn{10}{|l|}{ Algorithm 1} \\
\hline & {$[10,20]^{T}$} & 19 & 26 & 49 & 119 & 0.2391 & 0.3871 & 0.7716 & 1.6781 \\
\hline & {$[-10,-10]^{T}$} & 25 & 39 & 64 & 123 & 0.2991 & 0.5192 & 0.9981 & 1.7021 \\
\hline & {$[10,20]^{T}$} & 31 & 45 & 73 & 189 & 0.3018 & 0.7610 & 1.1012 & 2.4071 \\
\hline
\end{tabular}

Author Contributions: Data curation, N.W.; formal analysis, M.Y.; funding acquisition, N.P. (Nuttapol Pakkaranang) and N.P. (Nattawut Pholasa); investigation, N.W., N.P. (Nuttapol Pakkaranang) and H.u.R.; methodology, H.u.R.; project administration, H.u.R., N.P. (Nattawut Pholasa) and M.Y.; resources, N.P. (Nattawut Pholasa); software, H.u.R.; supervision, H.u.R. and N.P. (Nuttapol Pakkaranang); Writing-original draft, N.W. and H.u.R.; Writing-review and editing, N.P. (Nuttapol Pakkaranang). All authors have read and agreed to the published version of the manuscript.

Funding: This research was funded by School of Science, University of Phayao, Phayao, Thailand (Grant No. UoE 63002).

Acknowledgments: We are very grateful to the editor and the anonymous referees for their valuable and useful comments, which helps in improving the quality of this work. N. Wairojjana would like to thank Valaya Alongkorn Rajabhat University under the Royal Patronage (VRU). N. Pholasa was partially supported by University of Phayao.

Conflicts of Interest: The authors declare no conflict of interest.

\section{References}

1. Stampacchia, G. Formes bilinéaires coercitives sur les ensembles convexes. Comptes Rendus Hebd. Des Seances De L Acad. Des Sci. 1964, 258, 4413.

2. Konnov, I.V. On systems of variational inequalities. Russ. Math. C/C-Izv.-Vyss. Uchebnye Zaved. Mat. 1997, 41, 77-86.

3. Kassay, G.; Kolumbán, J.; Páles, Z. On Nash stationary points. Publ. Math. 1999, 54, 267-279.

4. Kassay, G.; Kolumbán, J.; Páles, Z. Factorization of Minty and Stampacchia variational inequality systems. Eur. J. Oper. Res. 2002, 143, 377-389. [CrossRef]

5. Kinderlehrer, D.; Stampacchia, G. An Introduction to Variational Inequalities and Their Applications; Society for Industrial and Applied Mathematics: Philadelphia, PA, USA, 2000; doi:10.1137/1.9780898719451. [CrossRef]

6. Konnov, I. Equilibrium Models and Variational Inequalities; Elsevier: Amsterdam, The Netherlands, 2007; Volume 210.

7. Takahashi, W. Introduction to Nonlinear and Convex Analysis; Yokohama Publishers, Yokohama, Japan, 2009.

8. Korpelevich, G. The extragradient method for finding saddle points and other problems. Matecon 1976, 12, 747-756.

9. Noor, M.A. Some iterative methods for nonconvex variational inequalities. Comput. Math. Model. 2010, 21, 97-108. [CrossRef]

10. Censor, Y.; Gibali, A.; Reich, S. The subgradient extragradient method for solving variational inequalities in Hilbert space. J. Optim. Theory Appl. 2010, 148, 318-335. [CrossRef]

11. Censor, Y.; Gibali, A.; Reich, S. Extensions of Korpelevich's extragradient method for the variational inequality problem in Euclidean space. Optimization 2012, 61, 1119-1132. [CrossRef] 
12. Malitsky, Y.V.; Semenov, V.V. An Extragradient Algorithm for Monotone Variational Inequalities. Cybern. Syst. Anal. 2014, 50, 271-277. [CrossRef]

13. Tseng, P. A Modified Forward-Backward Splitting Method for Maximal Monotone Mappings. SIAM J. Control Optim. 2000, 38, 431-446. [CrossRef]

14. Moudafi, A. Viscosity Approximation Methods for Fixed-Points Problems. J. Math. Anal. Appl. 2000, 241, 46-55. [CrossRef]

15. Zhang, L.; Fang, C.; Chen, S. An inertial subgradient-type method for solving single-valued variational inequalities and fixed point problems. Numer. Algorithms 2018, 79, 941-956. [CrossRef]

16. Iusem, A.N.; Svaiter, B.F. A variant of korpelevich's method for variational inequalities with a new search strategy. Optimization 1997, 42, 309-321. [CrossRef]

17. Thong, D.V.; Hieu, D.V. Modified subgradient extragradient method for variational inequality problems. Numer. Algorithms 2017, 79, 597-610. [CrossRef]

18. Thong, D.V.; Hieu, D.V. Weak and strong convergence theorems for variational inequality problems. Numer. Algorithms 2017, 78, 1045-1060. [CrossRef]

19. Marino, G.; Scardamaglia, B.; Karapinar, E. Strong convergence theorem for strict pseudo-contractions in Hilbert spaces. J. Inequal. Appl. 2016, 2016. [CrossRef]

20. Ur Rehman, H.; Kumam, P.; Cho, Y.J.; Yordsorn, P. Weak convergence of explicit extragradient algorithms for solving equilibirum problems. J. Inequal. Appl. 2019, 2019. [CrossRef]

21. Ur Rehman, H.; Kumam, P.; Je Cho, Y.; Suleiman, Y.I.; Kumam, W. Modified Popov's explicit iterative algorithms for solving pseudomonotone equilibrium problems. Optim. Methods Softw. 2020, 1-32. [CrossRef]

22. Ur Rehman, H.; Kumam, P.; Abubakar, A.B.; Cho, Y.J. The extragradient algorithm with inertial effects extended to equilibrium problems. Comput. Appl. Math. 2020, 39. [CrossRef]

23. Ur Rehman, H.; Kumam, P.; Kumam, W.; Shutaywi, M.; Jirakitpuwapat, W. The inertial sub-gradient extra-gradient method for a class of pseudo-monotone equilibrium problems. Symmetry 2020, 12, 463. [CrossRef]

24. Ur Rehman, H.; Kumam, P.; Argyros, I.K.; Deebani, W.; Kumam, W. Inertial extra-gradient method for solving a family of strongly pseudomonotone equilibrium problems in real Hilbert spaces with application in variational inequality problem. Symmetry 2020, 12, 503. [CrossRef]

25. Ur Rehman, H.; Kumam, P.; Argyros, I.K.; Alreshidi, N.A.; Kumam, W.; Jirakitpuwapat, W. A self-adaptive extra-gradient methods for a family of pseudomonotone equilibrium programming with application in different classes of variational inequality problems. Symmetry 2020, 12, 523. [CrossRef]

26. Ur Rehman, H.; Kumam, P.; Argyros, I.K.; Shutaywi, M.; Shah, Z. Optimization based methods for solving the equilibrium problems with applications in variational inequality problems and solution of Nash equilibrium models. Mathematics 2020, 8, 822. [CrossRef]

27. Ur Rehman, H.; Kumam, P.; Shutaywi, M.; Alreshidi, N.A.; Kumam, W. Inertial optimization based two-step methods for solving equilibrium problems with applications in variational inequality problems and growth control equilibrium models. Energies 2020, 13, 3292. [CrossRef]

28. Rehman, H.U.; Kumam, P.; Dong, Q.L.; Peng, Y.; Deebani, W. A new Popov's subgradient extragradient method for two classes of equilibrium programming in a real Hilbert space. Optimization 2020, 1-36. [CrossRef]

29. Antipin, A.S. On a method for convex programs using a symmetrical modification of the Lagrange function. Ekon. I Mat. Metod. 1976, 12, 1164-1173.

30. Yang, J.; Liu, H.; Liu, Z. Modified subgradient extragradient algorithms for solving monotone variational inequalities. Optimization 2018, 67, 2247-2258. [CrossRef]

31. Kraikaew, R.; Saejung, S. Strong Convergence of the Halpern Subgradient Extragradient Method for Solving Variational Inequalities in Hilbert Spaces. J. Optim. Theory Appl. 2013, 163, 399-412. [CrossRef]

32. Heinz, H.; Bauschke, P.L.C. Convex Analysis and Monotone Operator Theory in Hilbert Spaces, 2nd ed.; CMS Books in Mathematics; Springer International Publishing: New York, NY, USA, 2017.

33. Kreyszig, E. Introductory Functional Analysis with Applications, 1st ed.; Wiley Classics Library: Hoboken, NJ, USA, 1989.

34. Xu, H.K. Another control condition in an iterative method for nonexpansive mappings. Bull. Aust. Math. Soc. 2002, 65, 109-113. [CrossRef] 
35. Maingé, P.E. Strong Convergence of Projected Subgradient Methods for Nonsmooth and Nonstrictly Convex Minimization. Set-Valued Anal. 2008, 16, 899-912. [CrossRef]

36. Takahashi, W. Nonlinear Functional Analysis; Yokohama Publisher, Yokohama, Japan, 2000.

37. Liu, Z.; Zeng, S.; Motreanu, D. Evolutionary problems driven by variational inequalities. J. Differ. Equ. 2016, 260, 6787-6799. [CrossRef]

38. Liu, Z.; Migórski, S.; Zeng, S. Partial differential variational inequalities involving nonlocal boundary conditions in Banach spaces. J. Differ. Equ. 2017, 263, 3989-4006. [CrossRef]

39. Harker, P.T.; Pang, J.S. for the Linear Complementarity Problem. Comput. Solut. Nonlinear Syst. Equ. 1990, $26,265$.

40. Solodov, M.V.; Svaiter, B.F. A New Projection Method for Variational Inequality Problems. SIAM J. Control Optim. 1999, 37, 765-776. [CrossRef]

41. Van Hieu, D.; Anh, P.K.; Muu, L.D. Modified hybrid projection methods for finding common solutions to variational inequality problems. Comput. Optim. Appl. 2016, 66, 75-96. [CrossRef]

42. Dong, Q.L.; Cho, Y.J.; Zhong, L.L.; Rassias, T.M. Inertial projection and contraction algorithms for variational inequalities. J. Glob. Optim. 2017, 70, 687-704. [CrossRef]

43. Dong, Q.L.; Lu, Y.Y.; Yang, J. The extragradient algorithm with inertial effects for solving the variational inequality. Optimization 2016, 65, 2217-2226. [CrossRef]

Publisher's Note: MDPI stays neutral with regard to jurisdictional claims in published maps and institutional affiliations.

(C) 2020 by the authors. Licensee MDPI, Basel, Switzerland. This article is an open access article distributed under the terms and conditions of the Creative Commons Attribution (CC BY) license (http://creativecommons.org/licenses/by/4.0/). 



\title{
Reconstruction of Piecewise Smooth Multivariate Functions from Fourier Data
}

\author{
David Levin \\ School of Mathematical Sciences, Tel-Aviv University, Tel Aviv 6997801, Israel; levin@tauex.tau.ac.il \\ Received: 24 June 2020; Accepted: 22 July 2020; Published: 24 July 2020
}

\begin{abstract}
In some applications, one is interested in reconstructing a function $f$ from its Fourier series coefficients. The problem is that the Fourier series is slowly convergent if the function is non-periodic, or is non-smooth. In this paper, we suggest a method for deriving high order approximation to $f$ using a Padé-like method. Namely, we do this by fitting some Fourier coefficients of the approximant to the given Fourier coefficients of $f$. Given the Fourier series coefficients of a function on a rectangular domain in $\mathbb{R}^{d}$, assuming the function is piecewise smooth, we approximate the function by piecewise high order spline functions. First, the singularity structure of the function is identified. For example in the 2D case, we find high accuracy approximation to the curves separating between smooth segments of $f$. Secondly, simultaneously we find the approximations of all the different segments of $f$. We start by developing and demonstrating a high accuracy algorithm for the 1D case, and we use this algorithm to step up to the multidimensional case.
\end{abstract}

Keywords: fourier data; reconstruction; multivariate approximation; piecewise smooth

\section{Introduction}

Fourier series expansion is a useful tool for representing and approximating functions, with applications in many areas of applied mathematics. The quality of the approximation depends on the smoothness of the approximated function and on whether or not it is periodic. For functions that are not periodic, the convergence rate is slow near the boundaries and the approximation by partial sums exhibits the Gibbs phenomenon. Several approaches have been used to improve the convergence rate, mostly for the one-dimensional case. One approach is to filter out the oscillations, as discussed in several papers [1,2]. Another useful approach is to transform the Fourier series into an expansion in a different basis. For the univariate case this approach is shown to be very efficient, as shown in [1] using Gegenbauer polynomials with suitably chosen parameters. Further improvement of this approach is presented in [3] using Freud polynomials, achieving very good results for univariate functions with singularities.

An algebraic approach for reconstructing a piecewise smooth univariate function from its first $N$ Fourier coefficients has been realized by Eckhoff in a series of papers [4-6]. There, the "jumps" are determined by a corresponding system of linear equations. A full analysis of this approach is presented by Betankov [7]. Nersessian and Poghosyan [8] have used a rational Padé type approximation strategy for approximating univariate non-periodic smooth functions. For multiple Fourier series of smooth non-periodic functions, a convergence acceleration approach was suggested by Levin and Sidi [9]. More challenging is the case of multivariate functions with discontinuities, i.e., functions that are piecewise smooth. Here again, the convergence rate is slow, and near the discontinuities, the approximation exhibits the Gibbs phenomenon. In this paper, we present a Padé-like approach consisting of finding a piecewise-defined spline whose Fourier coefficients match the given Fourier coefficients.

The main contribution of this paper is demonstrating that this approach can be successfully applied to the multivariate case. Namely, we present a strategy for approximating both non-periodic 
and non-smooth multivariate functions. We derive the numerical procedures involved and provide some interesting numerical results. We start by developing and demonstrating a high accuracy algorithm for the $1 \mathrm{D}$ case, and use this algorithm to step up to the multidimensional case.

\section{The 1D Case}

In this section, we present the main tools for function approximation using its Fourier series coefficients. We define the basis functions and describe the fitting strategy and develop the computation algorithm. After dealing with the smooth case we move on to approximate a piecewise smooth function with a jump singularity.

\subsection{Reconstructing Smooth Non-Periodic Functions}

Let $f \in C^{m}[0,1]$, and assume we know the Fourier series expansion of $f$

$$
f(x)=\sum_{n \in \mathbb{Z}} \hat{f}_{n} e^{2 \pi i n x}
$$

The series converge pointwise for any $x \in[0,1]$, however, if $f$ is not periodic, the convergence may be slow, and if $f(1) \neq f(0)$ the convergence is not uniform and the Gibbs phenomenon occurs near 0 and near 1 . As discussed in $[9,10]$, one can apply convergence acceleration techniques for improving the convergence rate of the series. Another convergence acceleration approach was suggested by Gottlieb and Shu [1] using Gegenbauer polynomials. Yet, in both approaches, the convergence rate is not much improved near 0 and near 1 . We suggest an approach in the spirit of Padé approximation. A Padé approximant is a rational function whose power series agrees as much as possible with the given power series of $f$. Here we look for approximations to $f$ whose Fourier coefficients agree with a subset of the given Fourier coefficients of $f$. The approximation space can be any favorable linear approximation space, such as polynomials or trigonometric functions.

We choose to build the approximation using $k$ th order spline functions, represented in the B-spline basis:

$$
S_{d}^{[k]}(x)=\sum_{j=1}^{N_{d}} a_{j} B_{d}^{[k]}(x-j d) .
$$

$B_{d}^{[k]}(x)$ is the B-spline of order $k$ with equidistant knots $\{-k d, \ldots,-2 d,-d, 0\}$, and $N_{d}=1 / d+k-1$ is the number of B-splines whose shifts do not vanish in $[0,1]$. The advantage of using spline functions is threefold:

- The locality of the B-spline basis functions.

- A closed form formula for their Fourier coefficients.

- Their approximation power, i.e., if $f \in C^{k}[0,1]$, there exists a spline $S_{d}^{[k]}$ such that $\left\|f-S_{d}^{[k]}\right\|_{\infty,[0,1]} \leq$ $C d^{k}$.

The B-splines basis functions used in the 1D case are shown in Figure 1 . We denote by $\left.S \equiv S_{d}^{[k]}\right|_{[0,1]}$ the restriction of $S_{d}^{[k]}$ to the interval $[0,1]$. We find the coefficients $\left\{a_{i}\right\}_{i=1}^{N_{d}}$ by least-squares fitting, matching the first $M+1$ Fourier coefficients of $S$ to the corresponding $M+1$ Fourier coefficients of $f$. That is,

$$
\left\{a_{i}\right\}_{i=1}^{N_{d}}=\arg \min \sum_{n=0}^{M}\left|\hat{f}_{n}-\hat{S}_{n}\right|^{2} .
$$

Notice that it is enough to consider the Fourier coefficients with non-negative indices. 


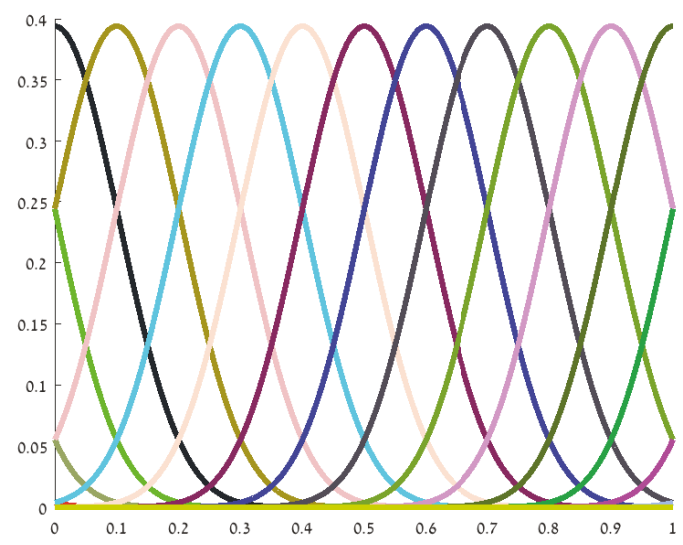

Figure 1. The B-splines used in Example 1.

We denote by $\left.B_{i} \equiv B_{d}^{[k]}(\cdot-i d)\right|_{[0,1]}$ the restriction of $B_{d}^{[k]}(\cdot-i d)$ to the interval $[0,1]$, and by $\left\{\hat{B}_{i, n}\right\}$ its Fourier coefficients. The normal equations for the least squares problem (3) induce the linear system $A a=b$ for $a=\left\{a_{i}\right\}_{i=1}^{N_{d}}$, where

$$
A_{i, j}=\sum_{n=0}^{M}\left[\operatorname{Re}\left(\hat{B}_{i, n}\right) \operatorname{Re}\left(\hat{B}_{j, n}\right)+\operatorname{Im}\left(\hat{B}_{i, n}\right) \operatorname{Im}\left(\hat{B}_{j, n}\right)\right], \quad 1 \leq i, j \leq N_{d},
$$

and

$$
b_{i}=\sum_{n=0}^{M}\left[\operatorname{Re}\left(\hat{B}_{i, n}\right) \operatorname{Re}\left(\hat{f}_{n}\right)+\operatorname{Im}\left(\hat{B}_{i, n}\right) \operatorname{Im}\left(\hat{f}_{n}\right)\right], \quad 1 \leq i \leq N_{d} .
$$

Numerical Example-The Smooth 1D Case

We consider the test function $f(x)=x \exp (x)+\sin (8 x)$, assuming only its Fourier coefficients are given. We have used only the 20 Fourier coefficients $\left\{\hat{f}_{n}\right\}_{n=0}^{19}$, and computed an approximation using 12th degree splines with equidistant knots' distance $d=0.1$. For this case, the matrix $A$ is of size $19 \times 19$, and $\operatorname{cond}(A)=5.75 \times 10^{20}$. We have employed an iterative refinement algorithm described below to obtain a high precision solution. The results are shown in the following two figures. In Figure 2 we see the test function on the left and the approximation error on the right. Figure 3 presents the graph of $\log _{10}\left(\hat{f}_{n}\right)$ in blue and the graph of $\log _{10}\left(\hat{f}_{n}-\hat{S}_{n}\right)$, showing eight orders of magnitude reduction in the Fourier coefficients. Notice the matching in the first Fourier coefficients reflected in the beginning of the red graph.

Remark 1. The powerful iterative refinement method described in $[11,12]$ is as follows:

For solving a system $A x=b$, we use some solver, e.g., the Matlab pinv function. We obtain the solution $x^{(0)}=\operatorname{pinv}(A) b$. Next we compute the residual $r^{(0)}=b-A x^{(0)}$. In case cond $(A)$ is very large, the residual will be large. Now we solve again the system with $r^{(0)}$ at the right hand side, and use the solution to correct $x^{(0)}$, to obtain

$$
x^{(1)}=x^{(0)}+\operatorname{pinv}(A) r^{(0)} .
$$

We repeat this correction steps a few times, i.e., $r^{(k)}=b-A x^{(k)}$, and

$$
x^{(k+1)}=x^{(k)}+\operatorname{pinv}(A) r^{(k)},
$$

until the resulting residual $r^{(k)}$ is small enough. 

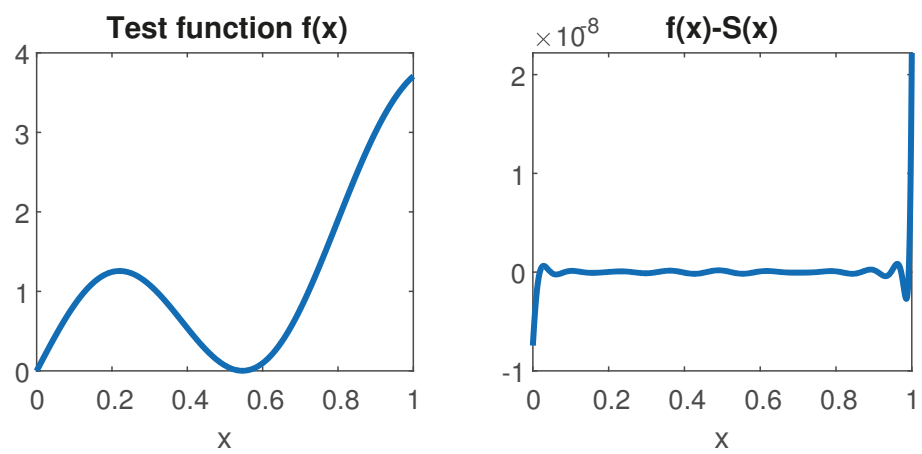

Figure 2. The test function (left) and the spline approximation error (right).

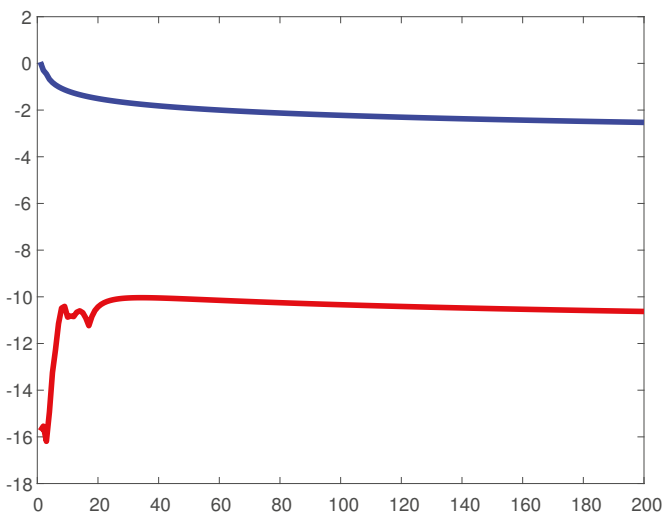

Figure 3. $\log _{10}$ of the given Fourier coefficients (blue), and of the Fourier coefficients of the approximation error (red).

\subsection{Reconstructing Non-Smooth Univariate Functions}

Let $f$ be a piecewise smooth function on $[0,1]$, defined by combined two pieces $f_{1} \in C^{m}\left[0, s^{*}\right]$ and $f_{2} \in C^{m}\left(s^{*}, 1\right]$, and assume that $f_{2}$ can be continuously extended to $\left[s^{*}, 1\right]$.

$$
f(x)= \begin{cases}f_{1}(x) & x \geq s^{*}, \\ f_{2}(x) & x<s^{*}\end{cases}
$$

Here again, we assume that all we know about $f$ is its Fourier series expansion. In particular, we do not know the position $s^{*} \in[0,1]$ of the singularity of $f$. As in the case of a non-periodic function, the existence of a singularity in $[0,1]$ significantly influences the Fourier series coefficients and implies their slow decay. As we demonstrate below, good matching of the Fourier coefficients requires a good approximation of the singularity location. The approach we suggest here involves finding approximations to $f_{1}$ and $f_{2}$ simultaneously with a high precision identification of $s^{*}$.

Let $s$ be an approximation of the singularity location $s^{*}$, and let us follow the algorithm suggested above for the smooth case. The difference here is that now we look for two separate spline approximations:

$$
\left.S_{1} \equiv S_{d}^{[k]}\right|_{[0, s]}(x)=\left.\sum_{i=1}^{N_{d}} a_{1 i} B_{d}^{[k]}(x-i d)\right|_{[0, s]} \sim f_{1}
$$


and

$$
\left.S_{2} \equiv S_{d}^{[k]}\right|_{(s, 1]}(x)=\left.\sum_{i=1}^{N_{d}} a_{2 i} B_{d}^{[k]}(x-i d)\right|_{(s, 1]} \sim f_{2} .
$$

The combination $S$ of $S_{1}$ and $S_{2}$ constitutes the approximation to $f$. Here again we aim at matching the first $M+1$ Fourier coefficients of $f$ and of $S$. Here $S$ depends on the $N_{d}$ coefficients $\left\{a_{1 i}\right\}$ of $S_{1}$, the $N_{d}$ coefficients $\left\{a_{2 i}\right\}$ of $S_{2}$ and on $s$. Therefore, the minimization process solves for all these unknowns:

$$
\left[\left\{a_{1 i}\right\}_{i=1}^{N_{d}},\left\{a_{2 i}\right\}_{i=1}^{N_{d}}, s\right]=\arg \min \sum_{n=0}^{M}\left|\hat{f}_{n}-\hat{S}_{n}\right|^{2} .
$$

The minimization is non-linear with respect to $s$, and linear with respect to the other unknowns. Therefore, the minimization problem is actually a one parameter non-linear minimization problem, the parameter $s$. Using the approximation power of $k$ th order splines $(k \leq m)$, and considering the value of the objective cost function for $s=s^{*}$, we can deduce that the minimal value of $\sum_{n=0}^{M}\left|\hat{f}_{n}-\hat{S}_{n}\right|^{2}$ is $O\left(d^{2 k}\right)$. We also observe that an $\epsilon$ deviation from $s^{*}$ implies a bounded deviation of the minimizing Fourier coefficients

$$
\max _{n \in \mathbb{Z}}\left|\hat{f}_{n}-\hat{S}_{n}\right| \leq c_{1} \epsilon+c_{2} d^{k}
$$

As shown below, these observations can be used for finding a good approximation to $s^{*}$.

We denote by $\left.B_{1 i} \equiv B_{d}^{[k]}(\cdot-i d)\right|_{[0, s]}$ the restriction of $B_{d}^{[k]}(\cdot-i d)$ to the interval $[0, s]$, and by $\left.B_{2 i} \equiv B_{d}^{[k]}(\cdot-i d)\right|_{(s, 1]}$ the restriction of $B_{d}^{[k]}(\cdot-i d)$ to the interval $(s, 1]$. We concatenate these two sequences of basis functions, $\left\{B_{1 i}\right\}$ and $\left\{B_{2 i}\right\}$ into one sequence $\left\{B_{i}\right\}_{i=1}^{2 N_{d}}$, and denote their Fourier coefficients by $\left\{\hat{B}_{i, n}\right\}_{n \in \mathbb{Z}}$. For a given $s$, the normal equations for the least squares problem (9) induce the linear system $A a=b$ for the splines' coefficients $a=\left(\left\{a_{1 i}\right\}_{i=1}^{N_{d}},\left\{a_{2 i}\right\}_{i=1}^{N_{d}}\right)$, where:

$$
A_{i, j}=\sum_{n=0}^{M}\left[\operatorname{Re}\left(\hat{B}_{i, n}\right) \operatorname{Re}\left(\hat{B}_{j, n}\right)+\operatorname{Im}\left(\hat{B}_{i, n}\right) \operatorname{Im}\left(\hat{B}_{j, n}\right)\right], \quad 1 \leq i, j \leq 2 N_{d}
$$

and

$$
b_{i}=\sum_{n=0}^{M}\left[\operatorname{Re}\left(\hat{B}_{i, n}\right) \operatorname{Re}\left(\hat{f}_{n}\right)+\operatorname{Im}\left(\hat{B}_{i, n}\right) \operatorname{Im}\left(\hat{f}_{n}\right)\right], \quad 1 \leq i \leq 2 N_{d}
$$

Remark 2. Due to the locality of the B-splines, some of the basis functions $\left\{B_{1 i}\right\}$ and $\left\{B_{2 i}\right\}$ may be identical 0 . It thus seems better to use only the non-zero basis functions. From our experience, since we use the generalized inverse approach for solving the system of equations, using all the basis functions gives the same solution.

The generalized inverse approach computes the least-squares solution to a system of linear equations that lacks a unique solution. It is also called the Moore-Penrose inverse, and is computed by Matlab pinv function.

The above construction can be carried out to the case of several singular points.

\subsubsection{Finding $s^{*}$}

We present the strategy for finding $s^{*}$ together with a specific numerical example. We consider a test function on $[0,1]$ with a jump discontinuity at $s^{*}=0.5$ :

$$
f(x)= \begin{cases}f_{1}(x)=\sin (5 x) & x \geq s^{*} \\ f_{2}(x)=\frac{1}{(x-0.5)^{2}+0.5} & x<s^{*} .\end{cases}
$$

As expected, the Fourier series of $f$ is slowly convergent, and it exhibits the Gibbs phenomenon near the ends of $[0,1]$ and near $s^{*}$. In Figure 4 , on the left, we present the sum of the first 200 terms of 
the Fourier series, computed at 20,000 points in $[0,1]$. This sum is not acceptable as an approximation to $f$, and yet we can use it to obtain a good initial approximation to $s_{0} \sim s^{*}$. On the right graph, we plot the first differences of the values in the left graph. The maximal difference is achieved at a distance of order $10^{-4}$ from $s^{*}$.
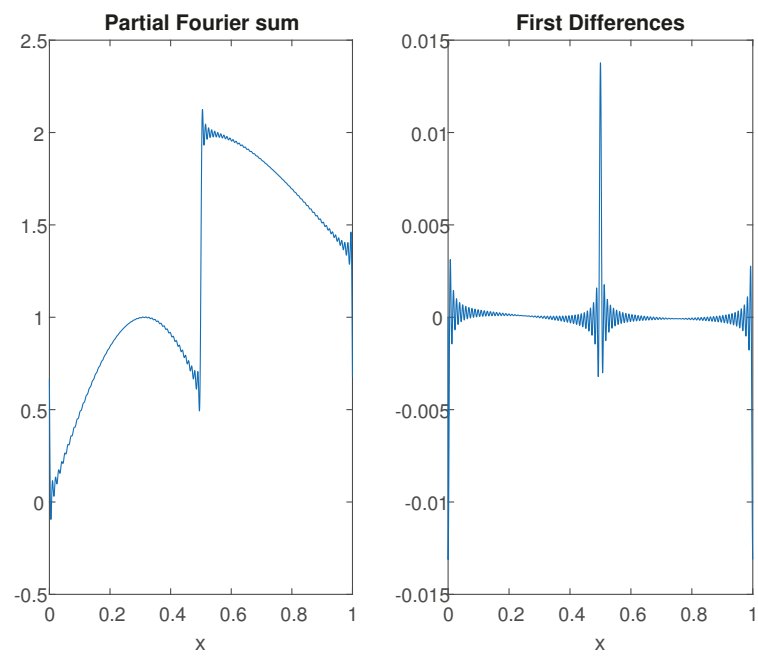

Figure 4. A partial Fourier sum (left) and its first differences (right).

Having a good approximation $s_{0} \sim s^{*}$ is not enough for achieving a good approximation to $f$. However, $s_{0}$ can be used as a starting point for an iterative method leading to a high precision approximation to $s^{*}$. To support this assertion we present the graph in Figure 5, depicting the maximum norm of the difference between 1000 of the given Fourier coefficients and the corresponding Fourier coefficients of the approximation $S$, as a function of $s$, near $s^{*}=0.5$. This function is almost linear on each side of $s^{*}$, and simple quasi-Newton iterations converge very fast to $s^{*}$. After obtaining a high accuracy approximation to $s^{*}$, we use it for deriving the piecewise spline approximation to $f$.

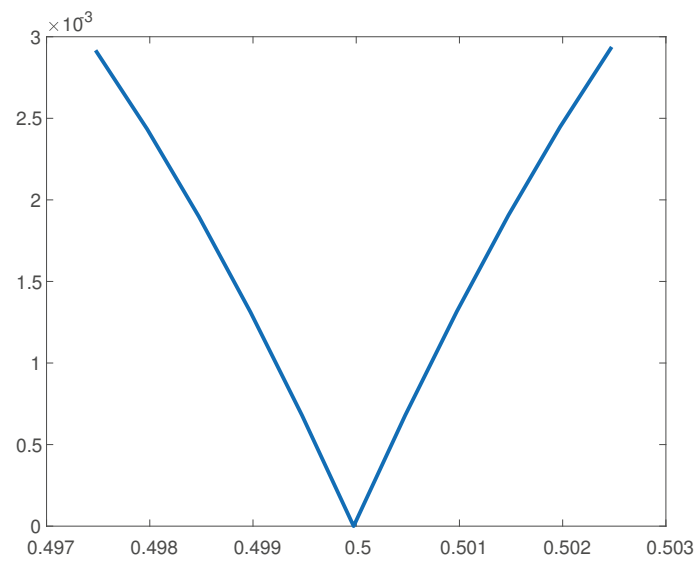

Figure 5. The graph of the error $\|\hat{f}-\hat{S}\|$ as a function of $s$ near $s^{*}=0.5$.

In the following, we present the numerical results obtained for the test function defined in (13). We have used only 20 Fourier coefficients of $f$, and the two approximating functions $S_{1}$ and $S_{2}$ are 
splines of order eight, with knots' distance $d=0.1$. Figure 6 depicts the approximation error, showing that $\|f-S\|_{\infty}=5.3 \times 10^{-8}$, and that the Gibbs phenomenon is completely removed. Figure 7 shows $\log _{10}$ of the absolute values of the given Fourier coefficients of $f$ (in blue), and the corresponding values for the Fourier coefficients of $f-S$ (in red). The graph shows a reduction of $\sim 7$ orders of magnitude. These results clearly demonstrate the high effectiveness of the proposed approach.

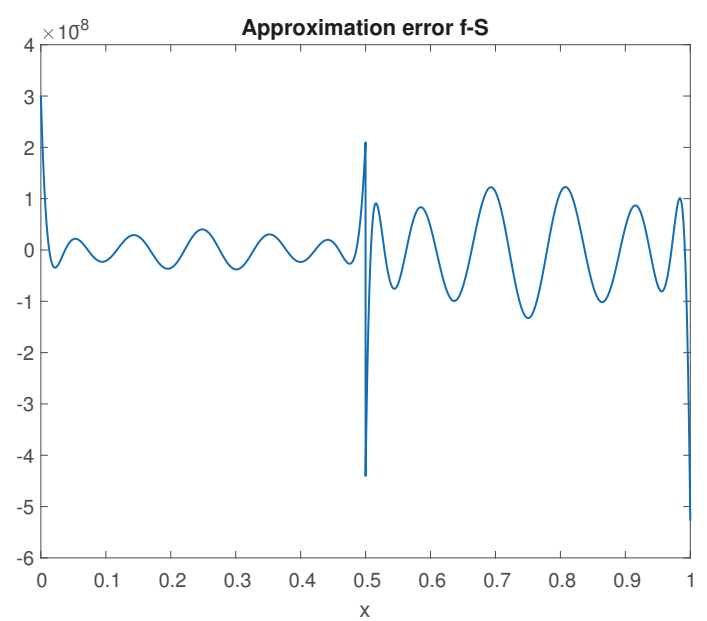

Figure 6. The approximation error for the 1D non-smooth case.

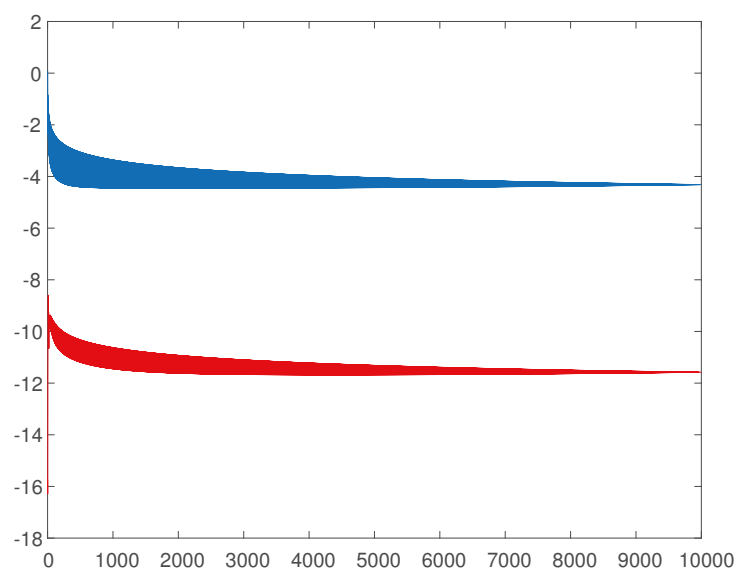

Figure 7. $\log _{10}$ of the given Fourier coefficients (blue), and of the Fourier coefficients of the approximation error (red).

2.2.2. The 1D Approximation Procedure

Let us sum up the suggested approximation procedure:

(1) Choose the approximation space $\Pi$ for approximating $f_{1}$ and $f_{2}$.

(2) Define the number of Fourier coefficients to be used for building the approximation such that

$$
M+1 \geq 2 \operatorname{dim}(\Pi)
$$


(3) Find first approximation to $s^{*}$ : Compute a partial Fourier sum and locate maximal first order difference.

(4) Calculate the first $M+1$ Fourier coefficients of the basis functions of $\Pi$, truncated at $s^{*}$.

(5) Use the above Fourier coefficients to compute the approximation to $f_{1}$ and $f_{2}$ by solving the system of linear equation defined by (11), (12).

(6) Update the approximation to $s^{*}$, by performing quasi-Newton iterations to reduce the objective function in (9).

(7) Go back to (4) to update the approximation.

\section{The 2D Case-Non-Periodic and Non-Smooth}

\subsection{The Smooth $2 D$ Case}

Let $f \in C^{m}[0,1]^{2}$, and assume we know its Fourier series expansion

$$
f(x, y)=\sum_{m \in \mathbb{Z}} \sum_{n \in \mathbb{Z}} \hat{f}_{m n} e^{2 \pi i m x} e^{2 \pi i n y} .
$$

Such series are obtained when solving PDE using spectral methods. However, if the function is not periodic, or, as in the case of hyperbolic equations, the function has a jump discontinuity along some curve in $[0,1]^{2}$, the convergence of the Fourier series is slow. Furthermore, the approximation of $f$ by its partial sums suffers from the Gibbs phenomenon near the boundaries and near the singularity curve.

We deal with the case of smooth non-periodic 2D functions in the same manner as we did for the univariate case. We look for a bivariate spline function $S$ whose Fourier coefficients match the Fourier coefficients of $f$. As in the univariate case, it is enough to match the coefficients of low frequency terms in the Fourier series. The technical difference in the 2D case is that we look for a tensor product spline approximation, using tensor product $k$ th order B-spline basis functions.

$$
S_{d}^{[k]}(x, y)=\sum_{i=1}^{N_{d}} \sum_{j=1}^{N_{d}} a_{i j} B_{d}^{[k]}(x-i d) B_{d}^{[k]}(y-j d) .
$$

The system of equations for the B-spline coefficients is the same as the system defined by (4) and (5) in the univariate case, only here we reshape the unknowns as a vector of $N_{d}^{2}$ unknowns.

Numerical Example-The Smooth 2D Case

We consider the test function

$$
f(x, y)=\frac{10}{1+10\left(x^{2}+(y-1)^{2}\right)}+\sin (10(x-y)),
$$

assuming only its Fourier coefficients are given. We have used only 160 Fourier coefficients, and constructed an approximation using 10th degree tensor product splines with equidistant knots' distance $d=0.1$ in each direction. For this case, the matrix $A$ is of size $361 \times 361$, and $\operatorname{cond}(A)=$ $6.2 \times 10^{30}$. Again, we have employed the iterative refinement algorithm to obtain a high precision solution (relative error $10^{-15}$ ). Computation time $\sim 18 \mathrm{~s}$.

In Figure 8 we plot the test function on $[0,1]^{2}$. Note that it has high derivatives near $(0,1)$.

The approximation error $f-S_{0.1}^{[10]}$ is shown in Figure 9. To demonstrate the convergence acceleration of the Fourier series achieved by subtracting the approximation from $f$, we present in Figure $10 \log _{10}$ of the absolute values of the Fourier coefficients of $f$ (in green) and of the Fourier coefficients of $f-S_{0.1}^{[10]}$ (in blue), for frequencies $0 \leq m, n \leq 200$. The magnitude of the Fourier coefficients is reduced by a factor of $10^{5}$, and even more so for the low frequencies due to the matching strategy used to derive the spline approximation. 


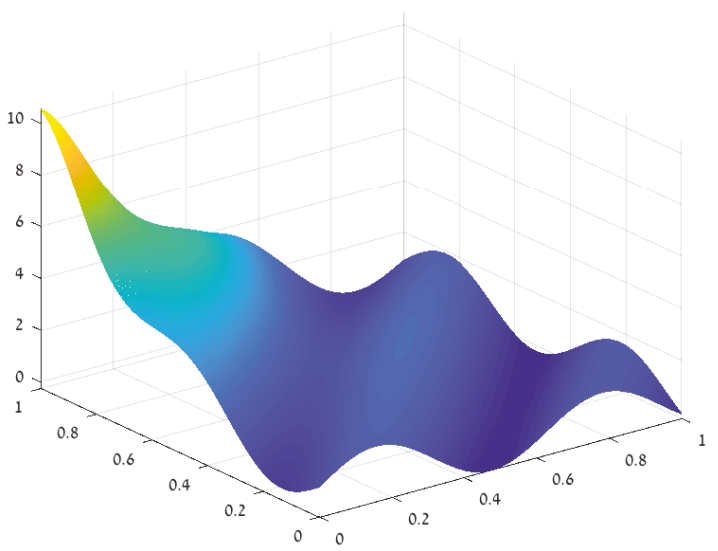

Figure 8. The test function for the smooth $2 \mathrm{D}$ case.

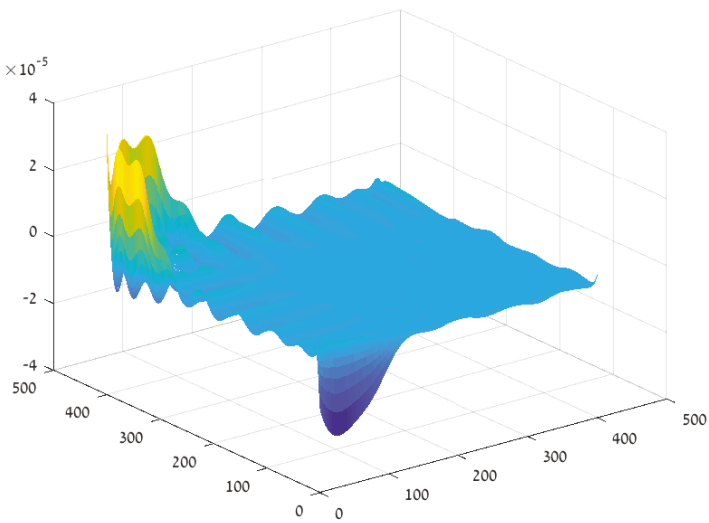

Figure 9. The approximation error $f-S_{0.1}^{[10]}$.

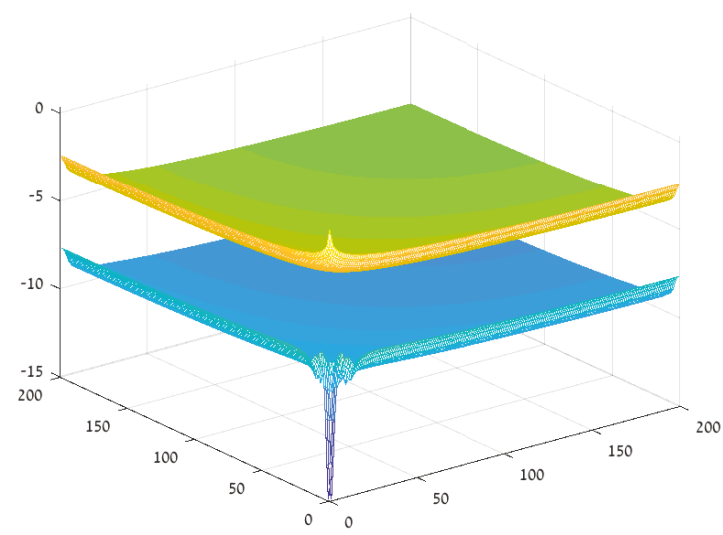

Figure 10. $\log _{10}$ of the Fourier coefficients before (green), and after (blue). 


\subsection{The Non-Smooth $2 D$ Case}

Let $\Omega_{1}, \Omega_{2} \subset[0,1]^{2}$ be open, simply connected domains with the properties

$$
\Omega_{1} \cap \Omega_{2}=\varnothing, \quad \bar{\Omega}_{1} \cup \bar{\Omega}_{2}=[0,1]^{2} .
$$

Let $\Gamma^{*}$ be the curve separating the two domains,

$$
\Gamma^{*}=\bar{\Omega}_{1} \cap \bar{\Omega},
$$

and assume $\Gamma^{*}$ is a $C^{m}$-smooth curve.

Let $f$ be a piecewise smooth function on $[0,1]^{2}$, defined by combined two pieces $f_{1} \in C^{m}\left[\Omega_{1}\right]$ and $f_{2} \in C^{m}\left[\Omega_{2}\right]$, and assume that each $f_{j}$ can be continuously extended to a function in $C^{m}\left(\bar{\Omega}_{j}\right)$, $j=1,2$. Here again, we assume that all we know about $f$ is its Fourier expansion. In particular, we do not know the position of the dividing curve separating $\Omega_{1}$ and $\Omega_{2}$. We denote this curve by $\Gamma^{*}$, and we assume that it is a $C^{m}$-smooth curve. As in the case of a non-periodic function, the existence of a singularity curve in $[0,1]^{2}$ significantly influences the Fourier series coefficients and implies their slow decay. In case of discontinuity of $f$ across $\Gamma^{*}$, partial sums of the Fourier series exhibit the Gibbs phenomenon near $\Gamma^{*}$. As demonstrated below, a good matching of the Fourier coefficients requires a good approximation of the singularity location. As in the univariate non-smooth case, the computation algorithm involves finding approximations to $f_{1}$ and $f_{2}$ simultaneously with a high precision identification of $\Gamma^{*}$.

Evidently, finding a high precision approximation of the singularity curve $\Gamma^{*}$ is more involved than finding a high precision approximation to the singularity point $s^{*}$ in the univariate case. Let $D_{\Gamma^{*}}(x, y)$ be the signed-distance function corresponding to the curve $\Gamma^{*}$ :

$$
D_{\Gamma^{*}}(x, y)=\left\{\begin{array}{cc}
\operatorname{dist}\left((x, y), \Gamma^{*}\right) & (x, y) \in \Omega_{1}, \\
-\operatorname{dist}\left((x, y), \Gamma^{*}\right) & (x, y) \in \Omega_{2} .
\end{array}\right.
$$

In looking for an approximation to $\Gamma^{*}$, we look for an approximation to $D_{\Gamma^{*}}$. Here again we are using a tensor product spline approximants, the same set of spline functions described in the previous section. Since the curve is $C^{m}$, it can be shown that one can construct a spline function $\tilde{D}$ of order $k \leq m$, with knots' distance $h$, which approximates $D_{\Gamma^{*}}$ near $\Gamma^{*}$ so that the Hausdorff distance between the zero level set of $\tilde{D}$ and $\Gamma^{*}$ is $O\left(h^{k}\right)$.

Let $D_{\bar{b}}$ be a spline approximation to $D_{\Gamma^{*}}$, with spline coefficients $\bar{b}=\left\{b_{i j}\right\}_{i, j=1}^{N_{h}}$ :

$$
D_{\bar{b}}(x, y)=\sum_{i=1}^{N_{h}} \sum_{j=1}^{N_{h}} b_{i j} B_{h}^{[k]}(x-i h) B_{h}^{[k]}(y-j h) .
$$

For a given $D_{\bar{b}}$ we define the approximation to $f$ similar to the construction in the univariate case by Equations (7)-(9). We look here for an approximation $S$ to $f$ which is a combination of two bivariate splines components:

$$
\begin{aligned}
& S(x, y)=\sum_{i=1}^{N_{d}} \sum_{j=1}^{N_{d}} a_{1 i j} B_{d}^{[k]}(x-i d) B_{d}^{[k]}(y-j d), \quad D_{\bar{b}}(x, y) \geq 0, \\
& S(x, y)=\sum_{i=1}^{N_{d}} \sum_{j=1}^{N_{d}} a_{2 i j} B_{d}^{[k]}(x-i d) B_{d}^{[k]}(y-j d), \quad D_{\bar{b}}(x, y)<0,
\end{aligned}
$$

such that $(2 M+1)^{2}$ Fourier coefficients of $f$ and $S$ are matched in the least-squares sense: 


$$
\left[\left\{a_{1 i j}\right\}_{i, j=1}^{N_{d}},\left\{a_{2 i j}\right\}_{i, j=1}^{N_{d}},\left\{b_{i j}\right\}_{i, j=1}^{N_{d}}\right]=\arg \min \left(\sum_{m, n=-M}^{M}\left|\hat{f}_{m n}-\hat{S}_{m n}\right|^{2}\right) .
$$

We denote by $B_{1 i j}(x, y)$ the restriction of $B_{d}^{[k]}(x-i d) B^{[k]}(y-j d)$ to the domain defined by $D_{\bar{b}}(x, y) \geq 0$, and by $B_{2 i j}(x, y)$ the restriction of $B_{d}^{[k]}(x-i d) B^{[k]}(y-j d)$ to the domain defined by $D_{\bar{b}}(x, y)<0$. We concatenate these two sequences of basis functions, $\left\{B_{1 i j}\right\}$ and $\left\{B_{2 i j}\right\}$ into one sequence $\left\{B_{i j}\right\}_{i=1, j=1}^{N_{d}, 2 N_{d}}$, denoting their Fourier coefficients by $\left\{\hat{B}_{i j, n}\right\}_{n \in \mathbb{Z}}$, and rearranging them (for each $n$ ) in vectors of length $2 N_{d}^{2},\left\{\hat{B}_{i, n}\right\}_{i=1, n \in \mathbb{Z}}^{2 N_{d}^{2}}$. For a given $D_{b}$, the normal equations for the least squares problem (21) induce the linear system $A a=b$ for the splines' coefficients $a=\left(\left\{a_{1 i j}\right\}_{i, j=1}^{N_{d}},\left\{a_{2 i j}\right\}_{i, j=1}^{N_{d}}\right)$, where:

$$
A_{i, j}=\sum_{m, n=-M}^{M}\left[\operatorname{Re}\left(\hat{B}_{i, n}\right) \operatorname{Re}\left(\hat{B}_{j, n}\right)+\operatorname{Im}\left(\hat{B}_{i, n}\right) \operatorname{Im}\left(\hat{B}_{j, n}\right)\right], \quad 1 \leq i \leq 2 N_{d}^{2}
$$

and

$$
b_{i}=\sum_{m, n=-M}^{M}\left[\operatorname{Re}\left(\hat{B}_{i, n}\right) \operatorname{Re}\left(\hat{f}_{n}\right)+\operatorname{Im}\left(\hat{B}_{i, n}\right) \operatorname{Im}\left(\hat{f}_{n}\right)\right], \quad 1 \leq i \leq 2 N_{d}^{2} .
$$

For a given choice of $\bar{b}=\left\{b_{i j}\right\}$, the coefficients $\left\{a_{1 i j}\right\}_{i, j=1}^{N_{d}},\left\{a_{2 i j}\right\}_{i, j=1}^{N_{d}}$ are obtained by solving a linear system of equations, and properly rearranging the solution. However, finding the optimal $\bar{b}$ is a non-linear problem that requires an iterative process and is much more expensive.

Remark 3. Representing the singularity curve of the approximation $S$ as the zero level set of the bivariate spline function $D_{\bar{b}}$ is the way to achieve a smooth control over the approximation. As a result, the objective function in (21) varies smoothly with respect to the spline coefficients $\left\{b_{i j}\right\}$.

Remark 4. In principle, the above framework is applicable to cases where $f$ is combined of $k$ functions defined on $k$ disjoint subdomains of $[0,1]^{2}$. The implementation, however, is more involved. The main challenge is to find a good first approximation to the curves separating the subdomains. In this context, for our case of two subdomains, we further assume for simplicity that the separating curve $\Gamma^{*}$ is bijective.

Here again we choose to demonstrate the whole approximation procedure alongside a specific numerical example.

\subsubsection{The Approximation Procedure-A Numerical Example}

Consider a piecewise smooth function on $[0,1]^{2}$ with a jump singularity across the curve $\Gamma^{*}$ which is the quarter circle defined by $x^{2}+y^{2}=0.5$. The test function is shown in Figure 11 and is defined as

$$
f(x, y)= \begin{cases}\left(x^{2}+y^{2}-0.5\right) \sin (10(x+y)) & x^{2}+y^{2} \geq 0.5, \\ \left(x^{2}+y^{2}-0.5\right) \sin (10(x+y))+2 x & x^{2}+y^{2}<0.5 .\end{cases}
$$

In the univariate case, in Section 2.2.1, we use the Gibbs phenomenon in order to find an initial approximation $s_{0}$ to the singularity location $s^{*}$. The same idea, with some modifications to the 2D case, is applied here. The truncated Fourier sum

$$
f_{50}(x, y)=\sum_{m, n=-50}^{50} \hat{f}_{m n} e^{2 \pi i m x} e^{2 \pi i n y} .
$$

gives an approximation to $f$, but the approximation suffers from a Gibbs phenomenon near the boundaries of the domain and near the singularity curve $\Gamma^{*}$. We evaluated $f_{50}$ on a $400 \times 400$ mesh 
on $[0,1]^{2}$, and enhanced the Gibbs effect by applying first order differences along the $x$-direction. The results are depicted in Figure 12. The locations of large $x$-direction differences and of large $y$-direction differences within $[0,1]^{2}$ indicate the location of $\Gamma^{*}$.

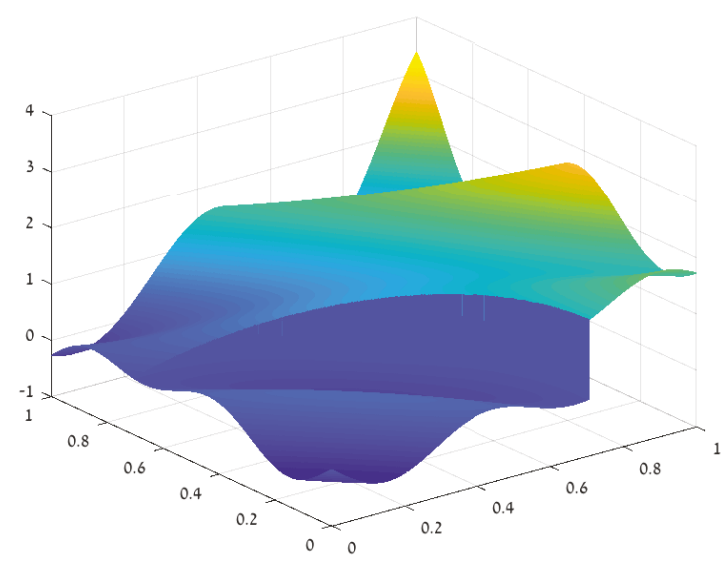

Figure 11. The test function for the 2D non-smooth case.

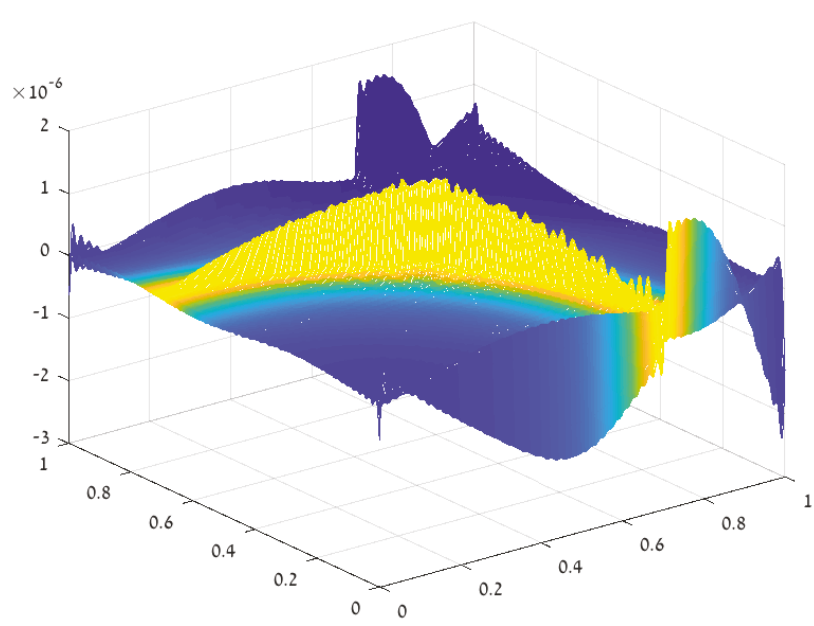

Figure 12. First order $x$-direction differences of a truncated Fourier sum-notice the relatively high values at the boundary and near the singularity curve.

\section{Building the initial approximation $D_{\bar{b}_{0}}$}

Searching along 50 horizontal lines ( $x$-direction) for maximal $x$-direction differences, and along 50 vertical lines ( $y$-direction) for maximal $y$ direction differences, we have found 72 such maximum points, which we denote by $P_{0}$. We display these points (in red) in Figure 13, on top of the curve $\Gamma^{*}$ (in blue). Now we use these points to construct the spline $D_{\bar{b}_{0}}$, whose zero level curve is taken as the initial approximation to $\Gamma^{*}$. To construct $D_{\bar{b}_{0}}$ we first overlay on $[0,1]^{2}$ a net of $11 \times 11$ points, $Q_{0}$. These are the green points displayed in Figure 14. 


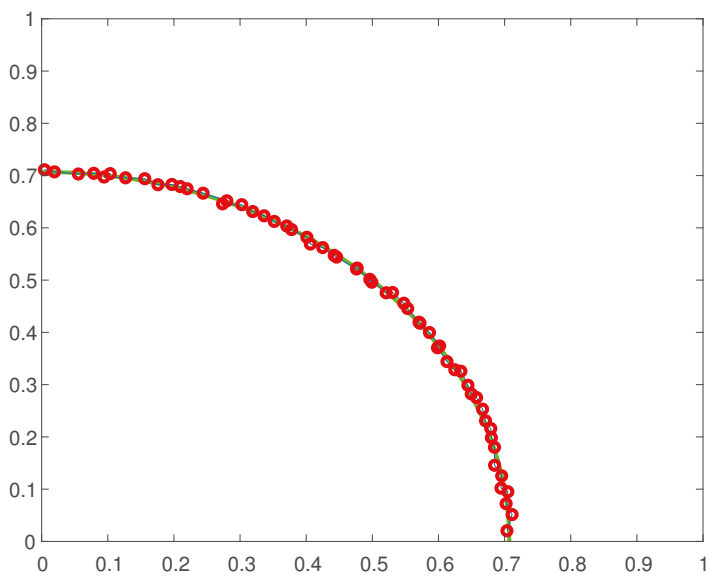

Figure 13. The singularity curve $\Gamma^{*}$ (blue) and points of maximal first differences of $f_{50}$.

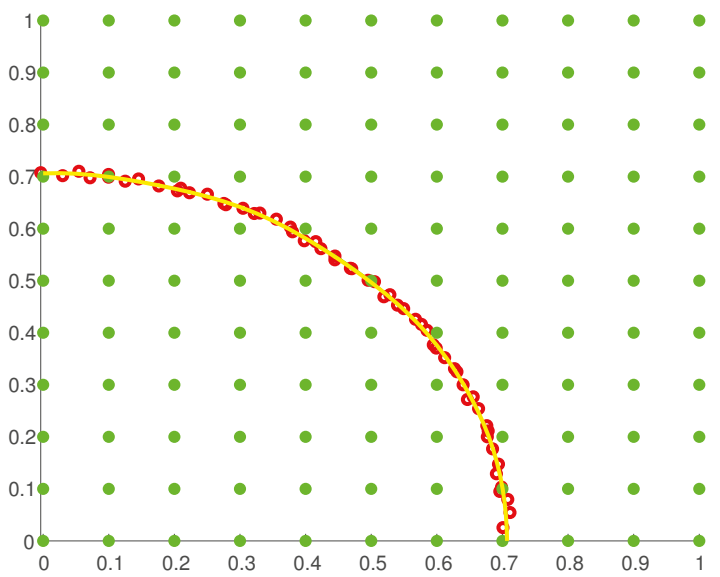

Figure 14. The singularity curve $\Gamma^{*}$ (blue) and points of maximal first differences of $f_{50}$.

To each point in $Q_{0}$ we assign the value of its distance from the set $P_{0}$, with a plus sign for points which are on the right or above $P_{0}$, and a minus sign for the other points. To each point in $P_{0}$ we assign the value zero. The spline function $D_{\bar{b}_{0}}$ is now defined by the least-squares approximation to the values at all the points $P_{0} \cup Q_{0}$. We have used here tensor product splines of order 10, on a uniform mesh with knots' distance $=0.1$. We denote the level curve zero of the resulting $D_{\bar{b}_{0}}$ as $\Gamma_{0}$, and this curve is depicted in yellow in Figure 14. It seems that $\Gamma_{0}$ is already a good approximation to $\Gamma^{*}$ (in blue), and thus it is a good starting point for achieving the minimization target (21).

\section{Improving the approximation to $\Gamma^{*}$, and building the two approximants}

Starting from $D_{\bar{b}_{0}}$ we use a quasi-Newton method for iterative improvement of the approximation to $\Gamma^{*}$. The expensive ingredient in the computation procedure is the need to recompute the Fourier coefficients of the $B$-splines for any new set of coefficients $\bar{b}$ of $D_{\bar{b}}$. We recall that we need $(2 M+1)^{2}$ of these coefficients for each $B$-spline, and we have $2 N_{d}^{2} B$-splines. In the numerical example we have used $M=40$ and $N_{d}=19$. To illustrate the issue we present in Figure 15 one of those $B$-spline whose support intersects the singularity curve. When the singularity curve is updated, the Fourier coefficients of this $B$-spline are recalculated. 
Remark 5. Calculating Fourier coefficients of the B-splines Calculating the Fourier coefficients of the $B$-splines is the most costly step in the approximation procedure. For the univariate case the Fourier coefficients of the B-splines can be computed analytically. For a smooth d-variate function $f:[0,1]^{d} \rightarrow \mathbb{R}$, with no singularity within the unit cube $[0,1]^{d}$, piecewise Gauss quadrature may be used to compute the Fourier coefficients with high precision. The non-smooth multivariate case is more difficult, and more expensive. However, we noticed that using low precision approximations for the Fourier coefficients of the B-splines is fine. For example, in the above example, we have employed a simple numerical quadrature combined with fast Fourier transform, and we obtained the Fourier coefficients with a relative error $\sim 10^{-5}$. Yet the resulting approximation error is small $\|f-S\|_{\infty}<5 \times 10^{-6}$, as seen in Figure 18 .

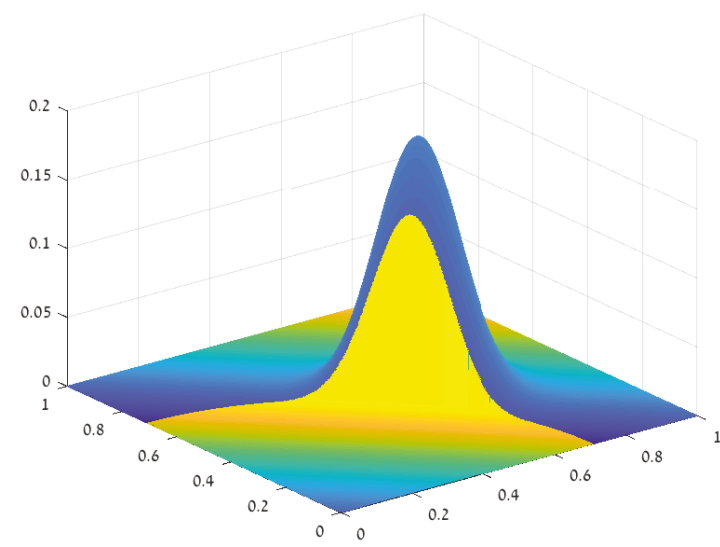

Figure 15. One of the tensor product B-splines used for the approximation of $f$, chopped off by the singularity curve.

Using one quasi-Newton step we obtained new spline coefficients $\bar{b}_{1}$ and an improved approximation $\Gamma_{1}$ to $\Gamma^{*}$ as the zero level set of $D_{\bar{b}_{1}}$. Stopping the procedure at this point yields approximation results as shown in the figures below. Figure 16 shows the approximation error $f-S$ on $[0,1]^{2} \backslash U$, where $U$ is a small neighborhood of $\Gamma^{*}$. Figure 17 shows, in green, $\log _{10}$ of the magnitude of the giver Fourier coefficients $\hat{f}_{m n}$ and, in blue, $\log _{10}$ of the Fourier coefficients of the difference $f-S$. We observe a reduction of three orders of magnitude between the two.

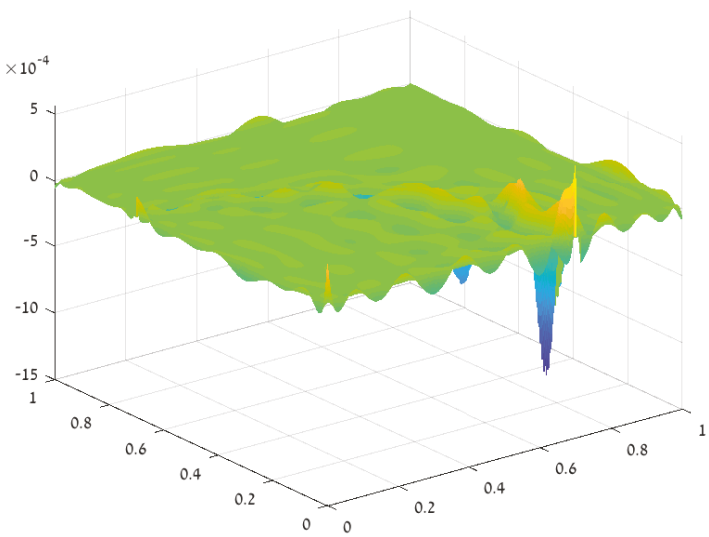

Figure 16. The approximation error with $D_{\bar{b}_{1}}$. 


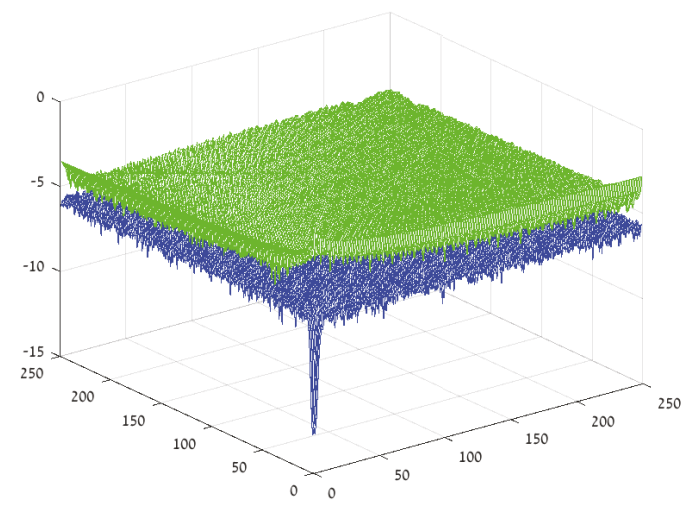

Figure 17. The magnitude reduction of the Fourier coefficients with $D_{\bar{b}_{1}}$.

Applying four quasi-Newton iterations took $\sim 24$ min execution time. The approximation of $\Gamma^{*}$ by the zero level set of $D_{\bar{b}_{4}}$ is now with an error of $10^{-9}$. The consequent approximation error to $f$ is reduced as shown in Figure 18, and the Fourier coefficients of the error are reduced by 5 orders of magnitude, as shown in Figure 19.

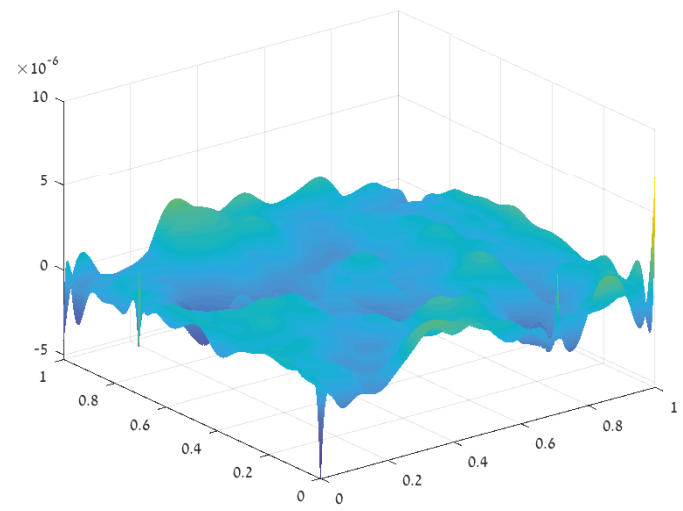

Figure 18. The approximation error with $D_{\bar{b}_{4}}$.

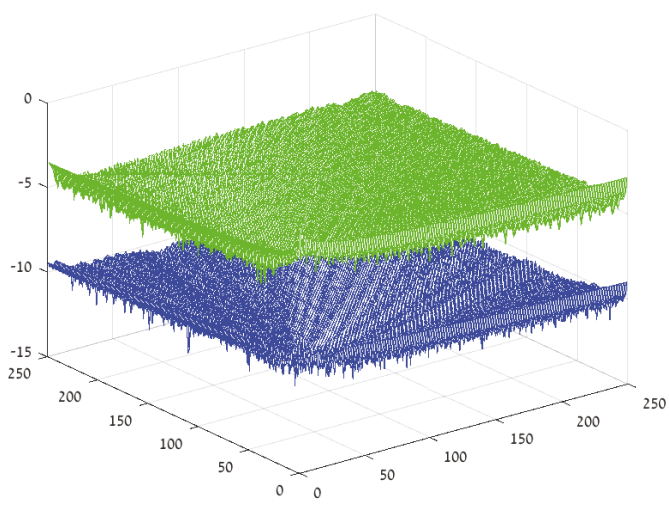

Figure 19. The magnitude reduction of the Fourier coefficients with $D_{\bar{b}_{4}}$. 


\subsubsection{The 2D Approximation Procedure}

Let us sum up the suggested approximation procedure:

(1) Choose the approximation space $\Pi_{1}$ for approximating $f_{1}$ and $f_{2}$ and the approximation space $\Pi_{2}$ for approximating $\Gamma^{*}$.

(2) Define the number of Fourier coefficients to be used for building the approximation such that

$$
(2 M+1)^{2} \geq 2 \operatorname{dim}\left(\Pi_{1}\right)+\operatorname{dim}\left(\Pi_{2}\right) .
$$

(3) Find first approximation to $\Gamma^{*}$ :

(a) Compute a partial Fourier sum and locate maximal first order differences along horizontal and vertical lines to find points $P_{0}$ near $\Gamma^{*}$, with assigned values 0 .

(b) Overlay a net of points $Q_{0}$ as in Figure 14, with assigned signed-distance values.

(c) Compute the least-squares approximation from $\Pi_{2}$ to the values at $P_{0} \cup Q_{0}$, denote it $D_{\bar{b}_{0}}$.

(4) Calculate the first $(2 M+1)^{2}$ Fourier coefficients of the basis functions of $\Pi_{1}$, truncated with respect to the zero level curve of $D_{\bar{b}_{0}}$.

(5) Use the above Fourier coefficients to compute the approximation to $f_{1}$ and $f_{2}$ by solving the system of linear equation defined by (22), (23).

(6) Update $D_{b}$ to improve the approximation to $\Gamma^{*}$, by performing quasi-Newton iterations to reduce the objective function in (21).

(7) Go back to (4) to update the approximation.

\subsubsection{Lower Order Singularities}

Let us assume that $f(x, y)$ is a continuous function, and that $f_{x}(x, y)$ is discontinuous across the singularity curve $\Gamma^{*}$. In this case we cannot use the Gibbs phenomenon effect to approximate the singularity curve. However, the Fourier coefficients

$$
\hat{g}_{m n}=i m \hat{f}_{m n}
$$

represent a function $g$ that has discontinuity across $\Gamma^{*}$, and the above procedure for approximating $\Gamma^{*}$ can be applied.

\subsection{Error Analysis}

We consider the non-smooth bivariate case, where $f$ is a combination of two smooth parts, $f_{1}$ on $\Omega_{1}$ and $f_{2}$ on $\Omega_{2}$, separated by a smooth curve $\Gamma^{*}$. Throughout the paper we approximate $f$ using spline functions. In this section we consider approximations by general approximation spaces. Let $\Pi_{1}$ be the approximation space for approximating the smooth pieces constituting $f$, and let $\Pi_{2}$ be the approximation space used for approximating the singularity curve. The following assumption characterize and quantify the assumptions about the function $f$ and its singularity curve $\Gamma^{*}$ in terms the ability to approximate them using the approximation spaces $\Pi_{1}, \Pi_{2}$.

Assumption 1. We assume that $\Pi_{1}$ and $\Pi_{2}$ are finite dimensional spaces of dimensions $N_{1}$ and $N_{2}$ respectively.

Assumption 2. We assume that $f_{1}$ and $f_{2}$ are smoothly extendable to $[0,1]^{2}$ and dist ${ }_{[0,1]^{2}}\left(f_{1}, \Pi_{1}\right) \leq$ $\epsilon$, $\operatorname{dist}_{[0,1]^{2}}\left(f_{2}, \Pi_{1}\right) \leq \epsilon$.

Assumption 3. For $p \in \Pi_{2}$, let us denote by $\Gamma_{0}(p)$ the zero level curve of $p$ within $[0,1]^{2}$. we assume there exists $p \in \Pi_{2}$ such that

$$
d_{H}\left(\Gamma^{*}, \Gamma_{0}(p)\right) \leq \delta,
$$


where $d_{H}$ denotes the Hausdorff distance.

We look for an approximation $S$ to $f$ which is a combination of two components, $p_{1} \in \Pi_{1}$ in $\tilde{\Omega}_{1}$ and $p_{2} \in \Pi_{1}$ in $\tilde{\Omega}_{2}$, separated by $\Gamma_{0}(p), p \in \Pi_{2}$, such that $(2 M+1)^{2}$ Fourier coefficients of $f$ and $S$ are matched in the least-squares sense:

$$
F\left(p_{1}, p_{2}, p\right)=\sum_{m, n=-M}^{M}\left|\hat{f}_{m n}-\hat{S}_{m n}\right|^{2} \rightarrow \text { minimum. }
$$

Assumption 4. Consider the above function $S$ constructed by a triple $\left(p_{1}, p_{2}, \Gamma_{0}(p)\right), p_{1}, p_{2} \in \Pi_{1}, p \in \Pi_{2}$. We assume that there is a Lipschitz continuous inverse mapping from the $(2 M+1)^{2}$ Fourier coefficients of $S$ to the triple $\left(p_{1}, p_{2}, \Gamma_{0}(p)\right)$ :

$$
\left\{\hat{S}_{m n}\right\}_{m, n=-M}^{M} \rightarrow\left(p_{1}, p_{2}, \Gamma_{0}(p)\right) .
$$

Remark 6. To enable the above property we choose $M$ so that

$$
(2 M+1)^{2}>2 N_{1}+N_{2} .
$$

The topology in the space of triples $\left(p_{1}, p_{2}, \Gamma_{0}(p)\right)$ is in terms of the maximum norm for the first two components and the Hausdorff distance for the third component.

Proposition 1. Let $f_{1}, f_{2}, \Gamma^{*}, \Pi_{1}$ and $\Pi_{2}$ satisfy Assumptions 1, 2, 3 and 4. Then the triple $\left(p_{1}^{*}, p_{2}^{*}, p^{*}\right)$ minimizing (27) provides the following approximation bounds:

$$
\begin{gathered}
\left\|f_{1}-p_{1}^{*}\right\|_{\infty, \Omega_{1}^{*}} \leq C_{1} M \epsilon+C_{2} M \delta, \\
\left\|f_{2}-p_{2}^{*}\right\|_{\infty, \Omega_{2}^{*}} \leq C_{1} M \epsilon+C_{2} M \delta,
\end{gathered}
$$

and

$$
d_{H}\left(\Gamma^{*}, \Gamma_{0}\left(p^{*}\right)\right) \leq C_{3} M \epsilon+C_{4} M \delta,
$$

where $\Omega_{1}^{*}$ and $\Omega_{2}^{*}$ are separated by $\Gamma_{0}\left(p^{*}\right)$.

Proof. By Assumptions 2, 3 it follows that there exists an approximation $S$ defined as above by a triple $\left(\bar{p}_{1}, \bar{p}_{2}, \bar{p}\right)$, such that

$$
\begin{aligned}
& \left\|f_{1}-\bar{p}_{1}\right\|_{\infty,[0,1]^{2}} \leq \epsilon, \\
& \left\|f_{2}-\bar{p}_{2}\right\|_{\infty,[0,1]^{2}} \leq \epsilon,
\end{aligned}
$$

and

$$
d_{H}\left(\Gamma^{*}, \Gamma_{0}(\bar{p})\right) \leq \delta
$$

Building an approximation $\bar{S}$ to $f$ as above by a triple $\left(\bar{p}_{1}, \bar{p}_{2}, \bar{p}\right)$, we can estimate its Fourier coefficients using the above bounds, and it follows that

$$
\left|\hat{f}_{m n}-\hat{S}_{m n}\right| \leq \epsilon+L \delta,-M \leq m, n \leq M .
$$

Therefore,

$$
\min \left\{F\left(p_{1}, p_{2}, p\right)\right\} \leq(2 M+1)^{2}(\epsilon+L \delta)^{2} .
$$

Let

$$
\left[p_{1}^{*}, p_{2}^{*}, p^{*}\right]=\arg \min \left\{\sum_{m, n=-M}^{M}\left|\hat{f}_{m n}-\hat{S}_{m n}\right|^{2}\right\} .
$$


The approximation $S^{*}$ to $f$ is the combination of the two components, $p_{1}^{*}$ in $\Omega_{1}^{*}$ and $p_{2}^{*}$ in $\Omega_{2}^{*}$, where $\Omega_{1}^{*}$ and $\Omega_{2}^{*}$ are separated by $\Gamma_{0}\left(p^{*}\right)$.

Using the bound in (37) it follows that

$$
\left|\hat{f}_{m n}-\hat{S}_{m n}^{*}\right| \leq(2 M+1)(\epsilon+L \delta),-M \leq m, n \leq M
$$

In view of (36) and (39) if follows that

$$
\left|\hat{\bar{S}}_{m n}-\hat{S}_{m n}^{*}\right| \leq(2 M+2)(\epsilon+L \delta),-M \leq m, n \leq M
$$

Using Assumption 4, the bound (40) implies

$$
\begin{aligned}
& \left\|p_{1}^{*}-\bar{p}_{1}\right\|_{\infty, \Omega_{1}^{*}} \leq C(2 M+2)(\epsilon+L \delta), \\
& \left\|p_{2}^{*}-\bar{p}_{2}\right\|_{\infty, \Omega_{2}^{*}} \leq C(2 M+2)(\epsilon+L \delta),
\end{aligned}
$$

and

$$
d_{H}\left(\Gamma_{0}\left(p^{*}\right), \Gamma_{0}(\bar{p})\right) \leq C(2 M+2)(\epsilon+L \delta) .
$$

The approximation result now follows by considering the inequalities (41)-(43), together with the inequalities (33)-(35), and applying the triangle inequality.

Validity of the Approximation Assumptions

Let us check the validity of Assumptions 1,2, 3 and 4 for the approximation tools suggested in Section 3.2 and used in the above numerical tests.

We assume that $f_{1}, f_{2} \in C^{m}[0,1]^{2}$, and that $\Gamma^{*}$ is a $C^{m}$ curve. To construct the approximation to $f_{1}$ and $f_{2}$ we use the space $\Pi_{1}$ of $k$ th degree tensor-product splines with equidistant knots' distance $d$ in each direction, $k \leq m$. The approximation to $\Gamma^{*}$ is obtained using the approximation space $\Pi_{2}$ of $\ell$ th degree tensor product splines with equidistant knots' distance $h$ in each direction, $\ell \leq m$. $\operatorname{dim}\left(\Pi_{1}\right)=(1 / d+k-1)^{2} \equiv N_{d}^{2}, \operatorname{dim}\left(\Pi_{2}\right)=(1 / h+\ell-1)^{2} \equiv N_{h}^{2}$, and for both spaces we use the $B$-spline basis functions. Assumptions 2 and 3 are fulfilled with $\epsilon=C_{1} d^{k}$ and $\delta=C_{2} h^{\ell}$.

Assumption 4 is more challenging. To define the mapping

$$
\left\{\hat{S}_{m n}\right\}_{m, n=-M}^{M} \rightarrow\left(p_{1}, p_{2}, \Gamma_{0}(p)\right)
$$

we use the same procedure Section 3.2.2 for defining the approximation to $f$ :

We represent $p$ and $p_{1}, p_{2}$ using the $B$-spline basis function as in (18), (19) and (20), respectively. Each triple $\left(p_{1}, p_{2}, p\right)$ defines a piecewise spline approximation $T(x, y)$, and we look for the approximation $\mathrm{T}(\mathrm{x}, \mathrm{y})$ such that $(2 M+1)^{2}$ Fourier coefficients of $T$ match the Fourier coefficients $\left\{\hat{S}_{m n}\right\}_{m, n=-M}^{M}$ in the least-squares sense:

$$
\left[\left\{a_{1 i j}\right\}_{i, j=1}^{N_{d}},\left\{a_{2 i j}\right\}_{i, j=1}^{N_{d}},\left\{b_{i j}\right\}_{i, j=1}^{N_{h}}\right]=\arg \min \left(\sum_{m, n=-M}^{M}\left|\hat{S}_{m n}-\hat{T}_{m n}\right|^{2}\right)
$$

Out of all the possible solutions of the above problem we look for the one with minimal coefficients' norm, i.e., minimizing

$$
\sum_{i, j=1}^{N_{d}} a_{1 i j}^{2}+\sum_{i, j=1}^{N_{d}} a_{2 i j}^{2}
$$

Following the procedure of Section 3.2.2, we observe that every step in the procedure is smooth with respect to its input. Possible non-uniqueness in solving the linear system of equations on step (5) is resolved by using the generalized inverse. Therefore, the composition of all the steps is also a smooth function of the input, which implies the validity of Assumption 4. 


\section{The 3D Case}

Numerical Example-The Smooth 3D Case

We consider the test function

$$
f(x, y, z)=\left(x^{2}+y^{2}+z^{2}-0.5\right) \sin (4(x+y-z))
$$

assuming only its Fourier coefficients are given. We have used only $10^{3}$ Fourier coefficients and constructed an approximation using 5th-degree tensor product splines with equidistant knots' distance $d=0.1$ in each direction. For this case, the matrix $A$ is of size $15^{3} \times 15^{3}$, and $\operatorname{cond}(A)=1.2 \times 10^{22}$. Again, we have employed the iterative refinement algorithm to obtain a high precision solution. The test function is shown in Figure 20. The error in the resulting approximation is displayed in Figure 21.

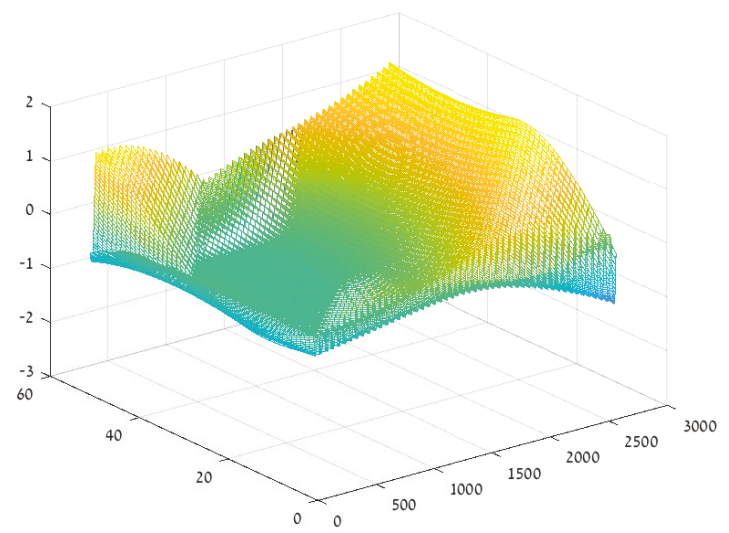

Figure 20. The 3D test function reshaped into 2D.

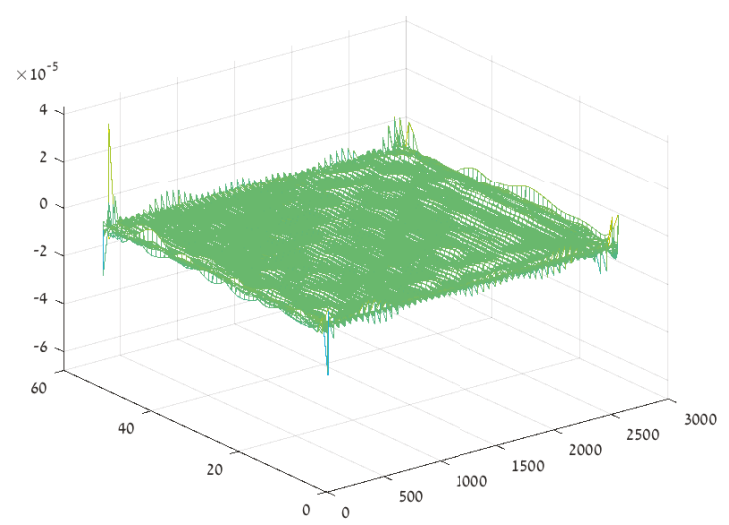

Figure 21. The approximation error graph, reshaped into 2D.

\section{Concluding Remarks}

The basic crucial assumption behind the presented Fourier acceleration strategy is that the underlying function is piecewise 'nice'. That is, piecewisely, the function can be well approximated by a suitable finite set of basis functions. The Fourier series of the function may be given to us as a result of the computational method dictated by the structure of the mathematical problem at hand. 
In itself, the Fourier series may not be the best tool for approximating the desired solution, and yet it contains all the information about the requested function. Utilizing this information we can derive high accuracy piecewise approximations to that function. The simple idea is to make the approximation match the coefficients of the given Fourier series. The suggested method is simple to implement for the approximation of smooth non-periodic functions in any dimension. The case of non-smooth functions is more challenging, and a special strategy is suggested and demonstrated for the univariate and bivariate cases. The paper contains a descriptive graphical presentation of the approximation procedure, together with a fundamental error analysis.

Funding: This research received no external funding.

Conflicts of Interest: The authors declare no conflicts of interest.

\section{References}

1. Gottlieb, D.; Shu, C.W. On the Gibbs phenomenon and its resolution. SIAM Rev. 1977, 39, 644-668. [CrossRef]

2. Tadmor, E. Filters, mollifiers and the computation of the Gibbs phenomenon. Acta Numer. 2007, 16, 305-378. [CrossRef]

3. Gelb, A.; Tanner, J. Robust reprojection methods for the resolution of the Gibbs phenomenon. Appl. Comput. Harmon. Anal. 2006, 20, 3-25. [CrossRef]

4. Eckhoff, K.S. Accurate and efficient reconstruction of discontinuous functions from truncated series expansions. Math. Comput. 1993, 61, 745-763. [CrossRef]

5. Eckhoff, K.S. Accurate reconstructions of functions of finite regularity from truncated Fourier series expansions. Math. Comput. 1995, 64, 671-690. [CrossRef]

6. Eckhoff, K.S. On a high order numerical method for functions with singularities. Math. Comput. 1998, 67, 1063-1087. [CrossRef]

7. Batenkov, D. Complete algebraic reconstruction of piecewise-smooth functions from Fourier data. Math. Comput. 2015, 84, 2329-2350. [CrossRef]

8. Nersessian, A.; Poghosyan, A. On a rational linear approximation of Fourier series for smooth functions. J. Sci. Comput. 2006, 26, 111-125. [CrossRef]

9. Levin, D.; Sidi, A. Extrapolation methods for infinite multiple series and integrals. J. Comput. Methods Sci. Eng. 2001, 1, 167-184. [CrossRef]

10. Sidi, A. Acceleration of convergence of (generalized) Fourier series by the d-transformation. Ann. Numer. Math. 1995, 2, 381-406.

11. Wilkinson, J.H. Rounding Errors in Algebraic Processes; Prentice-Hall: Englewood Cliffs, NJ, USA, 1963.

12. Moler, C.B. Iterative refinement in floating point. J. ACM 1967, 14, 316-321. [CrossRef]

(C) 2020 by the author. Licensee MDPI, Basel, Switzerland. This article is an open access article distributed under the terms and conditions of the Creative Commons Attribution (CC BY) license (http:/ / creativecommons.org/licenses/by/4.0/). 


\title{
Article
}

\section{A Discussion on the Existence of Best Proximity Points That Belong to the Zero Set}

\author{
Erdal Karapınar ${ }^{1,2, *,+}$, Mujahid Abbas ${ }^{3,4, *,+}$ and Sadia Farooq ${ }^{5,+}$ \\ 1 Department of Medical Research, China Medical University Hospital, China Medical University, \\ Taichung 40402, Taiwan \\ 2 Department of Mathematics, Çankaya University, Etimesgut 06790, Ankara, Turkey \\ 3 Department of Mathematics, Government College University, Lahore 54000, Pakistan \\ 4 Department of Mathematics and Applied Mathematics, University of Pretoria, Lynnwood Road, \\ Pretoria 0002, South Africa \\ 5 Department of Mathematics, University of Management and Technology, Lahore 54782, Pakistan; \\ farooqsadia389@gmail.com \\ * Correspondence: erdalkarapinar@yahoo.com or karapinar@mail.cmuh.org.tw (E.K.); \\ abbas.mujahid@gmail.com (M.A.) \\ $\dagger$ These authors contributed equally to this work.
}

Received: 13 December 2019; Accepted: 6 February 2020; Published: 11 February 2020

\begin{abstract}
In this paper, we investigate the existence of best proximity points that belong to the zero set for the $\alpha_{p}$-admissible weak $(F, \varphi)$-proximal contraction in the setting of $M$-metric spaces. For this purpose, we establish $\varphi$-best proximity point results for such mappings in the setting of a complete $M$-metric space. Some examples are also presented to support the concepts and results proved herein. Our results extend, improve and generalize several comparable results on the topic in the related literature.
\end{abstract}

Keywords: $m$-metric space; proximal $\alpha_{p}$-admissible; $\alpha_{p}$-admissible weak $(F, \varphi)$-proximal contraction; $G$-proximal graphic contraction; $\varphi$-best proximity point

MSC: 47H10; 54H25; 46J10

\section{Introduction and Preliminaries}

Several real-world problems can be reformulated as a fixed point problem. In other words, the solution of the real-world problem reduces to the solution of a fixed point problem. In some cases getting a fixed point for certain mapping is impossible. In this case, instead of exact solution, it is natural to consider the approximate solution. Roughly speaking, if the equation $F(\xi)=0$ has no exact solution where $F(\xi)=T(\xi)-\xi$, where $T$ is an opeator defined on a certain distance space. In 1969, Ky Fan [1] suggested an answer to the question that what happen if a given mapping does not possess a fixed point. More precisely, he proved that if $A$ is a compact, convex and nonempty subset of a Banach space $S$ and $T$ is continuous mapping from $A$ to $S$, then there exists a point $\xi^{*} \in A$ such that

$$
d\left(\xi^{*}, T \xi^{*}\right)=d\left(T \xi^{*}, A\right)=\inf \left\{d\left(\xi, T \xi^{*}\right), \xi \in A\right\} .
$$

This results is known as best approximation theorem. In the above statement, the point $\xi^{*} \in A$ is called as approximate fixed point of $T$ or an approximate solution of a fixed point equation $T \xi=\xi$. In general, if $A, B$ are nonempty subsets of a Banach space $S$ and $T: A \rightarrow B$, then $\xi^{*} \in A$ is called best proximity point of $T$ if it satisfies

$$
d\left(\xi^{*}, T \xi^{*}\right)=d(A, B)=\inf \{d(a, b): a \in A, b \in B\} .
$$


Note that $\xi^{*} \in A$ turns to be a fixed point of $T$, if the sets $A, B$ have non-empty intersection. Indeed, if $A \cap B \neq \phi$ or $A=B$, then $d(A, B)=0$ and hence the best proximity point $\xi^{*} \in A$ becomes the solution of a fixed point equation $T \xi=\xi$. Attendantly, best proximity point results are natural generalizations of metric fixed point results. For further discussion in this direction, we refer to [2-8].

We underline the fact that a best proximity point $\xi^{*} \in A$, indeed solves the following optimization problem:

$$
\min _{\xi \in A} d(\xi, T \xi) .
$$

On the other hand, fixed point theory has been extended in several directions. For instance, metric space structure has been changed by some new abstract space which is more general than the standard set-up. One of the significant examples of this trend was given by Matthews [9]. He defined the notion of partial metric space and characterized the Banach contraction principle in that space. Roughly speaking, despite the metric space, in partial metric space self-distance may not be zero. This notion especially provides some simplicity in computer science, in particular, domain theory. A number of authors have involved in this trend with interesting results, see e.g., [10-18] and related reference therein. For the sake of completeness, we recall the concept of partial metric space as follows:

Definition 1 ([9]). A distance function $p: S \times S \rightarrow[0, \infty)$, on a non-empty set $S$, is called partial metric if the followings are fulfilled:

(p1) $p(\xi, \xi)=p(\eta, \eta)=p(\xi, \eta) \Leftrightarrow \xi=\eta$,

(p2) $p(\xi, \xi) \leq p(\xi, \eta)$,

(p3) $p(\xi, \eta)=p(\eta, \xi)$

(p4) $p(\xi, \eta) \leq p(\xi, \zeta)+p(\zeta, \eta)-p(\zeta, \zeta)$

for all $\xi, \eta, \zeta \in S$. A corresponding pair $(S, p)$ is called a partial metric space.

It is evident that $p(\xi, \eta)=0$, yields $\xi=\eta$. The contrary of the statement is false.

Asadi et al. [19] introduced the notion of an $M$-metric space and obtained fixed point results in the setup of $M$-metric spaces. It was indicated that $M$-metric space is a real generalization of a partial metric space and they supported their claim by providing some constructive examples. For more results in this direction see e.g., [20,21].

The following notations are useful in the sequel.

(1) $m_{\xi \eta}=\min \{\rho(\xi, \xi), \rho(\eta, \eta)\}$,

(2) $M_{\xi \eta}=\max \{\rho(\xi, \xi), \rho(\eta, \eta)\}$.

Definition 2 ([19]). A distance function $\rho: S \times S \rightarrow[0, \infty)$, on a non-empty set $S$, is called $M$-metric if the followings are fulfilled:

(m1) $\rho(\xi, \xi)=\rho(\eta, \eta)=\rho(\xi, \eta) \Leftrightarrow \xi=\eta$,

(m2) $m_{\xi \eta} \leq \rho(\xi, \eta)$

(m3) $\rho(\xi, \eta)=\rho(\eta, \xi)$

(m4) $\rho(\xi, \eta)-m_{\xi \eta} \leq \rho(\xi, \zeta)-m_{\xi \zeta}+\rho(\zeta, \eta)-m_{\zeta \eta}$

for all $\xi, \eta, \zeta \in S$. A corresponding pair $(S, \rho)$ is called an $M$-metric space.

Lemma 1 ([19]). Each partial metric forms an M-metric. The converse is false.

Example 1. Let $S=\{\xi, \eta, \zeta\}$. Define

$$
\begin{aligned}
& \rho(\xi, \xi)=1, \quad \rho(\eta, \eta)=9, \quad \rho(\zeta, \zeta)=5, \\
& \rho(\xi, \eta)=\rho(\eta, \xi)=10, \quad \rho(\xi, \zeta)=\rho(\zeta, \xi)=7, \\
& \rho(\zeta, \eta)=\rho(\eta, \zeta)=7 .
\end{aligned}
$$


It is clear that $\rho$ is an M-metric. Notice that $\rho$ does not form a partial metric.

Definition 3 ([19]). Let $(S, \rho)$ be an $M$-metric space and $\xi \in S$. A sequence $\left\{\xi_{n}\right\}$ in $S$ is called:

(1) $M$-convergent to $\xi \in S$ if and only if

$$
\lim _{n \rightarrow \infty}\left(\rho\left(\xi_{n}, \xi\right)-m_{\xi_{n}, \xi}\right)=0,
$$

(2) M-Cauchy sequence if and only if

$$
\lim _{n, m \rightarrow \infty}\left(\rho\left(\xi_{n}, \xi_{m}\right)-m_{\xi_{n}, \xi_{m}}\right) \text { and } \lim _{n, m \rightarrow \infty}\left(M_{\xi_{n}, \xi_{m}}-m_{\xi_{n}, \xi_{m}}\right),
$$

exist (and are finite).

Definition 4 ([19]). An M-metric space is said to be $M$-complete if every $M$-Cauchy sequence $\left\{\xi_{n}\right\}$ in $S$ converges with respect to $\tau_{m}$ (topology induced by $m$ ) to a point $\xi \in S$ such that

$$
\lim _{n \rightarrow \infty}\left(\rho\left(\xi_{n}, \xi\right)-m_{\xi_{n}, \xi}\right)=0 \text { and } \lim _{n \rightarrow \infty}\left(M_{\xi_{n}, \xi}-m_{\xi_{n}, \xi}\right)=0
$$

Remark 1 ([19]). Let $(S, \rho)$ be an M-metric space and for every $\xi, \eta \in(S, \rho)$, we have

(r1) $0 \leq M_{\xi \eta}+m_{\xi \eta}=\rho(\xi, \xi)+\rho(\eta, \eta)$,

(r2) $0 \leq M_{\xi \eta}-m_{\xi \eta}=[\rho(\xi, \xi)-\rho(\eta, \eta)]$,

(r3) $M_{\xi \eta}-m_{\xi \eta} \leq\left(M_{\xi \zeta}-m_{\xi \zeta}\right)+\left(M_{\zeta \eta}-m_{\zeta \eta}\right)$.

The set $\left\{\xi^{*} \in A: \varphi\left(\xi^{*}\right)=0\right\}$ of all zeros of the function $\varphi: A \rightarrow[0, \infty)$ is denoted by $Z_{\varphi}$. By using this notion, Jleli et al. [22] introduced the notion of $\varphi$-fixed point as follows: If $S$ is a non empty set, $T: S \rightarrow S$ and $\varphi: S \rightarrow[0, \infty)$ is a given function, then $\xi^{*} \in S$ is said to be $\varphi$ - fixed point of $T$ if and only if $T\left(\xi^{*}\right)=\xi^{*}$ and $\varphi\left(\xi^{*}\right)=0$. We denote the set of all $\varphi$-fixed points of $T$ by $\varphi_{F}(S)$, that is,

$$
\varphi_{F}(S)=\left\{\xi^{*} \in S: T\left(\xi^{*}\right)=\xi^{*} \text { and } \varphi\left(\xi^{*}\right)=0\right\}
$$

In [22], the authors also considered the concept of control function $F:[0, \infty)^{3} \rightarrow[0, \infty)$ defined as follows:

(F1) $\max \{s, t\} \leq F(s, t, r)$, for all $s, t, r \in[0, \infty)$,

(F2) $F$ is continuous,

(F3) $F(0,0,0)=0$.

The set of such control functions is denoted by $\mathcal{F}$. An immediate examples of the control functions are collected below:

Example 2 ([22]). Let $i=\{1,2,3\}$. Define $F_{i}:[0, \infty)^{3} \rightarrow[0, \infty)$ as follows:

$$
F_{1}(a, b, c)=a+b+c, F_{2}(a, b, c)=\max \{a, b\}+c \text { and } F_{3}(a, b, c)=a+a^{2}+b+c,
$$

for all $a, b, c \in[0, \infty)$. Note that $F_{1}, F_{2}, F_{3} \in \mathcal{F}$.

In [22], the notion of $(F, \varphi)$-contraction mapping was defined and the existence of a fixed point for such mappings were considered.

Definition 5 ([22]). Let $(S, d)$ be a complete metric space and $\varphi: S \rightarrow[0, \infty)$. A mapping $T: S \rightarrow S$ is said to be an $(F, \varphi)$-contraction mapping if there exist $F \in \mathcal{F}$ and $k \in[0,1)$ such that

$$
F(d(T \xi, T \eta), \varphi(T \xi), \varphi(T \eta)) \leq k F(d(\xi, \eta), \varphi(\xi), \varphi(\eta)), \text { for all } \xi, \eta \in S \text {. }
$$


Later, this result has been followed by several authors, see e.g., [23-26].

Let $\Psi$ denote the set of nondecreasing functions $\psi:[0, \infty) \rightarrow[0, \infty)$ such that $\sum_{n=1}^{+\infty} \psi^{n}(t)<\infty$, for all $t>0$, where $\psi^{n}$ is an $n$-iterate of $\psi$. A function $\psi$ is called a $(c)$-comparison function if $\psi \in \Psi$. Note that if $\psi \in \Psi$, then $\psi(0)=0$ and $\psi(t)<t$, for all $t>0$ [27].

Remark 2 ([27]). Note that $\sum_{n=1}^{+\infty} \psi^{n}(t)<\infty$ implies $\lim _{n \rightarrow \infty} \psi^{n}(t)=0$, for all $t \in(0, \infty)$.

In what follows we introduce the notion of " $\varphi$-best proximity point".

Definition 6. Let $(S, \rho)$ be an M-metric space, $A, B$ are two subsets of $S$. An element $\xi^{*} \in Z_{\varphi}$ is said to be a $\varphi$-best proximity point of the operator $T: A \rightarrow B$ if and only if $\rho\left(\xi^{*}, T \xi^{*}\right)=\rho(A, B)$, where $\rho(A, B)=\inf \{\rho(a, b): a \in A, b \in B\}$ and $\varphi\left(\xi^{*}\right)=0$.

We denote the set of all $\varphi$-best proximity points of $T$ by $\varphi_{T}(A)$, that is,

$$
\varphi_{T}(A)=\left\{\xi^{*} \in A: \rho\left(\xi^{*}, T \xi^{*}\right)=\rho(A, B) \text { and } \varphi\left(\xi^{*}\right)=0\right\} .
$$

The following definitions are also needed in the sequel. Before we state the definition, we underline the following assumption: Throughout the paper, all sets and subsets are supposed non-empty. We characterize the following sets (that plays a crucial role in best proximity theory) in the setting of $M$-metric space.

Definition 7. Let $(S, \rho)$ be an M-metric space, and $A, B$ be two subsets of $S$. Define

$$
\begin{aligned}
A_{0} & =\{\xi \in A: \rho(\xi, \eta)=\rho(A, B), \text { for some } \eta \in B\} \text { and } \\
B_{0} & =\{\xi \in B: \rho(\xi, \eta)=\rho(A, B), \text { for some } \eta \in A\} .
\end{aligned}
$$

Definition 8. Let $(S, \rho)$ be an M-metric space, and let $A, B$ be two subsets of $S$. If $\alpha: A \times A \rightarrow[-\infty, \infty)$, then mapping $T: A \rightarrow B$ is said to be proximal $\alpha_{p}$-admissible if

$$
\left.\begin{array}{r}
\alpha(\xi, \eta) \geq 0 \\
\rho(u, T \xi)=\rho(A, B) \\
\rho(v, T \eta)=\rho(A, B)
\end{array}\right\} \Longrightarrow \alpha(u, v) \geq 0,
$$

for all $\xi, \eta, u, v \in A$.

Definition 9. Let $(S, \rho)$ be an $M$-metric space, and $T: A \rightarrow B$. In addition, let $A$ be a subset of $S$, and $\alpha: A \times A \rightarrow[-\infty, \infty)$. Then $A$ is said to be $\alpha-$ regular, if $\left\{\xi_{n}\right\}$ is a sequence in $A$ such that $\alpha\left(\xi_{n}, \xi_{n+1}\right) \geq 0$ and $\xi_{n} \rightarrow \xi \in A$ as $n \rightarrow \infty$, then $\alpha\left(\xi_{n}, \xi\right) \geq 0$ for all $n \in N$.

In this paper, we introduce the notion of $\varphi$-best proximity point and prove the $\varphi$-best proximity point result in the setting of $M$-metric space. We also present an example to support our result.

\section{Main Results}

We start the section by introducing the notion of $\alpha_{p}$-admissible weak $(F, \varphi)$-proximal contraction mappings as follows. 
Definition 10. Let $A, B$ be two subsets of $M$-metric space $(S, \rho)$ and $F \in \mathcal{F}$. An $\alpha_{p}$-admissible mapping $T: A \rightarrow B$ is called an $\alpha_{p}$-admissible weak $(F, \varphi)$-proximal contraction, if there exists a lower semi-continuous function $\varphi: A \rightarrow[0, \infty)$ such that

$$
\left.\begin{array}{c}
\alpha(\xi, \eta) \geq 0 \\
\rho(u, T \xi)=\rho(A, B) \\
\rho(v, T \eta)=\rho(A, B)
\end{array}\right\}
$$

for all $\xi, \eta, u, v \in A$ and $\psi \in \Psi$.

By taking $\alpha(\xi, \eta)=0$, we shall get the following definition:

Definition 11. Let $A, B$ be two subsets of $M$-metric space $(S, \rho)$ and $F \in \mathcal{F}$. A mapping $T: A \rightarrow B$ is said to be a weak $(F, \varphi)$-proximal contraction, if there exist two functions $\varphi: A \rightarrow[0, \infty)$ and $\psi \in \Psi$ such that

$$
\begin{aligned}
& \rho(u, T \xi)=\rho(A, B)\} \\
& \rho(v, T \eta)=\rho(A, B)\} \\
& \Longrightarrow F(\rho(u, v), \varphi(u), \varphi(v)) \leq \psi(F(\rho(\xi, \eta), \varphi(\xi), \varphi(\eta))) \text {, }
\end{aligned}
$$

for all $\xi, \eta, u, v \in A$ and $\psi \in \Psi$.

The main result of the article is below.

Theorem 1. Let $A, B$ be two subsets of an M-complete $M$-metric space $(S, \rho)$ and $F \in \mathcal{F}$. Suppose that a mapping $T: A \rightarrow B$ is an $\alpha_{p}$-admissible weak $(F, \varphi)$-proximal contraction. If $T\left(A_{0}\right) \subseteq B_{0}$ and $A_{0}$ is $\alpha$-regular closed set in $S$, then there exists a $\varphi$-best proximity point of T provided that there exist $\xi_{0}, \xi_{1} \in A_{0}$ such that

$$
\rho\left(\xi_{1}, T \xi_{0}\right)=\rho(A, B) \text { and } \alpha\left(\xi_{0}, \xi_{1}\right) \geq 0 .
$$

Moreover, if $\alpha(\xi, \eta) \geq 0$ for all $\xi, \eta \in \varphi_{T}(A)$, then $\xi^{*}$ is the unique $\varphi$-best proximity point of $T$.

Proof. Let $\xi_{0}, \xi_{1} \in A_{0}$ be such that $\rho\left(\xi_{1}, T \xi_{0}\right)=\rho(A, B)$ and $\alpha\left(\xi_{0}, \xi_{1}\right) \geq 0$. As $T \xi_{0} \in T\left(A_{0}\right) \subseteq B_{0}$, there exists $\xi_{2}$ in $A_{0}$ such that $\rho\left(\xi_{2}, T \xi_{1}\right)=\rho(A, B)$. Since $T$ is proximal $\alpha_{p}$-admissible, we have $\alpha\left(\xi_{1}, \xi_{2}\right) \geq 0$. Similarly, by $T\left(A_{0}\right) \subseteq B_{0}$, we obtain a point $\xi_{3} \in A_{0}$ such that $\rho\left(\xi_{3}, T \xi_{2}\right)=\rho(A, B)$ which further implies that $\alpha\left(\xi_{2}, \xi_{3}\right) \geq 0$. Continuing this way, we can obtain a sequence $\left\{\xi_{n}\right\}$ in $A_{0}$ such that

$$
\begin{aligned}
& \rho\left(\xi_{n}, T \xi_{n-1}\right)=\rho(A, B), \\
& \rho\left(\xi_{n+1}, T \xi_{n}\right)=\rho(A, B), \alpha\left(\xi_{n}, \xi_{n+1}\right) \geq 0, \text { for all } n \in \mathbb{N} \cup\{0\} .
\end{aligned}
$$

Since $T$ is $\alpha_{p}$-admissible weak $(F, \varphi)$-proximal contraction, we have

$$
\alpha\left(\xi_{n-1}, \xi_{n}\right)+F\left(\rho\left(\xi_{n}, \xi_{n+1}\right), \varphi\left(\xi_{n}\right), \varphi\left(\xi_{n+1}\right)\right) \leq \psi\left(F\left(\rho\left(\xi_{n-1}, \xi_{n}\right), \varphi\left(\xi_{n-1}\right), \varphi\left(\xi_{n}\right)\right)\right) .
$$

Since $\alpha(\xi, \eta) \geq 0$ for all $\xi, \eta \in A$, we obtain that

$$
F\left(\rho\left(\xi_{n}, \xi_{n+1}\right), \varphi\left(\xi_{n}\right), \varphi\left(\xi_{n+1}\right)\right) \leq \psi\left(F\left(\rho\left(\xi_{n-1}, \xi_{n}\right), \varphi\left(\xi_{n-1}\right), \varphi\left(\xi_{n}\right)\right)\right)
$$

By induction, we get

$$
F\left(\rho\left(\xi_{n}, \xi_{n+1}\right), \varphi\left(\xi_{n}\right), \varphi\left(\xi_{n+1}\right)\right) \leq \psi^{n}\left(F\left(\rho\left(\xi_{0}, \xi_{1}\right), \varphi\left(\xi_{0}\right), \varphi\left(\xi_{1}\right)\right)\right) .
$$


It follows from the condition (F1) that

$$
\max \left\{\rho\left(\xi_{n}, \xi_{n+1}\right), \varphi\left(\xi_{n}\right)\right\} \leq \psi^{n}\left(F\left(\rho\left(\xi_{0}, \xi_{1}\right), \varphi\left(\xi_{0}\right), \varphi\left(\xi_{1}\right)\right)\right)
$$

By (2), we obtain that

$$
\rho\left(\xi_{n}, \xi_{n+1}\right) \leq \psi^{n}\left(F\left(\rho\left(\xi_{0}, \xi_{1}\right), \varphi\left(\xi_{0}\right), \varphi\left(\xi_{1}\right)\right)\right) .
$$

On the other hand, we get

$$
\lim _{n \rightarrow \infty} \rho\left(\xi_{n}, \xi_{n+1}\right)=0
$$

Using (4) and the condition (m2), we have

$$
\begin{aligned}
\lim _{n \rightarrow \infty} \rho\left(\xi_{n}, \xi_{n}\right) & =\lim _{n \rightarrow \infty} \min \left\{\rho\left(\xi_{n}, \xi_{n}\right), \rho\left(\xi_{n+1}, \xi_{n+1}\right)\right\} \\
& =\lim _{n \rightarrow \infty} m_{\xi_{n}, \xi_{n+1}} \\
& \leq \lim _{n \rightarrow \infty} \rho\left(\xi_{n}, \xi_{n+1}\right)=0 .
\end{aligned}
$$

Since $\lim _{n \rightarrow \infty} \rho\left(\xi_{n}, \xi_{n}\right)=0$, we have

$$
\lim _{n, m \rightarrow \infty} m_{\xi_{n}, \xi_{m}}=0
$$

We shall indicate that $\left\{\xi_{n}\right\}$ is an $M$-Cauchy sequence. Consider $m, n \in \mathbb{N}$ such that $m>n$. On using (3) and the condition (m4), we have

$$
\begin{aligned}
\rho\left(\xi_{n}, \xi_{m}\right)-m_{\xi_{n}, \xi_{m} \leq} \leq & \rho\left(\xi_{n}, \xi_{n+1}\right)-m_{\xi_{n}, \xi_{n+1}}+\rho\left(\xi_{n+1}, \xi_{m}\right)-m_{\xi_{n+1}, \xi_{m}} \\
\leq & \rho\left(\xi_{n}, \xi_{n+1}\right)-m_{\xi_{n}, \xi_{n+1}}+\rho\left(\xi_{n+1}, \xi_{n+2}\right)-m_{\xi_{n+1}, \xi_{n+2}}+\rho\left(\xi_{n+2}, \xi_{m}\right)-m_{\xi_{n+2}, \xi_{m}} \\
\leq & \rho\left(\xi_{n}, \xi_{n+1}\right)-m_{\xi_{n}, \xi_{n+1}}+\rho\left(\xi_{n+1}, \xi_{n+2}\right)-m_{\xi_{n+1}, \xi_{n+2}} \\
& +\ldots+\rho\left(\xi_{m-1}, \xi_{m}\right)-m_{\xi_{m-1}, \xi_{m}} \\
\leq & \rho\left(\xi_{n}, \xi_{n+1}\right)+\rho\left(\xi_{n+1}, \xi_{n+2}\right)+\ldots+\rho\left(\xi_{m-1}, \xi_{m}\right) \\
\leq & \psi^{n}\left(F\left(\rho\left(\xi_{0}, \xi_{1}\right), \varphi\left(\xi_{0}\right), \varphi\left(\xi_{1}\right)\right)\right)+\psi^{n+1}\left(F\left(\rho\left(\xi_{0}, \xi_{1}\right), \varphi\left(\xi_{0}\right), \varphi\left(\xi_{1}\right)\right)\right)+ \\
& \ldots+\psi^{m-1}\left(F\left(\rho\left(\xi_{0}, \xi_{1}\right), \varphi\left(\xi_{0}\right), \varphi\left(\xi_{1}\right)\right)\right) \\
\leq & \sum_{i=1}^{m-1} \psi^{i}\left(F\left(\rho\left(\xi_{0}, \xi_{1}\right), \varphi\left(\xi_{0}\right), \varphi\left(\xi_{1}\right)\right)\right)-\sum_{j=1}^{n-1} \psi^{j}\left(F\left(\rho\left(\xi_{0}, \xi_{1}\right), \varphi\left(\xi_{0}\right), \varphi\left(\xi_{1}\right)\right)\right) .
\end{aligned}
$$

It follows from Remark 2 and (6) that $\rho\left(\xi_{n}, \xi_{m}\right)-m_{\xi_{n}, \xi_{m}} \rightarrow 0$ as $n \rightarrow \infty$. On the other hand, by (5), we obtain that

$$
\lim _{n, m \rightarrow \infty}\left(M_{\xi_{n}, \xi_{m}}-m_{\xi_{n}, \xi_{m}}\right)=0 .
$$

Thus $\left\{\xi_{n}\right\}$ is an $M$-Cauchy sequence in $A_{0} \subseteq A \subset S$. By the completeness of $S$ and closeness of $A_{0}$, there exists $\xi^{*} \in A_{0}$ such that

$$
\lim _{n \rightarrow \infty} \rho\left(\xi_{n}, \xi^{*}\right)-m_{\xi_{n}, \xi^{*}}=0 \text { and } \lim _{n \rightarrow \infty} M_{\xi_{n}, \xi^{*}}-m_{\xi_{n}, \zeta^{*}}=0
$$

Since $\lim _{n \rightarrow \infty} \rho\left(\xi_{n}, \xi_{n}\right)=0$, we have

$$
\lim _{n \rightarrow \infty} \rho\left(\xi_{n}, \xi^{*}\right)=0 \text { and } \lim _{n \rightarrow \infty} M_{\xi_{n}, \xi^{*}}=0
$$

Thus by Remark 1, we get that

$$
\lim _{n \rightarrow \infty} \rho\left(\xi^{*}, \xi^{*}\right)=\lim _{n \rightarrow \infty}\left[M_{\xi_{n}, \xi^{*}}+m_{\xi_{n}, \xi^{*}}-\rho\left(\xi_{n}, \xi_{n}\right)\right]=0 .
$$

This implies that

$$
\rho\left(\xi^{*}, \xi^{*}\right)=0
$$


Now we need to show that $\varphi\left(\tilde{\zeta}^{*}\right)=0$. Using (2), we have

$$
\varphi\left(\xi_{n}\right) \leq \psi^{n}\left(F\left(\rho\left(\xi_{0}, \xi_{1}\right), \varphi\left(\xi_{0}\right), \varphi\left(\xi_{1}\right)\right)\right) .
$$

Letting $n \rightarrow \infty$ on the inequality above, we obtain

$$
\lim _{n \rightarrow \infty} \varphi\left(\xi_{n}\right)=0
$$

Since $\varphi$ is lower semi continuous, it follows from (7) and (8) that

$$
0 \leq \varphi\left(\xi^{*}\right) \leq \lim _{n \rightarrow \infty} \inf \varphi\left(\xi_{n}\right)=0 .
$$

Hence $\varphi\left(\xi^{*}\right)=0$. Since $A_{0}$ is $\alpha$-regular, $\alpha\left(\xi_{n}, \xi^{*}\right) \geq 0$. As $\xi^{*} \in A_{0}, T\left(A_{0}\right) \subseteq B_{0}, T \xi^{*} \in B_{0}$, we may choose a point $z \in A_{0}$ such that $z \neq \xi^{*}$ and

$$
\rho\left(z, T \xi^{*}\right)=\rho(A, B)
$$

We shall prove that $z=\xi^{*}$. On the contrary suppose that $z \neq \xi^{*}$. Since $T$ is $\alpha_{p}$-admissible weak $(F, \varphi)$-proximal contraction, by using (1) and (9) we have

$$
\begin{aligned}
\rho\left(\xi_{n+1}, z\right) & \leq \max \left\{\rho\left(\xi_{n+1}, z\right), \varphi\left(\xi_{n+1}\right)\right\} \\
& \leq F\left(\rho\left(\xi_{n+1}, z\right), \varphi\left(\xi_{n+1}\right), \varphi(z)\right) \\
& \leq \alpha\left(\xi_{n}, \xi^{*}\right)+F\left(\rho\left(\xi_{n+1}, z\right), \varphi\left(\xi_{n+1}\right), \varphi(z)\right) \\
& \leq \psi\left(F\left(\rho\left(\xi_{n}, \xi^{*}\right), \varphi\left(\xi_{n}\right), \varphi\left(\xi^{*}\right)\right)\right) \\
& <F\left(\rho\left(\xi_{n}, \xi^{*}\right), \varphi\left(\xi_{n}\right), \varphi\left(\xi^{*}\right)\right) \\
& =F\left(\rho\left(\xi_{n}, \xi^{*}\right), \varphi\left(\xi_{n}\right), 0\right) .
\end{aligned}
$$

Letting $n \rightarrow \infty$ on the inequality above, we have

$$
\begin{aligned}
\lim _{n \rightarrow \infty} \rho\left(\xi_{n+1}, z\right) & =\lim _{n \rightarrow \infty} F\left(\rho\left(\xi_{n}, \xi^{*}\right), \varphi\left(\xi_{n}\right), 0\right) \\
& =F(0,0,0)=0
\end{aligned}
$$

which implies that

$$
\lim _{n \rightarrow \infty} \rho\left(\xi_{n+1}, z\right)=0 .
$$

By using the condition (m4), we have

$$
\begin{aligned}
\rho\left(\xi^{*}, z\right)-m_{\xi^{*}, z} & \leq \rho\left(\xi^{*}, \xi_{n+1}\right)-m_{\xi^{*}, \xi_{n+1}}+\rho\left(\xi_{n+1}, z\right)-m_{\xi_{n+1}, z} \\
\rho\left(\xi^{*}, z\right)-m_{\xi^{*}, z} & \leq \rho\left(\xi^{*}, \xi_{n+1}\right)+\rho\left(\xi_{n+1}, z\right) \\
\lim _{n \rightarrow \infty} \rho\left(\xi^{*}, z\right)-m_{\xi^{*}, z} & \leq \lim _{n \rightarrow \infty} \rho\left(\xi^{*}, \xi_{n+1}\right)+\lim _{n \rightarrow \infty} \rho\left(\xi_{n+1}, z\right) \\
\lim _{n \rightarrow \infty} \rho\left(\xi^{*}, z\right)-m_{\xi^{*}, z} & \leq 0 .
\end{aligned}
$$

Since $\rho\left(\xi^{*}, \xi^{*}\right)=0, \xi^{*}=z$. This is a contradiction. Attendantly, we have

$$
\rho\left(\xi^{*}, T \xi^{*}\right)=\rho(A, B)
$$

Uniqueness: Let $\alpha(\xi, \eta) \geq 0$, for all $\xi, \eta \in \varphi_{T}(A)$. Suppose that $\xi^{*}$ and $w$ are two $\varphi$-best proximity points of $T$ with $\xi^{*} \neq w$. Hence

$$
\rho(w, T w)=\rho(A, B),
$$


and

$$
\varphi\left(\xi^{*}\right)=\varphi(w)=0 .
$$

Since $T$ is $\alpha_{p}$-admissible weak $(F, \varphi)$-proximal contraction, we have

$$
\begin{aligned}
F\left(\rho\left(\xi^{*}, w\right), 0,0\right) & \leq \alpha\left(\xi^{*}, w\right)+F\left(\rho\left(\xi^{*}, w\right), \varphi\left(\xi^{*}\right), \varphi(w)\right) \\
& \leq \psi\left(F\left(\rho\left(\xi^{*}, w\right), \varphi\left(\xi^{*}\right), \varphi(w)\right)\right) \\
& <F\left(\rho\left(\xi^{*}, w\right), 0,0\right),
\end{aligned}
$$

a contradiction. Consequently, we find that $\xi^{*}$ is a unique $\varphi$-best proximity point of $T$.

Corollary 1. Let $A, B$ be two subsets of an $M$-complete $M$-metric space $(S, \rho)$ and $F \in \mathcal{F}$. Suppose that a mapping $T: A \rightarrow B$ is a weak $(F, \varphi)$-proximal contraction. If $T\left(A_{0}\right) \subseteq B_{0}$ and $A_{0}$ is closed set in $S$, then there exist a unique $\varphi$-best proximity point of $T$ provided that there exist $\xi_{0}, \xi_{1} \in A_{0}$ such that

$$
\rho\left(\xi_{1}, T \xi_{0}\right)=\rho(A, B) .
$$

Proof. It is derived from Theorem 1 by choosing $\alpha(\xi, \eta)=0$.

Since an $M$-metric space is a partial metric space, from the Theorem 1 we deduce immediately the following result. Note that in the following result we consider the notions in Definitions 10 and 11 in the setting of partial metric spaces.

Corollary 2. Let $A, B$ be two subsets of a complete partial metric space $(S, p)$ and $F \in \mathcal{F}$. Suppose that a mapping $T: A \rightarrow B$ is an $\alpha_{p}$-admissible weak $(F, \varphi)$-proximal contraction. If $T\left(A_{0}\right) \subseteq B_{0}$ and $A_{0}$ is $\alpha$-regular closed set in $S$, then there exists a $\varphi$-best proximity point of $T$ provided that there exist $\xi_{0}, \xi_{1} \in A_{0}$ such that

$$
p\left(\xi_{1}, T \xi_{0}\right)=p(A, B) \text { and } \alpha\left(\xi_{0}, \xi_{1}\right) \geq 0,
$$

$p(A, B)=\inf \{p(a, b): a \in A, b \in B\}$. Moreover, if $\alpha(\xi, \eta) \geq 0$ for all $\xi, \eta \in \varphi_{T}(A)$, then $\xi^{*}$ is the unique $\varphi$-best proximity point of $T$.

Proof. Since an $M$-metric space is a generalization of partial metric space, from Theorem 1 we deduce the result.

Corollary 3. Let $A, B$ be two subsets of a complete partial metric space $(S, p)$ and $F \in \mathcal{F}$. Suppose that a mapping $T: A \rightarrow B$ is a weak $(F, \varphi)$-proximal contraction. If $T\left(A_{0}\right) \subseteq B_{0}$ and $A_{0}$ is closed set in $S$, then there exist a unique $\varphi$-best proximity point of $T$ provided that there exist $\xi_{0}, \xi_{1} \in A_{0}$ such that

$$
p\left(\xi_{1}, T \xi_{0}\right)=p(A, B) .
$$

Proof. It is deduced from Corollary 2 by choosing $\alpha(\xi, \eta)=0$.

To support Corollary 1, we provide the following example.

Example 3. Let $S=[0,1]$ and $\rho: S \times S \rightarrow[0, \infty)$ be defined by

$$
\rho(\xi, \eta)=|\xi-\eta|
$$

otherwise. Then $(S, \rho)$ is an M-metric space. Suppose that $A=\{0,0.4,0.6,0.9\}$ and $B=\{0.1,0.3,0.7,1\}$. Note that $\rho(A, B)=0.1, A=A_{0}$ and $B=B_{0}$. Define a mapping $T: A \rightarrow B$ as:

$$
T(0)=0.1, T(0.4)=0.1, T(0.6)=0.1, T(0.9)=0.3 .
$$


Note that $T\left(A_{0}\right) \subseteq B_{0}$. Define functions $\psi:[0, \infty) \rightarrow[0, \infty), F:[0, \infty)^{3} \rightarrow[0, \infty)$ and $\varphi: A \rightarrow[0, \infty)$ by

$$
\begin{aligned}
\psi(t) & =\frac{2 t}{3}, \\
F(a, b, c) & =\max \{a, b\}+c, \text { for all } a, b, c \in[0, \infty) \\
\text { and } \varphi(\xi) & =\xi, \text { for all } \xi \in A .
\end{aligned}
$$

If we take $\xi=0.6, \eta=0.9, u=0$ and $v=0.4$, then we have

$$
\rho(u, T \xi)=\rho(v, T \eta)=0.1=\rho(A, B)
$$

which implies that

$$
F(\rho(u, v), \varphi(u), \varphi(v))=0.8 \leq 1=\psi(F(\rho(\xi, \eta), \varphi(\xi), \varphi(\eta))) .
$$

Hence $T$ forms a weak $(F, \varphi)$-proximal contraction. Thus, all the conditions of Corollary 1 are satisfied. Moreover, $\xi^{*}=0$ is a unique $\varphi$-best proximity point.

To support Corollary 3, we provide the following example.

Example 4. Let $S=[0,1] \cup[2,3]$. Define the mapping $p: S \times S \rightarrow[0, \infty)$ by

$$
p(\xi, \eta)=\left\{\begin{array}{cl}
\max \{\xi, \eta\}, & \{\tilde{\zeta}, \eta\} \cap[2,3] \neq \phi, \\
|\xi-\eta|, & \{\tilde{\xi}, \eta\} \subseteq[0,1] .
\end{array}\right.
$$

Then $(S, p)$ is a partial metric space. Suppose that $A=\{0,0.4,0.6,0.9\}$ and $B=\{0.1,0.3,0.7,1\}$. Note that $p(A, B)=0.1, A=A_{0}$ and $B=B_{0}$. Define a mapping $T: A \rightarrow B$ as:

$$
T(0)=0.1, T(0.4)=0.1, T(0.6)=0.1, T(0.9)=0.3 .
$$

Note that $T\left(A_{0}\right) \subseteq B_{0}$. Define mappings $\psi:[0, \infty) \rightarrow[0, \infty), F:[0, \infty)^{3} \rightarrow[0, \infty)$ and $\varphi: A \rightarrow[0, \infty)$ by

$$
\begin{aligned}
\psi(t) & =\frac{t}{2}, \\
F(a, b, c) & =a+b+c, \text { for all } a, b, c \in[0, \infty) \\
\text { and } \varphi(\xi) & =\xi, \text { for all } \xi \in A .
\end{aligned}
$$

If we take $\xi=0.6, \eta=0.9, u=0$ and $v=0.4$, then we have

$$
p(u, T \xi)=p(v, T \eta)=0.1=p(A, B)
$$

which implies that

$$
F(p(u, v), \varphi(u), \varphi(v))=0.8 \leq 0.9=\psi(F(p(\xi, \eta), \varphi(\xi), \varphi(\eta)))
$$

Hence, $T$ forms a weak $(F, \varphi)$-proximal contraction. Thus all the conditions of Corollary 3 are satisfied. Moreover $\xi^{*}=0$ is a unique $\varphi$-best proximity point.

\section{Application to Fixed Point Theory}

Let us take $A=B=S$, and suppose that $T$ is proximal $\alpha_{p}$-admissible mapping. Obviously

$$
\alpha(\xi, \eta) \geq 0
$$


and

$$
\rho(u, T \xi)=0 \text { and } \rho(v, T \eta)=0,
$$

implies that

$$
\alpha(T \xi, T \eta)=\alpha(u, v) \geq 0 .
$$

Hence $T$ is $\alpha_{p}$-admissible mapping.

Remark 3. If $\alpha: S \times S \rightarrow[-\infty, \infty), \varphi: S \rightarrow[0, \infty)$ and a selfmapping $T$ on $S$ is $\alpha_{p}$-admissible weak $(F, \varphi)$-contraction, then $\alpha(\xi, \eta) \geq 0$ implies that

$$
\alpha(\xi, \eta)+F(\rho(T \xi, T \eta), \varphi(T \xi), \varphi(T \eta)) \leq \psi(F(\rho(\xi, \eta), \varphi(\xi), \varphi(\eta))),
$$

where $F \in \mathcal{F}$, and $\psi \in \Psi$, for all $\xi, \eta \in S$. In other words, we consider the notions in Definitions 10 and 11 in the setting of standard metric spaces.

Definition 12. A self mapping $T: S \rightarrow S$ satisfying the above implication is called $\alpha_{p}$-admissible weak $(F, \varphi)$-contraction.

Corollary 4. Let $(S, d)$ be a $M$-complete $M$-metric space, $F \in \mathcal{F}$, and a self-mapping $T$ be an $\alpha_{p}$-admissible weak $(F, \varphi)$-contraction. If $\left\{\xi_{n}\right\}$ is a sequence in $S$ such that $\alpha\left(\xi_{n}, \xi_{n+1}\right) \geq 0$ and $\lim _{n \rightarrow \infty} \xi_{n}=\xi \in S$, then $\alpha\left(\xi_{n}, \xi\right) \geq 0$, for all $n \in N$. Then there exists a $\varphi$-fixed point of $T$ provided that there exists $\xi_{0} \in S$ such that $\alpha\left(\xi_{0}, T \xi_{0}\right) \geq 0$. Moreover, if $\alpha(\xi, \eta) \geq 0$ for all $\xi, \eta \in \varphi_{F}(S)$, then $\xi^{*}$ is the unique $\varphi$-fixed point of $T$.

Proof. Let us take $A=B=S$ in Theorem 1. We shall show that $T$ is $\alpha_{p}$-admissible weak $(F, \varphi)$-contraction. Suppose that $\xi, \eta, u, v \in S$ satisfies the following

$$
\begin{aligned}
\alpha(\xi, \eta) & \geq 0, \\
\rho(u, T \xi) & =\rho(A, B), \\
\rho(v, T \eta) & =\rho(A, B) .
\end{aligned}
$$

As $\rho(A, B)=0$, we have $u=T \xi$ and $v=T \eta$. Since $T$ satisfies the condition (10), so

$$
\alpha(\xi, \eta)+F(\rho(T \xi, T \eta), \varphi(T \xi), \varphi(T \eta)) \leq \psi(F(\rho(\xi, \eta), \varphi(\xi), \varphi(\eta))),
$$

that is,

$$
\alpha(\xi, \eta)+F(\rho(u, v), \varphi(u), \varphi(v)) \leq \psi(F(\rho(\xi, \eta), \varphi(\xi), \varphi(\eta))),
$$

which implies that $T$ is an $\alpha_{p}$-admissible weak $(F, \varphi)$-contraction Let $\xi_{0}$ be an arbitrary point in $S$. Define a sequence $\left\{\xi_{n}\right\}$ in $S$ by

$$
\xi_{n}=T \xi_{n-1}, \text { for all } n \in \mathbb{N} .
$$

As $T$ is $\alpha_{p}$-admissible mapping. So, we have

$$
\alpha\left(\xi_{0}, \xi_{1}\right)=\alpha\left(\xi_{0}, T \xi_{0}\right) \geq 0 \text { implies that } \alpha\left(T \xi_{0}, T \xi_{1}\right)=\alpha\left(\xi_{1}, \xi_{2}\right) \geq 0
$$

By induction, we get that

$$
\alpha\left(\xi_{n}, \xi_{n+1}\right)=\alpha\left(\xi_{n}, T \xi_{n}\right) \geq 0, \text { for all } n \in \mathbb{N} .
$$


Using (11) and the fact that $T$ is $\left(F, M, \varphi, \alpha_{p}, \psi\right)$-contraction, we obtain

$$
\begin{aligned}
F\left(\rho\left(\xi_{n}, \xi_{n+1}\right), \varphi\left(\xi_{n}\right), \varphi\left(\xi_{n+1}\right)\right) & =F\left(\rho\left(T \xi_{n-1}, T \xi_{n}\right), \varphi\left(T \xi_{n-1}\right), \varphi\left(T \xi_{n}\right)\right) \\
\leq & \alpha\left(\xi_{n-1}, \xi_{n}\right)+F\left(\rho\left(T \xi_{n-1}, T \xi_{n}\right), \varphi\left(T \xi_{n-1}\right), \varphi\left(T \xi_{n}\right)\right) \\
\leq & \psi\left(F\left(\rho\left(\xi_{n-1}, \xi_{n}\right), \varphi\left(\xi_{n}\right), \varphi\left(\xi_{n+1}\right)\right)\right), \text { for all } n \in \mathbb{N} .
\end{aligned}
$$

Using the arguments similar to those given in the proof of Theorem 1 , we obtain that $\left\{\xi_{n}\right\}_{n \in \mathbb{N}}$ is a Cauchy sequence in $S$. Since $(S, \rho)$ is $M$-complete $M$-metric space, there exists $\xi^{*} \in S$ such that

$$
\lim _{n \rightarrow \infty} \rho\left(\xi_{n}, \xi^{*}\right)=0 \text { and } \lim _{n \rightarrow \infty} M_{\tilde{\zeta}_{n}, \xi^{*}}=0 .
$$

We now show that $\varphi\left(\xi^{*}\right)=0$. From (2), we conclude that

$$
\varphi\left(\xi_{n}\right) \leq \psi^{n}\left(F\left(\rho\left(\xi_{0}, \xi_{1}\right), \varphi\left(\xi_{0}\right), \varphi\left(\xi_{1}\right)\right)\right) .
$$

Again by using the arguments similar to those given in the proof of Theorem 1, we obtain that $\varphi\left(\xi^{*}\right)=0$. In the view of (11) and (12) we have $\alpha\left(\xi_{n}, \xi^{*}\right) \geq 0$, for all $n \in \mathbb{N}$. By taking $\xi=\xi_{n}$ and $\eta=\xi^{*}$ in the condition (10), we have

$$
\begin{aligned}
\rho\left(\xi_{n+1}, T \xi^{*}\right) & =\rho\left(T \xi_{n}, T \xi^{*}\right) \\
& \leq \max \left\{\rho\left(T \xi_{n}, T \xi^{*}\right), \varphi\left(T \xi_{n}\right)\right\} \\
& \leq F\left(\rho\left(T \xi_{n}, T \xi^{*}\right), \varphi\left(T \xi_{n}\right), \varphi\left(T \xi^{*}\right)\right) \\
& \leq \alpha\left(\xi_{n}, \xi^{*}\right)+F\left(\rho\left(T \xi_{n}, T \xi^{*}\right), \varphi\left(T \xi_{n}\right), \varphi\left(T \xi^{*}\right)\right) \\
& \leq \psi\left(F\left(\rho\left(\xi_{n}, \xi^{*}\right), \varphi\left(\xi_{n}\right), \varphi\left(\xi^{*}\right)\right)\right) \\
& <F\left(\rho\left(\xi_{n}, \xi^{*}\right), \varphi\left(\xi_{n}\right), \varphi\left(\xi^{*}\right)\right) \\
& =F\left(\rho\left(\xi_{n}, \xi^{*}\right), \varphi\left(\xi_{n}\right), 0\right) .
\end{aligned}
$$

On taking limit as $n \rightarrow \infty$ on the both sides of the above inequality, we have

$$
\begin{aligned}
\lim _{n \rightarrow \infty} \rho\left(\xi_{n+1}, T \xi^{*}\right) & =\lim _{n \rightarrow \infty} F\left(\rho\left(\xi_{n}, \xi^{*}\right), \varphi\left(\xi_{n}\right), 0\right) \\
& =F(0,0,0)=0
\end{aligned}
$$

which implies that

$$
\lim _{n \rightarrow \infty} \rho\left(\xi_{n+1}, T \xi^{*}\right)=0 .
$$

By using the condition (m4), we have

$$
\begin{aligned}
\rho\left(\xi^{*}, T \xi^{*}\right)-m_{\xi^{*}, T \zeta^{*}} & \leq \rho\left(\xi^{*}, \xi_{n+1}\right)-m_{\xi^{*}, \xi_{n+1}}+\rho\left(\xi_{n+1}, T \xi^{*}\right)-m_{\xi_{n+1}, T \xi^{*}} \\
& \leq \rho\left(\xi^{*}, \xi_{n+1}\right)+\rho\left(\xi_{n+1}, T \xi^{*}\right) .
\end{aligned}
$$

Letting $n \rightarrow \infty$ in the inequality above, we deduce that

$$
\begin{aligned}
& \lim _{n \rightarrow \infty} \rho\left(\xi^{*}, T \xi^{*}\right)-m_{\mathcal{\zeta}^{*}, T \xi^{*}} \leq \lim _{n \rightarrow \infty} \rho\left(\xi^{*}, \xi_{n+1}\right)+\lim _{n \rightarrow \infty} \rho\left(\xi_{n+1}, T \xi^{*}\right) \\
& \lim _{n \rightarrow \infty} \rho\left(\xi^{*}, T \xi^{*}\right)-m_{\mathcal{\zeta}^{*}, T \xi^{*}} \leq 0 .
\end{aligned}
$$

Since $\rho\left(\xi^{*}, \xi^{*}\right)=0$, hence

$$
\rho\left(\mathcal{\zeta}^{*}, T \mathcal{\zeta}^{*}\right)=0,
$$

gives that $\xi^{*}$ is a $\varphi$-fixed point of $T$. 
Uniqueness: Let $\alpha(\xi, \eta) \geq 0$ for all $\xi, \eta \in \varphi_{F}(S)$. Suppose that $\xi^{*}$ and $w$ are two $\varphi$-fixed point of $T$ with $\xi^{*} \neq w$. Hence

$$
\rho(w, T w)=0
$$

and

$$
\varphi\left(\xi^{*}\right)=\varphi(w)=0 .
$$

Since $T$ is $\alpha_{p}$-admissible weak $(F, \varphi)$-contraction, we have

$$
\begin{aligned}
F\left(\rho\left(\xi^{*}, w\right), 0,0\right) & =F\left(\rho\left(T \xi^{*}, T w\right), \varphi\left(T \xi^{*}\right), \varphi(T w)\right) \\
& \leq \alpha\left(\xi^{*}, w\right)+F\left(\rho\left(T \xi^{*}, T w\right), \varphi\left(T \xi^{*}\right), \varphi(T w)\right) \\
& \leq \psi\left(F\left(\rho\left(\xi^{*}, w\right), \varphi\left(\xi^{*}\right), \varphi(w)\right)\right) \\
& <F\left(\rho\left(\xi^{*}, w\right), 0,0\right),
\end{aligned}
$$

a contradiction. Attendantly, we find that $\xi^{*}$ is a unique $\varphi$-fixed point of $T$.

\section{Application to Graph Theory}

Let $S$ be a set and $\Delta$ denotes the diagonal of $S \times S$. A graph is a pair $(V, E)$, where the set $V=V(G)$ of its vertices coincides with $S$ and set $E=E(G)$ of its edges which contains all loops, that is, $\Delta \subseteq S \times S$. Furthermore, we assume that the graph $G$ has no parallel edges. In a graph $G$, by reversing the direction of edges we get the graph $G^{-1}$ whose set of edges and set of vertices are defined as follows:

$$
E\left(G^{-1}\right)=\{(\xi, \eta) \in S \times S:(\eta, \xi) \in E(G)\} \text { and } V\left(G^{-1}\right)=V(G)
$$

We denote the undirected graph by $\widetilde{G}$ obtained from $G$ by ignoring the direction of edges.

Consider the graph $\widetilde{G}$ as a directed graph for which the set of its edges is symmetric, under this convention, we have

$$
E(\widetilde{G})=E(G) \cup E\left(G^{-1}\right)
$$

Definition 13 ([28]). 1. A graph's subgraph is a graph whose vertex set is a subset of $V(G)$ and whose edge set is a subset of $E(G)$.

2. Let $\xi$ and $\eta$ be two vertices of a graph $G$. A path from $\xi$ to $\eta$ of length $n$ (where $n \in \mathbb{N} \cup\{0\}$ ) in a graph $G$ is a sequence $\left\{\xi_{n}: n=0,1,2, \ldots, n\right\}$ of $n+1$ distinct vertices such that $\xi_{0}=\xi_{,}, \xi_{n}=\eta$ and $\left(\xi_{i}, \xi_{i+1}\right) \in E(G)$ for $i=1,2, \ldots, n$.

3. A graph $G$ is called connected graph if there exist a path between any two vertices of graph $G$ and if $\widetilde{G}$ is connected then $G$ is said to be weakly connected graph.

4. A path is called elementary if no vertices appear more than once in it.

Throughout this section, we suppose that $(S, \rho)$ is an $M$-metric space endowed with a directed graph $G$ and has no parallel edges.

We now introduce a notion of $G$-proximal graphic contraction.

Definition 14. Let $A, B$ be two subsets of an $M$-complete $M$-metric space $(S, \rho), \varphi: S \rightarrow[0, \infty), \psi \in \Psi$, $F \in \mathcal{F}$ and $G$ be a graph without parallel edges such that $V(G)=S$. A mapping $T: A \rightarrow B$ is said to be a $G$-proximal graphic contraction if for all $\xi, \eta, u, v \in A, \xi \neq \eta$, with $(\xi, \eta) \in E(G)$ we have

$$
\left.\begin{array}{l}
\rho(u, T \xi)=\rho(A, B) \\
\rho(v, T \eta)=\rho(A, B)
\end{array}\right\} \Longrightarrow F(\rho(u, v), \varphi(u), \varphi(v)) \leq \psi(F(\rho(\xi, \eta), \varphi(\xi), \varphi(\eta))),
$$

and

$$
(u, v) \in E(G)
$$


Theorem 2. Let $\varphi: A \rightarrow[0, \infty)$ be a lower semi continuous function and $T: A \rightarrow B$ a $G$-proximal graphic contraction. If $T\left(A_{0}\right) \subseteq B_{0}, A_{0}$ is closed set in $S$ and there exist a path $\left(\eta^{i}\right)_{i=0}^{N} \subseteq A_{0}$ in $G$ between any two elements $\xi$ and $\eta$. Then there exist a unique $\varphi$-best proximity point of T provided that there exist $\xi_{0}, \xi_{1} \in A_{0}$ and an elementary path between them in $A_{0}$ and

$$
\rho\left(\xi_{1}, T \xi_{0}\right)=\rho(A, B) .
$$

Proof. Let $\xi_{0}, \xi_{1} \in A_{0}$ such that $\rho\left(\xi_{1}, T \xi_{0}\right)=\rho(A, B)$. A path $\left\{s_{0}^{0}, s_{0}^{1}, s_{0}^{2}, \ldots, s_{0}^{N}\right\}$ in $G$ is a sequence containing points of $A_{0}$. Consequently, $s_{0}^{0}=\xi_{0}, s_{0}^{N}=\xi_{1}$ and $\left(s_{0}^{i}, s_{0}^{i+1}\right) \in E(G)$ for all $0 \leq i \leq N-$ 1. Given that $s_{0}^{1} \in A_{0}$, by $T\left(A_{0}\right) \subseteq B_{0}$ and the definition of $A_{0}$, there exist $s_{1}^{1} \in A_{0}$ such that $\rho\left(s_{1}^{1}, T s_{0}^{1}\right)=$ $\rho(A, B)$. Similarly, for each $i=2, \ldots, N$, there exists $s_{1}^{i} \in A_{0}$ such that $\rho\left(s_{1}^{i}, T s_{0}^{i}\right)=\rho(A, B)$. As $\left\{s_{0}^{0}, s_{0}^{1}, s_{0}^{2}, \ldots, s_{0}^{N}\right\}$ is a path in $G,\left(s_{0}^{0}, s_{0}^{1}\right)=\left(\xi_{0}, s_{0}^{1}\right) \in E(G)$. From the above argument, we have $\rho\left(\xi_{1}, T \xi_{0}\right)=\rho(A, B)$ and $\rho\left(s_{1}^{1}, T s_{0}^{1}\right)=\rho(A, B)$. Since, $T$ is $G$-proximal graphic contraction, it follows that $\left(\xi_{1}, s_{1}^{1}\right) \in E(G)$. In similar manner, we have the following:

$$
\left(s_{1}^{i-1}, s_{1}^{i}\right) \in E(G), \text { for all } 1 \leq i \leq N .
$$

If $\xi_{2}=s_{1}^{N}$, then $\left\{s_{1}^{0}, s_{1}^{1}, s_{1}^{2}, \ldots, s_{1}^{N}\right\}$ is a path from $\xi_{1}=s_{1}^{0}$ to $\xi_{2}=s_{1}^{N}$. As $s_{1}^{i} \in A_{0}$ and $T s_{1}^{i} \in T\left(A_{0}\right) \subseteq B_{0}$, or each $i=1,2,3, \ldots, N$, by the definition of $B_{0}$, there exists $s_{2}^{i} \in A_{0}$ such that $\rho\left(s_{2}^{i}, T s_{1}^{i}\right)=\rho(A, B)$. In addition, we have $\rho\left(\xi_{2}, T \xi_{1}\right)=\rho(A, B)$. As mentioned above, we have

$$
\left(\xi_{2}, s_{2}^{1}\right) \in E(G) \text { and }\left(s_{2}^{i-1}, s_{2}^{i}\right) \in E(G), \text { for all } 1 \leq i \leq N .
$$

Similarly, by $T\left(A_{0}\right) \subseteq B_{0}$, there exists a point $\xi_{3} \in A_{0}$ where $\xi_{3}=s_{2}^{N}$. Then $\left(s_{2}^{i}\right)_{i=0}^{N}$ is a path from $s_{2}^{0}=\xi_{2}$ and $s_{2}^{N}=\xi_{3}$. Continuing in this manner for all $n \in \mathbb{N}$, we obtain a sequence $\left\{\xi_{n}\right\}_{n \in \mathbb{N}}$ where $\xi_{n+1} \in\left[\xi_{n}\right]_{G}^{N}$ and $\rho\left(\xi_{n+1}, T \xi_{n}\right)=\rho(A, B)$ by producing a path $\left\{s_{n}^{0}, s_{n}^{1}, s_{n}^{2}, \ldots, s_{n}^{N}\right\}$ from $\xi_{n}=s_{n}^{0}$ and $\xi_{n+1}=s_{n}^{N}$ in such a way that

$$
\rho\left(s_{n+1}^{i}, T s_{n}^{i}\right)=\rho(A, B),
$$

for all $1 \leq i \leq N, n \in \mathbb{N}$. Thus we have

$$
\rho\left(s_{n}^{i-1}, T s_{n-1}^{i-1}\right)=\rho(A, B)=\rho\left(s_{n}^{i}, T s_{n-1}^{i}\right), \text { for all } 1 \leq i \leq N, n \in \mathbb{N} .
$$

Now for any positive integer $n$

$$
\begin{aligned}
\rho\left(\xi_{n}, \xi_{n+1}\right) & =\rho\left(s_{n}^{0}, s_{n}^{N}\right) \\
& \leq \rho\left(s_{n}^{0}, s_{n}^{1}\right)-m_{s_{n}^{0}, s_{n}^{1}}+\rho\left(s_{n}^{1}, s_{n}^{2}\right)-m_{s_{n}^{1}, s_{n}^{2}}+\ldots+\rho\left(s_{n}^{N-1}, s_{n}^{N}\right)-m_{s_{n}^{N-1}, s_{n}^{N}} \\
& \leq \rho\left(s_{n}^{0}, s_{n}^{1}\right)+\rho\left(s_{n}^{1}, s_{n}^{2}\right)+\ldots+\rho\left(s_{n}^{N-1}, s_{n}^{N}\right) \\
& =\sum_{i=1}^{N} \rho\left(s_{n}^{i-1}, s_{n}^{i}\right),
\end{aligned}
$$

for all $1 \leq i \leq N$ and $n \in \mathbb{N}$. Note that, $\left(s_{n-1}^{i-1}, s_{n-1}^{i}\right) \in E(G)$, and $T$ is $G$-proximal graphic contraction. It follows from (13), that

$$
F\left(\rho\left(s_{n}^{i-1}, s_{n}^{i}\right), \varphi\left(s_{n}^{i-1}\right), \varphi\left(s_{n}^{i}\right)\right) \leq \psi\left(F\left(\rho\left(s_{n-1}^{i-1}, s_{n-1}^{i}\right), \varphi\left(s_{n-1}^{i-1}\right), \varphi\left(s_{n-1}^{i}\right)\right)\right), \text { for all } 1 \leq i \leq N, n \in \mathbb{N} .
$$

Again by using the arguments similar to those given in the proof of Theorem 1, we obtain that

$$
\rho\left(s_{n}^{i-1}, s_{n}^{i}\right) \leq \psi^{n}\left(F\left(\rho\left(s_{0}^{i-1}, s_{0}^{i}\right), \varphi\left(s_{0}^{i-1}\right), \varphi\left(s_{0}^{i}\right)\right)\right) .
$$

From (14) and (15), we have

$$
\rho\left(\xi_{n}, \xi_{n+1}\right) \leq \psi^{n} M, \text { for all } n \in \mathbb{N}
$$


where $M=\sum_{i=1}^{N}\left(F\left(\rho\left(s_{0}^{i-1}, s_{1}^{i}\right), \varphi\left(s_{0}^{i-1}\right), \varphi\left(s_{1}^{i}\right)\right)\right)$. Again by using the arguments similar to those given in the proof of Theorem 1 , we obtain

$$
\varphi\left(\xi^{*}\right)=0 \text { and } \rho\left(\xi^{*}, T \xi^{*}\right)=\rho(A, B) .
$$

Hence $\xi^{*}$ is a unique $\varphi$-best proximity point of $T$.

\section{Conclusions}

In this paper, we defined $\varphi$-best proximity point and $\alpha_{p}$-admissible weak $(F, \varphi)$-contraction. We proved some $\varphi$-best proximity point results in the setting of $M$-metric spaces. As an application, we derived the $\varphi$-fixed point results for some self mappings. We also introduced the notions of $G$-proximal graphic contraction and provided an application to graph theory in the setting of $M$-complete $M$-metric space. Some examples are also presented to illustrate the novelty of the result proved herein.

Author Contributions: Writing—original draft, S.F.; Writing—review and editing, E.K. and M.A. All authors have read and agreed to the published version of the manuscript.

Funding: This research received no external funding.

Acknowledgments: Authors are thankful to the reviewers for their suggestions to improve the presentation of this paper.

Conflicts of Interest: The authors declare no conflict of interest.

\section{References}

1. Fan, K. Extensions of two fixed point Theorems of F. E. Browder. Math. Z. 1969, 112, 234-240. [CrossRef]

2. Abbas, M.; Saleem, N.; De la Sen, M. Optimal coincidence point results in partially ordered nonArchimedean fuzzy metric spaces. Fixed Point Theory Appl. 2016, 2016, 44. [CrossRef]

3. Eldred, A.A.; Veeramani, P. Existence and convergence of best proximity points. J. Math. Anal. Appl. 2006, 323, 1001-1006. [CrossRef]

4. Bilgili, N.; Karapinar, E.; Sadarangani, K. A generalization for the best proximity point of Geraghty-contractions. J. Inequalities Appl. 2013, 2013, 286. [CrossRef]

5. Karapinar, E.E.; Erhan, I.M. Best Proximity Point on Different Type Contractions. Appl. Math. Inf. Sci. 2011, 3, 342-353.

6. Karapinar, E. Fixed point theory for cyclic weak $\phi$-contraction. Appl. Math. Lett. 2011, 24, 822-825. [CrossRef]

7. Karapinar, E. Best proximity points of Kannan type cylic weak $\varphi$-contractions in ordered metric spaces. Analele Stiintifice Universitatii Ovidius Constanta 2012, 20, 51-64. [CrossRef]

8. Mongkolkeha, C.; Cho, Y.J.; Kumam, P. Best proximity points for generalized proximal contraction mappings in metric spaces with partial orders. J. Inequalities Appl. 2013, 2013, 534127. [CrossRef]

9. Matthews, S.G. Partial metric topology. N. Y. Acad. Sci. 1994, 728, 183-197. [CrossRef]

10. Karapinar, E.; Erhan, I.; Ozturk, A. Fixed point theorems on quasi-partial metric spaces. Math. Comput. Model. 2013, 57, 2442-2448. [CrossRef]

11. Karapinar, E.; Chi, K.P.; Thanh, T.D. A generalization of Ciric quasi-contractions. Abstr. Appl. Anal. 2012, 2012, 518734. [CrossRef]

12. Chi, K.P.; Karapinar, E.; Thanh, T.D. A Generalized Contraction Principle in Partial Metric Spaces. Math. Comput. Model. 2012, 55, 1673-1681, doi:10.1016/j.mcm.2011.11.005. [CrossRef]

13. Karapinar, E.; Erhan, I.M.; Ulus, A.Y. Fixed Point Theorem for Cyclic Maps on Partial Metric Spaces. Appl. Math. Inf. Sci. 2012, 6, 239-244.

14. Chi, K.P.; Karapinar, E.; Thanh, T.D. On the fixed point theorems in generalized weakly contractive mappings on partial metric spaces. Bull. Iranian Math. Soc. 2013, 39, 369-381.

15. Shatanawi, W.; Postolache, M. Coincidence and fixed point results for generalized weak contractions in the sense of Berinde on partial metric spaces. Fixed Point Theory Appl. 2013, 2013, 54. [CrossRef] 
16. Nastasi, A.; Vetro, P. Fixed point results on metric and partial metric spaces via simulation functions. J. Nonlinear Sci. Appl. 2015, 8, 1059-1069. [CrossRef]

17. Oltra, S.; Valero, O. Banach's fixed point theorem for partial metric spaces. Rend. Istit. Mat. Univ. Trieste 2004, $36,17-26$.

18. Rus, I.A. Fixed point theory in partial metric spaces. Univ. Vest. Timis. Ser. Mat. Inform. 2008, 46, 41-160.

19. Asadi, M.; Karapinar, E.; Salimi, P. New extension of $p$-metric spaces with fixed points results on $M$-metric spaces. J. Inequalities Appl. 2014, 2014, 18. [CrossRef]

20. Patle, P.R.; Patel, D.K.; Aydi, H.; Gopal, D.; Mlaiki, N. Nadler and Kannan type set valued mappings in M-metric spaces and an application. Mathematics 2019, 7, 373. [CrossRef]

21. Asadi, M.; Azhini, M.; Karapinar, E.; Monfared, H. Simulation Functions Over M-Metric Spaces. East Asian Math. J. 2017, 33, 559-570.

22. Jleli, M.; Samet, B.; Vetro, C. Fixed point theory in partial metric spaces via $\varphi$-fixed point's concept in metric spaces. J. Inequalities Appl. 2014, 2014, 426. [CrossRef]

23. Kumrod, P.; Sintunavara, W. A new contractive condition approach to $\varphi$-fixed point results in metric spaces and its applications. J. Comput. Appl. Math. 2017, 311, 194-204. [CrossRef]

24. Asadi, M. Discontinuity of control function in the $(F, \varphi, \theta)$-contraction in metric spaces. Filomat 2017, 31, 17. [CrossRef]

25. Imdad, M.; Khan, A.R.; Saleh, H.N.; Alfaqih, W.M. Some $\varphi$-fixed point results for $(F, \varphi, \alpha-\psi)-$ contractive type mappings with applications. Mathematics 2019, 7, 122. [CrossRef]

26. Samet, B.; Karapinar, E.; O'regan, D. On the existence of fixed points that belong to the zero set of a certain function. Fixed Point Theory Appl. 2015, 2015, 152, doi:10.1186/s13663-015-0401-7. [CrossRef]

27. Rus, I.A. Generalized Contractions and Applications; Cluj University Press: Clui-Napoca, Romania, 2001.

28. Jachymski, J. The contraction principle for mappings on a metric space with a graph. Proc. Am. Math. Soc. 2008, 136, 1359-1373. [CrossRef]

(C) 2020 by the authors. Licensee MDPI, Basel, Switzerland. This article is an open access article distributed under the terms and conditions of the Creative Commons Attribution (CC BY) license (http:/ / creativecommons.org/licenses/by/4.0/). 



\title{
Article \\ Admissible Hybrid $\mathcal{Z}$-Contractions in $b$-Metric Spaces
}

\author{
Ioan Cristian Chifu ${ }^{1}$ and Erdal Karapınar ${ }^{2, *}$ \\ 1 Department of Business, Babeş-Bolyai University Cluj-Napoca, Horea Street, No.7, 400000 Cluj-Napoca, \\ Romania; cristian.chifu@tbs.ubbcluj.ro \\ 2 Department of Medical Research, China Medical University Hospital, China Medical University, \\ Taichung 40402, Taiwan \\ * Correspondence: erdalkarapinar@yahoo.com or karapinar@mail.cmuh.org.tw
}

Received: 25 November 2019; Accepted: 19 December 2019; Published: 21 December 2019

\begin{abstract}
In this manuscript, we introduce a new notion, admissible hybrid $\mathcal{Z}$-contraction that unifies several nonlinear and linear contractions in the set-up of a $b$-metric space. In our main theorem, we discuss the existence and uniqueness result of such mappings in the context of complete $b$-metric space. The given result not only unifies the several existing results in the literature, but also extends and improves them. We express some consequences of our main theorem by using variant examples of simulation functions. As applications, the well-posedness and the Ulam-Hyers stability of the fixed point problem are also studied.
\end{abstract}

Keywords: admissible spaces; hybrid contraction; interpolative contraction; $b$-metric spaces; simulation function; interpolative contraction

MSC: 47H10; 54H25

\section{Introduction}

Metric fixed point theory can be settled in the intersection of two disciplines; (nonlinear) functional analysis and topology. From the fixed point researchers' aspect, the first application of the metric fixed point theory is on the solution of differential equations. However, according to the point of view of researchers in applied mathematics, metric fixed point theory is a tool in the solution of a first-order ordinary differential equation with an initial value. Indeed, fixed point theory appears, firstly, in the paper of Liouville in 1837, and, later, in the paper of Picard in 1890. In the paper of Picard, the method of the successive approaches was used to investigate the existence of the solution. In 1922, Banach reported the first metric fixed point result in the setting of complete norm space that would be called Banach space later. Examined enough and carefully, we realized that Banach's theorem is the abstraction of the successive approaches. The characterization of the nominated fixed point theorem of Banach, in the complete metric space, was reported by Caccioppoli in 1931. This can be accepted as the first generalization of Banach's theorem. After this, a huge number of papers, on the generalization and extension of Banach's fixed point theorem, has been released.

Extensions and generalizations of Banach's theorem are based on two elements: by changing the structure (abstract space) and by changing the conditions on the considered mappings. The immediate examples of these new structures are partial metric space, quasi-metric space, semi-metric space, b-metric space, etc. Among all of these, we shall consider the $b$-metric that is the most interesting and most general form of the distance. The notion of $b$-metric has been discovered by several authors, such as Bourbaki [1], Bakhtin [2], and Czerwik [3], in different periods of time. Roughly speaking, $b$-metric space is derived from metric space by relaxing the triangle inequality. 
As it was mentioned before, the theory has been advanced by reporting several new fixed point results that are obtained by changing the conditions on the given mappings. As a result, in the literature, there are so many different types of metric fixed point results that cause a disturbance, conflict, and disorder. For overcoming this problem, it needs to consider new theorems that cover several different results. One of the successful results in directions was given in [4] where admissible mappings were introduced to combine different structures. Other interesting results were given in [5] in which the notion of the simulation function was defined to combine many distinct contractions. The notion of the hybrid contractions can also be considered as a result of this trend: in two recent papers [6,7], the authors introduce a new type of contraction, namely admissible hybrid contraction, in order to unify several linear, nonlinear and interpolative contractions in the set-up of a complete metric and $b$-metric spaces.

One of the main aims of this paper is to unify the several existing results in the literature by combining the interesting notions: admissible mappings, simulation functions, and hybrid contractions. Besides unifying the results, we express our results in the most generalized form: in the setting of a complete $b$-metric space. Next, we shall consider applications for our obtained results. In particular, we shall consider the well-posedness and the Ulam-Hyers stability of the fixed point problem. We shall give some consequences and we shall indicate how one can get more consequences from the main theorem of the paper. In the next section, we shall give some basic notions and results to provide a self-contained, easily readable paper.

\section{Preliminaries}

In this section, we shall collect the necessary notations, notions, and results for the sake of the completeness of the paper. We first express the definition of the $b$-metric, as follows.

Definition 1 (See, e.g., Bourbaki [1], Bakhtin [2], and Czerwik [3]). Let $X$ be a nonempty set and let $s \geq 1$ be a given real number. A functional $d: X \times X \rightarrow[0, \infty)$ is said to be a b-metric with constant s, if

1. $d$ is symmetric, that is, $d(x, y)=d(y, x)$ for all $x, y$,

2. $d$ is self-distance, that is, $d(x, y)=0$ if and only if $x=y$,

3. $d$ provides s-weighted triangle inequality, that is

$$
d(x, z) \leq s[d(x, y)+d(y, z)], \text { for all } x, y, z \in X
$$

In this case, the triple $(X, d, s)$ is called a b-metric space with constant s.

It is evident that the notions of $b$-metric and standard metric coincide in case of $s=1$. For more details on $b$-metric spaces, see, e.g., [8-11] and corresponding references therein.

In what follows, we express the following immediate interesting examples of $b$-metric space to indicate the richness of this abstract space.

Example 1. Let $S$ be any set that has more than three elements. Suppose that $S_{1}, S_{2}$ are the subsets of $S$ such that $S_{1} \cap S_{2}=\varnothing$ and $S=S_{1} \cup S_{2}$ Let $s \geq 1$ be arbitrary. Consider the functional $d: X \times X \rightarrow[0, \infty)$, which is defined by:

$$
d(a, b):= \begin{cases}0, & a=b \\ 2 s, & a, b \in S_{1} \\ 1, & \text { otherwise. }\end{cases}
$$

It is obvious that $(X, d, s)$ forms a b-metric space.

Another simple, but interesting example is the following: 
Example 2. Let $X=\mathbb{R}$. The function $d: \mathbb{R} \times \mathbb{R} \rightarrow[0, \infty)$, defined as

$$
d(x, y)=|x-y|^{2}
$$

is a b-metric on $\mathbb{R}$ with $s=2$. Clearly, the first two conditions are satisfied. For the third condition, we have

$$
\begin{aligned}
|x-y|^{2} & =|x-z+z-y|^{2}=|x-z|^{2}+2|x-z||z-y|+|z-y|^{2} \\
& \leq 2\left[|x-z|^{2}+|z-y|^{2}\right]
\end{aligned}
$$

since

$$
2|x-z||z-y| \leq|x-z|^{2}+|z-y|^{2}
$$

Thus, $(X, d, 2)$ is a b-metric space.

Example 3. Let $X=\{a, b, c\}$ and $d: X \times X \rightarrow \mathbb{R}_{0}^{+}$such that

$$
\begin{aligned}
& d(a, b)=d(b, a)=d(a, c)=d(c, a)=1, \\
& d(b, c)=d(c, b)=\alpha \geq 2, \\
& d(a, a)=d(b, b)=d(c, c)=0 .
\end{aligned}
$$

Then,

$$
d(x, y) \leq \frac{\alpha}{2}[d(x, z)+d(z, y)], \text { for } a, b, c \in X
$$

Then, $\left(X, d, \frac{\alpha}{2}\right)$ is a b-metric space.

Example 4 ([8]). Let $B$ be a Banach space with the zero vector $0_{B}$. Suppose that $P$ be a cone whose interior is non-empty. Suppose also that $\preceq$ forms a partial order with respect to $P$.

For a non-empty set $S$, we consider the functional $d: X \times X \rightarrow B$ that fulfills

(M1) $0_{B} \preceq \delta(a, b)$,

(M2) $\delta(a, b)=0$ if and only if $x=y$,

(M3) $\delta(a, b) \preceq \delta(a, c)+\delta(c, b)$,

(M4) $\delta(a, b)=\delta(b, a)$,

for all $a, b, c \in S$. Then, $\delta$ is said to be a cone metric (or, Banach-valued metric). Furthermore, the pair $(S, \delta)$ is called a cone metric space (or Banach-valued metric space).

Let $E$ be a Banach space and $P$ be a normal cone in $E$ with the coefficient of normality denoted by $K$. Let $D: X \times X \rightarrow[0, \infty)$ be defined by $D(x, y)=\|d(x, y)\|$, where $d: X \times X \rightarrow E$ is a cone metric space. Then, $(X, D, K)$ forms a b-metric space.

Example 5 (See, e.g., [1]). Let $X=L^{p}[0,1]$ be the collections of all real functions $x(t)$ such that $\int_{0}^{1}|x(t)|^{p} d t<\infty$, where $t \in[0,1]$ and $0<p<1$. For the function $d: X \times X \rightarrow \mathbb{R}_{0}^{+}$defined by

$$
d(x, y):=\left(\int_{0}^{1}|x(t)-y(t)|^{p} d t\right)^{1 / p}, \text { for each } x, y \in L^{p}[0,1]
$$

the ordered triple $\left(X, d, 2^{1 / p}\right)$ forms a b-metric space.

Example 6 (See, e.g., [1]). Let $p \in(0,1)$ and let

$$
X=l_{p}(\mathbb{R})=\left\{x=\left\{x_{n}\right\} \subset \mathbb{R} \text { such that } \sum_{n=1}^{\infty}\left|x_{n}\right|^{p}<\infty\right\} .
$$


Define $d(x, y): X \times X \rightarrow[0, \infty)$ by

$$
d(x, y)=\left(\sum_{n=1}^{\infty}\left|x_{n}-y_{n}\right|^{p}\right)^{1 / p}
$$

Then, $\left(X, d, 2^{1 / p}\right)$ is a b-metric space.

Definition 2 ([12]). A mapping $\varphi:[0, \infty) \rightarrow[0, \infty)$ is called a comparison function if it is increasing and $\varphi^{n}(t) \rightarrow 0$, as $n \rightarrow \infty$, for any $t \in[0, \infty)$.

Example 7. Let $\gamma:[0, \infty) \rightarrow[0, \infty)$ be a function such that

$$
\gamma(t)=c t \text { for all } t \in[0, \infty) \text { where } c \in(0,1) \text {. }
$$

Then, $\gamma$ forms a comparison function.

Example 8. Let $\beta:[0, \infty) \rightarrow[0, \infty)$ be a function such that

$$
\beta(t)=\frac{t}{1+t} \text { for all } t \in[0, \infty) .
$$

Then, $\gamma$ forms a comparison function.

Lemma 1 ([10]). If $\varphi:[0, \infty) \rightarrow[0, \infty)$ is a comparison function, then:

(1) each iterate $\varphi^{k}$ of $\varphi, k \geq 1$, is also a comparison function;

(2) $\varphi$ is continuous at 0 ;

(3) $\varphi(t)<t$, for any $t>0$.

Definition $3([12])$. A function $\varphi:[0, \infty) \rightarrow[0, \infty)$ is said to be a c-comparison function if

(1) $\varphi$ is increasing;

(2) there exists $k_{0} \in \mathbb{N}, a \in(0,1)$ and a convergent series of nonnegative terms $\sum_{k=1}^{\infty} v_{k}$ such that $\varphi^{k+1}(t) \leq a \varphi^{k}(t)+v_{k}$, for $k \geq k_{0}$ and any $t \in[0, \infty)$.

Remark 1. Note that $\gamma$ in Example 7 is also c-comparison function. On the other hand, $\beta$ in Example 8 is not a c-comparison function.

It is evident that the $c$-comparison function is not useful to work in the setting of $b$-metric space due to the third axiom, $s$-weighted triangle inequality. In the setting of $b$-metric space, we should involve the $b$-metric constant " $s$ " in our analysis. That is why the $b$-comparison function was suggested by Berinde [10]. Indeed, the idea is so simple. In order to investigate fixed point results in the class of $b$-metric spaces, the notion of $c$-comparison function was extended to the $b$-comparison function by involving the $b$-metric constant " $s$ ".

In what follows, we remind readers about the formal definition of the $b$-comparison function:

Definition 4 ([10]). Let $s \geq 1$ be a real number. A mapping $\varphi:[0, \infty) \rightarrow[0, \infty)$ is called a b-comparison function if the following conditions are fulfilled:

(1) $\varphi$ is monotone increasing;

(2) there exist $k_{0} \in \mathbb{N}, a \in(0,1)$ and a convergent series of nonnegative terms $\sum_{k=1}^{\infty} v_{k}$ such that $s^{k+1} \varphi^{k+1}(t) \leq a s^{k} \varphi^{k}(t)+v_{k}$, for $k \geq k_{0}$ and any $t \in[0, \infty)$. 
Example 9. Let $s \geq 1$ be a real number and $\gamma:[0, \infty) \rightarrow[0, \infty)$ be a function such that

$$
\gamma(t)=c t \text { for all } t \in[0, \infty) \text { where } c \in\left(0, \frac{1}{s}\right) .
$$

Then, $\gamma$ forms a comparison function.

The following lemma is very important in the proof of our results.

Lemma 2 ([10]). If $\varphi:[0, \infty) \rightarrow[0, \infty)$ is a $b$-comparison function, then we have the following conclusions:

(1) the series $\sum_{k=0}^{\infty} s^{k} \varphi^{k}(t)$ converges for any $t \in[0, \infty)$;

(2) the function $S_{b}:[0, \infty) \rightarrow[0, \infty)$ defined by $S_{b}(t)=\sum_{k=0}^{\infty} s^{k} \varphi^{k}(t), t \in[0, \infty)$, is increasing and continuous at 0 .

Remark 2. Due to the Lemma 1.2., any b-comparison function is a comparison function.

Let $\alpha: X \times X \rightarrow[0, \infty)$ be a function. We say that a mapping $f: X \rightarrow X$ is $\alpha$-orbital admissible ([13]) if

$$
\alpha(x, f x) \geq 1 \Rightarrow \alpha\left(f x, f^{2}(x)\right) \geq 1 .
$$

An $\alpha$-orbital admissible mapping $f$ is called triangular $\alpha$-orbital admissible ([13]) if

$$
\alpha(x, y) \geq 1 \text { and } \alpha(y, f y) \geq 1 \Rightarrow \alpha(x, f y) \geq 1 \text {, for every } x, y \in X
$$

Lemma 3. Let $(X, d)$ be a b-metric space with constant $s \geq 1$, and let $f: X \rightarrow X$ be triangular $\alpha$-orbital admissible mapping having the property that there exists $x_{0} \in X$ such that $\alpha\left(x_{0}, f\left(x_{0}\right)\right) \geq 1$. Then,

$$
\alpha\left(x_{n}, x_{m}\right) \geq 1, \quad \text { for all } n, m \in \mathbb{N},
$$

where the sequence $\left(x_{n}\right)_{n \in \mathbb{N}}$ is defined by $x_{n+1}=f\left(x_{n}\right), n \in \mathbb{N}$.

Very recently, an interesting auxiliary function, to unify the different type contraction, was defined by Khojasteh [5] under the name of simulation function.

Definition 5 ([5]). A simulation function is a mapping $\zeta:[0, \infty) \times[0, \infty) \rightarrow \mathbb{R}$ satisfying the following conditions:

$\left(\zeta_{1}\right) \zeta(t, s)<s-t$ for all $t, s>0$;

$\left(\zeta_{2}\right)$ if $\left(t_{n}\right)_{n \in \mathbb{N}},\left(s_{n}\right)_{n \in \mathbb{N}}$ are sequences in $(0, \infty)$ such that $\lim _{n \rightarrow \infty} t_{n}=\lim _{n \rightarrow \infty} s_{n}>0$, then

$$
\limsup _{n \rightarrow \infty} \zeta\left(t_{n}, s_{n}\right)<0
$$

In the original definition, given in [5], there was an additional but a superfluous condition $\zeta(0,0)=0$. We underline the observation that a function $\zeta(t, s):=k s-t$, where $k \in[0,1)$ for all $s, t \in[0, \infty)$, is an instantaneous example of a simulation function. For further and more interesting examples, we refer e.g., [5,14-18] and relate references therein.

A self-mapping $f$, defined on a metric space $(X, d)$, is called a $\mathcal{Z}$-contraction with respect to $\zeta \in \mathcal{Z}[5]$, if

$$
\zeta(d(f x, f y), d(x, y)) \geq 0 \quad \text { for all } x, y \in X .
$$

The following is the main results of [5]: 
Theorem 1. Every $\mathcal{Z}$-contraction on a complete metric space has a unique fixed point.

As it is mentioned above, the immediate example $\zeta(t, s):=k s-t$ implies the outstanding Banach contraction mapping principle.

Definition 6 (cf. [7]). Let $(X, d)$ be a b-metric space with constant $s \geq 1$. A self-mapping $f$ is called an admissible hybrid contraction, if there exist $\varphi:[0, \infty) \rightarrow[0, \infty)$ a b-comparison function and $\alpha: X \times X \rightarrow$ $[0, \infty)$ such that

$$
\alpha(x, y) d(f x, f y) \leq \varphi\left(\mathcal{R}_{f}^{q}(x, y)\right)
$$

where $q \geq 0$ and $\lambda_{i} \geq 0, i=1,2,3,4,5$ such that $\sum_{i=1}^{5} \lambda_{i}=1$ and

$$
\mathcal{R}_{f}^{q} d(x, y)=\left\{\begin{aligned}
{[N(x, y)]^{1 / q}, } & \text { for } q>0, x, y \in X \\
P(x, y), & \text { for } q=0, x, y \in X
\end{aligned}\right.
$$

where

$$
\begin{aligned}
N(x, y):= & \lambda_{1} d^{q}(x, y)+\lambda_{2} d^{q}(x, f x)+\lambda_{3} d^{q}(y, f y) \\
& +\lambda_{4}\left(\frac{d(y, f y)(1+d(x, f x))}{1+d(x, y)}\right)^{q}+\lambda_{5}\left(\frac{d(y, f x)(1+d(x, f y))}{1+d(x, y)}\right)^{q},
\end{aligned}
$$

and

$$
\begin{aligned}
P(x, y):= & d^{\lambda_{1}}(x, y) \cdot d^{\lambda_{2}}(x, f x) \cdot d^{\lambda_{3}}(y, f y) \\
& \cdot\left(\frac{d(y, f y)(1+d(x, f x))}{1+d(x, y)}\right)^{\lambda_{4}} \cdot\left(\frac{d(x, f y)+d(y, f x)}{2 s}\right)^{\lambda_{5}} .
\end{aligned}
$$

Definition 7. Let $(X, d)$ be a b-metric space with constant $s \geq 1$. A mapping $f: X \rightarrow X$ is called admissible hybrid $\mathcal{Z}$-contraction mapping if there is $\varphi:[0, \infty) \rightarrow[0, \infty)$ a b-comparison function, $\alpha: X \times X \rightarrow[0, \infty)$ and $\zeta \in \mathcal{Z}$ such that

$$
\zeta\left(\alpha(x, y) d(f x, f y), \varphi\left(\mathcal{R}_{f}^{q}(x, y)\right)\right) \geq 0, \text { for all } x, y \in X
$$

where $\mathcal{R}_{f}^{q}(x, y)$ is as above.

\section{Existence and Uniqueness Results}

Theorem 2. Let $(X, d)$ be a complete b-metric space with constant $s \geq 1$ and let $f: X \rightarrow X$ be an admissible hybrid $\mathcal{Z}$-contraction. Suppose also that:

(i) $f$ is triangular $\alpha$-orbital admissible;

(ii) there exists $x_{0} \in X$ such that $\alpha\left(x_{0}, f\left(x_{0}\right)\right) \geq 1$;

(iii) either, $f$ is continuous or

(iv) $f^{2}$ is continuous and $\alpha(f x, x) \geq 1$ for any $x \in$ Fix $_{f^{2}}(X)$.

Then, $f$ has a fixed point.

Proof. Let $x_{0} \in X$ be an arbitrary point. Starting from here, we recursively construct the sequence $\left(x_{n}\right)_{n \in \mathbb{N}}$, as $x_{n}=f^{n}\left(x_{0}\right)$ for all $n \in \mathbb{N}$. Supposing that there exists some $m \in \mathbb{N}$ such that $f x_{m}=$ $x_{m+1}=x_{m}$, we find that $x_{m}$ is a fixed point of $f$ and the proof is finished. Thus, we can presume, from now on, that $x_{n} \neq x_{n-1}$ for any $n \in \mathbb{N}$. Under the assumption $(i), f$ is an admissible hybrid $\mathcal{Z}$-contraction, if we consider in (6) $x=x_{n-1}$ and $y=x_{n}$, we get

$$
\begin{aligned}
0 \leq & \zeta\left(\alpha\left(x_{n-1}, x_{n}\right) d\left(f\left(x_{n-1}\right), f\left(x_{n}\right)\right), \varphi\left(\mathcal{R}_{f}^{q}\left(x_{n-1}, x_{n}\right)\right)\right) \\
& <\varphi\left(\mathcal{R}_{f}^{q}\left(x_{n-1}, x_{n}\right)\right)-\alpha\left(x_{n-1}, x_{n}\right) d\left(f\left(x_{n-1}\right), f\left(x_{n}\right)\right),
\end{aligned}
$$


which is equivalent to

$$
\alpha\left(x_{n-1}, x_{n}\right) d\left(f\left(x_{n-1}\right), f\left(x_{n}\right)\right) \leq \varphi\left(\mathcal{R}_{f}^{q}\left(x_{n-1}, x_{n}\right)\right) .
$$

Taking into account that $f$ is triangular $\alpha$-orbital admissible, from (ii) and Lemma 1.3., we have $\alpha\left(x_{n-1}, x_{n}\right) \geq 1$. In this way, the above inequality becomes

$$
d\left(x_{n}, x_{n+1}\right) \leq \alpha\left(x_{n-1}, x_{n}\right) d\left(f\left(x_{n-1}\right), f\left(x_{n}\right)\right)<\varphi\left(\mathcal{R}_{f}^{q}\left(x_{n-1}, x_{n}\right)\right) .
$$

Case 1. For the case $q>0$, we have

$$
\begin{aligned}
\mathcal{R}_{f}^{q}\left(x_{n-1}, x_{n}\right)= & {\left[\lambda_{1} d^{q}\left(x_{n-1}, x_{n}\right)+\lambda_{2} d^{q}\left(x_{n-1}, f\left(x_{n-1}\right)\right)+\lambda_{3} d^{q}\left(x_{n}, f\left(x_{n}\right)\right)+\right.} \\
& \left.\quad+\lambda_{4}\left(\frac{d\left(x_{n}, f\left(x_{n}\right)\right)\left(1+d\left(x_{n-1}, f\left(x_{n-1}\right)\right)\right.}{1+d\left(x_{n-1}, x_{n}\right)}\right)^{q}+\lambda_{5}\left(\frac{d\left(x_{n}, f\left(x_{n-1}\right)\right)\left(1+d\left(x_{n-1}, f\left(x_{n}\right)\right)\right.}{1+d\left(x_{n-1}, x_{n}\right)}\right)^{q}\right]^{\frac{1}{q}} \\
= & {\left[\lambda_{1} d^{q}\left(x_{n-1}, x_{n}\right)+\lambda_{2} d^{q}\left(x_{n-1}, x_{n}\right)+\lambda_{3} d^{q}\left(x_{n}, x_{n+1}\right)+\right.} \\
& \left.\quad+\lambda_{4}\left(\frac{d\left(x_{n}, x_{n+1}\right)\left(1+d\left(x_{n-1}, x_{n}\right)\right)}{1+d\left(x_{n-1}, x_{n}\right)}\right)^{q}+\lambda_{5}\left(\frac{d\left(x_{n}, x_{n}\right)\left(1+d\left(x_{n-1}, x_{n+1}\right)\right)}{1+d\left(x_{n-1}, x_{n}\right)}\right)^{q}\right]^{\frac{1}{q}} \\
= & \left.\lambda_{1} d^{q}\left(x_{n-1}, x_{n}\right)+\lambda_{2} d^{q}\left(x_{n-1}, x_{n}\right)+\lambda_{3} d^{q}\left(x_{n}, x_{n+1}\right)+\lambda_{4}\left(d\left(x_{n}, x_{n+1}\right)\right)^{q}\right]^{\frac{1}{q}} \\
= & {\left[\left(\lambda_{1}+\lambda_{2}\right) d^{q}\left(x_{n-1}, x_{n}\right)+\left(\lambda_{3}+\lambda_{4}\right) d^{q}\left(x_{n}, x_{n+1}\right)\right]^{1 / q}, }
\end{aligned}
$$

and from (8) we get

$$
\begin{aligned}
d\left(x_{n}, x_{n+1}\right) & \leq \alpha\left(x_{n-1}, x_{n}\right) d\left(f\left(x_{n-1}\right), f\left(x_{n}\right)\right)<\varphi\left(\mathcal{R}_{f}^{q}\left(x_{n-1}, x_{n}\right)\right) \\
& =\varphi\left(\left[\left(\lambda_{1}+\lambda_{2}\right) d^{q}\left(x_{n-1}, x_{n}\right)+\left(\lambda_{3}+\lambda_{4}\right) d^{q}\left(x_{n}, x_{n+1}\right)\right]^{1 / q}\right) .
\end{aligned}
$$

Suppose that $d\left(x_{n-1}, x_{n}\right) \leq d\left(x_{n}, x_{n+1}\right)$. Since $\varphi$ is a nondecreasing function, Equation (9) can be estimated as follows:

$$
\begin{aligned}
d\left(x_{n}, x_{n+1}\right) & \leq \alpha\left(x_{n-1}, x_{n}\right) d\left(f\left(x_{n-1}\right), f\left(x_{n}\right)\right) \\
& \leq \varphi\left(\left[\left(\lambda_{1}+\lambda_{2}\right) d^{q}\left(x_{n-1}, x_{n}\right)+\left(\lambda_{3}+\lambda_{4}\right) d^{q}\left(x_{n}, x_{n+1}\right)\right]^{1 / q}\right) \\
& \text { due to assumption } d\left(x_{n-1}, x_{n}\right) \leq d\left(x_{n}, x_{n+1}\right) \text { we get } \\
& \left.\leq \varphi\left(\left[\lambda_{1}+\lambda_{2}+\lambda_{3}+\lambda_{4}\right) d^{q}\left(x_{n}, x_{n+1}\right)\right]^{1 / q}\right) \\
& \text { when we rearrange it, we get } \\
& =\varphi\left(\left(\lambda_{1}+\lambda_{2}+\lambda_{3}+\lambda_{4}\right)^{1 / q} d\left(x_{n}, x_{n+1}\right)\right) \\
& \text { on account of the fact that } \varphi(t)<t, \text { we find } \\
& <\left(\lambda_{1}+\lambda_{2}+\lambda_{3}+\lambda_{4}\right)^{1 / q} d\left(x_{n}, x_{n+1}\right) \\
& \text { since } \lambda_{1}+\lambda_{2}+\lambda_{3}+\lambda_{4} \leq 1, \text { we obtain } \\
& \leq d\left(x_{n}, x_{n+1}\right),
\end{aligned}
$$$$
\text { due to assumption } d\left(x_{n-1}, x_{n}\right) \leq d\left(x_{n}, x_{n+1}\right) \text { we get }
$$

which is a contradiction. Therefore, for every $n \in \mathbb{N}$, we have

$$
d\left(x_{n}, x_{n+1}\right)<d\left(x_{n-1}, x_{n}\right),
$$


in which case the inequality (8) yields

$$
\begin{aligned}
d\left(x_{n}, x_{n+1}\right) & \leq \varphi\left(\left[\left(\lambda_{1}+\lambda_{2}\right) d^{q}\left(x_{n-1}, x_{n}\right)+\left(\lambda_{3}+\lambda_{4}\right) d^{q}\left(x_{n}, x_{n+1}\right)\right]^{1 / q}\right) \\
& <\varphi\left(\left(\lambda_{1}+\lambda_{2}+\lambda_{3}+\lambda_{4}\right)^{1 / q} d\left(x_{n-1}, x_{n}\right)\right) \\
& \leq \varphi\left(d\left(x_{n-1}, x_{n}\right)\right) \leq \varphi^{2}\left(d\left(x_{n-2}, x_{n-1}\right)\right) \leq \ldots \leq \varphi^{n}\left(d\left(x_{0}, x_{1}\right)\right) .
\end{aligned}
$$

Now let $m, p \in \mathbb{N}$ such that $p>m$. Using the triangle inequality and (10), we have

$$
\begin{aligned}
d\left(x_{m}, x_{p}\right) & \leq s d\left(x_{m}, x_{m+1}\right)+s^{2} d\left(x_{m+1}, x_{m+2}\right)+\ldots+s^{p-m} \cdot d\left(x_{p-1}, x_{p}\right) \\
& \leq s \varphi^{m}\left(\left(d\left(x_{0}, x_{1}\right)\right)+s^{2} \varphi^{m+1}\left(d\left(x_{0}, x_{1}\right)\right)+\ldots+s^{p-m+1} \varphi^{p}\left(d\left(x_{0}, x_{1}\right)\right)\right. \\
& =\frac{1}{s^{m-1}}\left(s^{m} \varphi^{m}\left(\left(d\left(x_{0}, x_{1}\right)\right)+s^{m+1} \varphi^{m+1}\left(d\left(x_{0}, x_{1}\right)\right)+\ldots+s^{p} \varphi^{p}\left(d\left(x_{0}, x_{1}\right)\right)\right)\right. \\
& =\frac{1}{s^{m-1}} \sum_{j=m}^{p} s^{j} \varphi^{j}\left(\left(d\left(x_{0}, x_{1}\right)\right) .\right.
\end{aligned}
$$

Since $\varphi$ is a $b$-comparison function, the series $\sum_{j=0}^{\infty} \varphi^{j}\left(d\left(x_{0}, x_{1}\right)\right)$ is convergent. Denoting by $\mathcal{S}_{n}=$ $\sum_{j=0}^{n} \varphi^{j}\left(d\left(x_{0}, x_{1}\right)\right)$, the above inequality becomes

$$
d\left(x_{m}, x_{p}\right) \leq \frac{1}{s^{m-1}}\left(\mathcal{S}_{p-1}-\mathcal{S}_{m-1}\right)
$$

and as $m, p \rightarrow \infty$ we get

$$
d\left(x_{m}, x_{p}\right) \rightarrow 0,
$$

which tells us that $\left(x_{n}\right)_{n \in \mathbb{N}}$ is a Cauchy sequence on a complete $b$-metric space, so there exists $x^{*} \in X$ such that

$$
\lim _{n \rightarrow \infty} d\left(x_{n} x^{*}\right)=0 .
$$

We shall prove that $x^{*}$ is a fixed point of $f$. If $f$ is continuous, (due to assumption (iii))

$$
d\left(x^{*}, f\left(x^{*}\right)\right)=\lim _{n \rightarrow \infty} d\left(x_{n}, f\left(x_{n}\right)\right)=\lim _{n \rightarrow \infty} d\left(x_{n}, x_{n+1}\right)=0,
$$

so we get that $f\left(x^{*}\right)=x^{*}$, that is, $x^{*}$ is a fixed point of $f$.

Suppose now that $f^{2}$ is continuous. It follows that $f^{2}\left(x^{*}\right)=\lim _{n \rightarrow \infty} f^{2}\left(x_{n}\right)=x^{*}$. We shall prove that $f\left(x^{*}\right)=x^{*}$. Supposing that, on the contrary, $f\left(x^{*}\right) \neq x^{*}$, we have from (6)

$$
\begin{aligned}
0 & \leq \zeta\left(\alpha\left(f\left(x^{*}\right), x^{*}\right) d\left(f^{2}\left(x^{*}\right), f\left(x^{*}\right)\right), \varphi\left(\mathcal{R}_{f}^{q}\left(f\left(x^{*}\right), x^{*}\right)\right)\right) \\
& =\varphi\left(\mathcal{R}_{f}^{q}\left(f\left(x^{*}\right), x^{*}\right)\right)-\alpha\left(f\left(x^{*}\right), x^{*}\right) d\left(f^{2}\left(x^{*}\right), f\left(x^{*}\right)\right),
\end{aligned}
$$


which implies

$$
\begin{aligned}
d\left(x^{*}, f\left(x^{*}\right)\right) & =d\left(f^{2}\left(x^{*}\right), f\left(x^{*}\right)\right) \leq \alpha\left(f\left(x^{*}\right), x^{*}\right) d\left(f\left(x^{*}\right), x^{*}\right) \\
& \text { since } \varphi(t)<t, \text { we get } \\
& \leq \varphi\left(\mathcal{R}_{f}^{q}\left(f\left(x^{*}\right), x^{*}\right)\right)<\mathcal{R}_{f}^{q}\left(f\left(x^{*}\right), x^{*}\right) ; \text { due to (5), we have } \\
& =\left[\lambda_{1} d^{q}\left(f\left(x^{*}\right), x^{*}\right)+\lambda_{2} d^{q}\left(x^{*}, f\left(x^{*}\right)\right)+\lambda_{3} d^{q}\left(f\left(x^{*}\right), f^{2}\left(x^{*}\right)\right)+\right. \\
& \left.\lambda_{4}\left(\frac{d\left(x^{*}, f\left(x^{*}\right)\right)\left(1+d\left(f\left(x^{*}\right), f^{2}\left(x^{*}\right)\right)\right.}{1+d\left(x^{*}, f\left(x^{*}\right)\right)}\right)^{q}+\lambda_{5}\left(\frac{d\left(f\left(x^{*}\right), f\left(x^{*}\right)\right)\left(1+d\left(x^{*}, f^{2}\left(x^{*}\right)\right)\right.}{1+d\left(x^{*}, f\left(x^{*}\right)\right)}\right)^{q}\right]^{\frac{1}{q}} \\
& =\left[\lambda_{1} d^{q}\left(f\left(x^{*}\right), x^{*}\right)+\lambda_{2} d^{q}\left(x^{*}, f\left(x^{*}\right)\right)+\lambda_{3} d^{q}\left(f\left(x^{*}\right), x^{*}\right)+\right. \\
& \left.+\lambda_{4}\left(\frac{d\left(x^{*}, f\left(x^{*}\right)\right)\left(1+d\left(f\left(x^{*}\right), x^{*}\right)\right)}{1+d\left(x^{*}, f\left(x^{*}\right)\right)}\right)^{q}+\lambda_{5}\left(\frac{d\left(f\left(x^{*}\right), f\left(x^{*}\right)\right)\left(1+d\left(x^{*}, x^{*}\right)\right)}{1+d\left(x^{*}, f\left(x^{*}\right)\right)}\right)^{q}\right]^{\frac{1}{q}} \\
& =\left[\left(\lambda_{1}+\lambda_{2}+\lambda_{3}+\lambda_{4}\right) d^{q}\left(x^{*}, f\left(x^{*}\right)\right)\right]^{\frac{1}{q}} \\
& =\left[\left(\lambda_{1}+\lambda_{2}+\lambda_{3}+\lambda_{4}\right)\right]^{\frac{1}{q}} d\left(x^{*}, f\left(x^{*}\right)\right) \\
& \leq d\left(x^{*}, f\left(x^{*}\right)\right) .
\end{aligned}
$$

This is a contradiction, so that $f\left(x^{*}\right)=x^{*}$.

Case 2. For the case $q=0$, if we consider $x=x_{n-1}$ and $y=x_{n}$, we have

$$
\begin{aligned}
\mathcal{R}_{f}^{q}\left(x_{n-1}, x_{n}\right) & =d^{\lambda_{1}}\left(x_{n-1}, x_{n}\right) \cdot d^{\lambda_{2}}\left(x_{n-1}, f\left(x_{n-1}\right)\right) \cdot d^{\lambda_{3}}\left(x_{n}, f\left(x_{n}\right)\right) . \\
& \cdot\left[\frac{d\left(x_{n}, f\left(x_{n}\right)\right)\left(1+d\left(x_{n-1}, f x_{n-1}\right)\right)}{1+d\left(x_{n-1}, x_{n}\right)}\right]^{\lambda_{4}} \cdot\left[\frac{\left.d\left(x_{n-1}, f\left(x_{n}\right)\right)+d\left(x_{n}, f x_{n-1}\right)\right)}{2 s}\right]^{\lambda_{5}} \\
& =d^{\lambda_{1}}\left(x_{n-1}, x_{n}\right) \cdot d^{\lambda_{2}}\left(x_{n-1}, x_{n}\right) \cdot d^{\lambda_{3}}\left(x_{n}, x_{n+1}\right) \cdot \\
& \cdot\left[\frac{d\left(x_{n}, x_{n+1}\right)\left(1+d\left(x_{n-1}, x_{n}\right)\right)}{1+d\left(x_{n-1}, x_{n}\right)}\right]^{\lambda_{4}} \cdot\left[\frac{\left.d\left(x_{n-1}, x_{n+1}\right)+d\left(x_{n}, x_{n}\right)\right)}{2 s}\right]^{\lambda_{5}} \\
& =d^{\lambda_{1}}\left(x_{n-1}, x_{n}\right) \cdot d^{\lambda_{2}}\left(x_{n-1}, x_{n}\right) \cdot d^{\lambda_{3}}\left(x_{n}, x_{n+1}\right) \cdot d^{\lambda_{4}}\left(x_{n}, x_{n+1}\right) \cdot\left[\frac{d\left(x_{n-1}, x_{n+1}\right)}{2 s}\right]^{\lambda_{5}} .
\end{aligned}
$$

Employing the triangle inequality, we have

$$
\begin{array}{r}
\mathcal{R}_{f}^{q}\left(x_{n-1}, x_{n}\right) \leq d^{\lambda_{1}}\left(x_{n-1}, x_{n}\right) \cdot d^{\lambda_{2}}\left(x_{n-1}, x_{n}\right) \cdot d^{\lambda_{3}}\left(x_{n}, x_{n+1}\right) \cdot d^{\lambda_{4}}\left(x_{n}, x_{n+1}\right) \\
\cdot\left[\frac{d\left(x_{n-1}, x_{n}\right)+d\left(x_{n}, x_{n+1}\right)}{2}\right]^{\lambda_{5}} .
\end{array}
$$

Using the following inequality

$$
\left(\frac{a+b}{2}\right)^{k} \leq \frac{a^{k}+b^{k}}{2}, \text { for all } a, b, k>0,
$$

(13) becomes

$$
\begin{aligned}
\mathcal{R}_{f}^{q}\left(x_{n-1}, x_{n}\right) & \leq d^{\lambda_{1}}\left(x_{n-1}, x_{n}\right) \cdot d^{\lambda_{2}}\left(x_{n-1}, x_{n}\right) \cdot d^{\lambda_{3}}\left(x_{n}, x_{n+1}\right) \\
& \cdot d^{\lambda_{4}}\left(x_{n}, x_{n+1}\right) \cdot \frac{d^{\lambda_{5}}\left(x_{n-1}, x_{n}\right)+d^{\lambda_{5}}\left(x_{n}, x_{n+1}\right)}{2},
\end{aligned}
$$

and, from (6),

$$
\begin{aligned}
0 \leq & \zeta\left(\alpha\left(x_{n-1}, x_{n}\right) d\left(f\left(x_{n-1}\right), f\left(x_{n}\right)\right), \varphi\left(\mathcal{R}_{f}^{q}\left(x_{n-1}, x_{n}\right)\right)\right) \\
& <\varphi\left(\mathcal{R}_{f}^{q}\left(x_{n-1}, x_{n}\right)\right)-\alpha\left(x_{n-1}, x_{n}\right) d\left(f\left(x_{n-1}\right), f\left(x_{n}\right)\right),
\end{aligned}
$$

which yields that

$$
d\left(x_{n}, x_{n+1}\right) \leq \alpha\left(x_{n-1}, x_{n}\right) d\left(f\left(x_{n-1}\right), f\left(x_{n}\right)\right) \leq \varphi\left(\mathcal{R}_{f}^{q}\left(x_{n-1}, x_{n}\right)\right) .
$$


Supposing that $d\left(x_{n-1}, x_{n}\right) \leq d\left(x_{n}, x_{n+1}\right)$, since $\varphi$ is a nondecreasing function, we have

$$
d\left(x_{n}, x_{n+1}\right)<d^{\lambda_{1}+\lambda_{2}+\lambda_{3}+\lambda_{4}+\lambda_{5}}\left(x_{n}, x_{n+1}\right)=d\left(x_{n}, x_{n+1}\right),
$$

which is a contradiction. Then, from (14), inductively, we obtain

$$
d\left(x_{n}, x_{n+1}\right) \leq \varphi\left(\mathcal{R}_{f}^{q}\left(x_{n-1}, x_{n}\right)\right)<\varphi^{n}\left(d\left(x_{0}, x_{1}\right)\right) .
$$

By using the same arguments as the case $q>0$, we shall easily obtain that $\left(x_{n}\right)_{n \in \mathbb{N}}$ is a Cauchy sequence in a complete metric space and thus there exists $x^{*}$ such that $\lim _{n \rightarrow \infty} x_{n}=x^{*}$.

We claim that $x^{*}$ is a fixed point of $f$.

Under the assumption that $f$ is continuous, we have

$$
d\left(x^{*}, f\left(x^{*}\right)\right)=\lim _{n \rightarrow \infty} d\left(x_{n}, f\left(x_{n}\right)\right)=\lim _{n \rightarrow \infty} d\left(x_{n}, x_{n+1}\right)=0,
$$

and together with the uniqueness of limit, $f\left(x^{*}\right)=x^{*}$. In addition, if $f^{2}$ is continuous, as in case $\mathbf{1}$, we have that $f^{2}\left(x^{*}\right)=x^{*}$ and suppose that $f\left(x^{*}\right) \neq x^{*}$. Then, we get

$$
\begin{aligned}
0 & \leq \zeta\left(\alpha\left(f\left(x^{*}\right), x^{*}\right) d\left(f^{2}\left(x^{*}\right), f\left(x^{*}\right)\right), \varphi\left(\mathcal{R}_{f}^{q}\left(f^{2}\left(x^{*}\right), f\left(x^{*}\right)\right)\right)\right. \\
& =\varphi\left(\mathcal{R}_{f}^{q}\left(f^{2}\left(x^{*}\right), f\left(x^{*}\right)\right)\right)-\alpha\left(f\left(x^{*}\right), x^{*}\right) d\left(f^{2}\left(x^{*}\right), f\left(x^{*}\right)\right),
\end{aligned}
$$

which implies

$$
\begin{aligned}
d\left(x^{*}, f\left(x^{*}\right)\right) & =d\left(f^{2}\left(x^{*}\right), f\left(x^{*}\right)\right) \\
& \leq \alpha\left(f\left(x^{*}\right), x^{*}\right) d\left(f^{2}\left(x^{*}\right), f\left(x^{*}\right)\right) \\
& \leq \varphi\left(\mathcal{R}_{f}^{q}\left(f^{2}\left(x^{*}\right), f\left(x^{*}\right)\right)=\varphi\left(\mathcal{R}_{f}^{q}\left(x^{*}, f\left(x^{*}\right)\right),\right.\right.
\end{aligned}
$$

where

$$
\begin{aligned}
\mathcal{R}_{f}^{q}\left(x^{*}, f\left(x^{*}\right)\right) & =d^{\lambda_{1}+\lambda_{2}+\lambda_{3}}\left(x^{*}, f\left(x^{*}\right)\right) \cdot\left[\frac{d\left(x^{*}, f\left(x^{*}\right)\right)\left(1+d\left(x^{*}, f\left(x^{*}\right)\right)\right.}{1+d\left(x^{*}, f\left(x^{*}\right)\right)}\right]^{\lambda_{4}} \cdot\left[\frac{d\left(x^{*}, x^{*}\right)+d\left(f\left(x^{*}\right), f\left(x^{*}\right)\right)}{2 s}\right]^{\lambda_{5}} \\
& =d^{\lambda_{1}+\lambda_{2}+\lambda_{3}+\lambda_{4}}\left(x^{*}, f\left(x^{*}\right)\right)<d\left(x^{*}, f\left(x^{*}\right)\right) .
\end{aligned}
$$

Hence, we have

$$
d\left(x^{*}, f\left(x^{*}\right)\right) \leq \varphi\left(\mathcal{R}_{f}^{q}\left(x^{*}, f\left(x^{*}\right)\right)<\varphi\left(d\left(x^{*}, f\left(x^{*}\right)\right)<d\left(x^{*}, f\left(x^{*}\right)\right),\right.\right.
$$

which is a contradiction.

Theorem 3. In the hypothesis of Theorem 2, if we assume supplementary that

$$
\alpha\left(x^{*}, y^{*}\right) \geq 1,
$$

for any $x^{*}, y^{*} \in$ Fix $_{f}(X)$, then the fixed point of $f$ is unique.

Proof. Let $y^{*} \in X$ be another fixed point of $f$. Suppose that $x^{*} \neq y^{*}$. In the case that $q>0$, using (6), we have:

$$
\begin{aligned}
0 & \leq \zeta\left(\alpha\left(x^{*}, y^{*}\right) d\left(f\left(x^{*}\right), f\left(y^{*}\right)\right), \varphi\left(\mathcal{R}_{f}^{q}\left(x^{*}, y^{*}\right)\right)\right) \\
& <\varphi\left(\mathcal{R}_{f}^{q}\left(x^{*}, y^{*}\right)\right)-\alpha\left(x^{*}, y^{*}\right) d\left(f\left(x^{*}\right), f\left(y^{*}\right)\right),
\end{aligned}
$$


which yields that

$$
\begin{aligned}
d\left(x^{*}, y^{*}\right) & \leq \alpha\left(x^{*}, y^{*}\right) d\left(f\left(x^{*}\right), f\left(y^{*}\right)\right) \leq \varphi\left(\mathcal{R}_{f}^{q}\left(x^{*}, y^{*}\right)\right)<\mathcal{R}_{f}^{q}\left(x^{*}, y^{*}\right) \\
& =\left[\lambda_{1} d\left(x^{*}, y^{*}\right)+\lambda_{2} d^{q}\left(x^{*}, f\left(x^{*}\right)\right)+\lambda_{3} d^{q}\left(y^{*}, f\left(y^{*}\right)\right)+\lambda_{4}\left(\frac{d\left(y^{*}, f\left(y^{*}\right)\right)\left(1+d\left(x^{*}, f\left(x^{*}\right)\right)\right.}{1+d\left(x^{*}, y^{*}\right)}\right)^{q}+\right. \\
& \left.\lambda_{5}\left(\frac{d\left(y^{*}, f\left(x^{*}\right)\right)\left(1+d\left(x^{*}, f\left(y^{*}\right)\right)\right.}{1+d\left(x^{*}, y^{*}\right)}\right)^{q}\right]^{\frac{1}{q}} \\
& =\left(\lambda_{1}+\lambda_{5}\right)^{\frac{1}{q}} d\left(x^{*}, y^{*}\right)<d\left(x^{*}, y^{*}\right),
\end{aligned}
$$

which is a contradiction.

In the case that $q=0$, if we suppose that $x^{*} \neq y^{*}$, then we obtain that $0<d\left(x^{*}, y^{*}\right)<0$, which is a contradiction.

Thus, $x^{*}=y^{*}$, so that $f$ possesses exactly one fixed point.

Example 10. Let $X=[0,2], d: X \times X \rightarrow[0, \infty), d(x, y)=|x-y|^{2}$ for all $x, y \in X$. Consider that the mapping $f: X \rightarrow X$ is defined by $f(x)=\left\{\begin{array}{ll}1 / 2, & \text { if } x \in[0,1] \\ x / 2, & \text { if } x \in(1,2]\end{array}\right.$ and the function $\alpha(x, y)=$ $\begin{cases}2, & \text { if } x, y \in[0,1], \\ 1, & \text { if } x=0, y=2 \text { and the b-comparison function } \varphi:[0, \infty) \rightarrow[0, \infty), \varphi(t)=\frac{t}{2}, \zeta(t, s)=\frac{1}{2} s-t, \\ 0, & \text { otherwise. }\end{cases}$

We can easily observe that:

1. $(X, d)$ is a complete b-metric space with the constant $s=2$;

2. $f$ triangular $\alpha$-orbital admissible;

3. for $x_{0} \in[0,1], f\left(x_{0}\right)=\frac{1}{2} \in[0,1]$ and hence $\alpha\left(x_{0}, f\left(x_{0}\right)\right)=2>1$;

4. $f$ is continuous;

5. $f^{2}(x)=\frac{1}{2}$ is continuous. Moreover, for $x=\frac{1}{2} \in$ Fix $_{f^{2}}(X)$, we have $\alpha\left(f\left(\frac{1}{2}\right) \cdot \frac{1}{2}\right)=\alpha\left(\frac{1}{2}, \frac{1}{2}\right)=2>1$;

6. $\quad \zeta\left(\alpha(x, y) d(f x, f y), \varphi\left(\mathcal{R}_{f}^{q}(x, y)\right)\right) \geq 0$.

If $x, y \in[0,1]$, then $f x=f y=\frac{1}{2}$ and hence $d(f x, f y)=0$. We have

$$
\zeta\left(0, \varphi\left(\mathcal{R}_{f}^{q}(x, y)\right)\right)=\frac{1}{2} \varphi\left(\mathcal{R}_{f}^{q}(x, y)\right) \geq 0, \text { for all } x, y \in[0,1]
$$

and hence

$$
\zeta\left(\alpha(x, y) d(f x, f y), \varphi\left(\mathcal{R}_{f}^{q}(x, y)\right)\right) \geq 0, \text { for all } x, y \in[0,1] .
$$

If $x=0$ and $y=2$, then if we consider $q=2, \lambda_{1}=\lambda_{2}=\lambda_{3}=\lambda_{4}=\lambda_{5}=\frac{1}{5}$, then we have

$$
\begin{aligned}
\zeta\left(\alpha(0,2) d(f(0), f(2)), \varphi\left(\mathcal{R}_{f}^{q}(0,2)\right)\right) & =\frac{1}{2} \varphi\left(\mathcal{R}_{f}^{q}(0,2)\right)-\alpha(0,2) d(f(0), f(2))=<= \\
& =\frac{1}{4}\left[\frac{1}{5} d^{2}(0,2)+\frac{1}{5} d^{2}(0, f(0))+\frac{1}{5} d^{2}(2, f(2))+\right. \\
& \left.+\frac{1}{5}\left(\frac{d(2, f(2))(1+d(0, f(0)))}{1+d(0,2)}\right)^{2}+\frac{1}{5}\left(\frac{d(2, f(0))(1+d(0, f(2)))}{1+d(0,2)}\right)^{2}\right]^{\frac{1}{2}} \\
& -\alpha(0,2) d\left(\frac{1}{2}, 1\right) \\
& =\frac{1}{4}\left[\frac{1}{5}\left(16+\frac{1}{16}+1+\frac{1}{16}+\frac{81}{100}\right)\right]^{\frac{1}{2}} \\
& =\frac{1}{4}\left(\frac{3587}{1000}\right)^{\frac{1}{2}}-\frac{1}{4} \geq 0 .
\end{aligned}
$$

Hence,

$$
\zeta\left(\alpha(0,2) d(f(0), f(2)), \varphi\left(\mathcal{R}_{f}^{q}(0,2)\right)\right) \geq 0 .
$$


In all other cases, $\alpha(x, y)=0$ and

$$
\zeta\left(0, \varphi\left(\mathcal{R}_{f}^{q}(x, y)\right)\right)=\frac{1}{2} \varphi\left(\mathcal{R}_{f}^{q}(x, y)\right) \geq 0
$$

Thus, we obtain that $f$ is an admissible hybrid $\mathcal{Z}$-contraction which satisfies the assumptions of Theorem 2 and then $x=\frac{1}{2}$ is the fixed point of $f$.

Remark 3. If, in the above example, we consider $f(x)=\left\{\begin{array}{ll}1 / 3, & \text { if } x \in[0,1] \\ x / 2, & \text { if } x \in(1,2]\end{array}\right.$, then $f$ is not continuous, but $f^{2}(x)=\frac{1}{3}$ and for $x=\frac{1}{3} \in$ Fix $_{f^{2}}(X)$, we have $\alpha\left(f\left(\frac{1}{3}\right) \cdot \frac{1}{3}\right)=\alpha\left(\frac{1}{3}, \frac{1}{3}\right)=2>1$.

Let $\Phi$ be the collection of all auxiliary functions $\phi:[0, \infty) \rightarrow[0, \infty)$ which are continuous and $\phi(t)=0$ if and only if $t=0$.

Theorem 4. Let $(X, d)$ be a complete $b$-metric space with constant $s \geq 1, f: X \rightarrow X$ and $\alpha: X \times X \rightarrow[0, \infty)$. Suppose that there exist two functions $\phi_{1}, \phi_{2} \in \Phi$, with $\phi_{1}(t)<t \leq \phi_{2}(t)$, for all $t>0$, such that

$$
\phi_{2}(\alpha(x, y) d(f x, f y)) \leq \phi_{1}\left(\mathcal{R}_{f}^{q}(x, y)\right)
$$

Furthermore, we suppose that:

(i) $f$ is triangular $\alpha$-orbital admissible;

(ii) there exists $x_{0} \in X$ such that $\alpha\left(x_{0}, f\left(x_{0}\right)\right) \geq 1$;

(iii) either, $f$ is continuous or

(iv) $f^{2}$ is continuous and $\alpha(f x, x) \geq 1$ for any $x \in \operatorname{Fix}_{f^{2}}(X)$

(v) if $x^{*}, y^{*} \in \operatorname{Fix}_{f}(X)$, then $\alpha\left(x^{*}, y^{*}\right) \geq 1$.

Then, $f$ has a unique fixed point.

Proof. Let $\zeta(t, s)=\phi_{1}(s)-\phi_{2}(t)$. According to Example 10, if $\phi_{1}, \phi_{2} \in \Phi$ have the property $\phi_{1}(t)<$ $t \leq \phi_{2}(t)$ for all $t>0$, then $\zeta \in \mathcal{Z}$. Thus, the desired results follow from Theorems 2 and 3 .

Theorem 5. Let $(X, d)$ be a complete $b$-metric space with constant $s \geq 1, f: X \rightarrow X$ and $\alpha: X \times X \rightarrow[0, \infty)$. Suppose that there exists a function $\phi \in \Phi$, such that

$$
\alpha(x, y) d(f x, f y) \leq \mathcal{R}_{f}^{q}(x, y)-\phi\left(\mathcal{R}_{f}^{q}(x, y)\right)
$$

Furthermore, we suppose that

(i) $f$ is triangular $\alpha$-orbital admissible;

(ii) there exists $x_{0} \in X$ such that $\alpha\left(x_{0}, f\left(x_{0}\right)\right) \geq 1$;

(iii) either, $f$ is continuous or

(iv) $f^{2}$ is continuous and $\alpha(f x, x) \geq 1$ for any $x \in$ Fix $_{f^{2}}(X)$.

(v) if $x^{*}, y^{*} \in \operatorname{Fix}_{f}(X)$, then $\alpha\left(x^{*}, y^{*}\right) \geq 1$.

Then, $f$ has a unique fixed point.

Proof. Let $\zeta(t, s)=s-\phi(s))-t$. According to Example $10, \zeta \in \mathcal{Z}$. Thus, the desired results follow from Theorems 2 and 3 . 
Theorem 6. Let $(X, d)$ be a complete b-metric space with constant $s \geq 1, f: X \rightarrow X$ and $\alpha: X \times X \rightarrow[0, \infty)$. Suppose that there exists a function $\mu:[0, \infty) \rightarrow[0, \infty)$ such that $\int_{0}^{\varepsilon} \mu(u) d u$ exists and $\int_{0}^{\varepsilon} \mu(u) d u>\varepsilon$, for each $\varepsilon>0$, with the property that

$$
\alpha(x, y) d(f x, f y) \leq \int_{0}^{\mathcal{R}_{f}^{q}(x, y)} \mu(u) d u .
$$

Furthermore, we suppose that

(i) $f$ is triangular $\alpha$-orbital admissible;

(ii) there exists $x_{0} \in X$ such that $\alpha\left(x_{0}, f\left(x_{0}\right)\right) \geq 1$;

(iii) either, $f$ is continuous or

(iv) $f^{2}$ is continuous and $\alpha(f x, x) \geq 1$ for any $x \in$ Fix $_{f^{2}}(X)$.

(v) if $x^{*}, y^{*} \in \operatorname{Fix}_{f}(X)$, then $\alpha\left(x^{*}, y^{*}\right) \geq 1$.

Then, $f$ has a unique fixed point.

Proof. Let $\zeta(t, s)=s-\int_{0}^{t} \mu(u) d u$. According to Example 10, $\zeta \in \mathcal{Z}$. Thus, the desired results follow from Theorems 2 and 3.

Let $\Phi$ be the class of auxiliary functions $\phi:[0, \infty) \rightarrow[0, \infty)$ that are continuous functions and $\mu(t)=0$ if and only if, $t=0$.

The following example is derived from $[5,14,15]$.

Example 11. (See, e.g., $[5,14,15])$ Let $\phi_{i} \in \Phi$ for $i=1,2,3$ and $\sigma_{j}: \mathbb{R}_{0}^{+} \times \mathbb{R}_{0}^{+} \rightarrow \mathbb{R}$ for $j=1,2,3,4,5,6$. Each of the functions defined below is an example of simulation functions:

(E1) $\sigma_{2}(t, s)=s-\phi_{3}(s)-t$ for all $t, s \geq 0$.

(E2) $\sigma_{4}(t, s)=f(s)-t$ for all $t, s \geq 0, t, s \geq 0$, where the function $f:[0, \infty) \rightarrow[0, \infty)$ is upper semi-continuous and such that $f(t)<t$ for all $t>0$ and $f(0)=0$.

(E3) $\sigma_{5}(t, s)=s-\frac{g(t, s)}{h(t, s)}$ for all $t, s \geq 0$, where $g, h:[0, \infty)^{2} \rightarrow(0, \infty)$ are two continuous functions with respect to each variable such that $g(t, s)>h(t, s)$ for all $t, s>0$.

(E4) $\sigma_{6}(t, s)=s \eta(s)-t$ for all $t, s \geq 0$, where $\eta:[0, \infty) \rightarrow[0,1)$ is a function with the property $\lim \sup _{t \rightarrow r^{+}} \eta(t)<1$ for all $r>0$

Remark 4. By using the examples above, we may derive more consequences of our results.

\section{Well Posedness and Ulam-Hyers Stability}

Considered as a type of data dependence, the notion of Ulam stability was started by Ulam $[19,20]$ and developed by Hyers [21], Rassias [22], etc. In this section, we investigate the general Ulam type stability in sense of a fixed point problem as well the well posedness of the fixed point problem.

Suppose that $f: X \rightarrow X$ is a self-mapping on a $b$-metric space $(X, d)$ with the constant $s>1$ and let us consider the following fixed point problem:

$$
x=f(x) .
$$

Definition 8. The fixed point problem (19) is well-posed if

(i) Fix $_{f}(X)=\left\{x^{*}\right\}$;

(ii) If $\left(x_{n}\right)_{n \in \mathbb{N}}$ is a sequence such that $d\left(x_{n}, f\left(x_{n}\right)\right) \rightarrow 0$, as $n \rightarrow \infty$, then $x_{n} \rightarrow x^{*}$, as $n \rightarrow \infty$. 
Theorem 7. Let $(X, d)$ be a complete b-metric space with constant $s>1$. Suppose that all the hypotheses of Theorem 3 hold, and $q>0$. Additionally, we suppose that for any sequence $\left(x_{n}\right)_{n \in \mathbb{N}}$, with $d\left(x_{n}, f\left(x_{n}\right)\right) \rightarrow 0$, as $n \rightarrow \infty$, we have $\alpha\left(x_{n}, x^{*}\right) \geq 1$, for all $n \in \mathbb{N}$, where $x^{*} \in$ Fix $f(X)$. If $\lambda_{1}+\lambda_{5}<\frac{1}{\gamma^{2}(q)}$, where $\gamma(q)=\max \left\{1,2^{q-1} s^{q}\right\}$, then the fixed point problem (19) is well-posed.

Proof. Taking into account the supplementary condition, since Fix $f(X)=x^{*}, u \sin g(6)$, we have

$$
\begin{aligned}
0 & \leq \zeta\left(\alpha\left(x_{n}, x^{*}\right) d\left(f\left(x_{n}\right), f\left(x^{*}\right)\right), \varphi\left(\mathcal{R}_{f}^{q}\left(x_{n}, x^{*}\right)\right)\right) \\
& <\varphi\left(\mathcal{R}_{f}^{q}\left(x_{n}, x^{*}\right)\right)-\alpha\left(x_{n}, x^{*}\right) d\left(f\left(x_{n}\right), f\left(x^{*}\right)\right) .
\end{aligned}
$$

We have

$$
\begin{aligned}
d\left(x_{n}, x^{*}\right) \leq & s d\left(x_{n}, f\left(x_{n}\right)\right)+s d\left(f\left(x_{n}\right), f\left(x^{*}\right)\right) \leq s d\left(x_{n}, f\left(x_{n}\right)\right)+s \alpha\left(x_{n}, x^{*}\right) d\left(f\left(x_{n}\right), f\left(x^{*}\right)\right) \\
\leq & s d\left(x_{n}, f\left(x_{n}\right)\right)+s \varphi\left(\mathcal{R}_{f}^{q}\left(x_{n}, x^{*}\right)\right)<s d\left(x_{n}, f\left(x_{n}\right)\right)+s \mathcal{R}_{f}^{q}\left(x_{n}, x^{*}\right) \\
\leq & s\left[\lambda_{1} d^{q}\left(x_{n}, x^{*}\right)+\lambda_{2} d^{q}\left(x_{n}, f\left(x_{n}\right)\right)+\lambda_{3} d^{q}\left(x^{*}, f\left(x^{*}\right)\right)+\lambda_{4}\left(\frac{d\left(x^{*}, f\left(x^{*}\right)\right)\left(1+d\left(x_{n}, f\left(x_{n}\right)\right)\right)}{1+d\left(x_{n}, x^{*}\right)}\right)^{q}+\right. \\
& \left.\quad+\lambda_{5}\left(\frac{d\left(x^{*}, f\left(x_{n}\right)\right)\left(1+d\left(x_{n}, f\left(x^{*}\right)\right)\right)}{1+d\left(x_{n}, x^{*}\right)}\right)^{q}\right]^{\frac{1}{q}}+s d\left(x_{n}, f\left(x_{n}\right)\right) \\
= & s\left[\lambda_{1} d^{q}\left(x_{n}, x^{*}\right)+\lambda_{2} d^{q}\left(x_{n}, f\left(x_{n}\right)\right)+\lambda_{5} d^{q}\left(x^{*}, f\left(x_{n}\right)\right]^{\frac{1}{q}}+s d\left(x_{n}, f\left(x_{n}\right)\right)\right. \\
\leq & s\left[\lambda_{1} d^{q}\left(x_{n}, x^{*}\right)+\lambda_{2} d^{q}\left(x_{n}, f\left(x_{n}\right)\right)+s^{q} \lambda_{5}\left(d\left(x^{*}, x_{n}\right)+d\left(x_{n}, f\left(x_{n}\right)\right)^{q}\right]^{\frac{1}{q}}+s d\left(x_{n}, f\left(x_{n}\right)\right)\right. \\
\leq & s\left[\lambda_{1} d^{q}\left(x_{n}, x^{*}\right)+\lambda_{2} d^{q}\left(x_{n}, f\left(x_{n}\right)\right)+2^{q-1} s^{q} \lambda_{5} d^{q}\left(x^{*}, x_{n}\right)+2^{q-1} s^{q} \lambda_{5} d^{q}\left(x_{n}, f\left(x_{n}\right)\right]^{\frac{1}{q}}+\right. \\
+ & s d\left(x_{n}, f\left(x_{n}\right)\right) .
\end{aligned}
$$

In this way, we obtain

$$
\begin{aligned}
d^{q}\left(x_{n}, x^{*}\right) & \leq 2^{q-1} s^{q} \lambda_{1} d^{q}\left(x_{n}, x^{*}\right)+2^{q-1} s^{q} \lambda_{2} d^{q}\left(x_{n}, f\left(x_{n}\right)\right)+2^{2 q-2} s^{2 q} \lambda_{5} d^{q}\left(x^{*}, x_{n}\right)+ \\
& +2^{2 q-2} s^{2 q} \lambda_{5} d^{q}\left(x_{n}, f\left(x_{n}\right)\right)+2^{q-1} s^{q} d^{q}\left(x_{n}, f\left(x_{n}\right)\right)
\end{aligned}
$$

or

$$
\left(1-2^{q-1} s^{q} \lambda_{1}-2^{2 q-2} s^{2 q} \lambda_{5}\right) d^{q}\left(x_{n}, x^{*}\right) \leq 2^{q-1} s^{q}\left(1+\lambda_{2}+2^{q-1} s^{q} \lambda_{5}\right)^{q} d^{q}\left(x_{n}, f\left(x_{n}\right)\right) .
$$

From here, we obtain

$$
d^{q}\left(x_{n}, x^{*}\right) \leq \frac{\left(1+\lambda_{2}+\gamma(q) \lambda_{5}\right) \gamma(q)}{1-\gamma(q) \lambda_{1}-\gamma^{2}(q) \lambda_{5}} d^{q}\left(x_{n}, f\left(x_{n}\right)\right) .
$$

Letting $n \rightarrow \infty$ in the above inequality and keeping in mind that $\lim _{n \rightarrow \infty} d\left(x_{n}, f\left(x_{n}\right)\right)=0$, we obtain

$$
\lim _{n \rightarrow \infty} d\left(x_{n}, x^{*}\right)=0
$$

that is, the fixed point Equation (19) is well-posed.

Definition 9. The fixed point problem (19) is called generalized Ulam-Hyers stable if and only if there exists $\rho:[0, \infty) \rightarrow[0, \infty)$ is increasing, continuous in 0 and $\rho(0)=0$, such that for each $\varepsilon>0$ and for each $y^{*} \in X$, which satisfy the inequality

$$
d(y, f(y)) \leq \varepsilon,
$$

there exists a solution $x^{*}$ of the fixed point problem (19) such that

$$
d\left(y^{*}, x^{*}\right) \leq \rho(\varepsilon) .
$$


If there exists $c>0$ such that $\rho(t):=c \cdot t$, for each $t \in \mathbb{R}_{+}$, then the fixed point problem (19) is said to be Ulam-Hyers stable.

Before stating our theorem, we underline that Ulam-Hyers stability can be potentially applicable to the study of switched dynamics, see e.g., [23], and the related references therein.

Theorem 8. Let $(X, d)$ be a complete b-metric space with constant $s>1$. Suppose that all the hypotheses of Theorem 3 hold, and $q>0$. Additionally, we suppose that $\alpha\left(y^{*}, x^{*}\right) \geq 1$, for all $y^{*} \in X$ verifying (20) and $x^{*} \in$ Fix $_{f}(X)$. If $\lambda_{1}+\lambda_{5}<\frac{1}{\gamma^{2}(q)}$, where $\gamma(q)=\max \left\{1,2^{q-1} s^{q}\right\}$, then the fixed point problem (19) is Ulam-Hyers stable.

Proof. Using (6),

$$
\begin{aligned}
0 & \leq \zeta\left(\alpha\left(y^{*}, x^{*}\right) d\left(f\left(y^{*}\right), f\left(x^{*}\right)\right), \varphi\left(\mathcal{R}_{f}^{q}\left(y^{*}, x^{*}\right)\right)\right) \\
& <\varphi\left(\mathcal{R}_{f}^{q}\left(y^{*}, x^{*}\right)\right)-\alpha\left(y^{*}, x^{*}\right) d\left(f\left(y^{*}\right), f\left(x^{*}\right)\right) \\
d\left(y^{*}, x^{*}\right)=d\left(y^{*}, f\left(x^{*}\right)\right) \leq & s d\left(f\left(y^{*}\right), f\left(x^{*}\right)\right)+s d\left(y^{*}, f\left(y^{*}\right)\right) \\
\leq & s \alpha\left(y^{*}, x^{*}\right) d\left(f\left(y^{*}\right), f\left(x^{*}\right)\right)+s d\left(y^{*}, f\left(y^{*}\right)\right) \\
\leq & \left.s \varphi\left(\mathcal{R}_{f}^{q}\left(y^{*}, x^{*}\right)\right)+s \varepsilon<s \mathcal{R}_{f}^{q}\left(y^{*}, x^{*}\right)\right)+s \varepsilon \\
\leq & s\left[\lambda_{1} d^{q}\left(y^{*}, x^{*}\right)+\lambda_{2} d^{q}\left(y^{*}, f\left(y^{*}\right)\right)+\lambda_{3} d^{q}\left(x^{*}, f\left(x^{*}\right)\right)+\right. \\
& \left.\lambda_{4}\left(\frac{d\left(x^{*}, f\left(x^{*}\right)\right)\left(1+d\left(x^{*}, f\left(x^{*}\right)\right)\right.}{1+d\left(y^{*}, x^{*}\right)}\right)^{q}+\lambda_{5}\left(\frac{d\left(x^{*}, f\left(y^{*}\right)\right)\left(1+d\left(y^{*}, f\left(x^{*}\right)\right)\right)}{1+d\left(y^{*}, x^{*}\right)}\right)^{q}\right]^{\frac{1}{q}}+s \varepsilon \\
= & s\left[\lambda_{1} d^{q}\left(y^{*}, x^{*}\right)+\lambda_{2} \varepsilon^{q}+\lambda_{5} d^{q}\left(x^{*}, f\left(y^{*}\right)\right]^{\frac{1}{q}}+s \varepsilon\right. \\
\leq & s\left[\lambda_{1} d^{q}\left(y^{*}, x^{*}\right)+\lambda_{2} \varepsilon^{q}+s^{q} \lambda_{5}\left(d\left(y^{*}, x^{*}\right)+d\left(y^{*}, f\left(y^{*}\right)\right)^{q}\right]^{\frac{1}{q}}+s \varepsilon\right. \\
\leq & s\left[\lambda_{1} d^{q}\left(y^{*}, x^{*}\right)+\lambda_{2} \varepsilon^{q}+2^{q-1} s^{q} \lambda_{5} d^{q}\left(y^{*}, x^{*}\right)+2^{q-1} s^{q} \lambda_{5} d^{q}\left(y^{*}, f\left(y^{*}\right)\right)\right]^{\frac{1}{q}}+s \varepsilon \\
\leq & s\left[\lambda_{1} d^{q}\left(y^{*}, x^{*}\right)+\lambda_{2} \varepsilon^{q}+2^{q-1} s^{q} \lambda_{5} d^{q}\left(y^{*}, x^{*}\right)+2^{q-1} s^{q} \lambda_{5} \varepsilon^{q}\right]^{\frac{1}{q}}+s \varepsilon .
\end{aligned}
$$

In this way, we obtain

$$
\begin{aligned}
d^{q}\left(y^{*}, x^{*}\right) & \leq 2^{q-1} s^{q} \lambda_{1} d^{q}\left(y^{*}, x^{*}\right)+2^{q-1} s^{q} \lambda_{2} \varepsilon^{q}+2^{2 q-2} s^{2 q} \lambda_{5} d^{q}\left(y^{*}, x^{*}\right)+ \\
& +2^{2 q-2} s^{2 q} \lambda_{5} \varepsilon^{q}+2^{q-1} s^{q} \varepsilon^{q}
\end{aligned}
$$

or

$$
\left(1-2^{q-1} s^{q} \lambda_{1}-2^{2 q-2} s^{2 q} \lambda_{5}\right) d^{q}\left(y^{*}, x^{*}\right) \leq 2^{q-1} s^{q}\left(1+\lambda_{2}+2^{q-1} s^{q} \lambda_{5}\right)^{q} \varepsilon^{q}
$$

From here, we obtain

$$
d^{q}\left(y^{*}, x^{*}\right) \leq \frac{\left(1+\lambda_{2}+\gamma(q) \lambda_{5}\right) \gamma(q)}{1-\gamma(q) \lambda_{1}-\gamma^{2}(q) \lambda_{5}} \varepsilon^{q}
$$

Hence,

$$
d^{q}\left(y^{*}, x^{*}\right) \leq c \varepsilon^{q},
$$

where $c=\frac{\left(1+\lambda_{2}+\gamma(q) \lambda_{5}\right) \gamma(q)}{1-\gamma(q) \lambda_{1}-\gamma^{2}(q) \lambda_{5}}$, for any $q>0$ and $\lambda_{1}, \lambda_{5} \in[0,1)$ such that $\lambda_{1}+\lambda_{5}<\frac{1}{\gamma^{2}(q)}$.

\section{Conclusions}

In this paper, we unify, extend, and improve several existing fixed point theorems by introducing the notion of admissible hybrid $\mathcal{Z}$-contraction in the setting of complete $b$-metric spaces. Consequently, all presented results valid in the setting of complete metric space by letting $s=1$. On the other hand, unifying several existing results in the literature, we have used admissible mappings, simulation functions, and hybrid contractions. We need to underline the fact that, by setting admissible function $\alpha$ in a proper way, one can get several new consequences of the existence results in the setting of (i) standard metric space, (ii) metric space endowed a partial order on it, and (iii) cyclic contraction. One can easily get these consequences by using the techniques in [4]. Furthermore, for the 
different examples of simulation functions (as we showed in Theorems 5 and 6), one can get more new corollaries. Lastly, by regarding hybrid contraction approaches, one can get several more consequences, by following the techniques in [21,24-26].

Besides expressing a more generalized result in the setting of a complete $b$-metric space, we also present some applications for our obtained results. In particular, we shall consider the well-posedness and the Ulam-Hyers stability of the fixed point problem. We note that the word 'hybrid' has been used in different ways, in particular, in applicable nonlinear fields, see, e.g., [27,28].

Author Contributions: Writing—original draft, I.C.C.; Writing—review and editing, E.K. All authors have read and agreed to the published version of the manuscript.

Funding: This research received no external funding.

Acknowledgments: The authors thank the anonymous referees for their remarkable comments, suggestions, and ideas that help to improve this paper.

Conflicts of Interest: The authors declare no conflicts of interest.

\section{References}

1. Bourbaki, N. Topologie Générale; Herman: Paris, France, 1974.

2. Bakhtin, I.A. The contraction mapping principle in quasimetric spaces. Funct. Anal. 1989, 30, $26-37$.

3. Czerwik, S. Nonlinear set-valued contraction mappings in $b$-metric spaces. Atti Sem. Mat. Univ. Modena 1998, 46, 263-276.

4. Karapinar, E.; Samet, B. Generalized $\alpha-\psi$ Contractive Type Mappings and Related Fixed Point Theorems with Applications. Abstr. Appl. Anal. 2012, 2012, 793486. [CrossRef]

5. Khojasteh, F.; Shukla, S.; Radenović, S. A new approach to the study of fixed point theorems via simulation functions. Filomat 2015, 29, 1189-1194. [CrossRef]

6. Karapinar, E.; Fulga, A. An Admissible Hybrid Contractions with an Ulam-Hyers Type Stability. Demonstr. Math. 2019, 52, 428-436. [CrossRef]

7. Karapinar, E.; Fulga, A. New Hybrid Contractions on b-Metric Spaces. Mathematics 2019, 7, 578. [CrossRef]

8. Aydi, H.; Bota, M.-F.; Karapinar, E.; Moradi, S. A Common Fixed Point For Weak -Phi-Contractions on b-Metric Spaces. Fixed Point Theory 2012, 13, 337-346.

9. Bota, M.; Chifu, C.; Karapinar, E. Fixed point theorems for generalized (alpha-psi)-Ciric-type contractive multivalued operators in b-metric spaces. J. Nonlinear Sci. Appl. 2016, 9, 1165-1177. [CrossRef]

10. Berinde, V. Generalized contractions in quasimetric spaces. Semin. Fixed Point Theory 1993, 3, 3-9.

11. Karapinar, E.; Khojasteh, F.; Mitrović, Z.D. A Proposal for Revisiting Banach and Caristi Type Theorems in b-Metric Spaces. Mathematics 2019, 7, 308. [CrossRef]

12. Rus, I.A. Generalized Contractions and Applications; Cluj University Press: Clui-Napoca, Romania, 2001.

13. Popescu, O. Some new fixed point theorems for $\alpha$-Geraghty contractive type maps in metric spaces. Fixed Point Theory Appl. 2014, 2014, 190. [CrossRef]

14. Alsulami, H.H.; Karapinar, E.; Khojasteh, F.; Roldán-López-de-Hierro, A.F. A proposal to the study of contractions in quasi-metric spaces. Discret. Dyn. Nat. Soc. 2014, 2014, 269286. [CrossRef]

15. Roldán-López-de-Hierro, A.F.; Karapınar, E.; Roldxaxn-Lxoxpez-de-Hierro, C.; Martínez-Moreno, J. Coincidence Point Theorems on Metric Spaces via Simulation Functions. J. Comput. Appl. Math. 2015, 275, 345-355. [CrossRef]

16. Alharbi, A.S.S.; Alsulami, H.H.; Karapinar, E. On the Power of Simulation and Admissible Functions in Metric Fixed Point Theory. J. Funct. Spaces 2017, 2017, 2068163. [CrossRef]

17. Alqahtani, B.; Fulga, A.; Karapinar, E. Fixed Point Results On $\Delta$-Symmetric Quasi-Metric Space Via Simulation Function With An Application To Ulam Stability. Mathematics 2018, 6, 208. [CrossRef]

18. Aydi, H.; Felhi, A.; Karapinar, E.; Alojail, F.A. Fixed points on quasi-metric spaces via simulation functions and consequences. J. Math. Anal. 2018, 9, 10-24.

19. Ulam, S.M. Problems in Modern Mathematics; John Wiley and Sons: New York, NY, USA, 1940.

20. Ulam, S.M. A Collection of Mathematical Problems; Interscience: New York, NY, USA, 1968.

21. Mitrović, Z.D.; Aydi, M.S. Noorani, H. Qawaqneh, The weight inequalities on Reich type theorem in b-metric spaces. J. Math. Comput. Sci. 2019, 19, 51-570. [CrossRef] 
22. Rassias, T.M. On the stability of linear mapping in Banach Spaces. Proc. Am. Math. Soc. 1978, 72, $297-300$. [CrossRef]

23. Shang, Y. Subspace confinement for switched linear systems. Forum Math. 2017, 29, 693-699. [CrossRef]

24. Karapinar, E. Revisiting the Kannan Type Contractions via Interpolation. Adv. Theory Nonlinear Anal. Appl. 2018, 2, 85-87. [CrossRef]

25. Karapinar, E.; Agarwal, R.; Aydi, H. Interpolative Reich-Rus-Ćirić Type Contractions on Partial Metric Spaces. Mathematics 2018, 6, 256. [CrossRef]

26. Abodayeh, K.; Karapınar, E.; Pitea, A.; Shatanawi, W. Hybrid Contractions on Branciari Type Distance Spaces. Mathematics 2019, 7, 994. [CrossRef]

27. Shang, Y. Consensus of hybrid multi-agent systems with malicious nodes. IEEE Trans. Circuits Syst. II Express Briefs 2019. [CrossRef]

28. Shang, Y. Hybrid consensus for averager-copier-voter networks with non-rational agents, Chaos. Solitons Fractals 2018, 110, 244-251. [CrossRef]

(C) 2019 by the authors. Licensee MDPI, Basel, Switzerland. This article is an open access article distributed under the terms and conditions of the Creative Commons Attribution (CC BY) license (http:/ / creativecommons.org/licenses/by/4.0/). 

Article

\title{
Exact Solutions for a Class of Wick-Type Stochastic (3+1)-Dimensional Modified Benjamin-Bona-Mahony Equations
}

\author{
Praveen Agarwal ${ }^{1}$, Abd-Allah Hyder ${ }^{2,3}$, M. Zakarya ${ }^{2,4}$, Ghada AlNemer ${ }^{5}$, Clemente Cesarano \\ ${ }^{6, *}$ and Dario Assante ${ }^{7}$ \\ 1 Department of Mathematics, Anand International College of Engineering, Jaipur 303012, India; \\ goyal.praveen2011@gmail.com \\ 2 Department of Mathematics, College of Science, King Khalid University, P.O. Box 9004, \\ Abha 61413, Saudi Arabia; abdallah.hyder@yahoo.com (A.-A.H.); \\ mohammed_zakaria1983@yahoo.com (M.Z.) \\ 3 Department of Engineering Mathematics and Physics, Faculty of Engineering Al-Azhar University, Cairo \\ P.O. Box 11371, Egypt \\ 4 Department of Mathematics, Faculty of Science, Al-Azhar University, Assiut P.O. Box 71524, Egypt \\ 5 Department of Mathematical Science, College of Science, Princess Nourah bint Abdulrahman University, \\ P.O. Box 105862, Riyadh 11656, Saudi Arabia; gnnemer@pnu.edu.sa \\ 6 Section of Mathematics, Università Telematica Internazionale Uninettuno, 00186 Rome, Italy \\ 7 Faculty of Engineering, Università Telematica Internazionale Uninettuno, 00186 Rome, Italy; \\ d.assante@uninettunouniversity.net \\ * Correspondence: c.cesarano@uninettunouniversity.net; Tel.: +39-06-69207675
}

Received: 9 October 2019; Accepted: 18 November 2019; Published: 3 December 2019

\begin{abstract}
In this paper, we investigate the Wick-type stochastic (3+1)-dimensional modified Benjamin-Bona-Mahony (BBM) equations. We present a generalised version of the modified tanh-coth method. Using the generalised, modified tanh-coth method, white noise theory, and Hermite transform, we produce a new set of exact travelling wave solutions for the (3+1)-dimensional modified BBM equations. This set includes solutions of exponential, hyperbolic, and trigonometric types. With the help of inverse Hermite transform, we obtained stochastic travelling wave solutions for the Wick-type stochastic (3+1)-dimensional modified BBM equations. Eventually, by application example, we show how the stochastic solutions can be given as white noise functional solutions.
\end{abstract}

Keywords: modified BBM equations; (3+1)-dimensional equations; white noise; Brownian motion; travelling wave solutions; wick-type stochastic

MSC: 60H15; 60H35; 35C07; 60H40

\section{Introduction}

In this paper, with the help of white noise theory, Hermite transform and a generalised, modified tanh-coth method, we deduce stochastic travelling wave solutions for the Wick-type stochastic $(3+1)$-dimensional modified BBM equations as the forms:

$$
\begin{aligned}
& U_{t}+R_{1}(t) \diamond U_{z}+R_{2}(t) \diamond U^{\diamond 2} \diamond U_{x}+R_{3}(t) \diamond U_{x y t}=0, \\
& V_{t}+R_{4}(t) \diamond V_{x}+R_{5}(t) \diamond V^{\diamond 2} \diamond V_{y}+R_{6}(t) \diamond V_{x z t}=0,
\end{aligned}
$$

and

$$
W_{t}+R_{7}(t) \diamond W_{y}+R_{8}(t) \diamond W^{\diamond 2} \diamond W_{z}+R_{9}(t) \diamond W_{x x t}=0,
$$


where $(x, y, z, t) \in \mathbb{R}^{3} \times \mathbb{R}^{+}, R_{i}(i=1,2, \ldots 9)$ are non-zero integrable functions from $\mathbb{R}^{+}$to the Kondrative distribution space $(\mathcal{S})_{-1}$, which was defined by Holden et al. in [1] as a Banach algebra with the Wick-product $\diamond$ Equations (1)-(3) are the perturbations of the (3+1)-dimensional modified BBM equations with variable coefficients:

$$
\begin{aligned}
& u_{t}+r_{1}(t) u_{z}+r_{2}(t) u^{2} u_{x}+r_{3}(t) u_{x y t}=0 \\
& v_{t}+r_{4}(t) v_{x}+r_{5}(t) v^{2} v_{y}+r_{6}(t) v_{x z t}=0
\end{aligned}
$$

and

$$
w_{t}+r_{7}(t) w_{y}+r_{8}(t) w^{2} w_{z}+r_{9}(t) w_{x x t}=0,
$$

where $r_{i}(i=1,2, \ldots 9)$ are non-zero integrable functions o $n \mathbb{R}^{+}$. The modified BBM equation:

$$
u_{t}+k(t) u_{x}+l(t) u^{2} u_{x}+m(t) u_{x x x}=0 .
$$

which describes the surface long waves in nonlinear dispersive media. It is also used as a character to acoustic-gravity waves in compressible fluids, hydromagnetic waves in cold plasma, and acoustic waves in anharmonic crystals [2]. The study of (3+1)-dimensional nonlinear equations is promising because these equations model the real features in a wide assortment of science, technology, fluid mechanics, wave propagations, electrodynamics, and engineering fields [3-6]. For this reason, Hereman [4,5] proposed the (3+1)-dimensional nonlinear modified KdV equation. Analogously, and by the same sense, Wazwaz [7] introduced Equations (4)-(6). Moreover, if Equations (4)-(6) are considered in a random environment, we have random (3+1)-dimensional modified BBM equations. In order to obtain the exact solutions of random $(3+1)$-dimensional modified BBM equations, we only consider them in a white noise environment; that is, we will discuss the Wick-type, stochastic, (3+1)-dimensional modified BBM Equations (1)-(3).

Recently, the study of solutions to nonlinear partial differential equations (PDEs) is prospering [8-10]. Many authors have researched the subject of the random travelling wave, which is a significant subject of stochastic partial differential equations (SPDEs). Wadati [11] first proposed and discussed the stochastic KdV equation and gave the propagation of soliton of the KdV equation under the effect of Gaussian noise. Furthermore, Ghany and Hyder [12-15], Ghany, Hyder and Zakarya [16,17], Chen and Xie [18-20], Hyder and Zakarya [21,22], Hyder [23,24], and Agarwal, Hyder and Zakarya [25] investigated a wide class of Wick-type stochastic evolution equations by using different extension methods and white noise analysis.

There are many methods to obtain travelling wave solutions to nonlinear PDEs, such as the inverse scattering method [26], the Newton's method [27], the tanh method [28], the Sinc-Galerkin method [29], the residual power series method [30], the semi-inverse variational principle and the first integral method [31], and the Daftardar-Gejji and Jafari method [32]. The tanh method, established by Malfliet [33], pursues a specially straightforward and effective algorithm to obtain exact solutions for a wide class of nonlinear PDEs. Moreover, a variety of research papers have focused on the different applications and extensions of the tanh method. Fan [34] has introduced an extended tanh method and gave new travelling wave solutions that cannot be obtained by the tanh method. Also, Wazwaz extended the tanh method and named it the tanh-coth method [35]. Furthermore, El-Wakil [36] and Soliman [37] modified the tanh-coth method and presented new, exact solutions for some nonlinear PDEs.

Our aim in this work was to obtain new stochastic travelling wave solutions for the Wick-type stochastic (3+1)-dimensional modified BBM equations. Firstly, we give a generalised version of the modified tanh-coth method to make it convenient for the nonlinear $(3+1)$-dimensional and multi dimensional PDEs. Secondly, we use the generalised, modified tanh-coth method, white noise theory, and Hermite transform to produce a new set of exact travelling wave solutions for the $(3+1)$-dimensional modified BBM equations, this set includes solutions of exponential, hyperbolic, and 
trigonometric types. Finally, we use the inverse Hermite transform to obtain stochastic travelling wave solutions for the Wick-type stochastic (3+1)-dimensional modified BBM equations. Moreover, by an application example, we show how the stochastic solutions can be given as white noise functional solutions. In our work, the modified BBM equation describes the surface long waves in nonlinear dispersive media. It is also used as a character of acoustic gravity waves in compressible fluids, hydromagnetic waves in cold plasma, and acoustic waves in harmonic crystals [2]. The study of $(3+1)$-dimensional nonlinear equations is prospering because these equations model the real features in a wide assortment of science, technology, fluid mechanics, wave propagations, electrodynamics, and engineering fields [3-6]. The origin and references of Equation (1) are given in Holden [1]. Ghany and Fathallah studied white-noise functional solutions for wick-type stochastic time-fractional Benjamin-Bona-Mahony (BBM) equation in [38]. Recently, Sahoo and Saha Ray studied by other methods the stochastic solutions of wick-type stochastic time-fractional BBM equation for modeling long surface gravity waves of small amplitude, in [39]. The PDE of Benjamin et al. [2] is now often called the BBM equation, although it is also known as the regularised long wave (RLW) equation. Morrison et al proposed the one-dimensional PDE, as an equally valid and accurate model for the same wave phenomena simulated by the KdV and RLW equations [40]. Random waves are an important subject of random PDEs. In essence, to investigate the exact solutions of random BBM equation, we restricted our attention to consider this problem in white noise environment [38].

This paper is organized as follows: In Section 2, we recall some requisites from Gaussian white noise analysis. In Section 3, we give a generalisation to the modified tanh-coth method to make it convenient for the nonlinear (3+1)-dimensional equations. In Section 4, we employ the generalised, modified tanh-coth method, white noise theory, and Hermite transform to obtain a new set of exact travelling wave solutions for the (3+1)-dimensional modified BBM equations. In Section 5, we apply the inverse Hermite transform to explore stochastic travelling wave solutions for the Wick-type stochastic (3+1)-dimensional modified BBM equations. In Section 5, we give some examples to show that the stochastic solutions can be given as Brownian motion functional solutions and white noise functional solutions. In Section 6, we give a summary and discussion.

\section{Requisites from Gaussian White Noise Analysis}

The Gaussian white noise analysis starts with the rigging $\mathcal{S}\left(\mathbb{R}^{d}\right) \subset L^{2}\left(\mathbb{R}^{d}\right) \subset \mathcal{S}^{*}\left(\mathbb{R}^{d}\right)$, where $\mathcal{S}\left(\mathbb{R}^{d}\right)$ is the Schwartz space of rapidly decreasing, infinite differentiable functions on $\mathbb{R}^{d}$, and $\mathcal{S}^{*}\left(\mathbb{R}^{d}\right)$ is the space of tempered distributions. From the Bochner-Minlos theorem [1], we have a unique white noise measure $\mu$, on $\left(\mathcal{S}^{*}\left(\mathbb{R}^{d}\right), \beta\left(\mathcal{S}^{*}\left(\mathbb{R}^{d}\right)\right)\right)$. Assume that $\xi_{n}(x)=\pi^{-1 / 4}((n-$ $1) !)^{-1 / 2} e^{-x^{2} / 2} h_{n-1}(\sqrt{2 x}), n \in \mathbb{N}$ are the Hermite functions, where $h_{n}(x)$ denotes the Hermite polynomials. It is well known that the collection $\left(\xi_{n}\right)_{n \in \mathbb{N}}$ forms an orthonormal basis for $L^{2}(\mathbb{R})$. Let $\alpha=\left(\alpha_{1}, \ldots, \alpha_{d}\right)$ be a $d$-dimensional multi-indices with $\alpha_{1}, \ldots, \alpha_{d} \in \mathbb{N}$; then, the family of tensor products $\xi_{\alpha}:=\xi_{\left(\alpha_{1}, \ldots, \alpha_{d}\right)}=\xi_{\alpha_{1}} \otimes \ldots \otimes \xi_{\alpha_{d}}, \alpha \in \mathbb{N}^{d}$ constitutes an orthonormal basis for $L^{2}\left(\mathbb{R}^{d}\right)$. Now, introduce an ordering in $\mathbb{N}^{d}$ by

$$
i<j \Rightarrow \sum_{k=1}^{d} \alpha_{k}^{(i)} \leq \sum_{k=1}^{d} \alpha_{k}^{(j)}, \text { where } \alpha^{(i)}=\left(\alpha_{k}^{(i)}\right)_{k=1}^{d}, \alpha^{(j)}=\left(\alpha_{k}^{(j)}\right)_{k=1}^{d} \in \mathbb{N}^{d} .
$$

Using this ordering, we define $\eta_{i}:=\xi_{\alpha^{(i)}}=\xi_{\alpha_{1}^{(i)}} \otimes \ldots \otimes \xi_{\alpha_{d}^{(i)}}, i \in \mathbb{N}$. Let $\mathbb{J}=\left(\mathbb{N}_{0}^{\mathbb{N}}\right)_{c}$ be the set of all sequences $\alpha=\left(\alpha_{i}\right)_{i \in \mathbb{N}}$ with $\alpha_{i} \in \mathbb{N}_{0}$ and with compact support. For $\alpha \in \mathbb{J}$, we define

$$
H_{\alpha}(\omega)=\prod_{i=1}^{\infty} h_{\alpha_{i}}\left(\left\langle\omega, \eta_{i}\right\rangle\right), \omega \in \mathcal{S}^{*}\left(\mathbb{R}^{d}\right)
$$


Let $n \in \mathbb{N}$, the Kondrative space of stochastic test functions $(\mathcal{S})_{1}^{n}$ is defined by:

$$
(\mathcal{S})_{1}^{n}=\left\{f=\sum_{\alpha} c_{\alpha} H_{\alpha} \in \bigoplus_{k=1}^{n} L^{2}(\mu): c_{\alpha} \in \mathbb{R}^{n} \text { and }\|f\|_{1, k}^{2}:=\sum_{\alpha} c_{\alpha}^{2}(\alpha !)^{2}(2 \mathbb{N})^{k \alpha}<\infty \forall k \in \mathbb{N}\right\},
$$

and the Kondrative space of stochastic distributions $(\mathcal{S})_{-1}^{n}$ is defined by:

$$
(\mathcal{S})_{-1}^{n}=\left\{F=\sum_{\alpha} b_{\alpha} H_{\alpha}: b_{\alpha} \in \mathbb{R}^{n} \text { and }\|F\|_{-1, k}^{2}:=\sum_{\alpha} b_{\alpha}^{2}(2 \mathbb{N})^{-q \alpha}<\infty \text { for some } q \in \mathbb{N}\right\} .
$$

The family of seminorms $\|f\|_{1, k}, k \in \mathbb{N}$ produces a topology on $(\mathcal{S})_{1}^{n}$ and $(\mathcal{S})_{-1}^{n}$ can be represented as the dual of $(\mathcal{S})_{1}^{n}$ under the action $\langle F, f\rangle=\sum_{\alpha}\left(b_{\alpha}, c_{\alpha}\right) \alpha$ !, where $F=\sum_{\alpha} b_{\alpha} H_{\alpha} \in(\mathcal{S})_{-1}^{n}, f=\sum_{\alpha} c_{\alpha} H_{\alpha} \in$ $(\mathcal{S})_{1}^{n}$ and $\left(b_{\alpha}, c_{\alpha}\right)$ is the usual scalar product on $\mathbb{R}^{n}$.

The Wick product of two distributions $F=\sum_{\alpha} a_{\alpha} H_{\alpha}, G=\sum_{\beta} b_{\beta} H_{\beta} \in(\mathcal{S})_{-1}^{n}$ with $a_{\alpha}, b_{\beta} \in \mathbb{R}^{n}$ is defined by:

$$
F \diamond G=\sum_{\alpha, \beta}\left(a_{\alpha}, b_{\beta}\right) H_{\alpha+\beta} .
$$

Let $F=\sum_{\alpha} a_{\alpha} H_{\alpha} \in(\mathcal{S})_{-1}^{n}$ with $a_{\alpha} \in \mathbb{R}^{n}$. The Hermite transform of $F$ is defined by:

$$
\mathcal{H} F(w)=\widetilde{F}(w)=\sum_{\alpha} a_{\alpha} w^{\alpha} \in \mathbb{C}^{n} \quad \text { (when convergent) }
$$

where $w=\left(w_{1}, w_{2}, \ldots\right) \in \mathbb{C}^{\mathbb{N}}$ and $w^{\alpha}=\prod_{i=1}^{\infty} w_{i}^{\alpha_{i}}$, with $\alpha=\left(\alpha_{1}, \alpha_{2}, \ldots\right) \in \mathbb{J}$ and $w_{i}^{0}=1$.

For $F, G \in(S)_{-1}^{n}$, by the definition of Hermite transform, we get:

$$
\widetilde{F \diamond G}(w)=\widetilde{F}(w) \widetilde{G}(w),
$$

for all $w$ such that $\widetilde{F}(w)$ and $\widetilde{G}(w)$ exist. The multiplication on the right hand side of the above equality is the complex bilinear multiplication in $\mathbb{C}^{n}$ which is defined by $\left(w_{1}^{1}, \ldots w_{n}^{1}\right)\left(w_{1}^{2}, \ldots, w_{n}^{2}\right)=\sum_{i=1}^{n} w_{i}^{1} w_{i}^{2}$, where $w_{i}^{k} \in \mathbb{C}$. Hence, The Hermite transform converts the Wick product into the usual product and convergence in $(\mathcal{S})_{-1}^{n}$ into pointwise and bounded convergence in a specific neighbourhood of zero in $\mathbb{C}^{n}$. For more details about stochastic Kondrative spaces, Wick product, and Hermite transform we refer the reader to [1].

\section{Generalization of the Modified Tanh-Coth Method}

Consider a multi dimensional, nonlinear PDE of wave propagation:

$$
P\left(u, u_{t}, u_{x_{i}}, u_{x_{i} x_{j}}, u_{x_{i} x_{j} x_{k}}, \ldots\right)=0,
$$

where $u$ is the dependent variable and $t=x_{0}, x_{1}, x_{2}, \ldots, x_{m}$ are the independent variables. Introduce the wave transformation:

$$
u=u(\xi), \quad \xi=\sum_{i=0}^{m} a_{i} x_{i},
$$

where $a_{i}(i=0,1,2, \ldots, m)$ are unknown constants. Therefore, Equation (15) can be transformed into a nonlinear ordinary differential equation (NODE):

$$
Q\left(u, u^{\prime}, u^{\prime \prime}, u^{\prime \prime \prime}, \ldots\right)=0 .
$$


For simplicity, we integrate the NODE (17), provided that all terms include derivatives, and set the integration constants to be zero. Subsequently, the transformed Equation (17) can be solved by expanding its general solution in finite series as follows:

$$
u(\xi)=\sum_{k=0}^{N} A_{k} \Phi^{k}(\xi)+\sum_{k=1}^{N} B_{k} \Phi^{-k}(\xi),
$$

where $\Phi$ solves the first order Riccati equation [41]:

$$
\Phi^{\prime}(\xi)=\alpha_{0}+\alpha_{1} \Phi(\xi)+\alpha_{2} \Phi^{2}(\xi)
$$

where $\alpha_{0}, \alpha_{1}$, and $\alpha_{2}$ are constants to be determined. The positive constant $N$ can be specified by balancing the linear and nonlinear terms of highest order in Equation (17). Inserting Equations (18) and (19) into Equation (17), yields an algebraic equation in $\Phi$ and its powers. Equating the coefficients of $\Phi^{k}$ to zero, gives an algebraic system of equations in $A_{k}$ and $B_{k}$. With the help of the computer symbolic system Mathematica, we can obtain $A_{k}$ and $B_{k}$. The Riccati Equation (19) has the following particular solutions [42]:

$$
\begin{cases}\Phi(\xi)=e^{\xi}-1, & \alpha_{0}=1, \alpha_{1}=1, \alpha_{2}=0, \\ \Phi(\xi)=\operatorname{coth}(\xi) \pm(\xi), \tanh (\xi) \pm i(\xi), & \alpha_{0}=\frac{1}{2}, \alpha_{1}=0, \alpha_{2}=-\frac{1}{2} \\ \Phi(\xi)=\tan (\xi),-\cot (\xi), & \alpha_{0}=1, \alpha_{1}=0, \alpha_{2}=1, \\ \Phi(\xi)=\frac{1}{2} \cot (2 \xi), \frac{1}{2} \tan (2 \xi), & \alpha_{0}=1, \alpha_{1}=0, \alpha_{2}=4 .\end{cases}
$$

\section{The Wick-Type, Stochastic, (3+1)-Dimensional Modified BBM Equations}

We first investigate the model (1) of the Wick-Type, stochastic, (3+1)-dimensional modified BBM equations. Applying Hermite transform to Equation (1), gets the deterministic equation:

$$
\begin{aligned}
\widetilde{U}_{t}(x, y, z, t, w) & +\widetilde{R}_{1}(t, w) \widetilde{U}_{z}(x, y, z, t, w)+\widetilde{R}_{2}(t, w) \widetilde{U}^{2}(x, y, z, t, w) U_{x}(x, y, z, t, w)+ \\
& +\widetilde{R}_{3}(t, w) \widetilde{U}_{x y t}(x, y, z, t, w)=0,
\end{aligned}
$$

where $w=\left(w_{1}, w_{2}, \ldots\right) \in\left(\mathbb{C}^{\mathbb{N}}\right)$. To obtain travelling wave solutions to Equation (21), we introduce the transformations $\widetilde{R_{1}}(t, w)=r_{1}(t, w), \widetilde{R_{2}}(t, w)=r_{2}(t, w), \widetilde{R_{3}}(t, w)=r_{3}(t, w)$, and $\widetilde{U}(x, y, z, t, w)=$ $u(x, y, z, t, w)=u(\xi(x, y, z, t, w))$ with

$$
\xi(x, y, z, t, w)=a_{1} x+a_{2} y+a_{3} z+b \int_{0}^{t} \chi(\tau, w) d \tau,
$$

where $a_{i}(i=1,2,3), b$, and $c$ are arbitrary constants satisfying $a_{i} b \neq 0$ and $\chi$ is a non-zero function to be determined. Hence, Equation (21) can be converted to the following NODE:

$$
\left(b \chi+a_{3} r_{1}\right) u+\frac{1}{3} a_{1} r_{2} u^{3}+a_{1} a_{2} b \chi r_{2} u^{\prime \prime}=0 .
$$

Balancing $u^{3}$ with $u^{\prime \prime}$, gives $N=1$. Therefore, we put the solution of Equation (21) in the form:

$$
u(x, y, z, t, w)=A_{0}(t, w)+A_{1}(t, w) \Phi(\xi)+\frac{B_{1}(t, w)}{\Phi(\xi)},
$$

where $\Phi$ is the solution of Equation (19). Substituting Equations (24) and (19) into Equation (23), collecting the coefficients of $\Phi^{k}(k=-3,-2,-1,0,1,2,3)$, and equating them to zero, gives the following system of seven algebraic equations in $A_{0}, A_{1}, B_{1}$, and $\chi$. 


$$
\left\{\begin{array}{l}
\left(b \chi+a_{3} r_{1}\right) A_{0}+\frac{1}{3} a_{1} r_{2} I_{0}+a_{1} a_{2} b \chi r_{3} E_{0}=0 \\
\left(b \chi+a_{3} r_{1}\right) A_{1}+\frac{1}{3} a_{1} r_{2} I_{1}+a_{1} a_{2} b \chi r_{3} E_{1}=0 \\
\left(b \chi+a_{3} r_{1}\right) B_{1}+\frac{1}{3} a_{1} r_{2} J_{1}+a_{1} a_{2} b \chi r_{3} F_{1}=0 \\
\frac{1}{3} a_{1} r_{2} I_{2}+a_{1} a_{2} b \chi r_{3} E_{2}=0 \\
\frac{1}{3} a_{1} r_{2} I_{3}+a_{1} a_{2} b \chi r_{3} E_{3}=0 \\
\frac{1}{3} a_{1} r_{2} J_{2}+a_{1} a_{2} b \chi r_{3} F_{2}=0 \\
\frac{1}{3} a_{1} r_{2} J_{3}+a_{1} a_{2} b \chi r_{3} F_{3}=0 .
\end{array}\right.
$$

where $I_{0}=A_{0} G_{0}+A_{1} H_{1}+B_{1} G_{1}, I_{1}=A_{0} G_{1}+A_{1} G_{0}+B_{1} G_{2}, I_{2}=A_{0} G_{2}+A_{1} G_{1}, I_{3}=A_{1} G_{2}$ ，

$J_{1}=A_{0} H_{1}+A_{1} H_{2}+B_{1} G_{0}, J_{2}=A_{0} H_{2}+B_{1} H_{1}, J_{3}=B_{1} H_{2}, G_{0}=A_{0}^{2}+2 A_{1} B_{1}, G_{1}=2 A_{0} A_{1}$,

$G_{2}=A_{1}^{2}, H_{1}=2 A_{0} B_{1}, H_{2}=B_{1}^{2}, E_{0}=\alpha_{0} C_{1}-\alpha_{2} D_{1}, E_{1}=\alpha_{1} C_{1}+2 \alpha_{0} C_{2}, E_{2}=\alpha_{2} C_{1}+2 \alpha_{1} C_{2}$,

$E_{3}=2 \alpha_{2} C_{2}, F_{1}=-\alpha_{1} D_{1}-2 \alpha_{2} D_{2}, F_{2}=-\alpha_{0} D_{1}-2 \alpha_{1} D_{2}, F_{3}=-2 \alpha_{0} D_{2}, C_{0}=\alpha_{0} A_{1}-\alpha_{2} B_{1}$,

$C_{1}=\alpha_{1} A_{1}, C_{2}=\alpha_{2} A_{1}, D_{1}=-\alpha_{1} B_{1}, D_{2}=-\alpha_{0} B_{1}$.

Now, we solve the system (25) for some cases relating to the Riccati equation (19).

\subsection{Case I}

We reduce the system (25) by using $\alpha_{0}=\alpha_{1}=1$ and $\alpha_{2}=0$. By using Mathematica, we can find a set of solutions for the reduced system as follows:

$$
A_{0}= \pm i \sqrt{\frac{3 a_{3} r_{1}}{a_{1} r_{2}}}, \quad A_{1}=0, \quad B_{1}= \pm \sqrt{\frac{3 a_{2} a_{3} r_{1}}{a_{1} a_{2} r_{2} r_{3}-2}}, \quad \chi=\frac{2 a_{3} r_{1}}{b\left(a_{1} a_{2} r_{3}-2\right)} .
$$

Substituting the values (26) in Equation (24) and using (20), yields a travelling wave solution of Equation (21) of exponential type:

$$
\begin{aligned}
& u_{1}(x, y, z, t, w)= \\
& =\frac{ \pm i \sqrt{3 a_{3} r_{1}(t, w)\left(a_{1} a_{2} r_{2}(t, w) r_{3}(t, w)-2\right)}(\exp (\xi 1(x, y, z, t, w))-1) \pm \sqrt{3 a_{1} a_{2} a_{3} r_{1}(t, w) r_{2}(t, w)}}{\sqrt{a_{1} r_{2}(t, w)\left(a_{1} a_{2} r_{2}(t, w) r_{3}(t, w)-2\right)}\left(\exp \left(\xi_{1}(x, y, z, t, w)\right)-1\right)},
\end{aligned}
$$

where

$$
\xi_{1}(x, y, z, t, w)=a_{1} x+a_{2} y+a_{3} z+2 a_{3} \int_{0}^{t} \frac{r_{1}(\tau, w)}{a_{1} a_{2} r_{3}(\tau, w)-2} d \tau
$$

\subsection{Case II}

We reduce the system (25) by using $\alpha_{0}=\frac{1}{2}, \alpha_{1}=0$, and $\alpha_{2}=-\frac{1}{2}$. By using Mathematica, we can find a set of solutions for the reduced system as follows:

$$
A_{0}=0, \quad A_{1}= \pm \sqrt{\frac{3 a_{2} a_{3} r_{1} r_{3}}{r_{2}\left(2-a_{1} a_{2} r_{3}\right)}}, \quad B_{1}= \pm i \sqrt{\frac{3 a_{1} a_{2} r_{1}}{2 r_{2} r_{3}}}, \quad \chi=-\frac{4 a_{3} r_{1}}{b\left(4+a_{1} a_{2} r_{3}\right)} .
$$


Substituting the values (29) in Equation (24) and using (20), yields travelling wave solutions of Equation (21) of hyperbolic type:

$$
\begin{aligned}
u_{2}(x, y, z, t, w)= & \pm \sqrt{\frac{3 a_{2} a_{3} r_{1}(t, w) r_{3}(t, w)}{r_{2}(t, w)\left(2-a_{1} a_{2} r_{3}(t, w)\right)}}\left(\operatorname{coth}\left(\xi_{2}(x, y, z, t, w)\right) \pm\left(\xi_{2}(x, y, z, t, w)\right)\right) \\
& \pm i \frac{\sqrt{3 b a_{2} r_{1}(t, w)}}{\sqrt{2 r_{2}(t, w) r_{3}(t, w)}\left(\operatorname{coth}\left(\xi_{2}(x, y, z, t, w)\right) \pm\left(\xi_{2}(x, y, z, t, w)\right)\right)}
\end{aligned}
$$

where

$$
\xi_{2}(x, y, z, t, w)=a_{1} x+a_{2} y+a_{3} z-4 a_{3} \int_{0}^{t} \frac{r_{1}(\tau, w)}{4+a_{1} a_{2} r_{3}(\tau, w)} d \tau
$$

\subsection{Case III}

We reduce the system (25) by putting $\alpha_{0}=\alpha_{2}=1$ and $\alpha_{1}=0$. By using Mathematica, we can find a set of solutions for the reduced system as follows:

$$
A_{0}= \pm \sqrt{\frac{3 a_{2} a_{3} r_{1}}{1-a_{1} a_{2} r_{2} r_{3}}}, \quad A_{1}=B_{1}= \pm \sqrt{\frac{6 a_{2} a_{3} r_{1} r_{3}}{1+2 a_{1} a_{2} r_{2} r_{3}}}, \quad \chi=\frac{-a_{3} r_{1}}{b\left(1+2 a_{1} a_{2} r_{3}\right)} .
$$

Substituting the values (33) in Equation (24) and using (20), yields travelling wave solutions of Equation (21) of trigonometric type:

$$
\begin{aligned}
& u_{4}(x, y, z, t, w)=u_{5}(x, y, z, t, w)= \\
& = \pm \sqrt{\frac{3 a_{2} a_{3} r_{1}(t, w)}{1-a_{1} a_{2} r_{2}(t, w) r_{3}(t, w)}} \pm \sqrt{\frac{6 a_{2} a_{3} r_{1}(t, w) r_{3}(t, w)}{1+2 a_{1} a_{2} r_{2}(t, w) r_{3}(t, w)}}\left(\sec \left(\xi_{3}(x, y, z, t, w)\right) \csc \left(\xi_{3}(x, y, z, t, w)\right)\right)
\end{aligned}
$$

where

$$
\xi_{3}(x, y, z, t, w)=a_{1} x+a_{2} y+a_{3} z-a_{3} \int_{0}^{t} \frac{r_{1}(\tau, w)}{1+2 a_{1} a_{2} r_{3}(\tau, w)} d \tau
$$

\subsection{Case IV}

We reduce the system (25) by putting $\alpha_{0}=1, \alpha_{1}=0$ and $\alpha_{2}=4$. By using Mathematica, we can find a set of solutions for the reduced system as follows:

$$
A_{0}= \pm 3 \sqrt{\frac{3 a_{2} a_{3} r_{1}}{9 a_{1} a_{2} r_{2} r_{3}-2}}, \quad A_{1}= \pm 8 \sqrt{\frac{3 a_{2} a_{3} r_{1} r_{3}}{2+15 a_{1} a_{2} r_{2} r_{3}}}, \quad B_{1}= \pm i \sqrt{\frac{3 a_{3} r_{1}}{a_{1} r_{2} r_{3}}}, \quad \chi=-\frac{2 a_{3} r_{1}}{b\left(9 a_{1} a_{2} r_{2} r_{3}-2\right)} .
$$

Substituting the values (36) in Equation (24) and using (20), yields travelling wave solutions of Equation (21) of trigonometric type:

$$
\begin{aligned}
u_{6}(x, y, z, t, w)= \pm & 3 \sqrt{\frac{3 a_{2} a_{3} r_{1}(t, w)}{9 a_{1} a_{2} r_{2}(t, w) r_{3}(t, w)-2}} \pm 4 \sqrt{\frac{3 a_{2} a_{3} r_{1}(t, w) r_{3}(t, w)}{2+15 a_{1} a_{2} r_{2}(t, w) r_{3}(t, w)}}\left(\cot \left(2 \xi_{4}(x, y, z, t, w)\right)\right. \\
& \pm i \frac{\sqrt{3 a_{3} r_{1}(t, w)}}{2 \sqrt{a_{1} r_{2}(t, w) r_{3}(t, w)}\left(\cot \left(2 \xi_{4}(x, y, z, t, w)\right)\right)}
\end{aligned}
$$




$$
\begin{aligned}
u_{7}(x, y, z, t, w)= \pm & 3 \sqrt{\frac{3 a_{2} a_{3} r_{1}(t, w)}{9 a_{1} a_{2} r_{2}(t, w) r_{3}(t, w)-2}} \pm 4 \sqrt{\frac{3 a_{2} a_{3} r_{1}(t, w) r_{3}(t, w)}{2+15 a_{1} a_{2} r_{2}(t, w) r_{3}(t, w)}}\left(\tan \left(2 \xi_{4}(x, y, z, t, w)\right)\right. \\
& \pm i \frac{\sqrt{3 a_{3} r_{1}(t, w)}}{2 \sqrt{a_{1} r_{2}(t, w) r_{3}(t, w)}\left(\tan \left(2 \xi_{4}(x, y, z, t, w)\right)\right)}
\end{aligned}
$$

where

$$
\xi_{4}(x, y, z, t, w)=a_{1} x+a_{2} y+a_{3} z+2 a_{3} \int_{0}^{t} \frac{r_{1}(\tau, w)}{9 a_{1} a_{2} r_{3}(\tau, w)-2} d \tau .
$$

Obviously, there are several particular solutions for the system (25) with the Riccati equation (19), coming from many different cases. In the above cases we just clarified how far our technique is applicable.

Now, for $q<\infty, r>0$, consider the infinite dimensional neighbourhoods $K_{q}(r)=\left\{\left(w_{1}, w_{2}, \ldots\right) \in\right.$ $\left.\mathbb{C}^{\mathbb{N}}: \sum_{\alpha \neq 0}\left|w^{\alpha}\right|^{2}(2 \mathbb{N})^{q \alpha}<r^{2}\right\}$ of zero in $\mathbb{C}^{\mathbb{N}}$ [1]. The properties of exponential, hyperbolic, and trigonometric functions yield that there exists a bounded open set $\mathbf{D} \subset \mathbb{R}^{3} \times \mathbb{R}_{+}, q<\infty, r>0$, such that the solution $u(x, y, z, t, w)$ of Equation (21) and all its derivatives which are involved in Equation (21) are uniformly bounded for $(x, y, z, t, w) \in \mathbf{D} \times K_{q}(r)$, continuous with respect to $(x, y, z, t) \in \mathbf{D}$ for all $w \in K_{q}(r)$ and analytic with respect to $w \in K_{q}(r)$, for all $(x, y, z, t) \in \mathbf{D}$. From Theorem 4.1.1 in [1], there exists $U(x, y, z, t) \in(\mathcal{S})_{-1}$ such that $u(x, y, z, t, w)=\widetilde{U}(x, y, z)(w)$ for all $(x, y, z, t, w) \in \mathbf{D} \times K_{q}(r)$ and $U(x, y, z, t)$ solves Equation $(1)$ in $(\mathcal{S})_{-1}$. Hence, by applying the inverse Hermite transform to Equations (27), (30), (31), (34), (37), and (38), we obtain the solutions of Equation (1) as follows:

(I) Stochastic Travelling Wave Solution of Exponential Type:

$$
\begin{aligned}
& U_{1}(x, y, z, t)= \\
& \frac{ \pm i \sqrt{3 a_{3} R_{1}(t) \diamond\left(a_{1} a_{2} R_{2}(t) \diamond R_{3}(t)-2\right)} \diamond\left(\exp ^{\diamond}\left(\Xi_{1}(x, y, z, t)\right)-1\right) \pm \sqrt{3 a_{1} a_{2} a_{3} R_{1}(t) \diamond R_{2}(t)}}{\sqrt{a_{1} R_{2}(t) \diamond\left(a_{1} a_{2} R_{2}(t) \diamond R_{3}(t)-2\right)}\left(\exp ^{\diamond}\left(\Xi_{1}(x, y, z, t)\right)-1\right)},
\end{aligned}
$$

with

$$
\Xi_{1}(x, y, z, t)=a_{1} x+a_{2} y+a_{3} z+2 a_{3} \int_{0}^{t} \frac{R_{1}(\tau)}{a_{1} a_{2} R_{3}(\tau)-2} d \tau .
$$

(II) Stochastic Travelling Wave Solutions of Hyperbolic Type:

$$
\begin{aligned}
U_{2}(x, y, z, t)= & \pm \sqrt{\frac{3 a_{2} a_{3} R_{1}(t) \diamond R_{3}(t)}{R_{2}(t) \diamond\left(2-a_{1} a_{2} R_{3}(t)\right)}}\left(\operatorname{coth}^{\diamond}\left(\Xi_{2}(x, y, z, t)\right) \pm \diamond\left(\Xi_{2}(x, y, z, t)\right)\right) \\
& \pm i \frac{\sqrt{3 b a_{2} R_{1}(t)}}{\sqrt{2 R_{2}(t) \diamond R_{3}(t)} \diamond\left(\operatorname{coth}^{\diamond}\left(\Xi_{2}(x, y, z, t)\right) \pm \diamond\left(\Xi_{2}(x, y, z, t)\right)\right)}
\end{aligned}
$$

with

$$
\Xi_{2}(x, y, z, t)=a_{1} x+a_{2} y+a_{3} z-4 a_{3} \int_{0}^{t} \frac{R_{1}(\tau)}{4+a_{1} a_{2} R_{3}(\tau)} d \tau .
$$


(III) Stochastic Travelling Wave Solutions of Trigonometric Type:

$$
\begin{aligned}
U_{4}(x, y, z, t)=U_{5}(x, y, z, t)= & \pm \sqrt{\frac{3 a_{2} a_{3} R_{1}(t)}{1-a_{1} a_{2} R_{2}(t) \diamond R_{3}(t)}} \pm \sqrt{\frac{6 a_{2} a_{3} R_{1}(t) \diamond R_{3}(t)}{1+2 a_{1} a_{2} R_{2}(t) \diamond R_{3}(t)}} \\
& \diamond\left(\sec ^{\diamond}\left(\Xi_{3}(x, y, z, t)\right) \diamond \csc ^{\diamond}\left(\Xi_{3}(x, y, z, t)\right)\right),
\end{aligned}
$$

with

$$
\begin{aligned}
& \Xi_{3}(x, y, z, t)=a_{1} x+a_{2} y+a_{3} z-a_{3} \int_{0}^{t} \frac{R_{1}(\tau)}{1+2 a_{1} a_{2} R_{3}(\tau)} d \tau \\
& U_{6}(x, y, z, t)= \pm 3 \sqrt{\frac{3 a_{2} a_{3} R_{1}(t)}{9 a_{1} a_{2} R_{2}(t) \diamond R_{3}(t)-2}} \pm 4 \sqrt{\frac{3 a_{2} a_{3} R_{1}(t) \diamond R_{3}(t)}{2+15 a_{1} a_{2} R_{2}(t) \diamond R_{3}(t)}} \diamond\left(\cot ^{\diamond}\left(2 \Xi_{4}(x, y, z, t)\right)\right. \\
& \pm i \frac{\sqrt{3 a_{3} R_{1}(t)}}{2 \sqrt{a_{1} R_{2}(t) \diamond R_{3}(t)} \diamond\left(\cot ^{\diamond}\left(2 \Xi_{4}(x, y, z, t)\right)\right.}, \\
& U_{7}(x, y, z, t)= \pm 3 \sqrt{\frac{3 a_{2} a_{3} R_{1}(t)}{9 a_{1} a_{2} R_{2}(t) \diamond R_{3}(t)-2}} \pm 4 \sqrt{\frac{3 a_{2} a_{3} R_{1}(t) \diamond R_{3}(t)}{2+15 a_{1} a_{2} R_{2}(t) \diamond R_{3}(t)}} \diamond\left(\tan ^{\diamond}\left(2 \Xi_{4}(x, y, z, t)\right)\right) \\
& \pm i \frac{\sqrt{3 a_{3} R_{1}(t)}}{2 \sqrt{a_{1} R_{2}(t) \diamond R_{3}(t)} \diamond\left(\tan ^{\diamond}\left(2 \Xi_{4}(x, y, z, t)\right)\right)},
\end{aligned}
$$

with

$$
\Xi_{4}(x, y, z, t)=a_{1} x+a_{2} y+a_{3} z+2 a_{3} \int_{0}^{t} \frac{R_{1}(\tau)}{9 a_{1} a_{2} R_{3}(\tau)-2} d \tau .
$$

For the other two forms of the Wick-type, stochastic, (3+1)-dimensional modified BBM equations (2) and (3), we can follow the same technique as presented for the first form (1). Therefore, we just list the stochastic travelling wave solutions for each form. For Equation (2) one obtains the following stochastic travelling wave solution:

(I) Stochastic Travelling Wave Solution of Exponential Type:

$$
\begin{aligned}
& V_{1}(x, y, z, t)= \\
& \frac{ \pm i \sqrt{3 b_{3} R_{4}(t) \diamond\left(b_{1} b_{2} R_{5}(t) \diamond R_{6}(t)-2\right)}\left(\exp ^{\diamond}\left(\Lambda_{1}(x, y, z, t)\right)-1\right) \pm \sqrt{3 b_{1} b_{2} b_{3} R_{4}(t) \diamond R_{5}(t)}}{\sqrt{b_{1} R_{5}(t) \diamond\left(b_{1} b_{2} R_{5}(t) \diamond R_{6}(t)-2\right)}\left(\exp ^{\diamond}\left(\Lambda_{1}(x, y, z, t)\right)-1\right)}
\end{aligned}
$$

with

$$
\Lambda_{1}(x, y, z, t)=b_{1} x+b_{2} y+b_{3} z+2 b_{3} \int_{0}^{t} \frac{R_{4}(\tau)}{b_{1} b_{2} R_{6}(\tau)-2} d \tau .
$$

(II) Stochastic Travelling Wave Solutions of Hyperbolic Type:

$$
\begin{aligned}
V_{2}(x, y, z, t)= & \pm \sqrt{\frac{3 b_{2} b_{3} R_{4}(t) \diamond R_{6}(t)}{R_{5}(t) \diamond\left(2-b_{1} b_{2} R_{6}(t)\right)}} \diamond\left(\operatorname{coth}^{\diamond}\left(\Lambda_{2}(x, y, z, t)\right) \pm\left(\Lambda_{2}(x, y, z, t)\right)\right) \\
& \pm i \frac{\sqrt{3 b^{*} b_{2} R_{4}(t)}}{\sqrt{2 R_{5}(t) \diamond R_{6}(t)} \diamond\left(\operatorname{coth}^{\diamond}\left(\Lambda_{2}(x, y, z, t)\right) \pm\left(\Lambda_{2}(x, y, z, t)\right)\right)}, \\
V_{3}(x, y, z, t)= & \pm \sqrt{\frac{3 b_{2} b_{3} R_{4}(t) \diamond R_{6}(t)}{R_{5}(t) \diamond\left(2-b_{1} b_{2} R_{6}(t)\right)}} \diamond\left(\tanh ^{\diamond}\left(\Lambda_{2}(x, y, z, t)\right) \pm i^{\diamond}\left(\Lambda_{2}(x, y, z, t)\right)\right) \\
& \pm i \frac{\sqrt{3 b^{*} b_{2} R_{4}(t)}}{\sqrt{2 R_{5}(t) \diamond R_{6}(t)} \diamond\left(\tanh ^{\diamond}\left(\Lambda_{2}(x, y, z, t)\right) \pm i \diamond\left(\Lambda_{2}(x, y, z, t)\right)\right)}
\end{aligned}
$$


with

$$
\Lambda_{2}(x, y, z, t)=b_{1} x+b_{2} y+b_{3} z-4 b_{3} \int_{0}^{t} \frac{R_{4}(\tau)}{4+b_{1} b_{2} R_{6}(\tau)} d \tau .
$$

(III) Stochastic Travelling Wave Solutions of Trigonometric Type:

$$
\begin{aligned}
V_{4}(x, y, z, t)=V_{5}(x, y, z, t)= & \pm \sqrt{\frac{3 b_{2} b_{3} R_{4}(t)}{1-b_{1} b_{2} R_{5}(t) \diamond R_{6}(t)}} \pm \sqrt{\frac{6 b_{2} b_{3} R_{4}(t) \diamond R_{6}(t)}{1+2 b_{1} b_{2} R_{5}(t) \diamond R_{6}(t)}} \\
& \diamond\left(\sec ^{\diamond}\left(\Lambda_{3}(x, y, z, t)\right) \diamond \csc ^{\diamond}\left(\Lambda_{3}(x, y, z, t)\right)\right),
\end{aligned}
$$

with

$$
\begin{gathered}
\Lambda_{3}(x, y, z, t)=b_{1} x+b_{2} y+a_{3} z-b_{3} \int_{0}^{t} \frac{R_{4}(\tau)}{1+2 b_{1} b_{2} R_{6}(\tau)} d \tau \\
V_{6}(x, y, z, t)= \pm 3 \sqrt{\frac{3 b_{2} b_{3} R_{4}(t)}{9 b_{1} b_{2} R_{5}(t) \diamond R_{6}(t)-2}} \pm 4 \sqrt{\frac{3 b_{2} b_{3} R_{4}(t) \diamond R_{6}(t)}{2+15 b_{1} b_{2} R_{5}(t) \diamond R_{6}(t)}} \diamond\left(\cot ^{\diamond}\left(2 \Lambda_{4}(x, y, z, t)\right)\right. \\
\pm i \frac{\sqrt{3 b_{3} R_{4}(t)}}{2 \sqrt{b_{1} R_{5}(t) \diamond R_{6}(t) \diamond\left(\cot ^{\diamond}\left(2 \Lambda_{4}(x, y, z, t)\right)\right)}} \\
V_{7}(x, y, z, t)= \pm 3 \sqrt{\frac{3 b_{2} b_{3} R_{4}(t)}{9 b_{1} b_{2} R_{5}(t) \diamond R_{6}(t)-2}} \pm 4 \sqrt{\frac{3 b_{2} b_{3} R_{4}(t) \diamond R_{6}(t)}{2+15 b_{1} b_{2} R_{5}(t) \diamond R_{6}(t)}} \diamond\left(\tan ^{\diamond}\left(2 \Lambda_{4}(x, y, z, t)\right)\right. \\
\pm i \frac{\sqrt{3 b_{3} R_{4}(t)}}{2 \sqrt{b_{1} R_{5}(t) \diamond R_{6}(t) \diamond\left(\tan ^{\circ}\left(2 \Lambda_{4}(x, y, z, t)\right)\right)}}
\end{gathered}
$$

with

$$
\Lambda_{4}(x, y, z, t)=b_{1} x+b_{2} y+b_{3} z+2 b_{3} \int_{0}^{t} \frac{R_{4}(\tau)}{9 b_{1} b_{2} R_{6}(\tau)-2} d \tau,
$$

where $b_{i}(i=1,2,3)$ and $b^{*}$ are arbitrary constants satisfying $b_{i} b \neq 0$.

For Equation (3) one obtains the following stochastic travelling wave solution:

(I) Stochastic Travelling Wave Solution of Exponential Type:

$$
\begin{aligned}
& W_{1}(x, y, z, t)= \\
& \frac{ \pm i \sqrt{3 c_{1} R_{7}(t) \diamond\left(c_{1} c_{2} R_{8}(t) \operatorname{diamond}_{9}(t)-2\right)}\left(\exp ^{\diamond}\left(\Delta_{1}(x, y, z, t)\right)-1\right) \pm \sqrt{3 c_{1}^{2} c_{2} R_{7}(t) \diamond R_{8}(t)}}{\sqrt{c_{1} R_{8}(t) \diamond\left(c_{1} c_{2} R_{8}(t) \diamond R_{9}(t)-2\right)}\left(\exp ^{\diamond}\left(\Delta_{1}(x, y, z, t)\right)-1\right)}
\end{aligned}
$$

with

$$
\Delta_{1}(x, y, z, t)=c_{1} x+c_{2} y+c_{3} z+2 c_{1} \int_{0}^{t} \frac{R_{7}(\tau)}{c_{1} c_{2} R_{9}(\tau)-2} d \tau .
$$

(II) Stochastic Travelling Wave Solutions of Hyperbolic Type:

$$
\begin{aligned}
W_{2}(x, y, z, t)= & \pm \sqrt{\frac{3 c_{1} c_{2} R_{7}(t) \diamond R_{9}(t)}{R_{8}(t) \diamond\left(2-c_{1} c_{2} R_{9}(t)\right)}} \diamond\left(\operatorname{coth}^{\diamond}\left(\Delta_{2}(x, y, z, t)\right) \pm^{\diamond}\left(\Delta_{2}(x, y, z, t)\right)\right) \\
& \pm i \frac{\sqrt{3 b^{* *} c_{2} R_{7}(t)}}{\sqrt{2 R_{8}(t) \diamond R_{9}(t) \diamond\left(\operatorname{coth}^{\diamond}\left(\Delta_{2}(x, y, z, t)\right) \pm\left(\Delta_{2}(x, y, z, t)\right)\right)}}
\end{aligned}
$$




$$
\begin{aligned}
W_{3}(x, y, z, t)= & \pm \sqrt{\frac{3 c_{1} c_{2} R_{7}(t) \diamond R_{9}(t)}{R_{8}(t) \diamond\left(2-c_{1} c_{2} R_{9}(t)\right)}} \diamond\left(\tanh ^{\diamond}\left(\Delta_{2}(x, y, z, t)\right) \pm i^{\diamond}\left(\Delta_{2}(x, y, z, t)\right)\right) \\
& \pm i \frac{\sqrt{3 b^{* *} c_{2} R_{7}(t)}}{\sqrt{2 R_{8}(t) \diamond R_{9}(t)} \diamond\left(\tanh ^{\diamond}\left(\Delta_{2}(x, y, z, t)\right) \pm i \diamond\left(\Delta_{2}(x, y, z, t)\right)\right)}
\end{aligned}
$$

with

$$
\Delta_{2}(x, y, z, t)=c_{1} x+c_{2} y+c_{3} z-4 c_{1} \int_{0}^{t} \frac{R_{7}(\tau)}{4+c_{1} c_{2} R_{9}(\tau)} d \tau .
$$

(III) Stochastic Travelling Wave Solutions of Trigonometric Type:

$$
\begin{aligned}
W_{4}(x, y, z, t)=W_{5}(x, y, z, t)= & \pm \sqrt{\frac{3 c_{1} c_{2} R_{7}(t)}{1-c_{1} c_{2} R_{8}(t) \diamond R_{9}(t)}} \pm \sqrt{\frac{6 c_{1} c_{2} R_{7}(t) \diamond R_{9}(t)}{1+2 c_{1} c_{2} R_{8}(t) \diamond R_{9}(t)}} \\
& \diamond\left(\sec ^{\diamond}\left(\Delta_{3}(x, y, z, t)\right) \diamond \csc ^{\diamond}\left(\Delta_{3}(x, y, z, t)\right)\right)
\end{aligned}
$$

with

$$
\Delta_{3}(x, y, z, t)=c_{1} x+c_{2} y+c_{3} z-c_{1} \int_{0}^{t} \frac{R_{7}(\tau)}{1+2 c_{1} c_{2} R_{9}(\tau)} d \tau
$$

$$
\begin{aligned}
W_{6}(x, y, z, t)= & \pm 3 \sqrt{\frac{3 c_{1} c_{2} R_{7}(t)}{9 c_{1} c_{2} R_{8}(t) \diamond R_{9}(t)-2}} \pm 4 \sqrt{\frac{3 c_{1} c_{2} R_{7}(t) \diamond R_{9}(t)}{2+15 c_{1} c_{2} R_{8}(t) \diamond R_{9}(t)}} \diamond\left(\cot ^{\diamond}\left(2 \Delta_{4}(x, y, z, t)\right)\right. \\
& \pm i \frac{\sqrt{3 c_{1} R_{7}(t)}}{2 \sqrt{c_{1} R_{8}(t) \diamond R_{9}(t)} \diamond\left(\cot ^{\diamond}\left(2 \Delta_{4}(x, y, z, t)\right)\right)}
\end{aligned}
$$

$$
\begin{aligned}
W_{7}(x, y, z, t)= & \pm 3 \sqrt{\frac{3 c_{1} c_{2} R_{7}(t)}{9 c_{1} c_{2} R_{8}(t) \diamond R_{9}(t)-2}} \pm 4 \sqrt{\frac{3 c_{1} c_{2} R_{7}(t) \diamond R_{9}(t)}{2+15 c_{1} c_{2} R_{8}(t) \diamond R_{9}(t)}} \diamond\left(\tan ^{\diamond}\left(2 \Delta_{4}(x, y, z, t)\right)\right. \\
& \pm i \frac{\sqrt{3 c_{1} R_{7}(t)}}{2 \sqrt{c_{1} R_{8}(t) \diamond R_{9}(t) \diamond\left(\tan ^{\circ}\left(2 \Delta_{4}(x, y, z, t)\right)\right)}}
\end{aligned}
$$

with

$$
\Delta_{4}(x, y, z, t)=c_{1} x+c_{2} y+c_{3} z+2 c_{1} \int_{0}^{t} \frac{R_{7}(\tau)}{9 c_{1} c_{2} R_{9}(\tau)-2} d \tau,
$$

where $c_{i}(i=1,2,3)$ and $b^{* *}$ are arbitrary constants satisfying $c_{i} b \neq 0$.

\section{Example}

In this section, we provide a specific application example to demonstrate the effectiveness of our results and to justify the real contribution of these results. We focus our attention on Equation (1). Concerning the other two equations, Equations (2) and (3), the procedure is similar. We observe that the solutions of Equation (1) are strongly depend on the shape of the given functions $R_{1}(t)$ and $R_{2}(t)$. So, for dissimilar forms of $R_{1}(t)$ and $R_{2}(t)$, we can find dissimilar solutions of Equation (1) which come from Equations (70)-(78). We illustrate this by giving the following example.

Assume that $R_{2}(t)=\delta_{1} R_{1}(t), R_{3}(t)=\delta_{2} R_{1}(t)$ and $R_{1}(t)=f(t)+\delta_{3} W_{t}$, where $\delta_{1}, \delta_{2}$, and $\delta_{3}$ are arbitrary constants, $f(t)$ is a bounded measurable function on $\mathbb{R}_{+}$, and $W_{t}$ is the Gaussian white noise, which is the time derivative (in the strong sense in $(\mathcal{S})_{-1}$ ) of the Brownian motion $B_{t}$. The Hermite transform of $W_{t}$ is given by $\widetilde{W}_{t}(w)=\sum_{i=1}^{\infty} w_{i} \int_{0}^{t} \eta_{i}(\tau) d \tau$ [1]. Using the definition of $\widetilde{W}_{t}(w)$, Equations (70)-(78) yield the white noise functional solution of Equation (1) as follows: 


$$
\begin{aligned}
& U_{W_{1}}(x, y, z, t)= \\
& \frac{ \pm i \sqrt{3 a_{3}\left(a_{1} a_{2} \delta_{1} \delta_{2}\left(f(t)+\delta_{3} W_{t}\right)^{2}-2\right)}\left(\exp \left(\Omega_{1}(x, y, z, t)\right)-1\right) \pm \sqrt{3 a_{1} a_{2} a_{3} \delta_{1}}\left(f(t)+\delta_{3} W_{t}\right)}{\sqrt{a_{1} \delta_{1}\left(a_{1} a_{2} \delta_{1} \delta_{2}\left(f(t)+\delta_{3} W_{t}\right)^{2}-2\right)}\left(\exp \left(\Omega_{1}(x, y, z, t)\right)-1\right)}
\end{aligned}
$$

with

$$
\begin{gathered}
\Omega_{1}(x, y, z, t)=a_{1} x+a_{2} y+a_{3} z+2 a_{3} \int_{0}^{t} \frac{f(\tau)+\delta_{3} W_{\tau}}{a_{1} a_{2} \delta_{2}\left(f(\tau)+\delta_{3} W_{\tau}\right)-2} d \tau \\
\begin{aligned}
U_{W_{2}}(x, y, z, t)= & \pm \sqrt{\frac{3 a_{2} a_{3} \delta_{2}\left(f(t)+\delta_{3} W_{t}\right)}{\delta_{1}\left(2-a_{1} a_{2} \delta_{2}\left(f(t)+\delta_{3} W_{t}\right)\right)}}\left(\operatorname{coth}\left(\Omega_{2}(x, y, z, t)\right) \pm\left(\Omega_{2}(x, y, z, t)\right)\right) \\
& \pm i \frac{\sqrt{3 b a_{2}}}{\sqrt{2 \delta_{1} \delta_{2}\left(f(t)+\delta_{3} W_{t}\right)}\left(\operatorname{coth}\left(\Omega_{2}(x, y, z, t)\right) \pm\left(\Omega_{2}(x, y, z, t)\right)\right)} \\
U_{W_{3}}(x, y, z, t)= & \pm \sqrt{\frac{3 a_{2} a_{3} \delta_{2}\left(f(t)+\delta_{3} W_{t}\right)}{\delta_{1}\left(2-a_{1} a_{2} \delta_{2}\left(f(t)+\delta_{3} W_{t}\right)\right)}}\left(\tanh \left(\Omega_{2}(x, y, z, t)\right) \pm i\left(\Omega_{2}(x, y, z, t)\right)\right) \\
& \pm i \frac{\sqrt{3 b a_{2}}}{\sqrt{2 \delta_{1} \delta_{2}\left(f(t)+\delta_{3} W_{t}\right)}\left(\tanh \left(\Omega_{2}(x, y, z, t)\right) \pm i\left(\Omega_{2}(x, y, z, t)\right)\right)}
\end{aligned}
\end{gathered}
$$

with

$$
\begin{gathered}
\Omega_{2}(x, y, z, t)=a_{1} x+a_{2} y+a_{3} z-4 a_{3} \int_{0}^{t} \frac{f(\tau)+\delta_{3} W_{\tau}}{4+a_{1} a_{2} \delta_{2}\left(f(\tau)+\delta_{3} W_{\tau}\right)} d \tau \\
U_{W_{4}}(x, y, z, t)=U_{W_{5}}(x, y, z, t)= \pm \sqrt{\frac{3 a_{2} a_{3}\left(f(t)+\delta_{3} W_{t}\right)}{1-a_{1} a_{2} \delta_{1} \delta_{2}\left(f(t)+\delta_{3} W_{t}\right)^{2}}} \pm\left(f(t)+\delta_{3} W_{t}\right) \\
\times \sqrt{\frac{6 a_{2} a_{3} \delta_{2}}{1+2 a_{1} a_{2} \delta_{1} \delta_{2}\left(f(t)+\delta_{3} W_{t}\right)^{2}}}\left(\sec \left(\Omega_{3}(x, y, z, t)\right) \csc \left(\Omega_{3}(x, y, z, t)\right)\right),
\end{gathered}
$$

with

$$
\begin{aligned}
\Omega_{3}(x, y, z, t)= & a_{1} x+a_{2} y+a_{3} z-a_{3} \int_{0}^{t} \frac{f(\tau)+\delta_{3} W_{\tau}}{1+2 a_{1} a_{2} \delta_{2}\left(f(\tau)+\delta_{3} W_{\tau}\right)} d \tau \\
U_{W_{6}}(x, y, z, t)= & \pm 3 \sqrt{\frac{3 a_{2} a_{3}\left(f(t)+\delta_{3} W_{t}\right)}{9 a_{1} a_{2} \delta_{1} \delta_{2}\left(f(t)+\delta_{3} W_{t}\right)^{2}-2}} \pm 4\left(f(t)+\delta_{3} W_{t}\right) \\
& \times \sqrt{\frac{3 a_{2} a_{3} \delta_{2}}{2+15 a_{1} a_{2} \delta_{1} \delta_{2}\left(f(t)+\delta_{3} W_{t}\right)^{2}}}\left(\cot \left(2 \Omega_{4}(x, y, z, t)\right)\right) \\
& \pm i \frac{\sqrt{3 a_{3}}}{2 \sqrt{a_{1} \delta_{1} \delta_{2}\left(\cot \left(2 \Omega_{4}(x, y, z, t)\right)\right)}}, \\
U_{W_{6}}(x, y, z, t)= & \pm 3 \sqrt{\frac{3 a_{2} a_{3}\left(f(t)+\delta_{3} W_{t}\right)}{9 a_{1} a_{2} \delta_{1} \delta_{2}\left(f(t)+\delta_{3} W_{t}\right)^{2}-2}} \pm 4\left(f(t)+\delta_{3} W_{t}\right) \\
& \times \sqrt{\frac{3 a_{2} a_{3} \delta_{2}}{2+15 a_{1} a_{2} \delta_{1} \delta_{2}\left(f(t)+\delta_{3} W_{t}\right)^{2}}}\left(\tan \left(2 \Omega_{4}(x, y, z, t)\right)\right) \\
& \pm i \frac{\sqrt{3 a_{3}}}{2 \sqrt{a_{1} \delta_{1} \delta_{2}}\left(\tan \left(2 \Omega_{4}(x, y, z, t)\right)\right)}
\end{aligned}
$$


with

$$
\Omega_{4}(x, y, z, t)=a_{1} x+a_{2} y+a_{3} z+2 a_{3} \int_{0}^{t} \frac{f(\tau)+\delta_{3} W_{\tau}}{9 a_{1} a_{2} \delta_{2}\left(f(\tau)+\delta_{3} W_{\tau}\right)-2} d \tau
$$

\section{Conclusions}

Due to the fact that the stochastic models are more realistic than the deterministic models, we concentrated our study in this paper on the Wick-type, stochastic, (3+1)-dimensional modified BBM equations. Besides that, we investigated and solve the deterministic, (3+1)-dimensional modified BBM equations. In this paper, we set up a new and general version of the modified tanh-coth method to deal with the nonlinear multi dimensional PDEs. By using this generalisation of the modified tanh-coth method, Hermite transform, and white noise theory, we produced a new set of exact travelling wave solutions for the variable coefficients and (3+1)-dimensional modified BBM equations. This set includes solutions of exponential, hyperbolic, and trigonometric types. In [7], Wazwaz has solved the deterministic, (3+1)-dimensional modified BBM equations with constant coefficients, So, our results for this model are more general than the results obtained by him. With the aid of inverse Hermite transform, we obtained stochastic travelling wave solutions for the Wick-type, stochastic, (3+1)-dimensional modified BBM equations. Furthermore, we showed by an example how the stochastic solutions can be given as white noise functional solutions. Note that, the schema proposed in this paper can be used for solving several nonlinear evolution equations in mathematical physics, both Wick-type stochastic and deterministic. Moreover, the Riccati equation (19) has different solutions if we chose different values of $\alpha_{0}, \alpha_{1}$, and $\alpha_{2}$. Therefore, we can find many other solutions of the Wick-type stochastic and deterministic (3+1)-dimensional modified BBM equations.

The PDE of Benjamin et al. [2] is now often called the BBM equation, although it is also known as the regularised long wave (RLW) equation. Morrison et al. proposed the one-dimensional PDE, as an equally valid and accurate model for the same wave phenomena simulated by the KdV and RLW equations [40]. Random waves are an important subject of random PDEs. In essence, to investigate the exact solutions of random Benjamin-Bona-Mahony equation, we restricted our attention to consider this problem in a white noise environment [38]. The propagation of nonlinear wave in systems with polarity symmetry can be described by the (3+1)-dimensional modified Benjamin-Bona-Mahony Equation (7). If the problem is considered in a non-Gaussian stochastic environment, we can get non-Gaussian, stochastic, (2+1)-dimensional coupled KdV equation. Obviously, the planner which we have proposed in this paper can be also applied to other non-linear PDEs in mathematical physics such as KdV-Burgers, modified KdV-Burgers, Zhiber-Shabat and Benjamin-Bona-Mahony equations. We observe that the F-expansion method we used has many other particular solutions; this in turn gives many other exact solutions for the considered stochastic, $(3+1)$-dimensional modified Benjamin-Bona-Mahony equations. Additionally, in this work, we discussed the solutions of SPDEs driven by non-Gaussian white noise; this discussion is less detailed than the Gaussian discussion but more general, because it deals with the dual pairing generated by integration with respect to a non-Gaussian measure. Furthermore, in future work, we will discuss the solutions of SPDEs driven by non-Gaussian white noise to get exact stochastic solutions of the non-Gaussian, stochastic, (3+1)-dimensional modified Benjamin-Bona-Mahony equations; we only considered this problem in a non-Gaussian white noise environment; that is, we investigated the variable coefficients of stochastic, (3+1)-dimensional modified Benjamin-Bona-Mahony equations. For this aim, we developed a non-Gaussian Wick calculus based on the theory of hyper-complex systems to get exact travelling wave solutions of (3+1)-dimensional modified Benjamin-Bona-Mahony equations and non-Gaussian white noise functional solutions of Wick-type stochastic (3+1)-dimensional modified Benjamin-Bona-Mahony equations.

Author Contributions: The authors have equally contributed to the research.

Funding: This research was funded by the Deanship of Scientific Research at Princess Nourah bint Abdulrahman University through the Fast-track Research Funding Program. 
Conflicts of Interest: The authors declare no conflict of interest.

\section{References}

1. Holden, H.; Øsendal, B.; Ubøe, J.; Zhang, T. Stochastic Partial Differential Equations; Springer Science+Business Media, LLC: New York, NY, USA, 2010.

2. Benjamin, T.B.; Bona, J.L.; Mahony, J.J. Model equations for long waves in nonlinear dispersive systems. Trans. R. Soc. Lond. Ser. A 1972, 272, 47-78. [CrossRef]

3. Dehghan, M.; Shokri, A. A numerical method for solution of the two-dimensional sine-Gordon equation using the radial basis functions. Comput. Math. Simul. 2008, 79, 700-715. [CrossRef]

4. Hereman, W. Exact Solutions of Nonlinear Partial Differential Equations. The tanh/sech Method; Wolfram Research Academic Intern Program Inc.: Champaign, IL, USA, 2000; pp. 1-14.

5. Hereman, W. Shallow water waves and solitary waves. In Encyclopedia of Complexity and Systems Science; Meryers, R.A., Ed.; Springer: Heibelberg, Germany, 2009; pp. 1536-1620.

6. Khalique, C.M. Exact solutions and conservation laws of a coupled integrable dispersionless system. Filomat 2012, 26, 957-964. [CrossRef]

7. Wazwaz, A.M. Exact soliton and kink solutions for new (3+1)-dimensional nonlinear modified equations of wave propagation. Open Eng. 2017, 7, 169-174. [CrossRef]

8. Dai, C.Q.; Xu, Y.J. Exact solutions for a Wick-type stochastic reaction Duffing equation. Appl. Math. Model. 2015, 39, 7420-7426. [CrossRef]

9. Kong, L.Q.; Dai, C.Q. Some discussions about variable separation of nonlinear models using Riccati equation expansion method. Nonlinear Dyn. 2015, 81, 1553-1561. [CrossRef]

10. Wang, Y.Y.; Zhang, Y.P.; Dai, C.Q. Re-study on localized structures based on variable separation solutions from the modified tanh-function method. Nonlinear Dyn. 2015, 83, 1331-1339. [CrossRef]

11. Wadati, M. Stochastic Korteweg-de Vries equation. J. Phys. Soc. Jpn. 1983, 52, 2642-2648. [CrossRef]

12. Ghany, H.A.; Hyder, A. White noise functional solutions for the Wick-type two dimensional stochastic Zakharov-Kuznetsov equations. Int. Rev. Phys. 2012, 6, 153-157.

13. Ghany, H.A.; Hyder, A. The fractional coupled KdV equations: Exact solutions and white noise functional approach. Chin. Phys. B 2013, 22, 0805011. [CrossRef]

14. Ghany, H.A.; Hyder, A. Exact solutions for the Wick-type stochastic time-fractional KdV equations. Kuwait J. Sci. 2014, 41, 75-84.

15. Ghany, H.A.; Hyder, A. Abundant solutions of Wick-type stochastic fractional 2D KdV equations. Chin. Phys. B 2014, 23, 0605031. [CrossRef]

16. Ghany, H.A.; Hyder, A.; Zakarya, M. Non-gaussian white noise functional solutions of $\chi$-Wick-type stochastic KdV equations. Appl. Math. Inf. Sci. 2017, 11, 915-924. [CrossRef]

17. Ghany, H.A.; Hyder, A.; Zakarya, M. Exact Solutions of Stochastic Fractional Korteweg de-Vries Equation with Conformable Derivatives. Chin. Phys. B 2019, 28, 191092.

18. Chen, B.; Xie, Y.C. Exact solutions for generalized stochastic Wick- type KdV-mKdV equations. Chaos Solitons Fractals 2005, 23, 281-288. [CrossRef]

19. Chen, B.; Xie, Y.C. White noise functional solutions of Wick-type stochastic generalized Hirota-Satsuma coupled KdV equations. J. Comput. Appl. Math. 2006, 157, 345-354. [CrossRef]

20. Chen, B.; Xie, Y.C. Periodic-like solutions of variable coefficient and Wick-type stochastic NLS equations. J. Comput. Appl. Math. 2007, 203, 249-263. [CrossRef]

21. Hyder, A.; Zakarya, M. Non-Gaussian Wick calculus based on hypercomplex systems. Int. J. Pure Appl. Math. 2016, 109, 539-556. [CrossRef]

22. Hyder, A.; Zakarya, M. The Well-Posedness of Stochastic Kawahara Equation: Fixed Point Argument and Fourier Restriction Method. J. Egypt. Math. Soc. 2019, 27, 1-10.

23. Hyder, A. Wick-type stochastic KdV equation based on Lévy white noise. J. Math. Sci. Adv. Appl. 2017, 45, 1-20. [CrossRef]

24. Hyder, A. White noise analysis combined with hypercomplex systems for solving stochastic modified KdV equations with non-Gaussian parameters. Pioneer J. Adv. Appl. Math. 2018, 24, 39-61.

25. Agarwal, P.; Hyder, A.; Zakarya, M. Well-Posedness of Stochastic Modified Kawahara Equation. Adv. Differ. Equ. 2019, 2019, 423. 
26. Liu, X.Q.; Jiang, S.; Fan, W.B.; Liu, W.M. Soliton solutions in linear magnetic field and time-dependent laser field. Commun. Nonlinear Sci. Numer. Simul. 2004, 9, 361-365. [CrossRef]

27. Ivaz, K.; Mostahkam, B.S. Newton-Tau numerical solution of a system of nonlinear Fredholm integral equations of second kind. Appl. Comput. Math. 2006, 5, 201-208.

28. Wazwaz, A.M. New solitons and kinks solutions for the Gardner equation. Commun. Nonlinear Sci. Numer. Simul. 2007, 12, 1395-1404. [CrossRef]

29. Pourgholi, R.; Esfahani, A.; Houlari, T.; Foadian, S. An application of Sinc-Galerkin method for solving the Tzou equation. Appl. Comput. Math. 2017, 16, 257-268.

30. Momani, S.; Arqub, O.A.; Maayah, B.; Yousef, F.; Alsaedi, A. A reliable algorithm for solving linear and nonlinear Schrödinger equation. Appl. Comput. Math. 2018, 17, 151-160.

31. El-Ganaini, S.I.A.; Mirzazadeh, M.; Biswas, A. Solitons and other solutions to long-short wave resonance equation. Appl. Comput. Math. 2015, 14, 248-259.

32. Bhalekar, S.; Patade, J. Analytical solutions of nonlinear equations with proportional delays. Appl. Comput. Math. 2016, 15, 331-345.

33. Malfeit, W. Solitary wave solutions of nonlinear wave equations. Am. J. Phys. 1992, 60, 650-654.

34. Fan, E.G. Extended tanh-function method and its applications to nonlinear equations. Phys. Lett. A 2000, 277, 212-218. [CrossRef]

35. Wazwaz, A.M. The tanh-coth method for solitons and kink solutions for nonlinear parabolic equations. Appl. Math. Comput. 2007, 188, 1467-1475. [CrossRef]

36. El-Wakil, S.A.; El-Labany, S.K.; Zahran, M.A.; Sabry, R. Modified extended tanh-function method and its applications to nonlinear equations. Appl. Math. Comput. 2005, 161, 403-412.

37. Soliman, A.A. The modified extended tanh-function method for solving Burgers-type equations. Phys. A 2006, 361, 394-404. [CrossRef]

38. Ghany, H.A.; Fathallah, A. White-noise functional solutions for wick-type stochastic time-fractional Benjamin-Bona-Mahony equation. Int. J. Diff. Equ. Appl. 2014, 13, 37-49.

39. Sahoo, S.; Saha Ray, S.A. Novel approach for stochastic solutions of wick-type stochastic time-fractional Benjamin-Bona-Mahony equation for modeling long surface gravity waves of small amplitude. Stoch. Anal. Appl. 2019, 2019, 1-12. [CrossRef]

40. Korteweg, D.J.; de Vries, G. On the change of form of long waves advancing in a rectangular canal, and on a new type of long stationary waves. Phil. Mag. Ser. 1895, 39, 422-443. [CrossRef]

41. Larin, V.B. High-accuracy algorithms for solution of discrete periodic Riccati equations. Appl. Comput. Math. 2007, 6, 10-17.

42. Chen, H.; Zhang, H. New multiple soliton-like solutions to the generalized (2+1)-dimensional KP equation. Appl. Math. Comput. 2004, 157, 765-773. [CrossRef]

(C) 2019 by the authors. Licensee MDPI, Basel, Switzerland. This article is an open access article distributed under the terms and conditions of the Creative Commons Attribution (CC BY) license (http:/ / creativecommons.org/licenses/by/4.0/). 

Article

\title{
Best Proximity Points for Monotone Relatively Nonexpansive Mappings in Ordered Banach Spaces
}

\author{
Karim Chaira ${ }^{1,+}$, Mustapha Kabil ${ }^{1,+}$, Abdessamad Kamouss ${ }^{1, *,+}$ and Samih Lazaiz ${ }^{2,+}$ \\ 1 Laboratory of Mathematics and Applications, Department of Mathematics, Faculty of Sciences and \\ Technologies Mohammedia, University Hassan II Casablanca, Casablanca 20000, Morocco; \\ chaira_karim@yahoo.fr (K.C.); kabilfstm@gmail.com (M.K.) \\ 2 Laboratory of Mathematical Analysis and Applications, Department of Mathematics, Faculty of Sciences \\ Dhar El Mahraz, University Sidi Mohamed Ben Abdellah, Fes 30050, Morocco; samih.lazaiz@gmail.com \\ * Correspondence: akamouss@gmail.com \\ $\dagger$ These authors contributed equally to this work.
}

Received: 23 September 2019; Accepted: 25 October 2019; Published: 1 November 2019

\begin{abstract}
In this paper, we give sufficient conditions to ensure the existence of the best proximity point of monotone relatively nonexpansive mappings defined on partially ordered Banach spaces. An example is given to illustrate our results.
\end{abstract}

Keywords: best proximity point; fixed point; monotone mappings; relatively cyclic nonexpansive mappings; partially ordered Banach spaces

\section{Introduction}

Let $X$ be a Banach space and $(A, B)$ a pair of nonempty subsets of $X$. A cyclic mapping on $A \cup B$ is a mapping $T: A \cup B \rightarrow A \cup B$ such that $T(A) \subseteq B$ and $T(B) \subseteq A$. In case $A \cap B=\varnothing, T$ does not possess a fixed point, that is, a solution to the equation $T x=x$. Therefore, one can consider the following minimization problem:

$$
(P):\left\{\begin{array}{l}
\text { find }(x, y) \in A \times B \text { such that } \\
\|x-T x\|=\|y-T y\|=\operatorname{dist}(A, B) .
\end{array}\right.
$$

A point $x \in A \cup B$ is a best proximity point of $T$ if $x$ is a solution of the minimization problem $(P)$. The best proximity point notion can be seen as a generalization of fixed point notion since most fixed point theorems can be derived as corollaries of best proximity point theorems.

The first significant result of best proximity points was studied in [1], using the proximal normal structure, the authors proved that every cyclic relatively nonexpansive mapping from $A \cup B$ to itself has a best proximity point provided that $A$ and $B$ are weakly compact and convex. Furthermore, we find in [2] a similar result without invoking Zorn's lemma, i.e., without proximal normal structure. Recently, Chaira and Lazaiz [3] gave an extension of this last result in modular spaces. For a recent account of the theory we refer the reader to [4-6]. We can also find in ([7], pp. 27-31) an application of a best proximity point theorem to a system of differential equations.

On the other hand, the combination of metric fixed point theory and order theory allows Ran and Reurings in [8] to give a Banach Contraction Principle in partially ordered metric spaces. As consequence, they solved a matrix equation. Nieto and Rodríguez-López [9], extended the Ran-Reurings theorem in order to obtain a periodic solution for a first-order ordinary differential equation with periodic boundary conditions.

Recently, many authors studied the existence of fixed points of monotone nonexpansive mappings defined on partially ordered Banach spaces (see for example [10-15]). Recall that a self mapping $T$ 
on $X$ is said to be monotone nonexpansive if $T$ is monotone and $\|T x-T y\| \leq\|x-y\|$, for every comparable elements $x$ and $y$. We should mention that monotone nonexpansive mappings may not be continuous. The interested reader can consult the book of Carl and Heikkilä [16] for many applications of fixed point results of monotone mappings.

In this work, motivated by the recent study of a fixed point for monotone mappings, we investigate the existence of the best proximity point of monotone relatively nonexpansive mappings in partially ordered Banach spaces.

\section{Preliminaries and Basic Results}

Let $(X,\|\|$.$) be a Banach space endowed with a partial order \preceq$. Throughout, we assume that the order intervals are closed and convex. Recall that an order interval is any of the subsets

$$
[a, \rightarrow)=\{x \in X ; a \preceq x\} \quad, \quad(\leftarrow, a]=\{x \in X ; x \preceq a\}
$$

for any $a \in X$. As a direct consequence of this, the subset

$$
[a, b]=\{x \in X ; a \preceq x \preceq b\}=[a, \rightarrow) \cap(\leftarrow, b]
$$

is also closed and convex for any $a, b \in X$.

We will say that $x, y \in X$ are comparable whenever $x \preceq y$ or $y \preceq x$. The linear structure of $X$ is assumed to be compatible with the order structure in the following sense:

(i) $\quad x \preceq y$ implies $x+z \preceq y+z$ for all $x, y, z \in X$;

(ii) $\quad x \preceq y$ implies $\alpha x \preceq \alpha y$ for all $x, y \in X$ and $\alpha \in \mathbb{R}^{+}$.

Let us recall the definition of a uniformly convex Banach space.

Definition 1. Let $(X,\|\|$.$) be a Banach space. We say that X$ is uniformly convex (in short, UC) if for every $\epsilon>0$ we have $\delta(\epsilon)>0$ such that

$$
\delta(\epsilon)=\inf \left\{1-\left\|\frac{x+y}{2}\right\| ;\|x\| \leq 1 ;\|y\| \leq 1 ;\|x-y\| \geq \epsilon\right\} .
$$

The function $\delta$ is known as the modulus of uniform convexity of X. Note that any UC Banach space is reflexive.

A sequence $\left\{x_{n}\right\}_{n \in \mathbb{N}}$ in a partially ordered set $(X, \preceq)$ is said to be

(i) monotone increasing if $x_{n} \preceq x_{n+1}$, for all $n \in \mathbb{N}$;

(ii) monotone decreasing if $x_{n+1} \preceq x_{n}$, for all $n \in \mathbb{N}$;

(iii) monotone sequence if it is either monotone increasing or decreasing.

The following technical lemmas will be useful to establish the main results.

Lemma 1. Let $X$ be a Banach space endowed with a partial order $\preceq$. Assume that $\left\{x_{n}\right\}$ and $\left\{y_{n}\right\}$ are two sequences on $X$ which are weakly convergent to $\bar{x}$ and $\bar{y}$ respectively and $x_{n} \preceq y_{n}$ for any $n \in \mathbb{N}$, then

$$
\bar{x} \preceq \bar{y} .
$$

Proof. Note that the positive sequence $\left\{y_{n}-x_{n}\right\}_{n}$ converges weakly to $\bar{y}-\bar{x}$. Since closed convex subsets are also weakly closed, the positive cone is weakly closed and so we conclude that $\bar{y}-\bar{x}$ is positive.

Lemma 2. [17] Let $\left\{x_{n}\right\}$ be a bounded monotone sequence in $X$, and assume that $X$ is reflexive. Then $\left\{x_{n}\right\}$ is weakly convergent. 
Lemma 3. [18] Let $C$ be a nonempty closed convex subset of a UC Banach space $(X,\|\|$.$) . Let \tau: C \rightarrow[0, \infty)$ be a type function, i.e., there exists a bounded sequence $\left\{x_{n}\right\} \in X$ such that

$$
\tau(x)=\limsup _{n \rightarrow \infty}\left\|x_{n}-x\right\| \text {, }
$$

for every $x \in C$. Then $\tau$ has a unique minimum point $z \in C$ such that

$$
\tau(z)=\inf \{\tau(x) ; x \in C\}=\tau_{0} .
$$

Moreover, if $\left\{z_{n}\right\}$ is a minimizing sequence in $C$, i.e., $\lim _{n \rightarrow \infty} \tau\left(z_{n}\right)=\tau_{0}$, then $\left\{z_{n}\right\}$ converges strongly to $z$.

The norm $\|$.$\| of X$ is said to be monotone if

$$
u \preceq v \preceq w \text { implies } \max \{\|w-v\|,\|v-u\|\} \leq\|w-u\|,
$$

for any $u, v, w \in X$. If the norm is monotone and $\left\{x_{n}\right\}$ is monotone increasing (respectively, decreasing), then the sequence $\left\{\left\|x_{n}-y\right\|\right\}$ is decreasing for any $y$ such that $x_{n} \preceq y$ (respectively, $y \preceq x_{n}$ ), for any $n \in \mathbb{N}$. In this case,

$$
\liminf _{n \rightarrow \infty}\left\|x_{n}-y\right\|=\lim _{n \rightarrow \infty}\left\|x_{n}-y\right\|=\inf _{n \in \mathbb{N}}\left\|x_{n}-y\right\| .
$$

Recall that a mapping $T: X \rightarrow X$ is said to be

(i) monotone increasing if $x \preceq y$ implies $T(x) \preceq T(y)$, for all $x, y \in X$;

(ii) monotone decreasing if $x \preceq y$ implies $T(y) \preceq T(x)$, for all $x, y \in X$.

We conclude this section by extending the concept of relatively cyclic nonexpansive mapping to monotone relatively cyclic nonexpansive mapping as follows:

Definition 2. Let $(X,\|\|,. \preceq)$ be a Banach space endowed with a partially order and $(A, B)$ a pair of nonempty subset of $X$. The mapping $T: A \cup B \rightarrow A \cup B$ is said to be monotone increasing (respectively decreasing) relatively cyclic nonexpansive if

1. $T(A) \subseteq B$ and $T(B) \subseteq A$,

2. $T$ is monotone increasing (respectively decreasing),

3. $\|T x-T y\| \leq\|x-y\|$, whenever $x \in A$ and $y \in B$ are comparables.

\section{Main Result}

Throughout we assumed that $(X,\|\cdot\|, \preceq)$ is a Banach space endowed with a partial order for which order intervals are convex and closed and the linear structure of $X$ is assumed to be compatible with the order structure.

The following result gives sufficient conditions to obtain a fixed point theorem for a monotone increasing relatively cyclic nonexpansive mapping.

Theorem 1. Let $(A, B)$ be a nonempty bounded closed convex pair in a partially ordered Banach space $(X,\|\cdot\|, \preceq$ ). Assume that $(X,\|\|$.$) is UC. Let T: A \cup B \rightarrow A \cup B$ be a monotone increasing relatively cyclic nonexpansive mapping such that $x_{0} \preceq T x_{0}$ for some $x_{0} \in A$, then $A \cap B \neq \varnothing$ and there exists $a^{*} \in A \cap B$ such that $T a^{*}=a^{*}$.

Proof. We assume that $x_{0} \preceq T x_{0}$ and we define the sequence $\left\{x_{n}\right\}$ by $x_{n+1}=T x_{n}$ for all $n \geq 0$. By using the monotonicity of $T$ we get

$$
x_{0} \preceq x_{1} \preceq \cdots \preceq x_{n} \preceq x_{n+1} \preceq \cdots .
$$


Since $A$ and $B$ are bounded and closed, the sequence $\left\{x_{n}\right\}$ is bounded increasing in the reflexive space $X$. By Lemma 2,

$$
x_{2 n} \stackrel{w}{\longrightarrow} \bar{x}_{1} \in A \quad \text { and } \quad x_{2 n+1} \stackrel{w}{\longrightarrow} \bar{x}_{2} \in B .
$$

By uniqueness of the weak limit, $\bar{x}=\bar{x}_{1}=\bar{x}_{2}$. We claim that $A \cap B \neq \varnothing$.

Let $K=\left\{x \in A \cap B, \quad x_{n} \preceq x\right.$ for all $\left.n \in \mathbb{N}\right\}$. It is clear that $K$ is nonempty, closed and convex set. Since $\left\{x_{n}\right\}$ is a bounded sequence in $X$, we can define the type function as follows

$$
\tau(x)=\limsup _{n \rightarrow \infty}\left\|x_{n}-x\right\|,
$$

for any $x \in K$. From Lemma 3, it follows that there exists a unique $a^{*} \in K$ such that

$$
\tau\left(a^{*}\right)=\inf _{x \in K} \tau(x)
$$

We have

$$
\tau\left(T a^{*}\right)=\limsup _{n \rightarrow \infty}\left\|x_{n}-T a^{*}\right\|=\limsup _{n \rightarrow \infty}\left\|T x_{n-1}-T a^{*}\right\| .
$$

Since $x_{n-1} \preceq a^{*}$ and $T$ is monotone relatively cyclic nonexpansive mapping,

$$
\tau\left(T a^{*}\right) \leq \limsup _{n \rightarrow \infty}\left\|x_{n-1}-a^{*}\right\|=\tau\left(a^{*}\right) .
$$

Hence, $\tau\left(T a^{*}\right)=\tau\left(a^{*}\right)$. Thus $T a^{*}=a^{*}$, which completes the proof.

If $B=A$, we get the next result for a monotone nonexpansive mapping.

Corollary 1. Let A be a nonempty bounded closed convex set in a partially ordered Banach space $(X,\|\|,. \preceq)$. Let $T: A \rightarrow A$ be a monotone increasing nonexpansive mapping. Assume that $(X,\|\|$.$) is U C$ and there exists $x_{0} \in A$ such that $x_{0} \preceq T x_{0}$, then there exists $a^{*} \in A$ such that $T a^{*}=a^{*}$.

Now let $\left(\mathcal{A}_{0}^{\preceq}, \mathcal{B}_{0}^{\preceq}\right)$ denotes the pair obtained from $(A, B)$ upon setting

$$
\begin{aligned}
& \mathcal{A}_{0}^{\preceq}=\{x \in A ;\|x-y\|=\operatorname{dist}(A, B) \text { for some } y \in B \cap[x, \rightarrow)\} \\
& \mathcal{B}_{0}^{\preceq}=\{y \in B ;\|y-x\|=\operatorname{dist}(A, B) \text { for some } x \in A \cap(\leftarrow, y]\} .
\end{aligned}
$$

Lemma 4. Let $(A, B)$ be a nonempty bounded closed convex pair in a partially ordered reflexive Banach space $(X,\|\cdot\|, \preceq)$. Then,

(i) $\mathcal{A}_{0}^{\prec} \neq \varnothing$ if and only if $\mathcal{B}_{0}^{\preceq} \neq \varnothing$;

(ii) $\operatorname{dist}\left(\mathcal{A}_{0}^{\preceq}, \mathcal{B}_{0}^{\preceq}\right)=\operatorname{dist}(A, B)$;

(iii) $\left(\mathcal{A}_{0}^{\preceq}, \mathcal{B}_{0}^{\preceq}\right)$ is a closed pair;

(iv) $\left(\mathcal{A}_{0}^{\preceq}, \mathcal{B}_{0}^{\preceq}\right)$ is a convex pair.

Proof. Using the definitions of $\mathcal{A}_{0}^{\prec}$ and $\mathcal{B}_{0}^{\preceq}$, we can easily derive $(i)$ and $(i i)$.

(iii) Let $\left\{x_{n}\right\} \subset \mathcal{A}_{0}^{\prec}$ be a sequence which converges to some $\bar{x}$ in $A$. Then there exists a sequence $\left\{y_{n}\right\} \subset B$ such that

$$
\left\|x_{n}-y_{n}\right\|=\operatorname{dist}(A, B) \text { and } x_{n} \preceq y_{n}
$$

Since $B$ is closed and bounded in a reflexive Banach space, there exists a subsequence $\left\{y_{\varphi(n)}\right\}$ of $\left\{y_{n}\right\}$ such that $y_{\varphi(n)} \stackrel{w}{\rightarrow} \bar{y} \in B$. From Lemma 1 , it follows that $\bar{x} \preceq \bar{y}$. On the other hand,

$$
\|\bar{x}-\bar{y}\| \leq \liminf _{n \rightarrow \infty}\left\|x_{\varphi(n)}-y_{\varphi(n)}\right\|=\operatorname{dist}(A, B) .
$$


Therefore, we have $\bar{x} \in \mathcal{A}_{0}^{\preceq}$, and hence, $\mathcal{A}_{0}^{\preceq}$ is closed. By the same arguments we get that $\mathcal{B}_{0}^{\preceq}$ is also closed.

(iv) Let $x$ and $x^{\prime}$ in $\mathcal{A}_{0}^{\preceq}$. Then there exist $y$ and $y^{\prime}$ in $B$ such that

$$
\left\{\begin{array}{c}
\|x-y\|=\operatorname{dist}(A, B) \quad \text { and } \quad x \preceq y, \\
\left\|x^{\prime}-y^{\prime}\right\|=\operatorname{dist}(A, B) \text { and } \quad x^{\prime} \preceq y^{\prime} .
\end{array}\right.
$$

By using the fact that the linear structure of $X$ is compatible with the order structure, we get for any $t \in[0,1]$

$$
\begin{aligned}
\left\|t x+(1-t) x^{\prime}-t y-(1-t) y^{\prime}\right\| & =\left\|t(x-y)+(1-t)\left(x^{\prime}-y^{\prime}\right)\right\| \\
& \leq t\|x-y\|+(1-t)\left\|x^{\prime}-y^{\prime}\right\| \\
& =\operatorname{dist}(A, B) .
\end{aligned}
$$

This implies that $t x+(1-t) x^{\prime} \in \mathcal{A}_{0}^{\preceq}$. It follows that $\mathcal{A}_{0}^{\prec}$ is convex, as claimed. Similarly we prove that $\mathcal{B}_{0}^{\preceq}$ is also convex.

Remark 1. Note that if $T$ is a monotone decreasing relatively cyclic nonexpansive mapping, we have $T\left(\mathcal{A}_{0}^{\preceq}\right) \subset$ $\mathcal{B}_{0}^{\preceq}$ and $T\left(\mathcal{B}_{0}^{\preceq}\right) \subset \mathcal{A}_{0}^{\preceq}$. Indeed, let $x \in \mathcal{A}_{0}^{\preceq}$ then there exists $y \in B$ such that

$$
\|x-y\|=\operatorname{dist}(A, B) \quad \text { and } x \preceq y .
$$

Thus,

$$
\|T x-T y\| \leq\|x-y\|=\operatorname{dist}(A, B) \text { and } T y \preceq T x .
$$

This implies $T x \in \mathcal{B}_{0}^{\preceq}$. Consequently $T\left(\mathcal{A}_{0}^{\preceq}\right) \subset \mathcal{B}_{0}^{\preceq}$.

For the sake of simplicity, we use the following notation

$$
\mathcal{A}_{T}=\left\{\left(x_{0}, x_{0}^{\prime}\right) \in A \times A ; x_{0} \preceq T x_{0}^{\prime} ;\left\|x_{0}-T x_{0}^{\prime}\right\|=\operatorname{dist}(A, B)\right\} .
$$

The next lemma gives sufficient conditions such that $\mathcal{A}_{T}$ is nonempty.

Lemma 5. Let $(A, B)$ be a nonempty bounded closed convex pair in a partially ordered Banach space $(X,\|\cdot\|, \preceq)$ such that $\mathcal{A}_{0}^{\prec}$ is nonempty. Let $T: A \cup B \rightarrow A \cup B$ be a monotone relatively cyclic nonexpansive mapping. Then $\mathcal{A}_{T}$ is nonempty.

Proof. Suppose that $T$ is a monotone decreasing relatively cyclic nonexpansive mapping. Since $\mathcal{A}_{0}^{\prec} \neq$ $\varnothing$, we can find a $x_{0}^{\prime}$ in $\mathcal{A}_{0}^{\prec}$ such that there exists an $y \in B \cap\left[x_{0}^{\prime}, \rightarrow\right)$ satisfying $\left\|x_{0}^{\prime}-y\right\|=\operatorname{dist}(A, B)$.

Since $x_{0}^{\prime} \preceq y$ and $T$ is monotone decreasing relatively cyclic nonexpansive mapping, $T y \preceq T x_{0}^{\prime}$ and $\left\|T x_{0}^{\prime}-T y\right\| \leq\left\|x_{0}^{\prime}-y\right\|=\operatorname{dist}(A, B)$, give that $T x_{0}^{\prime} \in \mathcal{B}_{0}^{\preceq}$.

Next, for $T x_{0}^{\prime}$ there exists an element $x_{0} \in \mathcal{A}_{0}^{\prec}$ such that

$$
x_{0} \preceq T x_{0}^{\prime} \quad \text { and } \quad\left\|x_{0}-T x_{0}^{\prime}\right\|=\operatorname{dist}(A, B) .
$$

Now, suppose that $T$ is a monotone increasing relatively cyclic nonexpansive mapping. Since $\mathcal{A}_{0}^{\preceq} \neq \varnothing$, we can find a $x$ in $\mathcal{A}_{0}^{\preceq}$ such that there exists an $y \in \mathcal{B}_{0}^{\preceq}$ satisfying $x \preceq y$ and $\|x-y\|=\operatorname{dist}(A, B)$. 
Since $T$ is monotone increasing, $T x \preceq T y$ and

$$
\left\|T^{2} x-T^{2} y\right\| \leq\|T x-T y\| \leq\|x-y\|=\operatorname{dist}(A, B) .
$$

Take $x_{0}=T^{2} x \in A$ and $x_{0}^{\prime}=T y \in A$. We have clearly,

$$
x_{0} \preceq T x_{0}^{\prime} \quad \text { and } \quad\left\|x_{0}-T x_{0}^{\prime}\right\|=\operatorname{dist}(A, B) .
$$

Thus $\mathcal{A}_{T} \neq \varnothing$.

In the following, we give a best proximity result for monotone increasing relatively cyclic nonexpansive mapping.

Theorem 2. Let $(X,\|\|,. \preceq)$ be a partially ordered Banach space. Assume that $(X,\|\|$.$) is UC. Let (A, B)$ be a nonempty bounded closed convex pair in $X$. Let $T: A \cup B \rightarrow A \cup B$ be a monotone increasing relatively cyclic nonexpansive mapping. Assume that $T$ is weakly sequentially continuous, the norm $\|$.$\| of X$ is monotone and there exists $\left(x_{0}, x_{0}^{\prime}\right) \in \mathcal{A}_{T}$ such that $x_{0} \preceq x_{0}^{\prime} \preceq T^{2} x_{0}$ then there exist $\bar{x} \in A$ and $\bar{y} \in B$ such that $\|\bar{x}-T \bar{x}\|=\|\bar{y}-T \bar{y}\|=\operatorname{dist}(A, B)$.

Proof. Suppose that there exists $\left(x_{0}, x_{0}^{\prime}\right) \in A \times A$ such that

$$
\left\|x_{0}-T x_{0}^{\prime}\right\|=\operatorname{dist}(A, B) \quad \text { and } \quad x_{0} \preceq x_{0}^{\prime} \preceq T^{2} x_{0} .
$$

Let $\left\{x_{n}\right\}$ and $\left\{y_{n}\right\}$ be two sequences defined as follows:

$$
\left\{\begin{array}{l}
x_{n}=T^{2 n} x_{0} \\
y_{n}=T^{2 n+1} x_{0}^{\prime}
\end{array} \quad \text { for all } n \in \mathbb{N} .\right.
$$

Note that, since $x_{0} \preceq T x_{0}^{\prime}$ we get $T^{2 n} x_{0} \preceq T^{2 n+1} x_{0}^{\prime}$ for all $n \geq 0$, that is, $x_{n} \preceq y_{n}$ for all $n \geq 0$.

Since $T$ is monotone increasing relatively cyclic nonexpansive mapping, we get

$$
\left\|x_{n}-y_{n}\right\|=\left\|T^{2 n} x_{0}-T^{2 n+1} x_{0}^{\prime}\right\| \leq\left\|x_{0}-T x_{0}^{\prime}\right\|=\operatorname{dist}(A, B),
$$

that is, $\left\|x_{n}-y_{n}\right\|=\operatorname{dist}(A, B)$, for all $n \in \mathbb{N}$.

Since $x_{0} \preceq T^{2} x_{0}, x_{1}=T^{2} x_{0} \preceq T^{4} x_{0}=x_{2}$ and by induction on $n$, we can get

$$
x_{n} \preceq x_{n+1} \text { for all } n \in \mathbb{N} \text {. }
$$

In the same manner, we get

$$
y_{n} \preceq y_{n+1} \quad \text { for all } n \in \mathbb{N} \text {. }
$$

Since $\left\{x_{n}\right\}$ and $\left\{y_{n}\right\}$ are bounded increasing sequences in reflexive space, we get from Lemma 2, $x_{n} \stackrel{w}{\rightarrow} \bar{x}$ and $y_{n} \stackrel{w}{\rightarrow} \bar{y}$.

Note that $\bar{x}=\sup \left\{x_{n} ; n \in \mathbb{N}\right\}$ and $\bar{y}=\sup \left\{y_{n} ; n \in \mathbb{N}\right\}$.

Let $K=\left\{y \in B ; y_{n} \preceq y, \quad\right.$ for any $\left.n \in \mathbb{N}\right\}$ and define the type function $\tau: K \rightarrow[0, \infty)$ generated by the sequence $\left\{x_{n}\right\}$, that is,

$$
\tau(y)=\limsup _{n \rightarrow \infty}\left\|x_{n}-y\right\|,
$$

for $y \in K$. Using the fact that $\tau$ is increasing function, we get

$$
\tau(\bar{y})=\inf _{y \in K} \tau(y)
$$


Indeed, let $z_{1}, z_{2} \in K$ such that $z_{1} \preceq z_{2}$ then for all $n \in \mathbb{N}$ we have

$$
x_{n} \preceq y_{n} \preceq z_{1} \preceq z_{2} .
$$

Using the fact that the norm $\|$.$\| is monotone, we get$

$$
\left\|x_{n}-z_{1}\right\| \leq\left\|x_{n}-z_{2}\right\|
$$

hence,

$$
\tau\left(z_{1}\right) \leq \tau\left(z_{2}\right)
$$

From Lemma 3, it follows that there exists a unique $b^{*} \in K$ such that :

$$
\tau\left(b^{*}\right)=\inf _{y \in K} \tau(y)
$$

Since $\bar{y}=\sup \left\{y_{n} ; n \in \mathbb{N}\right\}$ and $b^{*} \in K, \bar{y} \preceq b^{*}$, that is, $\tau(\bar{y}) \preceq \tau\left(b^{*}\right)$.

Thus, $\tau(\bar{y})=\tau\left(b^{*}\right)$, i.e., $\bar{y}=b^{*}$.

We have also

$$
\begin{aligned}
\tau\left(T^{2} \bar{y}\right) & =\limsup _{n \rightarrow \infty}\left\|x_{n}-T^{2} \bar{y}\right\| \\
& =\limsup _{n \rightarrow \infty}\left\|T^{2} x_{n-1}-T^{2} \bar{y}\right\| \\
& \leq \limsup _{n \rightarrow \infty}\left\|x_{n-1}-\bar{y}\right\| \\
& =\tau(\bar{y})
\end{aligned}
$$

hence, $T^{2} \bar{y}=\bar{y}$.

Furthermore, $T$ is weakly sequentially continuous then $T x_{n} \stackrel{w}{\rightarrow} T \bar{x}$ and $T y_{n} \stackrel{w}{\rightarrow} T \bar{y}$. By the lower semi continuity of the norm, we get

$$
\|\bar{x}-\bar{y}\| \leq \liminf _{n \rightarrow \infty}\left\|x_{n}-y_{n}\right\|=\operatorname{dist}(A, B) .
$$

Let $\left\{x_{n}^{\prime}\right\}$ be a sequence defined by $x_{n}^{\prime}=T^{2 n} x_{0}^{\prime}$, for all $n \in \mathbb{N}$. We have

$$
y_{n}=T^{2 n+1} x_{0}^{\prime}=T\left(T^{2 n} x_{0}^{\prime}\right)=T x_{n}^{\prime} .
$$

Since $x_{0}^{\prime} \preceq T^{2} x_{0}^{\prime}, T^{2 n} x_{0}^{\prime} \preceq T^{2 n+2} x_{0}^{\prime}$, that is, $x_{n}^{\prime} \preceq x_{n+1}^{\prime}$, for all $n \in \mathbb{N}$. Since $\left\{x_{n}^{\prime}\right\}$ is bounded increasing sequence in reflexive space, we get by using Lemma $2 x_{n}^{\prime} \stackrel{w}{\longrightarrow} \bar{x}^{\prime}$. Since $T$ is weakly sequentially continuous, $y_{n}=T x_{n}^{\prime} \stackrel{w}{\rightarrow} T \bar{x}^{\prime}$. By the uniqueness of the limit, $T \bar{x}{ }^{\prime}=\bar{y}$, that is,

$$
\left\|\bar{x}-T \overline{x^{\prime}}\right\|=\operatorname{dist}(A, B) .
$$

Note that $x_{0} \preceq x_{0}^{\prime} \preceq T^{2} x_{0} \preceq T^{2} x_{0}^{\prime}$, that is, $x_{0} \preceq x_{0}^{\prime} \preceq x_{1} \preceq x_{1}^{\prime}$. Then, by induction on $n$, we can get

$$
x_{n} \preceq x_{n}^{\prime} \preceq x_{n+1} \preceq x_{n+1}^{\prime} .
$$

Define the sequence $\left\{z_{n}\right\}$ as follows

$$
z_{n}=\left\{\begin{array}{cc}
x_{n} & \text { if } n \text { is even } \\
x_{n-1}^{\prime} & \text { if } n \text { is odd }
\end{array}\right.
$$


Since $\left\{z_{n}\right\}$ is bounded increasing sequence in reflexive space, by using Lemma 2, we get $z_{n} \stackrel{w}{\longrightarrow}$. In particular, the subsequences $\left\{z_{2 n}\right\}$ and $\left\{z_{2 n+1}\right\}$ also converge to $\bar{z}$, that is, $\bar{z}=\bar{x}=\bar{x}^{\prime}$. Thus, by using (3) we get $\|\bar{x}-T \bar{x}\|=\operatorname{dist}(A, B)$.

In the following, we give a best proximity result for monotone decreasing relatively cyclic nonexpansive mapping without assuming the monotonicity of the norm $\|\cdot\|$.

Theorem 3. Let $(A, B)$ be a nonempty bounded closed convex pair in a partially ordered Banach space $(X,\|\|,. \preceq)$. Let $T: A \cup B \rightarrow A \cup B$ be a monotone decreasing relatively cyclic nonexpansive mapping. Assume that $(X,\|\|$.$) is UC, T$ is weakly sequentially continuous and there exists $\left(x_{0}, x_{0}^{\prime}\right) \in \mathcal{A}_{T}$ such that $x_{0}^{\prime} \preceq x_{0} \preceq T^{2} x_{0}^{\prime}$, then there exists $(\bar{x}, \bar{y}) \in A \times B$ such that

$$
\|\bar{x}-T \bar{x}\|=\|\bar{y}-T \bar{y}\|=\operatorname{dist}(A, B) .
$$

Proof. Let $\left(x_{0}, x_{0}^{\prime}\right) \in \mathcal{A}_{T}$ such that

$$
x_{0}^{\prime} \preceq x_{0} \preceq T^{2} x_{0}^{\prime} .
$$

If $A \cap B \neq \varnothing$ then $x_{0}=T x_{0}^{\prime}$ by Lemma 5. Since $x_{0}^{\prime} \preceq x_{0} \preceq T^{2} x_{0}^{\prime}$ and $T$ is decreasing, we get $x_{0} \preceq T x_{0}$ and $T x_{0} \preceq T x_{0}^{\prime}=x_{0}$. Thus, $T x_{0}=x_{0}$.

If $A \cap B=\varnothing$, then we consider the sequences $\left\{x_{n}\right\}$ and $\left\{z_{n}\right\} \subset A$ defined by

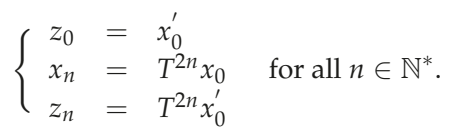

Since $x_{0}^{\prime} \preceq x_{0} \preceq T^{2} x_{0}^{\prime}=z_{1}$ and $T^{2}$ is a monotone increasing mapping, by induction on $n$, we get $T^{2 n} x_{0}^{\prime} \preceq T^{2 n} x_{0} \preceq T^{2 n+2} x_{0}^{\prime}$, which implies

$$
z_{n} \preceq x_{n} \preceq z_{n+1},
$$

for all $n \geq 0$. Also, since $x_{0} \preceq T x_{0}^{\prime}=T z_{0}$ and $T^{2}$ is a monotone increasing mapping, by induction on $n$, we get $T^{2 n} x_{0} \preceq T\left(T^{2 n} x_{0}^{\prime}\right)$, which implies

$$
x_{n} \preceq T z_{n},
$$

for all $n \geq 0$. The sequences $\left\{x_{n}\right\}$ and $\left\{z_{n}\right\}$ are increasing. Indeed, $x_{0} \preceq T^{2} x_{0}^{\prime} \preceq T^{2} x_{0}$ implies by induction on $n$ that $T^{2 n} x_{0} \preceq T^{2 n+2} x_{0}$. Thus,

$$
x_{n} \preceq x_{n+1}
$$

for all $n \in \mathbb{N}$. Since $\left\{x_{n}\right\}$ and $\left\{z_{n}\right\}$ are bounded increasing sequences in a reflexive space, we get by Lemma $2, x_{n} \stackrel{w}{\longrightarrow} \bar{x}$ and $z_{n} \stackrel{w}{\longrightarrow} \bar{z}$. Using the fact that $T$ is weakly sequentially continuous we conclude that $T z_{n} \stackrel{w}{\longrightarrow} T \bar{z}$.

Since $T$ is relatively cyclic nonexpansive mapping, we get

$$
\begin{aligned}
\left\|x_{n}-T z_{n}\right\| & =\left\|T^{2} x_{n-1}-T^{3} z_{n-1}\right\| \\
& \leq\left\|T x_{n-1}-T^{2} z_{n-1}\right\| \\
& \leq\left\|x_{n-1}-T z_{n-1}\right\|
\end{aligned}
$$

for all $n$ in $\mathbb{N}^{*}$. By induction on $n$, we prove that

$$
\left\|x_{n}-T z_{n}\right\| \leq\left\|x_{0}-T x_{0}^{\prime}\right\|=\operatorname{dist}(A, B),
$$


for all $n \in \mathbb{N}$. By the lower semi continuity of the norm, we get

$$
\|\bar{x}-T \bar{z}\| \leq \liminf _{n \rightarrow \infty}\left\|x_{n}-T z_{n}\right\|=\operatorname{dist}(A, B) .
$$

It follows from the Lemma 1 and the inequality (4) that $\bar{z} \preceq \bar{x} \preceq \bar{z}$, and hence, $\bar{z}=\bar{x}$.

Finally, by Equation (6) it follows that

$$
\|\bar{x}-T \bar{x}\|=\operatorname{dist}(A, B) .
$$

Let $\bar{y}=T \bar{x}$, then by inequality (5) and Lemma 1 we have $\bar{x} \preceq \bar{y}$ and

$$
\|\bar{y}-T \bar{y}\|=\|T \bar{x}-T \bar{y}\| \leq\|\bar{x}-\bar{y}\|=\operatorname{dist}(A, B) .
$$

So the proof is complete.

We claim that $T^{2} \bar{x}=\bar{x}$ and $T^{2} \bar{y}=\bar{y}$. Indeed, since $x_{n+1}=T^{2} x_{n} \stackrel{w}{\longrightarrow} \bar{x}$ and $x_{n+1}=T^{2} x_{n} \stackrel{w}{\longrightarrow} T^{2} \bar{x}$, the uniqueness of the weak limit implies that $T^{2} \bar{x}=\bar{x}$. Furthermore, $T \bar{x}=\bar{y}$ then

$$
T^{2} \bar{x}=\bar{x} \Longrightarrow T\left(T^{2} \bar{x}\right)=T \bar{x} \Longrightarrow T^{2}(T \bar{x})=\bar{y} \Longrightarrow T^{2} \bar{y}=\bar{y} .
$$

The following example illustrates Theorem 3.

Example 1. Consider $X=\mathbb{R}^{2}$ with usual norm and the partially order defined by:

$$
(a, b) \preceq(c, d) \quad \text { iff } \quad(a \leq c \quad \text { and } \quad b \leq d),
$$

for any $(a, b),(c, d)$ in $\mathbb{R}^{2}$. Suppose that

$$
\begin{gathered}
A=\left\{(x, 0) \in \mathbb{R}^{2} ; x \in[0,2]\right\} \text { and } \\
B=\left\{(x, 1) \in \mathbb{R}^{2} ; x \in[2,4]\right\},
\end{gathered}
$$

we can show that $\operatorname{dist}(A, B)=1, \mathcal{A}_{0}^{\prec}=\{(2,0)\}$ and $\mathcal{B}_{0}^{\prec}=\{(2,1)\}$. Suppose that a mapping $T: A \cup B \rightarrow$ $A \cup B$ is defined as follows

$$
\left\{\begin{array}{l}
T(x, 0)=(2,1) ; \quad \text { for all } \quad(x, 0) \in A \\
T(x, 1)=(4-x, 0) ; \text { for all } \quad(x, 1) \in B .
\end{array}\right.
$$

We have $T(A) \subset B, T(B) \subset A$ and $T$ is a decreasing mapping. Also, for any $\left((x, 0),\left(x^{\prime}, 1\right)\right) \in A \times B$ we have $(x, 0) \preceq\left(x^{\prime}, 1\right)$ and

$$
\left\{\begin{array}{rlr}
\left\|T(x, 0)-T\left(x^{\prime}, 1\right)\right\| & =\left\|(2,1)-\left(4-x^{\prime}, 0\right)\right\| \\
& =\sqrt{\left(x^{\prime}-2\right)^{2}+1} \\
\left\|(x, 0)-\left(x^{\prime}, 1\right)\right\| & =\left\|\left(x^{\prime}-x, 1\right)\right\| \\
& =\sqrt{\left(x^{\prime}-x\right)^{2}+1}
\end{array}\right.
$$


thus, $\left\|T(x, 0)-T\left(x^{\prime}, 1\right)\right\| \leq\left\|(x, 0)-\left(x^{\prime}, 1\right)\right\|$. Then $T$ is a monotone decreasing relatively cyclic nonexpansive mapping.

If we choose $x_{0}^{\prime}=(0,0)$ and $x_{0}=(2,0)$ in $A$ we get

$$
x_{0} \preceq T x_{0}^{\prime}, \quad\left\|x_{0}-T x_{0}^{\prime}\right\|=\operatorname{dist}(A, B) \quad \text { and } \quad x_{0}^{\prime} \preceq x_{0} \preceq T^{2} x_{0}^{\prime} .
$$

Then there exist $\bar{x}=(2,0) \in A$ and $\bar{y}=(2,1) \in B$ such that $T^{2} \bar{x}=\bar{x}, T^{2} \bar{y}=\bar{y}$ and

$$
\|\bar{x}-T \bar{x}\|=\|\bar{y}-T \bar{y}\|=\operatorname{dist}(A, B) .
$$

Author Contributions: The authors contributed equally to this work.

Funding: This research received no external funding.

Conflicts of Interest: The authors declare no conflict of interest.

\section{References}

1. Eldred, A.; Kirk, W.; Veeramani, P. Proximal normal structure and relatively nonexpansive mappings. Studia Math. 2005, 3, 283-293. [CrossRef]

2. Sankar Raj, V.; Veeramani, P. Best proximity pair theorems for relatively nonexpansive mappings. Appl. Gen. Topol. 2009, 10, 21-28. [CrossRef]

3. Chaira, K.; Lazaiz, S. Best proximity point theorems for cyclic relatively nonexpansive mappings in modular spaces. Abstr. Appl. Anal. 2018, 2018, 8084712. [CrossRef]

4. Espinola, R. A new approach to relatively nonexpansive mappings. Proc. Am. Math. Soc. 2008, 136, 1987-1995. [CrossRef]

5. Ggabeleh, M. Cyclic relatively nonexpansive mappings in convex metric spaces. Miskolc Math. Notes 2015, 16, 133-144. [CrossRef]

6. Chaira, K.; Lazaiz, S. Best proximity pair and fixed point results for noncyclic mappings in modular spaces. Arab J. Math. Sci. 2018, 24, 147-165. [CrossRef]

7. Veeramani, P.; Rajesh, S. Best proximity points. In Nonlinear Analysis: Approximation Theory, Optimization and Applications, 3rd ed.; Ansari, Q.H., Ed.; Springer Science + Business Media: New York, NY, USA, 2014; pp. 1-32, ISBN 978-81-322-1882-1.

8. Ran, A.C.M.; Reurings, M.C.B. A fixed point theorem in partially ordered sets and some applications to matrix equations. Proc. Am. Math. Soc. 2004, 132, 1435-1443. [CrossRef]

9. Nieto, J.J.; Rodríguez-López, R. Contractive mapping theorems in partially ordered sets and applications to ordinary differential equations. Order 2005, 22, 223-239. [CrossRef]

10. Zhang, X. Fixed point theorems of multivalued monotone mappings in ordered metric spaces. Appl. Math. Lett. 2010, 23, 235-240. [CrossRef]

11. Bachar, M.; Khamsi, M.A. Fixed points of monotone mappings and application to integral equations. Fixed Point Theory Appl. 2015, 2015, 110. [CrossRef]

12. Pandey, R.; Pant, R.; Al-Rawashdeh, A. Fixed point results for a class of monotone nonexpansive type mappings in hyperbolic spaces. J. Funct. Spaces 2018, 2018, 5850181. [CrossRef]

13. Alfuraidan, M.R.; Jorquera, E.D.; Khamsi, M.A. Fixed point theorems for monotone Caristi inward mappings. Numer. Funct. Anal. Optim. 2018, 39, 1092-1101. [CrossRef]

14. Van Dung, N.; Hieu, N.T.; Radojević, S. Fixed point theorems for g-monotone maps on partially ordered S-metric spaces. Filomat 2014, 28, 1885-1898. [CrossRef]

15. Dehaish, B.A.B.; Khamsi, M.A. Mann iteration process for monotone nonexpansive mappings. Fixed Point Theory Appl. 2015, 2015, 177. [CrossRef]

16. Carl, S.; Heikkilä, S. Fixed Point Theory in Ordered Sets and Applications: From Differential and Integral Equations to Game Theory, 1st ed.; Springer Science + Business Media: New York, NY, USA, 2011; ISBN 978-1-4419-7584-3.

17. Alfuraidan, M.R.; Khamsi, M.A. Fibonacci Mann iteration for monotone asymptotically nonexpansive mappings. Bull. Aust. Math. Soc. 2017, 96, 307-316. [CrossRef] 
18. Alfuraidan, M.; Khamsi, M.A. A fixed point theorem for monotone asymptotically nonexpansive mappings. Proc. Am. Math. Soc. 2018, 146, 2451-2456. [CrossRef]

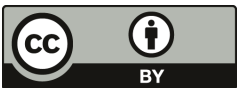

(C) 2019 by the authors. Licensee MDPI, Basel, Switzerland. This article is an open access article distributed under the terms and conditions of the Creative Commons Attribution (CC BY) license (http:/ / creativecommons.org/licenses/by/4.0/). 

MDPI

St. Alban-Anlage 66

4052 Basel

Switzerland

Tel. +41616837734

Fax +41 613028918

www.mdpi.com

Axioms Editorial Office

E-mail: axioms@mdpi.com

www.mdpi.com/journal/axioms

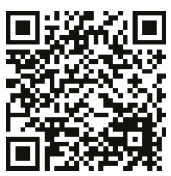





\section{MDPI}

St. Alban-Anlage 66

4052 Basel

Switzerland

Tel: +41 616837734

Fax: +41 613028918 